\title{
Feasibility of Genome-wide Association Analysis Using a Small Single Nucleotide Polymorphism Panel in an Apple Breeding Population Segregating for Fruit Skin Color
}

\author{
Lidia Lozano ${ }^{1}$ and Ignasi Iglesias \\ Institut de Recerca i Tecnologia Agroalimentàries, Av. Alcalde Rovira Roure, 191, E-25198, Lleida, \\ Spain
}

\author{
Diego Micheletti and Michela Troggio \\ Research and Innovation Centre, Foundation Edmund Mach, San Michele all'Adige, 38010, Trento, \\ Italy \\ Satish Kumar and Richard K. Volz \\ New Zealand Institute for Plant \& Food Research Ltd., Hawkes Bay Research Centre, Havelock North \\ 4157, New Zealand
}

\begin{abstract}
Andrew C. Allan
New Zealand Institute for Plant \& Food Research Ltd., Mount Albert Research Centre, Auckland 1142, New Zealand

David Chagné ${ }^{2}$ and Susan E. Gardiner

New Zealand Institute for Plant \& Food Research Ltd., Palmerston North Research Centre, Palmerston North 4442, New Zealand
\end{abstract}

\begin{abstract}
AdDitional INDEX wORDs. Malus $\times$ domestica, MYB, red color, SNP
Abstract. Single-nucleotide polymorphisms (SNPs) have been used for a range of genetic studies and are now starting to be applied for marker-assisted selection in plant breeding programs. To identify SNP markers associated with red fruit skin color, we conducted a genome-wide association (GWA) analysis in an apple (Malus $\times$ domestica Borkh.) breeding population comprising 94 phenotyped individuals using a 384-plex SNP assay. Linkage disequilibrium (LD) analysis indicated that LD extends over a long physical distance in the population (17 Mbp), indicating that a small number of generations separates the individuals. No significant association of anthocyanin content, overcolor, and colorimetric measures $\left(a^{*}, b^{*}, L^{*}, a / b^{*}\right.$, and hue angle) with a marker was identified, although the apple fruit skin color locus has been previously located on apple linkage group 9. Our trial of a small SNP panel for GWA in apple breeding material has demonstrated the limitation of this approach for marker trait association.
\end{abstract}

Plant breeders worldwide are focusing on improving the prediction of phenotype from genotype. A range of genomic tools is now available to address this issue, including dense genetic maps and whole genome sequences. Large sets of molecular markers are being developed and our knowledge of the molecular control of a wide range of plant traits is growing speedily. Molecular markers are now widely used in plant breeding for the acceleration of genetic gain. Systems include marker-assisted selection (MAS) using either markers that are candidate genes for the trait of interest or are closely linked to chromosomal segments (Collard and Mackill, 2008) as well as

Received for publication 23 Jan. 2014. Accepted for publication 7 July 2014. This work is part of an European Union funded project in the "7th Framework Programme: Marie Curie Actions, People International Research Staff Exchange Scheme (FP7-PEOPLE-IRSES-2008)" proposal 130857 and was also funded by Spain's Instituto Nacional de Investigación y Tecnología Agraria y Alimentaria (INIA) through the project RTA 2009-00069-00-00. L.L. was supported by a doctoral fellowship from INIA.

${ }^{1}$ Current address: Research Centre for Agriculture and Forestry Laimburg, I-39040 Ora/Auer (BZ), Italy.

${ }^{2}$ Corresponding author. E-mail: David.Chagne@plantandfood.co.nz. selection at the whole genome level (e.g., Kumar et al., 2012). The use of molecular markers for phenotypic prediction is especially attractive for long-lived woody crop plants, where characters related to fruiting are often expressed several years after crossing, the cost of raising plants in research orchards is high, and where some traits are difficult to assess.

In recent years, the application of SNP markers has gained much attention in both the scientific and plant breeding communities (Rafalski, 2002). SNPs are abundant and evenly distributed throughout the genomes of most plant species and their main advantage over other molecular markers is that they are the most abundant form of genetic variation within plant genomes. Considerable progress has been made both in the area of SNP discovery and SNP assay development. SNP discovery in silico is now feasible thanks to the availability of whole genome sequences of rosaceous species including strawberry [Fragaria vesca L. (Shulaev et al., 2011)], apple (Velasco et al., 2010), pear [Pyrus bretschneideri Rehd. (Wu et al., 2013) and Pyrus communis (Chagné et al., 2014)], and peach [Prunus persica (L.) Batch (Verde et al., 2013)]. In apple, SNP markers 
were developed from large sets of cDNA sequences before the whole genome sequence became available (Chagné et al., 2008) and more recently have been identified using whole genome resequencing (Chagné et al., 2012a). Once SNPs linked to a trait have been identified and validated, they can be used by breeders to screen large sets of seedlings. The range of techniques for high-throughput SNP genotyping has evolved rapidly in recent years thanks to technological progress and several high-throughput platforms allow rapid and simultaneous genotyping of hundreds or thousands of plants with up to hundreds of thousands of SNPs. This includes high-throughput SNP arrays (Chagné et al., 2012a; Verde et al., 2012) and more recently genotyping by sequencing as the throughput of next-generation sequencing techniques (Elshire et al., 2011) has increased. The GoldenGate $^{\circledR}$ assay (Illumina, San Diego, CA) has been the method of choice for researchers screening lower numbers of SNP markers, because it is capable of medium-density genotyping with 96 to 1536 SNPs per array (Fan et al., 2006). The GoldenGate ${ }^{\circledR}$ assay has been used for genetic analysis in several crop species: wheat [Triticum aestivum L. (Akhunov et al., 2009)], maize [Zea mays L. (Yan et al., 2010)], soybean [Glycine max (L.) Merr. (Hyten et al., 2008)], barley [Hordeum vulgare L. (Rostoks et al., 2006)], loblolly pine [Pinus taeda L. (Eckert et al., 2009)], and recently in apple (Khan et al., 2012) and peach (Martinez-Garcia et al., 2013).

SNP arrays have the potential to be used for a variety of applications in crop improvement, including genetic mapping and quantitative trait locus (QTL) analysis, diversity analysis, germplasm management and MAS as well as association studies (Yan et al., 2010). One methodology used for association analysis is GWA, which involves spanning the genome with a sufficient number of markers to enable detection of regions associated with the phenotype of interest. GWA is based on LD mapping and identifies markers that show a statistically significant association with phenotype in natural populations or germplasm collections (Myles et al., 2009). Once molecular markers linked to a trait of interest have been identified and validated, they can be used for MAS in wider breeding populations (Varshney et al., 2009). LD varies as a function of recombination frequency along the genome, history of the species, mating system as well as population structure (Gaut, 2003). Because the decay of LD is dependent on the history of the population used and its diversity, it is likely that plant material from breeding programs, which often use a limited number of founder individuals, will exhibit LD decay at a longer physical distance than more broadly based germplasm populations that are sampled over extensive natural ranges. GWA was recently reported for apple using an 8000SNP array and a total population size of 1200 individuals, enabling the identification of SNPs associated to a range of traits (Kumar et al., 2013).

The accumulation of anthocyanin pigments in apple fruit is an important determinant of fruit quality, because red coloration of apple skin is a key factor for the acceptance of apple fruit by the marketplace and individual consumers. In general, redcolored varieties achieve higher market prices (Baugher et al., 1990; Iglesias and Alegre, 2006). Furthermore, red pigment content is implicated in the health attributes of apple fruit (Boyer and Liu, 2004). The main pigment responsible for red color in apples is cyanidin-3-galactoside, which belongs to the anthocyanin family (McGhie et al., 2005). Anthocyanins are derived from cinnamic acid by L-phenylalanine deamination, a reaction catalyzed by phenylalanine ammonia-lyase (PAL) (Lancaster and Dougall, 1992). Both PAL activity and the development of red color are directly regulated by environmental factors such as light (Arakawa, 1991; Lancaster and Dougall, 1992; Saure, 1990; Ubi et al., 2006) and temperature (Faragher, 1983; Lin-Wang et al., 2011; Ubi et al., 2006; Xie et al., 2012) and are variable across cultivars (Curry, 1997; Dickinson and White, 1986; Iglesias et al., 2008). Although most of the genes encoding enzymes involved in anthocyanin biosynthesis are under the regulation of ultraviolet-B light and temperature, recent studies indicate that the overall regulation of expression of the anthocyanin biosynthetic genes is by a transcription factor belonging to the MYB family: $M d M Y B 1 / M Y B 10$ (Espley et al., 2007; Takos et al., 2006). This study investigating the use of a panel of 384 SNPs for identification of markers associated with red skin coloration using GWA analysis is a pilot study set up to evaluate the usefulness of this density of markers for trait mapping.

\section{Materials and Methods}

Plant material and DNA isolation. A total of 94 individuals segregating for skin color (derived from a single maternal parent and an unknown number of paternal parents) was selected from the joint Institut de Recerca i Tecnologia Agroalimentàries (IRTA) and New Zealand Plant \& Food Research Ltd. pipfruit breeding program in Lleida, Spain. The trees were planted at the Gimenells (Spain) IRTA research orchards in 2006 and grown at $1 \times 3.4-\mathrm{m}$ spacing using standard commercial management practices recommended for the area, including fertilizer application and disease and pest control. Leaf tissue was harvested from each genotype, freeze-dried, and stored at $-80{ }^{\circ} \mathrm{C}$ until required. The leaf samples were ground to fine powder in liquid nitrogen and DNA was extracted using the DNeasy Plant Mini Kit (Qiagen, Hilden, Germany) according to the manufacturer's instructions.

PhenotyPiC DATA FOR SKIN COLOR AND ANTHOCYANIN CONTENT. Five fruit from each tree/genotype were collected at optimum maturity (an average of approximately three) in a starch pattern index scale of 0 (low maturity) to 6 (over mature) (ENZA Fruit, Hastings, New Zealand) from the beginning of Aug. 2008 to the end of Oct. 2008. A total of 480 fruit was assessed within the $24 \mathrm{~h}$ after fruit harvest. Apple skin color was determined using three methods: visual assessment, colorimetry, and anthocyanin content. Overcolor was measured by a trained panel as the percentage of surface color using a visual scale from $0 \%$ (no blush) to $100 \%$ (fully colored) and expressed as the mean value of all fruits of the same genotype. Other assessments were at an equatorial location on the blushed side of each fruit. Colorimetric values were measured using a portable tristimulus colorimeter (Chroma Meter CR-200; Minolta Corp., Osaka, Japan). Chromaticity was expressed in $\mathrm{L}^{*}, \mathrm{a}^{*}$, and $\mathrm{b}^{*}$ color space coordinates according to Commission Internationale de l'Eclairage. The $\mathrm{L}^{*}$ value represents the relative lightness of colors with a range from 0 (black) to 100 (white), being small for dark colors and large for light colors. Both $\mathrm{a}^{*}$ and $\mathrm{b}^{*}$ scales extend from -60 to $60 ; a^{*}$ ranges from green $\left(-a^{*}\right)$ to red $\left(+a^{*}\right)$ and $b^{*}$ ranges from blue $\left(-b^{*}\right)$ to yellow $\left(+b^{*}\right)$. Hue angle was calculated as described by McGuire (1992) and expressed in degrees; $a^{*} / b^{*}$ was also calculated. 
Total anthocyanins were extracted from 11-mm-diameter skin disks taken separately for each color measurement. The skin disks were held at $4{ }^{\circ} \mathrm{C}$ for $24 \mathrm{~h}$ in the dark in $10 \mathrm{~mL}$ of a solution of 50 methanol $(26.4 \mathrm{M}): 1 \mathrm{HCl}(35 \%): 49$ water and absorbance of the extracts was measured with a spectrophotometer (series 1000; Cecil Instruments, Cambridge, U.K.) at $532 \mathrm{~nm}$. The anthocyanin concentration was subsequently determined using a molar extinction coefficient of $3.43 \times 10^{4}$ and data were expressed in nanomoles per square centimeter.

SNP MARKER DEVELOPMENT AND GENOTYPING. The 94 segregating individuals were genotyped with a panel of evenly spaced SNP markers spanning the entire apple genome using a 384-plex assay developed from a set of 1679 SNPs. These SNPs have been mapped in an integrated apple genetic map (Velasco et al., 2010) and are included in the International RosBREED SNP Consortium 8000-SNP array (Chagné et al., 2012a). Data were analyzed using GenomeStudio Version 1.0 software Genotyping Module (Illumina) with a Gencall threshold of 0.5 .

Statistical analysis. Descriptive statistics regarding phenotypic data (chromaticity values, anthocyanin content, and overcolor), including means, distributions and correlations, were generated using JMP 8.0 software (SAS Institute, Cary, NC).

The genome wide decay of the LD was calculated after removing alleles with minor frequency (MAF) lower than 5\%. GDA 1.1 software (Lewis and Zaykin, 2001) was used to compute the composite disequilibrium coefficient $\left(\Delta_{\mathrm{ab}}\right)$ between pairs of allele A and B at two different loci, either in the same or different LG, according to Weir (1996). For normalization, the interallelic correlation coefficient, $r^{2}$ ab, was calculated as in Weir (1996). The significance threshold, based on the null hypothesis of no linkage, was calculated by comparison with a $\chi^{2}$ test with 1 df for $\alpha \leq 0.01\left(\chi^{2}{ }_{1 \mathrm{df}}=6.635\right)$ as in Zaykin (2004).

The association analysis was carried out using the GAPIT R package (Lipka et al., 2012). The genome-wide significance threshold was $\alpha \leq 0.01$ (which roughly equates to a comparisonwise probability value of $3.4 \times 10^{-5}$ assuming 295 SNPs were tested). A regular mixed linear model that takes into account population structure ( $\mathrm{Q}$ matrix) and kinship (K matrix) was used for the association analysis. The $Q$ matrix takes into account the first six principal components of a principal component analysis (PCA). The $K$ matrix is a measure of relative kinship and quantifies the probability that two homologous genes are identical by descent. The $K$ matrix was generated according to the method of VanRaden (2008). Both the kinship and the structure were computed directly using GAPIT.

\section{Results}

PhenotyPic VARIATION FOR SKIN COLOR AND ANTHOCYANIN CONCENTRATION. Half of the individuals in the germplasm set exhibited colored fruit (orange-red, pink, red, or dark red) and the other half had non-colored fruit when the data were expressed as the mean percentage of overcolor in fruit harvested from an individual genotype. Fruit coloration was distributed from blushed fruit (10\% to $20 \%$ surface covered), bicolor fruits $(20 \%$ to $60 \%)$ to colored fruits $(60 \%$ to $100 \%)$ (Supplemental Fig. 1). Of all colored fruit, 58.1\% exhibited a blush pattern, whereas $41.9 \%$ had a striped pattern. Colorimeter $L^{*}$ values (lightness/darkness) varied from 35.51 (dark colors) to 80.13 (light colors), $\mathrm{a}^{*}$ had a range from green $(-17.63$, negative value) to red colors (41.27, positive value), and $b^{*}$ values showed a range of different yellow colors between 14 and
59.48. Hue angle value varied from a minimum of 22.5 to a maximum of 111.7; where lower values of hue angle indicated higher fruit coloration. Anthocyanin concentration varied from 6.02 to $77.66 \mathrm{nmol} \cdot \mathrm{cm}^{-2}$ (Supplemental Table 1).

The regressions for anthocyanin concentration vs. L*, hue angle, and overcolor provided coefficients of determination $\left(R^{2}\right)$ of $0.97,0.97$, and $0.94(P \leq 0.001)$, respectively (Fig. 1). The phenotypic correlations between color traits (chromaticity values, anthocyanin concentration, and overcolor) were highly significant for all traits $[P<0.001$ (Table 1)].
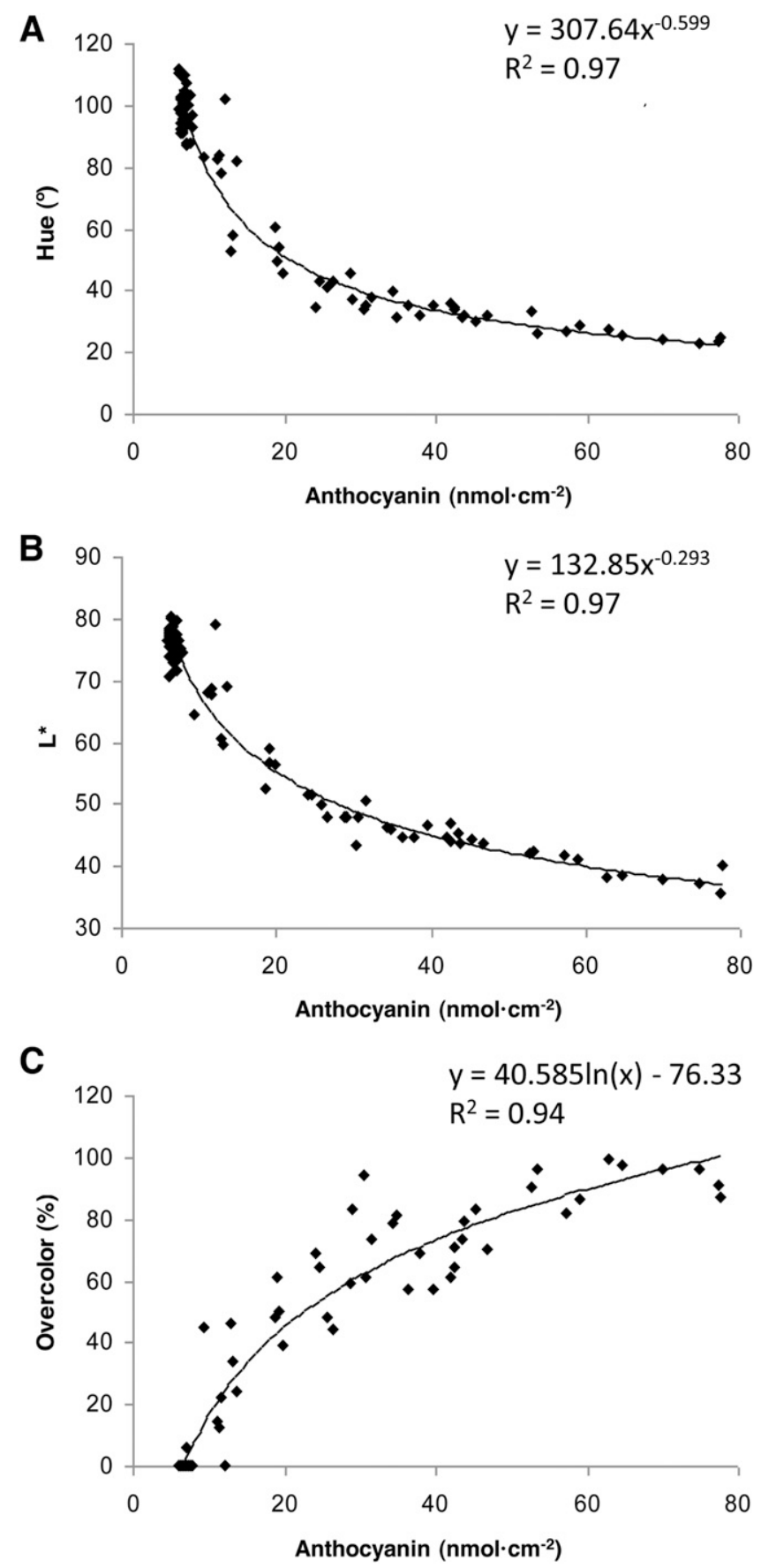

Fig. 1. Relationship between extractable anthocyanin content and hue angle (A) $L^{*}$ value (B), and overcolor (C) measured for fruit from 94 apple genotypes harvested in 2008. Regression significance is at $P \leq 0.001$. 
Table 1. Pearson's correlation coefficients for skin color phenotypic measurements $\left(\mathrm{L}^{*}\right.$ trait, $\mathrm{a}^{*}$ trait, $\mathrm{b}^{*}$ trait, hue angle, $\mathrm{a}^{*} / \mathrm{b}^{*}$ trait, and anthocyanin content) determined on 96 apple genotypes in a breeding population from the Institut de Recerca i Tecnologia AgroalimentàriesNew Zealand Plant \& Food Research Ltd. breeding program.

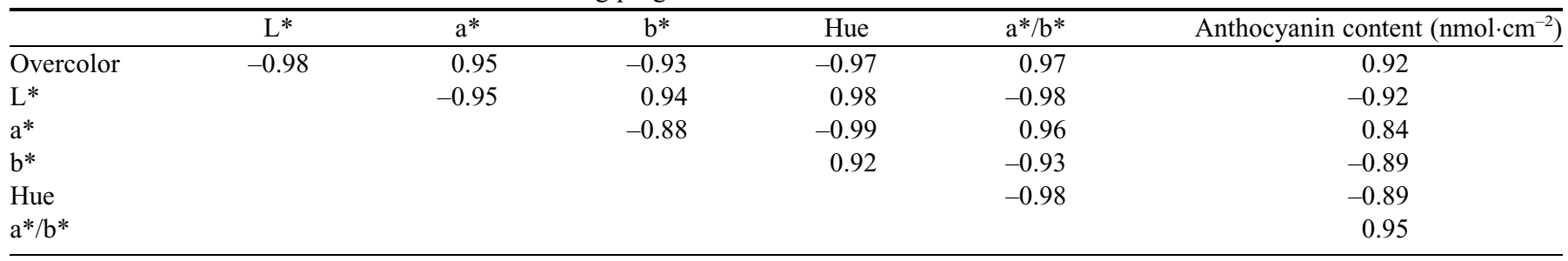

SNP POLYMORPHISM IN THE GERMPLASM SET. Of the 384 SNP markers screened over the 94 individuals (Supplemental Fig. 2), $309(80.5 \%)$ were polymorphic, displaying three clear clusters corresponding to the expected genotypes (e.g., AA homozygote, $\mathrm{BB}$ homozygote, and $\mathrm{AB}$ heterozygote). Ambiguous data points located between clusters were scored as missing data. Of the remaining markers, $53(13.8 \%)$ were monomorphic and $22(5.7 \%)$ failed to amplify.

LiNKAGE DiSEQUILIBRIUM DECAY ANALYSIS. The 295 SNPs with a MAF higher than 0.05 (Supplemental Table 2; Supplemental Figs. 2 and 3) were considered for the LD analysis, whereby the interallelic $r^{2}$ values, namely the association between each of the alleles at two loci, were calculated for a total of 43,345 pairs of alleles, 2,565 of which involved intrachromosome comparison. When the decay of $r^{2}$ against the physical distance between the pairs of loci based on the apple genome assembly (Velasco et al., 2010) was calculated (Fig. 2), the curves that describe the LD decay with distance reach the $r^{2}$ significance value at $17 \mathrm{Mbp}\left(r^{2} \geq 0.07\right)$. When pairs of SNPs with a distance shorter than $10 \mathrm{Mbp}$ were considered, the average $r^{2}$ and the

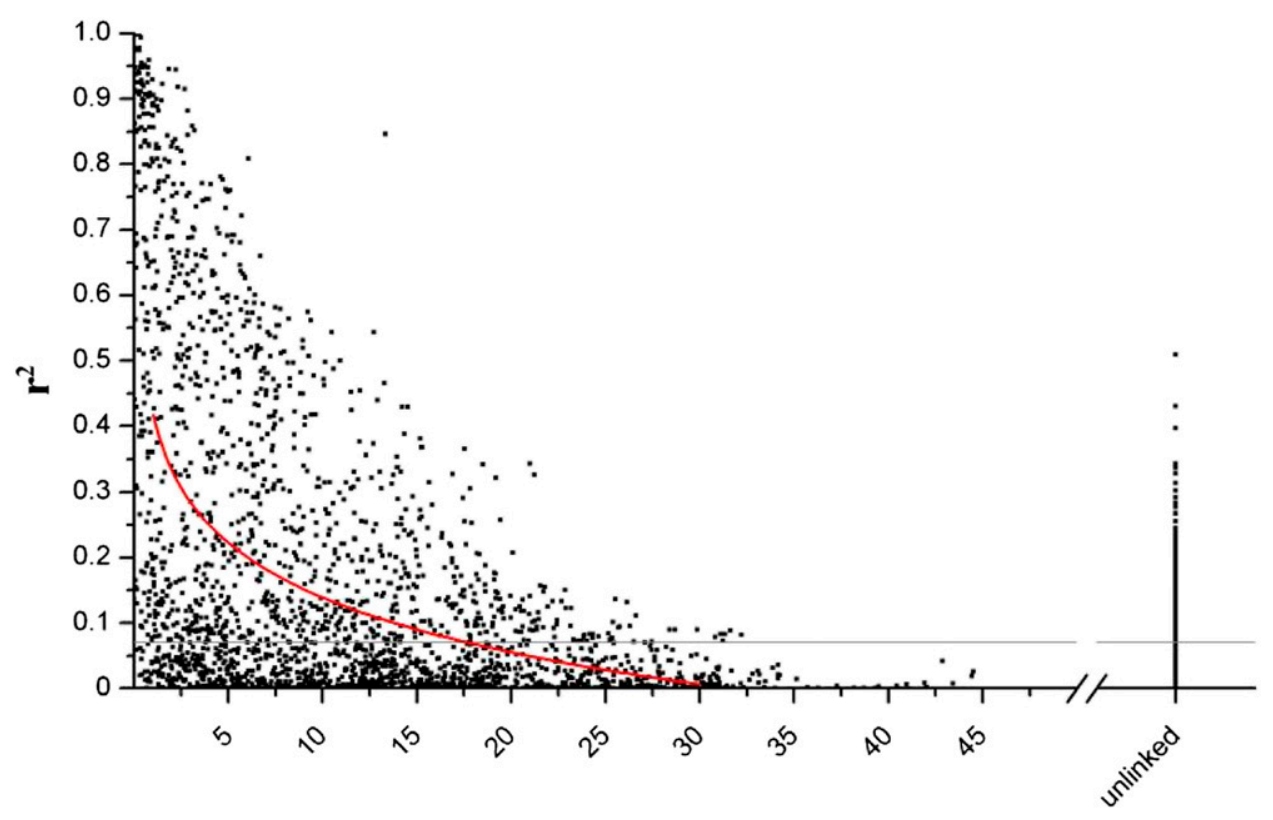

Distance (Mbp)

Fig. 2. Variation of linkage disequilibrium (LD) against physical distance in an apple population of 94 seedlings. The variation of $r^{2}$ value between alleles of marker placed either in same and different linkage groups with physical distance in megabase pairs. All the interchromosome comparison was reported in relation to the unlinked label on the x-axis. The trend line indicating the decay of the $r^{2}$ with the distance was the logarithmic fitting of the intrachromosome comparison. The horizontal line represents the significance threshold at $\alpha \leq 0.01$. percentage of comparison with $r^{2} \geq 0.07$ were $0.29 \%$ and $67.8 \%$, respectively. These values decrease to $0.18 \%$ and $49.4 \%$ considering all the intrachromosomal comparison and $0.02 \%$ and $4.1 \%$ considering the interchromosomal comparisons.

KinSHIP ANALYSIS AND POPULATION STRUCTURE. The kinship matrix (Supplemental Fig. 4) shows that the great majority of the individuals share between one-fourth and half of the genome.

The population structure (Supplemental Fig. 5) was evaluated through a PCA analysis. The first principal component highlights the presence of two clusters (perhaps indicating the involvement of at least two pollen parents), whereas the separation on the second component was not as clear with the individuals being evenly distributed.

MARKer-Trait associations. The genotypic data obtained for the 295 polymorphic SNP markers with MAF greater than 0.05 were used to test the association with the phenotypic data. When the $\log 10$ of the probability value was plotted against the position of the SNPs based on the 'Golden Delicious' apple genetic map (Velasco et al., 2010) (Fig. 3), no association was found for a genome-wide threshold of $\alpha<0.01$. Nevertheless, a weak association was found with marker GDsnp00766 and overcolor $(P=0.006)$ with anthocyanin concentration $(P=0.019)$ as well as colorimetric traits $\mathrm{L}^{*}(P=0.006), \mathrm{b} *$ $(P=0.005), \mathrm{a}^{*} / \mathrm{b}^{*}(P=0.008)$, and hue $(P=0.005)$ (Table 2$)$. However, such $P$ values were not significant at the genome-wide or marker level. Based on the LG9 assembly, marker GDsnp00766 is located 1.4 Mbp upstream of the anthocyanin regulating MYB10 gene.

\section{Discussion}

Our use of a 384-plex SNP assay for GWA analysis is a trial of the application of a low-throughput multiplexed SNP assay in an apple breeding population. The panel of 384 SNPs used for the assay had been previously validated in 'Golden Delicious' apple using the lower-throughput 48-plex SNPlex ${ }^{\mathrm{TM}}$ technique (Applied Biosystems, Foster City, CA) (Micheletti et al., 2011; Velasco et al., 2010). We found that 309 SNPs $(80.5 \%)$ were 
A) anthocyanin content

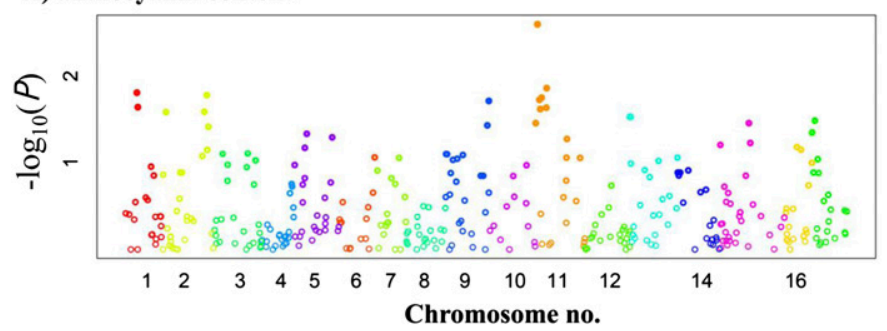

B) $L^{*}$

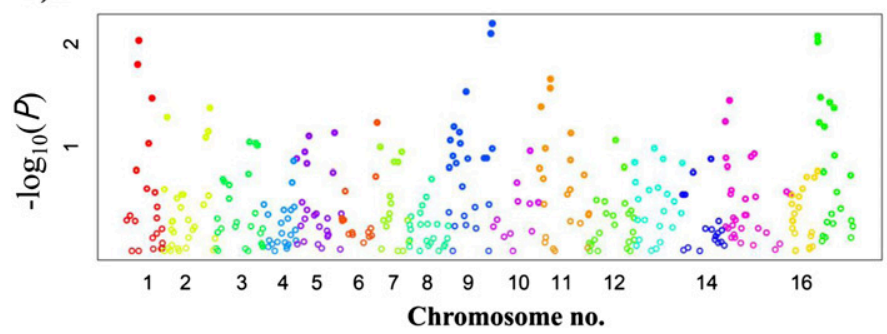

C) Hue angle

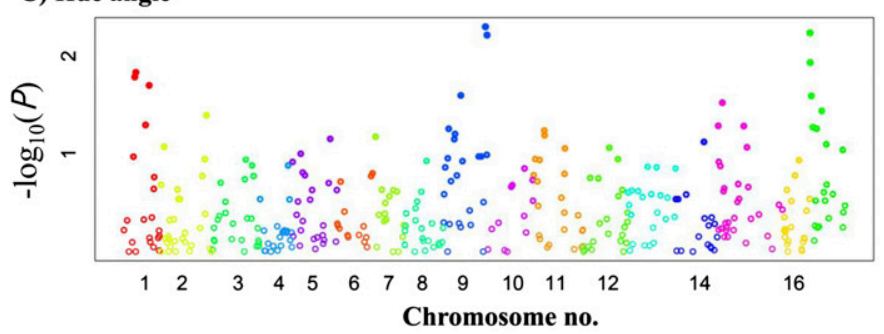

D) $\mathbf{a}^{*}$

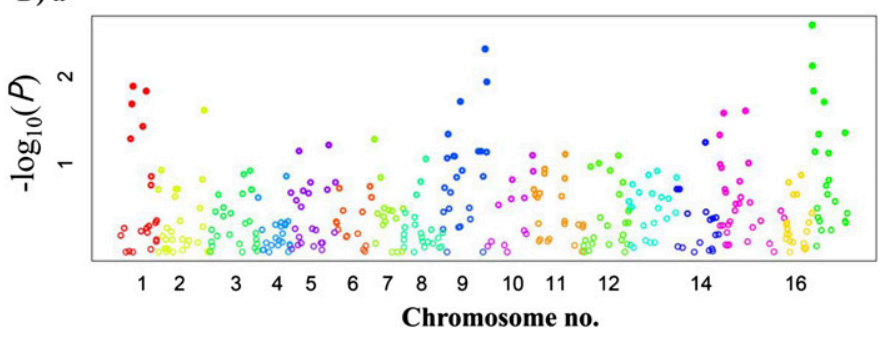

E) $\mathbf{b}^{*}$

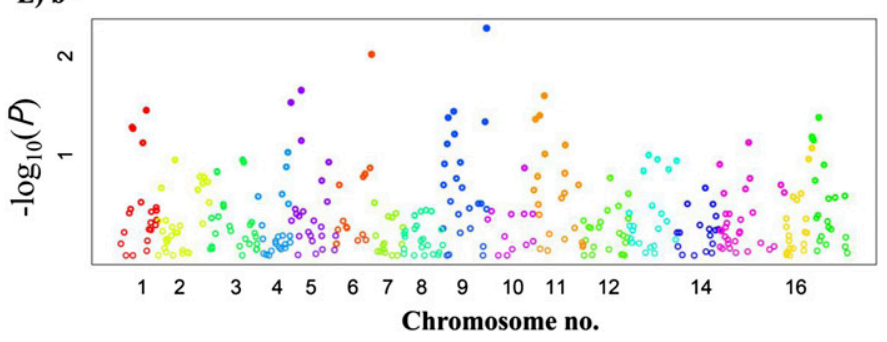

F) $\mathbf{a} * / \mathbf{b} *$

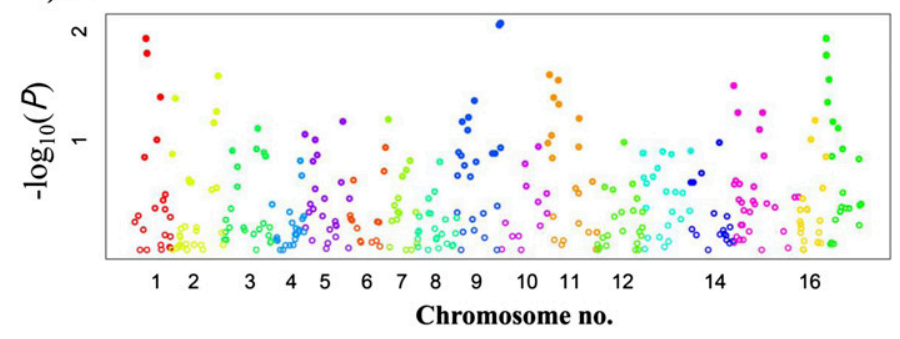

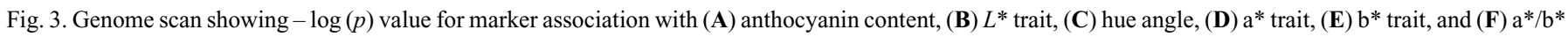
trait determined on fruit from 94 apple genotypes in a breeding population in Lleida, Spain, in 2008.

polymorphic, of which 295 with MAF greater than 0.05 were useful for association analysis in our study. This high degree of SNP transferability between 'Golden Delicious' and our set of individuals is similar to that obtained by Micheletti et al. (2011) (84\%) in a broader-based set of $123 \mathrm{M}$. xdomestica accessions that included 119 elite selections, founders and old cultivars, and four rootstock cultivars.

The number of polymorphic SNPs we used enabled us to cover the apple genome at an approximate density of one marker every $4 \mathrm{cM}$ based on a genetic length of $1200 \mathrm{cM}$ (Velasco et al., 2010) and one marker every $2.5 \mathrm{Mbp}$ based on an approximate genome size of $740 \mathrm{Mbp}$ for apple. This density is a significant increase compared with the microsatellite marker systems previously used for genome scans in apple (Patocchi et al., 2005); however, it is of lower density than the SNP array used by Kumar et al. (2013). Target marker density for GWA studies is dictated by the rate of LD decay across the genome; if the level of LD is low, GWA analysis may be difficult because of the high density of markers required; however, the resolution will be very high. On the other hand, if the LD is extensive, whole genome scans are feasible using a lower density of markers; however, conversely the resolution will be limited (Rafalski, 2002). Our analysis indicated that LD extends a large physical distance in our population, which might have enabled scanning of the genome for a marker-trait association using the 384-plex SNP array. However, a GWA analysis for red skin coloration in our population did not identify any significant marker association with red skin color.
Although associations with a marker on LG9 and phenotypes were weakly significant $(P>0.001)$, they were below the genome-wide threshold. However, the weak association suggested on LG9 is consistent with previous studies on the position of loci controlling red coloration in apple. A major locus for skin color was mapped in a segregating population at the distal end of LG 9 (Cheng et al., 1996; Maliepaard et al., 1998) and furthermore, recent research on the molecular control of anthocyanin biosynthesis in apple has shown that a transcription factor that controls fruit skin and flesh and foliage color (MYB10/MYB1) (Espley et al., 2007; Takos et al., 2006) maps at the distal end of LG 9 (Chagné et al., 2007). In addition, alleles of $M Y B 10 / M Y B 1$ were associated with skin color in a candidate gene-based association study in the U.S. breeding material (Zhu et al., 2011). Based on the apple genome assembly (Velasco et al., 2010), MYB10 is located $1.4 \mathrm{Mbp}$ from the closest marker we found to be weakly associated with skin color (GDsnp00766). Although a significant association between MYB10 and red skin color was expected in this study, our GWA strategy using a population of 94 individuals and 309 markers could not retrieve the association that had been demonstrated previously using the candidate gene approach.

Our GWA analysis is a pilot study demonstrating that a marker density of 309 polymorphic SNPs is insufficient to identify linkage between markers and a trait governed by a strong effect QTL in an apple breeding population material derived from a narrow genetic base. This indicates that careful consideration of the size of the effect is needed before using 
Table 2. List of single-nucleotide polymorphisms (SNPs) that showed significant association with skin color traits [L* trait, $a^{*}$ trait, $b^{*}$ trait, hue angle, $\mathrm{a}^{*} / \mathrm{b}^{*}$ trait, type, and anthocyanin content $\left(\mathrm{nmol} \cdot \mathrm{cm}^{-2}\right)$ ] determined for the 96 apple genotypes from the Institut de Recerca i Tecnologia Agroalimentàries-New Zealand Plant \& Food Research Ltd. breeding program.

\begin{tabular}{|c|c|c|c|c|c|c|c|}
\hline Skin color trait & Marker & Linkage group & Position (bp) & $P$ value & $\log 10(\mathrm{P})$ & $R^{2}$ without SNP & $R^{2}$ with SNP \\
\hline Anthocyanin concentration & GDsnp00120 & 2 & 31832613 & 0.017 & 1.78 & 0.27 & 0.32 \\
\hline Anthocyanin concentration & GDsnp00351 & 11 & 3079914 & 0.019 & 1.72 & 0.27 & 0.31 \\
\hline Anthocyanin concentration & GDsnp00766 & 9 & 30472702 & 0.019 & 1.71 & 0.27 & 0.31 \\
\hline Overcolor & GDsnp00766 & 9 & 30472702 & 0.006 & 2.20 & 0.27 & 0.31 \\
\hline Overcolor & GDsnp00192 & 17 & 244794 & 0.012 & 1.92 & 0.35 & 0.40 \\
\hline Overcolor & GDsnp01470 & 1 & 10713804 & 0.013 & 1.89 & 0.35 & 0.40 \\
\hline $\mathrm{L}^{*}$ & GDsnp00766 & 9 & 30472702 & 0.019 & 1.71 & 0.35 & 0.41 \\
\hline $\mathrm{L}^{*}$ & GDsnp00091 & 17 & 50402 & 0.008 & 2.07 & 0.35 & 0.40 \\
\hline $\mathrm{L}^{*}$ & GDsnp01500 & 1 & 11455127 & 0.009 & 2.03 & 0.35 & 0.40 \\
\hline$b^{*}$ & GDsnp02138 & 6 & 24959354 & 0.010 & 2.02 & 0.32 & 0.38 \\
\hline$a^{*}$ & GDsnp00091 & 17 & 50402 & 0.003 & 2.59 & 0.33 & 0.41 \\
\hline$a^{*}$ & GDsnp00766 & 9 & 30472702 & 0.011 & 1.94 & 0.33 & 0.38 \\
\hline$a^{*} / b^{*}$ & GDsnp00766 & 9 & 30472702 & 0.008 & 2.08 & 0.32 & 0.38 \\
\hline$a^{*} / b^{*}$ & GDsnp01470 & 1 & 10713804 & 0.012 & 1.93 & 0.32 & 0.37 \\
\hline Type & GDsnp00178 & 17 & 8360963 & 0.007 & 2.14 & 0.11 & 0.19 \\
\hline
\end{tabular}

such a GWA strategy. It is well known that a number of other polygenic quantitative traits of high agronomic importance in apple are controlled by a few large to medium effects as well as a multitude of small effect QTLs (Chagné et al., 2012b; Gardiner et al., 2012; Khan et al., 2007; Kumar et al., 2012; Soufflet-Freslon et al., 2008) and we advise the use of higherdensity SNP arrays for GWA analysis of such traits. Although the results from GWA analysis in a breeding population comprising seven full-sib families suggested that medium- to large-sized QTLs can be detected using an 8000-SNP array (Chagné et al., 2012a; Kumar et al., 2013), we believe that a larger array [e.g., the recent 20,000-SNP array developed by the FruitBreedomics project (Laurens et al., 2010)] will be needed for identifying smaller effect QTLs.

\section{Conclusion}

This study is an example of a whole genome scan performed for association analysis in apple. The scan was not successful in identifying markers associated with a large effect QTL of which we had prior knowledge. We have demonstrated that screening with high-density SNP arrays is required to identify an association between markers and large effect QTLs in a breeding population. For identification of marker associations with agronomically significant traits that are controlled by few large effect QTLs, we suggest GWA analysis of an existing breeding population using a SNP panel of 20,000 markers as an alternative approach to QTL analysis of full-sib populations.

\section{Literature Cited}

Akhunov, E., C. Nicolet, and J. Dvorak. 2009. Single nucleotide polymorphism genotyping in polyploid wheat with the Illumina GoldenGate assay. Theor. Appl. Genet. 119:507-517.
Arakawa, O. 1991. Effect of temperature on anthocyanin accumulation in apple fruit as affected by cultivar, stage of fruit ripening and bagging. J. Hort. Sci. 66:763-768.

Baugher, T.A., H.W.J. Hogmire, and G.W. Lightner. 1990. Determining apple packout losses and impact on profitability. Appl. Agr. Res. 5:343-349.

Boyer, J. and R. Liu. 2004. Apple phytochemicals and their health benefits. Nutr. J. 3:5.

Chagné, D., C.M. Carlisle, C. Blond, R.K. Volz, C.J. Whitworth, N.C. Oraguzie, R.N. Crowhurst, A.C. Allan, R.V. Espley, R.P. Hellens, and S.E. Gardiner. 2007. Mapping a candidate gene (MdMYB10) for red flesh and foliage colour in apple. BMC Genomics 8:212.

Chagné, D., R.N. Crowhurst, M. Pindo, A. Thrimawithana, C. Deng, H. Ireland, M. Fiers, H. Dzierzon, A. Cestaro, P. Fontana, L. Bianco, A. Lu, R. Storey, M. Knäbel, M. Saeed, S. Montanari, Y.K. Kim, D. Nicolini, S. Larger, E. Stefani, A.C. Allan, J. Bowen, I. Harvey, J. Johnston, M. Malnoy, M. Troggio, L. Perchepied, G. Sawyer, C. Wiedow, K. Won, R. Viola, R.P Hellens, L. Brewer, V.G.M. Bus, R.J. Schaffer, S.E. Gardiner, and R. Velasco. 2014. The draft genome sequence of European pear (Pyrus communis L. 'Bartlett'). PLoS ONE 9(4):e92644.

Chagné, D., R.N. Crowhurst, M. Troggio, M.W. Davey, B. Gilmore, C. Lawley, S. Vanderzande, R.P. Hellens, S. Kumar, A. Cestaro, R. Velasco, D. Main, J.D. Rees, A. Iezzoni, T. Mockler, L. Wilhelm, E. van de Weg, S.E. Gardiner, N. Bassil, C. Peace, and M. Bendahmane. 2012a. Genome-wide SNP detection, validation, and development of an 8K SNP array for apple. PLoS One 7:e31745.

Chagné, D., K. Lin-Wang, R.V. Espley, R.K. Volz, N.M. How, S. Rouse, C. Brendolise, C.M. Carlisle, S. Kumar, N. de Silva, D. Micheletti, T. McGhie, R.N. Crowhurst, R.D. Storey, R. Velasco, R.P. Hellens, S.E. Gardiner, and A.C. Allan. 2012b. An ancient duplication of apple MYB transcription factors is responsible for novel red fruitflesh phenotypes. Plant Physiol. 161:225-239.

Chagné, D., K. Gasic, R.N. Crowhurst, Y. Han, H.C. Bassett, D.R. Bowatte, T.J. Lawrence, E.H. Rikkerink, S.E. Gardiner, and S.S. 
Korban. 2008. Development of a set of SNP markers present in expressed genes of the apple. Genomics 92:353-358.

Cheng, F.S., N.F. Weeden, and S.K. Brown. 1996. Identification of codominant RAPD markers tightly linked to fruit skin color in apple. Theor. Appl. Genet. 933:222-227.

Collard, B.C. and D.J. Mackill. 2008. Marker-assisted selection: An approach for precision plant breeding in the twenty-first century. Phil. Trans. Royal Soc. B Biol. Sci. 363:557-572.

Curry, E.A. 1997. Temperatures for optimum anthocyanin accumulation in apple tissue. J. Hort. Sci. 72:723-729.

Dickinson, J.P. and A.G. White. 1986. Red colour distribution in the skin of Gala apple and some of its sports. N. Z. J. Agr. Res. 29:695698.

Eckert, A.J., B. Pande, E.S. Ersoz, M.H. Wright, V.K. Rashbrook, C.M. Nicolet, and D.B. Neale. 2009. High-throughput genotyping and mapping of single nucleotide polymorphisms in loblolly pine (Pinus taeda L.). Tree Genet. Genomes 5:225-234.

Elshire, R.J., J.C. Glaubitz, Q. Sun, J.A. Poland, K. Kawamoto, E.S. Buckler, S.E. Mitchell, and L. Orban. 2011. A robust, simple genotyping-by-sequencing (GBS) approach for high diversity species. PLoS One 6:e19379.

Espley, R.V., R.P. Hellens, J. Putterill, D.E. Stevenson, S. KuttyAmma, and A.C. Allan. 2007. Red colouration in apple fruit is due to the activity of the MYB transcription factor, MdMYB10. Plant J. 49:414-427.

Fan, J.B., M.S. Chee, and K.L. Gunderson. 2006. Highly parallel genomic assays. Nat. Rev. Genet. 7:632-644.

Faragher, J.D. 1983. Temperature regulation of anthocyanin accumulation in apple skin. J. Expt. Bot. 34:1291-1298.

Gardiner, S.E., J.L. Norelli, N. de Silva, G. Fazio, A. Peil, M. Malnoy, M. Horner, D. Bowatte, C. Carlisle, C. Wiedow, Y. Wan, C.L. Bassett, A.M. Baldo, J.M. Celton, K. Richter, H.S. Aldwinckle, and V.G.M. Bus. 2012. Putative resistance gene markers associated with quantitative trait loci for fire blight resistance in Malus 'Robusta 5' accessions. BMC Genet. 13:25.

Gaut, B.S. 2003. The lowdown on linkage disequilibrium. Plant Cell 15:1502-1506.

Hyten, D.L., Q. Song, I.Y. Choi, M.S. Yoon, J.E. Specht, L.K. Matukumalli, R.L. Nelson, R.C. Shoemaker, N.D. Young, and P.B. Cregan. 2008. High-throughput genotyping with the GoldenGate assay in the complex genome of soybean. Theor. Appl. Genet. 116:945-952.

Iglesias, I. and S. Alegre. 2006. The effect of anti-hail nets on fruit protection, radiation, temperature, quality and profitability of 'Mondial Gala' apples. J. Appl. Hort. 8:91-100.

Iglesias, I., G. Echeverría, and Y. Soria. 2008. Differences in fruit colour development, anthocyanin content, fruit quality and consumer acceptability of eight 'Gala' apple strains. Sci. Hort. 119:32-40.

Khan, M.A., C.E. Durel, B. Duffy, D. Drouet, M. Kellerhals, C. Gessler, and A. Patocchi. 2007. Development of molecular markers linked to the 'Fiesta' linkage group 7 major QTL for fire blight resistance and their application for marker-assisted selection. Genome 50:568-577.

Khan, M.A., Y. Han, Y.F. Zhao, and S.S. Korban. 2012. A highthroughput apple SNP genotyping platform using the GoldenGate ${ }^{\mathrm{TM}}$ assay. Gene 494:196-201.

Kumar, S., M.C.A.M. Bink, R.K. Volz, V.G.M. Bus, and D. Chagné. 2012. Towards genomic selection in apple (Malus $\times$ domestica Borkh.) breeding programmes: Prospects, challenges and strategies. Tree Genet. Genomes 8:1-14.

Kumar, S., D.J. Garrick, M.C. Bink, C. Whitworth, D. Chagné, and R.K. Volz. 2013. Novel genomic approaches unravel genetic architecture of complex traits in apple. BMC Genomics 14:393.

Lancaster, J. and D.K. Dougall. 1992. Regulation of skin color in apples. Crit. Rev. Plant Sci. 10:487-502.

Laurens, F., M.J. Aranzana, P. Arus, J. Bonany, L. Corelli, B. Diep, A. Patocchi, A. Peil, B. Quilot, S. Salvi, E. Van de Weg, and A. Vecchietti. 2010. FruitBreedomics: A new European initiative to bridge the gap between scientific research and breeding
Rosaceae fruit tree crops. Intl. Hort. Congr. Lisbon, Portugal. Intl. Soc. Hort. Sci., Louvain, Belgium. p. 242 (abstr.).

Lewis, P.O. and D. Zaykin. 2001. Genetic data analysis: Computer program for the analysis of allelic data, Version 1.0 (d16c). 15 Nov. 2012. <http:alleyn.eeb.uconn.edugda $>$.

Lin-Wang, K.U., D. Micheletti, J. Palmer, R. Volz, L. Lozano, R. Espley, R.P. Hellens, D. Chagnè, D.D. Rowan, M. Troggio, I. Iglesias, and A.C. Allan. 2011. High temperature reduces apple fruit colour via modulation of the anthocyanin regulatory complex. Plant Cell Environ. 34:11761190 .

Lipka, A.E., F. Tian, Q. Wang, J. Peiffer, M. Li, P.J. Bradbury, M.A. Gore, E.S. Buckler, and Z. Zhang. 2012. GAPIT: Genome association and prediction integrated tool. Bioinformatics 28:23972399.

Maliepaard, C., F.H. Alston, G. van Arkel, L.M. Brown, E. Chevreau, F. Dunemann, K.M. Evans, S. Gardiner, P. Guilford, A.W. van Heusden, J. Janse, F. Laurens, J.R. Lynn, A.G. Manganaris, A.P.M. den Nijs, N. Periam, E. Rikkerink, P. Roche, C. Ryder, S. Sansavini, H. Schmidt, S. Tartarini, J.J. Verhaegh, M. Vrielink-van Ginkel, and G.J. King. 1998. Aligning male and female linkage maps of apple (Malus pumila Mill.) using multi-allelic markers. Theor. Appl. Genet. 97:60-73.

Martinez-Garcia, P.J., J. Fresnedo-Ramirez, D.E. Parfitt, T.M. Gradziel, and C.H. Crisosto. 2013. Effect prediction of identified SNPs linked to fruit quality and chilling injury in peach [Prunus persica (L.) Batsch]. Plant Mol. Biol. 81:161-174.

McGhie, T.K., M. Hunt, and L.E. Barnett. 2005. Cultivar and growing region determine the antioxidant polyphenolic concentration and composition of apples grown in New Zealand. J. Agr. Food Chem. 53:3065-3070.

McGuire, R.G. 1992. Reporting of objective color measurements. HortScience 27:1254-1255.

Micheletti, D., M. Troggio, A. Zharkikh, F. Costa, M. Malnoy, R. Velasco, and S. Salvi. 2011. Genetic diversity of the genus Malus and implications for linkage mapping with SNPs. Tree Genet. Genomes 7:857-868.

Myles, S., J. Peiffer, P.J. Brown, E.S. Ersoz, Z. Zhang, D.E. Costich, and E.S. Buckler. 2009. Association mapping: Critical considerations shift from genotyping to experimental design. Plant Cell 21:2194-2202.

Patocchi, A., M. Walser, S. Tartarini, G.A.L. Broggini, F. Gennari, S. Sansavini, and C. Gessler. 2005. Identification by genome scanning approach (GSA) of a microsatellite tightly associated with the apple scab resistance gene Vm. Genome 48:630-636.

Rafalski, A. 2002. Applications of single nucleotide polymorphisms in crop genetics. Curr. Opin. Plant Biol. 5:94-100.

Rostoks, N., L. Ramsay, K. MacKenzie, L. Cardle, P.R. Bhat, M.L. Roose, J.T. Svensson, N. Stein, R.K. Varshney, D.F. Marshall, A. Graner, T.J. Close, and R. Waugh. 2006. Recent history of artificial outcrossing facilitates whole-genome association mapping in elite inbred crop varieties. Proc. Natl. Acad. Sci. USA 103:1865618661.

Saure, M.C. 1990. External control of anthocyanin formation in apple. Sci. Hort. 42:181-218.

Shulaev, V., D.J. Sargent, R.N. Crowhurst, T.C. Mockler, O. Folkerts, A.L. Delcher, P. Jaiswal, K. Mockaitis, A. Liston, S.P. Mane, P. Burns, T.M. Davis, J.P. Slovin, N. Bassil, R.P. Hellens, C. Evans, T. Harkins, C. Kodira, B. Desany, O.R. Crasta, R.V. Jensen, A.C. Allan, T.P. Michael, J.C. Setubal, J.M. Celton, D.J.G. Rees, K.P. Williams, S.H. Holt, J.J.R. Rojas, M. Chatterjee, B. Liu, H. Silva, L. Meisel, A. Adato, S.A. Filichkin, M. Troggio, R. Viola, T.L. Ashman, H. Wang, P. Dharmawardhana, J. Elser, R. Raja, H.D. Priest, D.W. Bryant, S.E. Fox, S.A. Givan, L.J. Wilhelm, S. Naithani, A. Christoffels, D.Y. Salama, J. Carter, E.L. Girona, A. Zdepski, W. Wang, R.A. Kerstetter, W. Schwab, S.S. Korban, J. Davik, A. Monfort, B. DenoyesRothan, P. Arus, R. Mittler, B. Flinn, A. Aharoni, J.L. Bennetzen, S.L. Salzberg, A.W. Dickerman, R. Velasco, M. Borodovsky, R.E. Veilleux, and K.M. Folta. 2011. The genome of woodland strawberry (Fragaria vesca). Nat. Genet. 43:109-116. 
Soufflet-Freslon, V., L. Gianfranceschi, A. Patocchi, and C.E. Durel. 2008. Inheritance studies of apple scab resistance and identification of Rvi14 a new major gene that acts together with other broadspectrum QTL. Genome 51:657-667.

Takos, A.M., F.W. Jaffe, S.R. Jacob, J. Bogs, S.P. Robinson, and A.R. Walker. 2006. Light-induced expression of a MYB gene regulates anthocyanin biosynthesis in red apples. Plant Physiol. 142:12161232.

Ubi, B.E., C. Honda, H. Bessho, S. Kondo, M. Wada, S. Kobayashi, and T. Moriguchi. 2006. Expression analysis of anthocyanin biosynthetic genes in apple skin: Effect of UV-B and temperature. Plant Sci. 170:571-578.

VanRaden, P.M. 2008. Efficient methods to compute genomic predictions. J. Dairy Sci. 91:4414-4423.

Varshney, R.K., S.N. Nayak, G.D. May, and S.A. Jackson. 2009. Nextgeneration sequencing technologies and their implications for crop genetics and breeding. Trends Biotechnol. 27:522-530.

Velasco, R., A. Zharkikh, J. Affourtit, A. Dhingra, A. Cestaro, A. Kalyanaraman, P. Fontana, S.K. Bhatnagar, M. Troggio, D. Pruss, S. Salvi, M. Pindo, P. Baldi, S. Castelletti, M. Cavaiuolo, G. Coppola, F. Costa, V. Cova, A. Dal Ri, V. Goremykin, M. Komjanc, S. Longhi, P. Magnago, G. Malacarne, M. Malnoy, D. Micheletti, M. Moretto, M. Perazzolli, A. Si-Ammour, S. Vezzulli, E. Zini, G. Eldredge, L.M. Fitzgerald, N. Gutin, J. Lanchbury, T. Macalma, J.T. Mitchell, J. Reid, B. Wardell, C. Kodira, Z. Chen, B. Desany, F. Niazi, M. Palmer, T. Koepke, D. Jiwan, S. Schaeffer, V. Krishnan, C. Wu, V.T. Chu, S.T. King, J. Vick, Q. Tao, A. Mraz, A. Stormo, K. Stormo, R. Bogden, D. Ederle, A. Stella, A. Vecchietti, M.M. Kater, S. Masiero, P. Lasserre, Y. Lespinasse, A.C. Allan, V. Bus, D. Chagné, R.N. Crowhurst, A.P. Gleave, E. Lavezzo, J.A. Fawcett, S. Proost, P. Rouzé, L. Sterck, S. Toppo, B. Lazzari, R.P. Hellens, C.E. Durel, A. Gutin, R.E. Bumgarner, S.E. Gardiner, M. Skolnick, M. Egholm, Y. van de Peer, F. Salamini, and R. Viola. 2010. The genome of the domesticated apple (Malus $\times$ domestica Borkh.). Nat. Genet. 42:833-839.

Verde, I., A.G. Abbott, S. Scalabrin, S. Jung, S. Shu, F. Marroni, T. Zhebentyayeva, M.T. Dettori, J. Grimwood, F. Cattonaro, A. Zuccolo, L. Rossini, J. Jenkins, E. Vendramin, L.A. Meisel, V. Decroocq, B. Sosinski, S. Prochnik, T. Mitros, A. Policriti, G. Cipriani, L. Dondini, S. Ficklin, D.M. Goodstein, P. Xuan, C.D.
Fabbro, V. Aramini, D. Copetti, S. Gonzalez, D.S. Horner, R. Falchi, S. Lucas, E. Mica, J. Maldonado, B. Lazzari, D. Bielenberg, R. Pirona, M. Miculan, A. Barakat, R. Testolin, A. Stella, S. Tartarini, P. Tonutti, P. Arús, A. Orellana, C. Wells, D. Main, G. Vizzotto, H. Silva, F. Salamini, J. Schmutz, M. Morgante, and D.S. Rokhsar. 2013. The high-quality draft genome of peach (Prunus persica) identifies unique patterns of genetic diversity, domestication and genome evolution. Nat. Genet. 45:487-494.

Verde, I., N. Bassil, S. Scalabrin, B. Gilmore, C.T. Lawley, K. Gasic, D. Micheletti, U.R. Rosyara, F. Cattonaro, E. Vendramin, D. Main, V. Aramini, A.L. Blas, T.C. Mockler, D.W. Bryant, L. Wilhelm, M. Troggio, B. Sosinski, M.J. Aranzana, P. Arús, A. Iezzoni, M. Morgante, C. Peace, and R. Wu. 2012. Development and evaluation of a 9K SNP array for peach by internationally coordinated SNP detection and validation in breeding germplasm. PLoS ONE 7:e35668.

Weir, S.B. 1996. Genetic data analysis II. Methods for discrete population genetics data. 2nd Ed. Sinauer Assoc., Sunderland, MA. Wu, J., Z. Wang, Z. Shi, S. Zhang, R. Ming, S. Zhu, M.A. Khan, S. Tao, S.S. Korban, H. Wang, N.J. Chen, T. Nishio, X. Xu, L. Cong, K. Qi, X. Huang, Y. Wang, X. Zhao, C. Deng, C. Gou, W. Zhou, H. Yin, G. Qin, Y. Sha, Y. Tao, H. Chen, Y. Yang, Y. Song, D. Zhan, J. Wang, L. Li, M. Dai, C. Gu, D. Shi, X. Wang, H. Zhang, L. Zeng, D. Zheng, C. Wang, M. Chen, G. Wang, L. Xie, V. Sovero, S. Sha, W. Huang, M. Zhang, J. Sun, L. Xu, Y. Li, X. Liu, Q. Li, J. Shen, R.E. Paull, and J.L. Bennetzen. 2013. The genome of the pear (Pyrus bretschneideri Rehd.). Genome Res. 23:396-408.

Xie, X.B., S. Li, R.F. Zhang, J. Zhao, Y.C. Chen, Q. Zhao, Y.X. Yao, C.X. You, X.S. Zhang, and Y.J. Hao. 2012. The bHLH transcription factor MdbHLH3 promotes anthocyanin accumulation and fruit colouration in response to low temperature in apples. Plant Cell Environ. 35:1884-1897.

Yan, J., X. Yang, T. Shah, H. Sánchez-Villeda, J. Li, M. Warburton, Y. Zhou, J.H. Crouch, and Y. Xu. 2010. High-throughput SNP genotyping with the GoldenGate assay in maize. Mol. Breed. 25:441-451.

Zaykin, D.V. 2004. Bounds and normalization of the composite linkage disequilibrium coefficient. Genet. Epidemiol. 27:252-257.

Zhu, Y., K. Evans, and C. Peace. 2011. Utility testing of an apple skin color MdMYB1 marker in two progenies. Mol. Breed. 27:525-532. 
Supplemental Table 1. Phenotypic data for red skin coloration in an apple breeding population of 94 seedlings. Red skin coloration was measured as percentage of overcolor, colorimetric measurement, anthocyanin concentration.

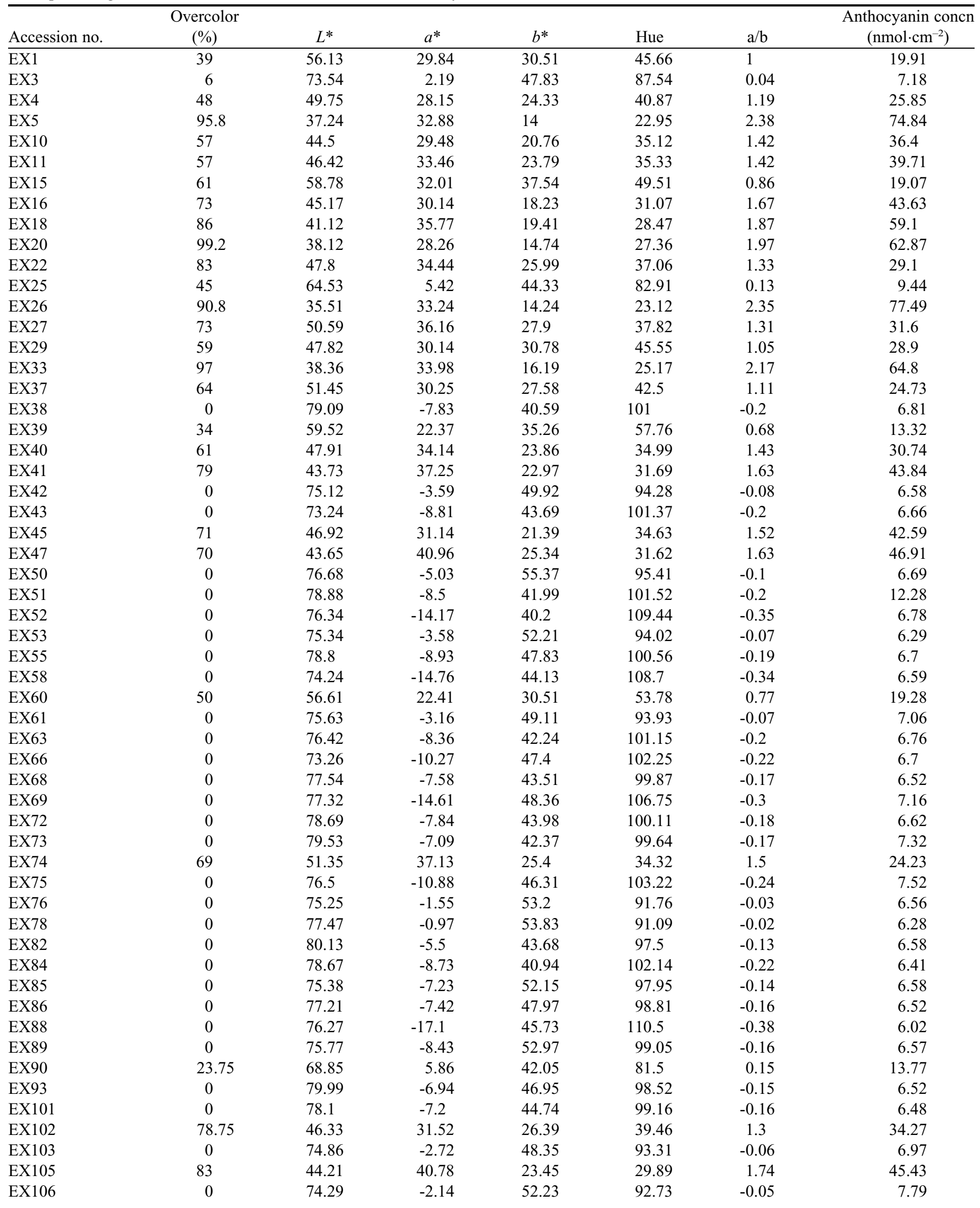


Supplemental Table 1. Continued.

\begin{tabular}{|c|c|c|c|c|c|c|c|}
\hline Accession no. & $\begin{array}{c}\text { Overcolor } \\
(\%)\end{array}$ & $L^{*}$ & $a^{*}$ & $b^{*}$ & Hue & $\mathrm{a} / \mathrm{b}$ & $\begin{array}{c}\text { Anthocyanin concn } \\
\left(\mathrm{nmol} \cdot \mathrm{cm}^{-2}\right)\end{array}$ \\
\hline$\overline{\text { EX107 }}$ & 69 & 44.69 & 32.94 & 20.5 & 31.96 & 1.61 & 37.95 \\
\hline EX109 & 0 & 76.92 & -9.46 & 43.35 & 102.59 & -0.23 & 6.32 \\
\hline EX110 & 48 & 52.37 & 19.03 & 33.83 & 60.32 & 0.58 & 18.8 \\
\hline EX115 & 0 & 77.29 & -9.57 & 45.85 & 101.8 & -0.21 & 6.35 \\
\hline EX117 & 0 & 72.89 & -12.18 & 46.56 & 104.66 & -0.26 & 6.77 \\
\hline EX120 & 0 & 70.42 & -17.63 & 43.77 & 111.78 & -0.4 & 6.23 \\
\hline EX121 & 90 & 41.84 & 32.01 & 20.73 & 32.88 & 1.56 & 52.86 \\
\hline EX123 & 0 & 71.16 & -5.13 & 54.5 & 95.46 & -0.1 & 6.62 \\
\hline EX124 & 0 & 71.47 & -4.64 & 49.37 & 95.62 & -0.1 & 7.28 \\
\hline EX125 & 12.5 & 67.62 & 5.33 & 50.33 & 83.7 & 0.11 & 11.59 \\
\hline EX128 & 0 & 75.99 & -0.57 & 59.48 & 90.57 & -0.01 & 6.51 \\
\hline EX130 & 0 & 75.25 & -2.37 & 52.62 & 92.58 & -0.05 & 6.79 \\
\hline EX133 & 0 & 74.96 & 2.88 & 57.72 & 87.41 & 0.05 & 7.75 \\
\hline EX135 & 22 & 68.71 & 9.79 & 44.72 & 77.66 & 0.22 & 11.67 \\
\hline EX136 & 0 & 77.45 & -2.19 & 57.4 & 92.15 & -0.04 & 6.45 \\
\hline EX137 & 0 & 77.73 & -3.72 & 58.08 & 93.75 & -0.07 & 6.82 \\
\hline EX139 & 0 & 74.42 & -5.8 & 52.24 & 96.44 & -0.11 & 7.9 \\
\hline EX141 & 0 & 75.5 & -6.82 & 53.38 & 97.22 & -0.13 & 6.74 \\
\hline EX142 & 0 & 73.72 & -3.23 & 44.89 & 94.17 & -0.07 & 6.35 \\
\hline EX143 & 0 & 75.32 & -5.6 & 53.65 & 96 & -0.11 & 7.09 \\
\hline EX145 & 14 & 68.03 & 5.66 & 43.04 & 82.41 & 0.14 & 11.26 \\
\hline EX146 & 0 & 75.01 & -7.45 & 42.34 & 100 & -0.18 & 6.5 \\
\hline EX157 & 95.8 & 42.32 & 41.27 & 19.96 & 25.83 & 2.07 & 53.38 \\
\hline EX162 & 46 & 60.5 & 21.36 & 27.65 & 52.62 & 0.78 & 13.08 \\
\hline EX164 & 0 & 77.62 & -7.43 & 50.29 & 98.43 & -0.15 & 6.12 \\
\hline EX165 & 96 & 37.93 & 33.15 & 14.83 & 24.04 & 2.25 & 69.99 \\
\hline EX168 & 0 & 73.09 & -6.48 & 46.56 & 97.98 & -0.14 & 6.68 \\
\hline EX169 & 94 & 43.43 & 31.61 & 21.21 & 33.96 & 1.5 & 30.46 \\
\hline EX173 & 61 & 44.68 & 33.73 & 24.21 & 35.73 & 1.44 & 42.16 \\
\hline EX174 & 0 & 78.4 & -6.59 & 45.98 & 98.19 & -0.14 & 6.35 \\
\hline EX178 & 87 & 39.9 & 36.48 & 16.87 & 24.77 & 2.18 & 77.66 \\
\hline EX182 & 81.25 & 45.84 & 35.39 & 20.99 & 30.86 & 1.69 & 34.93 \\
\hline EX187 & 82 & 41.75 & 33.19 & 16.46 & 26.35 & 2.03 & 57.31 \\
\hline EX188 & 0 & 73.14 & 1.09 & 44.47 & 86.91 & 0.07 & 7.22 \\
\hline EX189 & 0 & 77.42 & -7.74 & 51.37 & 98.66 & -0.15 & 6.17 \\
\hline EX191 & 44 & 47.84 & 29.4 & 26.97 & 42.79 & 1.1 & 26.55 \\
\hline EX192 & 0 & 78.06 & -5.22 & 43.21 & 97.33 & -0.13 & 6.26 \\
\hline EX193 & 64 & 43.98 & 33.7 & 22.32 & 33.55 & 1.57 & 42.51 \\
\hline
\end{tabular}


Supplemental Table 2. Genotypic data and marker information used for association analysis in an apple breeding population of 94 seedlings. The physical location of single nucleotide polymorphisms (SNPs) is given based on the 'Golden Delicious' genome assembly (“-” = missing genotypic data).

\begin{tabular}{|c|c|c|c|c|c|c|c|c|c|c|}
\hline SNP name & \multicolumn{10}{|c|}{ Linkage group Physical location (bp) Individuals } \\
\hline EX41 & EX42 & EX43 & EX45 & EX47 & EX50 & EX51 & EX52 & EX53 & EX55 & EX58 \\
\hline EX78 & EX82 & EX84 & EX85 & EX86 & EX88 & EX89 & EX90 & EX93 & EX101 & EX102 \\
\hline EX103 & EX105 & EX106 & EX107 & EX109 & EX110 & EX115 & EX117 & EX120 & EX121 & EX123 \\
\hline EX124 & EX125 & EX128 & EX130 & EX133 & EX135 & EX136 & EX137 & EX139 & EX141 & EX142 \\
\hline GDsnp00014 & 1 & \multicolumn{2}{|c|}{3056859} & GG & GG & CG & GG & CG & GG & GG \\
\hline $\mathrm{CG}$ & $\mathrm{CC}$ & CG & CG & GG & CG & CG & $\mathrm{CG}$ & CG & GG & CG \\
\hline $\mathrm{CG}$ & GG & $\mathrm{CG}$ & CG & CG & $\mathrm{CC}$ & - & $\mathrm{CC}$ & $\mathrm{CC}$ & $\mathrm{CG}$ & $\mathrm{CG}$ \\
\hline GG & $\mathrm{CC}$ & GG & CG & $\mathrm{CC}$ & GG & GG & GG & $\mathrm{CC}$ & $\mathrm{CC}$ & $\mathrm{CC}$ \\
\hline GG & $\mathrm{CG}$ & $\mathrm{CG}$ & GG & GG & CG & - & GG & GG & $\mathrm{CC}$ & GG \\
\hline CG & $\mathrm{CG}$ & GG & $\mathrm{CC}$ & $\mathrm{CC}$ & GG & $\mathrm{CG}$ & CG & CG & $\mathrm{CC}$ & \\
\hline GDsnp00152 & 1 & \multicolumn{2}{|c|}{5384623} & $\mathrm{AA}$ & $\mathrm{AA}$ & $\mathrm{AG}$ & $\mathrm{AA}$ & $\mathrm{AG}$ & AA & AA \\
\hline $\mathrm{AA}$ & $\mathrm{AG}$ & $\mathrm{AG}$ & AG & $\mathrm{AA}$ & $\mathrm{AG}$ & AG & $\mathrm{AG}$ & $\mathrm{AG}$ & AA & $\mathrm{AG}$ \\
\hline $\mathrm{AG}$ & $\mathrm{AA}$ & $\mathrm{AG}$ & $\mathrm{AG}$ & $\mathrm{AG}$ & GG & $\mathrm{AG}$ & GG & GG & $\mathrm{AG}$ & $\mathrm{AG}$ \\
\hline $\mathrm{AA}$ & GG & $\mathrm{AA}$ & $\mathrm{AG}$ & GG & $\mathrm{AA}$ & $\mathrm{AA}$ & $\mathrm{AA}$ & GG & GG & GG \\
\hline $\mathrm{AA}$ & $\mathrm{AG}$ & $\mathrm{AG}$ & $\mathrm{AA}$ & $\mathrm{AA}$ & $\mathrm{AG}$ & $\mathrm{AG}$ & AA & AA & GG & AA \\
\hline $\mathrm{AG}$ & $\mathrm{AG}$ & $\mathrm{AA}$ & GG & GG & GG & AA & $\mathrm{AG}$ & $\mathrm{AG}$ & $\mathrm{AG}$ & $\mathrm{AG}$ \\
\hline $\mathrm{AG}$ & $\mathrm{AG}$ & $\mathrm{AG}$ & $\mathrm{AG}$ & $\mathrm{AA}$ & AG & $\mathrm{AA}$ & $\mathrm{AG}$ & GG & AA & $\mathrm{AG}$ \\
\hline $\mathrm{AG}$ & $\mathrm{AG}$ & $\mathrm{AA}$ & GG & GG & $\mathrm{AG}$ & $\mathrm{AA}$ & $\mathrm{AA}$ & $\mathrm{AG}$ & $\mathrm{AG}$ & $\mathrm{AG}$ \\
\hline $\mathrm{AG}$ & $\mathrm{AG}$ & $\mathrm{AA}$ & GG & GG & AA & $\mathrm{AG}$ & GG & $\mathrm{AG}$ & GG & \\
\hline GDsnp02428 & 1 & \multicolumn{2}{|c|}{6628041} & $\mathrm{CC}$ & $\mathrm{CC}$ & $\mathrm{CC}$ & $\mathrm{CC}$ & $\mathrm{CC}$ & $\mathrm{CC}$ & $\mathrm{CC}$ \\
\hline $\mathrm{CC}$ & $\mathrm{CC}$ & $\mathrm{CC}$ & $\mathrm{CC}$ & $\mathrm{CC}$ & $\mathrm{CC}$ & $\mathrm{CC}$ & $\mathrm{CC}$ & $\mathrm{CC}$ & $\mathrm{CC}$ & \\
\hline GDsnp00533 & 1 & \multicolumn{2}{|c|}{8879872} & $\mathrm{AG}$ & GG & $\mathrm{AG}$ & GG & $A G$ & GG & $\mathrm{AG}$ \\
\hline GG & $\mathrm{AA}$ & $\mathrm{AA}$ & $\mathrm{AG}$ & GG & AG & $\mathrm{AG}$ & $\mathrm{AG}$ & $A G$ & GG & $\mathrm{AG}$ \\
\hline $\mathrm{AG}$ & $\mathrm{AG}$ & $\mathrm{AA}$ & $\mathrm{AA}$ & $\mathrm{AG}$ & $\mathrm{AA}$ & $\mathrm{AG}$ & $\mathrm{AA}$ & AA & GG & $\mathrm{AG}$ \\
\hline GG & $\mathrm{AG}$ & GG & $\mathrm{AG}$ & $\mathrm{AA}$ & $\mathrm{AG}$ & GG & GG & AA & AA & AA \\
\hline GG & AA & $\mathrm{AG}$ & $\mathrm{AG}$ & GG & AA & AA & GG & GG & AA & GG \\
\hline $\mathrm{AG}$ & $\mathrm{AG}$ & GG & $\mathrm{AA}$ & $\mathrm{AA}$ & AG & GG & $\mathrm{AG}$ & $A G$ & $\mathrm{AG}$ & $\mathrm{AG}$ \\
\hline $\mathrm{AG}$ & $\mathrm{AG}$ & AG & GG & GG & $\mathrm{AG}$ & $\mathrm{AG}$ & $\mathrm{AA}$ & AA & GG & $\mathrm{AG}$ \\
\hline $\mathrm{AG}$ & $\mathrm{AG}$ & GG & $\mathrm{AA}$ & $\mathrm{AA}$ & $\mathrm{AG}$ & $\mathrm{AG}$ & GG & $\mathrm{AG}$ & $\mathrm{AG}$ & AG \\
\hline $\mathrm{AA}$ & $\mathrm{AG}$ & GG & $\mathrm{AG}$ & $\mathrm{AA}$ & $\mathrm{AA}$ & AA & $\mathrm{AA}$ & $\mathrm{AG}$ & AA & \\
\hline GDsnp00631 & 1 & \multicolumn{2}{|c|}{9692952} & $\mathrm{AA}$ & $\mathrm{AA}$ & $\mathrm{AA}$ & $\mathrm{AA}$ & AA & AA & AA \\
\hline $\mathrm{AA}$ & AT & $\mathrm{AA}$ & $\mathrm{AA}$ & $\mathrm{AA}$ & $\mathrm{AA}$ & $\mathrm{AA}$ & $\mathrm{AA}$ & $\mathrm{AA}$ & AA & AA \\
\hline AA & AA & AT & $\mathrm{AA}$ & AA & AA & AA & $\mathrm{AA}$ & AA & AA & AA \\
\hline $\mathrm{AA}$ & $\mathrm{AA}$ & $\mathrm{AA}$ & $\mathrm{AA}$ & $\mathrm{AA}$ & $\mathrm{AA}$ & AA & $\mathrm{AA}$ & AA & AA & $\mathrm{AT}$ \\
\hline $\mathrm{AA}$ & $\mathrm{AA}$ & $\mathrm{AA}$ & $\mathrm{AA}$ & $\mathrm{AA}$ & AA & AA & $\mathrm{AA}$ & AA & AA & AA \\
\hline $\mathrm{AA}$ & $\mathrm{AA}$ & $\mathrm{AA}$ & AT & $\mathrm{AA}$ & $\mathrm{AA}$ & AA & $\mathrm{AA}$ & AA & AA & AA \\
\hline $\mathrm{AA}$ & AA & $\mathrm{AA}$ & $\mathrm{AA}$ & AA & AA & AA & AA & AA & AA & AA \\
\hline $\mathrm{AA}$ & AA & $\mathrm{AA}$ & $\mathrm{AA}$ & AA & AA & AA & $\mathrm{AA}$ & AA & AA & AA \\
\hline $\mathrm{AA}$ & AA & $\mathrm{AA}$ & AA & AA & AA & AA & AA & AA & AA & \\
\hline
\end{tabular}


Supplemental Table 2. Continued.

\begin{tabular}{|c|c|c|c|c|c|c|c|c|c|c|}
\hline \multirow{3}{*}{$\begin{array}{l}\text { SNP name } \\
\text { GDsnp01470 } \\
\text { CG }\end{array}$} & \multicolumn{10}{|c|}{ Linkage group Physical location (bp) Individuals } \\
\hline & 1 & \multicolumn{2}{|c|}{10713804} & $\mathrm{CC}$ & $\mathrm{CC}$ & $\mathrm{CC}$ & $\mathrm{CC}$ & $\mathrm{CC}$ & $\mathrm{CC}$ & $\mathrm{CC}$ \\
\hline & $\mathrm{CG}$ & $\mathrm{CC}$ & $\mathrm{CC}$ & $\mathrm{CC}$ & $\mathrm{CC}$ & $\mathrm{CC}$ & $\mathrm{CC}$ & CG & $\mathrm{CC}$ & $\mathrm{CC}$ \\
\hline $\mathrm{CC}$ & $\mathrm{CC}$ & $\mathrm{CG}$ & $\mathrm{CC}$ & $\mathrm{CC}$ & $\mathrm{CC}$ & $\mathrm{CC}$ & $\mathrm{CC}$ & $\mathrm{CC}$ & $\mathrm{CC}$ & $\mathrm{CC}$ \\
\hline $\mathrm{CC}$ & $\mathrm{CC}$ & $\mathrm{CC}$ & $\mathrm{CC}$ & $\mathrm{CC}$ & $\mathrm{CC}$ & $\mathrm{CC}$ & $\mathrm{CC}$ & $\mathrm{CC}$ & $\mathrm{CC}$ & $\mathrm{CG}$ \\
\hline $\mathrm{CC}$ & $\mathrm{CC}$ & $\mathrm{CC}$ & $\mathrm{CC}$ & $\mathrm{CC}$ & $\mathrm{CC}$ & - & $\mathrm{CC}$ & $\mathrm{CC}$ & $\mathrm{CC}$ & $\mathrm{CC}$ \\
\hline $\mathrm{CC}$ & $\mathrm{CC}$ & $\mathrm{CC}$ & $\mathrm{CG}$ & $\mathrm{CC}$ & $\mathrm{CC}$ & $\mathrm{CC}$ & $\mathrm{CC}$ & $\mathrm{CC}$ & $\mathrm{CC}$ & $\mathrm{CC}$ \\
\hline $\mathrm{CC}$ & $\mathrm{CC}$ & $\mathrm{CC}$ & $\mathrm{CC}$ & $\mathrm{CC}$ & $\mathrm{CC}$ & $\mathrm{CC}$ & $\mathrm{CC}$ & $\mathrm{CC}$ & $\mathrm{CC}$ & $\mathrm{CC}$ \\
\hline $\mathrm{CC}$ & $\mathrm{CC}$ & $\mathrm{CC}$ & $\mathrm{CC}$ & $\mathrm{CC}$ & $\mathrm{CC}$ & $\mathrm{CC}$ & $\mathrm{CC}$ & $\mathrm{CC}$ & $\mathrm{CC}$ & $\mathrm{CC}$ \\
\hline $\mathrm{CC}$ & $\mathrm{CC}$ & $\mathrm{CC}$ & $\mathrm{CC}$ & $\mathrm{CC}$ & $\mathrm{CC}$ & $\mathrm{CC}$ & $\mathrm{CC}$ & $\mathrm{CC}$ & $\mathrm{CC}$ & \\
\hline GDsnp00697 & 1 & \multicolumn{2}{|c|}{11348920} & AA & AA & AA & AA & AA & AA & AA \\
\hline $\mathrm{AA}$ & $\mathrm{AA}$ & $\mathrm{AA}$ & - & AA & $\mathrm{AA}$ & AA & AA & AA & $\mathrm{AA}$ & AA \\
\hline AA & AA & AA & $\mathrm{AA}$ & AA & $\mathrm{AA}$ & AA & AA & AA & $\mathrm{AA}$ & AA \\
\hline $\mathrm{AA}$ & AA & $\mathrm{AA}$ & $\mathrm{AA}$ & $\mathrm{AA}$ & $\mathrm{AA}$ & $\mathrm{AA}$ & $\mathrm{AA}$ & $\mathrm{AA}$ & $\mathrm{AA}$ & AA \\
\hline AA & AA & AA & $\mathrm{AA}$ & $\mathrm{AA}$ & $\mathrm{AA}$ & $\mathrm{AA}$ & $\mathrm{AA}$ & AA & $\mathrm{AA}$ & AA \\
\hline $\mathrm{AA}$ & $\mathrm{AA}$ & $\mathrm{AA}$ & $\mathrm{AA}$ & $\mathrm{AA}$ & $\mathrm{AA}$ & $\mathrm{AA}$ & AA & $\mathrm{AA}$ & $\mathrm{AA}$ & AA \\
\hline $\mathrm{AA}$ & $\mathrm{AA}$ & $\mathrm{AA}$ & AA & AA & $\mathrm{AA}$ & AA & AA & AA & $\mathrm{AA}$ & $\mathrm{AA}$ \\
\hline AA & $\mathrm{AA}$ & $\mathrm{AA}$ & AA & - & $\mathrm{AA}$ & AA & AA & $\mathrm{AA}$ & $\mathrm{AA}$ & $\mathrm{AA}$ \\
\hline $\mathrm{AA}$ & AA & AA & - & $\mathrm{AA}$ & $\mathrm{AA}$ & - & $\mathrm{AA}$ & $\mathrm{AA}$ & $\mathrm{AA}$ & \\
\hline GDsnp01500 & 1 & \multicolumn{2}{|c|}{11455127} & AA & $\mathrm{AA}$ & AA & AA & AA & AA & AA \\
\hline $\mathrm{AG}$ & $\mathrm{AA}$ & $\mathrm{AA}$ & $\mathrm{AG}$ & $\mathrm{AA}$ & $\mathrm{AA}$ & $\mathrm{AA}$ & $\mathrm{AA}$ & $\mathrm{AA}$ & $\mathrm{AA}$ & AA \\
\hline AA & $\mathrm{AA}$ & $\mathrm{AA}$ & $\mathrm{AA}$ & $\mathrm{AA}$ & $\mathrm{AA}$ & $\mathrm{AA}$ & $\mathrm{AA}$ & $\mathrm{AA}$ & $\mathrm{AA}$ & $\mathrm{AA}$ \\
\hline $\mathrm{AA}$ & $\mathrm{AA}$ & $\mathrm{AA}$ & $\mathrm{AA}$ & AA & $\mathrm{AA}$ & $\mathrm{AA}$ & AA & $\mathrm{AA}$ & $\mathrm{AG}$ & AA \\
\hline $\mathrm{AA}$ & $\mathrm{AA}$ & $\mathrm{AA}$ & AA & AA & $\mathrm{AA}$ & $\mathrm{AA}$ & AA & AA & $\mathrm{AA}$ & $\mathrm{AA}$ \\
\hline AA & $\mathrm{AA}$ & $\mathrm{AA}$ & $\mathrm{AA}$ & AA & $\mathrm{AA}$ & $\mathrm{AA}$ & $\mathrm{AA}$ & $\mathrm{AA}$ & $\mathrm{AA}$ & $\mathrm{AA}$ \\
\hline AA & $\mathrm{AA}$ & $\mathrm{AA}$ & $\mathrm{AA}$ & $\mathrm{AA}$ & $\mathrm{AA}$ & $\mathrm{AA}$ & $\mathrm{AA}$ & $\mathrm{AA}$ & $\mathrm{AA}$ & $\mathrm{AA}$ \\
\hline $\mathrm{AA}$ & AA & AA & $\mathrm{AA}$ & $\mathrm{AG}$ & $\mathrm{AA}$ & $\mathrm{AA}$ & $\mathrm{AA}$ & $\mathrm{AA}$ & $\mathrm{AA}$ & AA \\
\hline $\mathrm{AA}$ & AA & AA & $\mathrm{AA}$ & AA & AA & $\mathrm{AG}$ & $\mathrm{AA}$ & $\mathrm{AA}$ & $\mathrm{AA}$ & \\
\hline GDsnp00250 & 1 & \multicolumn{2}{|c|}{16992156} & AA & AA & AA & AA & AA & AA & AA \\
\hline $\mathrm{AA}$ & $\mathrm{AA}$ & $\mathrm{AA}$ & AA & AA & $\mathrm{AA}$ & AA & AA & AA & $\mathrm{AA}$ & $\mathrm{AA}$ \\
\hline $\mathrm{AA}$ & AA & $\mathrm{AG}$ & $\mathrm{AA}$ & $\mathrm{AA}$ & $\mathrm{AA}$ & $\mathrm{AA}$ & $\mathrm{AA}$ & $\mathrm{AA}$ & $\mathrm{AA}$ & AA \\
\hline AA & $\mathrm{AA}$ & $\mathrm{AA}$ & $\mathrm{AA}$ & $\mathrm{AA}$ & $\mathrm{AA}$ & $\mathrm{AA}$ & $\mathrm{AA}$ & $\mathrm{AA}$ & $\mathrm{AG}$ & $\mathrm{AG}$ \\
\hline $\mathrm{AA}$ & $\mathrm{AA}$ & $\mathrm{AA}$ & $\mathrm{AA}$ & $\mathrm{AA}$ & $\mathrm{AA}$ & - & AA & $\mathrm{AA}$ & $\mathrm{AA}$ & AA \\
\hline $\mathrm{AA}$ & AA & AA & $\mathrm{AA}$ & $\mathrm{AA}$ & $\mathrm{AA}$ & AA & AA & AA & $\mathrm{AA}$ & AA \\
\hline AA & $\mathrm{AA}$ & $\mathrm{AA}$ & AA & AA & $\mathrm{AA}$ & AA & AA & $\mathrm{AA}$ & $\mathrm{AA}$ & $\mathrm{AA}$ \\
\hline AA & $\mathrm{AA}$ & AA & $\mathrm{AA}$ & $\mathrm{AA}$ & $\mathrm{AA}$ & $\mathrm{AA}$ & $\mathrm{AA}$ & $\mathrm{AA}$ & $\mathrm{AA}$ & AA \\
\hline $\mathrm{AA}$ & AA & AA & AA & AA & $\mathrm{AA}$ & $\mathrm{AG}$ & AA & $\mathrm{AA}$ & $\mathrm{AA}$ & \\
\hline GDsnp01676 & 1 & \multicolumn{2}{|c|}{18252811} & AA & AA & AA & AA & $\mathrm{AA}$ & $\mathrm{AA}$ & AA \\
\hline $\mathrm{AA}$ & $\mathrm{AA}$ & $\mathrm{AA}$ & $\mathrm{AA}$ & $\mathrm{AA}$ & $\mathrm{AA}$ & $\mathrm{AA}$ & $\mathrm{AA}$ & $\mathrm{AA}$ & $\mathrm{AA}$ & $\mathrm{AA}$ \\
\hline $\mathrm{AA}$ & $\mathrm{AA}$ & $\mathrm{AA}$ & $\mathrm{AA}$ & AA & $\mathrm{AA}$ & $\mathrm{AA}$ & $\mathrm{AT}$ & $\mathrm{AT}$ & $\mathrm{AA}$ & AA \\
\hline $\mathrm{AA}$ & AA & AA & $\mathrm{AA}$ & $\mathrm{AT}$ & $\mathrm{AT}$ & $\mathrm{AA}$ & AA & AA & AA & AA \\
\hline $\mathrm{AA}$ & $\mathrm{AA}$ & $\mathrm{AA}$ & AA & $\mathrm{AT}$ & $\mathrm{AA}$ & $\mathrm{AA}$ & $\mathrm{AA}$ & $\mathrm{AT}$ & $\mathrm{AA}$ & AT \\
\hline AA & $\mathrm{AA}$ & $\mathrm{AA}$ & $\mathrm{AA}$ & $\mathrm{AA}$ & $\mathrm{AA}$ & $\mathrm{AA}$ & $\mathrm{AA}$ & $\mathrm{AT}$ & AT & $\mathrm{AA}$ \\
\hline $\mathrm{AA}$ & $\mathrm{AA}$ & $\mathrm{AA}$ & $\mathrm{AA}$ & AA & $\mathrm{AT}$ & $\mathrm{AA}$ & $\mathrm{AA}$ & $\mathrm{AA}$ & $\mathrm{AA}$ & AA \\
\hline $\mathrm{AA}$ & $\mathrm{AA}$ & $\mathrm{AT}$ & $\mathrm{AA}$ & $\mathrm{AA}$ & $\mathrm{AA}$ & $\mathrm{AA}$ & $\mathrm{AA}$ & $\mathrm{AA}$ & $\mathrm{AA}$ & AA \\
\hline $\mathrm{AA}$ & $\mathrm{AA}$ & $\mathrm{AA}$ & $\mathrm{AA}$ & $\mathrm{AA}$ & $\mathrm{AA}$ & $\mathrm{AT}$ & $\mathrm{AA}$ & $\mathrm{AA}$ & $\mathrm{AA}$ & \\
\hline GDsnp00087 & 1 & \multicolumn{2}{|c|}{20622641} & AA & $\mathrm{AA}$ & AA & AA & AA & AA & AA \\
\hline $\mathrm{AG}$ & AA & $\mathrm{AA}$ & $\mathrm{AG}$ & $\mathrm{AA}$ & $\mathrm{AA}$ & $\mathrm{AA}$ & $\mathrm{AA}$ & $\mathrm{AG}$ & $\mathrm{AA}$ & AA \\
\hline AA & $\mathrm{AA}$ & $\mathrm{AG}$ & $\mathrm{AA}$ & $\mathrm{AA}$ & $\mathrm{AA}$ & $\mathrm{AA}$ & $\mathrm{AG}$ & $\mathrm{AG}$ & $\mathrm{AA}$ & AA \\
\hline AA & $\mathrm{AA}$ & AA & $\mathrm{AA}$ & $\mathrm{AG}$ & $\mathrm{AG}$ & $\mathrm{AA}$ & $\mathrm{AA}$ & $\mathrm{AA}$ & $\mathrm{AG}$ & $\mathrm{AG}$ \\
\hline AA & $\mathrm{AA}$ & $\mathrm{AA}$ & $\mathrm{AA}$ & $\mathrm{AG}$ & $\mathrm{AA}$ & $\mathrm{AG}$ & - & $\mathrm{AG}$ & $\mathrm{AA}$ & $\mathrm{AA}$ \\
\hline $\mathrm{AA}$ & $\mathrm{AA}$ & $\mathrm{AA}$ & $\mathrm{AA}$ & $\mathrm{AA}$ & $\mathrm{AA}$ & $\mathrm{AA}$ & $\mathrm{AA}$ & $\mathrm{AG}$ & $\mathrm{AG}$ & $\mathrm{AA}$ \\
\hline $\mathrm{AA}$ & $\mathrm{AA}$ & $\mathrm{AA}$ & $\mathrm{AA}$ & $\mathrm{AA}$ & $\mathrm{AG}$ & $\mathrm{AA}$ & $\mathrm{AG}$ & $\mathrm{AA}$ & $\mathrm{AA}$ & $\mathrm{AA}$ \\
\hline- & $\mathrm{AA}$ & $\mathrm{AG}$ & $\mathrm{AA}$ & $\mathrm{AG}$ & $\mathrm{AA}$ & $\mathrm{AA}$ & $\mathrm{AA}$ & $\mathrm{AA}$ & $\mathrm{AA}$ & $\mathrm{AA}$ \\
\hline $\mathrm{AA}$ & $\mathrm{AA}$ & $\mathrm{AA}$ & $\mathrm{AA}$ & $\mathrm{AA}$ & $\mathrm{AA}$ & $\mathrm{AG}$ & $\mathrm{AA}$ & $\mathrm{AA}$ & $\mathrm{AA}$ & \\
\hline
\end{tabular}

Continued next page 
Supplemental Table 2. Continued.

\begin{tabular}{|c|c|c|c|c|c|c|c|c|c|c|}
\hline \multirow{3}{*}{$\begin{array}{l}\text { SNP name } \\
\text { GDsnp00674 } \\
\text { AG }\end{array}$} & \multicolumn{10}{|c|}{ Linkage group Physical location (bp) Individuals } \\
\hline & 1 & \multicolumn{2}{|c|}{20963765} & GG & GG & AA & GG & $\mathrm{AG}$ & GG & GG \\
\hline & AA & AA & $\mathrm{AG}$ & GG & $\mathrm{AG}$ & $\mathrm{AG}$ & $\mathrm{AG}$ & AA & GG & $\mathrm{AG}$ \\
\hline $\mathrm{AG}$ & GG & AA & $\mathrm{AG}$ & $\mathrm{AG}$ & AA & AA & AA & AA & $\mathrm{AG}$ & AA \\
\hline AG & AG & $\mathrm{AG}$ & $\mathrm{AG}$ & AA & $\mathrm{AG}$ & AA & $\mathrm{AG}$ & $\mathrm{AG}$ & $\mathrm{AA}$ & $\mathrm{AG}$ \\
\hline GG & $\mathrm{AG}$ & $\mathrm{AA}$ & $\mathrm{AG}$ & $\mathrm{AG}$ & $\mathrm{AG}$ & $\mathrm{AG}$ & $\mathrm{AG}$ & $\mathrm{AG}$ & $\mathrm{AG}$ & $\mathrm{AG}$ \\
\hline $\mathrm{AG}$ & $\mathrm{AG}$ & GG & $\mathrm{AG}$ & AA & $\mathrm{AG}$ & GG & $\mathrm{AA}$ & $\mathrm{AG}$ & $\mathrm{AG}$ & $\mathrm{AG}$ \\
\hline $\mathrm{AG}$ & $\mathrm{AA}$ & $\mathrm{AG}$ & GG & GG & $\mathrm{AG}$ & GG & $\mathrm{AG}$ & $\mathrm{AG}$ & GG & $\mathrm{AG}$ \\
\hline GG & $\mathrm{AG}$ & $\mathrm{AA}$ & $\mathrm{AG}$ & $\mathrm{AG}$ & $\mathrm{AG}$ & $\mathrm{AG}$ & $\mathrm{AG}$ & $\mathrm{AG}$ & $\mathrm{AG}$ & $\mathrm{AG}$ \\
\hline $\mathrm{AG}$ & $\mathrm{AG}$ & $\mathrm{AG}$ & GG & AA & GG & $\mathrm{AG}$ & AA & $\mathrm{AG}$ & $\mathrm{AA}$ & \\
\hline GDsnp00252 & 1 & \multicolumn{2}{|c|}{21447381} & $\mathrm{CC}$ & $\mathrm{CC}$ & $\mathrm{AC}$ & $\mathrm{CC}$ & $\mathrm{AC}$ & $\mathrm{CC}$ & $\mathrm{CC}$ \\
\hline $\mathrm{CC}$ & $\mathrm{AC}$ & AA & $\mathrm{AC}$ & $\mathrm{CC}$ & $\mathrm{CC}$ & $\mathrm{CC}$ & $\mathrm{AC}$ & $\mathrm{AA}$ & $\mathrm{AC}$ & $\mathrm{AC}$ \\
\hline $\mathrm{CC}$ & $\mathrm{CC}$ & $\mathrm{AA}$ & $\mathrm{CC}$ & $\mathrm{AC}$ & $\mathrm{AA}$ & $\mathrm{AC}$ & $\mathrm{AC}$ & $\mathrm{AC}$ & $\mathrm{AC}$ & AA \\
\hline $\mathrm{AC}$ & $\mathrm{AC}$ & $\mathrm{AC}$ & $\mathrm{AC}$ & $\mathrm{AC}$ & $\mathrm{CC}$ & $\mathrm{AC}$ & $\mathrm{AC}$ & $\mathrm{AC}$ & $\mathrm{AC}$ & $\mathrm{AC}$ \\
\hline $\mathrm{AC}$ & $\mathrm{AC}$ & $\mathrm{AC}$ & $\mathrm{AC}$ & $\mathrm{AC}$ & $\mathrm{AC}$ & $\mathrm{AC}$ & $\mathrm{AC}$ & $\mathrm{CC}$ & $\mathrm{AC}$ & $\mathrm{CC}$ \\
\hline $\mathrm{CC}$ & $\mathrm{AC}$ & $\mathrm{CC}$ & $\mathrm{AC}$ & $\mathrm{AC}$ & $\mathrm{AC}$ & $\mathrm{CC}$ & AA & $\mathrm{CC}$ & $\mathrm{AC}$ & $\mathrm{AC}$ \\
\hline $\mathrm{CC}$ & $\mathrm{AA}$ & $\mathrm{AC}$ & $\mathrm{CC}$ & $\mathrm{CC}$ & $\mathrm{CC}$ & $\mathrm{CC}$ & $\mathrm{AC}$ & $\mathrm{AC}$ & $\mathrm{CC}$ & $\mathrm{AC}$ \\
\hline $\mathrm{CC}$ & $\mathrm{AC}$ & $\mathrm{AC}$ & $\mathrm{CC}$ & $\mathrm{AC}$ & $\mathrm{AC}$ & $\mathrm{AC}$ & $\mathrm{AC}$ & $\mathrm{AC}$ & $\mathrm{AC}$ & $\mathrm{CC}$ \\
\hline $\mathrm{AC}$ & $\mathrm{AC}$ & $\mathrm{AC}$ & $\mathrm{CC}$ & AA & $\mathrm{CC}$ & $\mathrm{CC}$ & AA & $\mathrm{CC}$ & $\mathrm{AC}$ & \\
\hline GDsnp00761 & 1 & \multicolumn{2}{|c|}{22565180} & $\mathrm{AT}$ & $\mathrm{AT}$ & $\mathrm{AT}$ & $\mathrm{AT}$ & $\mathrm{AT}$ & $\mathrm{AT}$ & AT \\
\hline AT & $\mathrm{AT}$ & $\mathrm{AT}$ & $\mathrm{AT}$ & $\mathrm{AT}$ & $\mathrm{AT}$ & $\mathrm{AT}$ & $\mathrm{AT}$ & AA & $\mathrm{AT}$ & AT \\
\hline AT & $\mathrm{AT}$ & $\mathrm{AA}$ & $\mathrm{AT}$ & $\mathrm{AT}$ & $\mathrm{AT}$ & $\mathrm{AT}$ & $\mathrm{AT}$ & $\mathrm{AT}$ & AT & AT \\
\hline $\mathrm{AT}$ & $\mathrm{AT}$ & $\mathrm{AT}$ & $\mathrm{AT}$ & $\mathrm{AT}$ & $\mathrm{AT}$ & $\mathrm{AT}$ & $\mathrm{AT}$ & $\mathrm{AT}$ & $\mathrm{AT}$ & AT \\
\hline $\mathrm{AT}$ & $\mathrm{AT}$ & $\mathrm{AT}$ & $\mathrm{AT}$ & $\mathrm{AT}$ & $\mathrm{AT}$ & $\mathrm{AT}$ & $\mathrm{AT}$ & $\mathrm{AT}$ & AT & AT \\
\hline AT & $\mathrm{AT}$ & $\mathrm{AT}$ & $\mathrm{AT}$ & $\mathrm{AT}$ & $\mathrm{AT}$ & $\mathrm{AT}$ & $\mathrm{AT}$ & $\mathrm{AT}$ & $\mathrm{AT}$ & AT \\
\hline AT & $\mathrm{AT}$ & $\mathrm{AT}$ & $\mathrm{AT}$ & AT & $\mathrm{AT}$ & $\mathrm{AT}$ & $\mathrm{AT}$ & $\mathrm{AT}$ & $\mathrm{AT}$ & AT \\
\hline AT & $\mathrm{AT}$ & $\mathrm{AT}$ & $\mathrm{AT}$ & $\mathrm{AT}$ & $\mathrm{AT}$ & $\mathrm{AT}$ & $\mathrm{AT}$ & $\mathrm{AT}$ & $\mathrm{AT}$ & AT \\
\hline AT & AT & AT & AT & AT & AT & AT & AT & AT & AT & \\
\hline GDsnp00361 & 1 & \multicolumn{2}{|c|}{23735335} & GG & GG & $\mathrm{CG}$ & GG & $\mathrm{CG}$ & $\mathrm{CG}$ & GG \\
\hline CG & $\mathrm{CG}$ & $\mathrm{CC}$ & $\mathrm{CG}$ & GG & GG & GG & $\mathrm{CG}$ & $\mathrm{CC}$ & $\mathrm{CG}$ & $\mathrm{CG}$ \\
\hline GG & GG & $\mathrm{CC}$ & GG & $\mathrm{CG}$ & $\mathrm{CC}$ & $\mathrm{CC}$ & $\mathrm{CG}$ & $\mathrm{CG}$ & $\mathrm{CG}$ & $\mathrm{CC}$ \\
\hline $\mathrm{CG}$ & $\mathrm{CG}$ & $\mathrm{CG}$ & $\mathrm{CG}$ & $\mathrm{CG}$ & GG & $\mathrm{CG}$ & $\mathrm{CG}$ & $\mathrm{CG}$ & $\mathrm{CG}$ & $\mathrm{CG}$ \\
\hline $\mathrm{CG}$ & $\mathrm{CG}$ & $\mathrm{CG}$ & $\mathrm{CG}$ & $\mathrm{CG}$ & $\mathrm{CG}$ & - & $\mathrm{CG}$ & GG & $\mathrm{CG}$ & GG \\
\hline GG & $\mathrm{CG}$ & GG & $\mathrm{CG}$ & $\mathrm{CG}$ & $\mathrm{CG}$ & $\mathrm{CG}$ & $\mathrm{CC}$ & GG & $\mathrm{CG}$ & $\mathrm{CG}$ \\
\hline GG & $\mathrm{CC}$ & $\mathrm{CG}$ & GG & $\mathrm{CG}$ & GG & GG & $\mathrm{CG}$ & $\mathrm{CG}$ & GG & $\mathrm{CG}$ \\
\hline GG & $\mathrm{CG}$ & $\mathrm{CG}$ & GG & - & $\mathrm{CG}$ & $\mathrm{CG}$ & $\mathrm{CG}$ & $\mathrm{CG}$ & $\mathrm{CC}$ & GG \\
\hline $\mathrm{CG}$ & CG & CG & GG & $\mathrm{CC}$ & GG & GG & $\mathrm{CC}$ & CG & CG & \\
\hline GDsnp01035 & 1 & \multicolumn{2}{|c|}{23879768} & GG & GG & $\mathrm{AG}$ & GG & $\mathrm{AG}$ & $\mathrm{AG}$ & $\mathrm{AG}$ \\
\hline $\mathrm{AG}$ & $\mathrm{AG}$ & GG & $\mathrm{AG}$ & $\mathrm{AG}$ & $\mathrm{AG}$ & $\mathrm{AG}$ & GG & GG & $\mathrm{AG}$ & GG \\
\hline $\mathrm{AG}$ & $\mathrm{AG}$ & GG & $\mathrm{AG}$ & GG & GG & $\mathrm{AG}$ & GG & GG & GG & GG \\
\hline GG & GG & $\mathrm{AG}$ & GG & $\mathrm{AG}$ & $\mathrm{AG}$ & $\mathrm{AG}$ & GG & GG & $\mathrm{AG}$ & GG \\
\hline GG & $\mathrm{AG}$ & GG & GG & $\mathrm{AG}$ & $\mathrm{AG}$ & GG & GG & $\mathrm{AG}$ & $\mathrm{AG}$ & $\mathrm{AG}$ \\
\hline $\mathrm{AG}$ & GG & GG & GG & $\mathrm{AG}$ & GG & GG & GG & $\mathrm{AG}$ & GG & GG \\
\hline $\mathrm{AG}$ & GG & GG & GG & GG & $\mathrm{AG}$ & $\mathrm{AG}$ & $\mathrm{AG}$ & GG & GG & GG \\
\hline $\mathrm{AG}$ & $\mathrm{AG}$ & $\mathrm{AG}$ & GG & GG & GG & - & $\mathrm{AG}$ & GG & GG & $\mathrm{AG}$ \\
\hline AG & $\mathrm{AG}$ & GG & $\mathrm{AG}$ & GG & GG & $\mathrm{AG}$ & GG & $\mathrm{AG}$ & $\mathrm{AG}$ & \\
\hline GDsnp00782 & 1 & \multicolumn{2}{|c|}{24113589} & GG & GG & $\mathrm{AG}$ & GG & $\mathrm{AG}$ & $\mathrm{AG}$ & $\mathrm{AG}$ \\
\hline $\mathrm{AG}$ & $\mathrm{AG}$ & GG & GG & $\mathrm{AG}$ & $\mathrm{AG}$ & $\mathrm{AG}$ & GG & GG & $\mathrm{AG}$ & GG \\
\hline $\mathrm{AG}$ & $\mathrm{AG}$ & GG & $\mathrm{AG}$ & GG & GG & GG & GG & GG & GG & GG \\
\hline GG & GG & $\mathrm{AG}$ & GG & $\mathrm{AG}$ & $\mathrm{AG}$ & $\mathrm{AG}$ & GG & GG & $\mathrm{AG}$ & $\mathrm{AG}$ \\
\hline GG & $\mathrm{AG}$ & GG & $\mathrm{AG}$ & $\mathrm{AG}$ & $\mathrm{AG}$ & GG & GG & $\mathrm{AG}$ & $\mathrm{AG}$ & $\mathrm{AG}$ \\
\hline $\mathrm{AG}$ & GG & GG & $\mathrm{AG}$ & $\mathrm{AG}$ & GG & GG & GG & $\mathrm{AG}$ & GG & GG \\
\hline $\mathrm{AG}$ & GG & GG & GG & GG & $\mathrm{AG}$ & $\mathrm{AG}$ & GG & GG & GG & GG \\
\hline $\mathrm{AG}$ & $\mathrm{AG}$ & $\mathrm{AG}$ & $\mathrm{GG}$ & $\mathrm{GG}$ & GG & GG & $\mathrm{AG}$ & GG & GG & $\mathrm{AG}$ \\
\hline $\mathrm{AG}$ & $\mathrm{AG}$ & GG & $\mathrm{AG}$ & $\mathrm{GG}$ & GG & $\mathrm{AG}$ & GG & $\mathrm{AG}$ & $\mathrm{AG}$ & \\
\hline
\end{tabular}


Supplemental Table 2. Continued.

\begin{tabular}{|c|c|c|c|c|c|c|c|c|c|c|}
\hline \multirow{3}{*}{$\begin{array}{l}\text { SNP name } \\
\text { GDsnp01257 } \\
\text { AA }\end{array}$} & \multicolumn{10}{|c|}{ Linkage group Physical location (bp) Individuals } \\
\hline & 1 & \multicolumn{2}{|c|}{27026185} & AA & $\mathrm{AA}$ & AT & AT & AT & AT & $\mathrm{AA}$ \\
\hline & TT & TT & AT & AA & AA & AA & AT & $\mathrm{TT}$ & AT & AT \\
\hline AA & AT & TT & AA & $\mathrm{AT}$ & TT & AT & $\mathrm{TT}$ & TT & AT & $\mathrm{TT}$ \\
\hline AT & AT & AT & $\mathrm{AT}$ & $\mathrm{TT}$ & AT & AT & AT & AT & $\mathrm{TT}$ & AT \\
\hline $\mathrm{AT}$ & $\mathrm{AA}$ & $\mathrm{AT}$ & $\mathrm{TT}$ & $\mathrm{TT}$ & $\mathrm{AT}$ & $\mathrm{TT}$ & $\mathrm{AT}$ & AT & AT & AT \\
\hline AA & $\mathrm{AT}$ & $\mathrm{AT}$ & $\mathrm{AT}$ & AT & $\mathrm{AA}$ & $\mathrm{AT}$ & $\mathrm{TT}$ & $\mathrm{AT}$ & $\mathrm{AA}$ & AT \\
\hline AA & $\mathrm{TT}$ & $\mathrm{AT}$ & $\mathrm{AA}$ & $\mathrm{AT}$ & $\mathrm{AT}$ & $\mathrm{AA}$ & $\mathrm{AA}$ & $\mathrm{AT}$ & $\mathrm{AA}$ & AT \\
\hline AA & $\mathrm{AT}$ & TT & $\mathrm{AA}$ & $\mathrm{AT}$ & $\mathrm{AT}$ & $\mathrm{AT}$ & $\mathrm{AT}$ & $\mathrm{AT}$ & $\mathrm{TT}$ & AT \\
\hline AT & $\mathrm{AT}$ & $\mathrm{AT}$ & $\mathrm{AA}$ & $\mathrm{TT}$ & $\mathrm{AT}$ & $\mathrm{AT}$ & $\mathrm{TT}$ & $\mathrm{AT}$ & $\mathrm{AT}$ & \\
\hline GDsnp01678 & 1 & \multicolumn{2}{|c|}{27529888} & GG & GG & AA & $\mathrm{AG}$ & $\mathrm{AA}$ & $\mathrm{AA}$ & GG \\
\hline AG & AA & AA & - & $\mathrm{AG}$ & $\mathrm{AG}$ & $\mathrm{AG}$ & $\mathrm{AG}$ & $\mathrm{AA}$ & - & $\mathrm{AG}$ \\
\hline AA & $\mathrm{AG}$ & $\mathrm{AA}$ & $\mathrm{AG}$ & $\mathrm{AG}$ & $\mathrm{AA}$ & $\mathrm{AA}$ & AA & $\mathrm{AA}$ & $\mathrm{AG}$ & $\mathrm{AA}$ \\
\hline$A G$ & $\mathrm{AG}$ & - & $\mathrm{AG}$ & - & $\mathrm{AG}$ & AA & $\mathrm{AG}$ & $\mathrm{AG}$ & $\mathrm{AA}$ & $\mathrm{AA}$ \\
\hline $\mathrm{AG}$ & $\mathrm{AG}$ & $\mathrm{AG}$ & $\mathrm{AA}$ & - & $\mathrm{AA}$ & - & $\mathrm{AG}$ & $\mathrm{AG}$ & - & $\mathrm{AG}$ \\
\hline $\mathrm{AG}$ & $\mathrm{AG}$ & $\mathrm{AG}$ & AA & AA & GG & $\mathrm{AG}$ & AA & $\mathrm{AG}$ & $\mathrm{AG}$ & $\mathrm{AG}$ \\
\hline $\mathrm{AG}$ & $\mathrm{AG}$ & $\mathrm{AG}$ & GG & $\mathrm{AG}$ & $\mathrm{AG}$ & GG & $\mathrm{AG}$ & $\mathrm{AG}$ & GG & $\mathrm{AA}$ \\
\hline GG & AA & $\mathrm{AA}$ & GG & $\mathrm{AG}$ & $\mathrm{AG}$ & $\mathrm{AG}$ & AA & $\mathrm{AG}$ & $\mathrm{AA}$ & $\mathrm{AA}$ \\
\hline AA & AA & $\mathrm{AG}$ & $\mathrm{AG}$ & AA & $\mathrm{AG}$ & $\mathrm{AG}$ & AA & AA & - & \\
\hline GDsnp00575 & 1 & \multicolumn{2}{|c|}{27682472} & GG & GG & $\mathrm{AA}$ & $\mathrm{AG}$ & $\mathrm{AG}$ & $\mathrm{AG}$ & GG \\
\hline $\mathrm{AG}$ & AA & AA & $\mathrm{AG}$ & GG & $\mathrm{AG}$ & $\mathrm{AG}$ & $\mathrm{AG}$ & AA & $\mathrm{AG}$ & $\mathrm{AG}$ \\
\hline AA & $\mathrm{AG}$ & $\mathrm{AA}$ & $\mathrm{AG}$ & $\mathrm{AG}$ & $\mathrm{AA}$ & $\mathrm{AG}$ & $\mathrm{AA}$ & $\mathrm{AA}$ & $\mathrm{AG}$ & $\mathrm{AA}$ \\
\hline $\mathrm{AG}$ & $\mathrm{AG}$ & $\mathrm{AG}$ & $\mathrm{AG}$ & AA & $\mathrm{AG}$ & AA & $\mathrm{AG}$ & $\mathrm{AG}$ & $\mathrm{AA}$ & AA \\
\hline $\mathrm{AG}$ & GG & $\mathrm{AG}$ & $\mathrm{AA}$ & AA & $\mathrm{AG}$ & $\mathrm{AA}$ & $\mathrm{AG}$ & $\mathrm{AG}$ & $\mathrm{AG}$ & $\mathrm{AG}$ \\
\hline AA & $\mathrm{AG}$ & $\mathrm{AG}$ & $\mathrm{AG}$ & AA & GG & $\mathrm{AG}$ & AA & $\mathrm{AG}$ & $\mathrm{AG}$ & $\mathrm{AG}$ \\
\hline AG & $\mathrm{AG}$ & $\mathrm{AG}$ & GG & $\mathrm{AG}$ & $\mathrm{AG}$ & GG & GG & $\mathrm{AG}$ & GG & AA \\
\hline GG & $\mathrm{AG}$ & AA & GG & $\mathrm{AG}$ & $\mathrm{AG}$ & $\mathrm{AG}$ & $\mathrm{AG}$ & $\mathrm{AG}$ & $\mathrm{AA}$ & $\mathrm{AG}$ \\
\hline AG & AA & $\mathrm{AG}$ & $\mathrm{AG}$ & AA & $\mathrm{AG}$ & $\mathrm{AG}$ & $\mathrm{AA}$ & $\mathrm{AG}$ & $\mathrm{AA}$ & \\
\hline GDsnp02371 & 1 & \multicolumn{2}{|c|}{27787322} & AA & $\mathrm{AA}$ & $\mathrm{AG}$ & $\mathrm{AG}$ & $\mathrm{AG}$ & $\mathrm{AG}$ & $\mathrm{AA}$ \\
\hline $\mathrm{AA}$ & $\mathrm{AG}$ & GG & $\mathrm{AA}$ & AA & $\mathrm{AA}$ & $\mathrm{AA}$ & $\mathrm{AG}$ & GG & $\mathrm{AG}$ & $\mathrm{AG}$ \\
\hline $\mathrm{AG}$ & $\mathrm{AG}$ & $\mathrm{AG}$ & $\mathrm{AA}$ & $\mathrm{AG}$ & GG & $\mathrm{AG}$ & GG & GG & $\mathrm{AG}$ & GG \\
\hline $\mathrm{AG}$ & $\mathrm{AG}$ & $\mathrm{AG}$ & $\mathrm{AG}$ & GG & $\mathrm{AG}$ & $\mathrm{AG}$ & $\mathrm{AG}$ & $\mathrm{AG}$ & GG & GG \\
\hline $\mathrm{AG}$ & AA & $\mathrm{AG}$ & GG & GG & $\mathrm{AG}$ & GG & $\mathrm{AG}$ & $\mathrm{AG}$ & $\mathrm{AG}$ & $\mathrm{AG}$ \\
\hline AG & $\mathrm{AG}$ & $\mathrm{AG}$ & $\mathrm{AG}$ & $\mathrm{AG}$ & $\mathrm{AA}$ & $\mathrm{AG}$ & GG & $\mathrm{AG}$ & $\mathrm{AA}$ & $\mathrm{AG}$ \\
\hline AA & $\mathrm{AG}$ & $\mathrm{AG}$ & $\mathrm{AA}$ & $\mathrm{AG}$ & $\mathrm{AG}$ & AA & AA & $\mathrm{AG}$ & $\mathrm{AA}$ & $\mathrm{AG}$ \\
\hline AA & $\mathrm{AG}$ & GG & AA & $\mathrm{AG}$ & $\mathrm{AG}$ & $\mathrm{AG}$ & $\mathrm{AG}$ & $\mathrm{AG}$ & GG & $\mathrm{AG}$ \\
\hline $\mathrm{AG}$ & $\mathrm{AG}$ & $\mathrm{AG}$ & $\mathrm{AA}$ & GG & $\mathrm{AG}$ & $\mathrm{AG}$ & GG & $\mathrm{AG}$ & $\mathrm{AG}$ & \\
\hline GDsnp00348 & 2 & \multicolumn{2}{|c|}{1260526} & GG & GG & GG & GG & GG & GG & GG \\
\hline GG & GG & GG & GG & GG & GG & GG & GG & GG & GG & GG \\
\hline GG & GG & GG & GG & GG & GG & - & GG & GG & GG & GG \\
\hline GG & GG & GG & GG & GG & GG & GG & GG & GG & GG & GG \\
\hline GG & GG & GG & GG & GG & GG & GG & GG & GG & GG & GG \\
\hline GG & GG & GG & GG & GG & GG & GG & GG & GG & GG & GG \\
\hline GG & GG & GG & GG & GG & GG & GG & GG & GG & GG & GG \\
\hline GG & GG & GG & GG & GG & GG & GG & GG & GG & GG & GG \\
\hline GG & GG & GG & GG & GG & GG & GG & GG & GG & GG & \\
\hline GDsnp01134 & 2 & \multicolumn{2}{|c|}{1273219} & AA & $\mathrm{AC}$ & $\mathrm{AA}$ & $\mathrm{AA}$ & $\mathrm{AA}$ & $\mathrm{AC}$ & $\mathrm{AC}$ \\
\hline $\mathrm{AC}$ & $\mathrm{AC}$ & $\mathrm{AC}$ & $\mathrm{AA}$ & $\mathrm{AC}$ & $\mathrm{AA}$ & $\mathrm{AC}$ & AA & $\mathrm{CC}$ & $\mathrm{AC}$ & $\mathrm{CC}$ \\
\hline AA & $\mathrm{CC}$ & $\mathrm{AC}$ & AA & AA & $\mathrm{AA}$ & AA & $\mathrm{AC}$ & $\mathrm{AC}$ & $\mathrm{AC}$ & $\mathrm{AC}$ \\
\hline $\mathrm{AC}$ & $\mathrm{AC}$ & $\mathrm{CC}$ & $\mathrm{AC}$ & $\mathrm{AC}$ & $\mathrm{AC}$ & $\mathrm{AC}$ & $\mathrm{CC}$ & $\mathrm{AC}$ & $\mathrm{AC}$ & AA \\
\hline $\mathrm{AC}$ & $\mathrm{CC}$ & $\mathrm{AC}$ & $\mathrm{AC}$ & $\mathrm{CC}$ & $\mathrm{AC}$ & $\mathrm{CC}$ & $\mathrm{CC}$ & $\mathrm{CC}$ & $\mathrm{CC}$ & $\mathrm{AC}$ \\
\hline AA & $\mathrm{AC}$ & $\mathrm{CC}$ & $\mathrm{AA}$ & $\mathrm{AC}$ & $\mathrm{AC}$ & $\mathrm{CC}$ & $\mathrm{AC}$ & $\mathrm{AC}$ & $\mathrm{CC}$ & $\mathrm{AA}$ \\
\hline $\mathrm{AC}$ & $\mathrm{AC}$ & $\mathrm{AC}$ & $\mathrm{CC}$ & $\mathrm{AC}$ & $\mathrm{AC}$ & $\mathrm{AC}$ & $\mathrm{AA}$ & $\mathrm{AA}$ & $\mathrm{AC}$ & $\mathrm{AC}$ \\
\hline $\mathrm{AC}$ & $\mathrm{AC}$ & $\mathrm{AC}$ & $\mathrm{AC}$ & $\mathrm{AA}$ & $\mathrm{AA}$ & $\mathrm{CC}$ & $\mathrm{AA}$ & $\mathrm{AA}$ & $\mathrm{AA}$ & $\mathrm{AC}$ \\
\hline $\mathrm{AC}$ & $\mathrm{AC}$ & AA & $\mathrm{AC}$ & AA & $\mathrm{AC}$ & $\mathrm{CC}$ & $\mathrm{AC}$ & $\mathrm{AC}$ & $\mathrm{AA}$ & \\
\hline
\end{tabular}


Supplemental Table 2. Continued.

\begin{tabular}{|c|c|c|c|c|c|c|c|c|c|c|}
\hline \multirow{3}{*}{$\begin{array}{l}\text { SNP name } \\
\text { GDsnp00035 } \\
\text { AG }\end{array}$} & \multicolumn{10}{|c|}{ Linkage group Physical location (bp) Individuals } \\
\hline & 2 & \multicolumn{2}{|c|}{3267912} & $\mathrm{AG}$ & GG & GG & $\mathrm{AG}$ & GG & GG & GG \\
\hline & $\mathrm{AG}$ & GG & GG & GG & GG & GG & GG & $\mathrm{AG}$ & GG & GG \\
\hline GG & GG & GG & GG & GG & GG & $\mathrm{AG}$ & GG & GG & GG & GG \\
\hline AG & GG & GG & GG & GG & GG & GG & GG & GG & GG & GG \\
\hline GG & GG & GG & GG & GG & $\mathrm{AG}$ & GG & GG & GG & GG & GG \\
\hline GG & GG & GG & $\mathrm{AG}$ & GG & GG & GG & GG & GG & GG & GG \\
\hline GG & GG & GG & GG & GG & GG & $\mathrm{AG}$ & GG & GG & GG & GG \\
\hline GG & GG & GG & GG & GG & GG & GG & GG & GG & GG & $\mathrm{AG}$ \\
\hline GG & GG & GG & GG & GG & GG & GG & GG & GG & GG & \\
\hline GDsnp00203 & 2 & \multicolumn{2}{|c|}{4208368} & GG & GG & GG & $\mathrm{AG}$ & GG & GG & GG \\
\hline $\mathrm{AG}$ & GG & GG & GG & GG & GG & GG & GG & GG & $\mathrm{AG}$ & GG \\
\hline GG & $\mathrm{AG}$ & GG & GG & GG & $\mathrm{AG}$ & $\mathrm{AG}$ & $\mathrm{AG}$ & $\mathrm{AG}$ & $\mathrm{AG}$ & $\mathrm{AG}$ \\
\hline $\mathrm{AG}$ & $\mathrm{AG}$ & GG & GG & $\mathrm{AG}$ & GG & GG & GG & GG & $\mathrm{AG}$ & GG \\
\hline GG & $\mathrm{AG}$ & GG & GG & $\mathrm{AG}$ & $\mathrm{AG}$ & $\mathrm{AG}$ & $\mathrm{AG}$ & GG & $\mathrm{AG}$ & GG \\
\hline GG & GG & $\mathrm{AG}$ & $\mathrm{AG}$ & GG & GG & $\mathrm{AG}$ & GG & $\mathrm{AG}$ & $\mathrm{AG}$ & GG \\
\hline GG & GG & GG & $\mathrm{AG}$ & GG & GG & $\mathrm{AG}$ & GG & GG & GG & GG \\
\hline$A G$ & $\mathrm{AG}$ & GG & GG & $\mathrm{AG}$ & GG & $\mathrm{AG}$ & GG & GG & GG & GG \\
\hline$A G$ & GG & GG & GG & $\mathrm{AG}$ & $\mathrm{AG}$ & GG & GG & GG & GG & \\
\hline GDsnp00243 & 2 & \multicolumn{2}{|c|}{5554390} & GG & GG & GG & GG & GG & GG & GG \\
\hline GG & GG & GG & GG & GG & GG & GG & GG & GG & GG & GG \\
\hline GG & GG & GG & GG & GG & GG & $\mathrm{CG}$ & GG & GG & GG & GG \\
\hline GG & GG & GG & GG & GG & GG & GG & GG & GG & GG & GG \\
\hline GG & GG & GG & GG & GG & GG & GG & $\mathrm{CG}$ & GG & GG & GG \\
\hline GG & GG & GG & GG & GG & GG & GG & GG & GG & GG & GG \\
\hline GG & GG & GG & GG & GG & GG & GG & GG & GG & GG & GG \\
\hline GG & GG & GG & GG & GG & GG & GG & GG & GG & GG & GG \\
\hline GG & GG & GG & GG & GG & GG & GG & GG & GG & GG & \\
\hline GDsnp00308 & 2 & \multicolumn{2}{|c|}{6305739} & $\mathrm{AA}$ & $\mathrm{AA}$ & AA & AA & AA & AA & AA \\
\hline- & $\mathrm{AG}$ & $\mathrm{AG}$ & $\mathrm{AG}$ & AA & $\mathrm{AA}$ & AA & AA & AA & $\mathrm{AG}$ & $\mathrm{AA}$ \\
\hline AA & $\mathrm{AG}$ & $\mathrm{AA}$ & $\mathrm{AA}$ & AA & $\mathrm{AG}$ & $\mathrm{AG}$ & $\mathrm{AG}$ & $\mathrm{AG}$ & $\mathrm{AG}$ & $\mathrm{AG}$ \\
\hline AA & AA & AA & $\mathrm{AA}$ & $\mathrm{AG}$ & $\mathrm{AA}$ & $\mathrm{AA}$ & AA & $\mathrm{AA}$ & $\mathrm{AA}$ & $\mathrm{AA}$ \\
\hline AA & $\mathrm{AG}$ & $\mathrm{AA}$ & $\mathrm{AA}$ & AA & $\mathrm{AG}$ & $\mathrm{AG}$ & $\mathrm{AG}$ & $\mathrm{AA}$ & $\mathrm{AG}$ & AA \\
\hline AA & $\mathrm{AA}$ & $\mathrm{AA}$ & $\mathrm{AA}$ & AA & $\mathrm{AA}$ & $\mathrm{AG}$ & $\mathrm{AG}$ & $\mathrm{AG}$ & $\mathrm{AG}$ & $\mathrm{AA}$ \\
\hline AA & $\mathrm{AA}$ & $\mathrm{AA}$ & $\mathrm{AA}$ & AA & $\mathrm{AA}$ & AA & $\mathrm{AG}$ & $\mathrm{AA}$ & $\mathrm{AA}$ & $\mathrm{AA}$ \\
\hline AG & $\mathrm{AA}$ & AA & $\mathrm{AA}$ & $\mathrm{AG}$ & $\mathrm{AA}$ & $\mathrm{AG}$ & $\mathrm{AA}$ & $\mathrm{AA}$ & $\mathrm{AA}$ & AA \\
\hline $\mathrm{AG}$ & AA & AA & AA & $\mathrm{AG}$ & $\mathrm{AG}$ & $\mathrm{AA}$ & $\mathrm{AG}$ & $\mathrm{AA}$ & $\mathrm{AA}$ & \\
\hline GDsnp01162 & 2 & \multicolumn{2}{|c|}{6510261} & $\mathrm{CC}$ & $\mathrm{CC}$ & $\mathrm{CC}$ & $\mathrm{CC}$ & $\mathrm{CC}$ & $\mathrm{CC}$ & $\mathrm{CC}$ \\
\hline $\mathrm{AC}$ & $\mathrm{CC}$ & $\mathrm{AC}$ & $\mathrm{AC}$ & $\mathrm{CC}$ & $\mathrm{CC}$ & $\mathrm{CC}$ & $\mathrm{CC}$ & $\mathrm{CC}$ & $\mathrm{CC}$ & $\mathrm{CC}$ \\
\hline $\mathrm{CC}$ & $\mathrm{CC}$ & $\mathrm{CC}$ & $\mathrm{CC}$ & $\mathrm{CC}$ & $\mathrm{CC}$ & $\mathrm{AC}$ & $\mathrm{CC}$ & $\mathrm{CC}$ & $\mathrm{CC}$ & $\mathrm{CC}$ \\
\hline $\mathrm{CC}$ & $\mathrm{CC}$ & $\mathrm{CC}$ & $\mathrm{CC}$ & $\mathrm{CC}$ & $\mathrm{CC}$ & $\mathrm{CC}$ & $\mathrm{CC}$ & $\mathrm{CC}$ & $\mathrm{AC}$ & $\mathrm{CC}$ \\
\hline $\mathrm{CC}$ & $\mathrm{CC}$ & $\mathrm{CC}$ & $\mathrm{CC}$ & $\mathrm{CC}$ & $\mathrm{CC}$ & - & $\mathrm{CC}$ & $\mathrm{CC}$ & $\mathrm{CC}$ & $\mathrm{CC}$ \\
\hline $\mathrm{CC}$ & $\mathrm{CC}$ & $\mathrm{CC}$ & $\mathrm{CC}$ & $\mathrm{CC}$ & $\mathrm{CC}$ & $\mathrm{CC}$ & $\mathrm{CC}$ & $\mathrm{CC}$ & $\mathrm{CC}$ & $\mathrm{CC}$ \\
\hline $\mathrm{CC}$ & $\mathrm{CC}$ & $\mathrm{CC}$ & $\mathrm{CC}$ & $\mathrm{CC}$ & $\mathrm{CC}$ & $\mathrm{CC}$ & $\mathrm{AC}$ & $\mathrm{CC}$ & $\mathrm{CC}$ & $\mathrm{CC}$ \\
\hline $\mathrm{CC}$ & $\mathrm{CC}$ & $\mathrm{CC}$ & $\mathrm{CC}$ & $\mathrm{CC}$ & $\mathrm{CC}$ & $\mathrm{CC}$ & $\mathrm{CC}$ & $\mathrm{CC}$ & $\mathrm{CC}$ & $\mathrm{CC}$ \\
\hline $\mathrm{CC}$ & $\mathrm{CC}$ & $\mathrm{CC}$ & $\mathrm{CC}$ & $\mathrm{CC}$ & $\mathrm{CC}$ & $\mathrm{CC}$ & $\mathrm{CC}$ & $\mathrm{CC}$ & $\mathrm{CC}$ & \\
\hline GDsnp00333 & 2 & \multicolumn{2}{|c|}{6617211} & $\mathrm{CC}$ & $\mathrm{CC}$ & $\mathrm{CC}$ & $\mathrm{CC}$ & $\mathrm{CC}$ & $\mathrm{CC}$ & $\mathrm{CC}$ \\
\hline $\mathrm{CC}$ & $\mathrm{CC}$ & $\mathrm{CC}$ & $\mathrm{CC}$ & $\mathrm{CC}$ & $\mathrm{CC}$ & $\mathrm{CC}$ & $\mathrm{CC}$ & $\mathrm{CC}$ & $\mathrm{CC}$ & $\mathrm{CC}$ \\
\hline $\mathrm{CC}$ & $\mathrm{CC}$ & $\mathrm{CC}$ & $\mathrm{CC}$ & $\mathrm{CC}$ & $\mathrm{CC}$ & $\mathrm{AC}$ & $\mathrm{CC}$ & $\mathrm{CC}$ & $\mathrm{CC}$ & $\mathrm{CC}$ \\
\hline $\mathrm{CC}$ & $\mathrm{CC}$ & $\mathrm{CC}$ & $\mathrm{CC}$ & $\mathrm{CC}$ & $\mathrm{CC}$ & $\mathrm{CC}$ & $\mathrm{CC}$ & $\mathrm{CC}$ & $\mathrm{AC}$ & $\mathrm{CC}$ \\
\hline $\mathrm{CC}$ & $\mathrm{CC}$ & $\mathrm{CC}$ & $\mathrm{CC}$ & $\mathrm{CC}$ & $\mathrm{CC}$ & $\mathrm{CC}$ & $\mathrm{CC}$ & $\mathrm{CC}$ & $\mathrm{CC}$ & $\mathrm{CC}$ \\
\hline $\mathrm{CC}$ & $\mathrm{CC}$ & $\mathrm{CC}$ & $\mathrm{CC}$ & $\mathrm{CC}$ & $\mathrm{CC}$ & $\mathrm{CC}$ & $\mathrm{CC}$ & $\mathrm{CC}$ & $\mathrm{CC}$ & $\mathrm{CC}$ \\
\hline $\mathrm{CC}$ & $\mathrm{CC}$ & $\mathrm{CC}$ & $\mathrm{CC}$ & $\mathrm{CC}$ & $\mathrm{CC}$ & $\mathrm{CC}$ & $\mathrm{CC}$ & $\mathrm{CC}$ & $\mathrm{CC}$ & $\mathrm{CC}$ \\
\hline $\mathrm{CC}$ & $\mathrm{CC}$ & $\mathrm{CC}$ & $\mathrm{CC}$ & $\mathrm{CC}$ & $\mathrm{CC}$ & $\mathrm{CC}$ & $\mathrm{CC}$ & $\mathrm{CC}$ & $\mathrm{CC}$ & $\mathrm{CC}$ \\
\hline $\mathrm{CC}$ & $\mathrm{CC}$ & $\mathrm{CC}$ & $\mathrm{CC}$ & $\mathrm{CC}$ & $\mathrm{CC}$ & $\mathrm{CC}$ & $\mathrm{CC}$ & $\mathrm{CC}$ & $\mathrm{CC}$ & \\
\hline
\end{tabular}


Supplemental Table 2. Continued.

\begin{tabular}{|c|c|c|c|c|c|c|c|c|c|c|}
\hline \multirow{3}{*}{$\begin{array}{l}\text { SNP name } \\
\text { GDsnp00366 } \\
\text { CC }\end{array}$} & \multicolumn{10}{|c|}{ Linkage group Physical location (bp) Individuals } \\
\hline & 2 & \multicolumn{2}{|c|}{7138600} & $\mathrm{CC}$ & $\mathrm{CC}$ & $\mathrm{CC}$ & $\mathrm{CC}$ & $\mathrm{CC}$ & $\mathrm{CC}$ & $\mathrm{CC}$ \\
\hline & $\mathrm{AC}$ & $\mathrm{CC}$ & $\mathrm{CC}$ & $\mathrm{CC}$ & $\mathrm{CC}$ & $\mathrm{CC}$ & $\mathrm{CC}$ & $\mathrm{CC}$ & $\mathrm{CC}$ & $\mathrm{CC}$ \\
\hline $\mathrm{CC}$ & $\mathrm{CC}$ & $\mathrm{CC}$ & $\mathrm{CC}$ & $\mathrm{CC}$ & $\mathrm{CC}$ & $\mathrm{AC}$ & $\mathrm{CC}$ & $\mathrm{CC}$ & $\mathrm{CC}$ & $\mathrm{CC}$ \\
\hline $\mathrm{CC}$ & $\mathrm{CC}$ & $\mathrm{CC}$ & $\mathrm{CC}$ & $\mathrm{CC}$ & $\mathrm{CC}$ & $\mathrm{CC}$ & $\mathrm{CC}$ & $\mathrm{CC}$ & $\mathrm{AC}$ & $\mathrm{CC}$ \\
\hline $\mathrm{CC}$ & $\mathrm{CC}$ & $\mathrm{CC}$ & $\mathrm{CC}$ & $\mathrm{CC}$ & $\mathrm{CC}$ & $\mathrm{CC}$ & $\mathrm{AC}$ & $\mathrm{CC}$ & $\mathrm{CC}$ & $\mathrm{CC}$ \\
\hline $\mathrm{CC}$ & $\mathrm{CC}$ & $\mathrm{CC}$ & $\mathrm{CC}$ & $\mathrm{CC}$ & $\mathrm{CC}$ & $\mathrm{CC}$ & $\mathrm{CC}$ & $\mathrm{CC}$ & $\mathrm{CC}$ & $\mathrm{CC}$ \\
\hline $\mathrm{CC}$ & $\mathrm{CC}$ & $\mathrm{CC}$ & $\mathrm{CC}$ & $\mathrm{CC}$ & $\mathrm{CC}$ & $\mathrm{CC}$ & $\mathrm{CC}$ & $\mathrm{CC}$ & $\mathrm{CC}$ & $\mathrm{CC}$ \\
\hline $\mathrm{CC}$ & $\mathrm{CC}$ & $\mathrm{CC}$ & $\mathrm{CC}$ & $\mathrm{CC}$ & $\mathrm{CC}$ & $\mathrm{CC}$ & $\mathrm{CC}$ & $\mathrm{CC}$ & $\mathrm{CC}$ & $\mathrm{CC}$ \\
\hline $\mathrm{CC}$ & $\mathrm{CC}$ & $\mathrm{CC}$ & $\mathrm{CC}$ & $\mathrm{CC}$ & $\mathrm{CC}$ & $\mathrm{CC}$ & $\mathrm{CC}$ & $\mathrm{CC}$ & $\mathrm{CC}$ & \\
\hline GDsnp00289 & 2 & \multicolumn{2}{|c|}{7821054} & $\mathrm{TT}$ & $\mathrm{AT}$ & $\mathrm{AT}$ & $\mathrm{AT}$ & $\mathrm{AT}$ & AT & TT \\
\hline AT & $\mathrm{TT}$ & AT & $\mathrm{TT}$ & $\mathrm{AT}$ & $\mathrm{AT}$ & $\mathrm{AT}$ & $\mathrm{AT}$ & AA & $\mathrm{TT}$ & TT \\
\hline $\mathrm{TT}$ & $\mathrm{AT}$ & AA & $\mathrm{AT}$ & $\mathrm{TT}$ & $\mathrm{TT}$ & $\mathrm{AT}$ & $\mathrm{TT}$ & $\mathrm{TT}$ & $\mathrm{TT}$ & AT \\
\hline AA & $\mathrm{AT}$ & AA & $\mathrm{AT}$ & $\mathrm{TT}$ & $\mathrm{AA}$ & $\mathrm{AT}$ & $\mathrm{AT}$ & $\mathrm{AA}$ & AT & $\mathrm{AT}$ \\
\hline AT & $\mathrm{AT}$ & $\mathrm{TT}$ & $\mathrm{AT}$ & $\mathrm{TT}$ & $\mathrm{AT}$ & - & $\mathrm{AT}$ & $\mathrm{AA}$ & AT & AT \\
\hline AT & $\mathrm{AT}$ & AA & $\mathrm{AT}$ & AA & AT & $\mathrm{TT}$ & $\mathrm{AT}$ & $\mathrm{TT}$ & $\mathrm{AT}$ & AT \\
\hline AT & $\mathrm{AT}$ & AT & AA & $\mathrm{AT}$ & AT & $\mathrm{AT}$ & $\mathrm{AT}$ & $\mathrm{TT}$ & $\mathrm{AT}$ & AT \\
\hline TT & AA & $\mathrm{AT}$ & $\mathrm{TT}$ & $\mathrm{TT}$ & $\mathrm{TT}$ & $\mathrm{TT}$ & $\mathrm{TT}$ & $\mathrm{AT}$ & $\mathrm{TT}$ & AA \\
\hline TT & AA & $\mathrm{TT}$ & $\mathrm{AA}$ & $\mathrm{TT}$ & $\mathrm{TT}$ & $\mathrm{AA}$ & AT & $\mathrm{AT}$ & TT & \\
\hline GDsnp01223 & 2 & \multicolumn{2}{|c|}{10122263} & AA & $\mathrm{AG}$ & GG & $\mathrm{AG}$ & $\mathrm{AG}$ & $\mathrm{AA}$ & AA \\
\hline $\mathrm{AG}$ & AA & $\mathrm{AG}$ & $\mathrm{AA}$ & $\mathrm{AG}$ & $\mathrm{AG}$ & $\mathrm{AG}$ & $\mathrm{AG}$ & GG & $\mathrm{AG}$ & AA \\
\hline AA & GG & GG & $\mathrm{AG}$ & AA & $\mathrm{AG}$ & $\mathrm{AG}$ & $\mathrm{AG}$ & $\mathrm{AG}$ & $\mathrm{AG}$ & GG \\
\hline - & $\mathrm{AG}$ & $\mathrm{AG}$ & $\mathrm{AG}$ & $\mathrm{AG}$ & GG & $\mathrm{AG}$ & $\mathrm{AG}$ & GG & $\mathrm{AG}$ & $\mathrm{AG}$ \\
\hline $\mathrm{AG}$ & GG & AA & $\mathrm{AG}$ & $\mathrm{AG}$ & $\mathrm{AG}$ & - & $\mathrm{AG}$ & GG & GG & $\mathrm{AG}$ \\
\hline $\mathrm{AG}$ & $\mathrm{AG}$ & GG & $\mathrm{AG}$ & GG & $\mathrm{AG}$ & $\mathrm{AG}$ & GG & $\mathrm{AG}$ & GG & $\mathrm{AG}$ \\
\hline $\mathrm{AG}$ & $\mathrm{AG}$ & $\mathrm{AG}$ & GG & $\mathrm{AG}$ & $\mathrm{AG}$ & $\mathrm{AG}$ & $\mathrm{AG}$ & $\mathrm{AA}$ & $\mathrm{AG}$ & $\mathrm{AG}$ \\
\hline $\mathrm{AG}$ & $\mathrm{AG}$ & $\mathrm{AG}$ & $\mathrm{AA}$ & $\mathrm{AG}$ & $\mathrm{AA}$ & $\mathrm{AG}$ & AA & $\mathrm{AG}$ & $\mathrm{AA}$ & GG \\
\hline $\mathrm{AG}$ & GG & AA & GG & $\mathrm{AG}$ & $\mathrm{AG}$ & GG & GG & $\mathrm{AG}$ & $\mathrm{AA}$ & \\
\hline GDsnp00159 & 2 & \multicolumn{2}{|c|}{10837102} & $\mathrm{AA}$ & $\mathrm{AA}$ & $\mathrm{AG}$ & AA & $\mathrm{AA}$ & $\mathrm{AA}$ & AA \\
\hline AA & AA & AA & AA & AA & $\mathrm{AG}$ & $\mathrm{AG}$ & AA & AA & $\mathrm{AA}$ & AA \\
\hline AA & AA & $\mathrm{AG}$ & $\mathrm{AG}$ & AA & AA & AA & AA & AA & $\mathrm{AA}$ & AA \\
\hline AG & $\mathrm{AG}$ & AA & $\mathrm{AA}$ & $\mathrm{AG}$ & $\mathrm{AG}$ & $\mathrm{AA}$ & AA & $\mathrm{AG}$ & $\mathrm{AA}$ & $\mathrm{AG}$ \\
\hline AA & AA & AA & AA & AA & AA & AA & AA & $\mathrm{AG}$ & $\mathrm{AA}$ & AA \\
\hline $\mathrm{AG}$ & AA & $\mathrm{AG}$ & AA & $\mathrm{AG}$ & $\mathrm{AG}$ & AA & AA & AA & $\mathrm{AA}$ & AA \\
\hline AA & AA & AA & $\mathrm{AG}$ & $\mathrm{AG}$ & $\mathrm{AG}$ & AA & AA & AA & $\mathrm{AG}$ & AA \\
\hline AA & AA & $\mathrm{AG}$ & $\mathrm{AA}$ & AA & $\mathrm{AA}$ & $\mathrm{AA}$ & AA & $\mathrm{AA}$ & $\mathrm{AA}$ & $\mathrm{AG}$ \\
\hline AA & $\mathrm{AG}$ & AA & $\mathrm{AG}$ & AA & AA & $\mathrm{AG}$ & AA & $\mathrm{AG}$ & $\mathrm{AA}$ & \\
\hline GDsnp00985 & 2 & \multicolumn{2}{|c|}{11416692} & GG & GG & GG & GG & GG & GG & GG \\
\hline GG & GG & GG & GG & GG & GG & GG & GG & GG & GG & GG \\
\hline GG & GG & GG & GG & GG & GG & GG & GG & GG & GG & GG \\
\hline GG & GG & GG & GG & GG & GG & GG & GG & GG & GG & GG \\
\hline GG & GG & GG & GG & GG & GG & GG & GG & GG & GG & GG \\
\hline GG & GG & GG & GG & GG & GG & GG & GG & GG & GG & GG \\
\hline GG & GG & GG & GG & GG & GG & GG & GG & GG & GG & GG \\
\hline GG & GG & GG & GG & GG & GG & GG & GG & GG & GG & GG \\
\hline GG & GG & GG & GG & GG & GG & GG & GG & GG & GG & \\
\hline GDsnp00214 & 2 & \multicolumn{2}{|c|}{12711977} & AA & $\mathrm{AA}$ & AA & AA & AA & $\mathrm{AC}$ & AA \\
\hline- & $\mathrm{AC}$ & AA & AA & AA & $\mathrm{AA}$ & $\mathrm{AA}$ & AA & $\mathrm{AA}$ & $\mathrm{AC}$ & AA \\
\hline AA & $\mathrm{AC}$ & AA & $\mathrm{AA}$ & $\mathrm{AA}$ & $\mathrm{AC}$ & $\mathrm{AC}$ & $\mathrm{AC}$ & $\mathrm{AC}$ & $\mathrm{AC}$ & $\mathrm{AC}$ \\
\hline AA & AA & AA & $\mathrm{AA}$ & AA & $\mathrm{AA}$ & $\mathrm{AA}$ & AA & $\mathrm{AA}$ & $\mathrm{AC}$ & AA \\
\hline AA & $\mathrm{AC}$ & AA & $\mathrm{AC}$ & $\mathrm{AC}$ & $\mathrm{AC}$ & $\mathrm{AC}$ & AA & $\mathrm{AA}$ & $\mathrm{AC}$ & $\mathrm{AC}$ \\
\hline AA & AA & $\mathrm{AC}$ & AA & AA & AA & $\mathrm{AC}$ & $\mathrm{AC}$ & $\mathrm{AC}$ & $\mathrm{AC}$ & AA \\
\hline AA & AA & AA & $\mathrm{AA}$ & AA & AA & $\mathrm{AA}$ & AA & $\mathrm{AA}$ & $\mathrm{AA}$ & AA \\
\hline $\mathrm{AC}$ & AA & AA & AA & $\mathrm{AC}$ & AA & $\mathrm{AC}$ & AA & - & $\mathrm{AA}$ & $\mathrm{AA}$ \\
\hline $\mathrm{AC}$ & AA & AA & AA & $\mathrm{AC}$ & AA & $\mathrm{AA}$ & $\mathrm{AC}$ & $\mathrm{AA}$ & $\mathrm{AA}$ & \\
\hline
\end{tabular}


Supplemental Table 2. Continued.

\begin{tabular}{|c|c|c|c|c|c|c|c|c|c|c|}
\hline \multirow{3}{*}{$\begin{array}{l}\text { SNP name } \\
\text { GDsnp00174 } \\
\text { GG }\end{array}$} & \multicolumn{10}{|c|}{ Linkage group Physical location (bp) Individuals } \\
\hline & 2 & \multicolumn{2}{|c|}{13284241} & GG & GG & GG & GG & GG & GG & GG \\
\hline & $\mathrm{AG}$ & GG & GG & GG & GG & GG & GG & GG & GG & GG \\
\hline GG & GG & GG & GG & GG & GG & AG & GG & GG & GG & GG \\
\hline GG & GG & GG & GG & GG & GG & GG & GG & GG & GG & GG \\
\hline GG & GG & GG & GG & GG & GG & GG & GG & GG & GG & GG \\
\hline GG & GG & GG & GG & GG & GG & GG & GG & GG & GG & GG \\
\hline GG & GG & GG & GG & GG & GG & GG & GG & GG & GG & GG \\
\hline GG & GG & GG & GG & GG & GG & GG & GG & GG & GG & GG \\
\hline GG & GG & GG & GG & GG & GG & GG & GG & GG & GG & \\
\hline GDsnp02144 & 2 & \multicolumn{2}{|c|}{14461169} & $\mathrm{CC}$ & $\mathrm{CC}$ & $\mathrm{CC}$ & $\mathrm{CC}$ & $\mathrm{CC}$ & $\mathrm{CC}$ & $\mathrm{CC}$ \\
\hline $\mathrm{CC}$ & $\mathrm{CG}$ & $\mathrm{CC}$ & $\mathrm{CC}$ & $\mathrm{CC}$ & $\mathrm{CC}$ & $\mathrm{CC}$ & $\mathrm{CC}$ & $\mathrm{CC}$ & $\mathrm{CC}$ & $\mathrm{CC}$ \\
\hline $\mathrm{CC}$ & $\mathrm{CC}$ & $\mathrm{CC}$ & $\mathrm{CC}$ & $\mathrm{CC}$ & $\mathrm{CC}$ & CG & $\mathrm{CC}$ & $\mathrm{CC}$ & $\mathrm{CC}$ & $\mathrm{CC}$ \\
\hline $\mathrm{CC}$ & $\mathrm{CC}$ & $\mathrm{CC}$ & $\mathrm{CC}$ & $\mathrm{CC}$ & $\mathrm{CC}$ & $\mathrm{CC}$ & $\mathrm{CC}$ & $\mathrm{CC}$ & $\mathrm{CC}$ & $\mathrm{CC}$ \\
\hline $\mathrm{CC}$ & $\mathrm{CC}$ & $\mathrm{CC}$ & $\mathrm{CC}$ & $\mathrm{CC}$ & $\mathrm{CC}$ & $\mathrm{CC}$ & $\mathrm{CC}$ & $\mathrm{CC}$ & $\mathrm{CC}$ & $\mathrm{CC}$ \\
\hline $\mathrm{CC}$ & $\mathrm{CC}$ & $\mathrm{CC}$ & $\mathrm{CC}$ & $\mathrm{CC}$ & $\mathrm{CC}$ & $\mathrm{CC}$ & $\mathrm{CC}$ & $\mathrm{CC}$ & $\mathrm{CC}$ & $\mathrm{CC}$ \\
\hline $\mathrm{CC}$ & $\mathrm{CC}$ & $\mathrm{CC}$ & $\mathrm{CC}$ & $\mathrm{CC}$ & $\mathrm{CC}$ & $\mathrm{CC}$ & $\mathrm{CC}$ & $\mathrm{CC}$ & $\mathrm{CC}$ & $\mathrm{CC}$ \\
\hline $\mathrm{CC}$ & $\mathrm{CC}$ & $\mathrm{CC}$ & $\mathrm{CC}$ & $\mathrm{CC}$ & $\mathrm{CC}$ & $\mathrm{CC}$ & $\mathrm{CC}$ & $\mathrm{CC}$ & $\mathrm{CC}$ & $\mathrm{CC}$ \\
\hline $\mathrm{CC}$ & $\mathrm{CC}$ & $\mathrm{CC}$ & $\mathrm{CC}$ & $\mathrm{CC}$ & $\mathrm{CC}$ & $\mathrm{CC}$ & $\mathrm{CC}$ & $\mathrm{CC}$ & $\mathrm{CC}$ & \\
\hline GDsnp00425 & 2 & \multicolumn{2}{|c|}{16326525} & $\mathrm{AC}$ & $\mathrm{CC}$ & $\mathrm{CC}$ & $\mathrm{CC}$ & $\mathrm{AC}$ & $\mathrm{AC}$ & $\mathrm{AA}$ \\
\hline $\mathrm{AC}$ & $\mathrm{AA}$ & $\mathrm{AC}$ & $\mathrm{AA}$ & $\mathrm{AC}$ & $\mathrm{CC}$ & $\mathrm{AC}$ & $\mathrm{AA}$ & $\mathrm{CC}$ & $\mathrm{AC}$ & $\mathrm{AA}$ \\
\hline AA & $\mathrm{CC}$ & $\mathrm{AC}$ & $\mathrm{AC}$ & AA & $\mathrm{AC}$ & $\mathrm{AC}$ & $\mathrm{AC}$ & $\mathrm{AC}$ & $\mathrm{AC}$ & $\mathrm{CC}$ \\
\hline $\mathrm{CC}$ & $\mathrm{AC}$ & $\mathrm{CC}$ & $\mathrm{AC}$ & $\mathrm{AC}$ & $\mathrm{CC}$ & $\mathrm{AC}$ & $\mathrm{AC}$ & $\mathrm{CC}$ & $\mathrm{AC}$ & $\mathrm{AC}$ \\
\hline AA & $\mathrm{CC}$ & $\mathrm{AC}$ & $\mathrm{AC}$ & $\mathrm{AC}$ & $\mathrm{CC}$ & $\mathrm{CC}$ & $\mathrm{CC}$ & $\mathrm{CC}$ & $\mathrm{CC}$ & $\mathrm{AC}$ \\
\hline $\mathrm{AC}$ & $\mathrm{AC}$ & $\mathrm{CC}$ & $\mathrm{AC}$ & $\mathrm{CC}$ & $\mathrm{AC}$ & $\mathrm{AC}$ & $\mathrm{CC}$ & $\mathrm{AC}$ & $\mathrm{CC}$ & $\mathrm{AC}$ \\
\hline $\mathrm{AC}$ & $\mathrm{AC}$ & $\mathrm{AC}$ & $\mathrm{CC}$ & $\mathrm{AC}$ & $\mathrm{CC}$ & $\mathrm{CC}$ & $\mathrm{AC}$ & $\mathrm{AA}$ & $\mathrm{AC}$ & $\mathrm{AC}$ \\
\hline $\mathrm{CC}$ & $\mathrm{CC}$ & $\mathrm{AC}$ & $\mathrm{AA}$ & $\mathrm{AC}$ & $\mathrm{AA}$ & $\mathrm{AC}$ & $\mathrm{AC}$ & $\mathrm{AC}$ & $\mathrm{AC}$ & $\mathrm{CC}$ \\
\hline $\mathrm{AC}$ & $\mathrm{CC}$ & $\mathrm{AC}$ & $\mathrm{AC}$ & $\mathrm{AC}$ & $\mathrm{AC}$ & $\mathrm{CC}$ & $\mathrm{AC}$ & $\mathrm{AC}$ & $\mathrm{AC}$ & \\
\hline GDsnp00934 & 2 & \multicolumn{2}{|c|}{17909427} & $\mathrm{AG}$ & $\mathrm{AG}$ & $\mathrm{AG}$ & $\mathrm{AG}$ & $\mathrm{AA}$ & GG & GG \\
\hline $\mathrm{AA}$ & $\mathrm{AG}$ & $\mathrm{AG}$ & $\mathrm{AG}$ & $\mathrm{AG}$ & GG & GG & $\mathrm{AG}$ & $\mathrm{AG}$ & GG & GG \\
\hline GG & $\mathrm{AA}$ & $\mathrm{AA}$ & GG & GG & $\mathrm{AG}$ & AA & $\mathrm{AG}$ & $\mathrm{AG}$ & $\mathrm{AG}$ & $\mathrm{AG}$ \\
\hline $\mathrm{AG}$ & GG & $\mathrm{AA}$ & $\mathrm{AG}$ & GG & $\mathrm{AG}$ & $A G$ & $\mathrm{AG}$ & $\mathrm{AG}$ & $\mathrm{AG}$ & GG \\
\hline GG & AA & $\mathrm{AG}$ & $\mathrm{AG}$ & $\mathrm{AG}$ & $\mathrm{AA}$ & AA & $\mathrm{AG}$ & $\mathrm{AG}$ & $\mathrm{AA}$ & $\mathrm{AG}$ \\
\hline GG & $\mathrm{AG}$ & $\mathrm{AA}$ & $\mathrm{AG}$ & $\mathrm{AG}$ & GG & $A G$ & AA & $\mathrm{AG}$ & $\mathrm{AA}$ & $\mathrm{AG}$ \\
\hline $\mathrm{AG}$ & $\mathrm{AG}$ & $\mathrm{AG}$ & $\mathrm{AG}$ & $\mathrm{AG}$ & $\mathrm{AG}$ & AA & AA & GG & GG & $\mathrm{AG}$ \\
\hline AA & AA & GG & GG & GG & GG & $\mathrm{AG}$ & $\mathrm{AG}$ & $\mathrm{AG}$ & $\mathrm{AG}$ & $\mathrm{AG}$ \\
\hline AG & $\mathrm{AG}$ & $\mathrm{AG}$ & GG & $\mathrm{AG}$ & GG & $\mathrm{AG}$ & $\mathrm{AG}$ & GG & GG & \\
\hline GDsnp00633 & 2 & \multicolumn{2}{|c|}{18038089} & $\mathrm{AG}$ & $\mathrm{AA}$ & $\mathrm{AA}$ & $\mathrm{AA}$ & $\mathrm{AG}$ & $\mathrm{AG}$ & $\mathrm{AG}$ \\
\hline $\mathrm{AG}$ & $\mathrm{AG}$ & $\mathrm{AA}$ & $\mathrm{AG}$ & $\mathrm{AG}$ & $\mathrm{AA}$ & AA & $\mathrm{AG}$ & $\mathrm{AA}$ & $\mathrm{AG}$ & $\mathrm{AG}$ \\
\hline $\mathrm{AG}$ & $\mathrm{AG}$ & $\mathrm{AG}$ & $\mathrm{AA}$ & $\mathrm{AG}$ & $\mathrm{AG}$ & $\mathrm{AG}$ & $\mathrm{AG}$ & $\mathrm{AG}$ & $\mathrm{AG}$ & $\mathrm{AA}$ \\
\hline $\mathrm{AA}$ & $\mathrm{AA}$ & $\mathrm{AG}$ & $\mathrm{AG}$ & AA & $\mathrm{AA}$ & $A G$ & $\mathrm{AG}$ & AA & $\mathrm{AA}$ & $\mathrm{AA}$ \\
\hline $\mathrm{AG}$ & $\mathrm{AG}$ & $\mathrm{AG}$ & $\mathrm{AG}$ & $\mathrm{AG}$ & $\mathrm{AG}$ & $\mathrm{AG}$ & $\mathrm{AA}$ & $\mathrm{AA}$ & $\mathrm{AG}$ & $\mathrm{AG}$ \\
\hline AA & $\mathrm{AG}$ & $\mathrm{AG}$ & AA & AA & $\mathrm{AA}$ & $\mathrm{AG}$ & $\mathrm{AG}$ & $\mathrm{AG}$ & $\mathrm{AG}$ & $\mathrm{AG}$ \\
\hline $\mathrm{AG}$ & $\mathrm{AG}$ & $\mathrm{AG}$ & AA & AA & $\mathrm{AA}$ & $\mathrm{AG}$ & $\mathrm{AG}$ & $\mathrm{AG}$ & $\mathrm{AA}$ & $\mathrm{AG}$ \\
\hline $\mathrm{AG}$ & $\mathrm{AG}$ & AA & $\mathrm{AG}$ & $\mathrm{AG}$ & $\mathrm{AG}$ & $\mathrm{AG}$ & $\mathrm{AG}$ & $\mathrm{AG}$ & $\mathrm{AG}$ & AA \\
\hline AG & AA & AA & AA & $\mathrm{AG}$ & AA & AA & $\mathrm{AG}$ & $\mathrm{AA}$ & $\mathrm{AA}$ & \\
\hline GDsnp01090 & 2 & \multicolumn{2}{|c|}{21114028} & $\mathrm{AT}$ & $\mathrm{AT}$ & $\mathrm{AT}$ & $\mathrm{AT}$ & AA & $\mathrm{TT}$ & $\mathrm{TT}$ \\
\hline AA & $\mathrm{AT}$ & $\mathrm{AA}$ & $\mathrm{AT}$ & $\mathrm{AT}$ & $\mathrm{TT}$ & TT & $\mathrm{AT}$ & $\mathrm{AT}$ & $\mathrm{TT}$ & TT \\
\hline $\mathrm{TT}$ & AA & AA & $\mathrm{TT}$ & $\mathrm{TT}$ & $\mathrm{AT}$ & AA & $\mathrm{AT}$ & $\mathrm{AT}$ & $\mathrm{AA}$ & AT \\
\hline AT & $\mathrm{AT}$ & AA & $\mathrm{AT}$ & $\mathrm{TT}$ & $\mathrm{AT}$ & $\mathrm{AT}$ & $\mathrm{AT}$ & $\mathrm{AT}$ & $\mathrm{AA}$ & TT \\
\hline $\mathrm{TT}$ & $\mathrm{AA}$ & $\mathrm{AT}$ & $\mathrm{AT}$ & $\mathrm{AT}$ & $\mathrm{AA}$ & AA & $\mathrm{AT}$ & AT & $\mathrm{AA}$ & AT \\
\hline $\mathrm{TT}$ & $\mathrm{AT}$ & $\mathrm{AA}$ & $\mathrm{AT}$ & $\mathrm{AT}$ & $\mathrm{TT}$ & $\mathrm{AT}$ & $\mathrm{AA}$ & $\mathrm{AT}$ & $\mathrm{AA}$ & AT \\
\hline $\mathrm{AT}$ & $\mathrm{AT}$ & $\mathrm{AT}$ & $\mathrm{AT}$ & $\mathrm{AT}$ & $\mathrm{AT}$ & $\mathrm{AA}$ & $\mathrm{AA}$ & $\mathrm{TT}$ & $\mathrm{TT}$ & AT \\
\hline AA & $\mathrm{AA}$ & $\mathrm{AT}$ & $\mathrm{TT}$ & $\mathrm{TT}$ & $\mathrm{TT}$ & $\mathrm{AT}$ & $\mathrm{AT}$ & $\mathrm{AT}$ & $\mathrm{AA}$ & AT \\
\hline $\mathrm{AT}$ & $\mathrm{AT}$ & $\mathrm{AT}$ & $\mathrm{TT}$ & $\mathrm{AT}$ & $\mathrm{TT}$ & $\mathrm{TT}$ & $\mathrm{AT}$ & $\mathrm{TT}$ & $\mathrm{TT}$ & \\
\hline
\end{tabular}

Continued next page 
Supplemental Table 2. Continued.

\begin{tabular}{|c|c|c|c|c|c|c|c|c|c|c|}
\hline \multirow{3}{*}{$\begin{array}{l}\text { SNP name } \\
\text { GDsnp00833 } \\
\text { AG }\end{array}$} & \multicolumn{10}{|c|}{ Linkage group Physical location (bp) Individuals } \\
\hline & 2 & \multicolumn{2}{|c|}{22413796} & $\mathrm{AG}$ & $\mathrm{AG}$ & $\mathrm{AG}$ & $\mathrm{AG}$ & $\mathrm{AA}$ & GG & GG \\
\hline & $\mathrm{AG}$ & $\mathrm{AG}$ & GG & AA & GG & GG & $\mathrm{AG}$ & $\mathrm{AG}$ & GG & GG \\
\hline GG & AA & $\mathrm{AG}$ & GG & GG & $\mathrm{AG}$ & AA & $\mathrm{AG}$ & $\mathrm{AG}$ & AA & $\mathrm{AG}$ \\
\hline AG & AG & AA & AA & GG & $\mathrm{AG}$ & $\mathrm{AG}$ & AA & $\mathrm{AG}$ & $\mathrm{AG}$ & GG \\
\hline $\mathrm{AG}$ & $\mathrm{AA}$ & $\mathrm{AG}$ & $\mathrm{AG}$ & $\mathrm{AG}$ & $\mathrm{AA}$ & $\mathrm{AA}$ & $\mathrm{AG}$ & $\mathrm{AG}$ & $\mathrm{AA}$ & $\mathrm{AG}$ \\
\hline GG & $\mathrm{AG}$ & $\mathrm{AA}$ & $\mathrm{AG}$ & $\mathrm{AG}$ & GG & $\mathrm{AG}$ & $\mathrm{AA}$ & $\mathrm{AG}$ & $\mathrm{AA}$ & $\mathrm{AG}$ \\
\hline $\mathrm{AG}$ & $\mathrm{AG}$ & $\mathrm{AG}$ & $\mathrm{AG}$ & $\mathrm{AG}$ & $\mathrm{AG}$ & $\mathrm{AA}$ & $\mathrm{AG}$ & GG & GG & $\mathrm{AG}$ \\
\hline AA & $\mathrm{AA}$ & $\mathrm{AG}$ & GG & GG & GG & $\mathrm{AG}$ & $\mathrm{AG}$ & $\mathrm{AG}$ & $\mathrm{AA}$ & $\mathrm{AG}$ \\
\hline $\mathrm{AG}$ & $\mathrm{AG}$ & $\mathrm{AG}$ & GG & $\mathrm{AG}$ & GG & GG & $\mathrm{AG}$ & GG & $\mathrm{AG}$ & \\
\hline GDsnp01251 & 2 & \multicolumn{2}{|c|}{28641848} & AA & $\mathrm{AA}$ & $\mathrm{AC}$ & $\mathrm{AC}$ & $\mathrm{AA}$ & $\mathrm{AA}$ & $\mathrm{AA}$ \\
\hline AA & $\mathrm{AA}$ & AA & $\mathrm{AA}$ & AA & $\mathrm{AC}$ & $\mathrm{AC}$ & AA & $\mathrm{AC}$ & $\mathrm{AA}$ & $\mathrm{AA}$ \\
\hline AA & $\mathrm{AA}$ & $\mathrm{AC}$ & $\mathrm{AC}$ & $\mathrm{AC}$ & $\mathrm{AA}$ & $\mathrm{AA}$ & AA & $\mathrm{AA}$ & $\mathrm{AA}$ & $\mathrm{AC}$ \\
\hline $\mathrm{AC}$ & $\mathrm{AC}$ & $\mathrm{AA}$ & $\mathrm{AA}$ & $\mathrm{AC}$ & $\mathrm{AC}$ & $\mathrm{AC}$ & $\mathrm{AA}$ & $\mathrm{AC}$ & $\mathrm{AC}$ & $\mathrm{AC}$ \\
\hline AA & $\mathrm{AA}$ & AA & $\mathrm{AA}$ & AA & $\mathrm{AA}$ & $\mathrm{AC}$ & $\mathrm{AC}$ & $\mathrm{AC}$ & $\mathrm{AA}$ & AA \\
\hline $\mathrm{AC}$ & $\mathrm{AA}$ & $\mathrm{AA}$ & $\mathrm{AC}$ & $\mathrm{AC}$ & $\mathrm{AC}$ & $\mathrm{AC}$ & AA & AA & $\mathrm{AA}$ & $\mathrm{AA}$ \\
\hline AA & $\mathrm{AA}$ & $\mathrm{AA}$ & $\mathrm{AC}$ & $\mathrm{AC}$ & $\mathrm{AC}$ & $\mathrm{AA}$ & AA & $\mathrm{AA}$ & $\mathrm{AA}$ & $\mathrm{AA}$ \\
\hline AA & AA & $\mathrm{AA}$ & $\mathrm{AC}$ & $\mathrm{AC}$ & $\mathrm{AC}$ & AA & AA & $\mathrm{AC}$ & $\mathrm{AA}$ & $\mathrm{AC}$ \\
\hline AA & $\mathrm{AC}$ & $\mathrm{AC}$ & AA & AA & $\mathrm{AC}$ & $\mathrm{AC}$ & AA & $\mathrm{AC}$ & $\mathrm{AC}$ & \\
\hline GDsnp00629 & 2 & \multicolumn{2}{|c|}{30034626} & $\mathrm{AA}$ & $\mathrm{AA}$ & $\mathrm{AG}$ & $\mathrm{AG}$ & $\mathrm{AA}$ & $\mathrm{AA}$ & AA \\
\hline AA & AA & AA & AA & AA & $\mathrm{AG}$ & AA & AA & $\mathrm{AG}$ & $\mathrm{AA}$ & AA \\
\hline $\mathrm{AG}$ & $\mathrm{AA}$ & $\mathrm{AG}$ & $\mathrm{AG}$ & $\mathrm{AG}$ & $\mathrm{AA}$ & $\mathrm{AA}$ & $\mathrm{AA}$ & $\mathrm{AA}$ & $\mathrm{AA}$ & $\mathrm{AG}$ \\
\hline$A G$ & $\mathrm{AG}$ & $\mathrm{AA}$ & $\mathrm{AA}$ & $\mathrm{AG}$ & $\mathrm{AG}$ & $\mathrm{AG}$ & AA & $\mathrm{AG}$ & $\mathrm{AA}$ & $\mathrm{AG}$ \\
\hline AA & $\mathrm{AA}$ & $\mathrm{AA}$ & $\mathrm{AA}$ & AA & $\mathrm{AA}$ & $\mathrm{AG}$ & $\mathrm{AG}$ & $\mathrm{AG}$ & $\mathrm{AA}$ & $\mathrm{AA}$ \\
\hline $\mathrm{AG}$ & AA & AA & $\mathrm{AG}$ & $\mathrm{AG}$ & $\mathrm{AG}$ & $\mathrm{AG}$ & AA & AA & $\mathrm{AA}$ & AA \\
\hline AA & AA & AA & $\mathrm{AG}$ & $\mathrm{AG}$ & $\mathrm{AG}$ & AA & AA & $\mathrm{AA}$ & $\mathrm{AG}$ & AA \\
\hline AA & AA & AA & $\mathrm{AG}$ & AA & $\mathrm{AG}$ & AA & AA & $\mathrm{AG}$ & $\mathrm{AA}$ & $\mathrm{AG}$ \\
\hline AA & $\mathrm{AG}$ & $\mathrm{AG}$ & $\mathrm{AA}$ & $\mathrm{AA}$ & AG & $\mathrm{AG}$ & $\mathrm{AA}$ & $\mathrm{AG}$ & $\mathrm{AG}$ & \\
\hline GDsnp00120 & 2 & \multicolumn{2}{|c|}{31832613} & $\mathrm{TT}$ & $\mathrm{TT}$ & $\mathrm{AT}$ & $\mathrm{AT}$ & $\mathrm{TT}$ & $\mathrm{TT}$ & AT \\
\hline TT & $\mathrm{TT}$ & $\mathrm{TT}$ & $\mathrm{TT}$ & $\mathrm{TT}$ & $\mathrm{AT}$ & $\mathrm{TT}$ & $\mathrm{TT}$ & $\mathrm{AT}$ & $\mathrm{TT}$ & TT \\
\hline AT & $\mathrm{TT}$ & $\mathrm{AT}$ & $\mathrm{AT}$ & $\mathrm{AT}$ & $\mathrm{TT}$ & $\mathrm{TT}$ & $\mathrm{TT}$ & $\mathrm{TT}$ & $\mathrm{TT}$ & TT \\
\hline AT & $\mathrm{AT}$ & $\mathrm{TT}$ & $\mathrm{TT}$ & $\mathrm{AT}$ & $\mathrm{AT}$ & $\mathrm{AT}$ & $\mathrm{TT}$ & $\mathrm{AT}$ & $\mathrm{TT}$ & AT \\
\hline $\mathrm{TT}$ & $\mathrm{TT}$ & $\mathrm{TT}$ & $\mathrm{TT}$ & $\mathrm{TT}$ & $\mathrm{TT}$ & - & $\mathrm{TT}$ & $\mathrm{AT}$ & $\mathrm{TT}$ & TT \\
\hline AT & $\mathrm{TT}$ & $\mathrm{TT}$ & $\mathrm{AT}$ & $\mathrm{AT}$ & $\mathrm{AT}$ & $\mathrm{AT}$ & $\mathrm{TT}$ & $\mathrm{TT}$ & $\mathrm{TT}$ & TT \\
\hline TT & $\mathrm{TT}$ & $\mathrm{TT}$ & $\mathrm{AT}$ & $\mathrm{AT}$ & $\mathrm{AT}$ & $\mathrm{TT}$ & $\mathrm{TT}$ & $\mathrm{TT}$ & $\mathrm{AT}$ & TT \\
\hline TT & $\mathrm{TT}$ & $\mathrm{TT}$ & $\mathrm{AT}$ & $\mathrm{TT}$ & $\mathrm{AT}$ & $\mathrm{TT}$ & $\mathrm{TT}$ & AT & TT & TT \\
\hline TT & AT & AT & $\mathrm{TT}$ & $\mathrm{TT}$ & $\mathrm{TT}$ & $\mathrm{AT}$ & $\mathrm{TT}$ & AT & AT & \\
\hline GDsnp00267 & 2 & \multicolumn{2}{|c|}{32038429} & $\mathrm{CC}$ & $\mathrm{CC}$ & $\mathrm{AC}$ & $\mathrm{AC}$ & $\mathrm{CC}$ & $\mathrm{CC}$ & $\mathrm{AC}$ \\
\hline $\mathrm{CC}$ & $\mathrm{CC}$ & $\mathrm{CC}$ & $\mathrm{CC}$ & $\mathrm{CC}$ & $\mathrm{AC}$ & $\mathrm{CC}$ & $\mathrm{CC}$ & $\mathrm{CC}$ & $\mathrm{CC}$ & $\mathrm{CC}$ \\
\hline $\mathrm{AC}$ & $\mathrm{CC}$ & $\mathrm{AC}$ & $\mathrm{AC}$ & $\mathrm{AC}$ & $\mathrm{CC}$ & $\mathrm{CC}$ & $\mathrm{CC}$ & $\mathrm{CC}$ & $\mathrm{CC}$ & $\mathrm{CC}$ \\
\hline $\mathrm{AC}$ & $\mathrm{AC}$ & $\mathrm{CC}$ & $\mathrm{CC}$ & $\mathrm{AC}$ & $\mathrm{AC}$ & $\mathrm{AC}$ & $\mathrm{CC}$ & $\mathrm{AC}$ & $\mathrm{CC}$ & $\mathrm{CC}$ \\
\hline $\mathrm{CC}$ & $\mathrm{CC}$ & $\mathrm{CC}$ & $\mathrm{CC}$ & $\mathrm{CC}$ & $\mathrm{CC}$ & $\mathrm{AC}$ & $\mathrm{CC}$ & $\mathrm{AC}$ & $\mathrm{CC}$ & $\mathrm{CC}$ \\
\hline $\mathrm{AC}$ & $\mathrm{CC}$ & $\mathrm{CC}$ & $\mathrm{CC}$ & $\mathrm{AC}$ & $\mathrm{AC}$ & $\mathrm{AC}$ & $\mathrm{CC}$ & $\mathrm{CC}$ & $\mathrm{CC}$ & $\mathrm{CC}$ \\
\hline $\mathrm{CC}$ & $\mathrm{CC}$ & $\mathrm{CC}$ & $\mathrm{AC}$ & $\mathrm{AC}$ & $\mathrm{AC}$ & $\mathrm{CC}$ & $\mathrm{CC}$ & $\mathrm{CC}$ & $\mathrm{AC}$ & $\mathrm{CC}$ \\
\hline $\mathrm{CC}$ & $\mathrm{CC}$ & $\mathrm{CC}$ & $\mathrm{AC}$ & $\mathrm{CC}$ & $\mathrm{AC}$ & $\mathrm{CC}$ & $\mathrm{CC}$ & $\mathrm{AC}$ & $\mathrm{CC}$ & $\mathrm{CC}$ \\
\hline $\mathrm{CC}$ & $\mathrm{AC}$ & $\mathrm{AC}$ & $\mathrm{CC}$ & $\mathrm{CC}$ & $\mathrm{CC}$ & $\mathrm{AC}$ & $\mathrm{CC}$ & $\mathrm{AC}$ & $\mathrm{AC}$ & \\
\hline GDsnp01322 & 2 & \multicolumn{2}{|c|}{32907169} & GG & GG & GG & GG & GG & GG & GG \\
\hline GG & $\mathrm{AG}$ & GG & GG & GG & GG & GG & GG & $\mathrm{AG}$ & GG & GG \\
\hline GG & GG & GG & GG & GG & GG & $\mathrm{AG}$ & GG & GG & GG & GG \\
\hline GG & GG & GG & GG & GG & GG & GG & GG & GG & GG & $\mathrm{AG}$ \\
\hline GG & GG & GG & GG & $\mathrm{GG}$ & GG & GG & GG & GG & GG & GG \\
\hline GG & GG & GG & $\mathrm{AG}$ & GG & GG & GG & GG & GG & GG & GG \\
\hline GG & GG & GG & GG & GG & GG & GG & GG & GG & GG & GG \\
\hline GG & GG & GG & $\mathrm{GG}$ & $\mathrm{GG}$ & GG & GG & GG & GG & GG & GG \\
\hline GG & GG & GG & GG & $\mathrm{GG}$ & GG & GG & GG & GG & GG & \\
\hline
\end{tabular}

Continued next page 
Supplemental Table 2. Continued.

\begin{tabular}{|c|c|c|c|c|c|c|c|c|c|c|}
\hline \multirow{3}{*}{$\begin{array}{l}\text { SNP name } \\
\text { GDsnp00471 } \\
\text { TT }\end{array}$} & \multicolumn{10}{|c|}{ Linkage group Physical location (bp) Individuals } \\
\hline & 2 & \multicolumn{2}{|c|}{35430983} & $\mathrm{AT}$ & AT & AT & AT & AT & $\mathrm{AA}$ & $\mathrm{AA}$ \\
\hline & AA & $\mathrm{AT}$ & AT & AT & AA & AA & AT & AA & $\mathrm{AA}$ & AA \\
\hline AT & AT & TT & AT & AA & AT & AT & AT & AT & $\mathrm{TT}$ & TT \\
\hline AT & TT & $\mathrm{TT}$ & $\mathrm{TT}$ & AT & AT & AT & AA & $\mathrm{TT}$ & $\mathrm{AT}$ & AA \\
\hline AA & TT & $\mathrm{TT}$ & $\mathrm{AT}$ & $\mathrm{AT}$ & TT & $\mathrm{AT}$ & $\mathrm{AT}$ & $\mathrm{AT}$ & TT & AT \\
\hline AA & $\mathrm{AT}$ & $\mathrm{TT}$ & $\mathrm{AT}$ & AT & $\mathrm{AA}$ & $\mathrm{AT}$ & $\mathrm{TT}$ & $\mathrm{AT}$ & $\mathrm{AT}$ & AT \\
\hline AT & $\mathrm{TT}$ & AA & $\mathrm{TT}$ & $\mathrm{TT}$ & TT & $\mathrm{TT}$ & $\mathrm{AT}$ & $\mathrm{AT}$ & $\mathrm{AT}$ & AT \\
\hline $\mathrm{TT}$ & $\mathrm{TT}$ & AT & AA & AA & $\mathrm{AT}$ & $\mathrm{AT}$ & $\mathrm{AT}$ & $\mathrm{AT}$ & $\mathrm{AT}$ & TT \\
\hline AT & AT & AT & $\mathrm{AA}$ & AT & $\mathrm{AT}$ & AT & AT & $\mathrm{AA}$ & AT & \\
\hline GDsnp02535 & 2 & \multicolumn{2}{|c|}{36427906} & $\mathrm{AG}$ & $\mathrm{AG}$ & $\mathrm{AG}$ & GG & $\mathrm{AG}$ & $\mathrm{AA}$ & $\mathrm{AA}$ \\
\hline AG & AA & $\mathrm{AG}$ & $\mathrm{AG}$ & $\mathrm{AG}$ & $\mathrm{AA}$ & $\mathrm{AA}$ & $\mathrm{AG}$ & $\mathrm{AA}$ & $\mathrm{AA}$ & AA \\
\hline AG & $\mathrm{AG}$ & GG & $\mathrm{AG}$ & AA & $\mathrm{AG}$ & $\mathrm{AG}$ & $\mathrm{AG}$ & $\mathrm{AG}$ & GG & GG \\
\hline $\mathrm{AG}$ & GG & GG & GG & $\mathrm{AG}$ & $\mathrm{AG}$ & $\mathrm{AG}$ & AA & GG & $\mathrm{AG}$ & $\mathrm{AA}$ \\
\hline AA & GG & $\mathrm{AG}$ & $\mathrm{AG}$ & $\mathrm{AG}$ & GG & $\mathrm{AG}$ & $\mathrm{AG}$ & $\mathrm{AG}$ & GG & $\mathrm{AG}$ \\
\hline AA & $\mathrm{AG}$ & GG & $\mathrm{AG}$ & $\mathrm{AG}$ & $\mathrm{AA}$ & $\mathrm{AG}$ & GG & $\mathrm{AG}$ & $\mathrm{AG}$ & $\mathrm{AG}$ \\
\hline $\mathrm{AG}$ & GG & $\mathrm{AA}$ & GG & GG & GG & GG & $\mathrm{AG}$ & $\mathrm{AG}$ & $\mathrm{AG}$ & $\mathrm{AG}$ \\
\hline GG & GG & $\mathrm{AG}$ & $\mathrm{AA}$ & $\mathrm{AA}$ & $\mathrm{AG}$ & $\mathrm{AG}$ & $\mathrm{AG}$ & $\mathrm{AG}$ & $\mathrm{AG}$ & GG \\
\hline $\mathrm{AG}$ & $\mathrm{AG}$ & $\mathrm{AG}$ & AA & $\mathrm{AG}$ & $\mathrm{AG}$ & $\mathrm{AG}$ & $\mathrm{AG}$ & $\mathrm{AA}$ & $\mathrm{AG}$ & \\
\hline GDsnp01181 & 2 & \multicolumn{2}{|c|}{ unanchored } & $\mathrm{AA}$ & $\mathrm{AA}$ & $\mathrm{AA}$ & $\mathrm{AA}$ & $\mathrm{AA}$ & - & - \\
\hline AA & GG & $\mathrm{AA}$ & $\mathrm{AA}$ & $\mathrm{AA}$ & $\mathrm{AA}$ & $\mathrm{AA}$ & $\mathrm{AA}$ & $\mathrm{AA}$ & - & - \\
\hline- & AA & $\mathrm{AA}$ & AA & AA & $\mathrm{AA}$ & $\mathrm{AG}$ & AA & $\mathrm{AA}$ & $\mathrm{AA}$ & AA \\
\hline AA & AA & AA & AA & AA & $\mathrm{AA}$ & AA & AA & AA & $\mathrm{AG}$ & AA \\
\hline AA & AA & $\mathrm{AA}$ & $\mathrm{AA}$ & AA & $\mathrm{AA}$ & $\mathrm{AA}$ & $\mathrm{AA}$ & $\mathrm{AA}$ & $\mathrm{AA}$ & AA \\
\hline AA & $\mathrm{AA}$ & AA & $\mathrm{AA}$ & $\mathrm{AA}$ & $\mathrm{AA}$ & $\mathrm{AA}$ & $\mathrm{AA}$ & $\mathrm{AA}$ & $\mathrm{AA}$ & AA \\
\hline AA & AA & - & AA & AA & $\mathrm{AA}$ & AA & AA & - & $\mathrm{AA}$ & AA \\
\hline AA & AA & AA & - & - & $\mathrm{AA}$ & AA & AA & AA & $\mathrm{AA}$ & AA \\
\hline AA & AA & $\mathrm{AA}$ & - & $\mathrm{AA}$ & $\mathrm{AA}$ & $\mathrm{AA}$ & $\mathrm{AA}$ & $\mathrm{AA}$ & $\mathrm{AA}$ & \\
\hline GDsnp00322 & 3 & \multicolumn{2}{|c|}{866359} & $\mathrm{CG}$ & GG & $\mathrm{CG}$ & $\mathrm{CC}$ & GG & $\mathrm{CC}$ & $\mathrm{CG}$ \\
\hline- & $\mathrm{CG}$ & $\mathrm{CC}$ & $\mathrm{CG}$ & $\mathrm{CG}$ & GG & $\mathrm{CG}$ & GG & $\mathrm{CG}$ & $\mathrm{CG}$ & $\mathrm{CC}$ \\
\hline GG & $\mathrm{CC}$ & $\mathrm{CC}$ & $\mathrm{CG}$ & GG & $\mathrm{CG}$ & $\mathrm{CG}$ & $\mathrm{CG}$ & $\mathrm{CG}$ & $\mathrm{CC}$ & $\mathrm{CC}$ \\
\hline $\mathrm{CC}$ & $\mathrm{CC}$ & CG & $\mathrm{CG}$ & $\mathrm{CG}$ & $\mathrm{CC}$ & $\mathrm{CC}$ & $\mathrm{CG}$ & $\mathrm{CC}$ & $\mathrm{CG}$ & $\mathrm{CC}$ \\
\hline GG & $\mathrm{CG}$ & CG & $\mathrm{CC}$ & $\mathrm{CG}$ & $\mathrm{CC}$ & $\mathrm{CG}$ & $\mathrm{CG}$ & $\mathrm{CG}$ & $\mathrm{CG}$ & $\mathrm{CG}$ \\
\hline $\mathrm{CC}$ & $\mathrm{CG}$ & $\mathrm{CC}$ & GG & $\mathrm{CG}$ & GG & $\mathrm{CC}$ & $\mathrm{CC}$ & $\mathrm{CG}$ & $\mathrm{CG}$ & GG \\
\hline GG & $\mathrm{CC}$ & GG & $\mathrm{CC}$ & $\mathrm{CC}$ & $\mathrm{CG}$ & $\mathrm{CG}$ & GG & GG & $\mathrm{CG}$ & $\mathrm{CG}$ \\
\hline $\mathrm{CG}$ & GG & $\mathrm{CC}$ & $\mathrm{CG}$ & $\mathrm{CC}$ & GG & $\mathrm{CG}$ & $\mathrm{CG}$ & CG & GG & $\mathrm{CC}$ \\
\hline $\mathrm{CG}$ & $\mathrm{CC}$ & GG & $\mathrm{CG}$ & CG & CG & CG & $\mathrm{CC}$ & CG & $\mathrm{CC}$ & \\
\hline GDsnp00480 & 3 & \multicolumn{2}{|c|}{1667573} & GG & GG & $\mathrm{CG}$ & $\mathrm{CG}$ & $\mathrm{CG}$ & GG & GG \\
\hline CG & $\mathrm{CG}$ & $\mathrm{CG}$ & GG & $\mathrm{CG}$ & GG & GG & GG & GG & GG & $\mathrm{CG}$ \\
\hline GG & $\mathrm{CG}$ & $\mathrm{CG}$ & $\mathrm{CG}$ & GG & $\mathrm{CG}$ & $\mathrm{CG}$ & $\mathrm{CG}$ & $\mathrm{CG}$ & GG & GG \\
\hline $\mathrm{CG}$ & GG & GG & GG & GG & GG & $\mathrm{CG}$ & GG & GG & $\mathrm{CG}$ & $\mathrm{CG}$ \\
\hline GG & $\mathrm{CG}$ & GG & GG & GG & GG & GG & GG & GG & $\mathrm{CG}$ & GG \\
\hline $\mathrm{CG}$ & GG & $\mathrm{CG}$ & GG & $\mathrm{CG}$ & GG & $\mathrm{CG}$ & $\mathrm{CG}$ & GG & $\mathrm{CG}$ & GG \\
\hline GG & $\mathrm{CG}$ & GG & GG & GG & $\mathrm{CG}$ & $\mathrm{CG}$ & $\mathrm{CG}$ & GG & GG & $\mathrm{CG}$ \\
\hline $\mathrm{CG}$ & GG & $\mathrm{CG}$ & $\mathrm{CG}$ & $\mathrm{CG}$ & GG & $\mathrm{CG}$ & GG & GG & GG & GG \\
\hline CG & CG & GG & GG & CG & GG & CG & GG & GG & CG & \\
\hline GDsnp00078 & 3 & \multicolumn{2}{|c|}{1813618} & $\mathrm{TT}$ & $\mathrm{TT}$ & $\mathrm{TT}$ & $\mathrm{AT}$ & $\mathrm{TT}$ & $\mathrm{TT}$ & TT \\
\hline TT & $\mathrm{TT}$ & TT & $\mathrm{TT}$ & $\mathrm{TT}$ & TT & $\mathrm{TT}$ & $\mathrm{TT}$ & $\mathrm{TT}$ & $\mathrm{TT}$ & TT \\
\hline TT & AT & AT & $\mathrm{TT}$ & TT & $\mathrm{AT}$ & $\mathrm{TT}$ & $\mathrm{AT}$ & $\mathrm{AT}$ & TT & TT \\
\hline AT & TT & TT & $\mathrm{TT}$ & $\mathrm{TT}$ & TT & $\mathrm{TT}$ & $\mathrm{TT}$ & $\mathrm{TT}$ & $\mathrm{AT}$ & TT \\
\hline $\mathrm{TT}$ & $\mathrm{AT}$ & $\mathrm{TT}$ & $\mathrm{TT}$ & $\mathrm{TT}$ & $\mathrm{TT}$ & $\mathrm{TT}$ & $\mathrm{AT}$ & $\mathrm{TT}$ & $\mathrm{AT}$ & $\mathrm{TT}$ \\
\hline $\mathrm{TT}$ & $\mathrm{TT}$ & AT & $\mathrm{TT}$ & $\mathrm{TT}$ & $\mathrm{TT}$ & $\mathrm{AT}$ & $\mathrm{TT}$ & $\mathrm{TT}$ & $\mathrm{TT}$ & TT \\
\hline TT & $\mathrm{TT}$ & $\mathrm{TT}$ & $\mathrm{TT}$ & $\mathrm{TT}$ & $\mathrm{AT}$ & $\mathrm{AT}$ & $\mathrm{AT}$ & $\mathrm{TT}$ & $\mathrm{TT}$ & TT \\
\hline $\mathrm{TT}$ & $\mathrm{TT}$ & AT & $\mathrm{TT}$ & $\mathrm{AT}$ & $\mathrm{TT}$ & AT & $\mathrm{TT}$ & $\mathrm{TT}$ & $\mathrm{TT}$ & $\mathrm{TT}$ \\
\hline AT & $\mathrm{TT}$ & $\mathrm{TT}$ & $\mathrm{TT}$ & $\mathrm{TT}$ & $\mathrm{TT}$ & $\mathrm{TT}$ & $\mathrm{TT}$ & $\mathrm{TT}$ & $\mathrm{TT}$ & \\
\hline
\end{tabular}


Supplemental Table 2. Continued.

\begin{tabular}{|c|c|c|c|c|c|c|c|c|c|c|}
\hline \multirow{3}{*}{$\begin{array}{l}\text { SNP name } \\
\text { GDsnp00588 } \\
\text { AT }\end{array}$} & \multicolumn{10}{|c|}{ Linkage group Physical location (bp) Individuals } \\
\hline & 3 & \multicolumn{2}{|c|}{2130666} & AA & AA & AT & AT & AT & $\mathrm{AA}$ & $\mathrm{AA}$ \\
\hline & AT & AT & AA & AT & AA & AA & AA & AA & AA & AT \\
\hline AA & AT & AT & AT & AA & AT & AT & AT & AT & AA & AA \\
\hline AT & AA & AA & AA & AA & AA & AT & AA & AA & $\mathrm{AT}$ & AT \\
\hline AA & $\mathrm{AT}$ & AA & AA & AA & $\mathrm{AA}$ & $\mathrm{AA}$ & $\mathrm{AT}$ & $\mathrm{AA}$ & $\mathrm{AT}$ & AA \\
\hline AT & AA & $\mathrm{AT}$ & AA & AT & $\mathrm{AA}$ & $\mathrm{AT}$ & $\mathrm{AT}$ & AA & $\mathrm{AT}$ & AA \\
\hline AA & $\mathrm{AT}$ & AA & AA & $\mathrm{AA}$ & AT & $\mathrm{AT}$ & $\mathrm{AA}$ & $\mathrm{AA}$ & $\mathrm{AA}$ & AT \\
\hline AT & AA & $\mathrm{AT}$ & $\mathrm{AT}$ & AA & $\mathrm{AA}$ & $\mathrm{AT}$ & AA & $\mathrm{AA}$ & $\mathrm{AA}$ & AA \\
\hline AT & AT & AA & AA & AT & $\mathrm{AA}$ & AT & $\mathrm{AA}$ & $\mathrm{AA}$ & AT & \\
\hline GDsnp00477 & 3 & \multicolumn{2}{|c|}{4409802} & AA & $\mathrm{AA}$ & $\mathrm{AT}$ & $\mathrm{AT}$ & $\mathrm{AT}$ & $\mathrm{AA}$ & $\mathrm{AA}$ \\
\hline $\mathrm{AA}$ & $\mathrm{AT}$ & AT & AA & $\mathrm{AT}$ & $\mathrm{AA}$ & $\mathrm{AA}$ & $\mathrm{AA}$ & $\mathrm{AA}$ & $\mathrm{AA}$ & AT \\
\hline AA & $\mathrm{AT}$ & $\mathrm{AT}$ & AT & $\mathrm{AT}$ & AT & $\mathrm{AT}$ & $\mathrm{AT}$ & AT & $\mathrm{AA}$ & AA \\
\hline $\mathrm{AT}$ & AA & $\mathrm{AA}$ & $\mathrm{AA}$ & AA & $\mathrm{AA}$ & AA & AA & $\mathrm{AA}$ & $\mathrm{AT}$ & $\mathrm{AA}$ \\
\hline AA & $\mathrm{AT}$ & AA & $\mathrm{AT}$ & AA & $\mathrm{AA}$ & AA & $\mathrm{AT}$ & AA & $\mathrm{AT}$ & AA \\
\hline AT & AA & $\mathrm{AT}$ & AA & $\mathrm{AT}$ & $\mathrm{AA}$ & $\mathrm{AT}$ & AA & AA & $\mathrm{AT}$ & AT \\
\hline $\mathrm{AT}$ & $\mathrm{AT}$ & AA & $\mathrm{AA}$ & AA & $\mathrm{AT}$ & $\mathrm{AT}$ & AA & $\mathrm{AA}$ & $\mathrm{AA}$ & AT \\
\hline AT & AA & AT & AT & $\mathrm{AA}$ & $\mathrm{AA}$ & $\mathrm{AT}$ & $\mathrm{AA}$ & $\mathrm{AA}$ & $\mathrm{AA}$ & AA \\
\hline AT & $\mathrm{AT}$ & AA & $\mathrm{AA}$ & $\mathrm{AT}$ & $\mathrm{AA}$ & $\mathrm{AT}$ & AA & $\mathrm{AA}$ & $\mathrm{AT}$ & \\
\hline GDsnp02304 & 3 & \multicolumn{2}{|c|}{5461757} & $\mathrm{CG}$ & GG & $\mathrm{CG}$ & $\mathrm{CC}$ & $\mathrm{CG}$ & $\mathrm{CC}$ & GG \\
\hline $\mathrm{CG}$ & $\mathrm{CG}$ & $\mathrm{CC}$ & $\mathrm{CG}$ & $\mathrm{CC}$ & GG & $\mathrm{CG}$ & GG & $\mathrm{CG}$ & $\mathrm{CG}$ & $\mathrm{CC}$ \\
\hline GG & $\mathrm{CC}$ & $\mathrm{CC}$ & $\mathrm{CG}$ & $\mathrm{CG}$ & $\mathrm{CG}$ & $\mathrm{CG}$ & $\mathrm{CG}$ & $\mathrm{CG}$ & $\mathrm{CC}$ & $\mathrm{CC}$ \\
\hline $\mathrm{CC}$ & $\mathrm{CC}$ & $\mathrm{CG}$ & $\mathrm{CG}$ & $\mathrm{CG}$ & $\mathrm{CC}$ & $\mathrm{CG}$ & $\mathrm{CG}$ & $\mathrm{CC}$ & $\mathrm{CG}$ & $\mathrm{CG}$ \\
\hline $\mathrm{CG}$ & $\mathrm{CG}$ & $\mathrm{CG}$ & $\mathrm{CC}$ & $\mathrm{CG}$ & $\mathrm{CC}$ & - & $\mathrm{CC}$ & $\mathrm{CG}$ & $\mathrm{CG}$ & $\mathrm{CG}$ \\
\hline $\mathrm{CG}$ & GG & $\mathrm{CC}$ & GG & $\mathrm{CG}$ & $\mathrm{CG}$ & $\mathrm{CC}$ & $\mathrm{CG}$ & $\mathrm{CG}$ & $\mathrm{CG}$ & $\mathrm{CG}$ \\
\hline $\mathrm{CC}$ & $\mathrm{CC}$ & $\mathrm{CG}$ & $\mathrm{CC}$ & $\mathrm{CC}$ & $\mathrm{CG}$ & $\mathrm{CG}$ & GG & GG & $\mathrm{CG}$ & $\mathrm{CC}$ \\
\hline $\mathrm{CG}$ & GG & $\mathrm{CC}$ & $\mathrm{CG}$ & $\mathrm{CG}$ & GG & $\mathrm{CC}$ & $\mathrm{CG}$ & $\mathrm{CG}$ & GG & $\mathrm{CC}$ \\
\hline $\mathrm{CG}$ & $\mathrm{CC}$ & GG & $\mathrm{CG}$ & $\mathrm{CG}$ & $\mathrm{CG}$ & $\mathrm{CG}$ & $\mathrm{CC}$ & GG & $\mathrm{CC}$ & \\
\hline GDsnp01493 & 3 & \multicolumn{2}{|c|}{6394694} & GG & GG & GG & $\mathrm{AG}$ & GG & GG & GG \\
\hline GG & GG & GG & GG & GG & GG & GG & GG & GG & GG & GG \\
\hline GG & $\mathrm{AG}$ & $\mathrm{AG}$ & GG & GG & $\mathrm{AG}$ & GG & $\mathrm{AG}$ & $\mathrm{AG}$ & GG & GG \\
\hline $\mathrm{AG}$ & GG & GG & GG & GG & GG & GG & GG & GG & $\mathrm{AG}$ & GG \\
\hline GG & $\mathrm{AG}$ & GG & $\mathrm{AG}$ & GG & GG & - & $\mathrm{AG}$ & GG & $\mathrm{AG}$ & GG \\
\hline GG & GG & $\mathrm{AG}$ & GG & GG & GG & $\mathrm{AG}$ & GG & GG & GG & GG \\
\hline GG & GG & GG & GG & GG & $\mathrm{AG}$ & $\mathrm{AG}$ & GG & GG & GG & GG \\
\hline GG & GG & $\mathrm{AG}$ & GG & GG & GG & GG & GG & GG & GG & GG \\
\hline $\mathrm{AG}$ & GG & GG & - & GG & GG & GG & GG & GG & GG & \\
\hline GDsnp01749 & 3 & \multicolumn{2}{|c|}{9818804} & AA & $\mathrm{AC}$ & $\mathrm{CC}$ & $\mathrm{CC}$ & $\mathrm{AC}$ & $\mathrm{AA}$ & $\mathrm{CC}$ \\
\hline AA & $\mathrm{AC}$ & $\mathrm{AC}$ & AA & $\mathrm{CC}$ & $\mathrm{CC}$ & $\mathrm{AC}$ & $\mathrm{AC}$ & $\mathrm{AC}$ & $\mathrm{AC}$ & $\mathrm{AC}$ \\
\hline $\mathrm{CC}$ & $\mathrm{AC}$ & $\mathrm{AC}$ & $\mathrm{AC}$ & $\mathrm{AC}$ & $\mathrm{CC}$ & $\mathrm{AC}$ & $\mathrm{CC}$ & $\mathrm{CC}$ & $\mathrm{AC}$ & AA \\
\hline $\mathrm{AC}$ & AA & $\mathrm{AC}$ & $\mathrm{AC}$ & $\mathrm{CC}$ & $\mathrm{AA}$ & $\mathrm{AC}$ & $\mathrm{AC}$ & $\mathrm{AA}$ & $\mathrm{AC}$ & $\mathrm{AC}$ \\
\hline $\mathrm{AC}$ & $\mathrm{CC}$ & $\mathrm{AC}$ & $\mathrm{AC}$ & $\mathrm{CC}$ & $\mathrm{AA}$ & - & $\mathrm{AC}$ & $\mathrm{AC}$ & $\mathrm{CC}$ & $\mathrm{AC}$ \\
\hline $\mathrm{AC}$ & $\mathrm{CC}$ & $\mathrm{AC}$ & $\mathrm{CC}$ & AA & $\mathrm{AC}$ & $\mathrm{AC}$ & $\mathrm{CC}$ & $\mathrm{AC}$ & $\mathrm{AC}$ & $\mathrm{AC}$ \\
\hline AA & $\mathrm{AC}$ & AA & AA & AA & $\mathrm{CC}$ & $\mathrm{CC}$ & $\mathrm{CC}$ & $\mathrm{CC}$ & $\mathrm{AC}$ & AA \\
\hline $\mathrm{CC}$ & $\mathrm{CC}$ & $\mathrm{AC}$ & $\mathrm{AC}$ & AA & $\mathrm{CC}$ & $\mathrm{AA}$ & $\mathrm{AC}$ & $\mathrm{AC}$ & $\mathrm{CC}$ & $\mathrm{CC}$ \\
\hline $\mathrm{CC}$ & $\mathrm{AC}$ & $\mathrm{CC}$ & $\mathrm{AC}$ & $\mathrm{CC}$ & $\mathrm{AC}$ & $\mathrm{AC}$ & $\mathrm{CC}$ & $\mathrm{CC}$ & $\mathrm{AA}$ & \\
\hline GDsnp00194 & 3 & \multicolumn{2}{|c|}{9876267} & GG & $\mathrm{AG}$ & AA & AA & $\mathrm{AG}$ & GG & AA \\
\hline GG & $\mathrm{AG}$ & $\mathrm{AG}$ & GG & $\mathrm{AA}$ & $\mathrm{AA}$ & $\mathrm{AG}$ & $\mathrm{AG}$ & $\mathrm{AG}$ & $\mathrm{AG}$ & $\mathrm{AG}$ \\
\hline AA & $\mathrm{AG}$ & $\mathrm{AG}$ & $\mathrm{AG}$ & $\mathrm{AG}$ & $\mathrm{AA}$ & $\mathrm{AG}$ & AA & $\mathrm{AA}$ & $\mathrm{AG}$ & GG \\
\hline AG & GG & $\mathrm{AG}$ & $\mathrm{AG}$ & $\mathrm{AA}$ & GG & $\mathrm{AG}$ & $\mathrm{AG}$ & GG & $\mathrm{AG}$ & $\mathrm{AG}$ \\
\hline AG & AA & $\mathrm{AG}$ & $\mathrm{AG}$ & - & GG & $\mathrm{AG}$ & $\mathrm{AG}$ & $\mathrm{AG}$ & - & $\mathrm{AG}$ \\
\hline AG & $\mathrm{AA}$ & $\mathrm{AG}$ & AA & GG & $\mathrm{AG}$ & $\mathrm{AG}$ & $\mathrm{AG}$ & $\mathrm{AG}$ & $\mathrm{AG}$ & $\mathrm{AG}$ \\
\hline GG & $\mathrm{AG}$ & GG & GG & GG & $\mathrm{AA}$ & $\mathrm{AA}$ & $\mathrm{AG}$ & $\mathrm{AA}$ & $\mathrm{AG}$ & GG \\
\hline $\mathrm{AG}$ & $\mathrm{AG}$ & $\mathrm{AG}$ & $\mathrm{AG}$ & $\mathrm{GG}$ & $\mathrm{AA}$ & GG & $\mathrm{AG}$ & $\mathrm{AG}$ & $\mathrm{AA}$ & $\mathrm{AA}$ \\
\hline $\mathrm{AA}$ & $\mathrm{AG}$ & $\mathrm{AA}$ & $\mathrm{AG}$ & $\mathrm{AG}$ & $\mathrm{AG}$ & $\mathrm{AG}$ & $\mathrm{AG}$ & $\mathrm{AA}$ & GG & \\
\hline
\end{tabular}

Continued next page 
Supplemental Table 2. Continued.

\begin{tabular}{|c|c|c|c|c|c|c|c|c|c|c|}
\hline \multirow{3}{*}{$\begin{array}{l}\text { SNP name } \\
\text { GDsnp01821 } \\
\text { AA }\end{array}$} & \multicolumn{10}{|c|}{ Linkage group Physical location (bp) Individuals } \\
\hline & 3 & \multicolumn{2}{|c|}{14142041} & AT & AT & TT & TT & AT & AA & TT \\
\hline & TT & AA & AA & $\mathrm{TT}$ & TT & AT & AT & $\mathrm{TT}$ & AT & AT \\
\hline $\mathrm{TT}$ & TT & AT & AT & AT & $\mathrm{TT}$ & $\mathrm{TT}$ & $\mathrm{TT}$ & $\mathrm{TT}$ & AT & AA \\
\hline $\mathrm{TT}$ & AT & TT & AT & $\mathrm{TT}$ & AA & $\mathrm{AT}$ & AT & AA & $\mathrm{TT}$ & AT \\
\hline AT & $\mathrm{TT}$ & AT & AT & $\mathrm{TT}$ & $\mathrm{AA}$ & AA & AA & $\mathrm{AT}$ & $\mathrm{TT}$ & AT \\
\hline AT & $\mathrm{TT}$ & $\mathrm{AT}$ & $\mathrm{AT}$ & $\mathrm{AA}$ & $\mathrm{AT}$ & $\mathrm{AT}$ & $\mathrm{AT}$ & $\mathrm{AT}$ & $\mathrm{AT}$ & AT \\
\hline AA & $\mathrm{AT}$ & $\mathrm{AT}$ & $\mathrm{AT}$ & $\mathrm{AA}$ & $\mathrm{TT}$ & $\mathrm{AT}$ & $\mathrm{AT}$ & $\mathrm{TT}$ & $\mathrm{AT}$ & AA \\
\hline AT & $\mathrm{AT}$ & $\mathrm{AA}$ & AT & $\mathrm{AA}$ & $\mathrm{TT}$ & $\mathrm{AA}$ & $\mathrm{AT}$ & $\mathrm{AT}$ & TT & TT \\
\hline $\mathrm{TT}$ & $\mathrm{AT}$ & $\mathrm{TT}$ & $\mathrm{AT}$ & $\mathrm{AT}$ & $\mathrm{AT}$ & $\mathrm{TT}$ & $\mathrm{AT}$ & $\mathrm{TT}$ & $\mathrm{AA}$ & \\
\hline GDsnp00921 & 3 & \multicolumn{2}{|c|}{15053657} & $\mathrm{AC}$ & AA & AA & $\mathrm{AC}$ & AA & AA & $\mathrm{AC}$ \\
\hline AA & $\mathrm{AC}$ & AA & $\mathrm{AA}$ & AA & $\mathrm{AA}$ & AA & AA & $\mathrm{AC}$ & $\mathrm{AC}$ & $\mathrm{AC}$ \\
\hline AA & $\mathrm{AC}$ & $\mathrm{AC}$ & $\mathrm{AA}$ & AA & $\mathrm{AC}$ & $\mathrm{AC}$ & $\mathrm{AC}$ & $\mathrm{AC}$ & $\mathrm{AA}$ & AA \\
\hline $\mathrm{AC}$ & AA & $\mathrm{AC}$ & $\mathrm{AA}$ & $\mathrm{AC}$ & $\mathrm{AA}$ & $\mathrm{AA}$ & $\mathrm{AA}$ & $\mathrm{AA}$ & $\mathrm{AA}$ & $\mathrm{AC}$ \\
\hline AA & $\mathrm{AC}$ & $\mathrm{AA}$ & $\mathrm{AC}$ & $\mathrm{AC}$ & $\mathrm{AA}$ & $\mathrm{AA}$ & $\mathrm{AA}$ & $\mathrm{AA}$ & $\mathrm{AC}$ & AA \\
\hline $\mathrm{AA}$ & $\mathrm{AA}$ & $\mathrm{AC}$ & $\mathrm{AA}$ & AA & $\mathrm{AA}$ & $\mathrm{AC}$ & AA & $\mathrm{AA}$ & $\mathrm{AA}$ & AA \\
\hline $\mathrm{AA}$ & $\mathrm{AA}$ & $\mathrm{AA}$ & $\mathrm{AC}$ & AA & $\mathrm{AC}$ & AA & AA & AA & $\mathrm{AA}$ & $\mathrm{AA}$ \\
\hline AA & $\mathrm{AA}$ & $\mathrm{AA}$ & AA & AA & $\mathrm{AA}$ & AA & AA & $\mathrm{AA}$ & $\mathrm{AA}$ & $\mathrm{AC}$ \\
\hline AA & AA & AA & $\mathrm{AA}$ & AA & $\mathrm{AA}$ & $\mathrm{AC}$ & AA & $\mathrm{AA}$ & $\mathrm{AA}$ & \\
\hline GDsnp00955 & 3 & \multicolumn{2}{|c|}{21125269} & $\mathrm{AC}$ & $\mathrm{CC}$ & $\mathrm{CC}$ & $\mathrm{AC}$ & $\mathrm{CC}$ & $\mathrm{CC}$ & $\mathrm{AC}$ \\
\hline $\mathrm{CC}$ & $\mathrm{CC}$ & $\mathrm{CC}$ & $\mathrm{CC}$ & $\mathrm{CC}$ & $\mathrm{CC}$ & $\mathrm{CC}$ & $\mathrm{CC}$ & $\mathrm{AC}$ & $\mathrm{AC}$ & $\mathrm{AC}$ \\
\hline $\mathrm{CC}$ & $\mathrm{AC}$ & $\mathrm{AC}$ & $\mathrm{CC}$ & $\mathrm{CC}$ & $\mathrm{AC}$ & $\mathrm{CC}$ & $\mathrm{AC}$ & $\mathrm{AC}$ & $\mathrm{AC}$ & $\mathrm{CC}$ \\
\hline $\mathrm{AC}$ & $\mathrm{CC}$ & $\mathrm{AC}$ & $\mathrm{CC}$ & $\mathrm{AC}$ & $\mathrm{CC}$ & $\mathrm{CC}$ & $\mathrm{CC}$ & $\mathrm{CC}$ & $\mathrm{CC}$ & $\mathrm{AC}$ \\
\hline $\mathrm{CC}$ & $\mathrm{AC}$ & $\mathrm{CC}$ & $\mathrm{AC}$ & $\mathrm{AC}$ & $\mathrm{AC}$ & $\mathrm{CC}$ & $\mathrm{CC}$ & $\mathrm{CC}$ & $\mathrm{AC}$ & $\mathrm{CC}$ \\
\hline $\mathrm{CC}$ & $\mathrm{CC}$ & $\mathrm{AC}$ & $\mathrm{CC}$ & $\mathrm{CC}$ & $\mathrm{CC}$ & $\mathrm{AC}$ & $\mathrm{CC}$ & $\mathrm{CC}$ & $\mathrm{CC}$ & $\mathrm{CC}$ \\
\hline $\mathrm{CC}$ & $\mathrm{CC}$ & $\mathrm{CC}$ & $\mathrm{AC}$ & $\mathrm{CC}$ & $\mathrm{AC}$ & $\mathrm{CC}$ & $\mathrm{CC}$ & $\mathrm{CC}$ & $\mathrm{CC}$ & $\mathrm{CC}$ \\
\hline $\mathrm{CC}$ & $\mathrm{CC}$ & $\mathrm{CC}$ & $\mathrm{CC}$ & $\mathrm{CC}$ & $\mathrm{CC}$ & $\mathrm{CC}$ & $\mathrm{CC}$ & $\mathrm{CC}$ & $\mathrm{CC}$ & $\mathrm{AC}$ \\
\hline $\mathrm{CC}$ & $\mathrm{CC}$ & $\mathrm{CC}$ & $\mathrm{CC}$ & $\mathrm{CC}$ & $\mathrm{CC}$ & $\mathrm{AC}$ & $\mathrm{CC}$ & $\mathrm{CC}$ & $\mathrm{CC}$ & \\
\hline GDsnp01487 & 3 & \multicolumn{2}{|c|}{22945539} & GG & - & - & GG & - & - & GG \\
\hline- & GG & GG & - & - & - & - & - & - & GG & GG \\
\hline- & GG & GG & - & - & GG & GG & GG & GG & GG & GG \\
\hline GG & - & GG & - & GG & - & - & - & - & - & - \\
\hline- & GG & - & GG & GG & GG & GG & - & - & GG & - \\
\hline- & - & GG & - & - & - & - & - & - & - & - \\
\hline- & - & - & GG & - & GG & - & - & - & GG & - \\
\hline- & - & - & - & - & - & - & - & - & - & GG \\
\hline - & - & - & - & - & - & GG & - & - & - & \\
\hline GDsnp00278 & 3 & \multicolumn{2}{|c|}{23260704} & $\mathrm{AG}$ & GG & GG & $\mathrm{AG}$ & GG & GG & AG \\
\hline- & GG & GG & GG & GG & GG & GG & GG & GG & $\mathrm{AG}$ & $\mathrm{AG}$ \\
\hline GG & $\mathrm{AG}$ & $\mathrm{AG}$ & GG & GG & $\mathrm{AG}$ & GG & $\mathrm{AG}$ & $\mathrm{AG}$ & $\mathrm{AG}$ & $\mathrm{AG}$ \\
\hline $\mathrm{AG}$ & GG & $\mathrm{AG}$ & GG & $\mathrm{AG}$ & GG & GG & GG & GG & GG & GG \\
\hline GG & $\mathrm{AG}$ & GG & $\mathrm{AG}$ & $\mathrm{AG}$ & $\mathrm{AG}$ & $\mathrm{AG}$ & GG & GG & $\mathrm{AG}$ & GG \\
\hline GG & GG & $\mathrm{AG}$ & GG & GG & GG & GG & GG & GG & GG & GG \\
\hline GG & GG & GG & $\mathrm{AG}$ & GG & $\mathrm{AG}$ & GG & GG & GG & $\mathrm{AG}$ & GG \\
\hline GG & GG & GG & GG & GG & GG & GG & GG & GG & GG & $\mathrm{AG}$ \\
\hline GG & GG & GG & GG & GG & GG & $\mathrm{AG}$ & GG & GG & GG & \\
\hline GDsnp00317 & 3 & \multicolumn{2}{|c|}{24004818} & $\mathrm{AT}$ & $\mathrm{TT}$ & $\mathrm{TT}$ & $\mathrm{AT}$ & $\mathrm{TT}$ & $\mathrm{TT}$ & AT \\
\hline AT & $\mathrm{TT}$ & $\mathrm{TT}$ & $\mathrm{TT}$ & $\mathrm{TT}$ & $\mathrm{TT}$ & $\mathrm{TT}$ & $\mathrm{TT}$ & $\mathrm{TT}$ & AT & AT \\
\hline $\mathrm{TT}$ & $\mathrm{AT}$ & $\mathrm{AT}$ & $\mathrm{TT}$ & $\mathrm{TT}$ & $\mathrm{AT}$ & $\mathrm{TT}$ & $\mathrm{AT}$ & $\mathrm{AT}$ & $\mathrm{AT}$ & AT \\
\hline AT & $\mathrm{TT}$ & $\mathrm{AT}$ & $\mathrm{TT}$ & $\mathrm{AT}$ & $\mathrm{TT}$ & $\mathrm{TT}$ & $\mathrm{TT}$ & $\mathrm{TT}$ & $\mathrm{AT}$ & TT \\
\hline TT & $\mathrm{AT}$ & $\mathrm{TT}$ & $\mathrm{AT}$ & $\mathrm{AT}$ & $\mathrm{AT}$ & - & $\mathrm{TT}$ & $\mathrm{TT}$ & AT & TT \\
\hline $\mathrm{TT}$ & $\mathrm{TT}$ & $\mathrm{AT}$ & $\mathrm{TT}$ & $\mathrm{TT}$ & $\mathrm{TT}$ & $\mathrm{TT}$ & $\mathrm{TT}$ & $\mathrm{TT}$ & $\mathrm{TT}$ & TT \\
\hline $\mathrm{TT}$ & $\mathrm{TT}$ & $\mathrm{TT}$ & $\mathrm{AT}$ & $\mathrm{TT}$ & $\mathrm{AT}$ & $\mathrm{TT}$ & $\mathrm{TT}$ & $\mathrm{TT}$ & $\mathrm{AT}$ & $\mathrm{TT}$ \\
\hline $\mathrm{TT}$ & $\mathrm{TT}$ & TT & $\mathrm{TT}$ & $\mathrm{TT}$ & $\mathrm{TT}$ & $\mathrm{TT}$ & $\mathrm{TT}$ & $\mathrm{TT}$ & $\mathrm{TT}$ & AT \\
\hline $\mathrm{TT}$ & $\mathrm{TT}$ & $\mathrm{TT}$ & $\mathrm{TT}$ & $\mathrm{TT}$ & $\mathrm{TT}$ & AT & $\mathrm{TT}$ & $\mathrm{TT}$ & $\mathrm{TT}$ & \\
\hline
\end{tabular}


Supplemental Table 2. Continued.

\begin{tabular}{|c|c|c|c|c|c|c|c|c|c|c|}
\hline \multirow{3}{*}{$\begin{array}{l}\text { SNP name } \\
\text { GDsnp01969 } \\
\text { AA }\end{array}$} & \multicolumn{10}{|c|}{ Linkage group Physical location (bp) Individuals } \\
\hline & 3 & \multicolumn{2}{|c|}{25055516} & $\mathrm{AG}$ & $\mathrm{AG}$ & GG & GG & $\mathrm{AG}$ & $\mathrm{AG}$ & GG \\
\hline & $\mathrm{AG}$ & $\mathrm{AG}$ & $\mathrm{AG}$ & GG & GG & $\mathrm{AG}$ & $\mathrm{AG}$ & $\mathrm{AG}$ & $\mathrm{AG}$ & $\mathrm{AG}$ \\
\hline GG & GG & $\mathrm{AG}$ & $\mathrm{AG}$ & AA & GG & GG & GG & GG & GG & $\mathrm{AG}$ \\
\hline GG & AG & GG & $\mathrm{AG}$ & GG & AA & $\mathrm{AG}$ & $\mathrm{AG}$ & AA & GG & $\mathrm{AA}$ \\
\hline $\mathrm{AG}$ & GG & $\mathrm{AG}$ & $\mathrm{AG}$ & GG & $\mathrm{AG}$ & $\mathrm{AG}$ & $\mathrm{AG}$ & $\mathrm{AG}$ & GG & $\mathrm{AG}$ \\
\hline $\mathrm{AG}$ & $\mathrm{AG}$ & $\mathrm{AG}$ & $\mathrm{AG}$ & $\mathrm{AG}$ & $\mathrm{AG}$ & $\mathrm{AA}$ & $\mathrm{AG}$ & $\mathrm{AG}$ & $\mathrm{AG}$ & $\mathrm{AG}$ \\
\hline $\mathrm{AA}$ & $\mathrm{AG}$ & $\mathrm{AA}$ & $\mathrm{AG}$ & AA & $\mathrm{AG}$ & $\mathrm{AG}$ & $\mathrm{AG}$ & GG & GG & $\mathrm{AG}$ \\
\hline $\mathrm{AA}$ & GG & $\mathrm{AA}$ & $\mathrm{AG}$ & AA & GG & $\mathrm{AA}$ & GG & $\mathrm{AG}$ & GG & GG \\
\hline $\mathrm{AG}$ & $\mathrm{AG}$ & GG & $\mathrm{AG}$ & $\mathrm{AG}$ & $\mathrm{AG}$ & GG & GG & GG & $\mathrm{AG}$ & \\
\hline GDsnp01329 & 3 & \multicolumn{2}{|c|}{27949058} & $\mathrm{AG}$ & $\mathrm{AA}$ & AA & $\mathrm{AG}$ & $\mathrm{AA}$ & $\mathrm{AA}$ & $\mathrm{AG}$ \\
\hline- & $\mathrm{AA}$ & $\mathrm{AG}$ & $\mathrm{AG}$ & AA & $\mathrm{AA}$ & AA & AA & $\mathrm{AG}$ & $\mathrm{AA}$ & $\mathrm{AA}$ \\
\hline AA & $\mathrm{AG}$ & $\mathrm{AG}$ & $\mathrm{AA}$ & AA & $\mathrm{AG}$ & $\mathrm{AA}$ & $\mathrm{AG}$ & $\mathrm{AG}$ & $\mathrm{AG}$ & $\mathrm{AG}$ \\
\hline $\mathrm{AG}$ & AA & $\mathrm{AG}$ & $\mathrm{AA}$ & $\mathrm{AG}$ & $\mathrm{AA}$ & AA & AA & $\mathrm{AA}$ & $\mathrm{AA}$ & $\mathrm{AG}$ \\
\hline $\mathrm{AA}$ & $\mathrm{AG}$ & AA & $\mathrm{AG}$ & $\mathrm{AG}$ & $\mathrm{AG}$ & - & $\mathrm{AA}$ & $\mathrm{AA}$ & $\mathrm{AG}$ & AA \\
\hline $\mathrm{AA}$ & $\mathrm{AA}$ & $\mathrm{AG}$ & $\mathrm{AG}$ & AA & $\mathrm{AA}$ & AA & AA & AA & $\mathrm{AA}$ & $\mathrm{AA}$ \\
\hline AA & AA & AA & $\mathrm{AG}$ & AA & $\mathrm{AG}$ & $\mathrm{AA}$ & $\mathrm{AG}$ & $\mathrm{AA}$ & $\mathrm{AG}$ & AA \\
\hline AA & AA & $\mathrm{AA}$ & $\mathrm{AA}$ & AA & $\mathrm{AA}$ & - & AA & $\mathrm{AA}$ & $\mathrm{AA}$ & $\mathrm{AG}$ \\
\hline AA & AA & AA & AA & AA & - & AA & AA & AA & $\mathrm{AA}$ & \\
\hline GDsnp01667 & 3 & \multicolumn{2}{|c|}{28332036} & $\mathrm{AG}$ & $\mathrm{AA}$ & $\mathrm{AA}$ & $\mathrm{AG}$ & $\mathrm{AA}$ & $\mathrm{AA}$ & $\mathrm{AG}$ \\
\hline $\mathrm{AG}$ & AA & $\mathrm{AG}$ & - & AA & $\mathrm{AA}$ & AA & AA & $\mathrm{AG}$ & $\mathrm{AA}$ & AA \\
\hline $\mathrm{AA}$ & $\mathrm{AG}$ & $\mathrm{AG}$ & $\mathrm{AA}$ & AA & $\mathrm{AG}$ & $\mathrm{AG}$ & $\mathrm{AG}$ & $\mathrm{AG}$ & $\mathrm{AG}$ & $\mathrm{AG}$ \\
\hline $\mathrm{AA}$ & $\mathrm{AA}$ & $\mathrm{AG}$ & $\mathrm{AA}$ & $\mathrm{AG}$ & $\mathrm{AA}$ & AA & AA & AA & $\mathrm{AA}$ & $\mathrm{AG}$ \\
\hline AA & $\mathrm{AG}$ & $\mathrm{AA}$ & $\mathrm{AG}$ & $\mathrm{AG}$ & $\mathrm{AG}$ & - & $\mathrm{AA}$ & $\mathrm{AA}$ & $\mathrm{AG}$ & $\mathrm{AA}$ \\
\hline AA & AA & $\mathrm{AG}$ & $\mathrm{AG}$ & AA & $\mathrm{AA}$ & AA & AA & AA & $\mathrm{AA}$ & AA \\
\hline AA & AA & AA & $\mathrm{AG}$ & AA & $\mathrm{AG}$ & AA & - & $\mathrm{AA}$ & $\mathrm{AG}$ & AA \\
\hline AA & AA & AA & AA & AA & $\mathrm{AA}$ & AA & AA & AA & $\mathrm{AA}$ & $\mathrm{AG}$ \\
\hline $\mathrm{AA}$ & AA & AA & $\mathrm{AA}$ & AA & $\mathrm{AA}$ & $\mathrm{AA}$ & $\mathrm{AA}$ & $\mathrm{AA}$ & $\mathrm{AA}$ & \\
\hline GDsnp02030 & 3 & \multicolumn{2}{|c|}{29276414} & $\mathrm{AG}$ & $\mathrm{AA}$ & AA & $\mathrm{AG}$ & AA & $\mathrm{AG}$ & $\mathrm{AG}$ \\
\hline $\mathrm{AA}$ & $\mathrm{AG}$ & $\mathrm{AG}$ & $\mathrm{AA}$ & AA & $\mathrm{AA}$ & $\mathrm{AA}$ & AA & $\mathrm{AG}$ & $\mathrm{AA}$ & $\mathrm{AA}$ \\
\hline AA & $\mathrm{AG}$ & $\mathrm{AG}$ & $\mathrm{AA}$ & AA & $\mathrm{AG}$ & AA & $\mathrm{AG}$ & $\mathrm{AG}$ & $\mathrm{AG}$ & $\mathrm{AG}$ \\
\hline AA & AA & $\mathrm{AG}$ & AA & $\mathrm{AG}$ & $\mathrm{AA}$ & AA & AA & AA & $\mathrm{AA}$ & $\mathrm{AG}$ \\
\hline AA & $\mathrm{AG}$ & AA & $\mathrm{AG}$ & $\mathrm{AG}$ & $\mathrm{AG}$ & $\mathrm{AG}$ & AA & AA & $\mathrm{AG}$ & AA \\
\hline AA & AA & $\mathrm{AG}$ & $\mathrm{AG}$ & AA & $\mathrm{AA}$ & AA & AA & AA & $\mathrm{AA}$ & AA \\
\hline AA & AA & $\mathrm{AA}$ & $\mathrm{AG}$ & AA & $\mathrm{AG}$ & AA & AA & $\mathrm{AA}$ & $\mathrm{AG}$ & $\mathrm{AA}$ \\
\hline AA & AA & AA & AA & AA & $\mathrm{AA}$ & AA & AA & AA & $\mathrm{AA}$ & $\mathrm{AG}$ \\
\hline AA & AA & AA & $\mathrm{AA}$ & $\mathrm{AA}$ & $\mathrm{AA}$ & $\mathrm{AA}$ & $\mathrm{AA}$ & $\mathrm{AA}$ & $\mathrm{AA}$ & \\
\hline GDsnp01396 & 3 & \multicolumn{2}{|c|}{29562317} & $\mathrm{AG}$ & GG & GG & $\mathrm{AG}$ & GG & $\mathrm{AG}$ & $\mathrm{AG}$ \\
\hline GG & $\mathrm{AG}$ & $\mathrm{AG}$ & GG & GG & GG & GG & GG & $\mathrm{AG}$ & GG & GG \\
\hline GG & $\mathrm{AG}$ & $\mathrm{AG}$ & GG & GG & $\mathrm{AG}$ & GG & $\mathrm{AG}$ & $\mathrm{AG}$ & $\mathrm{AG}$ & $\mathrm{AG}$ \\
\hline GG & GG & $\mathrm{AG}$ & GG & $\mathrm{AG}$ & GG & GG & GG & GG & GG & $\mathrm{AG}$ \\
\hline GG & $\mathrm{AG}$ & GG & $\mathrm{AG}$ & $\mathrm{AG}$ & $\mathrm{AG}$ & $\mathrm{AG}$ & GG & GG & $\mathrm{AG}$ & GG \\
\hline GG & GG & $\mathrm{AG}$ & $\mathrm{AG}$ & GG & GG & GG & GG & GG & GG & GG \\
\hline GG & GG & GG & $\mathrm{AG}$ & GG & $\mathrm{AG}$ & GG & GG & GG & $\mathrm{AG}$ & GG \\
\hline GG & GG & GG & GG & GG & GG & GG & GG & GG & GG & $\mathrm{AG}$ \\
\hline GG & GG & GG & GG & GG & GG & GG & GG & GG & GG & \\
\hline GDsnp00664 & 3 & \multicolumn{2}{|c|}{31592370} & GG & $\mathrm{AG}$ & GG & GG & GG & $\mathrm{AG}$ & GG \\
\hline $\mathrm{AG}$ & GG & $\mathrm{AG}$ & $\mathrm{AG}$ & GG & GG & GG & $\mathrm{AG}$ & GG & $\mathrm{AG}$ & $\mathrm{AG}$ \\
\hline $\mathrm{AG}$ & GG & $\mathrm{AG}$ & $\mathrm{AG}$ & AA & $\mathrm{AG}$ & GG & $\mathrm{AG}$ & $\mathrm{AG}$ & GG & $\mathrm{AG}$ \\
\hline GG & $\mathrm{AG}$ & GG & $\mathrm{AG}$ & GG & $\mathrm{AA}$ & AA & AA & $\mathrm{AA}$ & $\mathrm{AG}$ & $\mathrm{AG}$ \\
\hline $\mathrm{AG}$ & GG & AA & GG & $\mathrm{GG}$ & $\mathrm{AG}$ & $\mathrm{AG}$ & $\mathrm{AG}$ & $\mathrm{AG}$ & GG & GG \\
\hline $\mathrm{AG}$ & $\mathrm{AG}$ & $\mathrm{AG}$ & $\mathrm{AG}$ & $\mathrm{AG}$ & $\mathrm{AG}$ & $\mathrm{AA}$ & $\mathrm{AG}$ & $\mathrm{AA}$ & $\mathrm{AG}$ & $\mathrm{AG}$ \\
\hline $\mathrm{AG}$ & $\mathrm{AG}$ & $\mathrm{AA}$ & $\mathrm{AG}$ & AA & $\mathrm{AG}$ & $\mathrm{AG}$ & GG & GG & GG & $\mathrm{AG}$ \\
\hline AA & GG & $\mathrm{AA}$ & $\mathrm{AG}$ & $\mathrm{AG}$ & GG & $\mathrm{AA}$ & GG & $\mathrm{AG}$ & GG & GG \\
\hline $\mathrm{AG}$ & $\mathrm{AG}$ & $\mathrm{AG}$ & - & $\mathrm{AG}$ & $\mathrm{AG}$ & GG & $\mathrm{AG}$ & GG & $\mathrm{AG}$ & \\
\hline
\end{tabular}


Supplemental Table 2. Continued.

\begin{tabular}{|c|c|c|c|c|c|c|c|c|c|c|}
\hline \multirow{3}{*}{$\begin{array}{l}\text { SNP name } \\
\text { GDsnp01073 } \\
\text { TT }\end{array}$} & \multicolumn{10}{|c|}{ Linkage group Physical location (bp) Individuals } \\
\hline & 3 & \multicolumn{2}{|c|}{32321745} & $\mathrm{AT}$ & $\mathrm{AT}$ & AT & AT & TT & AT & AT \\
\hline & $\mathrm{TT}$ & $\mathrm{AT}$ & TT & $\mathrm{TT}$ & $\mathrm{AT}$ & AT & AT & AT & TT & TT \\
\hline TT & $\mathrm{TT}$ & AT & $\mathrm{TT}$ & TT & TT & $\mathrm{TT}$ & AT & AT & AT & TT \\
\hline TT & $\mathrm{AT}$ & $\mathrm{AT}$ & $\mathrm{TT}$ & $\mathrm{AT}$ & $\mathrm{TT}$ & $\mathrm{TT}$ & $\mathrm{TT}$ & $\mathrm{TT}$ & $\mathrm{TT}$ & AT \\
\hline TT & $\mathrm{TT}$ & $\mathrm{TT}$ & AT & AT & $\mathrm{TT}$ & $\mathrm{AT}$ & $\mathrm{TT}$ & $\mathrm{TT}$ & $\mathrm{TT}$ & AT \\
\hline AT & $\mathrm{TT}$ & $\mathrm{AT}$ & $\mathrm{AT}$ & $\mathrm{AT}$ & $\mathrm{AT}$ & $\mathrm{TT}$ & $\mathrm{TT}$ & $\mathrm{TT}$ & $\mathrm{TT}$ & TT \\
\hline AT & $\mathrm{TT}$ & $\mathrm{TT}$ & $\mathrm{AT}$ & $\mathrm{AT}$ & $\mathrm{AT}$ & $\mathrm{TT}$ & $\mathrm{AT}$ & $\mathrm{AT}$ & $\mathrm{AT}$ & AT \\
\hline TT & $\mathrm{AT}$ & $\mathrm{TT}$ & $\mathrm{TT}$ & $\mathrm{AT}$ & AT & $\mathrm{AT}$ & $\mathrm{TT}$ & $\mathrm{AT}$ & TT & TT \\
\hline TT & $\mathrm{AT}$ & $\mathrm{TT}$ & $\mathrm{AT}$ & $\mathrm{TT}$ & $\mathrm{TT}$ & $\mathrm{TT}$ & $\mathrm{TT}$ & $\mathrm{AT}$ & $\mathrm{AT}$ & \\
\hline GDsnp00319 & 3 & \multicolumn{2}{|c|}{32713096} & $\mathrm{AG}$ & $\mathrm{AG}$ & $\mathrm{AG}$ & $\mathrm{AG}$ & GG & $\mathrm{AG}$ & $\mathrm{AG}$ \\
\hline- & GG & $\mathrm{AG}$ & $\mathrm{AG}$ & GG & $\mathrm{AG}$ & $\mathrm{AG}$ & $\mathrm{AG}$ & $\mathrm{AG}$ & GG & GG \\
\hline GG & GG & $\mathrm{AG}$ & GG & GG & GG & $\mathrm{AG}$ & $\mathrm{AG}$ & $\mathrm{AG}$ & $\mathrm{AG}$ & GG \\
\hline GG & $\mathrm{AG}$ & $\mathrm{AG}$ & GG & $\mathrm{AG}$ & GG & GG & GG & GG & GG & $\mathrm{AG}$ \\
\hline GG & GG & GG & $\mathrm{AG}$ & $\mathrm{AG}$ & GG & $\mathrm{AG}$ & GG & GG & GG & $\mathrm{AG}$ \\
\hline $\mathrm{AG}$ & GG & $\mathrm{AG}$ & $\mathrm{AG}$ & $\mathrm{AG}$ & $\mathrm{AG}$ & GG & GG & GG & GG & GG \\
\hline AG & GG & GG & $\mathrm{AG}$ & $\mathrm{AG}$ & $\mathrm{AG}$ & GG & $\mathrm{AG}$ & $\mathrm{AG}$ & $\mathrm{AG}$ & $\mathrm{AG}$ \\
\hline GG & $\mathrm{AG}$ & GG & GG & $\mathrm{AG}$ & $\mathrm{AG}$ & $\mathrm{AG}$ & GG & $\mathrm{AG}$ & GG & GG \\
\hline GG & $\mathrm{AG}$ & GG & $\mathrm{AG}$ & GG & GG & $\mathrm{AG}$ & GG & $\mathrm{AG}$ & $\mathrm{AG}$ & \\
\hline GDsnp01604 & 3 & \multicolumn{2}{|c|}{33113442} & $\mathrm{AC}$ & $\mathrm{AC}$ & $\mathrm{AC}$ & $\mathrm{AC}$ & $\mathrm{CC}$ & $\mathrm{AC}$ & $\mathrm{AC}$ \\
\hline $\mathrm{CC}$ & $\mathrm{CC}$ & $\mathrm{AC}$ & $\mathrm{CC}$ & $\mathrm{CC}$ & $\mathrm{AC}$ & $\mathrm{AC}$ & $\mathrm{AC}$ & $\mathrm{AC}$ & $\mathrm{CC}$ & $\mathrm{CC}$ \\
\hline $\mathrm{CC}$ & $\mathrm{CC}$ & $\mathrm{AC}$ & $\mathrm{CC}$ & $\mathrm{CC}$ & $\mathrm{CC}$ & $\mathrm{CC}$ & $\mathrm{AC}$ & $\mathrm{AC}$ & $\mathrm{AC}$ & $\mathrm{CC}$ \\
\hline $\mathrm{CC}$ & $\mathrm{AC}$ & $\mathrm{AC}$ & $\mathrm{CC}$ & $\mathrm{AC}$ & $\mathrm{CC}$ & $\mathrm{CC}$ & $\mathrm{CC}$ & $\mathrm{CC}$ & $\mathrm{CC}$ & $\mathrm{AC}$ \\
\hline $\mathrm{CC}$ & $\mathrm{CC}$ & $\mathrm{CC}$ & $\mathrm{AC}$ & $\mathrm{AC}$ & $\mathrm{CC}$ & $\mathrm{AC}$ & $\mathrm{CC}$ & $\mathrm{CC}$ & $\mathrm{CC}$ & $\mathrm{AC}$ \\
\hline $\mathrm{AC}$ & $\mathrm{CC}$ & $\mathrm{AC}$ & $\mathrm{AC}$ & $\mathrm{AC}$ & $\mathrm{AC}$ & $\mathrm{CC}$ & $\mathrm{CC}$ & $\mathrm{CC}$ & $\mathrm{CC}$ & $\mathrm{CC}$ \\
\hline $\mathrm{AC}$ & $\mathrm{CC}$ & $\mathrm{CC}$ & $\mathrm{AC}$ & $\mathrm{AC}$ & $\mathrm{AC}$ & $\mathrm{CC}$ & $\mathrm{AC}$ & $\mathrm{AC}$ & $\mathrm{AC}$ & $\mathrm{AC}$ \\
\hline $\mathrm{CC}$ & $\mathrm{AC}$ & $\mathrm{CC}$ & $\mathrm{CC}$ & $\mathrm{AC}$ & $\mathrm{AC}$ & $\mathrm{AC}$ & $\mathrm{CC}$ & $\mathrm{AC}$ & $\mathrm{CC}$ & $\mathrm{CC}$ \\
\hline $\mathrm{CC}$ & $\mathrm{AC}$ & $\mathrm{CC}$ & $\mathrm{AC}$ & $\mathrm{CC}$ & $\mathrm{CC}$ & $\mathrm{AC}$ & $\mathrm{CC}$ & $\mathrm{AC}$ & $\mathrm{AC}$ & \\
\hline GDsnp00168 & 3 & \multicolumn{2}{|c|}{33165007} & $\mathrm{CG}$ & $\mathrm{CG}$ & $\mathrm{CG}$ & $\mathrm{CG}$ & $\mathrm{CC}$ & $\mathrm{CG}$ & $\mathrm{CG}$ \\
\hline CG & $\mathrm{CC}$ & $\mathrm{CG}$ & $\mathrm{CC}$ & $\mathrm{CC}$ & $\mathrm{CG}$ & $\mathrm{CG}$ & $\mathrm{CG}$ & $\mathrm{CG}$ & $\mathrm{CC}$ & $\mathrm{CC}$ \\
\hline $\mathrm{CC}$ & $\mathrm{CC}$ & $\mathrm{CG}$ & $\mathrm{CC}$ & $\mathrm{CC}$ & $\mathrm{CC}$ & $\mathrm{CC}$ & $\mathrm{CG}$ & $\mathrm{CG}$ & $\mathrm{CG}$ & $\mathrm{CC}$ \\
\hline $\mathrm{CC}$ & $\mathrm{CG}$ & $\mathrm{CG}$ & $\mathrm{CC}$ & $\mathrm{CG}$ & $\mathrm{CC}$ & $\mathrm{CC}$ & $\mathrm{CC}$ & $\mathrm{CC}$ & $\mathrm{CC}$ & $\mathrm{CG}$ \\
\hline $\mathrm{CC}$ & $\mathrm{CC}$ & $\mathrm{CC}$ & $\mathrm{CG}$ & $\mathrm{CG}$ & $\mathrm{CC}$ & - & $\mathrm{CC}$ & $\mathrm{CC}$ & $\mathrm{CC}$ & $\mathrm{CG}$ \\
\hline CG & $\mathrm{CC}$ & $\mathrm{CG}$ & $\mathrm{CG}$ & $\mathrm{CG}$ & $\mathrm{CG}$ & $\mathrm{CC}$ & $\mathrm{CC}$ & $\mathrm{CC}$ & $\mathrm{CC}$ & $\mathrm{CC}$ \\
\hline CG & $\mathrm{CC}$ & $\mathrm{CC}$ & $\mathrm{CG}$ & $\mathrm{CG}$ & $\mathrm{CG}$ & $\mathrm{CC}$ & $\mathrm{CG}$ & $\mathrm{CG}$ & $\mathrm{CG}$ & $\mathrm{CG}$ \\
\hline $\mathrm{CC}$ & $\mathrm{CG}$ & $\mathrm{CC}$ & $\mathrm{CC}$ & $\mathrm{CG}$ & $\mathrm{CG}$ & $\mathrm{CG}$ & $\mathrm{CC}$ & $\mathrm{CG}$ & $\mathrm{CC}$ & $\mathrm{CC}$ \\
\hline $\mathrm{CC}$ & $\mathrm{CG}$ & $\mathrm{CC}$ & $\mathrm{CG}$ & $\mathrm{CC}$ & $\mathrm{CC}$ & CG & $\mathrm{CC}$ & CG & $\mathrm{CG}$ & \\
\hline GDsnp00237 & 4 & \multicolumn{2}{|c|}{796479} & GG & GG & GG & GG & GG & GG & GG \\
\hline GG & $\mathrm{CG}$ & GG & GG & GG & GG & GG & GG & GG & GG & GG \\
\hline GG & GG & GG & GG & GG & GG & GG & GG & GG & GG & GG \\
\hline GG & GG & GG & GG & GG & GG & GG & GG & GG & GG & GG \\
\hline GG & GG & GG & GG & GG & GG & GG & GG & GG & GG & GG \\
\hline GG & GG & GG & GG & GG & GG & GG & GG & GG & GG & GG \\
\hline GG & GG & GG & GG & GG & GG & GG & GG & GG & GG & GG \\
\hline GG & GG & GG & GG & GG & GG & GG & GG & GG & GG & GG \\
\hline GG & GG & GG & GG & GG & GG & $\mathrm{CG}$ & GG & GG & GG & \\
\hline GDsnp00904 & 4 & \multicolumn{2}{|c|}{3509721} & AA & $\mathrm{AA}$ & $\mathrm{AG}$ & AA & AA & AA & $\mathrm{AG}$ \\
\hline $\mathrm{AG}$ & AA & $\mathrm{AG}$ & $\mathrm{AG}$ & $\mathrm{AA}$ & $\mathrm{AG}$ & $\mathrm{AA}$ & $\mathrm{AG}$ & $\mathrm{AA}$ & $\mathrm{AA}$ & AA \\
\hline AG & AA & AA & $\mathrm{AG}$ & $\mathrm{AG}$ & $\mathrm{AG}$ & $\mathrm{AG}$ & AA & $\mathrm{AA}$ & $\mathrm{AA}$ & AA \\
\hline $\mathrm{AG}$ & AA & AA & $\mathrm{AA}$ & $\mathrm{AA}$ & $\mathrm{AA}$ & $\mathrm{AA}$ & $\mathrm{AA}$ & $\mathrm{AA}$ & $\mathrm{AG}$ & AA \\
\hline AA & AA & AA & $\mathrm{AA}$ & $\mathrm{AA}$ & $\mathrm{AA}$ & $\mathrm{AA}$ & $\mathrm{AA}$ & $\mathrm{AA}$ & $\mathrm{AA}$ & AA \\
\hline $\mathrm{AA}$ & $\mathrm{AG}$ & $\mathrm{AA}$ & $\mathrm{AA}$ & $\mathrm{AA}$ & $\mathrm{AA}$ & $\mathrm{AA}$ & $\mathrm{AA}$ & $\mathrm{AA}$ & $\mathrm{AA}$ & $\mathrm{AG}$ \\
\hline $\mathrm{AA}$ & AA & AA & $\mathrm{AA}$ & AA & $\mathrm{AA}$ & $\mathrm{AA}$ & $\mathrm{AA}$ & $\mathrm{AA}$ & $\mathrm{AA}$ & $\mathrm{AG}$ \\
\hline $\mathrm{AA}$ & AA & $\mathrm{AA}$ & $\mathrm{AG}$ & AA & $\mathrm{AA}$ & $\mathrm{AA}$ & $\mathrm{AA}$ & $\mathrm{AA}$ & $\mathrm{AA}$ & $\mathrm{AG}$ \\
\hline AG & AA & AA & $\mathrm{AA}$ & AA & $\mathrm{AG}$ & $\mathrm{AA}$ & $\mathrm{AA}$ & $\mathrm{AG}$ & $\mathrm{AG}$ & \\
\hline
\end{tabular}


Supplemental Table 2. Continued.

\begin{tabular}{|c|c|c|c|c|c|c|c|c|c|c|}
\hline \multirow{3}{*}{$\begin{array}{l}\text { SNP name } \\
\text { GDsnp01628 } \\
\text { AG }\end{array}$} & \multicolumn{10}{|c|}{ Linkage group Physical location (bp) Individuals } \\
\hline & 4 & \multicolumn{2}{|c|}{3963790} & AA & $\mathrm{AG}$ & $\mathrm{AG}$ & $\mathrm{AG}$ & GG & $\mathrm{AG}$ & GG \\
\hline & $\mathrm{AG}$ & $\mathrm{AG}$ & AA & GG & GG & $\mathrm{AA}$ & GG & $\mathrm{AG}$ & $\mathrm{AG}$ & GG \\
\hline GG & $\mathrm{AG}$ & GG & GG & GG & GG & $\mathrm{AA}$ & AA & AA & $\mathrm{AA}$ & GG \\
\hline $\mathrm{AG}$ & AA & $\mathrm{AG}$ & GG & AG & AA & $\mathrm{AG}$ & GG & $\mathrm{AG}$ & $\mathrm{AG}$ & $\mathrm{AA}$ \\
\hline GG & GG & GG & AA & $\mathrm{AG}$ & $\mathrm{AG}$ & $\mathrm{AA}$ & $\mathrm{AG}$ & $\mathrm{AG}$ & GG & AA \\
\hline AA & GG & $\mathrm{AG}$ & $\mathrm{AG}$ & AA & $\mathrm{AG}$ & $\mathrm{AG}$ & $\mathrm{AG}$ & $\mathrm{AG}$ & $\mathrm{AA}$ & $\mathrm{AG}$ \\
\hline $\mathrm{AG}$ & GG & $\mathrm{AA}$ & $\mathrm{AG}$ & $\mathrm{AG}$ & $\mathrm{AA}$ & GG & $\mathrm{AG}$ & $\mathrm{AA}$ & $\mathrm{AG}$ & GG \\
\hline GG & $\mathrm{AA}$ & $\mathrm{AG}$ & GG & $\mathrm{AG}$ & $\mathrm{AA}$ & $\mathrm{AG}$ & GG & $\mathrm{AA}$ & $\mathrm{AG}$ & $\mathrm{AG}$ \\
\hline AG & AA & AA & AA & $\mathrm{AG}$ & GG & $\mathrm{AG}$ & GG & GG & $\mathrm{AG}$ & \\
\hline GDsnp01656 & 4 & \multicolumn{2}{|c|}{4516066} & $\mathrm{AC}$ & $\mathrm{AC}$ & $\mathrm{CC}$ & $\mathrm{AC}$ & $\mathrm{CC}$ & $\mathrm{AC}$ & $\mathrm{CC}$ \\
\hline $\mathrm{CC}$ & $\mathrm{AC}$ & $\mathrm{CC}$ & $\mathrm{CC}$ & $\mathrm{CC}$ & $\mathrm{CC}$ & $\mathrm{AC}$ & $\mathrm{CC}$ & $\mathrm{AC}$ & $\mathrm{AC}$ & $\mathrm{CC}$ \\
\hline $\mathrm{CC}$ & $\mathrm{CC}$ & $\mathrm{CC}$ & $\mathrm{CC}$ & $\mathrm{CC}$ & $\mathrm{CC}$ & $\mathrm{CC}$ & $\mathrm{AC}$ & $\mathrm{AC}$ & $\mathrm{AC}$ & $\mathrm{CC}$ \\
\hline $\mathrm{CC}$ & $\mathrm{AC}$ & $\mathrm{CC}$ & $\mathrm{CC}$ & $\mathrm{AC}$ & $\mathrm{AC}$ & $\mathrm{AC}$ & $\mathrm{CC}$ & $\mathrm{AC}$ & $\mathrm{AC}$ & $\mathrm{AC}$ \\
\hline $\mathrm{CC}$ & $\mathrm{CC}$ & $\mathrm{CC}$ & $\mathrm{AC}$ & $\mathrm{CC}$ & $\mathrm{CC}$ & $\mathrm{AC}$ & $\mathrm{CC}$ & $\mathrm{CC}$ & $\mathrm{CC}$ & $\mathrm{AC}$ \\
\hline $\mathrm{AC}$ & $\mathrm{CC}$ & $\mathrm{AC}$ & $\mathrm{AC}$ & $\mathrm{AC}$ & $\mathrm{AC}$ & $\mathrm{AC}$ & $\mathrm{AC}$ & $\mathrm{CC}$ & $\mathrm{AC}$ & $\mathrm{CC}$ \\
\hline $\mathrm{AC}$ & $\mathrm{CC}$ & $\mathrm{AC}$ & $\mathrm{AC}$ & $\mathrm{CC}$ & $\mathrm{AC}$ & $\mathrm{CC}$ & $\mathrm{CC}$ & $\mathrm{AC}$ & $\mathrm{AC}$ & $\mathrm{CC}$ \\
\hline $\mathrm{CC}$ & $\mathrm{CC}$ & $\mathrm{AC}$ & $\mathrm{CC}$ & $\mathrm{AC}$ & $\mathrm{AC}$ & $\mathrm{CC}$ & $\mathrm{CC}$ & $\mathrm{AC}$ & $\mathrm{CC}$ & $\mathrm{CC}$ \\
\hline $\mathrm{CC}$ & $\mathrm{AC}$ & $\mathrm{AC}$ & $\mathrm{AC}$ & $\mathrm{CC}$ & $\mathrm{CC}$ & $\mathrm{CC}$ & $\mathrm{CC}$ & $\mathrm{CC}$ & $\mathrm{CC}$ & \\
\hline GDsnp00363 & 4 & \multicolumn{2}{|c|}{6434983} & $\mathrm{AG}$ & $\mathrm{AG}$ & $\mathrm{AG}$ & $\mathrm{AG}$ & GG & $\mathrm{AG}$ & $\mathrm{AG}$ \\
\hline $\mathrm{AG}$ & $\mathrm{AG}$ & $\mathrm{AG}$ & $\mathrm{AG}$ & GG & $\mathrm{AG}$ & $\mathrm{AG}$ & $\mathrm{AG}$ & $\mathrm{AG}$ & $\mathrm{AG}$ & GG \\
\hline $\mathrm{AG}$ & GG & $\mathrm{AG}$ & $\mathrm{AG}$ & $\mathrm{AG}$ & $\mathrm{AG}$ & $\mathrm{AG}$ & $\mathrm{AG}$ & $\mathrm{AG}$ & $\mathrm{AG}$ & GG \\
\hline GG & $\mathrm{AG}$ & GG & GG & $\mathrm{AG}$ & $\mathrm{AG}$ & $\mathrm{AG}$ & GG & $\mathrm{AG}$ & $\mathrm{AG}$ & $\mathrm{AG}$ \\
\hline AG & GG & GG & GG & GG & GG & $\mathrm{AG}$ & $\mathrm{AG}$ & GG & GG & $\mathrm{AG}$ \\
\hline $\mathrm{AG}$ & $\mathrm{AG}$ & $\mathrm{AG}$ & $\mathrm{AG}$ & $\mathrm{AG}$ & $\mathrm{AG}$ & $\mathrm{AG}$ & $\mathrm{AG}$ & GG & $\mathrm{AG}$ & $\mathrm{AG}$ \\
\hline $\mathrm{AG}$ & $\mathrm{AG}$ & $\mathrm{AG}$ & $\mathrm{AG}$ & GG & GG & GG & $\mathrm{AG}$ & $\mathrm{AG}$ & $\mathrm{AG}$ & $\mathrm{AG}$ \\
\hline GG & GG & $\mathrm{AG}$ & $\mathrm{AG}$ & $\mathrm{AG}$ & $\mathrm{AG}$ & GG & $\mathrm{AG}$ & $\mathrm{AG}$ & $\mathrm{AG}$ & $\mathrm{AG}$ \\
\hline $\mathrm{AG}$ & $\mathrm{AG}$ & $\mathrm{AG}$ & $\mathrm{AG}$ & GG & $\mathrm{AG}$ & $\mathrm{AG}$ & GG & $\mathrm{AG}$ & $\mathrm{AG}$ & \\
\hline GDsnp02646 & 4 & \multicolumn{2}{|c|}{8280892} & AA & $\mathrm{AA}$ & $\mathrm{AA}$ & AA & $\mathrm{AA}$ & $\mathrm{AA}$ & AA \\
\hline AA & AA & AA & AA & AA & $\mathrm{AA}$ & $\mathrm{AA}$ & AA & AA & $\mathrm{AA}$ & AA \\
\hline AA & $\mathrm{AA}$ & $\mathrm{AA}$ & $\mathrm{AA}$ & $\mathrm{AA}$ & $\mathrm{AA}$ & $\mathrm{AA}$ & AA & $\mathrm{AA}$ & $\mathrm{AA}$ & $\mathrm{AA}$ \\
\hline AA & $\mathrm{AA}$ & $\mathrm{AA}$ & $\mathrm{AA}$ & AA & $\mathrm{AA}$ & $\mathrm{AA}$ & $\mathrm{AA}$ & $\mathrm{AA}$ & - & AA \\
\hline AA & AA & AA & AA & AA & $\mathrm{AA}$ & - & AA & $\mathrm{AA}$ & $\mathrm{AA}$ & AA \\
\hline $\mathrm{AA}$ & $\mathrm{AA}$ & $\mathrm{AA}$ & $\mathrm{AA}$ & AA & $\mathrm{AA}$ & $\mathrm{AA}$ & AA & AA & $\mathrm{AA}$ & $\mathrm{AA}$ \\
\hline AA & $\mathrm{AA}$ & $\mathrm{AA}$ & $\mathrm{AA}$ & $\mathrm{AA}$ & $\mathrm{AA}$ & $\mathrm{AA}$ & AA & $\mathrm{AA}$ & $\mathrm{AA}$ & $\mathrm{AA}$ \\
\hline AA & AA & AA & $\mathrm{AA}$ & AA & $\mathrm{AA}$ & $\mathrm{AA}$ & AA & $\mathrm{AA}$ & $\mathrm{AA}$ & $\mathrm{AA}$ \\
\hline AA & AA & AA & AA & AA & AA & AA & AA & AA & $\mathrm{AA}$ & \\
\hline GDsnp00727 & 4 & \multicolumn{2}{|c|}{10031840} & $\mathrm{CG}$ & CG & CG & $\mathrm{CG}$ & $\mathrm{CC}$ & $\mathrm{CG}$ & $\mathrm{CG}$ \\
\hline $\mathrm{CC}$ & $\mathrm{CG}$ & $\mathrm{CG}$ & $\mathrm{CG}$ & $\mathrm{CC}$ & $\mathrm{CG}$ & $\mathrm{CG}$ & $\mathrm{CG}$ & $\mathrm{CG}$ & $\mathrm{CG}$ & $\mathrm{CC}$ \\
\hline $\mathrm{CG}$ & $\mathrm{CC}$ & $\mathrm{CG}$ & $\mathrm{CG}$ & CG & $\mathrm{CC}$ & $\mathrm{CC}$ & $\mathrm{CG}$ & $\mathrm{CG}$ & $\mathrm{CG}$ & $\mathrm{CC}$ \\
\hline $\mathrm{CC}$ & $\mathrm{CG}$ & $\mathrm{CC}$ & $\mathrm{CC}$ & CG & $\mathrm{CG}$ & $\mathrm{CG}$ & $\mathrm{CC}$ & $\mathrm{CG}$ & $\mathrm{CC}$ & $\mathrm{CG}$ \\
\hline $\mathrm{CG}$ & $\mathrm{CC}$ & $\mathrm{CC}$ & $\mathrm{CC}$ & $\mathrm{CC}$ & $\mathrm{CC}$ & $\mathrm{CG}$ & $\mathrm{CG}$ & $\mathrm{CC}$ & $\mathrm{CC}$ & $\mathrm{CG}$ \\
\hline $\mathrm{CG}$ & $\mathrm{CG}$ & $\mathrm{CG}$ & $\mathrm{CG}$ & $\mathrm{CG}$ & $\mathrm{CG}$ & $\mathrm{CG}$ & $\mathrm{CG}$ & $\mathrm{CC}$ & $\mathrm{CG}$ & $\mathrm{CG}$ \\
\hline $\mathrm{CG}$ & $\mathrm{CG}$ & $\mathrm{CG}$ & $\mathrm{CG}$ & $\mathrm{CC}$ & $\mathrm{CC}$ & $\mathrm{CC}$ & $\mathrm{CG}$ & $\mathrm{CG}$ & $\mathrm{CG}$ & $\mathrm{CG}$ \\
\hline $\mathrm{CC}$ & $\mathrm{CC}$ & $\mathrm{CG}$ & $\mathrm{CG}$ & $\mathrm{CG}$ & $\mathrm{CG}$ & $\mathrm{CG}$ & $\mathrm{CG}$ & $\mathrm{CG}$ & $\mathrm{CG}$ & $\mathrm{CG}$ \\
\hline $\mathrm{CG}$ & $\mathrm{CG}$ & $\mathrm{CG}$ & $\mathrm{CG}$ & $\mathrm{CC}$ & CG & $\mathrm{CG}$ & $\mathrm{CC}$ & $\mathrm{CG}$ & $\mathrm{CG}$ & \\
\hline GDsnp00928 & 4 & \multicolumn{2}{|c|}{12770142} & GG & $\mathrm{AG}$ & $\mathrm{AG}$ & $\mathrm{AG}$ & AA & $\mathrm{AG}$ & $\mathrm{AG}$ \\
\hline $\mathrm{AG}$ & $\mathrm{AG}$ & $\mathrm{AA}$ & $\mathrm{AA}$ & $\mathrm{AA}$ & $\mathrm{AG}$ & GG & $\mathrm{AG}$ & $\mathrm{AG}$ & $\mathrm{AG}$ & $\mathrm{AA}$ \\
\hline $\mathrm{AG}$ & $\mathrm{AG}$ & $\mathrm{AG}$ & $\mathrm{AG}$ & $\mathrm{AG}$ & $\mathrm{AA}$ & GG & $\mathrm{AA}$ & $\mathrm{AA}$ & $\mathrm{AG}$ & AA \\
\hline GG & $\mathrm{AG}$ & $\mathrm{AG}$ & $\mathrm{AA}$ & $\mathrm{AA}$ & $\mathrm{AG}$ & $\mathrm{AG}$ & AA & - & $\mathrm{AG}$ & GG \\
\hline AA & $\mathrm{AG}$ & $\mathrm{AA}$ & $\mathrm{AG}$ & $\mathrm{AG}$ & $\mathrm{AG}$ & $\mathrm{AG}$ & - & $\mathrm{AG}$ & $\mathrm{AA}$ & $\mathrm{AG}$ \\
\hline GG & $\mathrm{AG}$ & $\mathrm{AA}$ & $\mathrm{AG}$ & GG & $\mathrm{AG}$ & $\mathrm{AA}$ & $\mathrm{AG}$ & $\mathrm{AG}$ & GG & GG \\
\hline GG & $\mathrm{AG}$ & GG & $\mathrm{AA}$ & $\mathrm{AG}$ & GG & $\mathrm{AG}$ & $\mathrm{AG}$ & $\mathrm{AG}$ & $\mathrm{AG}$ & $\mathrm{AG}$ \\
\hline AA & GG & $\mathrm{AA}$ & $\mathrm{AG}$ & GG & GG & $\mathrm{AA}$ & $\mathrm{AA}$ & GG & $\mathrm{AG}$ & $\mathrm{AG}$ \\
\hline $\mathrm{AG}$ & $\mathrm{AG}$ & $\mathrm{AG}$ & GG & $\mathrm{AG}$ & $\mathrm{AG}$ & $\mathrm{AG}$ & $\mathrm{AA}$ & $\mathrm{AG}$ & GG & \\
\hline
\end{tabular}

Continued next page 
Supplemental Table 2. Continued.

\begin{tabular}{|c|c|c|c|c|c|c|c|c|c|c|}
\hline \multirow{3}{*}{$\begin{array}{l}\text { SNP name } \\
\text { GDsnp02296 } \\
\text { CC }\end{array}$} & \multicolumn{10}{|c|}{ Linkage group Physical location (bp) Individuals } \\
\hline & 4 & \multicolumn{2}{|c|}{13039943} & AA & $\mathrm{CC}$ & $\mathrm{CC}$ & $\mathrm{AC}$ & $\mathrm{CC}$ & $\mathrm{CC}$ & $\mathrm{AC}$ \\
\hline & $\mathrm{AC}$ & $\mathrm{CC}$ & $\mathrm{CC}$ & $\mathrm{CC}$ & $\mathrm{CC}$ & $\mathrm{CC}$ & $\mathrm{CC}$ & $\mathrm{CC}$ & $\mathrm{CC}$ & $\mathrm{CC}$ \\
\hline $\mathrm{CC}$ & $\mathrm{CC}$ & $\mathrm{CC}$ & $\mathrm{CC}$ & $\mathrm{CC}$ & $\mathrm{CC}$ & AA & $\mathrm{CC}$ & $\mathrm{CC}$ & $\mathrm{CC}$ & $\mathrm{CC}$ \\
\hline- & $\mathrm{CC}$ & $\mathrm{CC}$ & $\mathrm{CC}$ & $\mathrm{CC}$ & $\mathrm{CC}$ & $\mathrm{CC}$ & $\mathrm{CC}$ & $\mathrm{CC}$ & $\mathrm{AC}$ & $\mathrm{CC}$ \\
\hline $\mathrm{CC}$ & $\mathrm{CC}$ & $\mathrm{CC}$ & $\mathrm{CC}$ & $\mathrm{CC}$ & $\mathrm{CC}$ & $\mathrm{CC}$ & $\mathrm{CC}$ & $\mathrm{CC}$ & $\mathrm{CC}$ & $\mathrm{CC}$ \\
\hline- & $\mathrm{CC}$ & $\mathrm{CC}$ & $\mathrm{CC}$ & - & $\mathrm{CC}$ & $\mathrm{CC}$ & $\mathrm{CC}$ & $\mathrm{CC}$ & $\mathrm{CC}$ & $\mathrm{CC}$ \\
\hline $\mathrm{CC}$ & $\mathrm{CC}$ & $\mathrm{CC}$ & $\mathrm{CC}$ & $\mathrm{CC}$ & - & $\mathrm{CC}$ & $\mathrm{CC}$ & $\mathrm{CC}$ & $\mathrm{CC}$ & $\mathrm{CC}$ \\
\hline $\mathrm{CC}$ & - & $\mathrm{CC}$ & $\mathrm{CC}$ & $\mathrm{CC}$ & - & $\mathrm{CC}$ & $\mathrm{CC}$ & $\mathrm{CC}$ & $\mathrm{CC}$ & $\mathrm{CC}$ \\
\hline $\mathrm{CC}$ & $\mathrm{CC}$ & $\mathrm{CC}$ & - & $\mathrm{CC}$ & $\mathrm{CC}$ & $\mathrm{CC}$ & $\mathrm{CC}$ & $\mathrm{CC}$ & $\mathrm{CC}$ & \\
\hline GDsnp00063 & 4 & \multicolumn{2}{|c|}{14625711} & $\mathrm{AG}$ & $\mathrm{AG}$ & $\mathrm{AG}$ & GG & GG & $\mathrm{AG}$ & GG \\
\hline$A G$ & GG & $\mathrm{AG}$ & $\mathrm{AG}$ & GG & $\mathrm{AA}$ & AA & $\mathrm{AG}$ & $\mathrm{AG}$ & $\mathrm{AG}$ & GG \\
\hline AG & $\mathrm{AG}$ & $\mathrm{AG}$ & $\mathrm{AG}$ & $\mathrm{AG}$ & GG & $\mathrm{AG}$ & GG & GG & $\mathrm{AG}$ & GG \\
\hline AA & $\mathrm{AG}$ & $\mathrm{AG}$ & GG & GG & $\mathrm{AG}$ & $\mathrm{AA}$ & GG & GG & GG & AA \\
\hline AG & $\mathrm{AG}$ & GG & $\mathrm{AG}$ & $\mathrm{AG}$ & $\mathrm{AG}$ & $\mathrm{AG}$ & $\mathrm{AG}$ & $\mathrm{AG}$ & GG & $\mathrm{AG}$ \\
\hline AA & $\mathrm{AG}$ & GG & $\mathrm{AA}$ & AA & $\mathrm{AG}$ & $\mathrm{AG}$ & AA & $\mathrm{AG}$ & $\mathrm{AA}$ & AA \\
\hline AA & $\mathrm{AA}$ & $\mathrm{AA}$ & GG & $\mathrm{AG}$ & $\mathrm{AA}$ & $\mathrm{AG}$ & AA & $\mathrm{AG}$ & $\mathrm{AG}$ & $\mathrm{AG}$ \\
\hline GG & $\mathrm{AA}$ & GG & $\mathrm{AG}$ & AA & $\mathrm{AA}$ & GG & $\mathrm{AG}$ & $\mathrm{AA}$ & $\mathrm{AA}$ & $\mathrm{AA}$ \\
\hline$A G$ & $\mathrm{AG}$ & $\mathrm{AG}$ & $\mathrm{AA}$ & $\mathrm{AG}$ & $\mathrm{AA}$ & $\mathrm{AA}$ & $\mathrm{AG}$ & $\mathrm{AG}$ & $\mathrm{AA}$ & \\
\hline GDsnp00321 & 4 & \multicolumn{2}{|c|}{15355228} & $\mathrm{AG}$ & $\mathrm{AG}$ & $\mathrm{AG}$ & AA & AA & $\mathrm{AG}$ & AA \\
\hline $\mathrm{AG}$ & $\mathrm{AA}$ & $\mathrm{AG}$ & $\mathrm{AG}$ & $\mathrm{AA}$ & GG & GG & $\mathrm{AG}$ & $\mathrm{AG}$ & $\mathrm{AG}$ & AA \\
\hline $\mathrm{AG}$ & $\mathrm{AG}$ & $\mathrm{AG}$ & AG & $\mathrm{AG}$ & $\mathrm{AA}$ & $\mathrm{AG}$ & AA & AA & $\mathrm{AG}$ & AA \\
\hline GG & $\mathrm{AG}$ & $\mathrm{AG}$ & $\mathrm{AA}$ & AA & $\mathrm{AG}$ & GG & $\mathrm{AA}$ & $\mathrm{AA}$ & $\mathrm{AG}$ & GG \\
\hline$A G$ & $\mathrm{AG}$ & $\mathrm{AA}$ & $\mathrm{AG}$ & GG & $\mathrm{AG}$ & $\mathrm{AG}$ & $\mathrm{AG}$ & $\mathrm{AG}$ & $\mathrm{AA}$ & $\mathrm{AG}$ \\
\hline GG & $\mathrm{AG}$ & $\mathrm{AA}$ & GG & GG & $\mathrm{AG}$ & $\mathrm{AG}$ & GG & $\mathrm{AG}$ & GG & GG \\
\hline GG & GG & GG & $\mathrm{AA}$ & $\mathrm{AG}$ & GG & $\mathrm{AG}$ & GG & $\mathrm{AG}$ & $\mathrm{AG}$ & $\mathrm{AG}$ \\
\hline AA & GG & $\mathrm{AA}$ & $\mathrm{AG}$ & GG & GG & $\mathrm{AA}$ & $\mathrm{AG}$ & GG & GG & $\mathrm{AG}$ \\
\hline $\mathrm{AG}$ & $\mathrm{AG}$ & $\mathrm{AG}$ & GG & $\mathrm{AG}$ & GG & GG & $\mathrm{AG}$ & $\mathrm{AG}$ & GG & \\
\hline GDsnp00148 & 4 & \multicolumn{2}{|c|}{16599404} & $\mathrm{AG}$ & $\mathrm{AG}$ & $\mathrm{AG}$ & AA & AA & $\mathrm{AG}$ & - \\
\hline $\mathrm{AG}$ & $\mathrm{AA}$ & GG & $\mathrm{AG}$ & AA & GG & GG & $\mathrm{AG}$ & $\mathrm{AG}$ & $\mathrm{AG}$ & $\mathrm{AA}$ \\
\hline$A G$ & $\mathrm{AG}$ & $\mathrm{AG}$ & $\mathrm{AG}$ & GG & $\mathrm{AA}$ & AA & AA & $\mathrm{AA}$ & $\mathrm{AG}$ & $\mathrm{AA}$ \\
\hline GG & $\mathrm{AG}$ & $\mathrm{AG}$ & $\mathrm{AA}$ & AA & $\mathrm{AG}$ & GG & AA & $\mathrm{AA}$ & $\mathrm{AG}$ & GG \\
\hline AA & $\mathrm{AG}$ & $\mathrm{AA}$ & $\mathrm{AG}$ & GG & $\mathrm{AG}$ & $\mathrm{AG}$ & $\mathrm{AG}$ & $\mathrm{AG}$ & - & $\mathrm{AG}$ \\
\hline GG & $\mathrm{AG}$ & $\mathrm{AA}$ & GG & GG & $\mathrm{AG}$ & $\mathrm{AG}$ & GG & $\mathrm{AG}$ & GG & GG \\
\hline GG & $\mathrm{AG}$ & GG & AA & $\mathrm{AG}$ & GG & $\mathrm{AG}$ & GG & $\mathrm{AG}$ & $\mathrm{AG}$ & $\mathrm{AG}$ \\
\hline AA & GG & $\mathrm{AA}$ & $\mathrm{AG}$ & GG & GG & - & $\mathrm{AG}$ & GG & GG & $\mathrm{AG}$ \\
\hline $\mathrm{AG}$ & $\mathrm{AG}$ & $\mathrm{AG}$ & - & $\mathrm{AG}$ & GG & GG & $\mathrm{AG}$ & $\mathrm{AG}$ & GG & \\
\hline GDsnp00324 & 4 & \multicolumn{2}{|c|}{16640485} & $\mathrm{AG}$ & $\mathrm{AG}$ & $\mathrm{AG}$ & $\mathrm{AA}$ & $\mathrm{AA}$ & $\mathrm{AG}$ & AA \\
\hline $\mathrm{AA}$ & $\mathrm{AA}$ & GG & $\mathrm{AG}$ & $\mathrm{AA}$ & GG & GG & $\mathrm{AG}$ & $\mathrm{AA}$ & $\mathrm{AG}$ & $\mathrm{AA}$ \\
\hline$A G$ & $\mathrm{AG}$ & $\mathrm{AA}$ & $\mathrm{AG}$ & GG & $\mathrm{AA}$ & $\mathrm{AA}$ & $\mathrm{AA}$ & $\mathrm{AA}$ & $\mathrm{AG}$ & AA \\
\hline GG & $\mathrm{AG}$ & $\mathrm{AG}$ & AA & AA & $\mathrm{AG}$ & GG & AA & AA & $\mathrm{AA}$ & $\mathrm{AG}$ \\
\hline AA & $\mathrm{AG}$ & $\mathrm{AA}$ & $\mathrm{AG}$ & GG & $\mathrm{AG}$ & - & $\mathrm{AA}$ & $\mathrm{AG}$ & $\mathrm{AA}$ & $\mathrm{AG}$ \\
\hline GG & $\mathrm{AG}$ & $\mathrm{AA}$ & $\mathrm{AG}$ & GG & $\mathrm{AG}$ & $\mathrm{AG}$ & GG & $\mathrm{AG}$ & GG & GG \\
\hline GG & $\mathrm{AG}$ & GG & $\mathrm{AA}$ & $\mathrm{AG}$ & GG & $\mathrm{AG}$ & GG & $\mathrm{AG}$ & $\mathrm{AG}$ & $\mathrm{AG}$ \\
\hline AA & GG & $\mathrm{AA}$ & $\mathrm{AG}$ & GG & GG & $\mathrm{AA}$ & $\mathrm{AG}$ & GG & GG & $\mathrm{AG}$ \\
\hline $\mathrm{AG}$ & $\mathrm{AG}$ & $\mathrm{AG}$ & GG & $\mathrm{AG}$ & GG & $\mathrm{AG}$ & $\mathrm{AG}$ & $\mathrm{AG}$ & GG & \\
\hline GDsnp00881 & 4 & \multicolumn{2}{|c|}{17330929} & $\mathrm{CC}$ & $\mathrm{CC}$ & $\mathrm{CC}$ & $\mathrm{CC}$ & $\mathrm{CC}$ & $\mathrm{CC}$ & $\mathrm{CC}$ \\
\hline $\mathrm{AC}$ & $\mathrm{CC}$ & $\mathrm{AC}$ & $\mathrm{CC}$ & $\mathrm{CC}$ & $\mathrm{CC}$ & $\mathrm{CC}$ & $\mathrm{CC}$ & $\mathrm{CC}$ & $\mathrm{CC}$ & $\mathrm{CC}$ \\
\hline $\mathrm{CC}$ & $\mathrm{CC}$ & $\mathrm{AC}$ & $\mathrm{CC}$ & $\mathrm{CC}$ & $\mathrm{CC}$ & $\mathrm{CC}$ & $\mathrm{CC}$ & $\mathrm{CC}$ & $\mathrm{CC}$ & $\mathrm{CC}$ \\
\hline $\mathrm{CC}$ & $\mathrm{CC}$ & $\mathrm{CC}$ & $\mathrm{CC}$ & $\mathrm{CC}$ & $\mathrm{CC}$ & $\mathrm{CC}$ & $\mathrm{CC}$ & $\mathrm{CC}$ & $\mathrm{AC}$ & $\mathrm{AC}$ \\
\hline $\mathrm{CC}$ & $\mathrm{CC}$ & $\mathrm{CC}$ & $\mathrm{CC}$ & $\mathrm{CC}$ & $\mathrm{CC}$ & $\mathrm{CC}$ & $\mathrm{AC}$ & $\mathrm{CC}$ & $\mathrm{CC}$ & $\mathrm{CC}$ \\
\hline $\mathrm{CC}$ & $\mathrm{CC}$ & $\mathrm{CC}$ & $\mathrm{CC}$ & $\mathrm{CC}$ & $\mathrm{CC}$ & $\mathrm{CC}$ & $\mathrm{CC}$ & $\mathrm{CC}$ & $\mathrm{CC}$ & $\mathrm{CC}$ \\
\hline $\mathrm{CC}$ & $\mathrm{CC}$ & $\mathrm{CC}$ & $\mathrm{CC}$ & $\mathrm{CC}$ & $\mathrm{CC}$ & $\mathrm{CC}$ & $\mathrm{CC}$ & $\mathrm{CC}$ & $\mathrm{CC}$ & $\mathrm{CC}$ \\
\hline $\mathrm{CC}$ & $\mathrm{CC}$ & $\mathrm{CC}$ & $\mathrm{CC}$ & $\mathrm{CC}$ & $\mathrm{CC}$ & $\mathrm{CC}$ & $\mathrm{AC}$ & $\mathrm{CC}$ & $\mathrm{AC}$ & $\mathrm{AC}$ \\
\hline $\mathrm{AC}$ & $\mathrm{CC}$ & $\mathrm{CC}$ & $\mathrm{CC}$ & $\mathrm{CC}$ & $\mathrm{AC}$ & $\mathrm{CC}$ & $\mathrm{CC}$ & $\mathrm{CC}$ & $\mathrm{CC}$ & \\
\hline
\end{tabular}


Supplemental Table 2. Continued.

\begin{tabular}{|c|c|c|c|c|c|c|c|c|c|c|}
\hline \multirow{3}{*}{$\begin{array}{l}\text { SNP name } \\
\text { GDsnp00277 } \\
\text { GG }\end{array}$} & \multicolumn{10}{|c|}{ Linkage group Physical location (bp) Individuals } \\
\hline & 4 & \multicolumn{2}{|c|}{17724230} & $\mathrm{AA}$ & $\mathrm{AG}$ & $\mathrm{AG}$ & $\mathrm{AG}$ & GG & $\mathrm{AG}$ & $\mathrm{AG}$ \\
\hline & $\mathrm{AG}$ & $\mathrm{AG}$ & AG & GG & AA & AA & $\mathrm{AG}$ & $\mathrm{AG}$ & $\mathrm{AG}$ & GG \\
\hline AG & $\mathrm{AG}$ & $\mathrm{AG}$ & AG & AA & GG & $\mathrm{AG}$ & GG & GG & $\mathrm{AG}$ & GG \\
\hline AA & $\mathrm{AG}$ & $\mathrm{AG}$ & GG & GG & $\mathrm{AG}$ & AA & GG & GG & GG & $\mathrm{AG}$ \\
\hline GG & $\mathrm{AG}$ & GG & $\mathrm{AG}$ & - & GG & $\mathrm{AG}$ & $\mathrm{AG}$ & $\mathrm{AG}$ & GG & $\mathrm{AG}$ \\
\hline AA & $\mathrm{AG}$ & GG & $\mathrm{AA}$ & $\mathrm{AA}$ & $\mathrm{AG}$ & $\mathrm{AG}$ & AA & $\mathrm{AG}$ & $\mathrm{AG}$ & AA \\
\hline AA & $\mathrm{AG}$ & AA & GG & $\mathrm{AG}$ & $\mathrm{AA}$ & AG & $\mathrm{AA}$ & $\mathrm{AG}$ & $\mathrm{AG}$ & $\mathrm{AG}$ \\
\hline GG & AA & GG & $\mathrm{AG}$ & $\mathrm{AA}$ & $\mathrm{AA}$ & GG & GG & AA & $\mathrm{AG}$ & GG \\
\hline GG & $\mathrm{AG}$ & $\mathrm{AG}$ & $\mathrm{AA}$ & GG & $\mathrm{AG}$ & $\mathrm{AA}$ & $\mathrm{AG}$ & $\mathrm{AG}$ & $\mathrm{AA}$ & \\
\hline GDsnp01195 & 4 & \multicolumn{2}{|c|}{19373189} & $\mathrm{AG}$ & $\mathrm{AA}$ & AA & $\mathrm{AG}$ & $\mathrm{AA}$ & $\mathrm{AA}$ & $\mathrm{AG}$ \\
\hline AA & AA & AA & AA & $\mathrm{AA}$ & $\mathrm{AA}$ & $\mathrm{AA}$ & $\mathrm{AA}$ & AA & $\mathrm{AA}$ & AA \\
\hline AA & AA & AA & AA & $\mathrm{AA}$ & $\mathrm{AA}$ & $\mathrm{AA}$ & AA & AA & $\mathrm{AA}$ & AA \\
\hline AA & AA & AA & AA & $\mathrm{AA}$ & $\mathrm{AA}$ & $\mathrm{AA}$ & $\mathrm{AA}$ & AA & $\mathrm{AA}$ & AA \\
\hline AA & AA & AA & AA & $\mathrm{AA}$ & $\mathrm{AA}$ & AA & AA & AA & $\mathrm{AA}$ & AA \\
\hline AA & AA & AA & AA & $\mathrm{AA}$ & $\mathrm{AA}$ & AA & AA & AA & $\mathrm{AA}$ & AA \\
\hline AA & $\mathrm{AA}$ & AA & $\mathrm{AA}$ & $\mathrm{AA}$ & $\mathrm{AA}$ & $\mathrm{AA}$ & AA & AA & $\mathrm{AA}$ & AA \\
\hline AA & AA & AA & AA & $\mathrm{AA}$ & $\mathrm{AA}$ & $\mathrm{AA}$ & $\mathrm{AA}$ & AA & $\mathrm{AA}$ & AA \\
\hline AA & $\mathrm{AA}$ & AA & $\mathrm{AA}$ & $\mathrm{AA}$ & $\mathrm{AA}$ & $\mathrm{AA}$ & AA & $\mathrm{AA}$ & $\mathrm{AA}$ & \\
\hline GDsnp00619 & 4 & \multicolumn{2}{|c|}{19504268} & $\mathrm{AA}$ & $\mathrm{AA}$ & $\mathrm{AA}$ & $\mathrm{AA}$ & - & $\mathrm{AA}$ & AA \\
\hline GG & $\mathrm{AA}$ & $\mathrm{AG}$ & AA & - & $\mathrm{AA}$ & $\mathrm{AA}$ & $\mathrm{AA}$ & AA & $\mathrm{AA}$ & - \\
\hline AA & AA & AA & AA & $\mathrm{AA}$ & - & AA & AA & AA & $\mathrm{AA}$ & - \\
\hline AA & AA & AA & - & - & $\mathrm{AA}$ & AA & - & - & - & GG \\
\hline- & AA & GG & AA & $\mathrm{AA}$ & - & $\mathrm{AA}$ & $\mathrm{AA}$ & - & - & AA \\
\hline AA & $\mathrm{AA}$ & - & AA & $\mathrm{AA}$ & $\mathrm{AA}$ & $\mathrm{AA}$ & $\mathrm{AA}$ & AA & $\mathrm{AA}$ & AA \\
\hline AA & AA & AA & - & $\mathrm{AA}$ & $\mathrm{AA}$ & AA & AA & AA & $\mathrm{AA}$ & AA \\
\hline - & AA & - & $\mathrm{AA}$ & AA & AA & - & GG & AA & $\mathrm{AG}$ & AA \\
\hline AA & AA & AA & AA & - & $\mathrm{AA}$ & $\mathrm{AG}$ & $\mathrm{AA}$ & AA & $\mathrm{AA}$ & \\
\hline GDsnp01336 & 4 & \multicolumn{2}{|c|}{20287239} & $\mathrm{AG}$ & $\mathrm{AG}$ & $\mathrm{AG}$ & GG & GG & $\mathrm{AG}$ & $\mathrm{AG}$ \\
\hline GG & $\mathrm{AG}$ & $\mathrm{AG}$ & AG & GG & AA & AA & $\mathrm{AG}$ & $\mathrm{AG}$ & $\mathrm{AG}$ & GG \\
\hline $\mathrm{AG}$ & $\mathrm{AG}$ & $\mathrm{AA}$ & $\mathrm{AG}$ & $\mathrm{AA}$ & $\mathrm{AG}$ & $\mathrm{AG}$ & $\mathrm{AG}$ & $\mathrm{AG}$ & $\mathrm{AG}$ & GG \\
\hline AA & $\mathrm{AG}$ & $\mathrm{AG}$ & GG & GG & $\mathrm{AG}$ & $\mathrm{AA}$ & GG & GG & GG & $\mathrm{AG}$ \\
\hline GG & $\mathrm{AG}$ & GG & AG & $\mathrm{AA}$ & GG & $\mathrm{AG}$ & AA & $\mathrm{AG}$ & GG & $\mathrm{AG}$ \\
\hline AA & $\mathrm{AG}$ & GG & AA & $\mathrm{AA}$ & $\mathrm{AG}$ & $\mathrm{AG}$ & AA & $\mathrm{AG}$ & $\mathrm{AG}$ & AA \\
\hline AA & $\mathrm{AG}$ & AA & GG & $\mathrm{AG}$ & $\mathrm{AA}$ & $\mathrm{AG}$ & $\mathrm{AA}$ & AA & $\mathrm{AA}$ & $\mathrm{AG}$ \\
\hline GG & AA & GG & $\mathrm{AG}$ & $\mathrm{AA}$ & AA & GG & GG & AA & $\mathrm{AG}$ & $\mathrm{AG}$ \\
\hline GG & $\mathrm{AG}$ & $\mathrm{AG}$ & $\mathrm{AA}$ & GG & AA & $\mathrm{AA}$ & $\mathrm{AG}$ & $\mathrm{AA}$ & $\mathrm{AA}$ & \\
\hline GDsnp00226 & 4 & \multicolumn{2}{|c|}{21346225} & $\mathrm{AC}$ & $\mathrm{CC}$ & $\mathrm{CC}$ & $\mathrm{AC}$ & $\mathrm{AC}$ & $\mathrm{CC}$ & $\mathrm{CC}$ \\
\hline $\mathrm{AC}$ & $\mathrm{CC}$ & $\mathrm{AC}$ & $\mathrm{CC}$ & $\mathrm{AC}$ & $\mathrm{CC}$ & $\mathrm{CC}$ & $\mathrm{CC}$ & $\mathrm{CC}$ & $\mathrm{CC}$ & $\mathrm{AC}$ \\
\hline $\mathrm{CC}$ & $\mathrm{AC}$ & $\mathrm{CC}$ & $\mathrm{CC}$ & $\mathrm{CC}$ & $\mathrm{CC}$ & $\mathrm{CC}$ & $\mathrm{CC}$ & $\mathrm{CC}$ & $\mathrm{AC}$ & $\mathrm{CC}$ \\
\hline $\mathrm{CC}$ & $\mathrm{AC}$ & $\mathrm{CC}$ & $\mathrm{AC}$ & $\mathrm{AC}$ & $\mathrm{AC}$ & $\mathrm{CC}$ & $\mathrm{AC}$ & $\mathrm{AC}$ & $\mathrm{CC}$ & $\mathrm{CC}$ \\
\hline $\mathrm{AC}$ & $\mathrm{AC}$ & $\mathrm{AC}$ & $\mathrm{CC}$ & $\mathrm{CC}$ & $\mathrm{AC}$ & $\mathrm{AA}$ & $\mathrm{CC}$ & $\mathrm{AC}$ & $\mathrm{AC}$ & $\mathrm{CC}$ \\
\hline $\mathrm{CC}$ & $\mathrm{CC}$ & $\mathrm{AC}$ & $\mathrm{CC}$ & $\mathrm{CC}$ & $\mathrm{CC}$ & $\mathrm{CC}$ & $\mathrm{CC}$ & $\mathrm{CC}$ & $\mathrm{CC}$ & $\mathrm{CC}$ \\
\hline $\mathrm{CC}$ & $\mathrm{AC}$ & $\mathrm{CC}$ & $\mathrm{AC}$ & $\mathrm{CC}$ & $\mathrm{CC}$ & $\mathrm{AC}$ & $\mathrm{CC}$ & $\mathrm{CC}$ & $\mathrm{CC}$ & $\mathrm{CC}$ \\
\hline $\mathrm{AC}$ & $\mathrm{CC}$ & $\mathrm{AC}$ & $\mathrm{CC}$ & $\mathrm{CC}$ & $\mathrm{CC}$ & $\mathrm{AC}$ & $\mathrm{AC}$ & $\mathrm{CC}$ & $\mathrm{AC}$ & $\mathrm{CC}$ \\
\hline $\mathrm{AC}$ & $\mathrm{CC}$ & $\mathrm{CC}$ & - & $\mathrm{AC}$ & $\mathrm{CC}$ & $\mathrm{CC}$ & $\mathrm{AC}$ & $\mathrm{CC}$ & $\mathrm{CC}$ & \\
\hline GDsnp00232 & 4 & \multicolumn{2}{|c|}{21774407} & $\mathrm{AG}$ & AA & AA & $\mathrm{AG}$ & $\mathrm{AG}$ & $\mathrm{AA}$ & AA \\
\hline AA & AA & $\mathrm{AG}$ & AA & $\mathrm{AG}$ & $\mathrm{AA}$ & $\mathrm{AA}$ & $\mathrm{AA}$ & AA & $\mathrm{AA}$ & $\mathrm{AG}$ \\
\hline AA & $\mathrm{AG}$ & AA & $\mathrm{AA}$ & $\mathrm{AA}$ & AA & $\mathrm{AA}$ & $\mathrm{AG}$ & $\mathrm{AG}$ & $\mathrm{AG}$ & AA \\
\hline AA & $\mathrm{AG}$ & AA & $\mathrm{AG}$ & $\mathrm{AG}$ & $\mathrm{AG}$ & $\mathrm{AA}$ & $\mathrm{AG}$ & $\mathrm{AG}$ & $\mathrm{AA}$ & AA \\
\hline $\mathrm{AG}$ & $\mathrm{AG}$ & $\mathrm{AG}$ & $\mathrm{AG}$ & $\mathrm{AG}$ & $\mathrm{AG}$ & $\mathrm{AG}$ & AA & $\mathrm{AG}$ & $\mathrm{AG}$ & $\mathrm{AG}$ \\
\hline AA & $\mathrm{AA}$ & $\mathrm{AG}$ & AA & $\mathrm{AA}$ & $\mathrm{AA}$ & $\mathrm{AG}$ & $\mathrm{AA}$ & $\mathrm{AG}$ & $\mathrm{AA}$ & AA \\
\hline $\mathrm{AA}$ & $\mathrm{AG}$ & $\mathrm{AA}$ & $\mathrm{AG}$ & $\mathrm{AG}$ & $\mathrm{AG}$ & $\mathrm{AG}$ & $\mathrm{AA}$ & $\mathrm{AA}$ & $\mathrm{AG}$ & AA \\
\hline $\mathrm{AG}$ & $\mathrm{AA}$ & $\mathrm{AG}$ & AA & $\mathrm{AA}$ & AA & $\mathrm{AG}$ & $\mathrm{AG}$ & $\mathrm{AA}$ & $\mathrm{AG}$ & $\mathrm{AA}$ \\
\hline $\mathrm{AG}$ & AA & $\mathrm{AA}$ & $\mathrm{AA}$ & $\mathrm{AG}$ & $\mathrm{AA}$ & $\mathrm{AA}$ & $\mathrm{AG}$ & $\mathrm{AA}$ & $\mathrm{AA}$ & \\
\hline
\end{tabular}

Continued next page 
Supplemental Table 2. Continued.

\begin{tabular}{|c|c|c|c|c|c|c|c|c|c|c|}
\hline \multirow{3}{*}{$\begin{array}{l}\text { SNP name } \\
\text { GDsnp00136 } \\
\text { AC }\end{array}$} & \multicolumn{10}{|c|}{ Linkage group Physical location (bp) Individuals } \\
\hline & 4 & \multicolumn{2}{|c|}{22675179} & $\mathrm{AC}$ & $\mathrm{CC}$ & $\mathrm{CC}$ & $\mathrm{AC}$ & $\mathrm{AC}$ & $\mathrm{CC}$ & $\mathrm{CC}$ \\
\hline & $\mathrm{CC}$ & $\mathrm{AC}$ & $\mathrm{CC}$ & $\mathrm{AC}$ & $\mathrm{CC}$ & $\mathrm{CC}$ & $\mathrm{CC}$ & $\mathrm{CC}$ & $\mathrm{CC}$ & $\mathrm{CC}$ \\
\hline $\mathrm{CC}$ & $\mathrm{CC}$ & $\mathrm{CC}$ & $\mathrm{CC}$ & $\mathrm{CC}$ & $\mathrm{CC}$ & $\mathrm{CC}$ & $\mathrm{AC}$ & $\mathrm{AC}$ & $\mathrm{CC}$ & $\mathrm{CC}$ \\
\hline $\mathrm{CC}$ & $\mathrm{CC}$ & $\mathrm{CC}$ & $\mathrm{CC}$ & $\mathrm{CC}$ & $\mathrm{CC}$ & $\mathrm{CC}$ & $\mathrm{CC}$ & $\mathrm{AC}$ & $\mathrm{CC}$ & $\mathrm{CC}$ \\
\hline $\mathrm{CC}$ & $\mathrm{CC}$ & $\mathrm{AC}$ & $\mathrm{AC}$ & $\mathrm{AC}$ & $\mathrm{CC}$ & $\mathrm{CC}$ & $\mathrm{CC}$ & $\mathrm{CC}$ & $\mathrm{CC}$ & $\mathrm{AC}$ \\
\hline $\mathrm{CC}$ & $\mathrm{CC}$ & $\mathrm{CC}$ & $\mathrm{CC}$ & $\mathrm{CC}$ & $\mathrm{CC}$ & $\mathrm{AC}$ & $\mathrm{CC}$ & $\mathrm{AC}$ & $\mathrm{CC}$ & $\mathrm{CC}$ \\
\hline $\mathrm{CC}$ & $\mathrm{AC}$ & $\mathrm{CC}$ & $\mathrm{CC}$ & $\mathrm{AC}$ & $\mathrm{AC}$ & $\mathrm{AC}$ & $\mathrm{CC}$ & $\mathrm{CC}$ & $\mathrm{AC}$ & $\mathrm{CC}$ \\
\hline $\mathrm{CC}$ & $\mathrm{CC}$ & $\mathrm{CC}$ & $\mathrm{CC}$ & $\mathrm{CC}$ & $\mathrm{CC}$ & $\mathrm{CC}$ & $\mathrm{CC}$ & $\mathrm{CC}$ & $\mathrm{AC}$ & $\mathrm{CC}$ \\
\hline $\mathrm{CC}$ & $\mathrm{CC}$ & $\mathrm{CC}$ & $\mathrm{CC}$ & $\mathrm{CC}$ & $\mathrm{CC}$ & $\mathrm{CC}$ & $\mathrm{CC}$ & $\mathrm{CC}$ & $\mathrm{CC}$ & \\
\hline GDsnp00177 & 4 & \multicolumn{2}{|c|}{22900279} & $\mathrm{AC}$ & $\mathrm{AA}$ & AA & $\mathrm{AC}$ & $\mathrm{AC}$ & $\mathrm{AA}$ & $\mathrm{AA}$ \\
\hline $\mathrm{AC}$ & $\mathrm{AA}$ & $\mathrm{AC}$ & $\mathrm{AA}$ & $\mathrm{AC}$ & $\mathrm{AA}$ & AA & AA & $\mathrm{AA}$ & $\mathrm{AA}$ & $\mathrm{AA}$ \\
\hline AA & $\mathrm{AA}$ & $\mathrm{AA}$ & $\mathrm{AA}$ & AA & $\mathrm{AA}$ & $\mathrm{AA}$ & $\mathrm{AC}$ & $\mathrm{AC}$ & $\mathrm{AA}$ & $\mathrm{AA}$ \\
\hline AA & AA & $\mathrm{AA}$ & $\mathrm{AA}$ & AA & $\mathrm{AA}$ & AA & AA & $\mathrm{AC}$ & $\mathrm{AA}$ & $\mathrm{AA}$ \\
\hline AA & $\mathrm{AA}$ & $\mathrm{AC}$ & $\mathrm{AC}$ & $\mathrm{AC}$ & $\mathrm{AA}$ & $\mathrm{AA}$ & $\mathrm{AC}$ & $\mathrm{AA}$ & $\mathrm{AA}$ & $\mathrm{AC}$ \\
\hline AA & $\mathrm{AA}$ & $\mathrm{AA}$ & AA & AA & $\mathrm{AA}$ & $\mathrm{AC}$ & AA & $\mathrm{AC}$ & $\mathrm{AA}$ & $\mathrm{AA}$ \\
\hline AA & $\mathrm{AC}$ & $\mathrm{AA}$ & $\mathrm{AA}$ & $\mathrm{AC}$ & $\mathrm{AC}$ & $\mathrm{AC}$ & AA & $\mathrm{AA}$ & $\mathrm{AC}$ & $\mathrm{AA}$ \\
\hline AA & AA & $\mathrm{AA}$ & $\mathrm{AA}$ & AA & $\mathrm{AA}$ & AA & AA & $\mathrm{AA}$ & $\mathrm{AC}$ & $\mathrm{AA}$ \\
\hline AA & AA & AA & AA & AA & $\mathrm{AA}$ & AA & AA & AA & $\mathrm{AA}$ & \\
\hline GDsnp00737 & 5 & \multicolumn{2}{|c|}{600612} & $\mathrm{AC}$ & $\mathrm{AA}$ & $\mathrm{AC}$ & $\mathrm{AC}$ & $\mathrm{CC}$ & $\mathrm{AA}$ & AA \\
\hline- & AA & $\mathrm{CC}$ & $\mathrm{AC}$ & $\mathrm{CC}$ & $\mathrm{AA}$ & $\mathrm{AC}$ & $\mathrm{AC}$ & $\mathrm{CC}$ & $\mathrm{CC}$ & AA \\
\hline AA & $\mathrm{AC}$ & $\mathrm{AA}$ & $\mathrm{AA}$ & AA & $\mathrm{CC}$ & $\mathrm{CC}$ & $\mathrm{AA}$ & $\mathrm{AA}$ & $\mathrm{AC}$ & $\mathrm{AA}$ \\
\hline $\mathrm{AC}$ & $\mathrm{AA}$ & $\mathrm{AA}$ & $\mathrm{CC}$ & AA & $\mathrm{AC}$ & $\mathrm{AC}$ & $\mathrm{CC}$ & $\mathrm{AC}$ & $\mathrm{CC}$ & $\mathrm{CC}$ \\
\hline $\mathrm{AC}$ & $\mathrm{AA}$ & $\mathrm{AC}$ & $\mathrm{CC}$ & AA & $\mathrm{AA}$ & $\mathrm{AC}$ & $\mathrm{AC}$ & $\mathrm{AC}$ & $\mathrm{AC}$ & $\mathrm{CC}$ \\
\hline $\mathrm{AC}$ & $\mathrm{AC}$ & $\mathrm{AC}$ & $\mathrm{CC}$ & $\mathrm{AC}$ & $\mathrm{AC}$ & $\mathrm{AC}$ & AA & AA & $\mathrm{CC}$ & $\mathrm{AC}$ \\
\hline AA & $\mathrm{AC}$ & AA & $\mathrm{AC}$ & $\mathrm{AC}$ & $\mathrm{AA}$ & AA & $\mathrm{AC}$ & $\mathrm{AC}$ & $\mathrm{AA}$ & AA \\
\hline $\mathrm{AC}$ & $\mathrm{AC}$ & $\mathrm{AC}$ & $\mathrm{AC}$ & $\mathrm{AC}$ & $\mathrm{AA}$ & $\mathrm{CC}$ & $\mathrm{CC}$ & $\mathrm{CC}$ & $\mathrm{AC}$ & $\mathrm{AC}$ \\
\hline AA & $\mathrm{AC}$ & $\mathrm{CC}$ & $\mathrm{AA}$ & $\mathrm{CC}$ & $\mathrm{AC}$ & $\mathrm{CC}$ & $\mathrm{CC}$ & $\mathrm{AA}$ & $\mathrm{AC}$ & \\
\hline GDsnp01694 & 5 & \multicolumn{2}{|c|}{975873} & $\mathrm{AC}$ & $\mathrm{AA}$ & $\mathrm{AC}$ & $\mathrm{AC}$ & - & $\mathrm{AC}$ & $\mathrm{AA}$ \\
\hline $\mathrm{CC}$ & $\mathrm{AA}$ & $\mathrm{CC}$ & $\mathrm{AC}$ & $\mathrm{CC}$ & $\mathrm{AA}$ & $\mathrm{AC}$ & $\mathrm{AC}$ & $\mathrm{CC}$ & $\mathrm{CC}$ & $\mathrm{AC}$ \\
\hline $\mathrm{AC}$ & $\mathrm{AC}$ & $\mathrm{AA}$ & $\mathrm{AC}$ & $\mathrm{AC}$ & $\mathrm{CC}$ & $\mathrm{CC}$ & AA & $\mathrm{AA}$ & $\mathrm{AC}$ & $\mathrm{AC}$ \\
\hline $\mathrm{AC}$ & AA & $\mathrm{AC}$ & $\mathrm{CC}$ & AA & $\mathrm{AC}$ & $\mathrm{AC}$ & $\mathrm{CC}$ & $\mathrm{AC}$ & $\mathrm{CC}$ & $\mathrm{CC}$ \\
\hline $\mathrm{AC}$ & $\mathrm{AA}$ & $\mathrm{AC}$ & $\mathrm{CC}$ & AA & $\mathrm{AA}$ & $\mathrm{AC}$ & $\mathrm{AC}$ & $\mathrm{AC}$ & $\mathrm{AC}$ & $\mathrm{AC}$ \\
\hline $\mathrm{AC}$ & $\mathrm{AC}$ & $\mathrm{AC}$ & $\mathrm{CC}$ & $\mathrm{CC}$ & $\mathrm{AC}$ & $\mathrm{AC}$ & $\mathrm{AC}$ & $\mathrm{AA}$ & $\mathrm{CC}$ & $\mathrm{AC}$ \\
\hline AA & $\mathrm{AC}$ & $\mathrm{AC}$ & $\mathrm{AC}$ & $\mathrm{AC}$ & $\mathrm{AA}$ & AA & $\mathrm{AC}$ & $\mathrm{AC}$ & $\mathrm{AA}$ & $\mathrm{AC}$ \\
\hline $\mathrm{AC}$ & $\mathrm{AC}$ & $\mathrm{AC}$ & $\mathrm{AC}$ & $\mathrm{AC}$ & $\mathrm{AA}$ & $\mathrm{CC}$ & $\mathrm{CC}$ & $\mathrm{CC}$ & $\mathrm{AC}$ & $\mathrm{AC}$ \\
\hline AA & $\mathrm{AC}$ & $\mathrm{CC}$ & $\mathrm{AA}$ & $\mathrm{CC}$ & $\mathrm{AC}$ & $\mathrm{CC}$ & $\mathrm{AC}$ & $\mathrm{AA}$ & $\mathrm{AC}$ & \\
\hline GDsnp00244 & 5 & \multicolumn{2}{|c|}{3433402} & $\mathrm{AC}$ & $\mathrm{AA}$ & $\mathrm{AC}$ & $\mathrm{AC}$ & $\mathrm{CC}$ & $\mathrm{AA}$ & AA \\
\hline $\mathrm{AC}$ & AA & $\mathrm{AC}$ & $\mathrm{AC}$ & $\mathrm{AC}$ & $\mathrm{AA}$ & $\mathrm{AC}$ & $\mathrm{AC}$ & $\mathrm{AC}$ & $\mathrm{CC}$ & AA \\
\hline- & $\mathrm{AC}$ & $\mathrm{AC}$ & - & AA & $\mathrm{AC}$ & $\mathrm{AC}$ & AA & $\mathrm{AA}$ & $\mathrm{AC}$ & AA \\
\hline $\mathrm{AC}$ & $\mathrm{AA}$ & $\mathrm{AA}$ & $\mathrm{AC}$ & AA & $\mathrm{AC}$ & $\mathrm{AC}$ & $\mathrm{AC}$ & $\mathrm{AC}$ & $\mathrm{AC}$ & $\mathrm{CC}$ \\
\hline $\mathrm{AC}$ & - & $\mathrm{AC}$ & $\mathrm{CC}$ & AA & $\mathrm{AA}$ & $\mathrm{AA}$ & $\mathrm{AC}$ & $\mathrm{AC}$ & $\mathrm{AC}$ & $\mathrm{AC}$ \\
\hline $\mathrm{AC}$ & $\mathrm{AC}$ & $\mathrm{AC}$ & $\mathrm{AC}$ & $\mathrm{AC}$ & $\mathrm{AC}$ & $\mathrm{AC}$ & $\mathrm{AA}$ & $\mathrm{AA}$ & $\mathrm{CC}$ & $\mathrm{AC}$ \\
\hline AA & $\mathrm{AC}$ & AA & $\mathrm{AC}$ & AA & $\mathrm{AA}$ & AA & $\mathrm{AC}$ & $\mathrm{AC}$ & - & - \\
\hline $\mathrm{AC}$ & $\mathrm{AC}$ & $\mathrm{AC}$ & $\mathrm{AC}$ & $\mathrm{AC}$ & $\mathrm{AA}$ & $\mathrm{CC}$ & $\mathrm{AC}$ & $\mathrm{AC}$ & $\mathrm{AC}$ & $\mathrm{AC}$ \\
\hline AA & $\mathrm{AC}$ & $\mathrm{AC}$ & $\mathrm{AA}$ & $\mathrm{CC}$ & AA & $\mathrm{CC}$ & $\mathrm{AA}$ & $\mathrm{AA}$ & $\mathrm{AC}$ & \\
\hline GDsnp00284 & 5 & \multicolumn{2}{|c|}{4305491} & GG & $\mathrm{AG}$ & GG & GG & GG & $\mathrm{AG}$ & GG \\
\hline $\mathrm{AG}$ & GG & $\mathrm{AG}$ & GG & $\mathrm{AG}$ & GG & GG & GG & $\mathrm{AG}$ & GG & $\mathrm{AG}$ \\
\hline AG & $\mathrm{AG}$ & GG & $\mathrm{AG}$ & GG & $\mathrm{AG}$ & $\mathrm{AG}$ & GG & GG & GG & $\mathrm{AG}$ \\
\hline GG & GG & $\mathrm{AG}$ & $\mathrm{AG}$ & GG & GG & GG & $\mathrm{AG}$ & GG & $\mathrm{AG}$ & GG \\
\hline GG & GG & GG & GG & $\mathrm{GG}$ & GG & GG & $\mathrm{AG}$ & GG & GG & GG \\
\hline GG & GG & GG & $\mathrm{AG}$ & $\mathrm{AG}$ & GG & GG & $\mathrm{AG}$ & GG & GG & $\mathrm{AG}$ \\
\hline GG & GG & $\mathrm{AG}$ & GG & GG & GG & GG & GG & GG & GG & $\mathrm{AG}$ \\
\hline GG & GG & GG & $\mathrm{GG}$ & $\mathrm{GG}$ & GG & GG & GG & $\mathrm{AG}$ & GG & GG \\
\hline GG & GG & $\mathrm{AG}$ & GG & $\mathrm{GG}$ & GG & GG & $\mathrm{AG}$ & GG & GG & \\
\hline
\end{tabular}


Supplemental Table 2. Continued.

\begin{tabular}{|c|c|c|c|c|c|c|c|c|c|c|}
\hline \multirow{3}{*}{$\begin{array}{l}\text { SNP name } \\
\text { GDsnp00264 } \\
\text { CC }\end{array}$} & \multicolumn{10}{|c|}{ Linkage group Physical location (bp) Individuals } \\
\hline & 5 & \multicolumn{2}{|c|}{5559452} & $\mathrm{CC}$ & $\mathrm{AC}$ & $\mathrm{CC}$ & $\mathrm{CC}$ & $\mathrm{CC}$ & $\mathrm{AC}$ & $\mathrm{CC}$ \\
\hline & $\mathrm{CC}$ & $\mathrm{AC}$ & $\mathrm{CC}$ & $\mathrm{AC}$ & $\mathrm{CC}$ & $\mathrm{CC}$ & $\mathrm{CC}$ & $\mathrm{CC}$ & $\mathrm{CC}$ & $\mathrm{AC}$ \\
\hline $\mathrm{AC}$ & $\mathrm{AC}$ & $\mathrm{CC}$ & $\mathrm{AC}$ & $\mathrm{CC}$ & $\mathrm{AC}$ & $\mathrm{AC}$ & $\mathrm{CC}$ & $\mathrm{CC}$ & $\mathrm{CC}$ & $\mathrm{AC}$ \\
\hline $\mathrm{CC}$ & $\mathrm{CC}$ & $\mathrm{AC}$ & $\mathrm{CC}$ & $\mathrm{CC}$ & $\mathrm{CC}$ & $\mathrm{CC}$ & $\mathrm{AC}$ & $\mathrm{CC}$ & $\mathrm{CC}$ & $\mathrm{CC}$ \\
\hline $\mathrm{CC}$ & $\mathrm{CC}$ & $\mathrm{CC}$ & $\mathrm{CC}$ & $\mathrm{CC}$ & $\mathrm{CC}$ & $\mathrm{CC}$ & $\mathrm{AC}$ & $\mathrm{CC}$ & $\mathrm{CC}$ & $\mathrm{CC}$ \\
\hline $\mathrm{CC}$ & $\mathrm{CC}$ & $\mathrm{CC}$ & $\mathrm{AC}$ & $\mathrm{AC}$ & $\mathrm{CC}$ & $\mathrm{CC}$ & $\mathrm{AC}$ & $\mathrm{CC}$ & $\mathrm{CC}$ & $\mathrm{AC}$ \\
\hline $\mathrm{AC}$ & $\mathrm{CC}$ & $\mathrm{AC}$ & $\mathrm{CC}$ & $\mathrm{CC}$ & $\mathrm{CC}$ & $\mathrm{CC}$ & $\mathrm{CC}$ & $\mathrm{CC}$ & $\mathrm{CC}$ & $\mathrm{AC}$ \\
\hline $\mathrm{CC}$ & $\mathrm{CC}$ & $\mathrm{CC}$ & $\mathrm{CC}$ & $\mathrm{CC}$ & $\mathrm{AC}$ & $\mathrm{CC}$ & $\mathrm{CC}$ & $\mathrm{AC}$ & $\mathrm{CC}$ & $\mathrm{CC}$ \\
\hline $\mathrm{CC}$ & $\mathrm{CC}$ & $\mathrm{AC}$ & $\mathrm{CC}$ & $\mathrm{CC}$ & $\mathrm{CC}$ & $\mathrm{CC}$ & $\mathrm{AC}$ & $\mathrm{CC}$ & $\mathrm{CC}$ & \\
\hline GDsnp00238 & 5 & \multicolumn{2}{|c|}{6193587} & GG & GG & GG & GG & GG & GG & GG \\
\hline GG & GG & GG & GG & GG & GG & GG & GG & GG & GG & GG \\
\hline GG & GG & GG & GG & GG & GG & GG & GG & GG & GG & GG \\
\hline GG & GG & GG & GG & GG & GG & GG & GG & GG & $\mathrm{AG}$ & GG \\
\hline GG & GG & GG & GG & GG & GG & - & $\mathrm{AG}$ & GG & GG & GG \\
\hline GG & GG & GG & GG & GG & GG & GG & GG & GG & GG & GG \\
\hline GG & GG & GG & GG & GG & GG & GG & GG & GG & GG & GG \\
\hline GG & GG & GG & GG & GG & GG & GG & GG & GG & GG & GG \\
\hline GG & GG & GG & GG & GG & GG & GG & GG & GG & GG & \\
\hline GDsnp01333 & 5 & \multicolumn{2}{|c|}{6282548} & GG & $\mathrm{AG}$ & GG & GG & GG & $\mathrm{AG}$ & GG \\
\hline GG & GG & $\mathrm{AG}$ & GG & $\mathrm{AG}$ & $\mathrm{AG}$ & GG & GG & GG & GG & $\mathrm{AG}$ \\
\hline $\mathrm{AG}$ & $\mathrm{AG}$ & GG & $\mathrm{AG}$ & GG & $\mathrm{AG}$ & $\mathrm{AG}$ & GG & GG & GG & $\mathrm{AG}$ \\
\hline GG & GG & $\mathrm{AG}$ & GG & GG & GG & GG & $\mathrm{AG}$ & GG & GG & GG \\
\hline GG & GG & GG & GG & GG & GG & GG & $\mathrm{AG}$ & GG & GG & GG \\
\hline GG & GG & GG & $\mathrm{AG}$ & $\mathrm{AG}$ & GG & GG & $\mathrm{AG}$ & GG & GG & $\mathrm{AG}$ \\
\hline $\mathrm{AG}$ & GG & $\mathrm{AG}$ & GG & GG & GG & GG & GG & GG & GG & $\mathrm{AG}$ \\
\hline GG & GG & GG & GG & GG & $\mathrm{AG}$ & GG & GG & $\mathrm{AG}$ & GG & GG \\
\hline GG & GG & $\mathrm{AG}$ & GG & GG & GG & GG & $\mathrm{AG}$ & GG & GG & \\
\hline GDsnp00189 & 5 & \multicolumn{2}{|c|}{7624382} & $\mathrm{AG}$ & $\mathrm{AG}$ & $\mathrm{AG}$ & $\mathrm{AG}$ & $\mathrm{AA}$ & $\mathrm{AG}$ & GG \\
\hline AA & $\mathrm{AG}$ & AA & GG & $\mathrm{AG}$ & $\mathrm{AG}$ & $\mathrm{AG}$ & $\mathrm{AG}$ & $\mathrm{AG}$ & $\mathrm{AA}$ & $\mathrm{AG}$ \\
\hline $\mathrm{AG}$ & AA & $\mathrm{AG}$ & $\mathrm{AG}$ & GG & $\mathrm{AA}$ & AA & GG & GG & $\mathrm{AG}$ & $\mathrm{AG}$ \\
\hline $\mathrm{AG}$ & $\mathrm{AG}$ & $\mathrm{AG}$ & $\mathrm{AA}$ & GG & $\mathrm{AG}$ & $\mathrm{AG}$ & AA & $\mathrm{AG}$ & $\mathrm{AA}$ & $\mathrm{AG}$ \\
\hline $\mathrm{AG}$ & GG & $\mathrm{AG}$ & AA & GG & GG & GG & $\mathrm{AG}$ & $\mathrm{AG}$ & $\mathrm{AG}$ & $\mathrm{AG}$ \\
\hline $\mathrm{AG}$ & $\mathrm{AG}$ & $\mathrm{AG}$ & AA & AA & $\mathrm{AG}$ & $\mathrm{AG}$ & $\mathrm{AG}$ & GG & $\mathrm{AA}$ & AA \\
\hline $\mathrm{AG}$ & $\mathrm{AG}$ & $\mathrm{AG}$ & $\mathrm{AG}$ & GG & GG & GG & GG & $\mathrm{AG}$ & GG & $\mathrm{AG}$ \\
\hline $\mathrm{AG}$ & $\mathrm{AG}$ & AA & $\mathrm{AG}$ & $\mathrm{AG}$ & $\mathrm{AG}$ & $\mathrm{AA}$ & AA & $\mathrm{AA}$ & $\mathrm{AG}$ & $\mathrm{AG}$ \\
\hline GG & $\mathrm{AG}$ & AA & GG & $\mathrm{AG}$ & AG & $\mathrm{AG}$ & AG & GG & $\mathrm{AG}$ & \\
\hline GDsnp01942 & 5 & \multicolumn{2}{|c|}{7693959} & $\mathrm{AG}$ & $\mathrm{AA}$ & $\mathrm{AG}$ & $\mathrm{AG}$ & GG & $\mathrm{AA}$ & AA \\
\hline GG & $\mathrm{AG}$ & GG & AA & AA & $\mathrm{AA}$ & $\mathrm{AG}$ & $\mathrm{AG}$ & $\mathrm{AG}$ & GG & $\mathrm{AG}$ \\
\hline AA & GG & $\mathrm{AG}$ & AA & AA & GG & GG & AA & AA & $\mathrm{AG}$ & AA \\
\hline $\mathrm{AG}$ & $\mathrm{AG}$ & $\mathrm{AA}$ & GG & AA & $\mathrm{AG}$ & $\mathrm{AG}$ & GG & $\mathrm{AG}$ & GG & $\mathrm{AG}$ \\
\hline $\mathrm{AG}$ & AA & $\mathrm{AG}$ & GG & AA & $\mathrm{AA}$ & $\mathrm{AA}$ & $\mathrm{AG}$ & $\mathrm{AG}$ & $\mathrm{AG}$ & $\mathrm{AG}$ \\
\hline $\mathrm{AG}$ & $\mathrm{AG}$ & $\mathrm{AG}$ & GG & GG & $\mathrm{AG}$ & $\mathrm{AG}$ & $\mathrm{AA}$ & $\mathrm{AA}$ & GG & GG \\
\hline AA & $\mathrm{AG}$ & AA & $\mathrm{AG}$ & AA & $\mathrm{AA}$ & AA & AA & $\mathrm{AG}$ & $\mathrm{AA}$ & $\mathrm{AG}$ \\
\hline $\mathrm{AG}$ & $\mathrm{AG}$ & GG & $\mathrm{AG}$ & $\mathrm{AG}$ & $\mathrm{AA}$ & GG & GG & GG & $\mathrm{AG}$ & $\mathrm{AG}$ \\
\hline AA & $\mathrm{AG}$ & GG & $\mathrm{AA}$ & $\mathrm{AG}$ & $\mathrm{AA}$ & $\mathrm{AG}$ & $\mathrm{AA}$ & AA & AG & \\
\hline GDsnp00638 & 5 & \multicolumn{2}{|c|}{9082273} & GG & $\mathrm{AG}$ & GG & GG & $\mathrm{AG}$ & $\mathrm{AG}$ & GG \\
\hline GG & GG & $\mathrm{AG}$ & GG & $\mathrm{AG}$ & $\mathrm{AG}$ & GG & $\mathrm{AG}$ & $\mathrm{AG}$ & GG & GG \\
\hline $\mathrm{AG}$ & $\mathrm{AG}$ & $\mathrm{AG}$ & $\mathrm{AG}$ & GG & $\mathrm{AG}$ & $\mathrm{AG}$ & GG & GG & GG & $\mathrm{AG}$ \\
\hline GG & GG & $\mathrm{AG}$ & GG & GG & GG & GG & $\mathrm{AG}$ & GG & GG & GG \\
\hline GG & GG & GG & GG & GG & GG & GG & $\mathrm{AG}$ & GG & GG & GG \\
\hline GG & GG & GG & $\mathrm{AG}$ & $\mathrm{AG}$ & GG & GG & $\mathrm{AG}$ & GG & GG & $\mathrm{AG}$ \\
\hline $\mathrm{AG}$ & GG & $\mathrm{AG}$ & $\mathrm{GG}$ & GG & GG & GG & GG & GG & GG & $\mathrm{AG}$ \\
\hline $\mathrm{AG}$ & GG & GG & GG & GG & $\mathrm{AG}$ & GG & GG & $\mathrm{AG}$ & GG & $\mathrm{GG}$ \\
\hline GG & GG & $\mathrm{AG}$ & GG & $\mathrm{GG}$ & $\mathrm{AG}$ & GG & $\mathrm{AG}$ & GG & GG & \\
\hline
\end{tabular}


Supplemental Table 2. Continued.

\begin{tabular}{|c|c|c|c|c|c|c|c|c|c|c|}
\hline \multirow{3}{*}{$\begin{array}{l}\text { SNP name } \\
\text { GDsnp02834 } \\
\text { AA }\end{array}$} & \multicolumn{10}{|c|}{ Linkage group Physical location (bp) Individuals } \\
\hline & 5 & \multicolumn{2}{|c|}{12769386} & AA & $\mathrm{AA}$ & AA & AA & $\mathrm{AA}$ & $\mathrm{AA}$ & $\mathrm{AA}$ \\
\hline & AA & AA & AA & AA & AA & AA & AA & AA & AA & AA \\
\hline AA & AA & AA & AA & AA & AA & AA & AA & AA & AA & AA \\
\hline AA & AA & AA & AA & AA & AA & AA & AA & AA & $\mathrm{AA}$ & $\mathrm{AA}$ \\
\hline AA & $\mathrm{AA}$ & $\mathrm{AA}$ & $\mathrm{AA}$ & $\mathrm{AA}$ & $\mathrm{AA}$ & $\mathrm{AA}$ & $\mathrm{AG}$ & $\mathrm{AA}$ & $\mathrm{AA}$ & $\mathrm{AA}$ \\
\hline AA & AA & AA & AA & AA & $\mathrm{AA}$ & AA & AA & AA & $\mathrm{AA}$ & $\mathrm{AA}$ \\
\hline AA & $\mathrm{AA}$ & $\mathrm{AA}$ & $\mathrm{AA}$ & AA & $\mathrm{AA}$ & $\mathrm{AA}$ & $\mathrm{AA}$ & $\mathrm{AA}$ & $\mathrm{AA}$ & $\mathrm{AA}$ \\
\hline- & $\mathrm{AA}$ & $\mathrm{AA}$ & $\mathrm{AA}$ & AA & $\mathrm{AA}$ & $\mathrm{AA}$ & $\mathrm{AA}$ & $\mathrm{AA}$ & $\mathrm{AA}$ & $\mathrm{AA}$ \\
\hline AA & $\mathrm{AA}$ & - & $\mathrm{AA}$ & AA & $\mathrm{AA}$ & AA & AA & AA & $\mathrm{AA}$ & \\
\hline GDsnp01830 & 5 & \multicolumn{2}{|c|}{14199813} & GG & GG & GG & GG & GG & GG & GG \\
\hline GG & GG & GG & GG & GG & GG & GG & GG & GG & GG & GG \\
\hline GG & GG & GG & GG & GG & GG & GG & GG & GG & GG & GG \\
\hline GG & GG & GG & GG & GG & GG & GG & GG & GG & GG & GG \\
\hline GG & GG & GG & GG & GG & GG & GG & $\mathrm{AG}$ & GG & GG & GG \\
\hline GG & GG & GG & GG & GG & GG & GG & GG & GG & GG & GG \\
\hline GG & GG & GG & GG & GG & GG & GG & GG & GG & GG & GG \\
\hline GG & GG & GG & GG & GG & GG & GG & GG & GG & GG & GG \\
\hline GG & GG & GG & GG & GG & GG & GG & GG & GG & GG & \\
\hline GDsnp01993 & 5 & \multicolumn{2}{|c|}{15377178} & $\mathrm{AT}$ & $\mathrm{TT}$ & $\mathrm{AT}$ & $\mathrm{AT}$ & $\mathrm{AA}$ & $\mathrm{TT}$ & $\mathrm{TT}$ \\
\hline AA & AA & AA & $\mathrm{AT}$ & $\mathrm{TT}$ & $\mathrm{TT}$ & $\mathrm{AT}$ & $\mathrm{AT}$ & AA & $\mathrm{AA}$ & $\mathrm{AT}$ \\
\hline TT & TT & $\mathrm{AA}$ & $\mathrm{AT}$ & $\mathrm{TT}$ & $\mathrm{AT}$ & $\mathrm{AA}$ & $\mathrm{TT}$ & $\mathrm{TT}$ & $\mathrm{TT}$ & $\mathrm{TT}$ \\
\hline $\mathrm{AT}$ & $\mathrm{AT}$ & $\mathrm{TT}$ & $\mathrm{AT}$ & $\mathrm{TT}$ & $\mathrm{AT}$ & $\mathrm{AT}$ & AA & $\mathrm{AT}$ & $\mathrm{AA}$ & $\mathrm{AA}$ \\
\hline $\mathrm{TT}$ & $\mathrm{AT}$ & $\mathrm{TT}$ & $\mathrm{AA}$ & $\mathrm{TT}$ & $\mathrm{TT}$ & $\mathrm{TT}$ & $\mathrm{AT}$ & $\mathrm{AT}$ & AT & $\mathrm{TT}$ \\
\hline $\mathrm{AT}$ & $\mathrm{AT}$ & AA & AA & $\mathrm{AT}$ & $\mathrm{AT}$ & $\mathrm{AT}$ & $\mathrm{TT}$ & $\mathrm{TT}$ & $\mathrm{AA}$ & $\mathrm{AT}$ \\
\hline TT & $\mathrm{TT}$ & $\mathrm{TT}$ & $\mathrm{AT}$ & $\mathrm{TT}$ & $\mathrm{TT}$ & $\mathrm{TT}$ & $\mathrm{TT}$ & $\mathrm{AT}$ & $\mathrm{TT}$ & $\mathrm{TT}$ \\
\hline AT & $\mathrm{AT}$ & AA & $\mathrm{AT}$ & $\mathrm{AT}$ & $\mathrm{TT}$ & $\mathrm{TT}$ & $\mathrm{AT}$ & $\mathrm{AT}$ & $\mathrm{TT}$ & $\mathrm{AT}$ \\
\hline TT & AT & AT & $\mathrm{TT}$ & AT & $\mathrm{TT}$ & $\mathrm{AA}$ & $\mathrm{TT}$ & AT & $\mathrm{TT}$ & \\
\hline GDsnp00611 & 5 & \multicolumn{2}{|c|}{16418690} & $\mathrm{AG}$ & GG & GG & GG & $\mathrm{AG}$ & $\mathrm{AG}$ & GG \\
\hline GG & GG & $\mathrm{AG}$ & GG & $\mathrm{AG}$ & GG & GG & GG & $\mathrm{AG}$ & GG & GG \\
\hline GG & GG & GG & GG & GG & GG & GG & GG & GG & GG & GG \\
\hline GG & GG & $\mathrm{AG}$ & GG & GG & GG & GG & $\mathrm{AG}$ & GG & GG & GG \\
\hline GG & GG & GG & GG & GG & GG & - & $\mathrm{AG}$ & GG & GG & GG \\
\hline GG & GG & GG & $\mathrm{AG}$ & GG & GG & GG & GG & GG & GG & GG \\
\hline GG & GG & GG & GG & GG & GG & GG & $\mathrm{AG}$ & GG & GG & GG \\
\hline GG & GG & GG & GG & GG & GG & GG & GG & GG & GG & GG \\
\hline GG & GG & - & GG & GG & GG & GG & GG & GG & GG & \\
\hline GDsnp00231 & 5 & \multicolumn{2}{|c|}{17855045} & $\mathrm{AT}$ & $\mathrm{TT}$ & $\mathrm{TT}$ & $\mathrm{TT}$ & $\mathrm{AT}$ & $\mathrm{AT}$ & $\mathrm{TT}$ \\
\hline TT & $\mathrm{TT}$ & $\mathrm{AT}$ & $\mathrm{TT}$ & AT & $\mathrm{TT}$ & $\mathrm{TT}$ & $\mathrm{TT}$ & $\mathrm{AT}$ & $\mathrm{TT}$ & $\mathrm{TT}$ \\
\hline $\mathrm{TT}$ & $\mathrm{TT}$ & $\mathrm{TT}$ & $\mathrm{TT}$ & $\mathrm{TT}$ & $\mathrm{TT}$ & $\mathrm{TT}$ & $\mathrm{TT}$ & $\mathrm{TT}$ & $\mathrm{TT}$ & $\mathrm{TT}$ \\
\hline TT & $\mathrm{TT}$ & $\mathrm{AT}$ & $\mathrm{TT}$ & $\mathrm{TT}$ & TT & $\mathrm{TT}$ & $\mathrm{AT}$ & $\mathrm{TT}$ & $\mathrm{TT}$ & AT \\
\hline TT & $\mathrm{TT}$ & $\mathrm{TT}$ & $\mathrm{TT}$ & $\mathrm{TT}$ & $\mathrm{TT}$ & $\mathrm{TT}$ & $\mathrm{AT}$ & $\mathrm{TT}$ & $\mathrm{TT}$ & $\mathrm{TT}$ \\
\hline $\mathrm{TT}$ & TT & TT & $\mathrm{AT}$ & $\mathrm{TT}$ & $\mathrm{TT}$ & $\mathrm{TT}$ & $\mathrm{TT}$ & $\mathrm{TT}$ & $\mathrm{TT}$ & $\mathrm{TT}$ \\
\hline $\mathrm{TT}$ & $\mathrm{AT}$ & $\mathrm{TT}$ & $\mathrm{TT}$ & $\mathrm{TT}$ & $\mathrm{TT}$ & $\mathrm{TT}$ & $\mathrm{AT}$ & $\mathrm{TT}$ & $\mathrm{TT}$ & $\mathrm{TT}$ \\
\hline TT & $\mathrm{TT}$ & $\mathrm{TT}$ & $\mathrm{TT}$ & $\mathrm{TT}$ & TT & - & $\mathrm{AT}$ & $\mathrm{TT}$ & $\mathrm{TT}$ & $\mathrm{TT}$ \\
\hline TT & TT & TT & $\mathrm{TT}$ & $\mathrm{TT}$ & TT & AT & TT & $\mathrm{TT}$ & $\mathrm{TT}$ & \\
\hline GDsnp01304 & 5 & \multicolumn{2}{|c|}{21598682} & $\mathrm{AG}$ & GG & GG & $\mathrm{AG}$ & GG & GG & $\mathrm{AA}$ \\
\hline $\mathrm{AG}$ & GG & $\mathrm{AG}$ & $\mathrm{AG}$ & $\mathrm{AG}$ & GG & GG & GG & GG & GG & $\mathrm{AG}$ \\
\hline $\mathrm{AG}$ & $\mathrm{AG}$ & GG & $\mathrm{AG}$ & $\mathrm{AG}$ & GG & GG & AA & AA & $\mathrm{AA}$ & $\mathrm{AA}$ \\
\hline $\mathrm{AG}$ & $\mathrm{AG}$ & $\mathrm{AG}$ & GG & $\mathrm{AG}$ & $\mathrm{AG}$ & GG & GG & $\mathrm{AG}$ & GG & GG \\
\hline $\mathrm{AG}$ & GG & $\mathrm{AG}$ & GG & AA & $\mathrm{AA}$ & $\mathrm{AA}$ & - & $\mathrm{AG}$ & - & $\mathrm{AG}$ \\
\hline $\mathrm{AG}$ & GG & GG & GG & GG & GG & $\mathrm{AG}$ & $\mathrm{AG}$ & $\mathrm{AA}$ & GG & GG \\
\hline GG & $\mathrm{AG}$ & $\mathrm{AG}$ & $\mathrm{AG}$ & AA & $\mathrm{AG}$ & $\mathrm{AA}$ & $\mathrm{AG}$ & GG & $\mathrm{AA}$ & $\mathrm{AG}$ \\
\hline $\mathrm{AG}$ & $\mathrm{AG}$ & GG & $\mathrm{GG}$ & $\mathrm{GG}$ & $\mathrm{AG}$ & $\mathrm{AA}$ & GG & GG & $\mathrm{AG}$ & $\mathrm{AG}$ \\
\hline $\mathrm{AG}$ & GG & GG & $\mathrm{AG}$ & $\mathrm{AG}$ & $\mathrm{AA}$ & GG & $\mathrm{AG}$ & GG & $\mathrm{AG}$ & \\
\hline
\end{tabular}


Supplemental Table 2. Continued.

\begin{tabular}{|c|c|c|c|c|c|c|c|c|c|c|}
\hline \multirow{3}{*}{$\begin{array}{l}\text { SNP name } \\
\text { GDsnp00806 } \\
\text { AC }\end{array}$} & \multicolumn{10}{|c|}{ Linkage group Physical location (bp) Individuals } \\
\hline & 5 & \multicolumn{2}{|c|}{21951956} & $\mathrm{AC}$ & $\mathrm{AA}$ & AA & $\mathrm{AC}$ & $\mathrm{AA}$ & $\mathrm{AA}$ & $\mathrm{CC}$ \\
\hline & AA & $\mathrm{AC}$ & $\mathrm{AC}$ & $\mathrm{AC}$ & AA & AA & AA & AA & AA & $\mathrm{AC}$ \\
\hline $\mathrm{AC}$ & $\mathrm{AC}$ & AA & $\mathrm{AC}$ & $\mathrm{AC}$ & AA & AA & $\mathrm{CC}$ & $\mathrm{CC}$ & $\mathrm{CC}$ & $\mathrm{CC}$ \\
\hline $\mathrm{AC}$ & $\mathrm{AC}$ & $\mathrm{AC}$ & AA & $\mathrm{AC}$ & $\mathrm{AC}$ & AA & AA & $\mathrm{AC}$ & $\mathrm{AA}$ & $\mathrm{AC}$ \\
\hline $\mathrm{AC}$ & $\mathrm{AA}$ & $\mathrm{AC}$ & $\mathrm{AC}$ & $\mathrm{CC}$ & $\mathrm{CC}$ & $\mathrm{CC}$ & $\mathrm{CC}$ & $\mathrm{AC}$ & $\mathrm{CC}$ & $\mathrm{AC}$ \\
\hline $\mathrm{AC}$ & $\mathrm{AA}$ & $\mathrm{AA}$ & $\mathrm{AA}$ & AA & $\mathrm{AA}$ & $\mathrm{AC}$ & $\mathrm{AC}$ & $\mathrm{CC}$ & $\mathrm{AA}$ & AA \\
\hline $\mathrm{AA}$ & $\mathrm{AC}$ & $\mathrm{AC}$ & $\mathrm{AC}$ & $\mathrm{CC}$ & $\mathrm{AC}$ & $\mathrm{CC}$ & $\mathrm{CC}$ & $\mathrm{AA}$ & $\mathrm{CC}$ & $\mathrm{AC}$ \\
\hline $\mathrm{AC}$ & $\mathrm{AC}$ & $\mathrm{AC}$ & $\mathrm{AA}$ & AA & $\mathrm{AC}$ & $\mathrm{CC}$ & $\mathrm{AA}$ & $\mathrm{AA}$ & $\mathrm{AC}$ & $\mathrm{AC}$ \\
\hline $\mathrm{AC}$ & $\mathrm{AA}$ & - & $\mathrm{AC}$ & $\mathrm{AC}$ & $\mathrm{CC}$ & AA & $\mathrm{AC}$ & AA & $\mathrm{AC}$ & \\
\hline GDsnp00151 & 5 & \multicolumn{2}{|c|}{21958442} & $\mathrm{AG}$ & $\mathrm{AA}$ & $\mathrm{AG}$ & AA & $\mathrm{AG}$ & $\mathrm{AG}$ & $\mathrm{AA}$ \\
\hline $\mathrm{AG}$ & $\mathrm{AA}$ & $\mathrm{AG}$ & $\mathrm{AA}$ & $\mathrm{AG}$ & $\mathrm{AG}$ & $\mathrm{AG}$ & AA & $\mathrm{AG}$ & $\mathrm{AA}$ & $\mathrm{AA}$ \\
\hline $\mathrm{AG}$ & $\mathrm{AA}$ & $\mathrm{AA}$ & $\mathrm{AA}$ & $\mathrm{AG}$ & $\mathrm{AA}$ & $\mathrm{AA}$ & AA & $\mathrm{AA}$ & $\mathrm{AA}$ & $\mathrm{AA}$ \\
\hline AA & AA & $\mathrm{AG}$ & $\mathrm{AA}$ & AA & $\mathrm{AA}$ & AA & $\mathrm{AG}$ & $\mathrm{AA}$ & $\mathrm{AG}$ & $\mathrm{AA}$ \\
\hline $\mathrm{AG}$ & $\mathrm{AA}$ & AA & $\mathrm{AA}$ & AA & $\mathrm{AA}$ & $\mathrm{AA}$ & $\mathrm{AA}$ & $\mathrm{AA}$ & $\mathrm{AA}$ & AA \\
\hline $\mathrm{AA}$ & $\mathrm{AA}$ & $\mathrm{AA}$ & $\mathrm{AG}$ & AA & $\mathrm{AG}$ & AA & AA & AA & $\mathrm{AA}$ & $\mathrm{AA}$ \\
\hline AG & $\mathrm{AG}$ & AA & $\mathrm{AA}$ & AA & $\mathrm{AA}$ & $\mathrm{AA}$ & AA & $\mathrm{AG}$ & $\mathrm{AA}$ & AA \\
\hline AA & $\mathrm{AG}$ & $\mathrm{AA}$ & $\mathrm{AG}$ & AA & $\mathrm{AA}$ & AA & $\mathrm{AG}$ & $\mathrm{AA}$ & $\mathrm{AA}$ & $\mathrm{AA}$ \\
\hline AA & $\mathrm{AG}$ & AA & $\mathrm{AG}$ & AA & $\mathrm{AA}$ & AA & AA & $\mathrm{AG}$ & $\mathrm{AA}$ & \\
\hline GDsnp02701 & 5 & \multicolumn{2}{|c|}{22437370} & $\mathrm{AA}$ & GG & $\mathrm{AG}$ & $\mathrm{AG}$ & $\mathrm{AG}$ & $\mathrm{AG}$ & AA \\
\hline $\mathrm{AG}$ & $\mathrm{AG}$ & AA & $\mathrm{AG}$ & AA & $\mathrm{AG}$ & $\mathrm{AG}$ & GG & $\mathrm{AG}$ & GG & $\mathrm{AG}$ \\
\hline $\mathrm{AA}$ & $\mathrm{AG}$ & $\mathrm{AG}$ & $\mathrm{AG}$ & AA & GG & $\mathrm{AG}$ & $\mathrm{AA}$ & $\mathrm{AA}$ & $\mathrm{AA}$ & $\mathrm{AA}$ \\
\hline $\mathrm{AG}$ & $\mathrm{AG}$ & $\mathrm{AA}$ & GG & $\mathrm{AG}$ & $\mathrm{AG}$ & GG & $\mathrm{AG}$ & $\mathrm{AG}$ & $\mathrm{AG}$ & $\mathrm{AG}$ \\
\hline AA & GG & $\mathrm{AG}$ & $\mathrm{AG}$ & AA & $\mathrm{AA}$ & - & $\mathrm{AA}$ & $\mathrm{AG}$ & $\mathrm{AA}$ & $\mathrm{AG}$ \\
\hline $\mathrm{AG}$ & GG & GG & $\mathrm{AG}$ & GG & $\mathrm{AG}$ & $\mathrm{AG}$ & $\mathrm{AG}$ & AA & GG & GG \\
\hline $\mathrm{AG}$ & AA & $\mathrm{AG}$ & GG & AA & $\mathrm{AG}$ & AA & AA & $\mathrm{AG}$ & $\mathrm{AG}$ & $\mathrm{AG}$ \\
\hline AG & AA & $\mathrm{AG}$ & GG & GG & GG & AA & $\mathrm{AG}$ & GG & GG & $\mathrm{AG}$ \\
\hline AG & $\mathrm{AG}$ & GG & $\mathrm{AG}$ & $\mathrm{AG}$ & AA & GG & $\mathrm{AG}$ & $\mathrm{AG}$ & $\mathrm{AG}$ & \\
\hline GDsnp00382 & 5 & \multicolumn{2}{|c|}{25756567} & $\mathrm{AG}$ & $\mathrm{AG}$ & AA & $\mathrm{AG}$ & AA & $\mathrm{AA}$ & GG \\
\hline GG & $\mathrm{AA}$ & GG & $\mathrm{AG}$ & $\mathrm{AG}$ & $\mathrm{AA}$ & $\mathrm{AA}$ & $\mathrm{AG}$ & $\mathrm{AA}$ & $\mathrm{AA}$ & $\mathrm{AG}$ \\
\hline $\mathrm{AG}$ & $\mathrm{AG}$ & $\mathrm{AA}$ & GG & $\mathrm{AG}$ & $\mathrm{AG}$ & AA & GG & GG & GG & GG \\
\hline $\mathrm{AG}$ & $\mathrm{AG}$ & AA & $\mathrm{AG}$ & $\mathrm{AG}$ & $\mathrm{AG}$ & $\mathrm{AG}$ & AA & $\mathrm{AG}$ & $\mathrm{AA}$ & $\mathrm{AG}$ \\
\hline $\mathrm{AG}$ & $\mathrm{AG}$ & GG & $\mathrm{AG}$ & GG & GG & - & GG & $\mathrm{AG}$ & GG & $\mathrm{AG}$ \\
\hline GG & $\mathrm{AG}$ & AA & AA & $\mathrm{AG}$ & $\mathrm{AA}$ & $\mathrm{AG}$ & $\mathrm{AG}$ & $\mathrm{AG}$ & $\mathrm{AG}$ & $\mathrm{AG}$ \\
\hline AA & $\mathrm{AG}$ & GG & $\mathrm{AA}$ & $\mathrm{AG}$ & $\mathrm{AG}$ & $\mathrm{AG}$ & GG & $\mathrm{AA}$ & $\mathrm{AG}$ & GG \\
\hline $\mathrm{AG}$ & $\mathrm{AG}$ & $\mathrm{AG}$ & $\mathrm{AG}$ & AA & $\mathrm{AG}$ & GG & AA & $\mathrm{AG}$ & $\mathrm{AG}$ & $\mathrm{AG}$ \\
\hline $\mathrm{AG}$ & AA & $\mathrm{AG}$ & GG & $\mathrm{AG}$ & GG & $\mathrm{AG}$ & GG & $\mathrm{AA}$ & GG & \\
\hline GDsnp01539 & 5 & \multicolumn{2}{|c|}{26802751} & $\mathrm{AG}$ & $\mathrm{AA}$ & AA & $\mathrm{AG}$ & AA & $\mathrm{AA}$ & GG \\
\hline $\mathrm{AG}$ & AA & GG & $\mathrm{AG}$ & $\mathrm{AG}$ & $\mathrm{AA}$ & AA & AA & $\mathrm{AA}$ & $\mathrm{AA}$ & $\mathrm{AG}$ \\
\hline $\mathrm{AG}$ & $\mathrm{AA}$ & $\mathrm{AA}$ & $\mathrm{AG}$ & $\mathrm{AG}$ & $\mathrm{AA}$ & AA & GG & GG & GG & GG \\
\hline $\mathrm{AG}$ & $\mathrm{AG}$ & $\mathrm{AA}$ & $\mathrm{AA}$ & $\mathrm{AG}$ & $\mathrm{AG}$ & $\mathrm{AA}$ & $\mathrm{AA}$ & $\mathrm{AG}$ & $\mathrm{AA}$ & $\mathrm{AG}$ \\
\hline $\mathrm{AG}$ & $\mathrm{AA}$ & $\mathrm{AG}$ & $\mathrm{AG}$ & GG & GG & GG & GG & $\mathrm{AG}$ & $\mathrm{AG}$ & $\mathrm{AG}$ \\
\hline $\mathrm{AG}$ & AA & $\mathrm{AG}$ & AA & AA & $\mathrm{AA}$ & $\mathrm{AG}$ & $\mathrm{AG}$ & $\mathrm{AG}$ & $\mathrm{AA}$ & AA \\
\hline AA & $\mathrm{AG}$ & $\mathrm{AG}$ & $\mathrm{AG}$ & $\mathrm{AG}$ & $\mathrm{AG}$ & $\mathrm{AG}$ & $\mathrm{AG}$ & $\mathrm{AG}$ & $\mathrm{AG}$ & $\mathrm{AG}$ \\
\hline $\mathrm{AG}$ & $\mathrm{AG}$ & $\mathrm{AG}$ & AA & AA & $\mathrm{AA}$ & GG & AA & AA & $\mathrm{AA}$ & $\mathrm{AG}$ \\
\hline AA & AA & AA & $\mathrm{AG}$ & $\mathrm{AG}$ & GG & $\mathrm{AA}$ & $\mathrm{AG}$ & $\mathrm{AA}$ & $\mathrm{AG}$ & \\
\hline GDsnp00100 & 5 & \multicolumn{2}{|c|}{30768863} & AA & $\mathrm{AG}$ & $\mathrm{AG}$ & $\mathrm{AG}$ & $\mathrm{AG}$ & GG & AA \\
\hline AA & GG & $\mathrm{AG}$ & $\mathrm{AG}$ & AA & $\mathrm{AG}$ & $\mathrm{AG}$ & AA & $\mathrm{AG}$ & GG & $\mathrm{AG}$ \\
\hline AA & $\mathrm{AG}$ & $\mathrm{AG}$ & AA & AA & $\mathrm{AG}$ & AA & AA & AA & $\mathrm{AG}$ & AA \\
\hline GG & GG & GG & $\mathrm{AG}$ & AA & $\mathrm{AA}$ & $\mathrm{AG}$ & $\mathrm{AG}$ & $\mathrm{AG}$ & GG & AA \\
\hline AA & $\mathrm{AG}$ & GG & $\mathrm{AG}$ & $\mathrm{GG}$ & $\mathrm{AG}$ & $\mathrm{AG}$ & AA & $\mathrm{AA}$ & $\mathrm{AA}$ & $\mathrm{AG}$ \\
\hline $\mathrm{AA}$ & $\mathrm{AG}$ & GG & $\mathrm{AG}$ & $\mathrm{AG}$ & $\mathrm{AG}$ & $\mathrm{AA}$ & GG & $\mathrm{AA}$ & $\mathrm{AG}$ & $\mathrm{AG}$ \\
\hline $\mathrm{AG}$ & $\mathrm{AA}$ & $\mathrm{AG}$ & GG & AA & GG & $\mathrm{AG}$ & $\mathrm{AA}$ & $\mathrm{AA}$ & GG & $\mathrm{AA}$ \\
\hline AA & GG & GG & $\mathrm{AG}$ & $\mathrm{GG}$ & $\mathrm{AG}$ & $\mathrm{AA}$ & $\mathrm{AG}$ & $\mathrm{AG}$ & GG & $\mathrm{AG}$ \\
\hline $\mathrm{AG}$ & $\mathrm{AA}$ & $\mathrm{AG}$ & AA & $\mathrm{GG}$ & $\mathrm{AA}$ & GG & $\mathrm{AG}$ & $\mathrm{AG}$ & $\mathrm{AA}$ & \\
\hline
\end{tabular}

Continued next page 
Supplemental Table 2. Continued.

\begin{tabular}{|c|c|c|c|c|c|c|c|c|c|c|}
\hline \multirow{3}{*}{$\begin{array}{l}\text { SNP name } \\
\text { GDsnp02674 } \\
\text { CC }\end{array}$} & \multicolumn{10}{|c|}{ Linkage group Physical location (bp) Individuals } \\
\hline & 5 & \multicolumn{2}{|c|}{31470112} & $\mathrm{CC}$ & $\mathrm{CC}$ & $\mathrm{CC}$ & $\mathrm{CC}$ & $\mathrm{CC}$ & $\mathrm{CC}$ & $\mathrm{CC}$ \\
\hline & $\mathrm{CC}$ & $\mathrm{CC}$ & $\mathrm{CC}$ & $\mathrm{CC}$ & $\mathrm{CC}$ & $\mathrm{CC}$ & $\mathrm{CC}$ & $\mathrm{CC}$ & $\mathrm{CC}$ & $\mathrm{CC}$ \\
\hline $\mathrm{CC}$ & $\mathrm{CC}$ & $\mathrm{CC}$ & $\mathrm{CC}$ & $\mathrm{CC}$ & $\mathrm{CC}$ & $\mathrm{CC}$ & $\mathrm{CC}$ & $\mathrm{CC}$ & $\mathrm{CC}$ & $\mathrm{CC}$ \\
\hline $\mathrm{CC}$ & $\mathrm{CC}$ & $\mathrm{CC}$ & $\mathrm{CC}$ & $\mathrm{CC}$ & $\mathrm{CC}$ & $\mathrm{CC}$ & $\mathrm{CC}$ & $\mathrm{CC}$ & $\mathrm{CC}$ & $\mathrm{CC}$ \\
\hline $\mathrm{CC}$ & $\mathrm{CC}$ & $\mathrm{CC}$ & $\mathrm{CC}$ & $\mathrm{CC}$ & $\mathrm{CC}$ & $\mathrm{CC}$ & $\mathrm{AC}$ & $\mathrm{CC}$ & $\mathrm{CC}$ & $\mathrm{CC}$ \\
\hline $\mathrm{CC}$ & $\mathrm{CC}$ & $\mathrm{CC}$ & $\mathrm{CC}$ & $\mathrm{CC}$ & $\mathrm{CC}$ & $\mathrm{CC}$ & $\mathrm{CC}$ & $\mathrm{CC}$ & $\mathrm{CC}$ & $\mathrm{CC}$ \\
\hline $\mathrm{CC}$ & $\mathrm{CC}$ & $\mathrm{CC}$ & $\mathrm{CC}$ & $\mathrm{CC}$ & $\mathrm{CC}$ & $\mathrm{CC}$ & $\mathrm{CC}$ & $\mathrm{CC}$ & $\mathrm{CC}$ & $\mathrm{CC}$ \\
\hline $\mathrm{CC}$ & $\mathrm{CC}$ & $\mathrm{CC}$ & $\mathrm{CC}$ & $\mathrm{CC}$ & $\mathrm{CC}$ & $\mathrm{CC}$ & $\mathrm{CC}$ & $\mathrm{CC}$ & $\mathrm{CC}$ & $\mathrm{CC}$ \\
\hline $\mathrm{CC}$ & $\mathrm{CC}$ & $\mathrm{CC}$ & $\mathrm{CC}$ & $\mathrm{CC}$ & $\mathrm{CC}$ & $\mathrm{CC}$ & $\mathrm{CC}$ & $\mathrm{CC}$ & $\mathrm{CC}$ & \\
\hline GDsnp00347 & 6 & \multicolumn{2}{|c|}{410901} & $\mathrm{AG}$ & $\mathrm{AG}$ & $\mathrm{AG}$ & $\mathrm{AG}$ & GG & $\mathrm{AG}$ & $\mathrm{AG}$ \\
\hline GG & $\mathrm{AG}$ & $\mathrm{AG}$ & $\mathrm{AG}$ & GG & $\mathrm{AG}$ & $\mathrm{AG}$ & $\mathrm{AG}$ & GG & $\mathrm{AG}$ & $\mathrm{AG}$ \\
\hline AG & $\mathrm{AG}$ & GG & $\mathrm{AG}$ & $\mathrm{AG}$ & $\mathrm{AG}$ & GG & $\mathrm{AG}$ & $\mathrm{AG}$ & $\mathrm{AG}$ & GG \\
\hline GG & GG & $\mathrm{AG}$ & GG & $\mathrm{AG}$ & $\mathrm{AG}$ & $\mathrm{AG}$ & GG & GG & GG & GG \\
\hline GG & GG & GG & $\mathrm{AG}$ & $\mathrm{AG}$ & GG & - & GG & $\mathrm{AG}$ & $\mathrm{AG}$ & $\mathrm{AG}$ \\
\hline$A G$ & $\mathrm{AG}$ & $\mathrm{AG}$ & GG & $\mathrm{AG}$ & $\mathrm{AG}$ & GG & $\mathrm{AG}$ & $\mathrm{AG}$ & GG & $\mathrm{AG}$ \\
\hline $\mathrm{AG}$ & GG & $\mathrm{AG}$ & GG & GG & $\mathrm{AG}$ & GG & $\mathrm{AG}$ & $\mathrm{AG}$ & GG & $\mathrm{AG}$ \\
\hline GG & $\mathrm{AG}$ & GG & $\mathrm{AG}$ & $\mathrm{AG}$ & $\mathrm{AG}$ & GG & GG & $\mathrm{AG}$ & GG & $\mathrm{AG}$ \\
\hline GG & $\mathrm{AG}$ & $\mathrm{AG}$ & $\mathrm{AG}$ & GG & GG & $\mathrm{AG}$ & $\mathrm{AG}$ & $\mathrm{AG}$ & $\mathrm{AG}$ & \\
\hline GDsnp02001 & 6 & \multicolumn{2}{|c|}{790796} & $\mathrm{AG}$ & $\mathrm{AG}$ & $\mathrm{AG}$ & $\mathrm{AG}$ & AA & $\mathrm{AG}$ & $\mathrm{AG}$ \\
\hline $\mathrm{AA}$ & $\mathrm{AG}$ & $\mathrm{AG}$ & $\mathrm{AG}$ & $\mathrm{AA}$ & $\mathrm{AG}$ & $\mathrm{AG}$ & $\mathrm{AG}$ & $\mathrm{AA}$ & $\mathrm{AG}$ & $\mathrm{AG}$ \\
\hline $\mathrm{AG}$ & $\mathrm{AG}$ & AA & $\mathrm{AG}$ & $\mathrm{AG}$ & $\mathrm{AG}$ & AA & $\mathrm{AG}$ & $\mathrm{AG}$ & $\mathrm{AG}$ & AA \\
\hline AA & $\mathrm{AA}$ & $\mathrm{AG}$ & $\mathrm{AA}$ & $\mathrm{AG}$ & $\mathrm{AG}$ & $\mathrm{AG}$ & $\mathrm{AA}$ & $\mathrm{AA}$ & $\mathrm{AA}$ & AA \\
\hline AA & $\mathrm{AA}$ & $\mathrm{AA}$ & $\mathrm{AG}$ & $\mathrm{AG}$ & $\mathrm{AA}$ & $\mathrm{AG}$ & AA & $\mathrm{AG}$ & $\mathrm{AG}$ & $\mathrm{AG}$ \\
\hline $\mathrm{AG}$ & $\mathrm{AG}$ & $\mathrm{AG}$ & AA & $\mathrm{AG}$ & $\mathrm{AG}$ & $\mathrm{AA}$ & $\mathrm{AG}$ & $\mathrm{AG}$ & $\mathrm{AA}$ & $\mathrm{AG}$ \\
\hline AG & $\mathrm{AA}$ & $\mathrm{AG}$ & $\mathrm{AA}$ & $\mathrm{AA}$ & $\mathrm{AG}$ & $\mathrm{AA}$ & $\mathrm{AG}$ & $\mathrm{AG}$ & $\mathrm{AA}$ & $\mathrm{AG}$ \\
\hline AA & $\mathrm{AG}$ & $\mathrm{AA}$ & $\mathrm{AG}$ & $\mathrm{AG}$ & $\mathrm{AG}$ & $\mathrm{AA}$ & $\mathrm{AA}$ & $\mathrm{AG}$ & $\mathrm{AA}$ & $\mathrm{AG}$ \\
\hline AA & $\mathrm{AG}$ & $\mathrm{AG}$ & $\mathrm{AG}$ & $\mathrm{AA}$ & AA & $\mathrm{AG}$ & $\mathrm{AG}$ & $\mathrm{AG}$ & $\mathrm{AG}$ & \\
\hline GDsnp01996 & 6 & \multicolumn{2}{|c|}{1762063} & $\mathrm{AG}$ & $\mathrm{AG}$ & $\mathrm{AG}$ & $\mathrm{AG}$ & $\mathrm{AG}$ & $\mathrm{AG}$ & $\mathrm{AG}$ \\
\hline AA & $\mathrm{AG}$ & $\mathrm{AG}$ & $\mathrm{AG}$ & $\mathrm{AG}$ & $\mathrm{AG}$ & $\mathrm{AG}$ & $\mathrm{AG}$ & AA & $\mathrm{AG}$ & $\mathrm{AG}$ \\
\hline$A G$ & $\mathrm{AG}$ & $\mathrm{AA}$ & $\mathrm{AG}$ & $\mathrm{AG}$ & $\mathrm{AG}$ & AA & $\mathrm{AG}$ & $\mathrm{AG}$ & $\mathrm{AA}$ & $\mathrm{AG}$ \\
\hline AA & AA & $\mathrm{AG}$ & $\mathrm{AG}$ & $\mathrm{AG}$ & $\mathrm{AG}$ & $\mathrm{AG}$ & $\mathrm{AG}$ & $\mathrm{AA}$ & $\mathrm{AA}$ & $\mathrm{AA}$ \\
\hline AA & $\mathrm{AG}$ & $\mathrm{AG}$ & $\mathrm{AG}$ & $\mathrm{AG}$ & $\mathrm{AG}$ & $\mathrm{AG}$ & AA & $\mathrm{AG}$ & $\mathrm{AG}$ & $\mathrm{AG}$ \\
\hline $\mathrm{AG}$ & $\mathrm{AG}$ & $\mathrm{AG}$ & $\mathrm{AA}$ & $\mathrm{AG}$ & $\mathrm{AG}$ & $\mathrm{AA}$ & $\mathrm{AG}$ & $\mathrm{AG}$ & $\mathrm{AA}$ & $\mathrm{AG}$ \\
\hline$A G$ & $\mathrm{AG}$ & $\mathrm{AG}$ & $\mathrm{AA}$ & AA & $\mathrm{AG}$ & AA & $\mathrm{AG}$ & $\mathrm{AG}$ & $\mathrm{AA}$ & $\mathrm{AG}$ \\
\hline AA & $\mathrm{AG}$ & $\mathrm{AA}$ & $\mathrm{AG}$ & $\mathrm{AG}$ & $\mathrm{AG}$ & $\mathrm{AA}$ & $\mathrm{AG}$ & $\mathrm{AG}$ & $\mathrm{AA}$ & $\mathrm{AG}$ \\
\hline $\mathrm{AG}$ & $\mathrm{AG}$ & $\mathrm{AG}$ & $\mathrm{AG}$ & $\mathrm{AA}$ & $\mathrm{AG}$ & $\mathrm{AG}$ & $\mathrm{AG}$ & $\mathrm{AG}$ & $\mathrm{AG}$ & \\
\hline GDsnp02464 & 6 & \multicolumn{2}{|c|}{2660813} & $\mathrm{AA}$ & $\mathrm{AT}$ & $\mathrm{AT}$ & $\mathrm{AT}$ & $\mathrm{AT}$ & $\mathrm{AT}$ & AA \\
\hline $\mathrm{AA}$ & $\mathrm{AA}$ & $\mathrm{AA}$ & $\mathrm{AA}$ & $\mathrm{AT}$ & $\mathrm{AA}$ & $\mathrm{AT}$ & $\mathrm{AT}$ & $\mathrm{AA}$ & $\mathrm{AA}$ & $\mathrm{AA}$ \\
\hline AA & $\mathrm{AA}$ & $\mathrm{AA}$ & $\mathrm{AT}$ & $\mathrm{AT}$ & $\mathrm{AA}$ & $\mathrm{AA}$ & $\mathrm{AT}$ & $\mathrm{AT}$ & $\mathrm{AA}$ & AT \\
\hline AA & $\mathrm{AA}$ & $\mathrm{AA}$ & $\mathrm{AT}$ & $\mathrm{AT}$ & $\mathrm{AT}$ & $\mathrm{AT}$ & $\mathrm{AT}$ & AA & $\mathrm{AA}$ & $\mathrm{AA}$ \\
\hline AA & $\mathrm{AT}$ & $\mathrm{AT}$ & $\mathrm{AT}$ & $\mathrm{AT}$ & $\mathrm{AT}$ & $\mathrm{AT}$ & $\mathrm{AA}$ & $\mathrm{AT}$ & $\mathrm{AA}$ & AT \\
\hline AA & $\mathrm{AA}$ & $\mathrm{AT}$ & $\mathrm{AA}$ & $\mathrm{AA}$ & $\mathrm{AA}$ & $\mathrm{AA}$ & $\mathrm{AT}$ & $\mathrm{AT}$ & $\mathrm{AA}$ & AT \\
\hline AT & $\mathrm{AT}$ & $\mathrm{AT}$ & AA & AA & $\mathrm{AT}$ & AA & AA & $\mathrm{AT}$ & $\mathrm{AA}$ & AT \\
\hline AA & $\mathrm{AT}$ & $\mathrm{AA}$ & $\mathrm{AA}$ & AA & $\mathrm{AT}$ & $\mathrm{AA}$ & $\mathrm{AT}$ & $\mathrm{AA}$ & $\mathrm{AA}$ & AA \\
\hline $\mathrm{AT}$ & $\mathrm{AT}$ & $\mathrm{AA}$ & $\mathrm{AT}$ & $\mathrm{AA}$ & $\mathrm{AT}$ & $\mathrm{AA}$ & AT & AT & AA & \\
\hline GDsnp00197 & 6 & \multicolumn{2}{|c|}{5276296} & - & $\mathrm{CG}$ & $\mathrm{CC}$ & GG & GG & $\mathrm{CG}$ & - \\
\hline $\mathrm{CC}$ & GG & $\mathrm{CG}$ & - & GG & GG & GG & GG & $\mathrm{CG}$ & $\mathrm{CG}$ & - \\
\hline $\mathrm{CC}$ & $\mathrm{CC}$ & $\mathrm{CC}$ & - & GG & $\mathrm{CC}$ & $\mathrm{CC}$ & GG & GG & $\mathrm{CC}$ & GG \\
\hline $\mathrm{CC}$ & $\mathrm{CC}$ & - & $\mathrm{CG}$ & $\mathrm{CG}$ & GG & $\mathrm{CG}$ & GG & $\mathrm{CC}$ & $\mathrm{CG}$ & GG \\
\hline $\mathrm{CC}$ & $\mathrm{CG}$ & $\mathrm{CG}$ & GG & GG & GG & $\mathrm{CG}$ & $\mathrm{CC}$ & GG & $\mathrm{CC}$ & $\mathrm{CC}$ \\
\hline $\mathrm{CC}$ & $\mathrm{CC}$ & $\mathrm{CG}$ & GG & - & $\mathrm{CC}$ & $\mathrm{CC}$ & GG & GG & $\mathrm{CC}$ & GG \\
\hline $\mathrm{CG}$ & $\mathrm{CG}$ & $\mathrm{CG}$ & $\mathrm{CC}$ & $\mathrm{CC}$ & GG & $\mathrm{CC}$ & $\mathrm{CC}$ & GG & $\mathrm{CC}$ & GG \\
\hline $\mathrm{CC}$ & GG & $\mathrm{CC}$ & $\mathrm{CC}$ & $\mathrm{CC}$ & GG & $\mathrm{CC}$ & $\mathrm{CG}$ & - & $\mathrm{CC}$ & $\mathrm{CC}$ \\
\hline GG & $\mathrm{CG}$ & $\mathrm{CC}$ & $\mathrm{CG}$ & $\mathrm{CC}$ & $\mathrm{CG}$ & - & $\mathrm{CG}$ & $\mathrm{CG}$ & $\mathrm{CC}$ & \\
\hline
\end{tabular}


Supplemental Table 2. Continued.

\begin{tabular}{|c|c|c|c|c|c|c|c|c|c|c|}
\hline \multirow{3}{*}{$\begin{array}{l}\text { SNP name } \\
\text { GDsnp00434 } \\
\text { AC }\end{array}$} & \multicolumn{10}{|c|}{ Linkage group Physical location (bp) Individuals } \\
\hline & 6 & \multicolumn{2}{|c|}{7475532} & AA & $\mathrm{AC}$ & $\mathrm{AC}$ & AA & $\mathrm{AA}$ & $\mathrm{AC}$ & $\mathrm{AA}$ \\
\hline & $\mathrm{AC}$ & $\mathrm{AC}$ & AA & $\mathrm{AC}$ & AA & AA & AA & AA & $\mathrm{AC}$ & AA \\
\hline $\mathrm{AC}$ & $\mathrm{AC}$ & $\mathrm{AC}$ & AA & AA & $\mathrm{CC}$ & - & AA & AA & $\mathrm{AC}$ & AA \\
\hline $\mathrm{AC}$ & $\mathrm{AC}$ & AA & $\mathrm{AC}$ & $\mathrm{AC}$ & $\mathrm{CC}$ & $\mathrm{AC}$ & AA & $\mathrm{CC}$ & $\mathrm{CC}$ & $\mathrm{AA}$ \\
\hline $\mathrm{AC}$ & $\mathrm{AC}$ & $\mathrm{AC}$ & $\mathrm{AA}$ & $\mathrm{AA}$ & $\mathrm{AC}$ & $\mathrm{AC}$ & $\mathrm{CC}$ & $\mathrm{AA}$ & $\mathrm{AC}$ & $\mathrm{CC}$ \\
\hline $\mathrm{AC}$ & $\mathrm{AC}$ & $\mathrm{AC}$ & $\mathrm{AA}$ & AA & $\mathrm{AC}$ & $\mathrm{CC}$ & $\mathrm{AA}$ & $\mathrm{AA}$ & $\mathrm{AC}$ & $\mathrm{AC}$ \\
\hline $\mathrm{AC}$ & $\mathrm{CC}$ & $\mathrm{AC}$ & $\mathrm{AC}$ & $\mathrm{CC}$ & $\mathrm{AA}$ & $\mathrm{AC}$ & $\mathrm{AC}$ & $\mathrm{AA}$ & $\mathrm{AC}$ & $\mathrm{AA}$ \\
\hline $\mathrm{AC}$ & $\mathrm{AA}$ & $\mathrm{CC}$ & $\mathrm{AC}$ & $\mathrm{AC}$ & $\mathrm{AA}$ & $\mathrm{AC}$ & $\mathrm{AC}$ & $\mathrm{AA}$ & $\mathrm{AC}$ & $\mathrm{CC}$ \\
\hline AA & $\mathrm{AC}$ & $\mathrm{AC}$ & $\mathrm{AC}$ & AA & $\mathrm{CC}$ & AA & $\mathrm{AC}$ & $\mathrm{AC}$ & $\mathrm{AC}$ & \\
\hline GDsnp00967 & 6 & \multicolumn{2}{|c|}{7904258} & AA & $\mathrm{AG}$ & $\mathrm{AG}$ & AA & $\mathrm{AA}$ & $\mathrm{AG}$ & $\mathrm{AA}$ \\
\hline AA & $\mathrm{AG}$ & $\mathrm{AG}$ & AA & $\mathrm{AG}$ & $\mathrm{AA}$ & $\mathrm{AA}$ & $\mathrm{AA}$ & $\mathrm{AG}$ & $\mathrm{AG}$ & $\mathrm{AA}$ \\
\hline $\mathrm{AG}$ & $\mathrm{AG}$ & $\mathrm{AG}$ & $\mathrm{AA}$ & AA & $\mathrm{AG}$ & $\mathrm{AG}$ & AA & $\mathrm{AA}$ & $\mathrm{AG}$ & $\mathrm{AA}$ \\
\hline$A G$ & $\mathrm{AG}$ & $\mathrm{AA}$ & $\mathrm{AG}$ & $\mathrm{AG}$ & GG & $\mathrm{AG}$ & AA & GG & GG & $\mathrm{AA}$ \\
\hline $\mathrm{AG}$ & $\mathrm{AG}$ & $\mathrm{AG}$ & $\mathrm{AA}$ & AA & $\mathrm{AG}$ & $\mathrm{AG}$ & - & $\mathrm{AA}$ & $\mathrm{AG}$ & GG \\
\hline $\mathrm{AG}$ & $\mathrm{AG}$ & $\mathrm{AG}$ & $\mathrm{AA}$ & AA & $\mathrm{AG}$ & GG & AA & AA & $\mathrm{AG}$ & $\mathrm{AG}$ \\
\hline AG & $\mathrm{AG}$ & $\mathrm{AG}$ & $\mathrm{AG}$ & GG & $\mathrm{AA}$ & $\mathrm{AG}$ & $\mathrm{AG}$ & $\mathrm{AA}$ & $\mathrm{AG}$ & $\mathrm{AA}$ \\
\hline$A G$ & AA & GG & $\mathrm{AG}$ & $\mathrm{AG}$ & $\mathrm{AA}$ & $\mathrm{AG}$ & $\mathrm{AG}$ & $\mathrm{AA}$ & $\mathrm{AG}$ & GG \\
\hline AA & $\mathrm{AG}$ & $\mathrm{AG}$ & $\mathrm{AG}$ & AA & GG & AA & $\mathrm{AG}$ & $\mathrm{AG}$ & $\mathrm{AG}$ & \\
\hline GDsnp00282 & 6 & \multicolumn{2}{|c|}{15438638} & GG & $\mathrm{AG}$ & $\mathrm{AG}$ & GG & $\mathrm{AG}$ & $\mathrm{AG}$ & $\mathrm{AG}$ \\
\hline GG & GG & $\mathrm{AG}$ & GG & $\mathrm{AG}$ & GG & GG & GG & GG & $\mathrm{AG}$ & GG \\
\hline $\mathrm{AG}$ & $\mathrm{AG}$ & GG & GG & GG & $\mathrm{AA}$ & GG & $\mathrm{AG}$ & $\mathrm{AG}$ & $\mathrm{AG}$ & GG \\
\hline$A G$ & $\mathrm{AG}$ & GG & $\mathrm{AG}$ & $\mathrm{AG}$ & $\mathrm{AA}$ & $\mathrm{AG}$ & $\mathrm{AG}$ & AA & $\mathrm{AG}$ & $\mathrm{AG}$ \\
\hline $\mathrm{AG}$ & $\mathrm{AG}$ & $\mathrm{AG}$ & $\mathrm{AG}$ & $\mathrm{AG}$ & $\mathrm{AG}$ & - & - & GG & $\mathrm{AG}$ & $\mathrm{AA}$ \\
\hline AG & $\mathrm{AG}$ & AA & $\mathrm{AG}$ & GG & $\mathrm{AG}$ & AA & GG & GG & $\mathrm{AG}$ & $\mathrm{AG}$ \\
\hline AG & AA & $\mathrm{AG}$ & $\mathrm{AG}$ & AA & GG & $\mathrm{AG}$ & $\mathrm{AG}$ & $\mathrm{AG}$ & GG & GG \\
\hline AG & GG & AA & $\mathrm{AG}$ & GG & GG & $\mathrm{AG}$ & $\mathrm{AG}$ & GG & $\mathrm{AG}$ & $\mathrm{AG}$ \\
\hline GG & $\mathrm{AG}$ & $\mathrm{AG}$ & $\mathrm{AG}$ & GG & AG & GG & $\mathrm{AG}$ & GG & GG & \\
\hline GDsnp01556 & 6 & \multicolumn{2}{|c|}{18703773} & $\mathrm{AG}$ & $\mathrm{AG}$ & GG & $\mathrm{AG}$ & $\mathrm{AG}$ & $\mathrm{AG}$ & $\mathrm{AG}$ \\
\hline $\mathrm{AG}$ & $\mathrm{AA}$ & $\mathrm{AA}$ & $\mathrm{AA}$ & $\mathrm{AG}$ & $\mathrm{AA}$ & GG & $\mathrm{AG}$ & $\mathrm{AG}$ & $\mathrm{AG}$ & $\mathrm{AG}$ \\
\hline GG & $\mathrm{AG}$ & GG & $\mathrm{AG}$ & $\mathrm{AG}$ & GG & $\mathrm{AG}$ & $\mathrm{AG}$ & $\mathrm{AG}$ & $\mathrm{AG}$ & $\mathrm{AG}$ \\
\hline AG & $\mathrm{AG}$ & $\mathrm{AG}$ & $\mathrm{AG}$ & AA & GG & $\mathrm{AG}$ & GG & GG & $\mathrm{AG}$ & $\mathrm{AG}$ \\
\hline $\mathrm{AG}$ & $\mathrm{AG}$ & AA & $\mathrm{AG}$ & $\mathrm{AG}$ & $\mathrm{AG}$ & $\mathrm{AG}$ & GG & $\mathrm{AG}$ & $\mathrm{AG}$ & $\mathrm{AG}$ \\
\hline $\mathrm{AG}$ & GG & GG & $\mathrm{AG}$ & $\mathrm{AG}$ & GG & $\mathrm{AG}$ & AA & GG & $\mathrm{AG}$ & $\mathrm{AG}$ \\
\hline AA & GG & $\mathrm{AG}$ & GG & $\mathrm{AG}$ & $\mathrm{AG}$ & GG & GG & $\mathrm{AG}$ & $\mathrm{AG}$ & $\mathrm{AG}$ \\
\hline GG & $\mathrm{AA}$ & GG & GG & AA & $\mathrm{AA}$ & AA & $\mathrm{AG}$ & $\mathrm{AA}$ & $\mathrm{AG}$ & $\mathrm{AG}$ \\
\hline $\mathrm{AG}$ & $\mathrm{AG}$ & GG & GG & $\mathrm{AA}$ & $\mathrm{AG}$ & $\mathrm{AG}$ & $\mathrm{AG}$ & $\mathrm{AA}$ & $\mathrm{AG}$ & \\
\hline GDsnp00218 & 6 & \multicolumn{2}{|c|}{19291994} & $\mathrm{AT}$ & $\mathrm{AT}$ & AA & $\mathrm{AT}$ & $\mathrm{AT}$ & $\mathrm{AT}$ & $\mathrm{AT}$ \\
\hline $\mathrm{TT}$ & AA & $\mathrm{TT}$ & $\mathrm{TT}$ & AT & $\mathrm{TT}$ & AA & $\mathrm{AT}$ & $\mathrm{AT}$ & $\mathrm{AT}$ & $\mathrm{AT}$ \\
\hline AA & $\mathrm{AT}$ & AA & $\mathrm{AT}$ & $\mathrm{AT}$ & $\mathrm{AA}$ & $\mathrm{TT}$ & $\mathrm{AT}$ & $\mathrm{AT}$ & $\mathrm{AT}$ & $\mathrm{AT}$ \\
\hline $\mathrm{AT}$ & $\mathrm{AT}$ & $\mathrm{AT}$ & $\mathrm{AT}$ & $\mathrm{TT}$ & $\mathrm{AA}$ & $\mathrm{AT}$ & $\mathrm{AA}$ & $\mathrm{AA}$ & AT & AT \\
\hline $\mathrm{AT}$ & $\mathrm{AT}$ & $\mathrm{TT}$ & $\mathrm{AT}$ & $\mathrm{AT}$ & $\mathrm{AT}$ & - & $\mathrm{AA}$ & AT & AT & $\mathrm{AT}$ \\
\hline AT & AA & AA & AA & $\mathrm{AT}$ & $\mathrm{AA}$ & $\mathrm{AT}$ & $\mathrm{TT}$ & $\mathrm{AA}$ & AT & AT \\
\hline $\mathrm{TT}$ & AA & AA & AA & $\mathrm{AT}$ & $\mathrm{AT}$ & AA & AA & AA & $\mathrm{AT}$ & $\mathrm{AT}$ \\
\hline AA & $\mathrm{TT}$ & $\mathrm{AA}$ & $\mathrm{AA}$ & $\mathrm{TT}$ & $\mathrm{TT}$ & $\mathrm{TT}$ & $\mathrm{AT}$ & $\mathrm{TT}$ & $\mathrm{AA}$ & $\mathrm{AT}$ \\
\hline AT & AT & AA & - & $\mathrm{TT}$ & AT & AT & AT & $\mathrm{TT}$ & - & \\
\hline GDsnp01502 & 6 & \multicolumn{2}{|c|}{20473595} & GG & $\mathrm{AA}$ & $\mathrm{AA}$ & $\mathrm{AG}$ & $\mathrm{AG}$ & $\mathrm{AG}$ & $\mathrm{AG}$ \\
\hline $\mathrm{AG}$ & $\mathrm{AG}$ & GG & GG & $\mathrm{AG}$ & GG & AA & $\mathrm{AG}$ & GG & $\mathrm{AG}$ & $\mathrm{AG}$ \\
\hline AA & $\mathrm{AG}$ & $\mathrm{AG}$ & $\mathrm{AG}$ & $\mathrm{AG}$ & $\mathrm{AA}$ & $\mathrm{AG}$ & $\mathrm{AG}$ & $\mathrm{AG}$ & $\mathrm{AG}$ & $\mathrm{AG}$ \\
\hline AG & $\mathrm{AG}$ & GG & $\mathrm{AG}$ & $\mathrm{AG}$ & $\mathrm{AA}$ & $\mathrm{AG}$ & AA & $\mathrm{AA}$ & $\mathrm{AG}$ & $\mathrm{AG}$ \\
\hline $\mathrm{AG}$ & $\mathrm{AG}$ & GG & $\mathrm{AG}$ & $\mathrm{AG}$ & $\mathrm{AG}$ & $\mathrm{AG}$ & AA & $\mathrm{AG}$ & $\mathrm{AG}$ & $\mathrm{AG}$ \\
\hline $\mathrm{AG}$ & $\mathrm{AA}$ & $\mathrm{AA}$ & $\mathrm{AG}$ & $\mathrm{AG}$ & $\mathrm{AA}$ & $\mathrm{AG}$ & GG & $\mathrm{AA}$ & $\mathrm{AG}$ & $\mathrm{AG}$ \\
\hline GG & $\mathrm{AA}$ & $\mathrm{AA}$ & $\mathrm{AA}$ & $\mathrm{AG}$ & $\mathrm{AG}$ & $\mathrm{AA}$ & $\mathrm{AG}$ & $\mathrm{AA}$ & $\mathrm{AG}$ & $\mathrm{AG}$ \\
\hline AA & GG & $\mathrm{AA}$ & $\mathrm{AA}$ & $\mathrm{GG}$ & $\mathrm{AG}$ & GG & $\mathrm{AG}$ & GG & $\mathrm{AA}$ & $\mathrm{AG}$ \\
\hline $\mathrm{AG}$ & $\mathrm{AG}$ & AA & AA & $\mathrm{GG}$ & $\mathrm{AG}$ & $\mathrm{AA}$ & $\mathrm{AG}$ & GG & $\mathrm{AG}$ & \\
\hline
\end{tabular}

Continued next page 
Supplemental Table 2. Continued.

\begin{tabular}{|c|c|c|c|c|c|c|c|c|c|c|}
\hline \multirow{3}{*}{$\begin{array}{l}\text { SNP name } \\
\text { GDsnp01682 } \\
\text { AG }\end{array}$} & \multicolumn{10}{|c|}{ Linkage group Physical location (bp) Individuals } \\
\hline & 6 & \multicolumn{2}{|c|}{21054368} & GG & GG & GG & GG & GG & GG & GG \\
\hline & $\mathrm{AG}$ & $\mathrm{AG}$ & GG & GG & GG & GG & GG & GG & GG & GG \\
\hline GG & GG & $\mathrm{AG}$ & GG & GG & GG & GG & GG & GG & GG & GG \\
\hline GG & GG & GG & $\mathrm{AG}$ & GG & GG & GG & GG & GG & $\mathrm{AG}$ & $\mathrm{AG}$ \\
\hline GG & GG & $\mathrm{AG}$ & GG & GG & GG & GG & GG & GG & GG & GG \\
\hline GG & GG & GG & $\mathrm{AG}$ & GG & GG & GG & $\mathrm{AG}$ & GG & GG & GG \\
\hline GG & GG & GG & GG & GG & GG & GG & GG & GG & GG & GG \\
\hline GG & GG & GG & GG & GG & GG & GG & $\mathrm{AG}$ & GG & GG & GG \\
\hline GG & GG & GG & GG & GG & GG & $\mathrm{AG}$ & GG & GG & GG & \\
\hline GDsnp01574 & 6 & \multicolumn{2}{|c|}{23924169} & $\mathrm{AA}$ & GG & GG & $\mathrm{AG}$ & GG & $\mathrm{AG}$ & $\mathrm{AG}$ \\
\hline $\mathrm{AA}$ & $\mathrm{AG}$ & $\mathrm{AA}$ & $\mathrm{AG}$ & $\mathrm{AG}$ & $\mathrm{AG}$ & GG & AA & $\mathrm{AG}$ & - & GG \\
\hline GG & $\mathrm{AG}$ & - & $\mathrm{AG}$ & $\mathrm{AG}$ & GG & AA & $\mathrm{AG}$ & $\mathrm{AG}$ & GG & $\mathrm{AG}$ \\
\hline $\mathrm{AG}$ & $\mathrm{AG}$ & $\mathrm{AA}$ & $\mathrm{AG}$ & $\mathrm{AG}$ & $\mathrm{AG}$ & AG & GG & GG & $\mathrm{AG}$ & - \\
\hline GG & $\mathrm{AG}$ & AA & $\mathrm{AG}$ & $\mathrm{AG}$ & $\mathrm{AG}$ & $\mathrm{AG}$ & GG & $\mathrm{AG}$ & $\mathrm{AG}$ & $\mathrm{AG}$ \\
\hline $\mathrm{AG}$ & GG & GG & - & GG & GG & $\mathrm{AG}$ & AA & GG & $\mathrm{AG}$ & $\mathrm{AG}$ \\
\hline $\mathrm{AG}$ & GG & GG & GG & $\mathrm{AG}$ & $\mathrm{AG}$ & - & AA & GG & $\mathrm{AG}$ & GG \\
\hline- & AA & GG & GG & AA & $\mathrm{AG}$ & AA & $\mathrm{AG}$ & AA & GG & $\mathrm{AG}$ \\
\hline $\mathrm{AG}$ & $\mathrm{AG}$ & $\mathrm{AG}$ & $\mathrm{AG}$ & AA & $\mathrm{AG}$ & GG & $\mathrm{AA}$ & $\mathrm{AA}$ & $\mathrm{AG}$ & \\
\hline GDsnp02138 & 6 & \multicolumn{2}{|c|}{24959354} & $\mathrm{AC}$ & $\mathrm{AA}$ & AA & $\mathrm{AC}$ & AA & $\mathrm{AC}$ & $\mathrm{AC}$ \\
\hline $\mathrm{AC}$ & $\mathrm{AA}$ & $\mathrm{AC}$ & $\mathrm{AC}$ & $\mathrm{AC}$ & $\mathrm{AC}$ & AA & $\mathrm{CC}$ & $\mathrm{AC}$ & $\mathrm{AA}$ & $\mathrm{AA}$ \\
\hline AA & $\mathrm{AC}$ & $\mathrm{AA}$ & $\mathrm{AC}$ & $\mathrm{AC}$ & $\mathrm{AA}$ & $\mathrm{AC}$ & $\mathrm{AA}$ & $\mathrm{AA}$ & $\mathrm{AA}$ & $\mathrm{AC}$ \\
\hline $\mathrm{AC}$ & $\mathrm{AC}$ & $\mathrm{CC}$ & AA & $\mathrm{AC}$ & $\mathrm{AC}$ & $\mathrm{AC}$ & AA & AA & AA & AA \\
\hline AA & AA & $\mathrm{AC}$ & AA & $\mathrm{AC}$ & $\mathrm{AC}$ & AA & AA & $\mathrm{AC}$ & $\mathrm{AC}$ & $\mathrm{AC}$ \\
\hline $\mathrm{AC}$ & AA & AA & $\mathrm{AA}$ & AA & $\mathrm{AA}$ & $\mathrm{AC}$ & $\mathrm{AC}$ & $\mathrm{AA}$ & $\mathrm{AC}$ & $\mathrm{AC}$ \\
\hline $\mathrm{AC}$ & AA & AA & $\mathrm{AA}$ & $\mathrm{AA}$ & $\mathrm{AA}$ & AA & $\mathrm{AC}$ & $\mathrm{AA}$ & $\mathrm{AA}$ & $\mathrm{AA}$ \\
\hline AA & $\mathrm{AC}$ & $\mathrm{AA}$ & $\mathrm{AA}$ & $\mathrm{CC}$ & $\mathrm{AC}$ & $\mathrm{AC}$ & $\mathrm{AA}$ & $\mathrm{CC}$ & $\mathrm{AA}$ & $\mathrm{CC}$ \\
\hline $\mathrm{AC}$ & $\mathrm{AC}$ & $\mathrm{AC}$ & $\mathrm{AC}$ & $\mathrm{CC}$ & $\mathrm{AC}$ & AA & $\mathrm{CC}$ & $\mathrm{CC}$ & $\mathrm{AC}$ & \\
\hline GDsnp01994 & 7 & \multicolumn{2}{|c|}{2052413} & $\mathrm{AG}$ & $\mathrm{AA}$ & AA & AA & GG & AA & $\mathrm{AG}$ \\
\hline GG & GG & $\mathrm{AG}$ & GG & $\mathrm{AG}$ & $\mathrm{AG}$ & AG & AA & AA & $\mathrm{AA}$ & GG \\
\hline AA & AA & $\mathrm{AG}$ & $\mathrm{AG}$ & AA & $\mathrm{AG}$ & AG & AA & AA & $\mathrm{AA}$ & AA \\
\hline AA & $\mathrm{AG}$ & $\mathrm{AG}$ & $\mathrm{AG}$ & $\mathrm{AG}$ & $\mathrm{AA}$ & AA & $\mathrm{AG}$ & $\mathrm{AA}$ & GG & AA \\
\hline AA & AA & $\mathrm{AG}$ & AA & $\mathrm{AG}$ & $\mathrm{AA}$ & AA & - & $\mathrm{AA}$ & $\mathrm{AA}$ & $\mathrm{AG}$ \\
\hline $\mathrm{AA}$ & $\mathrm{AG}$ & $\mathrm{AG}$ & AA & AA & $\mathrm{AA}$ & AA & GG & $\mathrm{AG}$ & GG & $\mathrm{AG}$ \\
\hline $\mathrm{AG}$ & $\mathrm{AG}$ & $\mathrm{AG}$ & $\mathrm{AG}$ & $\mathrm{AG}$ & $\mathrm{AA}$ & AA & AA & $\mathrm{AG}$ & $\mathrm{AG}$ & $\mathrm{AG}$ \\
\hline $\mathrm{AG}$ & $\mathrm{AG}$ & $\mathrm{AG}$ & $\mathrm{AG}$ & GG & $\mathrm{AA}$ & $\mathrm{AG}$ & AA & $\mathrm{AG}$ & $\mathrm{AG}$ & $\mathrm{AG}$ \\
\hline AA & AA & $\mathrm{AG}$ & $\mathrm{AG}$ & - & AA & GG & $\mathrm{AG}$ & AA & $\mathrm{AG}$ & \\
\hline GDsnp00051 & 7 & \multicolumn{2}{|c|}{2871953} & $\mathrm{CC}$ & $\mathrm{CG}$ & GG & $\mathrm{CG}$ & $\mathrm{CC}$ & $\mathrm{CG}$ & $\mathrm{CC}$ \\
\hline $\mathrm{CG}$ & $\mathrm{CC}$ & $\mathrm{CC}$ & $\mathrm{CC}$ & $\mathrm{CG}$ & $\mathrm{CC}$ & $\mathrm{CG}$ & $\mathrm{CG}$ & $\mathrm{CG}$ & $\mathrm{CG}$ & $\mathrm{CC}$ \\
\hline GG & GG & $\mathrm{CG}$ & $\mathrm{CG}$ & GG & $\mathrm{CC}$ & $\mathrm{CG}$ & $\mathrm{CG}$ & $\mathrm{CG}$ & GG & $\mathrm{CG}$ \\
\hline GG & $\mathrm{CG}$ & $\mathrm{CG}$ & $\mathrm{CG}$ & $\mathrm{CC}$ & GG & GG & $\mathrm{CG}$ & $\mathrm{CG}$ & $\mathrm{CG}$ & $\mathrm{CG}$ \\
\hline GG & GG & $\mathrm{CG}$ & GG & $\mathrm{CG}$ & $\mathrm{CG}$ & - & - & GG & GG & $\mathrm{CG}$ \\
\hline $\mathrm{CG}$ & $\mathrm{CC}$ & $\mathrm{CC}$ & $\mathrm{CG}$ & GG & $\mathrm{CG}$ & GG & $\mathrm{CC}$ & $\mathrm{CC}$ & $\mathrm{CC}$ & $\mathrm{CG}$ \\
\hline $\mathrm{CC}$ & $\mathrm{CG}$ & $\mathrm{CG}$ & $\mathrm{CC}$ & $\mathrm{CG}$ & GG & $\mathrm{CG}$ & $\mathrm{CG}$ & $\mathrm{CC}$ & $\mathrm{CC}$ & $\mathrm{CC}$ \\
\hline $\mathrm{CC}$ & $\mathrm{CG}$ & $\mathrm{CC}$ & $\mathrm{CC}$ & $\mathrm{CC}$ & $\mathrm{CG}$ & $\mathrm{CG}$ & GG & $\mathrm{CG}$ & $\mathrm{CG}$ & $\mathrm{CG}$ \\
\hline $\mathrm{CC}$ & $\mathrm{CG}$ & $\mathrm{CG}$ & $\mathrm{CC}$ & $\mathrm{CG}$ & GG & $\mathrm{CC}$ & $\mathrm{CC}$ & GG & $\mathrm{CC}$ & \\
\hline GDsnp01170 & 7 & \multicolumn{2}{|c|}{4346176} & AA & $\mathrm{AG}$ & GG & $\mathrm{AG}$ & AA & $\mathrm{AG}$ & $\mathrm{AA}$ \\
\hline $\mathrm{AA}$ & $\mathrm{AA}$ & $\mathrm{AA}$ & $\mathrm{AG}$ & $\mathrm{AG}$ & $\mathrm{AA}$ & AG & $\mathrm{AG}$ & $\mathrm{AG}$ & $\mathrm{AG}$ & $\mathrm{AA}$ \\
\hline GG & GG & AA & $\mathrm{AG}$ & GG & $\mathrm{AA}$ & $\mathrm{AG}$ & $\mathrm{AG}$ & $\mathrm{AG}$ & GG & $\mathrm{AG}$ \\
\hline GG & $\mathrm{AG}$ & $\mathrm{AG}$ & $\mathrm{AG}$ & AA & GG & GG & $\mathrm{AG}$ & $\mathrm{AG}$ & $\mathrm{AG}$ & $\mathrm{AG}$ \\
\hline GG & GG & $\mathrm{AA}$ & GG & $\mathrm{AG}$ & $\mathrm{AG}$ & - & - & GG & GG & $\mathrm{AG}$ \\
\hline $\mathrm{AG}$ & $\mathrm{AA}$ & $\mathrm{AA}$ & $\mathrm{AG}$ & GG & $\mathrm{AG}$ & GG & $\mathrm{AA}$ & $\mathrm{AA}$ & $\mathrm{AA}$ & $\mathrm{AG}$ \\
\hline AA & $\mathrm{AG}$ & $\mathrm{AG}$ & $\mathrm{AA}$ & $\mathrm{AG}$ & GG & $\mathrm{AG}$ & $\mathrm{AG}$ & $\mathrm{AA}$ & $\mathrm{AA}$ & $\mathrm{AA}$ \\
\hline $\mathrm{AA}$ & $\mathrm{AG}$ & $\mathrm{AA}$ & AA & AA & $\mathrm{AG}$ & $\mathrm{AG}$ & GG & $\mathrm{AG}$ & $\mathrm{AG}$ & $\mathrm{AG}$ \\
\hline AA & $\mathrm{AG}$ & $\mathrm{AG}$ & $\mathrm{AA}$ & $\mathrm{AG}$ & GG & $\mathrm{AG}$ & $\mathrm{AA}$ & GG & $\mathrm{AA}$ & \\
\hline
\end{tabular}


Supplemental Table 2. Continued.

\begin{tabular}{|c|c|c|c|c|c|c|c|c|c|c|}
\hline SNP name & & & & inkag & Phy & catior & ndivi & & & \\
\hline$\overline{\text { GDsnp01872 }}$ & 7 & & & $\mathrm{AA}$ & $\mathrm{AC}$ & $\mathrm{AA}$ & $\mathrm{AA}$ & $\mathrm{AA}$ & $\mathrm{AC}$ & AA \\
\hline $\mathrm{AA}$ & $\mathrm{AA}$ & $\mathrm{AA}$ & $\mathrm{AC}$ & $\mathrm{AA}$ & $\mathrm{AC}$ & $\mathrm{AC}$ & $\mathrm{AC}$ & $\mathrm{AA}$ & $\mathrm{AC}$ & $\mathrm{AC}$ \\
\hline $\mathrm{AA}$ & $\mathrm{AA}$ & $\mathrm{AA}$ & $\mathrm{AA}$ & $\mathrm{AA}$ & $\mathrm{AC}$ & $\mathrm{AA}$ & $\mathrm{AC}$ & $\mathrm{AC}$ & AA & $\mathrm{AC}$ \\
\hline AA & $\mathrm{AA}$ & $\mathrm{AA}$ & $\mathrm{AA}$ & $\mathrm{AA}$ & $\mathrm{AC}$ & - & $\mathrm{AA}$ & $\mathrm{AA}$ & AA & $\mathrm{AA}$ \\
\hline $\mathrm{AC}$ & $\mathrm{AC}$ & $\mathrm{AC}$ & $\mathrm{AA}$ & $\mathrm{AA}$ & $\mathrm{AC}$ & $\mathrm{AA}$ & $\mathrm{AC}$ & $\mathrm{AC}$ & $\mathrm{AA}$ & AA \\
\hline $\mathrm{AC}$ & AA & $\mathrm{AA}$ & $\mathrm{AC}$ & $\mathrm{AA}$ & $\mathrm{AA}$ & $\mathrm{AA}$ & $\mathrm{AA}$ & $\mathrm{AC}$ & $\mathrm{AC}$ & $\mathrm{AC}$ \\
\hline $\mathrm{AC}$ & $\mathrm{AC}$ & $\mathrm{AA}$ & - & $\mathrm{AC}$ & $\mathrm{AA}$ & AA & $\mathrm{AC}$ & $\mathrm{AA}$ & $\mathrm{AC}$ & \\
\hline GDsnp00224 & 7 & & & $\mathrm{AG}$ & $\mathrm{AG}$ & $\mathrm{AG}$ & $\mathrm{AG}$ & $\mathrm{AG}$ & $\mathrm{AG}$ & $\mathrm{AG}$ \\
\hline $\mathrm{AG}$ & AA & AA & $\mathrm{AA}$ & $\mathrm{AG}$ & $\mathrm{AA}$ & $\mathrm{AG}$ & $\mathrm{AG}$ & $\mathrm{AG}$ & AA & AA \\
\hline GG & GG & $\mathrm{AG}$ & $\mathrm{AG}$ & GG & $\mathrm{AA}$ & $\mathrm{AG}$ & $\mathrm{AG}$ & $\mathrm{AG}$ & GG & $\mathrm{AG}$ \\
\hline GG & $\mathrm{AG}$ & $\mathrm{AG}$ & $\mathrm{AG}$ & $\mathrm{AA}$ & GG & GG & $\mathrm{AG}$ & $\mathrm{AG}$ & AA & $\mathrm{AG}$ \\
\hline $\mathrm{AA}$ & AG & $\mathrm{AG}$ & $\mathrm{AA}$ & $\mathrm{AG}$ & GG & $\mathrm{AG}$ & $\mathrm{AA}$ & GG & AA & \\
\hline GDsnp02274 & 7 & & & $\mathrm{AT}$ & $\mathrm{AT}$ & $\mathrm{AT}$ & $\mathrm{AT}$ & $\mathrm{AT}$ & $\mathrm{AT}$ & AT \\
\hline $\mathrm{AT}$ & $\mathrm{TT}$ & $\mathrm{TT}$ & $\mathrm{TT}$ & $\mathrm{AT}$ & $\mathrm{TT}$ & $\mathrm{AT}$ & $\mathrm{AT}$ & $\mathrm{AT}$ & $\mathrm{TT}$ & TT \\
\hline AA & AT & AT & AT & $\mathrm{AA}$ & $\mathrm{TT}$ & $\mathrm{AT}$ & $\mathrm{TT}$ & TT & $\mathrm{AA}$ & AT \\
\hline AA & $\mathrm{AT}$ & $\mathrm{AT}$ & $\mathrm{AT}$ & $\mathrm{TT}$ & $\mathrm{AA}$ & $\mathrm{AA}$ & AT & $\mathrm{AT}$ & $\mathrm{TT}$ & AT \\
\hline AA & AA & $\mathrm{TT}$ & $\mathrm{AA}$ & $\mathrm{AT}$ & $\mathrm{AT}$ & $\mathrm{AT}$ & $\mathrm{AT}$ & $\mathrm{AT}$ & AA & AT \\
\hline $\mathrm{AT}$ & $\mathrm{TT}$ & $\mathrm{TT}$ & $\mathrm{TT}$ & $\mathrm{AT}$ & $\mathrm{AT}$ & $\mathrm{AA}$ & $\mathrm{TT}$ & $\mathrm{TT}$ & $\mathrm{TT}$ & TT \\
\hline TT & AT & AT & TT & $\mathrm{AT}$ & $\mathrm{AA}$ & $\mathrm{AA}$ & $\mathrm{AT}$ & AT & $\mathrm{TT}$ & TT \\
\hline TT & $\mathrm{AT}$ & $\mathrm{TT}$ & $\mathrm{TT}$ & $\mathrm{AT}$ & $\mathrm{AT}$ & $\mathrm{AT}$ & $\mathrm{AA}$ & $\mathrm{AT}$ & $\mathrm{AT}$ & AT \\
\hline $\mathrm{TT}$ & $\mathrm{AT}$ & $\mathrm{AT}$ & TT & AT & AA & AT & TT & AA & TT & \\
\hline $\mathrm{AA}$ & $\mathrm{AG}$ & $\mathrm{AG}$ & $\mathrm{AA}$ & $\mathrm{AG}$ & GG & $\mathrm{AG}$ & $\mathrm{AA}$ & GG & AA & \\
\hline GDsnp00247 & 7 & & & $\mathrm{AG}$ & $\mathrm{AG}$ & $\mathrm{AG}$ & $\mathrm{AG}$ & $\mathrm{AG}$ & $\mathrm{AA}$ & $\mathrm{AG}$ \\
\hline $\mathrm{AG}$ & GG & GG & GG & $\mathrm{AG}$ & GG & $\mathrm{AG}$ & $\mathrm{AG}$ & $\mathrm{AG}$ & GG & $\mathrm{AG}$ \\
\hline AA & $\mathrm{AG}$ & $\mathrm{AG}$ & $\mathrm{AG}$ & $\mathrm{AA}$ & GG & $\mathrm{AG}$ & GG & GG & AA & $\mathrm{AG}$ \\
\hline AA & $\mathrm{AG}$ & AA & $\mathrm{AG}$ & GG & $\mathrm{AG}$ & $\mathrm{AA}$ & $\mathrm{AG}$ & $\mathrm{AG}$ & GG & $\mathrm{AG}$ \\
\hline $\mathrm{AA}$ & AA & GG & $\mathrm{AA}$ & $\mathrm{AG}$ & $\mathrm{AG}$ & $\mathrm{AA}$ & $\mathrm{AG}$ & $\mathrm{AG}$ & $\mathrm{AA}$ & AA \\
\hline $\mathrm{AG}$ & GG & GG & GG & $\mathrm{AG}$ & $\mathrm{AG}$ & $\mathrm{AA}$ & GG & GG & GG & GG \\
\hline GG & $\mathrm{AG}$ & $\mathrm{AG}$ & GG & $\mathrm{AG}$ & $\mathrm{AA}$ & $\mathrm{AA}$ & $\mathrm{AG}$ & $\mathrm{AG}$ & GG & GG \\
\hline GG & GG & GG & GG & $\mathrm{AG}$ & $\mathrm{AG}$ & $\mathrm{AG}$ & AA & $\mathrm{AG}$ & $\mathrm{AG}$ & $\mathrm{AG}$ \\
\hline GG & $\mathrm{AG}$ & $\mathrm{AG}$ & GG & $\mathrm{AG}$ & $\mathrm{AA}$ & $\mathrm{AG}$ & $\mathrm{GG}$ & $\mathrm{AA}$ & $\mathrm{GG}$ & \\
\hline GDsnp02840 & 7 & & & $\mathrm{AC}$ & $\mathrm{AC}$ & $\mathrm{AC}$ & $\mathrm{AC}$ & $\mathrm{AC}$ & $\mathrm{AC}$ & $\mathrm{AC}$ \\
\hline $\mathrm{AC}$ & $\mathrm{CC}$ & $\mathrm{CC}$ & $\mathrm{CC}$ & $\mathrm{AC}$ & $\mathrm{CC}$ & $\mathrm{AC}$ & $\mathrm{AA}$ & $\mathrm{AC}$ & $\mathrm{CC}$ & $\mathrm{AC}$ \\
\hline $\mathrm{AA}$ & $\mathrm{AC}$ & $\mathrm{AC}$ & $\mathrm{AC}$ & $\mathrm{AA}$ & $\mathrm{CC}$ & $\mathrm{AC}$ & $\mathrm{AC}$ & $\mathrm{AC}$ & AA & $\mathrm{AC}$ \\
\hline $\mathrm{AA}$ & $\mathrm{AC}$ & $\mathrm{AA}$ & $\mathrm{AC}$ & $\mathrm{CC}$ & $\mathrm{CC}$ & $\mathrm{AA}$ & $\mathrm{AC}$ & $\mathrm{AC}$ & $\mathrm{CC}$ & AA \\
\hline $\mathrm{AC}$ & AA & $\mathrm{CC}$ & $\mathrm{AA}$ & $\mathrm{AC}$ & $\mathrm{AC}$ & $\mathrm{AA}$ & $\mathrm{AC}$ & $\mathrm{AC}$ & $\mathrm{AA}$ & AA \\
\hline $\mathrm{AC}$ & $\mathrm{CC}$ & $\mathrm{AC}$ & $\mathrm{CC}$ & $\mathrm{AC}$ & $\mathrm{AC}$ & $\mathrm{AA}$ & $\mathrm{CC}$ & $\mathrm{CC}$ & $\mathrm{CC}$ & $\mathrm{CC}$ \\
\hline $\mathrm{CC}$ & $\mathrm{AC}$ & $\mathrm{AC}$ & $\mathrm{CC}$ & $\mathrm{AC}$ & $\mathrm{AA}$ & $\mathrm{AA}$ & $\mathrm{AC}$ & $\mathrm{AC}$ & $\mathrm{CC}$ & $\mathrm{CC}$ \\
\hline $\mathrm{CC}$ & $\mathrm{CC}$ & $\mathrm{CC}$ & $\mathrm{CC}$ & $\mathrm{AC}$ & $\mathrm{AC}$ & $\mathrm{AC}$ & $\mathrm{AA}$ & $\mathrm{AC}$ & $\mathrm{AC}$ & $\mathrm{AC}$ \\
\hline $\mathrm{CC}$ & $\mathrm{AC}$ & $\mathrm{AC}$ & $\mathrm{CC}$ & $\mathrm{AC}$ & $\mathrm{AA}$ & $\mathrm{AC}$ & $\mathrm{CC}$ & $\mathrm{AA}$ & $\mathrm{CC}$ & \\
\hline
\end{tabular}

Continued next page 
Supplemental Table 2. Continued.

\begin{tabular}{|c|c|c|c|c|c|c|c|c|c|c|}
\hline \multirow{3}{*}{$\begin{array}{l}\text { SNP name } \\
\text { GDsnp02657 } \\
\text { AA }\end{array}$} & \multicolumn{10}{|c|}{ Linkage group Physical location (bp) Individuals } \\
\hline & 7 & \multicolumn{2}{|c|}{14319903} & AA & $\mathrm{AA}$ & AA & AA & AA & AA & AA \\
\hline & AA & AA & AA & AA & AA & AA & AA & AA & $\mathrm{AA}$ & AA \\
\hline AA & AA & AA & AA & AA & AA & AA & AA & AA & AA & AA \\
\hline AA & AA & AA & AA & AA & AA & AA & AA & AA & $\mathrm{AA}$ & AA \\
\hline AA & AA & AA & AA & AA & $\mathrm{AA}$ & AA & AA & AA & $\mathrm{AA}$ & AA \\
\hline AA & AA & $\mathrm{AA}$ & $\mathrm{AA}$ & AA & $\mathrm{AA}$ & AA & AA & $\mathrm{AA}$ & $\mathrm{AA}$ & AA \\
\hline AA & AA & AA & AA & AA & $\mathrm{AA}$ & $\mathrm{AA}$ & AA & $\mathrm{AA}$ & $\mathrm{AA}$ & AA \\
\hline AA & $\mathrm{AA}$ & AA & $\mathrm{AA}$ & AA & $\mathrm{AA}$ & $\mathrm{AA}$ & AA & $\mathrm{AA}$ & $\mathrm{AA}$ & AA \\
\hline $\mathrm{AA}$ & AA & AA & AA & AA & AA & $\mathrm{AA}$ & $\mathrm{AA}$ & $\mathrm{AA}$ & $\mathrm{AA}$ & \\
\hline GDsnp01848 & 7 & \multicolumn{2}{|c|}{14397836} & AT & $\mathrm{AT}$ & $\mathrm{AT}$ & $\mathrm{AT}$ & $\mathrm{AT}$ & $\mathrm{AT}$ & AT \\
\hline $\mathrm{AA}$ & $\mathrm{AT}$ & AA & AA & $\mathrm{AT}$ & $\mathrm{AA}$ & $\mathrm{AT}$ & $\mathrm{TT}$ & $\mathrm{AT}$ & $\mathrm{AA}$ & AT \\
\hline $\mathrm{TT}$ & $\mathrm{AT}$ & $\mathrm{AT}$ & $\mathrm{AT}$ & $\mathrm{TT}$ & $\mathrm{AA}$ & $\mathrm{TT}$ & $\mathrm{AT}$ & AT & $\mathrm{TT}$ & AT \\
\hline $\mathrm{TT}$ & $\mathrm{AT}$ & $\mathrm{TT}$ & $\mathrm{AT}$ & AA & $\mathrm{AA}$ & $\mathrm{TT}$ & $\mathrm{AT}$ & $\mathrm{AT}$ & $\mathrm{AA}$ & TT \\
\hline AT & $\mathrm{TT}$ & AA & $\mathrm{TT}$ & $\mathrm{AT}$ & $\mathrm{AT}$ & $\mathrm{TT}$ & $\mathrm{AT}$ & $\mathrm{AT}$ & $\mathrm{TT}$ & TT \\
\hline AT & AA & $\mathrm{AT}$ & AA & $\mathrm{AT}$ & $\mathrm{AT}$ & $\mathrm{TT}$ & AA & $\mathrm{AA}$ & $\mathrm{AA}$ & AA \\
\hline $\mathrm{AA}$ & $\mathrm{AT}$ & $\mathrm{AT}$ & AA & $\mathrm{AT}$ & $\mathrm{TT}$ & $\mathrm{AT}$ & $\mathrm{AT}$ & $\mathrm{AT}$ & $\mathrm{AA}$ & $\mathrm{AA}$ \\
\hline AA & AA & AA & AA & $\mathrm{AT}$ & $\mathrm{AT}$ & $\mathrm{AT}$ & $\mathrm{TT}$ & $\mathrm{AT}$ & $\mathrm{AT}$ & AT \\
\hline $\mathrm{AA}$ & $\mathrm{AT}$ & $\mathrm{AT}$ & $\mathrm{AA}$ & $\mathrm{AT}$ & $\mathrm{TT}$ & $\mathrm{AT}$ & $\mathrm{AA}$ & $\mathrm{TT}$ & $\mathrm{AA}$ & \\
\hline GDsnp00256 & 7 & \multicolumn{2}{|c|}{17033576} & $\mathrm{AT}$ & $\mathrm{AT}$ & $\mathrm{AT}$ & $\mathrm{AT}$ & $\mathrm{AT}$ & AT & $\mathrm{AA}$ \\
\hline $\mathrm{TT}$ & $\mathrm{AT}$ & $\mathrm{TT}$ & $\mathrm{AT}$ & $\mathrm{AT}$ & $\mathrm{TT}$ & $\mathrm{AT}$ & $\mathrm{AA}$ & $\mathrm{TT}$ & $\mathrm{TT}$ & $\mathrm{AT}$ \\
\hline AA & $\mathrm{AT}$ & $\mathrm{AT}$ & $\mathrm{AT}$ & AA & $\mathrm{TT}$ & $\mathrm{AA}$ & $\mathrm{AT}$ & $\mathrm{AT}$ & $\mathrm{AA}$ & AT \\
\hline AA & $\mathrm{AT}$ & AA & $\mathrm{AT}$ & $\mathrm{TT}$ & $\mathrm{TT}$ & AA & AA & $\mathrm{AT}$ & $\mathrm{AT}$ & AA \\
\hline $\mathrm{AT}$ & $\mathrm{AA}$ & $\mathrm{TT}$ & AA & $\mathrm{AT}$ & $\mathrm{AT}$ & $\mathrm{AA}$ & $\mathrm{AT}$ & $\mathrm{AT}$ & $\mathrm{AA}$ & $\mathrm{AA}$ \\
\hline AT & $\mathrm{TT}$ & AA & $\mathrm{TT}$ & $\mathrm{AT}$ & $\mathrm{AT}$ & $\mathrm{AA}$ & $\mathrm{TT}$ & $\mathrm{TT}$ & $\mathrm{TT}$ & $\mathrm{TT}$ \\
\hline $\mathrm{TT}$ & $\mathrm{AT}$ & $\mathrm{AT}$ & $\mathrm{TT}$ & $\mathrm{AT}$ & $\mathrm{AA}$ & $\mathrm{TT}$ & $\mathrm{AT}$ & $\mathrm{AT}$ & $\mathrm{TT}$ & TT \\
\hline $\mathrm{TT}$ & $\mathrm{TT}$ & $\mathrm{AT}$ & $\mathrm{TT}$ & $\mathrm{TT}$ & $\mathrm{AT}$ & $\mathrm{AT}$ & $\mathrm{AT}$ & $\mathrm{AT}$ & $\mathrm{AT}$ & AT \\
\hline $\mathrm{TT}$ & $\mathrm{AT}$ & $\mathrm{AT}$ & $\mathrm{TT}$ & AT & $\mathrm{AA}$ & $\mathrm{AT}$ & $\mathrm{TT}$ & AA & $\mathrm{TT}$ & \\
\hline GDsnp02436 & 7 & \multicolumn{2}{|c|}{17901742} & AA & $\mathrm{AA}$ & $\mathrm{AA}$ & AA & $\mathrm{AA}$ & $\mathrm{AA}$ & $\mathrm{AA}$ \\
\hline AA & AA & AA & AA & AA & $\mathrm{AA}$ & AA & AA & AA & $\mathrm{AA}$ & AA \\
\hline AA & $\mathrm{AA}$ & $\mathrm{AA}$ & AA & AA & $\mathrm{AA}$ & AA & AA & $\mathrm{AA}$ & $\mathrm{AA}$ & AA \\
\hline AA & AA & AA & $\mathrm{AA}$ & $\mathrm{AA}$ & $\mathrm{AA}$ & $\mathrm{AA}$ & $\mathrm{AA}$ & $\mathrm{AA}$ & $\mathrm{AA}$ & AA \\
\hline AA & AA & AA & AA & AA & $\mathrm{AA}$ & - & AA & $\mathrm{AA}$ & $\mathrm{AA}$ & AA \\
\hline AA & AA & AA & AA & AA & $\mathrm{AA}$ & AA & AA & AA & $\mathrm{AA}$ & AA \\
\hline AA & AA & AA & AA & AA & $\mathrm{AA}$ & AA & AA & AA & $\mathrm{AA}$ & AA \\
\hline AA & AA & AA & $\mathrm{AA}$ & $\mathrm{AA}$ & $\mathrm{AA}$ & $\mathrm{AA}$ & $\mathrm{AA}$ & $\mathrm{AA}$ & $\mathrm{AA}$ & AA \\
\hline $\mathrm{AA}$ & AA & AA & $\mathrm{AA}$ & $\mathrm{AA}$ & AA & $\mathrm{AA}$ & $\mathrm{AA}$ & $\mathrm{AA}$ & $\mathrm{AA}$ & \\
\hline GDsnp02291 & 7 & \multicolumn{2}{|c|}{21085447} & $\mathrm{CC}$ & $\mathrm{AC}$ & $\mathrm{AC}$ & $\mathrm{AC}$ & $\mathrm{AC}$ & $\mathrm{AC}$ & $\mathrm{CC}$ \\
\hline $\mathrm{AA}$ & $\mathrm{AC}$ & AA & $\mathrm{AA}$ & $\mathrm{AC}$ & $\mathrm{AA}$ & $\mathrm{AC}$ & $\mathrm{CC}$ & $\mathrm{AA}$ & $\mathrm{AA}$ & $\mathrm{AC}$ \\
\hline $\mathrm{CC}$ & $\mathrm{AC}$ & $\mathrm{AC}$ & $\mathrm{AC}$ & $\mathrm{AC}$ & $\mathrm{AA}$ & $\mathrm{CC}$ & $\mathrm{AC}$ & $\mathrm{AC}$ & $\mathrm{CC}$ & $\mathrm{AC}$ \\
\hline $\mathrm{CC}$ & $\mathrm{AC}$ & $\mathrm{CC}$ & $\mathrm{AC}$ & AA & $\mathrm{AA}$ & $\mathrm{CC}$ & $\mathrm{CC}$ & $\mathrm{AC}$ & $\mathrm{AA}$ & $\mathrm{CC}$ \\
\hline $\mathrm{AC}$ & $\mathrm{CC}$ & AA & $\mathrm{CC}$ & $\mathrm{AC}$ & $\mathrm{AC}$ & $\mathrm{CC}$ & $\mathrm{CC}$ & $\mathrm{AC}$ & $\mathrm{CC}$ & $\mathrm{CC}$ \\
\hline AA & $\mathrm{AA}$ & $\mathrm{CC}$ & $\mathrm{AA}$ & $\mathrm{AC}$ & $\mathrm{AC}$ & $\mathrm{CC}$ & AA & $\mathrm{AC}$ & $\mathrm{AA}$ & AA \\
\hline AA & $\mathrm{AC}$ & $\mathrm{AC}$ & AA & $\mathrm{AC}$ & $\mathrm{CC}$ & AA & $\mathrm{AC}$ & $\mathrm{AC}$ & $\mathrm{AA}$ & AA \\
\hline $\mathrm{AC}$ & AA & $\mathrm{AC}$ & $\mathrm{AC}$ & AA & $\mathrm{AC}$ & $\mathrm{AC}$ & $\mathrm{AC}$ & $\mathrm{AC}$ & $\mathrm{AC}$ & $\mathrm{AC}$ \\
\hline $\mathrm{AA}$ & AA & $\mathrm{AC}$ & AA & $\mathrm{AC}$ & $\mathrm{CC}$ & $\mathrm{AC}$ & $\mathrm{AC}$ & $\mathrm{CC}$ & $\mathrm{AC}$ & \\
\hline GDsnp01040 & 7 & \multicolumn{2}{|c|}{21820259} & $\mathrm{AG}$ & $\mathrm{AA}$ & $\mathrm{AG}$ & $\mathrm{AG}$ & GG & $\mathrm{AG}$ & AA \\
\hline $\mathrm{AG}$ & $\mathrm{AG}$ & GG & $\mathrm{AG}$ & $\mathrm{AG}$ & GG & $\mathrm{AG}$ & AA & GG & $\mathrm{AG}$ & $\mathrm{AG}$ \\
\hline AA & $\mathrm{AG}$ & $\mathrm{AG}$ & $\mathrm{AG}$ & $\mathrm{AG}$ & GG & $\mathrm{AG}$ & GG & GG & $\mathrm{AG}$ & $\mathrm{AG}$ \\
\hline $\mathrm{AG}$ & $\mathrm{AA}$ & $\mathrm{AG}$ & $\mathrm{AG}$ & GG & GG & $\mathrm{AA}$ & AA & $\mathrm{AA}$ & $\mathrm{AG}$ & AA \\
\hline $\mathrm{AG}$ & $\mathrm{AA}$ & GG & $\mathrm{AG}$ & $\mathrm{AG}$ & $\mathrm{AG}$ & AA & AA & $\mathrm{AG}$ & $\mathrm{AA}$ & AA \\
\hline $\mathrm{AG}$ & $\mathrm{AG}$ & $\mathrm{AA}$ & $\mathrm{GG}$ & $\mathrm{AG}$ & $\mathrm{AG}$ & $\mathrm{AA}$ & $\mathrm{AG}$ & $\mathrm{AG}$ & GG & GG \\
\hline GG & $\mathrm{AG}$ & $\mathrm{AG}$ & GG & $\mathrm{AG}$ & $\mathrm{AA}$ & GG & $\mathrm{AG}$ & $\mathrm{AG}$ & GG & GG \\
\hline GG & GG & $\mathrm{AG}$ & $\mathrm{AG}$ & GG & $\mathrm{AG}$ & $\mathrm{AG}$ & GG & $\mathrm{AG}$ & $\mathrm{AG}$ & $\mathrm{AG}$ \\
\hline $\mathrm{AG}$ & GG & $\mathrm{AG}$ & $\mathrm{AG}$ & $\mathrm{AG}$ & $\mathrm{AA}$ & $\mathrm{AG}$ & $\mathrm{AG}$ & $\mathrm{AA}$ & $\mathrm{AG}$ & \\
\hline
\end{tabular}


Supplemental Table 2. Continued.

\begin{tabular}{|c|c|c|c|c|c|c|c|c|c|c|}
\hline \multirow{3}{*}{$\begin{array}{l}\text { SNP name } \\
\text { GDsnp00527 } \\
\text { AG }\end{array}$} & \multicolumn{10}{|c|}{ Linkage group Physical location (bp) Individuals } \\
\hline & 8 & \multicolumn{2}{|c|}{588425} & $\mathrm{AG}$ & $\mathrm{AG}$ & GG & $\mathrm{AG}$ & $\mathrm{AG}$ & GG & $\mathrm{AG}$ \\
\hline & AG & AA & $\mathrm{AG}$ & GG & GG & GG & AA & AA & $\mathrm{AG}$ & $\mathrm{AG}$ \\
\hline AA & $\mathrm{AG}$ & $\mathrm{AG}$ & GG & $\mathrm{AG}$ & AA & GG & AA & AA & GG & $\mathrm{AG}$ \\
\hline AG & $\mathrm{AG}$ & $\mathrm{AG}$ & AA & GG & GG & $\mathrm{AG}$ & AA & $\mathrm{AG}$ & $\mathrm{AA}$ & GG \\
\hline AG & AA & $\mathrm{AG}$ & $\mathrm{AG}$ & $\mathrm{AG}$ & $\mathrm{AA}$ & - & $\mathrm{AG}$ & $\mathrm{AG}$ & $\mathrm{AG}$ & $\mathrm{AG}$ \\
\hline AA & AA & $\mathrm{AG}$ & $\mathrm{AG}$ & GG & $\mathrm{AA}$ & $\mathrm{AG}$ & $\mathrm{AA}$ & $\mathrm{AA}$ & $\mathrm{AG}$ & GG \\
\hline AG & $\mathrm{AG}$ & $\mathrm{AG}$ & $\mathrm{AG}$ & $\mathrm{AG}$ & $\mathrm{AA}$ & GG & $\mathrm{AG}$ & GG & $\mathrm{AA}$ & $\mathrm{AG}$ \\
\hline $\mathrm{AG}$ & GG & GG & GG & AA & $\mathrm{AG}$ & $\mathrm{AG}$ & AA & $\mathrm{AG}$ & $\mathrm{AG}$ & $\mathrm{AG}$ \\
\hline $\mathrm{AA}$ & AA & $\mathrm{AG}$ & AA & GG & $\mathrm{AG}$ & $\mathrm{AG}$ & $\mathrm{AG}$ & GG & $\mathrm{AA}$ & \\
\hline GDsnp00503 & 8 & \multicolumn{2}{|c|}{669437} & $\mathrm{AG}$ & $\mathrm{AG}$ & GG & $\mathrm{AG}$ & $\mathrm{AG}$ & GG & $\mathrm{AG}$ \\
\hline GG & AA & AA & $\mathrm{AG}$ & GG & GG & GG & AA & AA & $\mathrm{AG}$ & $\mathrm{AG}$ \\
\hline AA & $\mathrm{AG}$ & AA & GG & $\mathrm{AG}$ & $\mathrm{AG}$ & $\mathrm{AG}$ & AA & AA & GG & GG \\
\hline $\mathrm{AG}$ & $\mathrm{AG}$ & $\mathrm{AG}$ & $\mathrm{AG}$ & GG & GG & $\mathrm{AG}$ & $\mathrm{AG}$ & $\mathrm{AG}$ & $\mathrm{AG}$ & GG \\
\hline GG & AA & $\mathrm{AG}$ & $\mathrm{AG}$ & $\mathrm{AG}$ & $\mathrm{AG}$ & $\mathrm{AG}$ & $\mathrm{AG}$ & $\mathrm{AG}$ & $\mathrm{AG}$ & $\mathrm{AG}$ \\
\hline AA & AA & $\mathrm{AG}$ & GG & GG & $\mathrm{AA}$ & $\mathrm{AG}$ & $\mathrm{AG}$ & $\mathrm{AA}$ & GG & GG \\
\hline $\mathrm{AG}$ & $\mathrm{AG}$ & $\mathrm{AG}$ & $\mathrm{AG}$ & $\mathrm{AG}$ & $\mathrm{AA}$ & GG & $\mathrm{AG}$ & GG & $\mathrm{AA}$ & $\mathrm{AG}$ \\
\hline AG & GG & GG & GG & AA & $\mathrm{AG}$ & $\mathrm{AG}$ & $\mathrm{AG}$ & $\mathrm{AG}$ & $\mathrm{AG}$ & $\mathrm{AG}$ \\
\hline $\mathrm{AA}$ & $\mathrm{AA}$ & $\mathrm{AG}$ & $\mathrm{AA}$ & GG & $\mathrm{AG}$ & $\mathrm{AA}$ & GG & GG & $\mathrm{AA}$ & \\
\hline GDsnp00175 & 8 & \multicolumn{2}{|c|}{697817} & $\mathrm{AG}$ & $\mathrm{AG}$ & $\mathrm{AA}$ & $\mathrm{AG}$ & $\mathrm{AG}$ & $\mathrm{AA}$ & $\mathrm{AG}$ \\
\hline AA & GG & GG & $\mathrm{AG}$ & AA & $\mathrm{AA}$ & $\mathrm{AA}$ & GG & GG & $\mathrm{AG}$ & $\mathrm{AG}$ \\
\hline GG & $\mathrm{AG}$ & GG & $\mathrm{AA}$ & $\mathrm{AG}$ & $\mathrm{AG}$ & $\mathrm{AG}$ & GG & GG & $\mathrm{AA}$ & $\mathrm{AA}$ \\
\hline AG & $\mathrm{AG}$ & $\mathrm{AG}$ & $\mathrm{AG}$ & AA & $\mathrm{AA}$ & $\mathrm{AG}$ & $\mathrm{AG}$ & $\mathrm{AG}$ & $\mathrm{AG}$ & AA \\
\hline $\mathrm{AA}$ & GG & $\mathrm{AG}$ & $\mathrm{AG}$ & $\mathrm{AG}$ & $\mathrm{AG}$ & $\mathrm{AG}$ & $\mathrm{AG}$ & $\mathrm{AG}$ & $\mathrm{AG}$ & $\mathrm{AG}$ \\
\hline GG & GG & $\mathrm{AG}$ & AA & AA & GG & $\mathrm{AG}$ & $\mathrm{AG}$ & GG & $\mathrm{AA}$ & AA \\
\hline $\mathrm{AG}$ & $\mathrm{AG}$ & $\mathrm{AG}$ & $\mathrm{AG}$ & $\mathrm{AG}$ & GG & $\mathrm{AA}$ & $\mathrm{AG}$ & $\mathrm{AA}$ & GG & $\mathrm{AG}$ \\
\hline AG & AA & AA & AA & GG & $\mathrm{AG}$ & $\mathrm{AG}$ & $\mathrm{AG}$ & $\mathrm{AG}$ & $\mathrm{AG}$ & $\mathrm{AG}$ \\
\hline GG & GG & $\mathrm{AG}$ & GG & AA & $\mathrm{AG}$ & $\mathrm{AG}$ & AA & AA & GG & \\
\hline GDsnp00487 & 8 & \multicolumn{2}{|c|}{1278221} & $\mathrm{AG}$ & $\mathrm{AG}$ & GG & $\mathrm{AG}$ & $\mathrm{AG}$ & GG & AA \\
\hline GG & $\mathrm{AG}$ & $\mathrm{AG}$ & $\mathrm{AG}$ & GG & GG & GG & AA & AA & $\mathrm{AG}$ & $\mathrm{AG}$ \\
\hline AA & $\mathrm{AG}$ & $\mathrm{AG}$ & GG & $\mathrm{AG}$ & $\mathrm{AG}$ & GG & AA & $\mathrm{AA}$ & GG & GG \\
\hline $\mathrm{AG}$ & $\mathrm{AG}$ & $\mathrm{AG}$ & $\mathrm{AG}$ & GG & GG & $\mathrm{AG}$ & $\mathrm{AG}$ & $\mathrm{AG}$ & $\mathrm{AG}$ & GG \\
\hline GG & AA & $\mathrm{AG}$ & $\mathrm{AG}$ & $\mathrm{AG}$ & $\mathrm{AG}$ & $\mathrm{AG}$ & $\mathrm{AG}$ & $\mathrm{AG}$ & $\mathrm{AG}$ & $\mathrm{AG}$ \\
\hline AA & AA & $\mathrm{AG}$ & GG & GG & $\mathrm{AA}$ & $\mathrm{AG}$ & GG & AA & GG & GG \\
\hline $\mathrm{AG}$ & $\mathrm{AG}$ & $\mathrm{AG}$ & $\mathrm{AG}$ & $\mathrm{AG}$ & $\mathrm{AA}$ & GG & $\mathrm{AG}$ & GG & $\mathrm{AA}$ & $\mathrm{AG}$ \\
\hline AG & GG & GG & GG & $\mathrm{AG}$ & $\mathrm{AG}$ & $\mathrm{AG}$ & $\mathrm{AG}$ & $\mathrm{AG}$ & $\mathrm{AG}$ & $\mathrm{AG}$ \\
\hline $\mathrm{AA}$ & AA & $\mathrm{AG}$ & AA & GG & $\mathrm{AG}$ & $\mathrm{AG}$ & GG & GG & $\mathrm{AA}$ & \\
\hline GDsnp00688 & 8 & \multicolumn{2}{|c|}{1674574} & $\mathrm{AT}$ & $\mathrm{AT}$ & $\mathrm{TT}$ & $\mathrm{AT}$ & $\mathrm{AT}$ & $\mathrm{TT}$ & $\mathrm{AA}$ \\
\hline $\mathrm{TT}$ & $\mathrm{AA}$ & $\mathrm{AT}$ & $\mathrm{AT}$ & $\mathrm{TT}$ & $\mathrm{TT}$ & $\mathrm{TT}$ & AA & $\mathrm{AA}$ & AT & AT \\
\hline AA & $\mathrm{AT}$ & $\mathrm{AT}$ & $\mathrm{TT}$ & AT & $\mathrm{AT}$ & $\mathrm{AT}$ & AA & AA & $\mathrm{TT}$ & TT \\
\hline AT & $\mathrm{AT}$ & $\mathrm{AT}$ & $\mathrm{AT}$ & $\mathrm{TT}$ & $\mathrm{TT}$ & $\mathrm{AT}$ & $\mathrm{AT}$ & $\mathrm{AT}$ & $\mathrm{AT}$ & TT \\
\hline $\mathrm{TT}$ & AA & $\mathrm{AT}$ & $\mathrm{AT}$ & $\mathrm{AT}$ & $\mathrm{AT}$ & $\mathrm{AT}$ & $\mathrm{AT}$ & $\mathrm{AT}$ & $\mathrm{AT}$ & AT \\
\hline AA & AA & AT & $\mathrm{TT}$ & $\mathrm{TT}$ & $\mathrm{AA}$ & $\mathrm{AT}$ & $\mathrm{TT}$ & $\mathrm{AA}$ & TT & TT \\
\hline AT & $\mathrm{AT}$ & $\mathrm{AT}$ & $\mathrm{AT}$ & AT & $\mathrm{AA}$ & $\mathrm{TT}$ & $\mathrm{AT}$ & $\mathrm{TT}$ & $\mathrm{AA}$ & AT \\
\hline AT & TT & TT & $\mathrm{TT}$ & AT & AT & $\mathrm{AT}$ & $\mathrm{AT}$ & $\mathrm{AT}$ & AT & AT \\
\hline AA & AA & AT & AA & $\mathrm{TT}$ & AT & AT & $\mathrm{TT}$ & $\mathrm{TT}$ & $\mathrm{AA}$ & \\
\hline GDsnp01059 & 8 & \multicolumn{2}{|c|}{7697889} & $\mathrm{CC}$ & $\mathrm{CC}$ & $\mathrm{CC}$ & $\mathrm{CC}$ & $\mathrm{CC}$ & $\mathrm{CC}$ & $\mathrm{CC}$ \\
\hline $\mathrm{CC}$ & $\mathrm{CC}$ & $\mathrm{CC}$ & $\mathrm{CC}$ & $\mathrm{CC}$ & $\mathrm{CC}$ & $\mathrm{CC}$ & $\mathrm{CC}$ & $\mathrm{CC}$ & $\mathrm{CC}$ & $\mathrm{CC}$ \\
\hline $\mathrm{CC}$ & $\mathrm{CC}$ & - & $\mathrm{CC}$ & $\mathrm{CC}$ & $\mathrm{CC}$ & $\mathrm{CC}$ & $\mathrm{CC}$ & $\mathrm{CC}$ & $\mathrm{CC}$ & $\mathrm{CC}$ \\
\hline $\mathrm{CC}$ & $\mathrm{CC}$ & $\mathrm{CC}$ & $\mathrm{CC}$ & $\mathrm{CC}$ & $\mathrm{CC}$ & $\mathrm{CC}$ & $\mathrm{CC}$ & $\mathrm{CC}$ & $\mathrm{CC}$ & $\mathrm{CC}$ \\
\hline $\mathrm{CC}$ & $\mathrm{CC}$ & $\mathrm{CC}$ & $\mathrm{CC}$ & $\mathrm{CC}$ & $\mathrm{CC}$ & - & - & $\mathrm{CC}$ & $\mathrm{CC}$ & $\mathrm{CC}$ \\
\hline $\mathrm{CC}$ & $\mathrm{CC}$ & $\mathrm{CC}$ & $\mathrm{CC}$ & $\mathrm{CC}$ & $\mathrm{CC}$ & $\mathrm{CC}$ & $\mathrm{CC}$ & $\mathrm{CC}$ & $\mathrm{CC}$ & $\mathrm{CC}$ \\
\hline $\mathrm{CC}$ & $\mathrm{CC}$ & $\mathrm{CC}$ & $\mathrm{CC}$ & $\mathrm{CC}$ & $\mathrm{CC}$ & $\mathrm{CC}$ & $\mathrm{CC}$ & $\mathrm{CC}$ & $\mathrm{CC}$ & $\mathrm{CC}$ \\
\hline $\mathrm{CC}$ & $\mathrm{CC}$ & $\mathrm{CC}$ & $\mathrm{CC}$ & $\mathrm{CC}$ & $\mathrm{CC}$ & $\mathrm{CC}$ & $\mathrm{CC}$ & $\mathrm{CC}$ & $\mathrm{CC}$ & $\mathrm{CC}$ \\
\hline $\mathrm{CC}$ & $\mathrm{CC}$ & $\mathrm{CC}$ & $\mathrm{CC}$ & $\mathrm{CC}$ & $\mathrm{CC}$ & $\mathrm{CC}$ & $\mathrm{CC}$ & $\mathrm{CC}$ & $\mathrm{CC}$ & \\
\hline
\end{tabular}


Supplemental Table 2. Continued.

\begin{tabular}{|c|c|c|c|c|c|c|c|c|c|c|}
\hline \multirow{3}{*}{$\begin{array}{l}\text { SNP name } \\
\text { GDsnp00316 } \\
\text { AT }\end{array}$} & \multicolumn{10}{|c|}{ Linkage group Physical location (bp) Individuals } \\
\hline & 8 & \multicolumn{2}{|c|}{8090809} & $\mathrm{AT}$ & AT & TT & AA & AT & TT & $\mathrm{AA}$ \\
\hline & AT & AT & AA & $\mathrm{TT}$ & TT & $\mathrm{TT}$ & AA & AA & AT & AT \\
\hline $\mathrm{AA}$ & AT & $\mathrm{AT}$ & AT & $\mathrm{AT}$ & AA & $\mathrm{TT}$ & AT & AT & TT & AT \\
\hline AT & AT & AT & AA & $\mathrm{TT}$ & TT & AA & AT & AT & $\mathrm{AA}$ & $\mathrm{TT}$ \\
\hline AT & $\mathrm{AA}$ & $\mathrm{AT}$ & $\mathrm{AT}$ & $\mathrm{AA}$ & AA & $\mathrm{AT}$ & $\mathrm{AT}$ & AT & AT & $\mathrm{AT}$ \\
\hline AA & AA & $\mathrm{AT}$ & $\mathrm{AT}$ & $\mathrm{TT}$ & AT & $\mathrm{AT}$ & $\mathrm{AT}$ & $\mathrm{AT}$ & $\mathrm{AT}$ & $\mathrm{TT}$ \\
\hline AT & $\mathrm{AT}$ & $\mathrm{AT}$ & $\mathrm{AT}$ & $\mathrm{AT}$ & AA & $\mathrm{AT}$ & $\mathrm{AT}$ & $\mathrm{TT}$ & $\mathrm{AT}$ & $\mathrm{TT}$ \\
\hline AT & TT & TT & TT & - & $\mathrm{AT}$ & $\mathrm{AT}$ & $\mathrm{AA}$ & $\mathrm{AT}$ & AT & $\mathrm{AT}$ \\
\hline $\mathrm{AA}$ & $\mathrm{AT}$ & $\mathrm{AT}$ & $\mathrm{AA}$ & $\mathrm{AT}$ & $\mathrm{AA}$ & AA & $\mathrm{AT}$ & $\mathrm{TT}$ & $\mathrm{AA}$ & \\
\hline GDsnp00188 & 8 & \multicolumn{2}{|c|}{8170368} & - & $\mathrm{AG}$ & GG & AA & $\mathrm{AG}$ & GG & $\mathrm{AA}$ \\
\hline GG & $\mathrm{AG}$ & $\mathrm{AG}$ & $\mathrm{AA}$ & GG & GG & GG & AA & $\mathrm{AA}$ & $\mathrm{AG}$ & $\mathrm{AG}$ \\
\hline AA & $\mathrm{AG}$ & $\mathrm{AG}$ & $\mathrm{AG}$ & $\mathrm{AG}$ & AA & GG & $\mathrm{AG}$ & $\mathrm{AG}$ & GG & $\mathrm{AG}$ \\
\hline $\mathrm{AG}$ & $\mathrm{AG}$ & $\mathrm{AG}$ & $\mathrm{AA}$ & GG & GG & AA & $\mathrm{AG}$ & $\mathrm{AG}$ & $\mathrm{AA}$ & GG \\
\hline $\mathrm{AG}$ & $\mathrm{AA}$ & $\mathrm{AG}$ & $\mathrm{AG}$ & AA & AA & $\mathrm{AG}$ & $\mathrm{AG}$ & $\mathrm{AG}$ & $\mathrm{AG}$ & $\mathrm{AG}$ \\
\hline $\mathrm{AA}$ & $\mathrm{AA}$ & $\mathrm{AG}$ & $\mathrm{AG}$ & GG & $\mathrm{AG}$ & $\mathrm{AG}$ & $\mathrm{AG}$ & $\mathrm{AG}$ & $\mathrm{AG}$ & GG \\
\hline AG & $\mathrm{AG}$ & $\mathrm{AG}$ & $\mathrm{AG}$ & $\mathrm{AG}$ & AA & $\mathrm{AG}$ & $\mathrm{AG}$ & GG & $\mathrm{AG}$ & GG \\
\hline $\mathrm{AG}$ & GG & GG & GG & $\mathrm{AG}$ & $\mathrm{AG}$ & $\mathrm{AG}$ & AA & $\mathrm{AG}$ & $\mathrm{AG}$ & $\mathrm{AG}$ \\
\hline AA & $\mathrm{AG}$ & $\mathrm{AG}$ & AA & $\mathrm{AG}$ & AA & AA & $\mathrm{AG}$ & GG & $\mathrm{AA}$ & \\
\hline GDsnp00204 & 8 & \multicolumn{2}{|c|}{9358626} & GG & GG & GG & GG & GG & GG & GG \\
\hline GG & GG & GG & GG & GG & GG & GG & GG & GG & GG & GG \\
\hline GG & GG & GG & GG & GG & $\mathrm{AG}$ & GG & GG & GG & GG & $\mathrm{AG}$ \\
\hline GG & GG & GG & $\mathrm{AG}$ & GG & GG & GG & $\mathrm{AG}$ & GG & $\mathrm{AG}$ & GG \\
\hline $\mathrm{AG}$ & GG & GG & GG & GG & $\mathrm{AG}$ & GG & $\mathrm{AG}$ & GG & GG & GG \\
\hline GG & GG & GG & - & GG & GG & GG & $\mathrm{AG}$ & GG & $\mathrm{AG}$ & GG \\
\hline GG & GG & GG & GG & GG & GG & GG & GG & GG & GG & GG \\
\hline GG & GG & GG & GG & GG & GG & GG & $\mathrm{AG}$ & GG & GG & GG \\
\hline GG & GG & GG & GG & $\mathrm{AG}$ & GG & GG & $\mathrm{AG}$ & GG & GG & \\
\hline GDsnp00209 & 8 & \multicolumn{2}{|c|}{12235175} & AA & $\mathrm{AA}$ & AA & AA & AA & $\mathrm{AA}$ & $\mathrm{AA}$ \\
\hline $\mathrm{AG}$ & $\mathrm{AA}$ & $\mathrm{AA}$ & $\mathrm{AG}$ & AA & AA & $\mathrm{AA}$ & AA & $\mathrm{AA}$ & $\mathrm{AA}$ & $\mathrm{AA}$ \\
\hline AA & AA & $\mathrm{AA}$ & $\mathrm{AA}$ & AA & $\mathrm{AA}$ & AA & AA & $\mathrm{AA}$ & $\mathrm{AA}$ & $\mathrm{AA}$ \\
\hline AA & AA & AA & AA & AA & AA & AA & AA & AA & $\mathrm{AA}$ & $\mathrm{AA}$ \\
\hline AA & AA & AA & AA & AA & AA & AA & $\mathrm{AG}$ & AA & $\mathrm{AA}$ & $\mathrm{AA}$ \\
\hline AA & AA & AA & AA & AA & AA & AA & AA & AA & $\mathrm{AA}$ & $\mathrm{AA}$ \\
\hline AA & AA & $\mathrm{AA}$ & $\mathrm{AA}$ & AA & $\mathrm{AA}$ & AA & AA & $\mathrm{AA}$ & $\mathrm{AA}$ & $\mathrm{AA}$ \\
\hline AA & AA & AA & AA & AA & AA & AA & AA & AA & $\mathrm{AA}$ & $\mathrm{AA}$ \\
\hline $\mathrm{AA}$ & AA & AA & AA & $\mathrm{AA}$ & AA & $\mathrm{AG}$ & $\mathrm{AA}$ & $\mathrm{AA}$ & $\mathrm{AA}$ & \\
\hline GDsnp00639 & 8 & \multicolumn{2}{|c|}{12360703} & AA & AA & AA & AA & AA & $\mathrm{AA}$ & $\mathrm{AA}$ \\
\hline $\mathrm{AC}$ & AA & AA & $\mathrm{AC}$ & AA & AA & AA & AA & $\mathrm{AA}$ & $\mathrm{AA}$ & $\mathrm{AA}$ \\
\hline AA & $\mathrm{AC}$ & $\mathrm{AC}$ & AA & AA & $\mathrm{AC}$ & AA & AA & AA & $\mathrm{AA}$ & $\mathrm{AC}$ \\
\hline AA & AA & AA & AA & $\mathrm{AA}$ & AA & $\mathrm{AA}$ & $\mathrm{AA}$ & $\mathrm{AA}$ & $\mathrm{AC}$ & $\mathrm{AA}$ \\
\hline AA & $\mathrm{AA}$ & $\mathrm{AA}$ & AA & AA & $\mathrm{AC}$ & $\mathrm{AA}$ & $\mathrm{AC}$ & $\mathrm{AA}$ & $\mathrm{AA}$ & $\mathrm{AA}$ \\
\hline AA & AA & AA & $\mathrm{AA}$ & $\mathrm{AA}$ & $\mathrm{AA}$ & $\mathrm{AA}$ & $\mathrm{AC}$ & $\mathrm{AA}$ & $\mathrm{AC}$ & $\mathrm{AA}$ \\
\hline AA & AA & AA & AA & AA & AA & $\mathrm{AC}$ & AA & AA & $\mathrm{AA}$ & $\mathrm{AA}$ \\
\hline $\mathrm{AA}$ & $\mathrm{AA}$ & $\mathrm{AA}$ & $\mathrm{AA}$ & AA & $\mathrm{AA}$ & AA & AA & AA & $\mathrm{AA}$ & $\mathrm{AA}$ \\
\hline $\mathrm{AA}$ & AA & AA & AA & $\mathrm{AC}$ & AA & $\mathrm{AC}$ & $\mathrm{AC}$ & $\mathrm{AA}$ & $\mathrm{AA}$ & \\
\hline GDsnp00354 & 8 & \multicolumn{2}{|c|}{13083911} & AA & AA & $\mathrm{AA}$ & $\mathrm{AA}$ & $\mathrm{AA}$ & $\mathrm{AA}$ & $\mathrm{AA}$ \\
\hline- & AA & $\mathrm{AA}$ & $\mathrm{AA}$ & AA & $\mathrm{AA}$ & AA & AA & $\mathrm{AA}$ & $\mathrm{AA}$ & $\mathrm{AA}$ \\
\hline AA & $\mathrm{AG}$ & $\mathrm{AG}$ & AA & AA & $\mathrm{AG}$ & AA & $\mathrm{AG}$ & $\mathrm{AG}$ & $\mathrm{AA}$ & $\mathrm{AA}$ \\
\hline AA & AA & AA & AA & AA & AA & AA & AA & $\mathrm{AG}$ & $\mathrm{AG}$ & $\mathrm{AA}$ \\
\hline AA & $\mathrm{AA}$ & AA & $\mathrm{AG}$ & $\mathrm{AG}$ & $\mathrm{AG}$ & - & $\mathrm{AG}$ & $\mathrm{AA}$ & $\mathrm{AA}$ & $\mathrm{AG}$ \\
\hline $\mathrm{AA}$ & $\mathrm{AA}$ & $\mathrm{AA}$ & $\mathrm{AA}$ & AA & $\mathrm{AA}$ & $\mathrm{AA}$ & $\mathrm{AG}$ & $\mathrm{AA}$ & $\mathrm{AG}$ & $\mathrm{AA}$ \\
\hline $\mathrm{AA}$ & $\mathrm{AA}$ & $\mathrm{AA}$ & $\mathrm{AA}$ & AA & $\mathrm{AG}$ & $\mathrm{AG}$ & $\mathrm{AA}$ & $\mathrm{AA}$ & $\mathrm{AA}$ & $\mathrm{AA}$ \\
\hline AA & $\mathrm{AA}$ & $\mathrm{AA}$ & $\mathrm{AA}$ & $\mathrm{AG}$ & $\mathrm{AA}$ & $\mathrm{AA}$ & $\mathrm{AA}$ & $\mathrm{AA}$ & $\mathrm{AA}$ & $\mathrm{AA}$ \\
\hline AA & $\mathrm{AA}$ & AA & AA & $\mathrm{AG}$ & AA & $\mathrm{AA}$ & $\mathrm{AG}$ & $\mathrm{AA}$ & $\mathrm{AA}$ & \\
\hline
\end{tabular}


Supplemental Table 2. Continued.

\begin{tabular}{|c|c|c|c|c|c|c|c|c|c|c|}
\hline \multirow{3}{*}{$\begin{array}{l}\text { SNP name } \\
\text { GDsnp00086 } \\
\text { AG }\end{array}$} & \multicolumn{10}{|c|}{ Linkage group Physical location (bp) Individuals } \\
\hline & 8 & \multicolumn{2}{|c|}{13722120} & GG & GG & GG & GG & GG & GG & GG \\
\hline & $\mathrm{AG}$ & GG & GG & GG & GG & GG & GG & GG & GG & GG \\
\hline GG & $\mathrm{AG}$ & $\mathrm{AG}$ & GG & GG & $\mathrm{AG}$ & $\mathrm{AG}$ & $\mathrm{AG}$ & $\mathrm{AG}$ & GG & GG \\
\hline GG & GG & GG & GG & GG & GG & GG & GG & $\mathrm{AG}$ & $\mathrm{AG}$ & GG \\
\hline GG & GG & GG & $\mathrm{AG}$ & $\mathrm{AG}$ & $\mathrm{AG}$ & - & $\mathrm{AG}$ & GG & GG & $\mathrm{AG}$ \\
\hline GG & GG & GG & GG & GG & GG & GG & $\mathrm{AG}$ & GG & $\mathrm{AG}$ & GG \\
\hline GG & GG & GG & GG & GG & $\mathrm{AG}$ & GG & GG & GG & GG & GG \\
\hline GG & GG & GG & GG & GG & GG & GG & GG & GG & GG & GG \\
\hline GG & GG & GG & GG & GG & GG & GG & $\mathrm{AG}$ & GG & GG & \\
\hline GDsnp01048 & 8 & \multicolumn{2}{|c|}{14914825} & GG & GG & GG & GG & GG & GG & GG \\
\hline $\mathrm{CG}$ & $\mathrm{CG}$ & GG & GG & GG & GG & GG & GG & GG & $\mathrm{CG}$ & GG \\
\hline GG & $\mathrm{CG}$ & $\mathrm{CG}$ & GG & GG & $\mathrm{CG}$ & $\mathrm{CG}$ & $\mathrm{CG}$ & $\mathrm{CG}$ & GG & GG \\
\hline GG & GG & GG & GG & GG & GG & GG & GG & $\mathrm{CG}$ & $\mathrm{CG}$ & GG \\
\hline GG & GG & GG & $\mathrm{CG}$ & $\mathrm{CG}$ & $\mathrm{CG}$ & - & $\mathrm{CG}$ & GG & GG & $\mathrm{CG}$ \\
\hline GG & GG & GG & GG & GG & GG & GG & $\mathrm{CG}$ & GG & $\mathrm{CG}$ & GG \\
\hline GG & GG & GG & GG & GG & $\mathrm{CG}$ & GG & GG & GG & - & GG \\
\hline GG & GG & GG & GG & $\mathrm{CG}$ & - & GG & GG & GG & GG & GG \\
\hline GG & GG & GG & GG & GG & GG & GG & $\mathrm{CG}$ & GG & GG & \\
\hline GDsnp00627 & 8 & \multicolumn{2}{|c|}{15854892} & $\mathrm{AG}$ & $\mathrm{AG}$ & GG & $\mathrm{AA}$ & $\mathrm{AA}$ & GG & AA \\
\hline $\mathrm{AG}$ & AA & $\mathrm{AG}$ & AA & GG & GG & GG & $\mathrm{AG}$ & $\mathrm{AG}$ & $\mathrm{AG}$ & GG \\
\hline $\mathrm{AA}$ & $\mathrm{AG}$ & $\mathrm{AA}$ & $\mathrm{AG}$ & $\mathrm{AG}$ & $\mathrm{AA}$ & $\mathrm{AG}$ & $\mathrm{AG}$ & $\mathrm{AG}$ & GG & $\mathrm{AG}$ \\
\hline $\mathrm{AG}$ & $\mathrm{AA}$ & $\mathrm{AG}$ & $\mathrm{AA}$ & GG & GG & $\mathrm{AA}$ & GG & $\mathrm{AG}$ & $\mathrm{AG}$ & GG \\
\hline GG & $\mathrm{AA}$ & $\mathrm{AA}$ & $\mathrm{AG}$ & AA & $\mathrm{AA}$ & $\mathrm{AG}$ & GG & $\mathrm{AG}$ & $\mathrm{AA}$ & $\mathrm{AG}$ \\
\hline AA & AA & $\mathrm{AG}$ & AA & GG & $\mathrm{AG}$ & $\mathrm{AG}$ & $\mathrm{AG}$ & $\mathrm{AG}$ & $\mathrm{AG}$ & GG \\
\hline $\mathrm{AG}$ & $\mathrm{AG}$ & GG & $\mathrm{AG}$ & $\mathrm{AG}$ & $\mathrm{AA}$ & $\mathrm{AA}$ & $\mathrm{AG}$ & GG & $\mathrm{AG}$ & GG \\
\hline $\mathrm{AG}$ & $\mathrm{AG}$ & GG & $\mathrm{AG}$ & AA & $\mathrm{AG}$ & $\mathrm{AG}$ & AA & $\mathrm{AG}$ & $\mathrm{AG}$ & AA \\
\hline AG & $\mathrm{AG}$ & $\mathrm{AG}$ & $\mathrm{AA}$ & $\mathrm{AG}$ & AA & $\mathrm{AA}$ & $\mathrm{AG}$ & GG & $\mathrm{AA}$ & \\
\hline GDsnp00862 & 8 & \multicolumn{2}{|c|}{17090019} & GG & GG & GG & GG & GG & GG & GG \\
\hline $\mathrm{AG}$ & $\mathrm{AG}$ & $\mathrm{AG}$ & $\mathrm{AG}$ & GG & GG & GG & GG & GG & $\mathrm{AG}$ & GG \\
\hline GG & $\mathrm{AG}$ & $\mathrm{AG}$ & GG & GG & $\mathrm{AG}$ & $\mathrm{AG}$ & $\mathrm{AG}$ & $\mathrm{AG}$ & GG & GG \\
\hline GG & $\mathrm{AG}$ & GG & GG & GG & GG & GG & GG & $\mathrm{AG}$ & GG & GG \\
\hline GG & GG & GG & $\mathrm{AG}$ & $\mathrm{AG}$ & $\mathrm{AG}$ & $\mathrm{AG}$ & GG & GG & GG & $\mathrm{AG}$ \\
\hline GG & GG & GG & $\mathrm{AG}$ & GG & GG & GG & $\mathrm{AG}$ & GG & $\mathrm{AG}$ & GG \\
\hline GG & GG & GG & GG & GG & $\mathrm{AG}$ & GG & $\mathrm{AG}$ & GG & GG & GG \\
\hline GG & $\mathrm{AG}$ & GG & GG & GG & GG & GG & GG & GG & GG & GG \\
\hline GG & GG & GG & GG & GG & GG & GG & GG & GG & GG & \\
\hline GDsnp00320 & 8 & \multicolumn{2}{|c|}{19659151} & AA & $\mathrm{AA}$ & $\mathrm{AA}$ & AA & AA & $\mathrm{AA}$ & AA \\
\hline AA & AA & $\mathrm{AG}$ & $\mathrm{AG}$ & AA & $\mathrm{AA}$ & $\mathrm{AA}$ & AA & $\mathrm{AA}$ & $\mathrm{AA}$ & AA \\
\hline AA & AA & $\mathrm{AG}$ & AA & AA & $\mathrm{AA}$ & $\mathrm{AA}$ & AA & AA & $\mathrm{AA}$ & AA \\
\hline AA & $\mathrm{AA}$ & $\mathrm{AA}$ & $\mathrm{AA}$ & AA & $\mathrm{AA}$ & $\mathrm{AA}$ & $\mathrm{AA}$ & $\mathrm{AA}$ & $\mathrm{AA}$ & $\mathrm{AA}$ \\
\hline AA & $\mathrm{AA}$ & $\mathrm{AA}$ & AA & AA & $\mathrm{AA}$ & $\mathrm{AA}$ & $\mathrm{AA}$ & $\mathrm{AA}$ & $\mathrm{AA}$ & $\mathrm{AA}$ \\
\hline AA & AA & AA & $\mathrm{AG}$ & AA & $\mathrm{AA}$ & AA & $\mathrm{AA}$ & $\mathrm{AA}$ & $\mathrm{AA}$ & $\mathrm{AA}$ \\
\hline AA & AA & AA & AA & AA & $\mathrm{AA}$ & $\mathrm{AA}$ & $\mathrm{AG}$ & AA & $\mathrm{AA}$ & AA \\
\hline $\mathrm{AA}$ & $\mathrm{AA}$ & $\mathrm{AA}$ & AA & AA & $\mathrm{AA}$ & $\mathrm{AA}$ & AA & AA & $\mathrm{AA}$ & $\mathrm{AA}$ \\
\hline AA & AA & AA & $\mathrm{AA}$ & $\mathrm{AA}$ & AA & $\mathrm{AG}$ & $\mathrm{AA}$ & $\mathrm{AA}$ & $\mathrm{AA}$ & \\
\hline GDsnp01598 & 8 & \multicolumn{2}{|c|}{20841241} & $\mathrm{CC}$ & $\mathrm{CC}$ & $\mathrm{CC}$ & - & - & $\mathrm{CC}$ & - \\
\hline $\mathrm{CC}$ & - & $\mathrm{AC}$ & $\mathrm{AA}$ & $\mathrm{CC}$ & $\mathrm{CC}$ & $\mathrm{CC}$ & $\mathrm{CC}$ & $\mathrm{CC}$ & $\mathrm{CC}$ & $\mathrm{CC}$ \\
\hline- & $\mathrm{CC}$ & - & $\mathrm{CC}$ & $\mathrm{CC}$ & - & $\mathrm{CC}$ & $\mathrm{CC}$ & $\mathrm{CC}$ & $\mathrm{CC}$ & $\mathrm{CC}$ \\
\hline $\mathrm{CC}$ & - & $\mathrm{CC}$ & $\mathrm{CC}$ & $\mathrm{CC}$ & $\mathrm{CC}$ & - & $\mathrm{CC}$ & $\mathrm{CC}$ & - & $\mathrm{CC}$ \\
\hline $\mathrm{CC}$ & - & - & $\mathrm{CC}$ & - & - & $\mathrm{CC}$ & $\mathrm{CC}$ & $\mathrm{CC}$ & - & $\mathrm{CC}$ \\
\hline- & - & $\mathrm{CC}$ & $\mathrm{AA}$ & $\mathrm{CC}$ & $\mathrm{CC}$ & $\mathrm{CC}$ & $\mathrm{CC}$ & $\mathrm{CC}$ & $\mathrm{CC}$ & $\mathrm{CC}$ \\
\hline $\mathrm{CC}$ & $\mathrm{CC}$ & $\mathrm{CC}$ & $\mathrm{CC}$ & $\mathrm{CC}$ & - & - & $\mathrm{AA}$ & $\mathrm{CC}$ & $\mathrm{CC}$ & $\mathrm{CC}$ \\
\hline $\mathrm{CC}$ & $\mathrm{CC}$ & $\mathrm{CC}$ & $\mathrm{CC}$ & - & $\mathrm{CC}$ & $\mathrm{CC}$ & - & $\mathrm{CC}$ & $\mathrm{CC}$ & - \\
\hline $\mathrm{CC}$ & $\mathrm{CC}$ & $\mathrm{CC}$ & - & $\mathrm{CC}$ & - & $\mathrm{CC}$ & $\mathrm{CC}$ & $\mathrm{CC}$ & $\mathrm{CC}$ & \\
\hline
\end{tabular}


Supplemental Table 2. Continued.

\begin{tabular}{|c|c|c|c|c|c|c|c|c|c|c|}
\hline \multirow{3}{*}{$\begin{array}{l}\text { SNP name } \\
\text { GDsnp01405 } \\
\text { GG }\end{array}$} & \multicolumn{10}{|c|}{ Linkage group Physical location (bp) Individuals } \\
\hline & 8 & \multicolumn{2}{|c|}{24394052} & GG & GG & GG & GG & GG & $\mathrm{AG}$ & GG \\
\hline & $\mathrm{AG}$ & GG & GG & GG & GG & GG & GG & GG & $\mathrm{AG}$ & GG \\
\hline GG & $\mathrm{AG}$ & $\mathrm{AG}$ & GG & GG & AG & $\mathrm{AG}$ & $\mathrm{AG}$ & $\mathrm{AG}$ & GG & GG \\
\hline GG & $\mathrm{AG}$ & GG & GG & $\mathrm{AG}$ & GG & GG & GG & $\mathrm{AG}$ & GG & GG \\
\hline GG & GG & GG & $\mathrm{AG}$ & GG & $\mathrm{AG}$ & $\mathrm{AG}$ & GG & $\mathrm{AG}$ & GG & $\mathrm{AG}$ \\
\hline GG & GG & $\mathrm{AG}$ & $\mathrm{AG}$ & GG & GG & GG & $\mathrm{AG}$ & GG & GG & GG \\
\hline GG & GG & GG & GG & GG & $\mathrm{AG}$ & GG & GG & GG & GG & GG \\
\hline GG & $\mathrm{AG}$ & GG & GG & $\mathrm{AG}$ & GG & $\mathrm{AG}$ & GG & GG & GG & $\mathrm{AG}$ \\
\hline GG & GG & GG & GG & GG & $\mathrm{AG}$ & $\mathrm{AG}$ & GG & GG & GG & \\
\hline GDsnp00342 & 8 & \multicolumn{2}{|c|}{25085251} & AA & $\mathrm{AG}$ & $\mathrm{AG}$ & $\mathrm{AA}$ & $\mathrm{AA}$ & $\mathrm{AA}$ & $\mathrm{AA}$ \\
\hline AA & AA & AA & $\mathrm{AG}$ & AA & $\mathrm{AG}$ & $\mathrm{AG}$ & $\mathrm{AG}$ & AA & $\mathrm{AG}$ & $\mathrm{AG}$ \\
\hline $\mathrm{AG}$ & $\mathrm{AA}$ & $\mathrm{AA}$ & AA & AA & $\mathrm{AA}$ & AA & AA & AA & $\mathrm{AG}$ & $\mathrm{AA}$ \\
\hline $\mathrm{AG}$ & $\mathrm{AA}$ & $\mathrm{AG}$ & $\mathrm{AG}$ & AA & $\mathrm{AG}$ & $\mathrm{AG}$ & $\mathrm{AG}$ & $\mathrm{AA}$ & $\mathrm{AA}$ & $\mathrm{AG}$ \\
\hline AG & AA & $\mathrm{AG}$ & $\mathrm{AA}$ & $\mathrm{AG}$ & AA & - & $\mathrm{AG}$ & $\mathrm{AA}$ & $\mathrm{AA}$ & $\mathrm{AA}$ \\
\hline AA & AA & AA & AA & $\mathrm{AG}$ & $\mathrm{AG}$ & $\mathrm{AG}$ & AA & $\mathrm{AG}$ & $\mathrm{AA}$ & $\mathrm{AG}$ \\
\hline $\mathrm{AG}$ & $\mathrm{AG}$ & $\mathrm{AG}$ & $\mathrm{AG}$ & $\mathrm{AG}$ & AA & AA & $\mathrm{AG}$ & AA & $\mathrm{AG}$ & $\mathrm{AG}$ \\
\hline AG & AA & $\mathrm{AG}$ & AA & AA & $\mathrm{AG}$ & AA & AA & AA & $\mathrm{AG}$ & AA \\
\hline AA & $\mathrm{AG}$ & $\mathrm{AG}$ & AG & $\mathrm{AA}$ & AA & $\mathrm{AA}$ & $\mathrm{AA}$ & $\mathrm{AA}$ & $\mathrm{AG}$ & \\
\hline GDsnp00975 & 8 & \multicolumn{2}{|c|}{26691557} & $\mathrm{AG}$ & GG & $\mathrm{AG}$ & $\mathrm{AG}$ & GG & $\mathrm{AA}$ & GG \\
\hline $\mathrm{AG}$ & $\mathrm{AG}$ & $\mathrm{AG}$ & GG & $\mathrm{AG}$ & $\mathrm{AG}$ & $\mathrm{AG}$ & $\mathrm{AG}$ & $\mathrm{AG}$ & $\mathrm{AG}$ & $\mathrm{AG}$ \\
\hline GG & $\mathrm{AA}$ & GG & GG & $\mathrm{AG}$ & $\mathrm{AA}$ & $\mathrm{AG}$ & $\mathrm{AA}$ & $\mathrm{AA}$ & $\mathrm{AG}$ & $\mathrm{AG}$ \\
\hline GG & GG & GG & $\mathrm{AG}$ & AA & $\mathrm{AG}$ & GG & $\mathrm{AG}$ & AA & $\mathrm{AG}$ & $\mathrm{AG}$ \\
\hline $\mathrm{AG}$ & $\mathrm{AG}$ & GG & AA & GG & GG & AA & GG & AA & $\mathrm{AG}$ & AA \\
\hline GG & $\mathrm{AG}$ & GG & $\mathrm{AG}$ & $\mathrm{AG}$ & GG & $\mathrm{AG}$ & $\mathrm{AA}$ & $\mathrm{AG}$ & $\mathrm{AG}$ & $\mathrm{AG}$ \\
\hline GG & GG & $\mathrm{AG}$ & GG & GG & GG & GG & GG & $\mathrm{AG}$ & $\mathrm{AG}$ & $\mathrm{AG}$ \\
\hline $\mathrm{AG}$ & GG & GG & GG & $\mathrm{AG}$ & GG & GG & $\mathrm{AG}$ & GG & GG & GG \\
\hline $\mathrm{AA}$ & GG & GG & $\mathrm{AG}$ & $\mathrm{AG}$ & GG & GG & $\mathrm{AG}$ & $\mathrm{AG}$ & GG & \\
\hline GDsnp01862 & 8 & \multicolumn{2}{|c|}{27487825} & $\mathrm{AG}$ & $\mathrm{AG}$ & $\mathrm{AG}$ & $\mathrm{AG}$ & GG & $\mathrm{AA}$ & $\mathrm{AG}$ \\
\hline AA & GG & $\mathrm{AG}$ & GG & $\mathrm{AG}$ & AA & AA & AA & $\mathrm{AG}$ & $\mathrm{AA}$ & $\mathrm{AG}$ \\
\hline AA & $\mathrm{AA}$ & GG & GG & $\mathrm{AG}$ & $\mathrm{AA}$ & $\mathrm{AA}$ & AA & $\mathrm{AA}$ & $\mathrm{AA}$ & $\mathrm{AG}$ \\
\hline $\mathrm{AG}$ & $\mathrm{AG}$ & $\mathrm{AG}$ & $\mathrm{AA}$ & AA & $\mathrm{AG}$ & $\mathrm{AG}$ & AA & $\mathrm{AA}$ & $\mathrm{AG}$ & $\mathrm{AA}$ \\
\hline AA & AA & $\mathrm{AG}$ & AA & $\mathrm{AG}$ & $\mathrm{AG}$ & AA & $\mathrm{AG}$ & $\mathrm{AA}$ & $\mathrm{AG}$ & AA \\
\hline GG & $\mathrm{AG}$ & $\mathrm{AG}$ & GG & AA & GG & AA & AA & AA & $\mathrm{AG}$ & $\mathrm{AA}$ \\
\hline GG & $\mathrm{AG}$ & $\mathrm{AA}$ & $\mathrm{AG}$ & $\mathrm{AG}$ & $\mathrm{AG}$ & GG & GG & $\mathrm{AG}$ & $\mathrm{AA}$ & $\mathrm{AA}$ \\
\hline AA & $\mathrm{AG}$ & $\mathrm{AG}$ & GG & AA & $\mathrm{AG}$ & $\mathrm{AA}$ & $\mathrm{AG}$ & GG & $\mathrm{AG}$ & $\mathrm{AG}$ \\
\hline $\mathrm{AA}$ & $\mathrm{AG}$ & $\mathrm{AG}$ & AA & $\mathrm{AG}$ & $\mathrm{AG}$ & $\mathrm{AG}$ & $\mathrm{AG}$ & $\mathrm{AG}$ & $\mathrm{AG}$ & \\
\hline GDsnp01386 & 9 & \multicolumn{2}{|c|}{298950} & $\mathrm{AA}$ & AA & $\mathrm{AC}$ & $\mathrm{AC}$ & $\mathrm{CC}$ & $\mathrm{AC}$ & AA \\
\hline $\mathrm{AA}$ & $\mathrm{AA}$ & $\mathrm{AC}$ & $\mathrm{AC}$ & $\mathrm{AC}$ & $\mathrm{AC}$ & $\mathrm{AA}$ & $\mathrm{CC}$ & $\mathrm{AC}$ & $\mathrm{AC}$ & $\mathrm{AC}$ \\
\hline $\mathrm{CC}$ & AA & $\mathrm{CC}$ & $\mathrm{AC}$ & AA & $\mathrm{AC}$ & $\mathrm{AC}$ & $\mathrm{CC}$ & $\mathrm{CC}$ & $\mathrm{CC}$ & $\mathrm{CC}$ \\
\hline $\mathrm{AC}$ & $\mathrm{AC}$ & $\mathrm{AC}$ & $\mathrm{AC}$ & $\mathrm{CC}$ & $\mathrm{CC}$ & $\mathrm{CC}$ & AA & $\mathrm{CC}$ & $\mathrm{AC}$ & $\mathrm{AC}$ \\
\hline $\mathrm{AC}$ & $\mathrm{AC}$ & $\mathrm{AC}$ & $\mathrm{AC}$ & $\mathrm{AC}$ & $\mathrm{AC}$ & $\mathrm{AA}$ & $\mathrm{AA}$ & $\mathrm{AA}$ & $\mathrm{CC}$ & $\mathrm{CC}$ \\
\hline $\mathrm{AC}$ & $\mathrm{CC}$ & $\mathrm{CC}$ & AA & $\mathrm{CC}$ & $\mathrm{AC}$ & $\mathrm{AA}$ & $\mathrm{CC}$ & $\mathrm{AA}$ & $\mathrm{CC}$ & $\mathrm{AC}$ \\
\hline $\mathrm{CC}$ & $\mathrm{AC}$ & $\mathrm{CC}$ & AA & $\mathrm{AC}$ & $\mathrm{AC}$ & $\mathrm{AC}$ & AA & AA & $\mathrm{AA}$ & $\mathrm{AC}$ \\
\hline $\mathrm{AC}$ & $\mathrm{AC}$ & $\mathrm{AC}$ & $\mathrm{AC}$ & $\mathrm{AC}$ & $\mathrm{AC}$ & $\mathrm{AC}$ & $\mathrm{AC}$ & $\mathrm{AC}$ & $\mathrm{AC}$ & $\mathrm{AC}$ \\
\hline $\mathrm{CC}$ & $\mathrm{AA}$ & $\mathrm{CC}$ & $\mathrm{AA}$ & $\mathrm{CC}$ & $\mathrm{AC}$ & $\mathrm{CC}$ & $\mathrm{AC}$ & $\mathrm{AA}$ & $\mathrm{AC}$ & \\
\hline GDsnp01189 & 9 & \multicolumn{2}{|c|}{588295} & AA & $\mathrm{AA}$ & $\mathrm{AG}$ & $\mathrm{AG}$ & GG & $\mathrm{AG}$ & $\mathrm{AA}$ \\
\hline $\mathrm{AG}$ & $\mathrm{AG}$ & $\mathrm{AG}$ & $\mathrm{AG}$ & $\mathrm{AG}$ & $\mathrm{AG}$ & $\mathrm{AA}$ & GG & $\mathrm{AG}$ & $\mathrm{AG}$ & $\mathrm{AG}$ \\
\hline GG & AA & GG & $\mathrm{AG}$ & AA & $\mathrm{AG}$ & $\mathrm{AG}$ & $\mathrm{AG}$ & $\mathrm{AG}$ & GG & GG \\
\hline $\mathrm{AG}$ & $\mathrm{AG}$ & $\mathrm{AG}$ & $\mathrm{AG}$ & GG & GG & GG & $\mathrm{AG}$ & GG & $\mathrm{AG}$ & $\mathrm{AG}$ \\
\hline $\mathrm{AG}$ & $\mathrm{AG}$ & $\mathrm{AG}$ & $\mathrm{AG}$ & $\mathrm{AG}$ & $\mathrm{AG}$ & $\mathrm{AA}$ & $\mathrm{AA}$ & $\mathrm{AA}$ & GG & $\mathrm{AG}$ \\
\hline $\mathrm{AG}$ & GG & GG & AA & $\mathrm{GG}$ & $\mathrm{AG}$ & $\mathrm{AA}$ & GG & $\mathrm{AA}$ & GG & $\mathrm{AG}$ \\
\hline GG & $\mathrm{AG}$ & GG & $\mathrm{AA}$ & $\mathrm{AG}$ & $\mathrm{AG}$ & $\mathrm{AG}$ & $\mathrm{AA}$ & $\mathrm{AA}$ & $\mathrm{AA}$ & $\mathrm{AG}$ \\
\hline- & $\mathrm{AG}$ & $\mathrm{AG}$ & $\mathrm{AG}$ & $\mathrm{AG}$ & $\mathrm{AG}$ & $\mathrm{AG}$ & $\mathrm{AG}$ & $\mathrm{AG}$ & $\mathrm{AG}$ & $\mathrm{AG}$ \\
\hline GG & $\mathrm{AA}$ & GG & AA & GG & $\mathrm{AG}$ & GG & $\mathrm{AG}$ & $\mathrm{AA}$ & $\mathrm{AG}$ & \\
\hline
\end{tabular}


Supplemental Table 2. Continued.

\begin{tabular}{|c|c|c|c|c|c|c|c|c|c|c|}
\hline SNP name & & & & inkage & Phys & cation & Indivi & & & \\
\hline$\overline{\text { GDsnp02013 }}$ & 9 & & & $\mathrm{AC}$ & $\mathrm{CC}$ & $\mathrm{AC}$ & $\mathrm{AC}$ & AA & $\mathrm{AC}$ & $\mathrm{CC}$ \\
\hline $\mathrm{AC}$ & $\mathrm{AC}$ & $\mathrm{AC}$ & $\mathrm{AC}$ & $\mathrm{AC}$ & $\mathrm{AC}$ & $\mathrm{CC}$ & AA & $\mathrm{AC}$ & $\mathrm{AC}$ & $\mathrm{AC}$ \\
\hline AA & $\mathrm{CC}$ & AA & $\mathrm{AC}$ & $\mathrm{CC}$ & $\mathrm{AC}$ & $\mathrm{AC}$ & $\mathrm{AC}$ & $\mathrm{AC}$ & AA & AA \\
\hline $\mathrm{AC}$ & $\mathrm{AC}$ & $\mathrm{AC}$ & $\mathrm{AC}$ & $\mathrm{AA}$ & AA & AA & $\mathrm{AC}$ & AA & $\mathrm{CC}$ & $\mathrm{AC}$ \\
\hline $\mathrm{AC}$ & $\mathrm{AC}$ & $\mathrm{AC}$ & $\mathrm{AC}$ & $\mathrm{AC}$ & $\mathrm{AC}$ & - & $\mathrm{CC}$ & $\mathrm{CC}$ & AA & $\mathrm{AC}$ \\
\hline $\mathrm{AC}$ & AA & $\mathrm{AA}$ & $\mathrm{CC}$ & $\mathrm{AA}$ & $\mathrm{AC}$ & $\mathrm{CC}$ & AA & $\mathrm{CC}$ & AA & $\mathrm{AC}$ \\
\hline AA & $\mathrm{AC}$ & $\mathrm{AA}$ & $\mathrm{CC}$ & $\mathrm{AC}$ & $\mathrm{AC}$ & $\mathrm{AC}$ & $\mathrm{CC}$ & $\mathrm{CC}$ & $\mathrm{CC}$ & $\mathrm{AC}$ \\
\hline $\mathrm{AC}$ & $\mathrm{AC}$ & $\mathrm{AC}$ & $\mathrm{AC}$ & $\mathrm{AC}$ & $\mathrm{AC}$ & $\mathrm{AC}$ & $\mathrm{AC}$ & $\mathrm{AC}$ & $\mathrm{AC}$ & $\mathrm{AC}$ \\
\hline AA & $\mathrm{CC}$ & $\mathrm{AA}$ & $\mathrm{AC}$ & $\mathrm{AA}$ & $\mathrm{AC}$ & $\mathrm{AA}$ & $\mathrm{AC}$ & $\mathrm{CC}$ & $\mathrm{AC}$ & \\
\hline GDsnp00298 & 9 & & & $\mathrm{CC}$ & $\mathrm{CC}$ & $\mathrm{CC}$ & $\mathrm{CC}$ & $\mathrm{CC}$ & $\mathrm{CC}$ & $\mathrm{CC}$ \\
\hline $\mathrm{CC}$ & $\mathrm{CC}$ & $\mathrm{CC}$ & $\mathrm{CC}$ & $\mathrm{CC}$ & $\mathrm{CC}$ & $\mathrm{CC}$ & $\mathrm{CC}$ & $\mathrm{CC}$ & $\mathrm{CC}$ & $\mathrm{CC}$ \\
\hline $\mathrm{CC}$ & $\mathrm{CC}$ & $\mathrm{CC}$ & $\mathrm{CC}$ & $\mathrm{CC}$ & $\mathrm{CC}$ & $\mathrm{CC}$ & $\mathrm{CC}$ & $\mathrm{CC}$ & $\mathrm{CC}$ & $\mathrm{CC}$ \\
\hline $\mathrm{CC}$ & $\mathrm{CC}$ & $\mathrm{CC}$ & $\mathrm{CC}$ & $\mathrm{CC}$ & $\mathrm{CC}$ & $\mathrm{CC}$ & $\mathrm{CC}$ & $\mathrm{CC}$ & $\mathrm{CC}$ & $\mathrm{CC}$ \\
\hline $\mathrm{CC}$ & $\mathrm{CC}$ & $\mathrm{CC}$ & $\mathrm{CC}$ & $\mathrm{CC}$ & $\mathrm{CC}$ & $\mathrm{CC}$ & $\mathrm{CC}$ & $\mathrm{CC}$ & $\mathrm{CC}$ & $\mathrm{CC}$ \\
\hline $\mathrm{CC}$ & $\mathrm{CC}$ & $\mathrm{CC}$ & $\mathrm{CC}$ & $\mathrm{CC}$ & $\mathrm{CC}$ & $\mathrm{CC}$ & $\mathrm{CC}$ & $\mathrm{CC}$ & $\mathrm{CC}$ & $\mathrm{CC}$ \\
\hline $\mathrm{CC}$ & $\mathrm{CC}$ & $\mathrm{CC}$ & $\mathrm{CC}$ & $\mathrm{CC}$ & $\mathrm{CC}$ & $\mathrm{CC}$ & $\mathrm{CC}$ & $\mathrm{CC}$ & $\mathrm{CC}$ & $\mathrm{CC}$ \\
\hline $\mathrm{CC}$ & $\mathrm{CC}$ & $\mathrm{CC}$ & $\mathrm{CC}$ & $\mathrm{CC}$ & $\mathrm{CC}$ & $\mathrm{CC}$ & $\mathrm{CC}$ & $\mathrm{CC}$ & $\mathrm{CC}$ & $\mathrm{CC}$ \\
\hline $\mathrm{CC}$ & $\mathrm{CC}$ & $\mathrm{CC}$ & $\mathrm{CC}$ & $\mathrm{CC}$ & $\mathrm{CC}$ & - & $\mathrm{CC}$ & $\mathrm{CC}$ & $\mathrm{CC}$ & \\
\hline GDsnp00033 & 9 & & & $\mathrm{CC}$ & $\mathrm{CC}$ & $\mathrm{CC}$ & $\mathrm{CC}$ & $\mathrm{CC}$ & $\mathrm{CC}$ & $\mathrm{CC}$ \\
\hline $\mathrm{CC}$ & $\mathrm{CC}$ & $\mathrm{CC}$ & $\mathrm{CC}$ & $\mathrm{CC}$ & $\mathrm{CC}$ & $\mathrm{CC}$ & $\mathrm{CC}$ & $\mathrm{CC}$ & $\mathrm{CC}$ & $\mathrm{CC}$ \\
\hline $\mathrm{CC}$ & $\mathrm{CC}$ & $\mathrm{CC}$ & $\mathrm{CC}$ & $\mathrm{CC}$ & $\mathrm{CC}$ & $\mathrm{CC}$ & $\mathrm{CC}$ & $\mathrm{CC}$ & $\mathrm{CC}$ & $\mathrm{CC}$ \\
\hline $\mathrm{CC}$ & $\mathrm{CC}$ & $\mathrm{CC}$ & $\mathrm{CC}$ & $\mathrm{CC}$ & $\mathrm{CC}$ & $\mathrm{CC}$ & $\mathrm{CC}$ & $\mathrm{CC}$ & $\mathrm{CC}$ & $\mathrm{CC}$ \\
\hline $\mathrm{CC}$ & $\mathrm{CC}$ & $\mathrm{CC}$ & $\mathrm{CC}$ & $\mathrm{CC}$ & $\mathrm{CC}$ & - & $\mathrm{CC}$ & $\mathrm{CC}$ & $\mathrm{CC}$ & $\mathrm{CC}$ \\
\hline $\mathrm{CC}$ & $\mathrm{CC}$ & $\mathrm{CC}$ & $\mathrm{CC}$ & $\mathrm{CC}$ & $\mathrm{CC}$ & $\mathrm{CC}$ & $\mathrm{CC}$ & $\mathrm{CC}$ & $\mathrm{CC}$ & $\mathrm{CC}$ \\
\hline $\mathrm{CC}$ & $\mathrm{CC}$ & $\mathrm{CC}$ & $\mathrm{CC}$ & $\mathrm{CC}$ & $\mathrm{CC}$ & $\mathrm{CC}$ & $\mathrm{CC}$ & $\mathrm{CC}$ & $\mathrm{CC}$ & $\mathrm{CC}$ \\
\hline $\mathrm{CC}$ & $\mathrm{CC}$ & $\mathrm{CC}$ & $\mathrm{CC}$ & $\mathrm{CC}$ & $\mathrm{CC}$ & $\mathrm{CC}$ & $\mathrm{CC}$ & $\mathrm{CC}$ & $\mathrm{CC}$ & $\mathrm{CC}$ \\
\hline $\mathrm{CC}$ & $\mathrm{CC}$ & $\mathrm{CC}$ & $\mathrm{CC}$ & $\mathrm{CC}$ & $\mathrm{CC}$ & $\mathrm{AC}$ & $\mathrm{CC}$ & $\mathrm{CC}$ & $\mathrm{CC}$ & \\
\hline GDsnp01524 & 9 & & & GG & GG & GG & GG & GG & GG & GG \\
\hline GG & GG & GG & GG & GG & GG & GG & GG & GG & GG & GG \\
\hline GG & GG & GG & GG & GG & GG & GG & GG & GG & GG & GG \\
\hline GG & GG & GG & GG & GG & GG & GG & GG & GG & GG & GG \\
\hline GG & GG & GG & GG & GG & GG & GG & GG & GG & GG & GG \\
\hline GG & GG & GG & GG & GG & GG & GG & GG & GG & GG & GG \\
\hline GG & GG & GG & GG & GG & GG & GG & GG & GG & GG & GG \\
\hline GG & GG & GG & GG & GG & GG & GG & GG & GG & GG & GG \\
\hline GG & GG & GG & GG & GG & GG & $\mathrm{AG}$ & GG & GG & GG & \\
\hline GDsnp01911 & 9 & & & $\mathrm{AT}$ & TT & $\mathrm{AT}$ & AT & TT & TT & AT \\
\hline AA & AT & - & AT & $\mathrm{TT}$ & TT & AT & TT & AT & TT & TT \\
\hline TT & TT & AT & AT & - & TT & $\mathrm{TT}$ & - & AT & TT & TT \\
\hline AT & - & $\mathrm{TT}$ & TT & $\mathrm{TT}$ & AT & $\mathrm{AT}$ & TT & AT & AA & TT \\
\hline TT & TT & TT & TT & $\mathrm{TT}$ & TT & - & AT & AT & TT & AT \\
\hline AT & TT & TT & - & $\mathrm{TT}$ & - & AT & TT & AT & TT & AT \\
\hline - & TT & TT & AT & $\mathrm{AT}$ & AT & $\mathrm{TT}$ & TT & AT & AT & - \\
\hline TT & TT & TT & TT & AT & AT & AT & TT & AT & TT & TT \\
\hline TT & $\mathrm{AT}$ & $\mathrm{TT}$ & $\mathrm{AT}$ & $\mathrm{TT}$ & $\mathrm{TT}$ & $\mathrm{AA}$ & TT & $\mathrm{AT}$ & - & \\
\hline GDsnp00514 & 9 & & & GG & $\mathrm{AG}$ & AG & AG & $\mathrm{AG}$ & $\mathrm{AG}$ & GG \\
\hline GG & $\mathrm{AG}$ & $\mathrm{AG}$ & AG & $\mathrm{AG}$ & $\mathrm{AG}$ & GG & $\mathrm{AG}$ & GG & AA & $\mathrm{GG}$ \\
\hline AA & GG & $\mathrm{AG}$ & GG & GG & $\mathrm{AG}$ & $\mathrm{AG}$ & $\mathrm{AG}$ & $\mathrm{AG}$ & AA & AA \\
\hline GG & AG & AG & AG & AA & AG & AG & GG & AG & GG & $\mathrm{AG}$ \\
\hline GG & $\mathrm{AG}$ & GG & AG & $\mathrm{AA}$ & AG & GG & - & $\mathrm{AG}$ & AA & $\mathrm{AG}$ \\
\hline AG & AA & AA & GG & AA & GG & GG & AA & GG & $\mathrm{AG}$ & $\mathrm{AG}$ \\
\hline AG & $\mathrm{AG}$ & AA & GG & $\mathrm{AG}$ & GG & $\mathrm{AG}$ & AG & GG & GG & GG \\
\hline AG & AG & AG & AG & GG & $\mathrm{AG}$ & AG & GG & AA & GG & $\mathrm{AG}$ \\
\hline AA & GG & AA & GG & AG & AG & $\mathrm{AG}$ & GG & GG & AG & \\
\hline
\end{tabular}

Continued next page 
Supplemental Table 2. Continued.

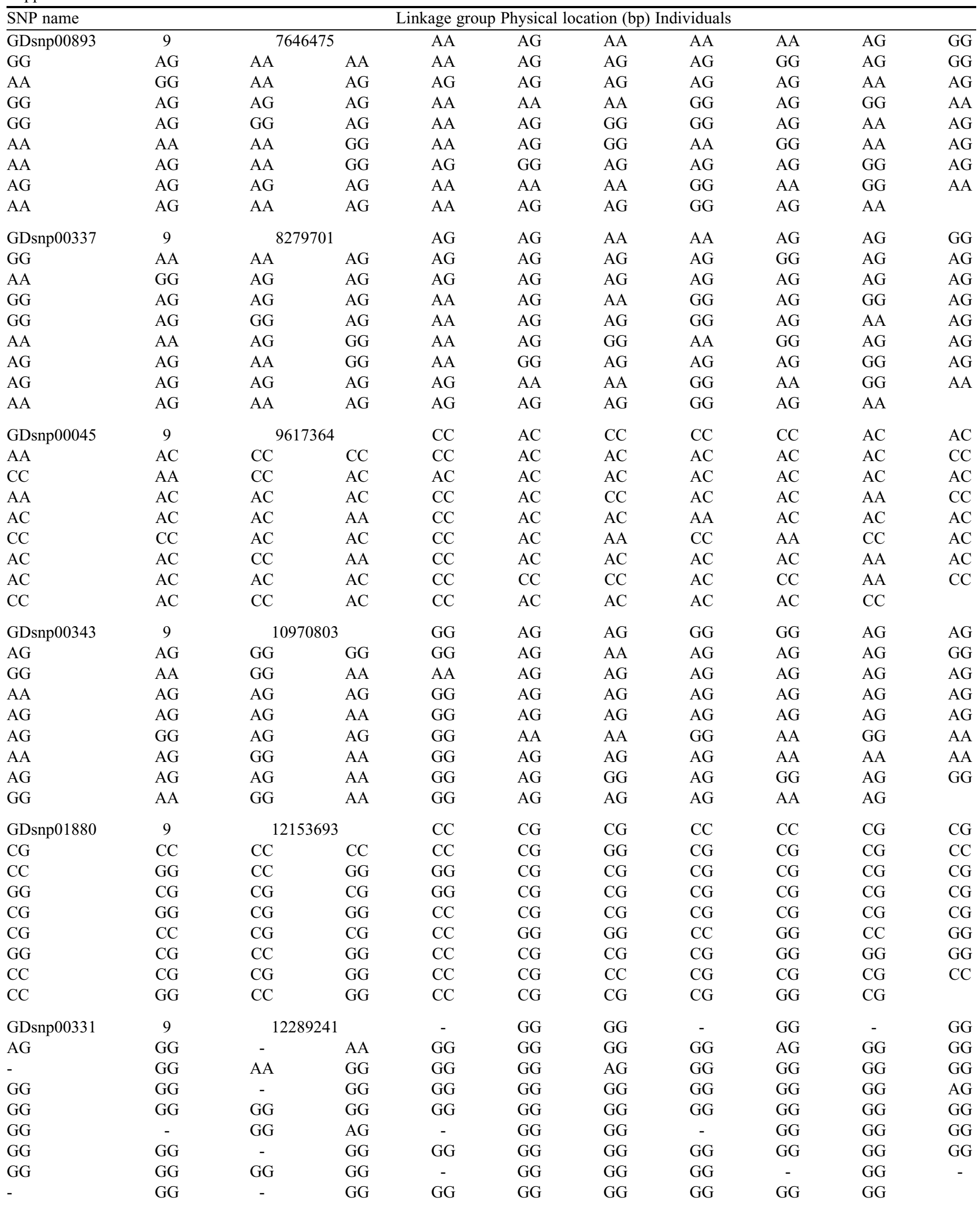


Supplemental Table 2. Continued.

\begin{tabular}{|c|c|c|c|c|c|c|c|c|c|c|}
\hline \multirow{3}{*}{$\begin{array}{l}\text { SNP name } \\
\text { GDsnp00958 } \\
\text { AC }\end{array}$} & \multicolumn{10}{|c|}{ Linkage group Physical location (bp) Individuals } \\
\hline & 9 & \multicolumn{2}{|c|}{13453035} & $\mathrm{AA}$ & $\mathrm{AC}$ & $\mathrm{AC}$ & AA & $\mathrm{AC}$ & AA & $\mathrm{AC}$ \\
\hline & $\mathrm{AC}$ & AA & AA & $\mathrm{AC}$ & $\mathrm{AC}$ & $\mathrm{CC}$ & $\mathrm{AC}$ & $\mathrm{AC}$ & $\mathrm{AC}$ & AA \\
\hline AA & $\mathrm{CC}$ & AA & $\mathrm{CC}$ & $\mathrm{CC}$ & $\mathrm{AC}$ & $\mathrm{AC}$ & $\mathrm{AC}$ & $\mathrm{AC}$ & $\mathrm{AC}$ & $\mathrm{AC}$ \\
\hline $\mathrm{CC}$ & $\mathrm{AC}$ & AA & $\mathrm{AC}$ & $\mathrm{CC}$ & $\mathrm{AC}$ & $\mathrm{AC}$ & $\mathrm{CC}$ & $\mathrm{AC}$ & $\mathrm{CC}$ & $\mathrm{AC}$ \\
\hline $\mathrm{CC}$ & $\mathrm{CC}$ & $\mathrm{CC}$ & $\mathrm{CC}$ & $\mathrm{AC}$ & $\mathrm{AC}$ & $\mathrm{CC}$ & $\mathrm{AC}$ & $\mathrm{AC}$ & $\mathrm{AC}$ & $\mathrm{AC}$ \\
\hline $\mathrm{AC}$ & $\mathrm{AA}$ & $\mathrm{AC}$ & $\mathrm{AC}$ & $\mathrm{AA}$ & $\mathrm{AC}$ & $\mathrm{CC}$ & $\mathrm{AA}$ & $\mathrm{CC}$ & $\mathrm{AC}$ & $\mathrm{CC}$ \\
\hline $\mathrm{CC}$ & $\mathrm{AC}$ & $\mathrm{AA}$ & $\mathrm{CC}$ & $\mathrm{AC}$ & $\mathrm{AC}$ & $\mathrm{AC}$ & $\mathrm{AC}$ & $\mathrm{CC}$ & $\mathrm{CC}$ & $\mathrm{CC}$ \\
\hline $\mathrm{AC}$ & $\mathrm{AC}$ & $\mathrm{CC}$ & $\mathrm{CC}$ & $\mathrm{AC}$ & $\mathrm{AC}$ & $\mathrm{AC}$ & $\mathrm{CC}$ & $\mathrm{AC}$ & $\mathrm{AC}$ & $\mathrm{AA}$ \\
\hline $\mathrm{AC}$ & $\mathrm{CC}$ & $\mathrm{AA}$ & $\mathrm{AC}$ & $\mathrm{AC}$ & $\mathrm{AC}$ & $\mathrm{AC}$ & $\mathrm{CC}$ & $\mathrm{CC}$ & $\mathrm{AC}$ & \\
\hline GDsnp02460 & 9 & \multicolumn{2}{|c|}{18384592} & GG & $\mathrm{AG}$ & GG & GG & $\mathrm{AG}$ & GG & $\mathrm{AA}$ \\
\hline AA & $\mathrm{AG}$ & $\mathrm{AG}$ & GG & $\mathrm{AG}$ & $\mathrm{AG}$ & $\mathrm{AG}$ & $\mathrm{AG}$ & AA & $\mathrm{AA}$ & GG \\
\hline $\mathrm{AG}$ & $\mathrm{AA}$ & GG & $\mathrm{AA}$ & $\mathrm{AA}$ & $\mathrm{AG}$ & $\mathrm{AG}$ & $\mathrm{AG}$ & $\mathrm{AG}$ & $\mathrm{AG}$ & $\mathrm{AG}$ \\
\hline AA & $\mathrm{AG}$ & GG & $\mathrm{AG}$ & $\mathrm{AA}$ & $\mathrm{AG}$ & $\mathrm{AG}$ & $\mathrm{AA}$ & $\mathrm{AG}$ & $\mathrm{AA}$ & $\mathrm{AG}$ \\
\hline AA & $\mathrm{AA}$ & $\mathrm{AA}$ & $\mathrm{AA}$ & $\mathrm{AG}$ & $\mathrm{AG}$ & $\mathrm{AA}$ & $\mathrm{AG}$ & $\mathrm{AG}$ & $\mathrm{AG}$ & $\mathrm{AG}$ \\
\hline $\mathrm{AG}$ & GG & $\mathrm{AG}$ & GG & GG & $\mathrm{AG}$ & $\mathrm{AA}$ & GG & $\mathrm{AA}$ & $\mathrm{AG}$ & AA \\
\hline AA & $\mathrm{AG}$ & GG & $\mathrm{AA}$ & $\mathrm{AA}$ & $\mathrm{AG}$ & $\mathrm{AA}$ & $\mathrm{AG}$ & $\mathrm{AA}$ & $\mathrm{AG}$ & $\mathrm{AA}$ \\
\hline $\mathrm{AG}$ & $\mathrm{AG}$ & $\mathrm{AA}$ & $\mathrm{AA}$ & GG & $\mathrm{AG}$ & $\mathrm{AG}$ & $\mathrm{AA}$ & $\mathrm{AG}$ & $\mathrm{AG}$ & GG \\
\hline $\mathrm{AG}$ & $\mathrm{AA}$ & GG & $\mathrm{AG}$ & $\mathrm{AG}$ & $\mathrm{AG}$ & $\mathrm{AG}$ & AA & AA & GG & \\
\hline GDsnp02845 & 9 & \multicolumn{2}{|c|}{19221884} & $\mathrm{CC}$ & $\mathrm{AC}$ & $\mathrm{CC}$ & $\mathrm{AC}$ & $\mathrm{AC}$ & $\mathrm{CC}$ & $\mathrm{AC}$ \\
\hline $\mathrm{AC}$ & $\mathrm{CC}$ & $\mathrm{AC}$ & $\mathrm{CC}$ & $\mathrm{AC}$ & $\mathrm{AC}$ & $\mathrm{AC}$ & $\mathrm{AC}$ & $\mathrm{AC}$ & AA & $\mathrm{CC}$ \\
\hline $\mathrm{AC}$ & $\mathrm{AC}$ & $\mathrm{CC}$ & $\mathrm{AA}$ & $\mathrm{AA}$ & $\mathrm{AC}$ & $\mathrm{AC}$ & $\mathrm{AC}$ & $\mathrm{AC}$ & $\mathrm{AC}$ & $\mathrm{AC}$ \\
\hline AA & $\mathrm{AC}$ & $\mathrm{CC}$ & $\mathrm{AC}$ & $\mathrm{AA}$ & $\mathrm{AC}$ & $\mathrm{AC}$ & AA & $\mathrm{AC}$ & $\mathrm{AC}$ & $\mathrm{AC}$ \\
\hline AA & $\mathrm{AA}$ & $\mathrm{AA}$ & $\mathrm{AA}$ & $\mathrm{AC}$ & $\mathrm{AC}$ & AA & $\mathrm{AC}$ & $\mathrm{AC}$ & $\mathrm{AC}$ & $\mathrm{AC}$ \\
\hline $\mathrm{AC}$ & $\mathrm{CC}$ & $\mathrm{AC}$ & $\mathrm{CC}$ & $\mathrm{CC}$ & $\mathrm{AC}$ & AA & $\mathrm{CC}$ & AA & $\mathrm{AC}$ & $\mathrm{AA}$ \\
\hline AA & $\mathrm{AC}$ & $\mathrm{CC}$ & $\mathrm{AA}$ & $\mathrm{AA}$ & $\mathrm{AC}$ & AA & $\mathrm{AC}$ & AA & $\mathrm{AC}$ & AA \\
\hline $\mathrm{AC}$ & $\mathrm{AC}$ & AA & $\mathrm{AA}$ & $\mathrm{CC}$ & $\mathrm{AA}$ & $\mathrm{AC}$ & $\mathrm{AC}$ & $\mathrm{AC}$ & $\mathrm{AC}$ & $\mathrm{CC}$ \\
\hline $\mathrm{AC}$ & $\mathrm{AA}$ & $\mathrm{CC}$ & $\mathrm{AC}$ & $\mathrm{AC}$ & $\mathrm{AC}$ & $\mathrm{AC}$ & $\mathrm{AA}$ & $\mathrm{AA}$ & $\mathrm{CC}$ & \\
\hline GDsnp02071 & 9 & \multicolumn{2}{|c|}{24723288} & GG & GG & GG & GG & GG & GG & GG \\
\hline $\mathrm{AG}$ & GG & GG & GG & GG & GG & GG & GG & GG & GG & GG \\
\hline GG & GG & $\mathrm{AG}$ & GG & GG & GG & $\mathrm{AG}$ & GG & GG & GG & GG \\
\hline GG & GG & GG & GG & GG & GG & GG & GG & GG & GG & GG \\
\hline GG & GG & GG & GG & GG & GG & GG & GG & GG & GG & GG \\
\hline GG & GG & GG & GG & GG & GG & GG & GG & GG & GG & GG \\
\hline GG & GG & GG & GG & GG & GG & GG & GG & GG & GG & GG \\
\hline GG & GG & GG & GG & GG & GG & GG & GG & GG & GG & GG \\
\hline GG & GG & GG & GG & GG & GG & GG & GG & GG & GG & \\
\hline GDsnp02437 & 9 & \multicolumn{2}{|c|}{26578275} & $\mathrm{AA}$ & AA & $\mathrm{AA}$ & $\mathrm{AA}$ & $\mathrm{AA}$ & $\mathrm{AA}$ & $\mathrm{AA}$ \\
\hline $\mathrm{AC}$ & $\mathrm{AA}$ & AA & $\mathrm{AA}$ & $\mathrm{AA}$ & $\mathrm{AA}$ & AA & AA & AA & AA & AA \\
\hline AA & $\mathrm{AA}$ & $\mathrm{AC}$ & $\mathrm{AA}$ & $\mathrm{AA}$ & $\mathrm{AA}$ & $\mathrm{AC}$ & AA & AA & AA & $\mathrm{AA}$ \\
\hline AA & $\mathrm{AA}$ & $\mathrm{AA}$ & $\mathrm{AA}$ & $\mathrm{AA}$ & $\mathrm{AA}$ & AA & $\mathrm{AA}$ & $\mathrm{AA}$ & AA & $\mathrm{AA}$ \\
\hline AA & $\mathrm{AA}$ & $\mathrm{AA}$ & $\mathrm{AA}$ & $\mathrm{AA}$ & $\mathrm{AA}$ & $\mathrm{AA}$ & $\mathrm{AA}$ & $\mathrm{AA}$ & $\mathrm{AA}$ & $\mathrm{AA}$ \\
\hline AA & $\mathrm{AA}$ & AA & $\mathrm{AA}$ & $\mathrm{AA}$ & $\mathrm{AA}$ & AA & AA & AA & AA & AA \\
\hline AA & $\mathrm{AA}$ & AA & $\mathrm{AA}$ & $\mathrm{AA}$ & $\mathrm{AA}$ & AA & AA & AA & AA & $\mathrm{AA}$ \\
\hline $\mathrm{AA}$ & $\mathrm{AA}$ & $\mathrm{AA}$ & $\mathrm{AA}$ & $\mathrm{AA}$ & $\mathrm{AA}$ & $\mathrm{AA}$ & $\mathrm{AA}$ & $\mathrm{AA}$ & AA & $\mathrm{AA}$ \\
\hline AA & AA & AA & AA & $\mathrm{AA}$ & AA & AA & $\mathrm{AA}$ & $\mathrm{AA}$ & AA & \\
\hline GDsnp02482 & 9 & \multicolumn{2}{|c|}{27905275} & $\mathrm{AA}$ & $\mathrm{AA}$ & $\mathrm{AA}$ & $\mathrm{AA}$ & $\mathrm{AA}$ & $\mathrm{AA}$ & $\mathrm{AA}$ \\
\hline AA & $\mathrm{AA}$ & $\mathrm{AA}$ & $\mathrm{AA}$ & $\mathrm{AA}$ & $\mathrm{AA}$ & AA & $\mathrm{AA}$ & AA & $\mathrm{AA}$ & $\mathrm{AA}$ \\
\hline AA & $\mathrm{AA}$ & AA & $\mathrm{AA}$ & $\mathrm{AA}$ & $\mathrm{AA}$ & AA & AA & AA & AA & AA \\
\hline AA & $\mathrm{AA}$ & AA & $\mathrm{AA}$ & $\mathrm{AA}$ & $\mathrm{AA}$ & AA & AA & AA & AA & AA \\
\hline AA & $\mathrm{AA}$ & $\mathrm{AA}$ & $\mathrm{AA}$ & $\mathrm{AA}$ & $\mathrm{AA}$ & AA & $\mathrm{AA}$ & AA & AA & $\mathrm{AA}$ \\
\hline $\mathrm{AA}$ & $\mathrm{AA}$ & $\mathrm{AA}$ & $\mathrm{AA}$ & $\mathrm{AA}$ & $\mathrm{AA}$ & $\mathrm{AA}$ & $\mathrm{AA}$ & $\mathrm{AA}$ & $\mathrm{AA}$ & $\mathrm{AA}$ \\
\hline $\mathrm{AA}$ & $\mathrm{AA}$ & $\mathrm{AA}$ & $\mathrm{AA}$ & $\mathrm{AA}$ & $\mathrm{AA}$ & $\mathrm{AA}$ & - & $\mathrm{AA}$ & $\mathrm{AA}$ & $\mathrm{AA}$ \\
\hline AA & $\mathrm{AA}$ & $\mathrm{AA}$ & $\mathrm{AA}$ & $\mathrm{AA}$ & $\mathrm{AA}$ & $\mathrm{AA}$ & $\mathrm{AA}$ & $\mathrm{AA}$ & $\mathrm{AA}$ & $\mathrm{AA}$ \\
\hline AA & $\mathrm{AA}$ & $\mathrm{AA}$ & $\mathrm{AA}$ & $\mathrm{AA}$ & $\mathrm{AA}$ & AA & $\mathrm{AA}$ & AA & AA & \\
\hline
\end{tabular}

Continued next page 
Supplemental Table 2. Continued.

\begin{tabular}{|c|c|c|c|c|c|c|c|c|c|c|}
\hline \multirow{3}{*}{$\begin{array}{l}\text { SNP name } \\
\text { GDsnp02581 } \\
\text { AA }\end{array}$} & \multicolumn{10}{|c|}{ Linkage group Physical location (bp) Individuals } \\
\hline & 9 & \multicolumn{2}{|c|}{28083974} & $\mathrm{AA}$ & AA & AA & AA & $\mathrm{AA}$ & AA & $\mathrm{AA}$ \\
\hline & AA & AA & AA & AA & $\mathrm{AA}$ & AA & AA & AA & AA & AA \\
\hline AA & AA & $\mathrm{AG}$ & $\mathrm{AA}$ & AA & $\mathrm{AA}$ & AG & AA & AA & AA & AA \\
\hline AA & AA & AA & $\mathrm{AA}$ & AA & $\mathrm{AA}$ & AA & AA & AA & AA & $\mathrm{AA}$ \\
\hline AA & $\mathrm{AA}$ & $\mathrm{AA}$ & $\mathrm{AA}$ & $\mathrm{AA}$ & $\mathrm{AA}$ & $\mathrm{AA}$ & $\mathrm{AA}$ & $\mathrm{AA}$ & $\mathrm{AA}$ & $\mathrm{AA}$ \\
\hline AA & $\mathrm{AA}$ & AA & $\mathrm{AA}$ & AA & $\mathrm{AA}$ & AA & AA & $\mathrm{AA}$ & AA & AA \\
\hline $\mathrm{AA}$ & $\mathrm{AA}$ & $\mathrm{AA}$ & $\mathrm{AA}$ & $\mathrm{AA}$ & $\mathrm{AA}$ & $\mathrm{AA}$ & $\mathrm{AA}$ & $\mathrm{AA}$ & $\mathrm{AA}$ & $\mathrm{AA}$ \\
\hline AA & $\mathrm{AA}$ & AA & $\mathrm{AA}$ & $\mathrm{AA}$ & $\mathrm{AA}$ & AA & AA & $\mathrm{AA}$ & AA & AA \\
\hline $\mathrm{AA}$ & $\mathrm{AA}$ & $\mathrm{AA}$ & $\mathrm{AA}$ & $\mathrm{AA}$ & $\mathrm{AA}$ & $\mathrm{AA}$ & AA & $\mathrm{AA}$ & AA & \\
\hline GDsnp00205 & 9 & \multicolumn{2}{|c|}{29375585} & $\mathrm{AA}$ & $\mathrm{AA}$ & $\mathrm{AA}$ & $\mathrm{AA}$ & $\mathrm{AA}$ & $\mathrm{AA}$ & $\mathrm{AA}$ \\
\hline AA & $\mathrm{AA}$ & $\mathrm{AA}$ & $\mathrm{AA}$ & $\mathrm{AA}$ & $\mathrm{AA}$ & AA & $\mathrm{AA}$ & $\mathrm{AA}$ & $\mathrm{AA}$ & AA \\
\hline AA & $\mathrm{AA}$ & $\mathrm{AA}$ & $\mathrm{AA}$ & $\mathrm{AA}$ & $\mathrm{AA}$ & $\mathrm{AA}$ & $\mathrm{AA}$ & $\mathrm{AA}$ & $\mathrm{AA}$ & $\mathrm{AA}$ \\
\hline AA & $\mathrm{AA}$ & $\mathrm{AA}$ & $\mathrm{AA}$ & $\mathrm{AA}$ & $\mathrm{AA}$ & AA & $\mathrm{AA}$ & $\mathrm{AA}$ & - & $\mathrm{AA}$ \\
\hline $\mathrm{AA}$ & $\mathrm{AA}$ & $\mathrm{AA}$ & $\mathrm{AA}$ & $\mathrm{AA}$ & $\mathrm{AA}$ & $\mathrm{AA}$ & $\mathrm{AG}$ & $\mathrm{AA}$ & $\mathrm{AA}$ & $\mathrm{AA}$ \\
\hline AA & $\mathrm{AA}$ & AA & $\mathrm{AA}$ & $\mathrm{AA}$ & $\mathrm{AA}$ & AA & AA & $\mathrm{AA}$ & AA & $\mathrm{AA}$ \\
\hline AA & $\mathrm{AA}$ & $\mathrm{AA}$ & $\mathrm{AA}$ & AA & $\mathrm{AA}$ & AA & $\mathrm{AG}$ & $\mathrm{AA}$ & AA & $\mathrm{AA}$ \\
\hline AA & $\mathrm{AA}$ & $\mathrm{AA}$ & $\mathrm{AA}$ & $\mathrm{AG}$ & $\mathrm{AA}$ & AA & $\mathrm{AA}$ & $\mathrm{AA}$ & $\mathrm{AA}$ & $\mathrm{AA}$ \\
\hline AA & $\mathrm{AA}$ & AA & $\mathrm{AA}$ & $\mathrm{AA}$ & $\mathrm{AA}$ & AA & AA & $\mathrm{AA}$ & AA & \\
\hline GDsnp00452 & 9 & \multicolumn{2}{|c|}{30412974} & $\mathrm{AC}$ & $\mathrm{AA}$ & $\mathrm{AC}$ & $\mathrm{AA}$ & $\mathrm{AC}$ & $\mathrm{AC}$ & $\mathrm{AA}$ \\
\hline $\mathrm{AC}$ & $\mathrm{AC}$ & $\mathrm{AC}$ & $\mathrm{AA}$ & $\mathrm{CC}$ & $\mathrm{AA}$ & AA & AA & $\mathrm{AA}$ & AA & $\mathrm{AC}$ \\
\hline AA & $\mathrm{AC}$ & $\mathrm{AC}$ & $\mathrm{AA}$ & $\mathrm{AA}$ & $\mathrm{AC}$ & $\mathrm{AA}$ & $\mathrm{CC}$ & $\mathrm{CC}$ & $\mathrm{AA}$ & $\mathrm{AC}$ \\
\hline $\mathrm{AC}$ & $\mathrm{AC}$ & $\mathrm{AC}$ & $\mathrm{CC}$ & $\mathrm{AA}$ & $\mathrm{AC}$ & $\mathrm{AC}$ & AA & $\mathrm{AC}$ & AA & $\mathrm{AA}$ \\
\hline $\mathrm{CC}$ & $\mathrm{AA}$ & $\mathrm{AC}$ & $\mathrm{AC}$ & $\mathrm{CC}$ & $\mathrm{AC}$ & $\mathrm{AC}$ & $\mathrm{CC}$ & $\mathrm{AC}$ & $\mathrm{AA}$ & $\mathrm{CC}$ \\
\hline $\mathrm{AC}$ & $\mathrm{AC}$ & $\mathrm{AC}$ & $\mathrm{AC}$ & $\mathrm{AC}$ & $\mathrm{AA}$ & $\mathrm{CC}$ & $\mathrm{AC}$ & $\mathrm{AC}$ & $\mathrm{CC}$ & $\mathrm{AA}$ \\
\hline AA & $\mathrm{AC}$ & $\mathrm{AC}$ & $\mathrm{AA}$ & $\mathrm{AC}$ & $\mathrm{AC}$ & AA & $\mathrm{AC}$ & $\mathrm{AA}$ & $\mathrm{CC}$ & AA \\
\hline $\mathrm{CC}$ & $\mathrm{CC}$ & $\mathrm{AC}$ & $\mathrm{AA}$ & $\mathrm{AC}$ & $\mathrm{AA}$ & $\mathrm{CC}$ & $\mathrm{CC}$ & $\mathrm{AA}$ & $\mathrm{AC}$ & $\mathrm{AC}$ \\
\hline $\mathrm{AC}$ & $\mathrm{AA}$ & $\mathrm{AC}$ & $\mathrm{AA}$ & $\mathrm{CC}$ & $\mathrm{AC}$ & $\mathrm{AC}$ & $\mathrm{AA}$ & $\mathrm{AA}$ & $\mathrm{AC}$ & \\
\hline GDsnp00766 & 9 & \multicolumn{2}{|c|}{30472702} & GG & $\mathrm{AA}$ & GG & $\mathrm{AG}$ & $\mathrm{AG}$ & GG & $\mathrm{AG}$ \\
\hline GG & GG & GG & $\mathrm{AG}$ & GG & AG & $\mathrm{AG}$ & $\mathrm{AG}$ & $\mathrm{AG}$ & $\mathrm{AG}$ & $\mathrm{AG}$ \\
\hline AA & GG & GG & $\mathrm{AA}$ & $\mathrm{AA}$ & GG & $\mathrm{AG}$ & GG & GG & $\mathrm{AA}$ & $\mathrm{AG}$ \\
\hline $\mathrm{AG}$ & $\mathrm{AG}$ & GG & GG & $\mathrm{AA}$ & $\mathrm{AG}$ & $\mathrm{AG}$ & AA & $\mathrm{AG}$ & AA & $\mathrm{AG}$ \\
\hline GG & $\mathrm{AA}$ & $\mathrm{AG}$ & $\mathrm{AG}$ & GG & AG & $\mathrm{AG}$ & GG & $\mathrm{AG}$ & $\mathrm{AG}$ & GG \\
\hline $\mathrm{AG}$ & GG & $\mathrm{AG}$ & GG & $\mathrm{AG}$ & AG & GG & GG & $\mathrm{AG}$ & GG & $\mathrm{AA}$ \\
\hline $\mathrm{AG}$ & $\mathrm{AG}$ & $\mathrm{AG}$ & $\mathrm{AA}$ & $\mathrm{AG}$ & AG & AA & $\mathrm{AG}$ & $\mathrm{AA}$ & GG & $\mathrm{AA}$ \\
\hline GG & GG & $\mathrm{AG}$ & $\mathrm{AA}$ & $\mathrm{AG}$ & $\mathrm{AA}$ & GG & GG & $\mathrm{AG}$ & $\mathrm{AG}$ & GG \\
\hline $\mathrm{AG}$ & $\mathrm{AA}$ & GG & $\mathrm{AG}$ & GG & $\mathrm{AG}$ & $\mathrm{AG}$ & $\mathrm{AG}$ & $\mathrm{AA}$ & GG & \\
\hline GDsnp00106 & 10 & \multicolumn{2}{|c|}{736211} & $\mathrm{AA}$ & $\mathrm{AA}$ & $\mathrm{AA}$ & $\mathrm{AA}$ & $\mathrm{AG}$ & $\mathrm{AG}$ & $\mathrm{AA}$ \\
\hline $\mathrm{AG}$ & $\mathrm{AA}$ & AA & $\mathrm{AG}$ & $\mathrm{AA}$ & $\mathrm{AA}$ & AA & AA & $\mathrm{AA}$ & $\mathrm{AG}$ & $\mathrm{AG}$ \\
\hline AA & $\mathrm{AA}$ & AA & $\mathrm{AA}$ & $\mathrm{AA}$ & $\mathrm{AA}$ & AA & AA & $\mathrm{AA}$ & AA & $\mathrm{AA}$ \\
\hline AA & $\mathrm{AA}$ & $\mathrm{AA}$ & $\mathrm{AG}$ & $\mathrm{AA}$ & $\mathrm{AA}$ & AA & $\mathrm{AG}$ & $\mathrm{AA}$ & AA & $\mathrm{AG}$ \\
\hline AA & $\mathrm{AA}$ & $\mathrm{AG}$ & $\mathrm{AA}$ & $\mathrm{AA}$ & $\mathrm{AA}$ & $\mathrm{AA}$ & $\mathrm{AA}$ & $\mathrm{AA}$ & $\mathrm{AA}$ & $\mathrm{AA}$ \\
\hline AA & $\mathrm{AA}$ & AA & $\mathrm{AG}$ & $\mathrm{AA}$ & $\mathrm{AA}$ & AA & AA & $\mathrm{AA}$ & AA & AA \\
\hline AA & $\mathrm{AG}$ & AA & $\mathrm{AA}$ & $\mathrm{AA}$ & $\mathrm{AA}$ & $\mathrm{AG}$ & $\mathrm{AG}$ & $\mathrm{AA}$ & AA & $\mathrm{AA}$ \\
\hline $\mathrm{AG}$ & $\mathrm{AA}$ & $\mathrm{AA}$ & $\mathrm{AA}$ & $\mathrm{AA}$ & $\mathrm{AA}$ & AA & $\mathrm{AG}$ & $\mathrm{AA}$ & AA & $\mathrm{AA}$ \\
\hline AA & AA & $\mathrm{AA}$ & AA & $\mathrm{AA}$ & $\mathrm{AA}$ & AA & $\mathrm{AA}$ & AA & AA & \\
\hline GDsnp01810 & 10 & \multicolumn{2}{|c|}{3310494} & $\mathrm{AA}$ & $\mathrm{AA}$ & $\mathrm{AA}$ & $\mathrm{AC}$ & $\mathrm{AC}$ & $\mathrm{AC}$ & $\mathrm{AC}$ \\
\hline $\mathrm{AC}$ & $\mathrm{AA}$ & $\mathrm{AA}$ & $\mathrm{AC}$ & $\mathrm{AC}$ & $\mathrm{AA}$ & AA & $\mathrm{AA}$ & $\mathrm{AC}$ & AA & $\mathrm{AC}$ \\
\hline AA & $\mathrm{AA}$ & $\mathrm{AC}$ & $\mathrm{AA}$ & $\mathrm{AA}$ & $\mathrm{AA}$ & AA & AA & $\mathrm{AA}$ & AA & AA \\
\hline AA & $\mathrm{AA}$ & $\mathrm{AA}$ & $\mathrm{AC}$ & $\mathrm{AA}$ & $\mathrm{AA}$ & AA & $\mathrm{AC}$ & $\mathrm{AA}$ & $\mathrm{AC}$ & $\mathrm{AC}$ \\
\hline AA & $\mathrm{AA}$ & $\mathrm{AC}$ & $\mathrm{AA}$ & $\mathrm{AA}$ & $\mathrm{AA}$ & AA & $\mathrm{AA}$ & $\mathrm{AA}$ & AA & $\mathrm{AA}$ \\
\hline AA & $\mathrm{AA}$ & $\mathrm{AA}$ & $\mathrm{AC}$ & $\mathrm{AA}$ & $\mathrm{AA}$ & AA & $\mathrm{AC}$ & $\mathrm{AA}$ & AA & $\mathrm{AA}$ \\
\hline $\mathrm{AA}$ & $\mathrm{AC}$ & $\mathrm{AA}$ & $\mathrm{AA}$ & $\mathrm{AA}$ & $\mathrm{AA}$ & $\mathrm{AC}$ & $\mathrm{AC}$ & $\mathrm{AA}$ & $\mathrm{AA}$ & $\mathrm{AA}$ \\
\hline $\mathrm{AC}$ & $\mathrm{AA}$ & $\mathrm{AA}$ & $\mathrm{AA}$ & $\mathrm{AC}$ & $\mathrm{AA}$ & $\mathrm{AA}$ & $\mathrm{AC}$ & $\mathrm{AA}$ & $\mathrm{AA}$ & $\mathrm{AA}$ \\
\hline AA & $\mathrm{AA}$ & $\mathrm{AA}$ & $\mathrm{AA}$ & $\mathrm{AA}$ & $\mathrm{AA}$ & AA & $\mathrm{AC}$ & $\mathrm{AA}$ & AA & \\
\hline
\end{tabular}


Supplemental Table 2. Continued.

\begin{tabular}{|c|c|c|c|c|c|c|c|c|c|c|}
\hline \multirow{3}{*}{$\begin{array}{l}\text { SNP name } \\
\text { GDsnp00875 } \\
\text { AC }\end{array}$} & \multicolumn{10}{|c|}{ Linkage group Physical location (bp) Individuals } \\
\hline & 10 & \multicolumn{2}{|c|}{8104933} & $\mathrm{AA}$ & $\mathrm{AA}$ & AA & $\mathrm{AC}$ & $\mathrm{AC}$ & $\mathrm{AC}$ & $\mathrm{AC}$ \\
\hline & AA & AA & $\mathrm{AC}$ & $\mathrm{AC}$ & AA & AA & AA & $\mathrm{AC}$ & $\mathrm{AC}$ & $\mathrm{AC}$ \\
\hline AA & AA & $\mathrm{AC}$ & $\mathrm{AA}$ & AA & AA & $\mathrm{AC}$ & AA & AA & AA & AA \\
\hline AA & AA & $\mathrm{AC}$ & $\mathrm{AC}$ & AA & AA & AA & AA & AA & $\mathrm{AC}$ & $\mathrm{AC}$ \\
\hline AA & $\mathrm{AA}$ & $\mathrm{AC}$ & $\mathrm{AA}$ & $\mathrm{AA}$ & $\mathrm{AA}$ & AA & $\mathrm{AA}$ & $\mathrm{AA}$ & AA & $\mathrm{AA}$ \\
\hline AA & $\mathrm{AA}$ & AA & $\mathrm{AC}$ & AA & $\mathrm{AA}$ & AA & $\mathrm{AC}$ & $\mathrm{AA}$ & AA & AA \\
\hline AA & $\mathrm{AC}$ & $\mathrm{AA}$ & $\mathrm{AA}$ & $\mathrm{AA}$ & $\mathrm{AA}$ & AA & $\mathrm{AC}$ & $\mathrm{AA}$ & AA & $\mathrm{AA}$ \\
\hline $\mathrm{AC}$ & $\mathrm{AC}$ & AA & $\mathrm{AA}$ & $\mathrm{AA}$ & $\mathrm{AA}$ & AA & $\mathrm{AC}$ & $\mathrm{AA}$ & AA & AA \\
\hline $\mathrm{AA}$ & AA & AA & AA & $\mathrm{AA}$ & AA & AA & $\mathrm{AC}$ & AA & AA & \\
\hline GDsnp01267 & 10 & \multicolumn{2}{|c|}{12373904} & $\mathrm{AA}$ & $\mathrm{AA}$ & AA & AA & $\mathrm{AA}$ & $\mathrm{AG}$ & AA \\
\hline AA & $\mathrm{AA}$ & $\mathrm{AA}$ & $\mathrm{AG}$ & $\mathrm{AA}$ & $\mathrm{AA}$ & AA & $\mathrm{AA}$ & $\mathrm{AG}$ & $\mathrm{AG}$ & $\mathrm{AA}$ \\
\hline AA & $\mathrm{AA}$ & $\mathrm{AG}$ & $\mathrm{AA}$ & $\mathrm{AA}$ & $\mathrm{AA}$ & AA & $\mathrm{AA}$ & $\mathrm{AA}$ & AA & $\mathrm{AA}$ \\
\hline AA & $\mathrm{AA}$ & $\mathrm{AG}$ & $\mathrm{AA}$ & $\mathrm{AA}$ & $\mathrm{AA}$ & AA & $\mathrm{AA}$ & $\mathrm{AA}$ & $\mathrm{AG}$ & $\mathrm{AG}$ \\
\hline AA & $\mathrm{AA}$ & AA & $\mathrm{AA}$ & $\mathrm{AA}$ & AA & AA & $\mathrm{AG}$ & $\mathrm{AA}$ & AA & AA \\
\hline AA & $\mathrm{AA}$ & AA & $\mathrm{AG}$ & $\mathrm{AA}$ & $\mathrm{AA}$ & AA & AA & $\mathrm{AA}$ & AA & $\mathrm{AA}$ \\
\hline AA & $\mathrm{AA}$ & AA & $\mathrm{AA}$ & $\mathrm{AA}$ & $\mathrm{AA}$ & AA & $\mathrm{AG}$ & $\mathrm{AA}$ & AA & $\mathrm{AA}$ \\
\hline AG & $\mathrm{AG}$ & $\mathrm{AA}$ & $\mathrm{AA}$ & $\mathrm{AA}$ & - & AA & $\mathrm{AA}$ & $\mathrm{AA}$ & AA & $\mathrm{AA}$ \\
\hline $\mathrm{AA}$ & $\mathrm{AA}$ & $\mathrm{AA}$ & - & $\mathrm{AA}$ & $\mathrm{AA}$ & $\mathrm{AG}$ & $\mathrm{AG}$ & $\mathrm{AA}$ & $\mathrm{AA}$ & \\
\hline GDsnp01710 & 10 & \multicolumn{2}{|c|}{14182739} & $\mathrm{AA}$ & $\mathrm{AA}$ & AA & $\mathrm{AA}$ & $\mathrm{AA}$ & AA & $\mathrm{AA}$ \\
\hline AA & $\mathrm{AA}$ & $\mathrm{AA}$ & $\mathrm{AA}$ & $\mathrm{AA}$ & $\mathrm{AA}$ & AA & $\mathrm{AA}$ & $\mathrm{AA}$ & AA & $\mathrm{AA}$ \\
\hline AA & $\mathrm{AA}$ & AA & $\mathrm{AA}$ & $\mathrm{AA}$ & AA & AA & $\mathrm{AA}$ & $\mathrm{AA}$ & AA & AA \\
\hline AA & $\mathrm{AA}$ & AA & $\mathrm{AA}$ & $\mathrm{AA}$ & $\mathrm{AA}$ & AA & AA & $\mathrm{AA}$ & AA & $\mathrm{AA}$ \\
\hline AA & $\mathrm{AA}$ & $\mathrm{AA}$ & $\mathrm{AA}$ & $\mathrm{AA}$ & $\mathrm{AA}$ & AA & $\mathrm{AA}$ & $\mathrm{AA}$ & AA & $\mathrm{AA}$ \\
\hline AA & $\mathrm{AA}$ & $\mathrm{AA}$ & $\mathrm{AA}$ & $\mathrm{AA}$ & $\mathrm{AA}$ & AA & $\mathrm{AA}$ & $\mathrm{AA}$ & AA & $\mathrm{AA}$ \\
\hline AA & $\mathrm{AA}$ & AA & $\mathrm{AA}$ & $\mathrm{AA}$ & $\mathrm{AA}$ & AA & AA & $\mathrm{AA}$ & AA & AA \\
\hline AA & $\mathrm{AA}$ & AA & AA & $\mathrm{AA}$ & AA & AA & AA & $\mathrm{AA}$ & AA & AA \\
\hline AA & $\mathrm{AA}$ & $\mathrm{AA}$ & $\mathrm{AA}$ & $\mathrm{AA}$ & $\mathrm{AA}$ & AA & $\mathrm{AA}$ & $\mathrm{AA}$ & AA & \\
\hline GDsnp02183 & 10 & \multicolumn{2}{|c|}{16995920} & $\mathrm{AA}$ & $\mathrm{AG}$ & $\mathrm{AG}$ & $\mathrm{AG}$ & $\mathrm{AG}$ & $\mathrm{AA}$ & $\mathrm{AG}$ \\
\hline GG & GG & AA & GG & $\mathrm{AG}$ & $\mathrm{AA}$ & $\mathrm{AG}$ & $\mathrm{AG}$ & $\mathrm{AG}$ & GG & $\mathrm{AG}$ \\
\hline GG & $\mathrm{AG}$ & GG & $\mathrm{AA}$ & $\mathrm{AA}$ & $\mathrm{AG}$ & $\mathrm{AG}$ & $\mathrm{AA}$ & $\mathrm{AA}$ & $\mathrm{AG}$ & $\mathrm{AA}$ \\
\hline AG & $\mathrm{AG}$ & GG & $\mathrm{AG}$ & $\mathrm{AG}$ & GG & $\mathrm{AG}$ & GG & $\mathrm{AG}$ & GG & - \\
\hline GG & $\mathrm{AA}$ & $\mathrm{AG}$ & $\mathrm{AG}$ & $\mathrm{AG}$ & $\mathrm{AG}$ & GG & GG & $\mathrm{AG}$ & GG & $\mathrm{AG}$ \\
\hline $\mathrm{AG}$ & $\mathrm{AA}$ & $\mathrm{AG}$ & GG & $\mathrm{AG}$ & $\mathrm{AG}$ & $\mathrm{AG}$ & GG & $\mathrm{AG}$ & GG & $\mathrm{AG}$ \\
\hline $\mathrm{AG}$ & $\mathrm{AG}$ & $\mathrm{AG}$ & $\mathrm{AA}$ & $\mathrm{AA}$ & $\mathrm{AA}$ & GG & GG & $\mathrm{AG}$ & $\mathrm{AG}$ & $\mathrm{AG}$ \\
\hline GG & $\mathrm{AG}$ & $\mathrm{AG}$ & $\mathrm{AG}$ & $\mathrm{AG}$ & AA & AA & $\mathrm{AG}$ & $\mathrm{AG}$ & $\mathrm{AG}$ & AA \\
\hline $\mathrm{AA}$ & $\mathrm{AA}$ & AA & - & $\mathrm{AG}$ & $\mathrm{AG}$ & $\mathrm{AG}$ & GG & GG & $\mathrm{AG}$ & \\
\hline GDsnp02072 & 10 & \multicolumn{2}{|c|}{17672554} & GG & GG & GG & GG & GG & GG & GG \\
\hline- & $\mathrm{AG}$ & GG & $\mathrm{AG}$ & GG & GG & GG & GG & $\mathrm{AG}$ & GG & GG \\
\hline GG & GG & $\mathrm{AG}$ & GG & GG & GG & $\mathrm{AG}$ & GG & GG & GG & GG \\
\hline GG & GG & GG & GG & GG & GG & GG & GG & GG & $\mathrm{AG}$ & GG \\
\hline GG & GG & GG & GG & GG & GG & GG & GG & GG & GG & GG \\
\hline GG & GG & GG & GG & GG & GG & GG & $\mathrm{AG}$ & GG & GG & GG \\
\hline GG & GG & GG & GG & GG & GG & $\mathrm{AG}$ & $\mathrm{AG}$ & GG & GG & GG \\
\hline AG & GG & GG & GG & GG & GG & GG & GG & GG & GG & GG \\
\hline GG & GG & GG & GG & $\mathrm{AG}$ & GG & GG & $\mathrm{AG}$ & GG & GG & \\
\hline GDsnp00355 & 10 & \multicolumn{2}{|c|}{25981281} & $\mathrm{AT}$ & $\mathrm{AT}$ & AA & AT & $\mathrm{AT}$ & AA & TT \\
\hline TT & $\mathrm{TT}$ & $\mathrm{AT}$ & $\mathrm{TT}$ & $\mathrm{AT}$ & $\mathrm{AA}$ & $\mathrm{AT}$ & $\mathrm{AT}$ & $\mathrm{TT}$ & $\mathrm{TT}$ & TT \\
\hline AT & $\mathrm{AT}$ & TT & $\mathrm{AT}$ & $\mathrm{AT}$ & AA & TT & $\mathrm{AA}$ & $\mathrm{AA}$ & $\mathrm{AT}$ & AT \\
\hline AT & $\mathrm{TT}$ & $\mathrm{AT}$ & $\mathrm{TT}$ & AT & $\mathrm{AT}$ & AT & AT & $\mathrm{AT}$ & $\mathrm{AT}$ & AT \\
\hline AT & $\mathrm{AT}$ & $\mathrm{AT}$ & $\mathrm{AT}$ & $\mathrm{AT}$ & $\mathrm{TT}$ & - & $\mathrm{TT}$ & $\mathrm{AT}$ & $\mathrm{TT}$ & TT \\
\hline AT & $\mathrm{TT}$ & $\mathrm{AT}$ & $\mathrm{AT}$ & $\mathrm{AT}$ & $\mathrm{AT}$ & $\mathrm{AT}$ & $\mathrm{TT}$ & $\mathrm{AT}$ & AT & AA \\
\hline AT & $\mathrm{AT}$ & $\mathrm{AT}$ & $\mathrm{AA}$ & $\mathrm{AA}$ & $\mathrm{AA}$ & $\mathrm{TT}$ & $\mathrm{AT}$ & $\mathrm{AT}$ & $\mathrm{AT}$ & AT \\
\hline $\mathrm{TT}$ & $\mathrm{AT}$ & AT & AT & $\mathrm{AT}$ & $\mathrm{AA}$ & $\mathrm{AA}$ & $\mathrm{TT}$ & AT & $\mathrm{AT}$ & $\mathrm{AA}$ \\
\hline AA & $\mathrm{AA}$ & $\mathrm{AA}$ & $\mathrm{TT}$ & $\mathrm{AT}$ & $\mathrm{AT}$ & AA & $\mathrm{TT}$ & $\mathrm{AT}$ & $\mathrm{AT}$ & \\
\hline
\end{tabular}

Continued next page 
Supplemental Table 2. Continued.

\begin{tabular}{|c|c|c|c|c|c|c|c|c|c|c|}
\hline \multirow{3}{*}{$\begin{array}{l}\text { SNP name } \\
\text { GDsnp00360 } \\
\text { AC }\end{array}$} & \multicolumn{10}{|c|}{ Linkage group Physical location (bp) Individuals } \\
\hline & 10 & \multicolumn{2}{|c|}{26173385} & $\mathrm{AC}$ & $\mathrm{AC}$ & $\mathrm{CC}$ & $\mathrm{AC}$ & $\mathrm{AC}$ & $\mathrm{CC}$ & $\mathrm{AA}$ \\
\hline & AA & $\mathrm{AC}$ & AA & $\mathrm{AC}$ & $\mathrm{CC}$ & $\mathrm{AC}$ & $\mathrm{AC}$ & AA & AA & AA \\
\hline $\mathrm{AC}$ & $\mathrm{AC}$ & AA & $\mathrm{AC}$ & $\mathrm{AC}$ & $\mathrm{CC}$ & AA & $\mathrm{CC}$ & $\mathrm{CC}$ & $\mathrm{AC}$ & $\mathrm{AC}$ \\
\hline $\mathrm{AC}$ & AA & $\mathrm{AC}$ & $\mathrm{AA}$ & $\mathrm{AC}$ & $\mathrm{AC}$ & $\mathrm{AC}$ & $\mathrm{AC}$ & $\mathrm{AC}$ & $\mathrm{AC}$ & $\mathrm{AC}$ \\
\hline $\mathrm{AC}$ & $\mathrm{AC}$ & $\mathrm{AC}$ & $\mathrm{AC}$ & $\mathrm{AC}$ & $\mathrm{AA}$ & - & $\mathrm{AA}$ & $\mathrm{AC}$ & $\mathrm{AA}$ & $\mathrm{AA}$ \\
\hline $\mathrm{AC}$ & $\mathrm{AA}$ & $\mathrm{AC}$ & $\mathrm{AC}$ & $\mathrm{AC}$ & $\mathrm{AC}$ & $\mathrm{AC}$ & $\mathrm{AA}$ & $\mathrm{AC}$ & $\mathrm{AC}$ & $\mathrm{CC}$ \\
\hline $\mathrm{AC}$ & $\mathrm{AC}$ & $\mathrm{AC}$ & $\mathrm{CC}$ & $\mathrm{CC}$ & $\mathrm{CC}$ & $\mathrm{AA}$ & $\mathrm{AA}$ & $\mathrm{AC}$ & $\mathrm{AC}$ & $\mathrm{AC}$ \\
\hline $\mathrm{AA}$ & $\mathrm{AC}$ & $\mathrm{AC}$ & $\mathrm{AC}$ & $\mathrm{AA}$ & $\mathrm{CC}$ & $\mathrm{CC}$ & $\mathrm{AA}$ & $\mathrm{AC}$ & $\mathrm{AC}$ & $\mathrm{CC}$ \\
\hline $\mathrm{CC}$ & $\mathrm{CC}$ & $\mathrm{CC}$ & $\mathrm{AA}$ & $\mathrm{AC}$ & $\mathrm{AC}$ & $\mathrm{CC}$ & $\mathrm{AA}$ & $\mathrm{AC}$ & $\mathrm{AC}$ & \\
\hline GDsnp00099 & 10 & \multicolumn{2}{|c|}{27409151} & $\mathrm{AA}$ & $\mathrm{AC}$ & $\mathrm{AA}$ & $\mathrm{AC}$ & $\mathrm{AA}$ & $\mathrm{AA}$ & $\mathrm{AC}$ \\
\hline $\mathrm{CC}$ & $\mathrm{AC}$ & $\mathrm{AC}$ & $\mathrm{CC}$ & $\mathrm{AC}$ & $\mathrm{AA}$ & $\mathrm{AC}$ & $\mathrm{AC}$ & $\mathrm{CC}$ & $\mathrm{CC}$ & AA \\
\hline $\mathrm{AC}$ & $\mathrm{AC}$ & $\mathrm{AC}$ & $\mathrm{AC}$ & $\mathrm{AC}$ & $\mathrm{AA}$ & $\mathrm{AC}$ & $\mathrm{AA}$ & AA & $\mathrm{AC}$ & $\mathrm{AC}$ \\
\hline $\mathrm{AC}$ & $\mathrm{CC}$ & $\mathrm{AC}$ & $\mathrm{AA}$ & $\mathrm{AC}$ & $\mathrm{AC}$ & $\mathrm{AC}$ & $\mathrm{AA}$ & $\mathrm{AC}$ & $\mathrm{AC}$ & $\mathrm{AC}$ \\
\hline $\mathrm{AC}$ & $\mathrm{AC}$ & $\mathrm{AC}$ & $\mathrm{AC}$ & $\mathrm{AC}$ & $\mathrm{CC}$ & $\mathrm{CC}$ & $\mathrm{AA}$ & $\mathrm{AC}$ & $\mathrm{CC}$ & $\mathrm{CC}$ \\
\hline $\mathrm{AC}$ & $\mathrm{CC}$ & $\mathrm{AC}$ & $\mathrm{AC}$ & $\mathrm{AC}$ & $\mathrm{AC}$ & $\mathrm{AC}$ & $\mathrm{AC}$ & $\mathrm{CC}$ & $\mathrm{AC}$ & AA \\
\hline $\mathrm{AC}$ & $\mathrm{AC}$ & $\mathrm{AC}$ & $\mathrm{AA}$ & $\mathrm{AA}$ & $\mathrm{AA}$ & $\mathrm{AC}$ & $\mathrm{AC}$ & AA & $\mathrm{AC}$ & $\mathrm{AC}$ \\
\hline- & $\mathrm{AC}$ & $\mathrm{AC}$ & $\mathrm{AC}$ & $\mathrm{AC}$ & $\mathrm{AA}$ & AA & $\mathrm{AC}$ & $\mathrm{AC}$ & $\mathrm{AA}$ & $\mathrm{AA}$ \\
\hline AA & $\mathrm{AA}$ & $\mathrm{AC}$ & $\mathrm{AC}$ & $\mathrm{AA}$ & $\mathrm{AC}$ & AA & $\mathrm{AC}$ & $\mathrm{AC}$ & $\mathrm{AC}$ & \\
\hline GDsnp01761 & 10 & \multicolumn{2}{|c|}{31910794} & $\mathrm{AA}$ & $\mathrm{AA}$ & $\mathrm{AA}$ & $\mathrm{AA}$ & $\mathrm{AA}$ & $\mathrm{AA}$ & $\mathrm{AA}$ \\
\hline AA & $\mathrm{AA}$ & $\mathrm{AG}$ & $\mathrm{AA}$ & $\mathrm{AA}$ & $\mathrm{AA}$ & AA & $\mathrm{AA}$ & AA & AA & $\mathrm{AA}$ \\
\hline $\mathrm{AA}$ & $\mathrm{AA}$ & $\mathrm{AA}$ & $\mathrm{AA}$ & $\mathrm{AA}$ & $\mathrm{AA}$ & $\mathrm{AA}$ & $\mathrm{AA}$ & $\mathrm{AA}$ & $\mathrm{AA}$ & $\mathrm{AA}$ \\
\hline $\mathrm{AA}$ & $\mathrm{AA}$ & $\mathrm{AA}$ & $\mathrm{AA}$ & $\mathrm{AA}$ & $\mathrm{AA}$ & $\mathrm{AA}$ & $\mathrm{AA}$ & $\mathrm{AA}$ & AA & $\mathrm{AA}$ \\
\hline AA & $\mathrm{AA}$ & $\mathrm{AA}$ & $\mathrm{AA}$ & $\mathrm{AA}$ & $\mathrm{AA}$ & AA & $\mathrm{AA}$ & $\mathrm{AA}$ & $\mathrm{AA}$ & $\mathrm{AA}$ \\
\hline AA & $\mathrm{AA}$ & AA & $\mathrm{AA}$ & $\mathrm{AA}$ & $\mathrm{AA}$ & AA & $\mathrm{AA}$ & AA & AA & $\mathrm{AA}$ \\
\hline AA & $\mathrm{AA}$ & AA & $\mathrm{AA}$ & $\mathrm{AA}$ & $\mathrm{AA}$ & AA & $\mathrm{AA}$ & AA & AA & AA \\
\hline AA & $\mathrm{AA}$ & AA & $\mathrm{AA}$ & $\mathrm{AA}$ & AA & AA & $\mathrm{AA}$ & AA & AA & $\mathrm{AA}$ \\
\hline AA & $\mathrm{AA}$ & $\mathrm{AA}$ & $\mathrm{AA}$ & $\mathrm{AA}$ & $\mathrm{AA}$ & $\mathrm{AG}$ & $\mathrm{AA}$ & $\mathrm{AA}$ & AA & \\
\hline GDsnp00405 & 10 & \multicolumn{2}{|c|}{32186209} & GG & GG & GG & GG & GG & GG & GG \\
\hline $\mathrm{AG}$ & GG & $\mathrm{AG}$ & GG & GG & GG & GG & GG & GG & GG & GG \\
\hline GG & GG & GG & GG & GG & GG & GG & GG & GG & GG & GG \\
\hline GG & GG & GG & GG & GG & GG & GG & GG & GG & $\mathrm{AG}$ & GG \\
\hline GG & GG & GG & GG & GG & GG & - & GG & GG & GG & GG \\
\hline GG & GG & GG & GG & GG & GG & GG & GG & GG & GG & GG \\
\hline GG & GG & GG & GG & GG & GG & GG & GG & GG & GG & GG \\
\hline GG & GG & GG & GG & GG & GG & GG & GG & GG & GG & GG \\
\hline GG & GG & GG & GG & GG & GG & $\mathrm{AG}$ & GG & GG & GG & \\
\hline GDsnp00253 & 11 & \multicolumn{2}{|c|}{545684} & AT & $\mathrm{TT}$ & $\mathrm{TT}$ & $\mathrm{TT}$ & $\mathrm{TT}$ & $\mathrm{TT}$ & AT \\
\hline AT & $\mathrm{AT}$ & $\mathrm{TT}$ & $\mathrm{TT}$ & $\mathrm{TT}$ & $\mathrm{AT}$ & AT & $\mathrm{AT}$ & $\mathrm{AT}$ & $\mathrm{TT}$ & TT \\
\hline $\mathrm{TT}$ & $\mathrm{TT}$ & $\mathrm{TT}$ & $\mathrm{TT}$ & $\mathrm{AT}$ & $\mathrm{TT}$ & $\mathrm{TT}$ & $\mathrm{TT}$ & $\mathrm{TT}$ & $\mathrm{TT}$ & TT \\
\hline AT & $\mathrm{TT}$ & $\mathrm{TT}$ & $\mathrm{TT}$ & $\mathrm{TT}$ & TT & $\mathrm{TT}$ & TT & TT & $\mathrm{TT}$ & TT \\
\hline $\mathrm{TT}$ & $\mathrm{TT}$ & $\mathrm{TT}$ & $\mathrm{TT}$ & $\mathrm{TT}$ & $\mathrm{TT}$ & $\mathrm{TT}$ & $\mathrm{TT}$ & $\mathrm{TT}$ & $\mathrm{TT}$ & TT \\
\hline $\mathrm{TT}$ & $\mathrm{TT}$ & $\mathrm{TT}$ & $\mathrm{AT}$ & $\mathrm{TT}$ & $\mathrm{AT}$ & $\mathrm{TT}$ & $\mathrm{TT}$ & $\mathrm{TT}$ & $\mathrm{TT}$ & TT \\
\hline AT & $\mathrm{TT}$ & $\mathrm{AT}$ & $\mathrm{TT}$ & $\mathrm{TT}$ & $\mathrm{TT}$ & $\mathrm{TT}$ & $\mathrm{TT}$ & $\mathrm{AT}$ & $\mathrm{TT}$ & TT \\
\hline AT & $\mathrm{TT}$ & $\mathrm{TT}$ & $\mathrm{TT}$ & $\mathrm{TT}$ & $\mathrm{AT}$ & $\mathrm{TT}$ & $\mathrm{TT}$ & $\mathrm{AT}$ & $\mathrm{TT}$ & TT \\
\hline TT & AT & AT & AT & TT & TT & AT & TT & AT & $\mathrm{AT}$ & \\
\hline GDsnp02281 & 11 & \multicolumn{2}{|c|}{1622012} & GG & $\mathrm{AG}$ & $\mathrm{AG}$ & GG & GG & $\mathrm{AG}$ & GG \\
\hline GG & GG & GG & GG & GG & GG & GG & GG & GG & GG & GG \\
\hline $\mathrm{AG}$ & GG & $\mathrm{AG}$ & $\mathrm{AG}$ & GG & GG & GG & GG & GG & GG & GG \\
\hline GG & GG & $\mathrm{AG}$ & GG & GG & GG & $\mathrm{AG}$ & GG & GG & GG & $\mathrm{AG}$ \\
\hline GG & GG & GG & GG & GG & GG & - & GG & GG & GG & GG \\
\hline $\mathrm{AG}$ & $\mathrm{AG}$ & GG & GG & $\mathrm{AG}$ & GG & GG & $\mathrm{AG}$ & GG & GG & $\mathrm{AG}$ \\
\hline GG & GG & GG & GG & GG & GG & GG & $\mathrm{AG}$ & GG & GG & $\mathrm{AG}$ \\
\hline GG & $\mathrm{AG}$ & GG & $\mathrm{AG}$ & $\mathrm{AG}$ & GG & GG & GG & GG & GG & GG \\
\hline GG & GG & GG & GG & GG & GG & GG & $\mathrm{AG}$ & GG & GG & \\
\hline
\end{tabular}


Supplemental Table 2. Continued.

\begin{tabular}{|c|c|c|c|c|c|c|c|c|c|c|}
\hline \multirow{3}{*}{$\begin{array}{l}\text { SNP name } \\
\text { GDsnp00351 } \\
\text { AA }\end{array}$} & \multicolumn{10}{|c|}{ Linkage group Physical location (bp) Individuals } \\
\hline & 11 & \multicolumn{2}{|c|}{3079914} & $\mathrm{AA}$ & $\mathrm{AG}$ & AA & AA & $\mathrm{AA}$ & AA & $\mathrm{AA}$ \\
\hline & $\mathrm{AG}$ & AA & AA & AA & $\mathrm{AG}$ & $\mathrm{AG}$ & $\mathrm{AG}$ & $\mathrm{AG}$ & AA & AA \\
\hline AA & AA & AA & AA & $\mathrm{AG}$ & AA & AA & AA & AA & AA & AA \\
\hline $\mathrm{AG}$ & AA & AA & AA & AA & AA & AA & AA & AA & $\mathrm{AG}$ & $\mathrm{AA}$ \\
\hline AA & $\mathrm{AA}$ & AA & AA & $\mathrm{AA}$ & $\mathrm{AA}$ & AA & $\mathrm{AA}$ & $\mathrm{AA}$ & AA & $\mathrm{AA}$ \\
\hline AA & $\mathrm{AG}$ & AA & $\mathrm{AG}$ & AA & $\mathrm{AG}$ & AA & AA & $\mathrm{AA}$ & AA & AA \\
\hline AG & $\mathrm{AA}$ & $\mathrm{AG}$ & $\mathrm{AA}$ & $\mathrm{AA}$ & $\mathrm{AA}$ & AA & $\mathrm{AA}$ & $\mathrm{AG}$ & AA & $\mathrm{AA}$ \\
\hline $\mathrm{AG}$ & $\mathrm{AA}$ & AA & AA & $\mathrm{AA}$ & $\mathrm{AG}$ & AA & AA & $\mathrm{AG}$ & AA & AA \\
\hline $\mathrm{AA}$ & $\mathrm{AG}$ & $\mathrm{AG}$ & $\mathrm{AG}$ & $\mathrm{AA}$ & AA & AA & $\mathrm{AA}$ & $\mathrm{AG}$ & $\mathrm{AG}$ & \\
\hline GDsnp01140 & 11 & \multicolumn{2}{|c|}{3624461} & $\mathrm{AA}$ & $\mathrm{AT}$ & AA & AA & $\mathrm{AA}$ & AA & AA \\
\hline AA & $\mathrm{AT}$ & AA & $\mathrm{AA}$ & $\mathrm{AA}$ & $\mathrm{AT}$ & AT & $\mathrm{AA}$ & $\mathrm{AT}$ & AA & $\mathrm{AA}$ \\
\hline AA & $\mathrm{AA}$ & $\mathrm{AA}$ & AA & AT & $\mathrm{AA}$ & AA & $\mathrm{AA}$ & $\mathrm{AA}$ & AA & $\mathrm{AA}$ \\
\hline AT & $\mathrm{AA}$ & AA & $\mathrm{AA}$ & $\mathrm{AA}$ & $\mathrm{AA}$ & AA & $\mathrm{AA}$ & $\mathrm{AA}$ & AA & $\mathrm{AA}$ \\
\hline AA & $\mathrm{AA}$ & AA & AA & $\mathrm{AA}$ & AA & AA & $\mathrm{AA}$ & $\mathrm{AA}$ & AA & AA \\
\hline AA & $\mathrm{AT}$ & AA & $\mathrm{AT}$ & $\mathrm{AA}$ & $\mathrm{AT}$ & AA & AA & $\mathrm{AA}$ & AA & $\mathrm{AA}$ \\
\hline AT & $\mathrm{AA}$ & $\mathrm{AT}$ & AA & $\mathrm{AA}$ & $\mathrm{AA}$ & AA & AA & $\mathrm{AT}$ & AA & $\mathrm{AA}$ \\
\hline AT & $\mathrm{AA}$ & AA & $\mathrm{AA}$ & $\mathrm{AA}$ & $\mathrm{AT}$ & AA & $\mathrm{AA}$ & $\mathrm{AT}$ & AA & $\mathrm{AA}$ \\
\hline $\mathrm{AA}$ & $\mathrm{AT}$ & $\mathrm{AT}$ & $\mathrm{AT}$ & $\mathrm{AA}$ & $\mathrm{AA}$ & $\mathrm{AT}$ & $\mathrm{AA}$ & $\mathrm{AT}$ & $\mathrm{AT}$ & \\
\hline GDsnp00225 & 11 & \multicolumn{2}{|c|}{4183094} & $\mathrm{AA}$ & $\mathrm{TT}$ & AA & AT & $\mathrm{TT}$ & AA & AT \\
\hline AT & $\mathrm{AT}$ & $\mathrm{AA}$ & TT & $\mathrm{AA}$ & $\mathrm{TT}$ & $\mathrm{AT}$ & $\mathrm{AA}$ & $\mathrm{AT}$ & $\mathrm{AT}$ & AT \\
\hline AA & $\mathrm{AT}$ & $\mathrm{AT}$ & AT & $\mathrm{TT}$ & $\mathrm{AT}$ & AT & AT & $\mathrm{AT}$ & $\mathrm{TT}$ & AT \\
\hline AT & $\mathrm{TT}$ & $\mathrm{AT}$ & $\mathrm{AT}$ & $\mathrm{AT}$ & $\mathrm{AT}$ & AA & AT & $\mathrm{AT}$ & $\mathrm{AT}$ & AT \\
\hline AA & $\mathrm{AA}$ & AT & $\mathrm{TT}$ & $\mathrm{AT}$ & AA & AA & $\mathrm{TT}$ & $\mathrm{AA}$ & AT & AT \\
\hline AA & $\mathrm{TT}$ & $\mathrm{TT}$ & $\mathrm{AT}$ & AT & $\mathrm{TT}$ & $\mathrm{AT}$ & $\mathrm{AA}$ & $\mathrm{AA}$ & $\mathrm{AT}$ & AT \\
\hline AT & $\mathrm{AT}$ & $\mathrm{TT}$ & $\mathrm{AT}$ & AT & $\mathrm{AT}$ & $\mathrm{TT}$ & $\mathrm{AT}$ & $\mathrm{AT}$ & $\mathrm{AT}$ & AT \\
\hline AT & $\mathrm{AT}$ & $\mathrm{TT}$ & $\mathrm{AA}$ & AT & $\mathrm{TT}$ & TT & AT & TT & AA & AA \\
\hline AT & $\mathrm{TT}$ & $\mathrm{TT}$ & $\mathrm{AT}$ & $\mathrm{AT}$ & $\mathrm{TT}$ & AT & $\mathrm{TT}$ & $\mathrm{TT}$ & $\mathrm{AT}$ & \\
\hline GDsnp00418 & 11 & \multicolumn{2}{|c|}{4580858} & GG & GG & $\mathrm{AG}$ & GG & GG & $\mathrm{AG}$ & $\mathrm{AG}$ \\
\hline- & GG & GG & GG & GG & GG & GG & $\mathrm{AG}$ & GG & GG & GG \\
\hline $\mathrm{AG}$ & GG & $\mathrm{AG}$ & $\mathrm{AG}$ & $\mathrm{AG}$ & GG & GG & GG & GG & GG & GG \\
\hline GG & GG & GG & GG & GG & GG & $\mathrm{AG}$ & GG & GG & GG & GG \\
\hline GG & GG & GG & GG & GG & GG & GG & GG & GG & GG & GG \\
\hline $\mathrm{AG}$ & GG & GG & GG & $\mathrm{AG}$ & GG & GG & $\mathrm{AG}$ & GG & GG & $\mathrm{AG}$ \\
\hline GG & GG & GG & GG & GG & GG & GG & $\mathrm{AG}$ & GG & GG & $\mathrm{AG}$ \\
\hline GG & $\mathrm{AG}$ & GG & $\mathrm{AG}$ & GG & GG & GG & GG & GG & GG & GG \\
\hline GG & GG & GG & GG & GG & GG & GG & GG & GG & GG & \\
\hline GDsnp01713 & 11 & \multicolumn{2}{|c|}{4891695} & $\mathrm{AA}$ & $\mathrm{AG}$ & AA & $\mathrm{AG}$ & GG & AA & $\mathrm{AG}$ \\
\hline AA & $\mathrm{AA}$ & AA & $\mathrm{AG}$ & $\mathrm{AA}$ & $\mathrm{AG}$ & AA & $\mathrm{AA}$ & $\mathrm{AA}$ & $\mathrm{AG}$ & $\mathrm{AG}$ \\
\hline AA & $\mathrm{AG}$ & $\mathrm{AG}$ & $\mathrm{AG}$ & $\mathrm{AG}$ & $\mathrm{AG}$ & $\mathrm{AG}$ & $\mathrm{AG}$ & $\mathrm{AG}$ & GG & $\mathrm{AG}$ \\
\hline AA & GG & $\mathrm{AG}$ & $\mathrm{AG}$ & $\mathrm{AG}$ & $\mathrm{AG}$ & AA & $\mathrm{AG}$ & $\mathrm{AG}$ & AA & $\mathrm{AA}$ \\
\hline AA & $\mathrm{AA}$ & $\mathrm{AG}$ & GG & GG & $\mathrm{AA}$ & AA & GG & $\mathrm{AA}$ & $\mathrm{AG}$ & $\mathrm{AG}$ \\
\hline AA & $\mathrm{AG}$ & GG & AA & $\mathrm{AG}$ & $\mathrm{AG}$ & $\mathrm{AG}$ & $\mathrm{AA}$ & $\mathrm{AA}$ & $\mathrm{AG}$ & $\mathrm{AG}$ \\
\hline AA & $\mathrm{AG}$ & $\mathrm{AG}$ & $\mathrm{AG}$ & $\mathrm{AG}$ & $\mathrm{AG}$ & GG & $\mathrm{AA}$ & $\mathrm{AA}$ & $\mathrm{AG}$ & $\mathrm{AG}$ \\
\hline- & $\mathrm{AG}$ & GG & AA & $\mathrm{AA}$ & $\mathrm{AG}$ & GG & $\mathrm{AG}$ & $\mathrm{AG}$ & AA & $\mathrm{AA}$ \\
\hline $\mathrm{AG}$ & $\mathrm{AG}$ & $\mathrm{AG}$ & $\mathrm{AA}$ & $\mathrm{AG}$ & GG & $\mathrm{AG}$ & GG & $\mathrm{AG}$ & $\mathrm{AA}$ & \\
\hline GDsnp00185 & 11 & \multicolumn{2}{|c|}{7848372} & $\mathrm{AA}$ & $\mathrm{AA}$ & AA & AA & $\mathrm{AA}$ & $\mathrm{AC}$ & $\mathrm{AC}$ \\
\hline AA & $\mathrm{AA}$ & AA & $\mathrm{AA}$ & $\mathrm{AA}$ & $\mathrm{AA}$ & AA & $\mathrm{AC}$ & $\mathrm{AA}$ & $\mathrm{AC}$ & $\mathrm{AC}$ \\
\hline $\mathrm{AC}$ & $\mathrm{AA}$ & AA & $\mathrm{AC}$ & $\mathrm{AC}$ & AA & AA & $\mathrm{AA}$ & $\mathrm{AA}$ & AA & AA \\
\hline AA & $\mathrm{AA}$ & AA & $\mathrm{AA}$ & $\mathrm{AA}$ & $\mathrm{AA}$ & $\mathrm{AC}$ & $\mathrm{AA}$ & $\mathrm{AA}$ & $\mathrm{AC}$ & $\mathrm{AC}$ \\
\hline AA & $\mathrm{AA}$ & AA & AA & $\mathrm{AA}$ & AA & AA & AA & $\mathrm{AA}$ & AA & AA \\
\hline $\mathrm{AC}$ & $\mathrm{AA}$ & AA & AA & $\mathrm{AC}$ & AA & AA & $\mathrm{AC}$ & $\mathrm{AA}$ & AA & $\mathrm{AC}$ \\
\hline AA & $\mathrm{AA}$ & AA & $\mathrm{AA}$ & $\mathrm{AA}$ & $\mathrm{AA}$ & AA & $\mathrm{AC}$ & $\mathrm{AA}$ & $\mathrm{AA}$ & $\mathrm{AC}$ \\
\hline $\mathrm{AA}$ & $\mathrm{AA}$ & $\mathrm{AA}$ & $\mathrm{AC}$ & $\mathrm{AA}$ & $\mathrm{AA}$ & AA & $\mathrm{AA}$ & $\mathrm{AA}$ & AA & $\mathrm{AA}$ \\
\hline $\mathrm{AA}$ & $\mathrm{AA}$ & $\mathrm{AC}$ & AA & $\mathrm{AA}$ & $\mathrm{AA}$ & $\mathrm{AC}$ & $\mathrm{AA}$ & $\mathrm{AA}$ & $\mathrm{AC}$ & \\
\hline
\end{tabular}

Continued next page 
Supplemental Table 2. Continued.

\begin{tabular}{|c|c|c|c|c|c|c|c|c|c|c|}
\hline \multirow{3}{*}{$\begin{array}{l}\text { SNP name } \\
\text { GDsnp00735 } \\
\text { AA }\end{array}$} & \multicolumn{10}{|c|}{ Linkage group Physical location (bp) Individuals } \\
\hline & 11 & \multicolumn{2}{|c|}{8120591} & $\mathrm{AA}$ & $\mathrm{AA}$ & AA & $\mathrm{AA}$ & AA & AA & $\mathrm{AA}$ \\
\hline & AA & AA & $\mathrm{AG}$ & AA & AA & AA & AA & AA & AA & AA \\
\hline AA & AA & AA & AA & AA & AA & AA & AA & AA & AA & AA \\
\hline AA & AA & AA & AA & AA & AA & AA & AA & AA & $\mathrm{AG}$ & $\mathrm{AG}$ \\
\hline AA & $\mathrm{AA}$ & $\mathrm{AA}$ & $\mathrm{AA}$ & $\mathrm{AA}$ & $\mathrm{AA}$ & $\mathrm{AA}$ & $\mathrm{AA}$ & $\mathrm{AA}$ & $\mathrm{AA}$ & $\mathrm{AA}$ \\
\hline AA & $\mathrm{AA}$ & AA & AA & AA & $\mathrm{AA}$ & AA & $\mathrm{AA}$ & AA & AA & AA \\
\hline $\mathrm{AA}$ & $\mathrm{AA}$ & $\mathrm{AA}$ & $\mathrm{AA}$ & $\mathrm{AA}$ & $\mathrm{AA}$ & $\mathrm{AA}$ & $\mathrm{AA}$ & $\mathrm{AA}$ & $\mathrm{AA}$ & $\mathrm{AA}$ \\
\hline- & $\mathrm{AA}$ & $\mathrm{AA}$ & $\mathrm{AA}$ & $\mathrm{AG}$ & $\mathrm{AA}$ & $\mathrm{AA}$ & $\mathrm{AA}$ & $\mathrm{AA}$ & $\mathrm{AA}$ & $\mathrm{AA}$ \\
\hline $\mathrm{AA}$ & $\mathrm{AA}$ & $\mathrm{AA}$ & $\mathrm{AA}$ & $\mathrm{AA}$ & $\mathrm{AA}$ & $\mathrm{AA}$ & $\mathrm{AA}$ & $\mathrm{AA}$ & AA & \\
\hline GDsnp00034 & 11 & \multicolumn{2}{|c|}{10059799} & GG & GG & GG & GG & GG & $\mathrm{AG}$ & $\mathrm{AG}$ \\
\hline $\mathrm{AG}$ & GG & GG & GG & GG & $\mathrm{AG}$ & GG & $\mathrm{AG}$ & GG & $\mathrm{AG}$ & $\mathrm{AG}$ \\
\hline $\mathrm{AG}$ & GG & $\mathrm{AG}$ & $\mathrm{AG}$ & $\mathrm{AG}$ & GG & GG & GG & GG & GG & GG \\
\hline GG & GG & GG & GG & GG & GG & $\mathrm{AG}$ & GG & GG & GG & $\mathrm{AG}$ \\
\hline GG & GG & GG & GG & GG & GG & GG & $\mathrm{AG}$ & GG & GG & GG \\
\hline $\mathrm{AG}$ & GG & GG & GG & $\mathrm{AG}$ & GG & GG & $\mathrm{AG}$ & GG & GG & $\mathrm{AG}$ \\
\hline GG & GG & GG & GG & GG & GG & GG & $\mathrm{AG}$ & GG & GG & $\mathrm{AG}$ \\
\hline GG & GG & GG & $\mathrm{AG}$ & GG & $\mathrm{AG}$ & GG & GG & GG & GG & GG \\
\hline GG & GG & $\mathrm{AG}$ & GG & GG & GG & GG & GG & GG & $\mathrm{AG}$ & \\
\hline GDsnp00240 & 11 & \multicolumn{2}{|c|}{11169491} & GG & $\mathrm{AG}$ & $\mathrm{AG}$ & GG & AA & GG & GG \\
\hline GG & $\mathrm{AG}$ & GG & GG & GG & GG & $\mathrm{AG}$ & GG & GG & GG & GG \\
\hline GG & GG & GG & GG & GG & GG & $\mathrm{AA}$ & GG & GG & GG & GG \\
\hline $\mathrm{AG}$ & GG & $\mathrm{AA}$ & GG & GG & $\mathrm{AG}$ & GG & $\mathrm{AA}$ & GG & GG & GG \\
\hline $\mathrm{AG}$ & GG & GG & GG & GG & GG & GG & $\mathrm{AA}$ & $\mathrm{AG}$ & GG & GG \\
\hline GG & $\mathrm{AA}$ & GG & GG & GG & AA & GG & GG & $\mathrm{AG}$ & GG & GG \\
\hline $\mathrm{AG}$ & GG & AA & AA & GG & GG & GG & GG & $\mathrm{AG}$ & GG & GG \\
\hline GG & $\mathrm{AA}$ & GG & GG & GG & GG & GG & $\mathrm{AA}$ & AA & $\mathrm{AG}$ & GG \\
\hline GG & $\mathrm{AA}$ & GG & $\mathrm{AG}$ & GG & GG & GG & GG & AA & GG & \\
\hline GDsnp00743 & 11 & \multicolumn{2}{|c|}{19798585} & $\mathrm{AA}$ & $\mathrm{AC}$ & $\mathrm{AC}$ & $\mathrm{AC}$ & $\mathrm{CC}$ & $\mathrm{AC}$ & $\mathrm{CC}$ \\
\hline $\mathrm{AA}$ & $\mathrm{CC}$ & $\mathrm{AC}$ & $\mathrm{AC}$ & $\mathrm{AC}$ & $\mathrm{CC}$ & $\mathrm{AC}$ & $\mathrm{AC}$ & $\mathrm{AC}$ & $\mathrm{CC}$ & $\mathrm{AC}$ \\
\hline $\mathrm{CC}$ & $\mathrm{CC}$ & $\mathrm{CC}$ & $\mathrm{AC}$ & $\mathrm{CC}$ & $\mathrm{AA}$ & $\mathrm{CC}$ & $\mathrm{AC}$ & $\mathrm{AC}$ & $\mathrm{CC}$ & $\mathrm{AC}$ \\
\hline $\mathrm{AC}$ & $\mathrm{CC}$ & $\mathrm{CC}$ & $\mathrm{CC}$ & $\mathrm{CC}$ & $\mathrm{AC}$ & $\mathrm{AC}$ & $\mathrm{CC}$ & $\mathrm{AC}$ & $\mathrm{AC}$ & $\mathrm{AC}$ \\
\hline $\mathrm{AC}$ & $\mathrm{CC}$ & $\mathrm{AC}$ & $\mathrm{CC}$ & $\mathrm{CC}$ & AA & - & $\mathrm{AC}$ & $\mathrm{AC}$ & $\mathrm{AC}$ & $\mathrm{AC}$ \\
\hline $\mathrm{AC}$ & $\mathrm{CC}$ & $\mathrm{CC}$ & $\mathrm{CC}$ & $\mathrm{CC}$ & $\mathrm{CC}$ & $\mathrm{AC}$ & $\mathrm{AC}$ & $\mathrm{AC}$ & $\mathrm{AC}$ & $\mathrm{CC}$ \\
\hline $\mathrm{AC}$ & $\mathrm{AC}$ & $\mathrm{CC}$ & $\mathrm{CC}$ & $\mathrm{CC}$ & $\mathrm{AC}$ & $\mathrm{AC}$ & $\mathrm{AC}$ & $\mathrm{AC}$ & $\mathrm{CC}$ & $\mathrm{AC}$ \\
\hline $\mathrm{CC}$ & $\mathrm{CC}$ & $\mathrm{CC}$ & $\mathrm{AC}$ & $\mathrm{AA}$ & $\mathrm{CC}$ & $\mathrm{CC}$ & $\mathrm{AC}$ & $\mathrm{CC}$ & $\mathrm{AC}$ & AA \\
\hline $\mathrm{AC}$ & $\mathrm{CC}$ & $\mathrm{CC}$ & $\mathrm{AC}$ & $\mathrm{CC}$ & $\mathrm{AC}$ & $\mathrm{AC}$ & $\mathrm{CC}$ & $\mathrm{CC}$ & $\mathrm{AC}$ & \\
\hline GDsnp00212 & 11 & \multicolumn{2}{|c|}{21829590} & GG & GG & GG & $\mathrm{AG}$ & AA & GG & $\mathrm{AG}$ \\
\hline $\mathrm{AG}$ & $\mathrm{AA}$ & $\mathrm{AG}$ & $\mathrm{AG}$ & $\mathrm{AG}$ & $\mathrm{AG}$ & GG & GG & GG & $\mathrm{AG}$ & GG \\
\hline AG & $\mathrm{AG}$ & $\mathrm{AG}$ & GG & $\mathrm{AG}$ & GG & AA & GG & GG & $\mathrm{AG}$ & $\mathrm{AG}$ \\
\hline $\mathrm{AG}$ & $\mathrm{AG}$ & $\mathrm{AA}$ & $\mathrm{AA}$ & $\mathrm{AG}$ & $\mathrm{AG}$ & GG & $\mathrm{AA}$ & $\mathrm{AG}$ & $\mathrm{AG}$ & GG \\
\hline $\mathrm{AG}$ & $\mathrm{AG}$ & $\mathrm{AG}$ & $\mathrm{AG}$ & $\mathrm{AG}$ & GG & $\mathrm{AG}$ & $\mathrm{AG}$ & $\mathrm{AG}$ & GG & GG \\
\hline GG & $\mathrm{AG}$ & $\mathrm{AG}$ & $\mathrm{AG}$ & $\mathrm{AG}$ & $\mathrm{AG}$ & GG & GG & $\mathrm{AG}$ & GG & $\mathrm{AG}$ \\
\hline GG & $\mathrm{AG}$ & $\mathrm{AG}$ & AA & $\mathrm{AG}$ & GG & $\mathrm{AG}$ & GG & GG & $\mathrm{AG}$ & GG \\
\hline $\mathrm{AA}$ & $\mathrm{AA}$ & $\mathrm{AG}$ & GG & GG & $\mathrm{AG}$ & $\mathrm{AG}$ & $\mathrm{AG}$ & $\mathrm{AG}$ & $\mathrm{AG}$ & GG \\
\hline AG & $\mathrm{AG}$ & $\mathrm{AG}$ & GG & $\mathrm{AA}$ & GG & $\mathrm{AG}$ & $\mathrm{AA}$ & $\mathrm{AG}$ & GG & \\
\hline GDsnp00123 & 11 & \multicolumn{2}{|c|}{21856103} & GG & GG & GG & $\mathrm{AG}$ & $\mathrm{AA}$ & $\mathrm{AG}$ & $\mathrm{AA}$ \\
\hline GG & $\mathrm{AA}$ & $\mathrm{AG}$ & $\mathrm{AA}$ & $\mathrm{AG}$ & $\mathrm{AA}$ & $\mathrm{AG}$ & $\mathrm{AG}$ & $\mathrm{AG}$ & $\mathrm{AA}$ & $\mathrm{AG}$ \\
\hline AA & $\mathrm{AA}$ & AA & GG & $\mathrm{AA}$ & GG & AA & $\mathrm{AG}$ & $\mathrm{AG}$ & AA & $\mathrm{AG}$ \\
\hline $\mathrm{AG}$ & $\mathrm{AA}$ & AA & AA & $\mathrm{AA}$ & $\mathrm{AG}$ & $\mathrm{AG}$ & $\mathrm{AA}$ & $\mathrm{AG}$ & $\mathrm{AG}$ & $\mathrm{AG}$ \\
\hline AG & $\mathrm{AA}$ & $\mathrm{AG}$ & $\mathrm{AA}$ & - & GG & AA & - & $\mathrm{AG}$ & $\mathrm{AG}$ & $\mathrm{AG}$ \\
\hline $\mathrm{AG}$ & $\mathrm{AG}$ & $\mathrm{AA}$ & $\mathrm{AA}$ & $\mathrm{AA}$ & $\mathrm{AG}$ & $\mathrm{AG}$ & $\mathrm{AG}$ & $\mathrm{AG}$ & $\mathrm{AG}$ & $\mathrm{AA}$ \\
\hline GG & $\mathrm{AG}$ & $\mathrm{AG}$ & $\mathrm{AA}$ & $\mathrm{AA}$ & $\mathrm{AG}$ & $\mathrm{AG}$ & $\mathrm{AG}$ & $\mathrm{AG}$ & $\mathrm{AA}$ & $\mathrm{AG}$ \\
\hline AA & $\mathrm{AA}$ & $\mathrm{AA}$ & $\mathrm{AG}$ & - & $\mathrm{AA}$ & $\mathrm{AA}$ & $\mathrm{AG}$ & $\mathrm{AG}$ & $\mathrm{AG}$ & GG \\
\hline $\mathrm{AG}$ & $\mathrm{AG}$ & $\mathrm{AA}$ & $\mathrm{AG}$ & $\mathrm{AA}$ & $\mathrm{AG}$ & $\mathrm{AG}$ & $\mathrm{AA}$ & $\mathrm{AG}$ & $\mathrm{AG}$ & \\
\hline
\end{tabular}


Supplemental Table 2. Continued.

\begin{tabular}{|c|c|c|c|c|c|c|c|c|c|c|}
\hline \multirow{3}{*}{$\begin{array}{l}\text { SNP name } \\
\text { GDsnp01486 } \\
\text { AG }\end{array}$} & \multicolumn{10}{|c|}{ Linkage group Physical location (bp) Individuals } \\
\hline & 11 & \multicolumn{2}{|c|}{21955480} & $\mathrm{AG}$ & GG & GG & AA & $\mathrm{AA}$ & $\mathrm{AG}$ & $\mathrm{AA}$ \\
\hline & AA & $\mathrm{AG}$ & $\mathrm{AG}$ & $\mathrm{AG}$ & AA & $\mathrm{AG}$ & $\mathrm{AG}$ & $\mathrm{AG}$ & AA & $\mathrm{AG}$ \\
\hline $\mathrm{AA}$ & $\mathrm{AA}$ & $\mathrm{AG}$ & GG & $\mathrm{AA}$ & GG & AA & $\mathrm{AG}$ & $\mathrm{AG}$ & AA & $\mathrm{AG}$ \\
\hline $\mathrm{AG}$ & $\mathrm{AA}$ & AA & $\mathrm{AA}$ & $\mathrm{AA}$ & $\mathrm{AG}$ & AG & AA & $\mathrm{AG}$ & $\mathrm{AG}$ & $\mathrm{AG}$ \\
\hline $\mathrm{AG}$ & $\mathrm{AA}$ & $\mathrm{AG}$ & $\mathrm{AA}$ & $\mathrm{AA}$ & GG & $\mathrm{AA}$ & GG & $\mathrm{AG}$ & $\mathrm{AG}$ & $\mathrm{AG}$ \\
\hline $\mathrm{AG}$ & $\mathrm{AG}$ & AA & $\mathrm{AA}$ & AA & $\mathrm{AG}$ & $\mathrm{AG}$ & $\mathrm{AG}$ & $\mathrm{AG}$ & $\mathrm{AG}$ & AA \\
\hline GG & $\mathrm{AG}$ & $\mathrm{AG}$ & $\mathrm{AA}$ & $\mathrm{AA}$ & $\mathrm{AG}$ & $\mathrm{AG}$ & $\mathrm{AG}$ & $\mathrm{AG}$ & $\mathrm{AA}$ & $\mathrm{AG}$ \\
\hline AA & $\mathrm{AA}$ & $\mathrm{AA}$ & $\mathrm{AG}$ & GG & $\mathrm{AA}$ & $\mathrm{AA}$ & $\mathrm{AG}$ & $\mathrm{AG}$ & $\mathrm{AG}$ & GG \\
\hline $\mathrm{AG}$ & $\mathrm{AG}$ & $\mathrm{AA}$ & $\mathrm{AG}$ & $\mathrm{AA}$ & $\mathrm{AG}$ & $\mathrm{AG}$ & AA & $\mathrm{AG}$ & $\mathrm{AG}$ & \\
\hline GDsnp01857 & 11 & \multicolumn{2}{|c|}{22232357} & $\mathrm{AG}$ & $\mathrm{AA}$ & $\mathrm{AA}$ & GG & GG & $\mathrm{AG}$ & GG \\
\hline $\mathrm{AG}$ & GG & $\mathrm{AG}$ & $\mathrm{AG}$ & $\mathrm{AG}$ & GG & $\mathrm{AG}$ & $\mathrm{AG}$ & $\mathrm{AG}$ & GG & $\mathrm{AG}$ \\
\hline GG & GG & $\mathrm{AG}$ & $\mathrm{AA}$ & GG & $\mathrm{AA}$ & GG & $\mathrm{AG}$ & $\mathrm{AG}$ & GG & $\mathrm{AG}$ \\
\hline $\mathrm{AG}$ & GG & GG & GG & GG & $\mathrm{AG}$ & $\mathrm{AG}$ & GG & $\mathrm{AG}$ & $\mathrm{AG}$ & $\mathrm{AG}$ \\
\hline $\mathrm{AG}$ & GG & $\mathrm{AG}$ & GG & GG & $\mathrm{AA}$ & GG & $\mathrm{AA}$ & $\mathrm{AG}$ & $\mathrm{AG}$ & $\mathrm{AG}$ \\
\hline $\mathrm{AG}$ & $\mathrm{AG}$ & GG & GG & GG & $\mathrm{AG}$ & $\mathrm{AG}$ & $\mathrm{AG}$ & $\mathrm{AG}$ & $\mathrm{AG}$ & GG \\
\hline AA & $\mathrm{AG}$ & $\mathrm{AG}$ & GG & GG & $\mathrm{AG}$ & $\mathrm{AG}$ & $\mathrm{AG}$ & $\mathrm{AG}$ & GG & $\mathrm{AG}$ \\
\hline GG & GG & GG & $\mathrm{AG}$ & $\mathrm{AA}$ & GG & GG & $\mathrm{AG}$ & $\mathrm{AG}$ & $\mathrm{AG}$ & $\mathrm{AA}$ \\
\hline $\mathrm{AG}$ & $\mathrm{AG}$ & GG & $\mathrm{AG}$ & GG & $\mathrm{AG}$ & AA & GG & $\mathrm{AG}$ & $\mathrm{AG}$ & \\
\hline GDsnp00004 & 11 & \multicolumn{2}{|c|}{28781443} & $\mathrm{AG}$ & GG & GG & $\mathrm{AA}$ & $\mathrm{AA}$ & GG & - \\
\hline GG & - & GG & GG & GG & - & GG & GG & GG & - & GG \\
\hline- & - & GG & GG & - & $\mathrm{AG}$ & - & GG & GG & $\mathrm{AA}$ & GG \\
\hline GG & - & $\mathrm{AA}$ & $\mathrm{AA}$ & - & $\mathrm{AG}$ & GG & AA & $\mathrm{AG}$ & GG & GG \\
\hline GG & - & GG & - & - & $\mathrm{AG}$ & - & GG & $\mathrm{AG}$ & GG & GG \\
\hline GG & GG & AA & GG & - & GG & GG & GG & $\mathrm{AG}$ & GG & - \\
\hline GG & GG & GG & $\mathrm{AA}$ & - & GG & GG & GG & - & - & GG \\
\hline- & $\mathrm{AA}$ & - & GG & GG & GG & GG & $\mathrm{AG}$ & GG & $\mathrm{AG}$ & GG \\
\hline- & GG & - & GG & - & GG & GG & - & GG & GG & \\
\hline GDsnp00795 & 11 & \multicolumn{2}{|c|}{31482449} & $\mathrm{AG}$ & GG & GG & $\mathrm{AA}$ & $\mathrm{AA}$ & $\mathrm{AG}$ & AA \\
\hline GG & $\mathrm{AG}$ & $\mathrm{AG}$ & $\mathrm{AG}$ & $\mathrm{AG}$ & $\mathrm{AA}$ & $\mathrm{AG}$ & $\mathrm{AG}$ & $\mathrm{AG}$ & $\mathrm{AA}$ & $\mathrm{AA}$ \\
\hline $\mathrm{AG}$ & $\mathrm{AA}$ & $\mathrm{AG}$ & GG & $\mathrm{AA}$ & $\mathrm{AG}$ & $\mathrm{AG}$ & $\mathrm{AG}$ & $\mathrm{AG}$ & $\mathrm{AA}$ & $\mathrm{AG}$ \\
\hline $\mathrm{AG}$ & $\mathrm{AA}$ & AA & $\mathrm{AA}$ & $\mathrm{AA}$ & $\mathrm{AA}$ & $\mathrm{AG}$ & AA & $\mathrm{AA}$ & $\mathrm{AG}$ & $\mathrm{AG}$ \\
\hline $\mathrm{AG}$ & $\mathrm{AA}$ & $\mathrm{AG}$ & $\mathrm{AA}$ & $\mathrm{AA}$ & $\mathrm{AA}$ & AA & $\mathrm{AG}$ & $\mathrm{AG}$ & $\mathrm{AG}$ & $\mathrm{AG}$ \\
\hline $\mathrm{AG}$ & $\mathrm{AG}$ & AA & $\mathrm{AA}$ & $\mathrm{AG}$ & $\mathrm{AG}$ & $\mathrm{AG}$ & $\mathrm{AG}$ & $\mathrm{AG}$ & $\mathrm{AG}$ & $\mathrm{AA}$ \\
\hline GG & $\mathrm{AG}$ & $\mathrm{AG}$ & $\mathrm{AA}$ & $\mathrm{AA}$ & $\mathrm{AG}$ & $\mathrm{AG}$ & $\mathrm{AG}$ & $\mathrm{AA}$ & $\mathrm{AA}$ & $\mathrm{AG}$ \\
\hline AA & $\mathrm{AG}$ & AA & $\mathrm{AG}$ & GG & $\mathrm{AG}$ & $\mathrm{AG}$ & $\mathrm{AG}$ & $\mathrm{AG}$ & AA & $\mathrm{AG}$ \\
\hline AA & $\mathrm{AG}$ & AA & $\mathrm{AG}$ & $\mathrm{AA}$ & $\mathrm{AG}$ & GG & $\mathrm{AA}$ & $\mathrm{AG}$ & $\mathrm{AG}$ & \\
\hline GDsnp01465 & 11 & \multicolumn{2}{|c|}{33973340} & GG & - & - & $\mathrm{AG}$ & $\mathrm{AG}$ & AA & $\mathrm{AG}$ \\
\hline- & $\mathrm{AA}$ & AA & $\mathrm{AG}$ & GG & $\mathrm{AG}$ & GG & GG & GG & $\mathrm{AG}$ & $\mathrm{AG}$ \\
\hline AA & $\mathrm{AA}$ & GG & - & $\mathrm{AG}$ & GG & - & GG & GG & $\mathrm{AG}$ & $\mathrm{AA}$ \\
\hline AA & $\mathrm{AG}$ & $\mathrm{AG}$ & $\mathrm{AG}$ & $\mathrm{AG}$ & $\mathrm{AG}$ & GG & $\mathrm{AG}$ & $\mathrm{AG}$ & - & $\mathrm{AA}$ \\
\hline GG & $\mathrm{AA}$ & GG & $\mathrm{AG}$ & $\mathrm{AG}$ & $\mathrm{AG}$ & $\mathrm{AG}$ & - & GG & $\mathrm{AA}$ & GG \\
\hline- & GG & $\mathrm{AG}$ & $\mathrm{AG}$ & $\mathrm{AA}$ & $\mathrm{AA}$ & GG & AA & GG & AA & $\mathrm{AG}$ \\
\hline- & GG & AA & $\mathrm{AG}$ & $\mathrm{AG}$ & GG & GG & AA & $\mathrm{AG}$ & $\mathrm{AG}$ & GG \\
\hline $\mathrm{AG}$ & GG & $\mathrm{AG}$ & GG & - & GG & GG & GG & $\mathrm{AA}$ & $\mathrm{AG}$ & $\mathrm{AA}$ \\
\hline AA & AA & $\mathrm{AG}$ & - & $\mathrm{AG}$ & AA & GG & $\mathrm{AA}$ & AA & GG & \\
\hline GDsnp00646 & 11 & \multicolumn{2}{|c|}{34425216} & $\mathrm{AC}$ & $\mathrm{AA}$ & $\mathrm{AA}$ & $\mathrm{AC}$ & $\mathrm{AC}$ & $\mathrm{AA}$ & $\mathrm{AC}$ \\
\hline AA & $\mathrm{AA}$ & $\mathrm{AA}$ & $\mathrm{AA}$ & $\mathrm{AA}$ & $\mathrm{AA}$ & AA & $\mathrm{AA}$ & $\mathrm{AA}$ & $\mathrm{AC}$ & $\mathrm{AA}$ \\
\hline AA & $\mathrm{AA}$ & AA & $\mathrm{AA}$ & $\mathrm{AA}$ & $\mathrm{AC}$ & $\mathrm{AC}$ & AA & $\mathrm{AA}$ & $\mathrm{AC}$ & AA \\
\hline AA & $\mathrm{AA}$ & $\mathrm{AC}$ & $\mathrm{AC}$ & $\mathrm{AA}$ & $\mathrm{AC}$ & AA & AA & $\mathrm{AC}$ & AA & AA \\
\hline AA & $\mathrm{AA}$ & $\mathrm{AA}$ & $\mathrm{AA}$ & $\mathrm{AA}$ & $\mathrm{AC}$ & AA & $\mathrm{AA}$ & $\mathrm{AA}$ & AA & $\mathrm{AA}$ \\
\hline AA & $\mathrm{AA}$ & $\mathrm{AC}$ & $\mathrm{AA}$ & $\mathrm{AA}$ & $\mathrm{AA}$ & AA & $\mathrm{AA}$ & $\mathrm{AA}$ & AA & $\mathrm{AA}$ \\
\hline AA & $\mathrm{AC}$ & $\mathrm{AA}$ & $\mathrm{AC}$ & $\mathrm{AA}$ & $\mathrm{AC}$ & $\mathrm{AA}$ & $\mathrm{AA}$ & $\mathrm{AA}$ & $\mathrm{AA}$ & $\mathrm{AA}$ \\
\hline AA & $\mathrm{AC}$ & $\mathrm{AC}$ & $\mathrm{AA}$ & $\mathrm{AA}$ & $\mathrm{AA}$ & $\mathrm{AA}$ & $\mathrm{AA}$ & $\mathrm{AA}$ & $\mathrm{AC}$ & $\mathrm{AA}$ \\
\hline AA & $\mathrm{AA}$ & $\mathrm{AA}$ & $\mathrm{AA}$ & $\mathrm{AA}$ & $\mathrm{AA}$ & AA & $\mathrm{AA}$ & $\mathrm{AA}$ & AA & \\
\hline
\end{tabular}

Continued next page 
Supplemental Table 2. Continued.

\begin{tabular}{|c|c|c|c|c|c|c|c|c|c|c|}
\hline \multirow{3}{*}{$\begin{array}{l}\text { SNP name } \\
\text { GDsnp00029 } \\
\text { CC }\end{array}$} & \multicolumn{10}{|c|}{ Linkage group Physical location (bp) Individuals } \\
\hline & 11 & \multicolumn{2}{|c|}{34714193} & $\mathrm{CG}$ & $\mathrm{CC}$ & $\mathrm{CC}$ & $\mathrm{CG}$ & $\mathrm{CG}$ & $\mathrm{CC}$ & $\mathrm{CG}$ \\
\hline & $\mathrm{CC}$ & $\mathrm{CC}$ & $\mathrm{CC}$ & $\mathrm{CC}$ & $\mathrm{CC}$ & $\mathrm{CC}$ & $\mathrm{CC}$ & $\mathrm{CC}$ & $\mathrm{CG}$ & $\mathrm{CC}$ \\
\hline $\mathrm{CC}$ & $\mathrm{CC}$ & $\mathrm{CC}$ & $\mathrm{CC}$ & $\mathrm{CC}$ & $\mathrm{CG}$ & $\mathrm{CG}$ & $\mathrm{CC}$ & $\mathrm{CC}$ & $\mathrm{CG}$ & $\mathrm{CC}$ \\
\hline $\mathrm{CC}$ & $\mathrm{CC}$ & CG & $\mathrm{CG}$ & $\mathrm{CC}$ & CG & $\mathrm{CC}$ & $\mathrm{CC}$ & $\mathrm{CG}$ & $\mathrm{CC}$ & $\mathrm{CC}$ \\
\hline $\mathrm{CC}$ & $\mathrm{CC}$ & $\mathrm{CC}$ & $\mathrm{CC}$ & $\mathrm{CC}$ & $\mathrm{CG}$ & $\mathrm{CC}$ & $\mathrm{CC}$ & $\mathrm{CC}$ & $\mathrm{CG}$ & $\mathrm{CC}$ \\
\hline $\mathrm{CC}$ & $\mathrm{CC}$ & $\mathrm{CG}$ & $\mathrm{CC}$ & $\mathrm{CC}$ & $\mathrm{CC}$ & $\mathrm{CC}$ & $\mathrm{CC}$ & $\mathrm{CC}$ & $\mathrm{CC}$ & $\mathrm{CC}$ \\
\hline $\mathrm{CC}$ & $\mathrm{CG}$ & $\mathrm{CC}$ & $\mathrm{CG}$ & $\mathrm{CC}$ & CG & $\mathrm{CC}$ & $\mathrm{CC}$ & $\mathrm{CC}$ & $\mathrm{CC}$ & $\mathrm{CC}$ \\
\hline $\mathrm{CC}$ & $\mathrm{CG}$ & $\mathrm{CG}$ & $\mathrm{CC}$ & $\mathrm{CC}$ & $\mathrm{CC}$ & $\mathrm{CC}$ & $\mathrm{CC}$ & $\mathrm{CC}$ & $\mathrm{CG}$ & $\mathrm{CC}$ \\
\hline $\mathrm{CC}$ & $\mathrm{CC}$ & $\mathrm{CC}$ & $\mathrm{CC}$ & $\mathrm{CC}$ & $\mathrm{CC}$ & $\mathrm{CC}$ & $\mathrm{CC}$ & $\mathrm{CC}$ & $\mathrm{CC}$ & \\
\hline GDsnp00173 & 12 & \multicolumn{2}{|c|}{304525} & GG & GG & GG & GG & GG & GG & $\mathrm{AG}$ \\
\hline GG & GG & $\mathrm{AG}$ & GG & $\mathrm{AG}$ & GG & GG & GG & $\mathrm{AG}$ & GG & GG \\
\hline GG & GG & $\mathrm{AG}$ & GG & GG & AG & GG & GG & GG & $\mathrm{AG}$ & $\mathrm{AG}$ \\
\hline $\mathrm{AG}$ & GG & GG & $\mathrm{AG}$ & GG & GG & GG & GG & $\mathrm{AG}$ & $\mathrm{AG}$ & $\mathrm{AG}$ \\
\hline $\mathrm{AG}$ & $\mathrm{AG}$ & GG & GG & $\mathrm{AG}$ & $\mathrm{AG}$ & $\mathrm{AA}$ & GG & $\mathrm{AG}$ & GG & $\mathrm{AG}$ \\
\hline GG & GG & GG & GG & GG & GG & GG & GG & GG & GG & GG \\
\hline GG & $\mathrm{AG}$ & GG & GG & $\mathrm{AG}$ & $\mathrm{AG}$ & GG & $\mathrm{AG}$ & GG & $\mathrm{AG}$ & GG \\
\hline GG & GG & $\mathrm{AG}$ & GG & GG & GG & $\mathrm{AG}$ & $\mathrm{AG}$ & GG & GG & GG \\
\hline $\mathrm{AG}$ & GG & GG & GG & GG & GG & $\mathrm{AG}$ & GG & GG & GG & \\
\hline GDsnp01070 & 12 & \multicolumn{2}{|c|}{688053} & $\mathrm{TT}$ & $\mathrm{TT}$ & $\mathrm{TT}$ & $\mathrm{TT}$ & $\mathrm{TT}$ & $\mathrm{TT}$ & TT \\
\hline $\mathrm{TT}$ & $\mathrm{TT}$ & TT & $\mathrm{TT}$ & $\mathrm{TT}$ & $\mathrm{TT}$ & $\mathrm{TT}$ & $\mathrm{TT}$ & $\mathrm{TT}$ & $\mathrm{TT}$ & TT \\
\hline TT & $\mathrm{TT}$ & TT & $\mathrm{TT}$ & $\mathrm{TT}$ & $\mathrm{TT}$ & $\mathrm{TT}$ & $\mathrm{TT}$ & $\mathrm{TT}$ & $\mathrm{TT}$ & TT \\
\hline $\mathrm{TT}$ & $\mathrm{TT}$ & $\mathrm{TT}$ & $\mathrm{TT}$ & $\mathrm{TT}$ & $\mathrm{TT}$ & $\mathrm{TT}$ & $\mathrm{TT}$ & $\mathrm{TT}$ & - & TT \\
\hline $\mathrm{TT}$ & $\mathrm{TT}$ & $\mathrm{TT}$ & $\mathrm{TT}$ & $\mathrm{TT}$ & $\mathrm{TT}$ & - & $\mathrm{TT}$ & $\mathrm{TT}$ & $\mathrm{TT}$ & TT \\
\hline $\mathrm{TT}$ & $\mathrm{TT}$ & TT & $\mathrm{TT}$ & $\mathrm{TT}$ & $\mathrm{TT}$ & $\mathrm{TT}$ & $\mathrm{TT}$ & $\mathrm{TT}$ & $\mathrm{TT}$ & TT \\
\hline $\mathrm{TT}$ & $\mathrm{TT}$ & TT & $\mathrm{TT}$ & $\mathrm{TT}$ & $\mathrm{TT}$ & $\mathrm{TT}$ & $\mathrm{TT}$ & $\mathrm{TT}$ & $\mathrm{TT}$ & TT \\
\hline $\mathrm{TT}$ & $\mathrm{TT}$ & $\mathrm{TT}$ & $\mathrm{TT}$ & $\mathrm{TT}$ & $\mathrm{TT}$ & $\mathrm{TT}$ & $\mathrm{TT}$ & $\mathrm{TT}$ & $\mathrm{TT}$ & TT \\
\hline TT & $\mathrm{TT}$ & $\mathrm{TT}$ & $\mathrm{TT}$ & $\mathrm{TT}$ & $\mathrm{TT}$ & TT & TT & $\mathrm{TT}$ & $\mathrm{TT}$ & \\
\hline GDsnp00116 & 12 & \multicolumn{2}{|c|}{1640777} & GG & GG & GG & GG & $\mathrm{AA}$ & GG & AA \\
\hline $\mathrm{AA}$ & GG & $\mathrm{AG}$ & $\mathrm{AG}$ & $\mathrm{AG}$ & $\mathrm{AG}$ & $\mathrm{AG}$ & GG & $\mathrm{AG}$ & $\mathrm{AG}$ & $\mathrm{AG}$ \\
\hline $\mathrm{AG}$ & $\mathrm{AG}$ & $\mathrm{AG}$ & $\mathrm{AG}$ & $\mathrm{AG}$ & $\mathrm{AG}$ & $\mathrm{AG}$ & $\mathrm{AA}$ & $\mathrm{AA}$ & $\mathrm{AA}$ & $\mathrm{AA}$ \\
\hline $\mathrm{AG}$ & $\mathrm{AG}$ & AA & $\mathrm{AA}$ & $\mathrm{AG}$ & AA & GG & $\mathrm{AA}$ & $\mathrm{AG}$ & AA & GG \\
\hline $\mathrm{AG}$ & $\mathrm{AA}$ & $\mathrm{AG}$ & $\mathrm{AA}$ & $\mathrm{AA}$ & AG & $\mathrm{AG}$ & $\mathrm{AG}$ & $\mathrm{AG}$ & $\mathrm{AG}$ & $\mathrm{AA}$ \\
\hline $\mathrm{AG}$ & GG & $\mathrm{AA}$ & GG & $\mathrm{AG}$ & $\mathrm{AG}$ & $\mathrm{AG}$ & $\mathrm{AG}$ & $\mathrm{AG}$ & GG & $\mathrm{AG}$ \\
\hline $\mathrm{AG}$ & $\mathrm{AA}$ & GG & $\mathrm{AG}$ & $\mathrm{AG}$ & $\mathrm{AG}$ & $\mathrm{AG}$ & GG & GG & $\mathrm{AA}$ & $\mathrm{AG}$ \\
\hline GG & $\mathrm{AG}$ & $\mathrm{AG}$ & $\mathrm{AG}$ & GG & GG & $\mathrm{AA}$ & $\mathrm{AG}$ & $\mathrm{AG}$ & AA & AA \\
\hline $\mathrm{AG}$ & GG & $\mathrm{AG}$ & GG & GG & $\mathrm{AG}$ & $\mathrm{AG}$ & $\mathrm{AG}$ & GG & $\mathrm{AG}$ & \\
\hline GDsnp01690 & 12 & \multicolumn{2}{|c|}{3897839} & $\mathrm{AG}$ & GG & GG & GG & $\mathrm{AG}$ & GG & $\mathrm{AG}$ \\
\hline GG & $\mathrm{AG}$ & $\mathrm{AG}$ & GG & $\mathrm{AG}$ & GG & GG & GG & GG & GG & GG \\
\hline GG & GG & GG & GG & GG & $\mathrm{AG}$ & $\mathrm{AG}$ & GG & GG & $\mathrm{AG}$ & $\mathrm{AG}$ \\
\hline $\mathrm{AG}$ & GG & GG & $\mathrm{AG}$ & GG & GG & GG & GG & $\mathrm{AG}$ & $\mathrm{AG}$ & GG \\
\hline $\mathrm{AG}$ & $\mathrm{AG}$ & GG & GG & $\mathrm{AG}$ & $\mathrm{AG}$ & $\mathrm{AG}$ & GG & $\mathrm{AG}$ & GG & $\mathrm{AG}$ \\
\hline GG & GG & GG & GG & GG & GG & GG & GG & GG & GG & GG \\
\hline GG & $\mathrm{AG}$ & GG & GG & $\mathrm{AG}$ & AG & GG & $\mathrm{AG}$ & GG & $\mathrm{AG}$ & GG \\
\hline GG & GG & $\mathrm{AG}$ & GG & GG & GG & $\mathrm{AG}$ & $\mathrm{AG}$ & GG & GG & GG \\
\hline AG & GG & GG & - & GG & GG & $\mathrm{AG}$ & GG & GG & GG & \\
\hline GDsnp02537 & 12 & \multicolumn{2}{|c|}{5280715} & GG & GG & GG & GG & GG & GG & GG \\
\hline GG & GG & GG & GG & GG & GG & GG & GG & GG & GG & GG \\
\hline GG & GG & $\mathrm{CG}$ & GG & GG & GG & GG & GG & GG & GG & GG \\
\hline GG & GG & GG & GG & GG & GG & GG & GG & GG & GG & $\mathrm{CG}$ \\
\hline GG & GG & GG & GG & GG & GG & - & GG & GG & GG & GG \\
\hline GG & GG & GG & GG & GG & GG & GG & GG & GG & GG & GG \\
\hline GG & GG & GG & GG & GG & GG & GG & GG & GG & GG & GG \\
\hline GG & GG & GG & GG & GG & GG & GG & GG & GG & GG & GG \\
\hline GG & GG & GG & GG & GG & GG & GG & GG & GG & GG & \\
\hline
\end{tabular}


Supplemental Table 2. Continued.

\begin{tabular}{|c|c|c|c|c|c|c|c|c|c|c|}
\hline \multirow{3}{*}{$\begin{array}{l}\text { SNP name } \\
\text { GDsnp00272 } \\
\text { AT }\end{array}$} & \multicolumn{10}{|c|}{ Linkage group Physical location (bp) Individuals } \\
\hline & 12 & \multicolumn{2}{|c|}{6266912} & TT & TT & TT & TT & AT & TT & AT \\
\hline & TT & TT & AT & TT & AT & AT & TT & AT & AT & TT \\
\hline TT & $\mathrm{AT}$ & AT & AT & $\mathrm{AT}$ & AT & AT & AT & AT & AT & AT \\
\hline $\mathrm{TT}$ & TT & AA & AT & $\mathrm{TT}$ & AT & TT & AA & TT & $\mathrm{AT}$ & TT \\
\hline TT & $\mathrm{TT}$ & AT & AT & $\mathrm{AT}$ & AT & $\mathrm{TT}$ & AT & TT & $\mathrm{AT}$ & AT \\
\hline AT & $\mathrm{TT}$ & $\mathrm{AT}$ & $\mathrm{TT}$ & AT & AT & $\mathrm{TT}$ & AT & $\mathrm{TT}$ & $\mathrm{TT}$ & AT \\
\hline AT & $\mathrm{AT}$ & $\mathrm{AT}$ & $\mathrm{TT}$ & $\mathrm{TT}$ & $\mathrm{TT}$ & $\mathrm{AT}$ & TT & $\mathrm{TT}$ & $\mathrm{AT}$ & $\mathrm{AT}$ \\
\hline $\mathrm{TT}$ & $\mathrm{AT}$ & $\mathrm{AT}$ & $\mathrm{AT}$ & AT & $\mathrm{TT}$ & AT & $\mathrm{TT}$ & $\mathrm{AT}$ & AA & AA \\
\hline $\mathrm{TT}$ & $\mathrm{TT}$ & TT & $\mathrm{TT}$ & $\mathrm{TT}$ & AT & $\mathrm{TT}$ & AT & $\mathrm{TT}$ & $\mathrm{AT}$ & \\
\hline GDsnp02857 & 12 & \multicolumn{2}{|c|}{7284234} & GG & GG & GG & GG & $\mathrm{AG}$ & GG & $\mathrm{AG}$ \\
\hline- & GG & GG & $\mathrm{AG}$ & GG & $\mathrm{AG}$ & $\mathrm{AG}$ & GG & $\mathrm{AG}$ & $\mathrm{AG}$ & GG \\
\hline GG & $\mathrm{AG}$ & $\mathrm{AG}$ & $\mathrm{AG}$ & GG & AG & $\mathrm{AG}$ & $\mathrm{AG}$ & $\mathrm{AG}$ & $\mathrm{AG}$ & $\mathrm{AG}$ \\
\hline GG & GG & $\mathrm{AA}$ & $\mathrm{AG}$ & GG & GG & GG & AA & GG & $\mathrm{AA}$ & GG \\
\hline GG & GG & $\mathrm{AG}$ & $\mathrm{AG}$ & $\mathrm{AG}$ & $\mathrm{AG}$ & GG & $\mathrm{AG}$ & GG & $\mathrm{AG}$ & $\mathrm{AG}$ \\
\hline $\mathrm{AG}$ & GG & $\mathrm{AG}$ & GG & $\mathrm{AG}$ & $A G$ & GG & $\mathrm{AG}$ & GG & GG & $\mathrm{AG}$ \\
\hline $\mathrm{AG}$ & $\mathrm{AG}$ & $\mathrm{AG}$ & GG & GG & GG & $\mathrm{AG}$ & - & GG & $\mathrm{AG}$ & $\mathrm{AG}$ \\
\hline GG & $\mathrm{AG}$ & $\mathrm{AG}$ & $\mathrm{AG}$ & - & GG & $\mathrm{AG}$ & GG & $\mathrm{AG}$ & $\mathrm{AA}$ & $\mathrm{AA}$ \\
\hline GG & GG & GG & GG & GG & AG & GG & $\mathrm{AG}$ & GG & $\mathrm{AG}$ & \\
\hline GDsnp02502 & 12 & \multicolumn{2}{|c|}{10923118} & GG & GG & GG & GG & GG & GG & GG \\
\hline GG & GG & GG & GG & GG & GG & GG & GG & GG & GG & GG \\
\hline GG & GG & $\mathrm{AG}$ & GG & GG & GG & GG & GG & GG & GG & GG \\
\hline GG & GG & GG & GG & GG & GG & GG & GG & GG & GG & $\mathrm{AG}$ \\
\hline GG & GG & GG & GG & GG & GG & GG & GG & GG & GG & GG \\
\hline GG & GG & GG & GG & GG & GG & GG & GG & GG & GG & GG \\
\hline GG & GG & GG & GG & GG & GG & GG & GG & GG & GG & GG \\
\hline GG & GG & GG & GG & GG & GG & GG & GG & GG & GG & GG \\
\hline GG & GG & GG & GG & GG & GG & GG & GG & GG & GG & \\
\hline GDsnp00318 & 12 & \multicolumn{2}{|c|}{11958909} & $\mathrm{CC}$ & $\mathrm{CC}$ & $\mathrm{CC}$ & $\mathrm{CC}$ & $\mathrm{AC}$ & $\mathrm{CC}$ & $\mathrm{AC}$ \\
\hline $\mathrm{AC}$ & $\mathrm{AC}$ & $\mathrm{CC}$ & $\mathrm{AC}$ & $\mathrm{CC}$ & $\mathrm{AC}$ & $\mathrm{AC}$ & $\mathrm{CC}$ & $\mathrm{AC}$ & $\mathrm{AC}$ & $\mathrm{CC}$ \\
\hline $\mathrm{CC}$ & $\mathrm{AC}$ & $\mathrm{AC}$ & $\mathrm{AC}$ & $\mathrm{CC}$ & $\mathrm{AC}$ & $\mathrm{AC}$ & $\mathrm{AC}$ & $\mathrm{AC}$ & $\mathrm{AC}$ & $\mathrm{AC}$ \\
\hline $\mathrm{CC}$ & $\mathrm{CC}$ & AA & $\mathrm{AC}$ & $\mathrm{CC}$ & $\mathrm{CC}$ & $\mathrm{CC}$ & AA & $\mathrm{CC}$ & $\mathrm{AC}$ & $\mathrm{CC}$ \\
\hline $\mathrm{CC}$ & $\mathrm{CC}$ & $\mathrm{AC}$ & $\mathrm{AC}$ & $\mathrm{AC}$ & $\mathrm{AC}$ & $\mathrm{AC}$ & $\mathrm{AC}$ & $\mathrm{CC}$ & $\mathrm{AC}$ & $\mathrm{AC}$ \\
\hline $\mathrm{AC}$ & $\mathrm{CC}$ & $\mathrm{AC}$ & $\mathrm{CC}$ & $\mathrm{AC}$ & $\mathrm{AC}$ & $\mathrm{CC}$ & $\mathrm{AC}$ & $\mathrm{CC}$ & $\mathrm{AC}$ & $\mathrm{AC}$ \\
\hline $\mathrm{AC}$ & $\mathrm{AC}$ & $\mathrm{AC}$ & $\mathrm{CC}$ & $\mathrm{CC}$ & $\mathrm{CC}$ & $\mathrm{AC}$ & $\mathrm{AC}$ & $\mathrm{CC}$ & $\mathrm{AC}$ & $\mathrm{AC}$ \\
\hline $\mathrm{CC}$ & $\mathrm{AC}$ & $\mathrm{AC}$ & $\mathrm{AC}$ & $\mathrm{AC}$ & $\mathrm{CC}$ & $\mathrm{AC}$ & $\mathrm{CC}$ & $\mathrm{AC}$ & $\mathrm{AC}$ & $\mathrm{AA}$ \\
\hline $\mathrm{CC}$ & $\mathrm{AC}$ & $\mathrm{CC}$ & $\mathrm{CC}$ & $\mathrm{CC}$ & $\mathrm{AC}$ & $\mathrm{AC}$ & $\mathrm{AC}$ & $\mathrm{CC}$ & $\mathrm{AC}$ & \\
\hline GDsnp02326 & 12 & \multicolumn{2}{|c|}{16794961} & $\mathrm{AA}$ & AA & AA & AA & AA & AA & $\mathrm{AA}$ \\
\hline AA & $\mathrm{AA}$ & AA & AA & $\mathrm{AA}$ & AA & AA & AA & AA & AA & AA \\
\hline AA & $\mathrm{AA}$ & AT & AA & $\mathrm{AA}$ & AA & AA & AA & AA & AA & $\mathrm{AA}$ \\
\hline AA & $\mathrm{AA}$ & $\mathrm{AA}$ & $\mathrm{AA}$ & $\mathrm{AA}$ & AA & AA & AA & $\mathrm{AA}$ & AA & AT \\
\hline AA & $\mathrm{AA}$ & $\mathrm{AA}$ & AA & $\mathrm{AA}$ & AA & $\mathrm{AA}$ & AT & AA & $\mathrm{AA}$ & $\mathrm{AA}$ \\
\hline $\mathrm{AA}$ & $\mathrm{AA}$ & $\mathrm{AA}$ & AA & $\mathrm{AA}$ & AA & AA & AA & AA & AA & AA \\
\hline AA & $\mathrm{AA}$ & AA & AA & $\mathrm{AA}$ & AA & AA & AA & AA & AA & $\mathrm{AA}$ \\
\hline $\mathrm{AA}$ & $\mathrm{AA}$ & $\mathrm{AA}$ & $\mathrm{AA}$ & $\mathrm{AA}$ & AA & $\mathrm{AA}$ & AA & $\mathrm{AA}$ & AA & $\mathrm{AA}$ \\
\hline $\mathrm{AA}$ & AA & AA & AA & $\mathrm{AA}$ & AA & AT & AA & AA & AA & \\
\hline GDsnp01029 & 12 & \multicolumn{2}{|c|}{17824232} & $\mathrm{AT}$ & AA & $\mathrm{AA}$ & AT & $\mathrm{AT}$ & $\mathrm{AA}$ & AT \\
\hline $\mathrm{AT}$ & $\mathrm{AA}$ & $\mathrm{AT}$ & $\mathrm{AA}$ & $\mathrm{AT}$ & AA & AA & AA & $\mathrm{AA}$ & $\mathrm{AA}$ & $\mathrm{AA}$ \\
\hline AA & $\mathrm{AA}$ & AA & AA & $\mathrm{AA}$ & AA & AA & AT & $\mathrm{AT}$ & AA & AT \\
\hline AT & $\mathrm{AT}$ & AA & $\mathrm{AT}$ & $\mathrm{AA}$ & AA & AA & AA & AA & AA & AA \\
\hline AT & $\mathrm{AT}$ & $\mathrm{AA}$ & $\mathrm{AA}$ & $\mathrm{AT}$ & AT & AT & AA & $\mathrm{AT}$ & AA & AT \\
\hline $\mathrm{AA}$ & $\mathrm{AA}$ & $\mathrm{AA}$ & $\mathrm{AA}$ & $\mathrm{AA}$ & AA & $\mathrm{AA}$ & $\mathrm{AA}$ & $\mathrm{AA}$ & $\mathrm{AA}$ & $\mathrm{AA}$ \\
\hline $\mathrm{AA}$ & $\mathrm{AA}$ & $\mathrm{AA}$ & $\mathrm{AA}$ & $\mathrm{AT}$ & AA & $\mathrm{AA}$ & AA & $\mathrm{AA}$ & $\mathrm{AT}$ & $\mathrm{AA}$ \\
\hline AA & $\mathrm{AA}$ & AT & AA & $\mathrm{AA}$ & AA & $\mathrm{AT}$ & $\mathrm{AT}$ & AA & $\mathrm{AA}$ & $\mathrm{AT}$ \\
\hline $\mathrm{AT}$ & $\mathrm{AA}$ & $\mathrm{AA}$ & $\mathrm{AA}$ & $\mathrm{AA}$ & AT & AA & AA & $\mathrm{AA}$ & AA & \\
\hline
\end{tabular}

Continued next page 
Supplemental Table 2. Continued.

\begin{tabular}{|c|c|c|c|c|c|c|c|c|c|c|}
\hline \multirow{3}{*}{$\begin{array}{l}\text { SNP name } \\
\text { GDsnp00193 } \\
\text { GG }\end{array}$} & \multicolumn{10}{|c|}{ Linkage group Physical location (bp) Individuals } \\
\hline & 12 & \multicolumn{2}{|c|}{18610313} & GG & $\mathrm{AG}$ & GG & GG & $\mathrm{AG}$ & GG & $\mathrm{AG}$ \\
\hline & AA & GG & AA & GG & AA & $\mathrm{AG}$ & $\mathrm{AG}$ & AA & $\mathrm{AG}$ & $\mathrm{AG}$ \\
\hline $\mathrm{AG}$ & $\mathrm{AG}$ & AA & $\mathrm{AG}$ & $\mathrm{AG}$ & $\mathrm{AG}$ & AA & $\mathrm{AG}$ & $\mathrm{AG}$ & AA & $\mathrm{AG}$ \\
\hline GG & GG & AA & AG & $\mathrm{AG}$ & $\mathrm{AG}$ & AA & AA & AA & $\mathrm{AG}$ & $\mathrm{AG}$ \\
\hline GG & GG & $\mathrm{AG}$ & $\mathrm{AA}$ & $\mathrm{AG}$ & $\mathrm{AG}$ & $\mathrm{AG}$ & - & GG & $\mathrm{AG}$ & $\mathrm{AG}$ \\
\hline AA & $\mathrm{AG}$ & AA & $\mathrm{AG}$ & $\mathrm{AG}$ & GG & $\mathrm{AG}$ & AA & $\mathrm{AG}$ & AA & $\mathrm{AG}$ \\
\hline $\mathrm{AG}$ & $\mathrm{AA}$ & $\mathrm{AA}$ & $\mathrm{AA}$ & GG & $\mathrm{AG}$ & $\mathrm{AG}$ & $\mathrm{AG}$ & $\mathrm{AG}$ & $\mathrm{AG}$ & $\mathrm{AG}$ \\
\hline $\mathrm{AG}$ & GG & $\mathrm{AG}$ & $\mathrm{AG}$ & $\mathrm{AA}$ & $\mathrm{AG}$ & $\mathrm{AG}$ & GG & $\mathrm{AA}$ & $\mathrm{AG}$ & $\mathrm{AG}$ \\
\hline GG & $\mathrm{AG}$ & GG & $\mathrm{AG}$ & $\mathrm{AG}$ & GG & $\mathrm{AG}$ & $\mathrm{AG}$ & $\mathrm{AG}$ & $\mathrm{AG}$ & \\
\hline GDsnp01769 & 12 & \multicolumn{2}{|c|}{24505274} & GG & - & GG & GG & $\mathrm{AG}$ & GG & $\mathrm{AG}$ \\
\hline GG & $\mathrm{AG}$ & GG & $\mathrm{AG}$ & GG & $\mathrm{AA}$ & - & GG & $\mathrm{AG}$ & AA & - \\
\hline- & GG & - & - & - & GG & $\mathrm{AG}$ & - & - & $\mathrm{AA}$ & $\mathrm{AG}$ \\
\hline GG & GG & $\mathrm{AA}$ & $\mathrm{AG}$ & - & - & AA & $\mathrm{AA}$ & $\mathrm{AA}$ & GG & GG \\
\hline GG & GG & - & - & - & $\mathrm{AG}$ & $\mathrm{AA}$ & $\mathrm{AA}$ & - & GG & $\mathrm{AA}$ \\
\hline- & - & $\mathrm{AA}$ & GG & - & GG & - & $\mathrm{AA}$ & - & $\mathrm{AA}$ & - \\
\hline- & $\mathrm{AA}$ & $\mathrm{AA}$ & AA & GG & - & - & - & GG & - & - \\
\hline- & GG & - & - & $\mathrm{AG}$ & $\mathrm{AA}$ & - & - & $\mathrm{AA}$ & - & $\mathrm{AG}$ \\
\hline GG & - & - & - & - & GG & $\mathrm{AG}$ & GG & - & - & \\
\hline GDsnp00747 & 12 & \multicolumn{2}{|c|}{24953914} & $\mathrm{AT}$ & $\mathrm{AT}$ & $\mathrm{AA}$ & $\mathrm{AT}$ & $\mathrm{TT}$ & $\mathrm{AA}$ & TT \\
\hline AT & $\mathrm{AT}$ & AT & $\mathrm{AT}$ & AT & $\mathrm{TT}$ & $\mathrm{AT}$ & AA & $\mathrm{AT}$ & $\mathrm{TT}$ & AT \\
\hline AT & $\mathrm{AA}$ & $\mathrm{TT}$ & $\mathrm{AT}$ & $\mathrm{AT}$ & $\mathrm{AA}$ & $\mathrm{AT}$ & $\mathrm{AT}$ & $\mathrm{AT}$ & $\mathrm{TT}$ & TT \\
\hline AT & $\mathrm{AA}$ & $\mathrm{TT}$ & $\mathrm{TT}$ & $\mathrm{AT}$ & $\mathrm{AT}$ & $\mathrm{TT}$ & $\mathrm{TT}$ & $\mathrm{TT}$ & $\mathrm{AT}$ & AT \\
\hline $\mathrm{AT}$ & $\mathrm{AA}$ & $\mathrm{AT}$ & $\mathrm{AT}$ & $\mathrm{AT}$ & $\mathrm{TT}$ & $\mathrm{AT}$ & $\mathrm{AT}$ & $\mathrm{AT}$ & $\mathrm{AA}$ & TT \\
\hline AT & $\mathrm{AT}$ & $\mathrm{TT}$ & AA & AT & AA & $\mathrm{AT}$ & $\mathrm{TT}$ & $\mathrm{AT}$ & $\mathrm{TT}$ & AT \\
\hline AT & $\mathrm{TT}$ & $\mathrm{TT}$ & $\mathrm{TT}$ & $\mathrm{AA}$ & $\mathrm{AT}$ & AT & $\mathrm{AT}$ & AA & $\mathrm{AT}$ & AT \\
\hline AT & $\mathrm{AA}$ & $\mathrm{AT}$ & $\mathrm{AT}$ & $\mathrm{AT}$ & $\mathrm{TT}$ & $\mathrm{AT}$ & AT & $\mathrm{TT}$ & $\mathrm{AT}$ & TT \\
\hline AT & $\mathrm{AT}$ & AT & $\mathrm{AT}$ & AT & AT & TT & $\mathrm{AA}$ & $\mathrm{AT}$ & AT & \\
\hline GDsnp01426 & 12 & \multicolumn{2}{|c|}{26511747} & $\mathrm{AG}$ & $\mathrm{AG}$ & GG & $\mathrm{AG}$ & $\mathrm{AA}$ & $\mathrm{AG}$ & $\mathrm{AG}$ \\
\hline $\mathrm{AG}$ & $\mathrm{AA}$ & $\mathrm{AG}$ & $\mathrm{AG}$ & $\mathrm{AG}$ & $\mathrm{AA}$ & $\mathrm{AG}$ & GG & GG & $\mathrm{AA}$ & GG \\
\hline $\mathrm{AG}$ & $\mathrm{AG}$ & $\mathrm{AA}$ & $\mathrm{AG}$ & $\mathrm{AG}$ & $\mathrm{AG}$ & AA & $\mathrm{AG}$ & $\mathrm{AG}$ & $\mathrm{AA}$ & $\mathrm{AA}$ \\
\hline $\mathrm{AG}$ & GG & AA & AA & $\mathrm{AG}$ & $\mathrm{AG}$ & $\mathrm{AG}$ & AA & AA & $\mathrm{AG}$ & GG \\
\hline $\mathrm{AG}$ & $\mathrm{AG}$ & $\mathrm{AG}$ & $\mathrm{AG}$ & $\mathrm{AG}$ & $\mathrm{AA}$ & - & $\mathrm{AG}$ & $\mathrm{AG}$ & $\mathrm{AG}$ & $\mathrm{AA}$ \\
\hline $\mathrm{AG}$ & $\mathrm{AA}$ & AA & $\mathrm{AG}$ & $\mathrm{AG}$ & GG & $\mathrm{AG}$ & AA & $\mathrm{AG}$ & AA & $\mathrm{AG}$ \\
\hline $\mathrm{AG}$ & $\mathrm{AA}$ & $\mathrm{AA}$ & $\mathrm{AA}$ & GG & $\mathrm{AG}$ & $\mathrm{AG}$ & $\mathrm{AG}$ & GG & $\mathrm{AG}$ & $\mathrm{AG}$ \\
\hline $\mathrm{AG}$ & $\mathrm{AG}$ & $\mathrm{AG}$ & GG & $\mathrm{AA}$ & $\mathrm{AA}$ & $\mathrm{AG}$ & $\mathrm{AG}$ & AA & $\mathrm{AG}$ & AA \\
\hline $\mathrm{AG}$ & $\mathrm{AG}$ & $\mathrm{AG}$ & $\mathrm{AG}$ & $\mathrm{AG}$ & $\mathrm{AG}$ & AA & $\mathrm{AG}$ & $\mathrm{AG}$ & $\mathrm{AG}$ & \\
\hline GDsnp00762 & 12 & \multicolumn{2}{|c|}{27587472} & GG & GG & GG & GG & GG & GG & GG \\
\hline GG & GG & GG & GG & GG & GG & GG & GG & GG & GG & GG \\
\hline GG & GG & GG & GG & GG & GG & GG & GG & GG & GG & GG \\
\hline GG & GG & GG & GG & GG & GG & GG & GG & GG & GG & GG \\
\hline GG & GG & GG & GG & GG & GG & GG & $\mathrm{AG}$ & GG & GG & GG \\
\hline GG & GG & GG & GG & GG & GG & GG & GG & GG & GG & GG \\
\hline GG & GG & GG & GG & GG & GG & GG & GG & GG & GG & GG \\
\hline GG & GG & GG & GG & GG & GG & GG & GG & GG & GG & GG \\
\hline GG & GG & GG & GG & GG & GG & GG & GG & GG & GG & \\
\hline GDsnp00391 & 12 & \multicolumn{2}{|c|}{27698739} & GG & $\mathrm{AG}$ & GG & GG & $\mathrm{AA}$ & GG & $\mathrm{AG}$ \\
\hline- & $\mathrm{AA}$ & GG & $\mathrm{AA}$ & GG & $\mathrm{AA}$ & $\mathrm{AG}$ & $\mathrm{AG}$ & $\mathrm{AG}$ & $\mathrm{AA}$ & GG \\
\hline $\mathrm{AG}$ & $\mathrm{AG}$ & $\mathrm{AG}$ & $\mathrm{AG}$ & $\mathrm{AG}$ & $\mathrm{AG}$ & AA & $\mathrm{AG}$ & $\mathrm{AG}$ & AA & $\mathrm{AG}$ \\
\hline GG & GG & AA & AA & $\mathrm{AG}$ & - & $\mathrm{AG}$ & $\mathrm{AG}$ & AA & $\mathrm{AG}$ & GG \\
\hline AG & $\mathrm{AG}$ & $\mathrm{AG}$ & $\mathrm{AG}$ & $\mathrm{AA}$ & $\mathrm{AG}$ & - & $\mathrm{AG}$ & $\mathrm{AA}$ & $\mathrm{AG}$ & $\mathrm{AA}$ \\
\hline $\mathrm{AG}$ & $\mathrm{AA}$ & $\mathrm{AA}$ & $\mathrm{AG}$ & GG & GG & $\mathrm{AG}$ & $\mathrm{AA}$ & $\mathrm{AG}$ & $\mathrm{AA}$ & $\mathrm{AG}$ \\
\hline $\mathrm{AG}$ & $\mathrm{AA}$ & $\mathrm{AA}$ & $\mathrm{AA}$ & GG & $\mathrm{AG}$ & GG & $\mathrm{AA}$ & GG & $\mathrm{AG}$ & $\mathrm{AG}$ \\
\hline $\mathrm{AG}$ & GG & $\mathrm{AG}$ & GG & $\mathrm{AA}$ & $\mathrm{AA}$ & $\mathrm{AG}$ & $\mathrm{AG}$ & AA & $\mathrm{AG}$ & $\mathrm{AG}$ \\
\hline GG & $\mathrm{AG}$ & $\mathrm{AG}$ & - & $\mathrm{AG}$ & GG & AA & $\mathrm{AG}$ & $\mathrm{AG}$ & GG & \\
\hline
\end{tabular}

Continued next page 
Supplemental Table 2. Continued.

\begin{tabular}{|c|c|c|c|c|c|c|c|c|c|c|}
\hline \multirow{3}{*}{$\begin{array}{l}\text { SNP name } \\
\text { GDsnp01051 } \\
\text { TT }\end{array}$} & \multicolumn{10}{|c|}{ Linkage group Physical location (bp) Individuals } \\
\hline & 12 & \multicolumn{2}{|c|}{28635275} & TT & TT & TT & TT & TT & TT & TT \\
\hline & TT & $\mathrm{TT}$ & $\mathrm{TT}$ & TT & TT & $\mathrm{TT}$ & TT & TT & $\mathrm{TT}$ & TT \\
\hline $\mathrm{TT}$ & $\mathrm{TT}$ & $\mathrm{TT}$ & TT & $\mathrm{TT}$ & TT & TT & TT & $\mathrm{TT}$ & $\mathrm{TT}$ & $\mathrm{TT}$ \\
\hline $\mathrm{TT}$ & TT & TT & TT & $\mathrm{TT}$ & TT & TT & TT & TT & $\mathrm{TT}$ & TT \\
\hline TT & $\mathrm{TT}$ & $\mathrm{TT}$ & $\mathrm{TT}$ & $\mathrm{TT}$ & TT & $\mathrm{TT}$ & - & $\mathrm{TT}$ & $\mathrm{TT}$ & TT \\
\hline $\mathrm{TT}$ & $\mathrm{TT}$ & $\mathrm{TT}$ & $\mathrm{TT}$ & $\mathrm{TT}$ & TT & $\mathrm{TT}$ & TT & $\mathrm{TT}$ & $\mathrm{TT}$ & TT \\
\hline $\mathrm{TT}$ & $\mathrm{TT}$ & $\mathrm{TT}$ & $\mathrm{TT}$ & $\mathrm{TT}$ & TT & $\mathrm{TT}$ & TT & $\mathrm{TT}$ & $\mathrm{TT}$ & TT \\
\hline- & $\mathrm{TT}$ & $\mathrm{TT}$ & $\mathrm{TT}$ & $\mathrm{TT}$ & TT & $\mathrm{TT}$ & TT & $\mathrm{TT}$ & $\mathrm{TT}$ & TT \\
\hline $\mathrm{TT}$ & $\mathrm{TT}$ & $\mathrm{TT}$ & $\mathrm{TT}$ & $\mathrm{TT}$ & TT & $\mathrm{TT}$ & TT & $\mathrm{TT}$ & $\mathrm{TT}$ & \\
\hline GDsnp00334 & 12 & \multicolumn{2}{|c|}{29260634} & $\mathrm{AG}$ & $\mathrm{AA}$ & $\mathrm{AA}$ & $\mathrm{AG}$ & $\mathrm{AA}$ & $\mathrm{AG}$ & $\mathrm{AA}$ \\
\hline AA & $\mathrm{AA}$ & $\mathrm{AG}$ & $\mathrm{AA}$ & $\mathrm{AG}$ & $\mathrm{AA}$ & AA & $\mathrm{AA}$ & $\mathrm{AA}$ & $\mathrm{AA}$ & AA \\
\hline AA & $\mathrm{AG}$ & $\mathrm{AG}$ & $\mathrm{AA}$ & $\mathrm{AA}$ & AG & $\mathrm{AG}$ & $\mathrm{AA}$ & $\mathrm{AA}$ & $\mathrm{AA}$ & $\mathrm{AG}$ \\
\hline $\mathrm{AG}$ & $\mathrm{AA}$ & $\mathrm{AA}$ & $\mathrm{AA}$ & $\mathrm{AA}$ & $\mathrm{AA}$ & AA & $\mathrm{AA}$ & $\mathrm{AA}$ & $\mathrm{AG}$ & $\mathrm{AA}$ \\
\hline $\mathrm{AA}$ & $\mathrm{AG}$ & $\mathrm{AA}$ & $\mathrm{AA}$ & $\mathrm{AA}$ & $\mathrm{AG}$ & - & $\mathrm{AA}$ & $\mathrm{AA}$ & $\mathrm{AG}$ & $\mathrm{AA}$ \\
\hline $\mathrm{AA}$ & $\mathrm{AA}$ & $\mathrm{AA}$ & $\mathrm{AA}$ & $\mathrm{AA}$ & $\mathrm{AA}$ & $\mathrm{AA}$ & $\mathrm{AG}$ & $\mathrm{AA}$ & $\mathrm{AG}$ & AA \\
\hline AA & $\mathrm{AA}$ & $\mathrm{AA}$ & $\mathrm{AA}$ & AA & $\mathrm{AA}$ & AA & $\mathrm{AA}$ & $\mathrm{AA}$ & AA & $\mathrm{AA}$ \\
\hline AA & $\mathrm{AG}$ & $\mathrm{AA}$ & $\mathrm{AA}$ & $\mathrm{AA}$ & $\mathrm{AA}$ & AA & $\mathrm{AA}$ & $\mathrm{AA}$ & $\mathrm{AA}$ & $\mathrm{AG}$ \\
\hline $\mathrm{AG}$ & $\mathrm{AA}$ & AA & $\mathrm{AA}$ & $\mathrm{AA}$ & $\mathrm{AG}$ & $\mathrm{AG}$ & $\mathrm{AG}$ & $\mathrm{AA}$ & AA & \\
\hline GDsnp01665 & 12 & \multicolumn{2}{|c|}{30578149} & $\mathrm{AG}$ & $\mathrm{AG}$ & $\mathrm{AG}$ & $\mathrm{AG}$ & AA & $\mathrm{AG}$ & AA \\
\hline GG & $\mathrm{AG}$ & $\mathrm{AG}$ & $\mathrm{AG}$ & $\mathrm{AG}$ & $\mathrm{AA}$ & AA & $\mathrm{AG}$ & $\mathrm{AG}$ & AA & GG \\
\hline $\mathrm{AG}$ & GG & $\mathrm{AG}$ & $\mathrm{AA}$ & $\mathrm{AG}$ & GG & $\mathrm{AG}$ & $\mathrm{AG}$ & $\mathrm{AG}$ & $\mathrm{AA}$ & $\mathrm{AA}$ \\
\hline GG & $\mathrm{AG}$ & $\mathrm{AA}$ & $\mathrm{AA}$ & $\mathrm{AG}$ & AG & $\mathrm{AG}$ & $\mathrm{AG}$ & $\mathrm{AA}$ & GG & GG \\
\hline $\mathrm{AG}$ & $\mathrm{AG}$ & $\mathrm{AG}$ & $\mathrm{AG}$ & $\mathrm{AA}$ & AG & $\mathrm{AG}$ & GG & $\mathrm{AA}$ & GG & $\mathrm{AG}$ \\
\hline $\mathrm{AG}$ & $\mathrm{AA}$ & AA & GG & GG & GG & $\mathrm{AG}$ & $\mathrm{AG}$ & $\mathrm{AG}$ & $\mathrm{AG}$ & $\mathrm{AG}$ \\
\hline $\mathrm{AG}$ & $\mathrm{AA}$ & AA & $\mathrm{AA}$ & $\mathrm{AG}$ & $\mathrm{AG}$ & GG & $\mathrm{AG}$ & GG & $\mathrm{AG}$ & $\mathrm{AG}$ \\
\hline AA & $\mathrm{AG}$ & $\mathrm{AG}$ & GG & $\mathrm{AG}$ & $\mathrm{AA}$ & $\mathrm{AG}$ & $\mathrm{AG}$ & $\mathrm{AG}$ & $\mathrm{AG}$ & $\mathrm{AA}$ \\
\hline $\mathrm{AG}$ & $\mathrm{AG}$ & $\mathrm{AG}$ & $\mathrm{AA}$ & $\mathrm{AA}$ & $\mathrm{AG}$ & $\mathrm{AG}$ & GG & $\mathrm{AA}$ & GG & \\
\hline GDsnp00915 & 12 & \multicolumn{2}{|c|}{31276432} & $\mathrm{AG}$ & $\mathrm{AA}$ & $\mathrm{AA}$ & $\mathrm{AG}$ & GG & $\mathrm{AG}$ & GG \\
\hline AA & $\mathrm{AG}$ & $\mathrm{AG}$ & $\mathrm{AG}$ & $\mathrm{AG}$ & AG & $\mathrm{AG}$ & $\mathrm{AG}$ & $\mathrm{AG}$ & GG & $\mathrm{AA}$ \\
\hline AA & $\mathrm{AA}$ & $\mathrm{AG}$ & $\mathrm{AG}$ & $\mathrm{AA}$ & $\mathrm{AA}$ & $\mathrm{AG}$ & $\mathrm{AG}$ & $\mathrm{AG}$ & $\mathrm{AG}$ & GG \\
\hline AA & $\mathrm{AG}$ & GG & GG & $\mathrm{AA}$ & $\mathrm{AA}$ & $\mathrm{AG}$ & $\mathrm{AG}$ & $\mathrm{AG}$ & AA & AA \\
\hline GG & $\mathrm{AG}$ & $\mathrm{AG}$ & $\mathrm{AG}$ & $\mathrm{AG}$ & GG & $\mathrm{AG}$ & $\mathrm{AA}$ & $\mathrm{AG}$ & AA & $\mathrm{AA}$ \\
\hline AA & $\mathrm{AG}$ & $\mathrm{AG}$ & $\mathrm{AA}$ & $\mathrm{AA}$ & $\mathrm{AA}$ & AA & $\mathrm{AG}$ & $\mathrm{AG}$ & $\mathrm{AG}$ & $\mathrm{AG}$ \\
\hline $\mathrm{AG}$ & GG & $\mathrm{AG}$ & $\mathrm{AG}$ & $\mathrm{AG}$ & $\mathrm{AA}$ & AA & GG & $\mathrm{AA}$ & $\mathrm{AG}$ & $\mathrm{AA}$ \\
\hline GG & $\mathrm{AG}$ & $\mathrm{AG}$ & $\mathrm{AA}$ & GG & - & AA & $\mathrm{AG}$ & $\mathrm{AG}$ & $\mathrm{AG}$ & GG \\
\hline $\mathrm{AG}$ & $\mathrm{AG}$ & AA & $\mathrm{AG}$ & GG & $\mathrm{AG}$ & $\mathrm{AG}$ & $\mathrm{AA}$ & $\mathrm{AG}$ & AA & \\
\hline GDsnp00296 & 12 & \multicolumn{2}{|c|}{31353204} & $\mathrm{AA}$ & AT & $\mathrm{AT}$ & $\mathrm{AA}$ & $\mathrm{AA}$ & AA & $\mathrm{AA}$ \\
\hline AT & $\mathrm{AT}$ & AA & $\mathrm{AA}$ & $\mathrm{AA}$ & AT & AT & $\mathrm{AA}$ & $\mathrm{AA}$ & AA & AA \\
\hline AT & $\mathrm{AT}$ & $\mathrm{AT}$ & $\mathrm{AT}$ & $\mathrm{AT}$ & AT & $\mathrm{AT}$ & $\mathrm{AA}$ & $\mathrm{AA}$ & $\mathrm{AT}$ & $\mathrm{AA}$ \\
\hline AA & $\mathrm{AA}$ & $\mathrm{AA}$ & $\mathrm{AA}$ & $\mathrm{AT}$ & AT & AA & $\mathrm{AA}$ & $\mathrm{AT}$ & $\mathrm{AT}$ & $\mathrm{AA}$ \\
\hline AA & $\mathrm{AT}$ & $\mathrm{AA}$ & $\mathrm{AA}$ & $\mathrm{AT}$ & $\mathrm{AA}$ & $\mathrm{AA}$ & AT & AT & $\mathrm{AT}$ & AT \\
\hline AT & $\mathrm{AT}$ & $\mathrm{AT}$ & $\mathrm{AA}$ & $\mathrm{AA}$ & $\mathrm{AA}$ & AT & AT & $\mathrm{AT}$ & $\mathrm{AT}$ & AA \\
\hline AA & $\mathrm{AA}$ & $\mathrm{AT}$ & $\mathrm{AT}$ & $\mathrm{AA}$ & AT & AA & $\mathrm{AA}$ & $\mathrm{AA}$ & AA & AT \\
\hline AA & $\mathrm{AA}$ & $\mathrm{AT}$ & $\mathrm{AA}$ & $\mathrm{AA}$ & AT & $\mathrm{AT}$ & $\mathrm{AA}$ & $\mathrm{AA}$ & AA & $\mathrm{AA}$ \\
\hline AA & AA & AT & AT & $\mathrm{AA}$ & $\mathrm{AA}$ & AT & AT & AT & AA & \\
\hline GDsnp01190 & 13 & \multicolumn{2}{|c|}{131962} & $\mathrm{AA}$ & $\mathrm{AA}$ & $\mathrm{AA}$ & $\mathrm{AA}$ & $\mathrm{AA}$ & $\mathrm{AA}$ & $\mathrm{AA}$ \\
\hline AA & $\mathrm{AG}$ & $\mathrm{AA}$ & $\mathrm{AA}$ & $\mathrm{AA}$ & $\mathrm{AA}$ & AA & $\mathrm{AA}$ & $\mathrm{AG}$ & $\mathrm{AA}$ & $\mathrm{AA}$ \\
\hline AA & $\mathrm{AA}$ & AA & $\mathrm{AA}$ & $\mathrm{AA}$ & $\mathrm{AA}$ & $\mathrm{AG}$ & $\mathrm{AA}$ & $\mathrm{AA}$ & AA & AA \\
\hline AA & $\mathrm{AA}$ & $\mathrm{AA}$ & $\mathrm{AA}$ & $\mathrm{AA}$ & $\mathrm{AA}$ & AA & $\mathrm{AA}$ & $\mathrm{AA}$ & $\mathrm{AA}$ & $\mathrm{AA}$ \\
\hline AA & $\mathrm{AA}$ & $\mathrm{AA}$ & $\mathrm{AA}$ & $\mathrm{AA}$ & $\mathrm{AA}$ & AA & $\mathrm{AA}$ & $\mathrm{AA}$ & AA & $\mathrm{AA}$ \\
\hline $\mathrm{AA}$ & $\mathrm{AA}$ & $\mathrm{AA}$ & $\mathrm{AA}$ & $\mathrm{AA}$ & $\mathrm{AA}$ & AA & $\mathrm{AA}$ & $\mathrm{AA}$ & AA & $\mathrm{AA}$ \\
\hline $\mathrm{AA}$ & $\mathrm{AA}$ & $\mathrm{AA}$ & $\mathrm{AA}$ & $\mathrm{AA}$ & $\mathrm{AA}$ & $\mathrm{AA}$ & $\mathrm{AA}$ & $\mathrm{AA}$ & $\mathrm{AA}$ & $\mathrm{AA}$ \\
\hline AA & $\mathrm{AA}$ & $\mathrm{AA}$ & $\mathrm{AA}$ & $\mathrm{AA}$ & $\mathrm{AA}$ & $\mathrm{AA}$ & $\mathrm{AA}$ & $\mathrm{AA}$ & $\mathrm{AA}$ & $\mathrm{AA}$ \\
\hline AA & $\mathrm{AA}$ & $\mathrm{AA}$ & $\mathrm{AA}$ & $\mathrm{AA}$ & $\mathrm{AA}$ & AA & $\mathrm{AA}$ & $\mathrm{AA}$ & AA & \\
\hline
\end{tabular}

Continued next page 
Supplemental Table 2. Continued.

\begin{tabular}{|c|c|c|c|c|c|c|c|c|c|c|}
\hline \multirow{3}{*}{$\begin{array}{l}\text { SNP name } \\
\text { GDsnp01655 } \\
\text { AA }\end{array}$} & \multicolumn{10}{|c|}{ Linkage group Physical location (bp) Individuals } \\
\hline & 13 & \multicolumn{2}{|c|}{365863} & AA & AA & AA & $\mathrm{AA}$ & AA & AA & $\mathrm{AA}$ \\
\hline & AT & AA & AA & AA & AA & AA & AA & AT & AA & AA \\
\hline AA & AA & AA & AA & AA & AA & AT & AA & AA & AA & AA \\
\hline AA & AA & AA & AA & AA & AA & AA & AA & AA & AA & $\mathrm{AA}$ \\
\hline AA & $\mathrm{AA}$ & $\mathrm{AA}$ & $\mathrm{AA}$ & $\mathrm{AA}$ & $\mathrm{AA}$ & $\mathrm{AA}$ & $\mathrm{AA}$ & $\mathrm{AA}$ & $\mathrm{AA}$ & $\mathrm{AA}$ \\
\hline AA & $\mathrm{AA}$ & AA & AA & AA & $\mathrm{AA}$ & AA & $\mathrm{AA}$ & AA & AA & AA \\
\hline $\mathrm{AA}$ & $\mathrm{AA}$ & $\mathrm{AA}$ & $\mathrm{AA}$ & $\mathrm{AA}$ & $\mathrm{AA}$ & $\mathrm{AA}$ & $\mathrm{AA}$ & $\mathrm{AA}$ & $\mathrm{AA}$ & $\mathrm{AA}$ \\
\hline AA & $\mathrm{AA}$ & AA & AA & $\mathrm{AA}$ & $\mathrm{AA}$ & AA & $\mathrm{AA}$ & AA & AA & AA \\
\hline $\mathrm{AA}$ & $\mathrm{AA}$ & $\mathrm{AA}$ & $\mathrm{AA}$ & $\mathrm{AA}$ & $\mathrm{AA}$ & $\mathrm{AA}$ & $\mathrm{AA}$ & $\mathrm{AA}$ & AA & \\
\hline GDsnp01917 & 13 & \multicolumn{2}{|c|}{623002} & $\mathrm{CC}$ & $\mathrm{CC}$ & $\mathrm{CC}$ & $\mathrm{CC}$ & $\mathrm{CC}$ & $\mathrm{CC}$ & $\mathrm{CC}$ \\
\hline $\mathrm{CC}$ & $\mathrm{AC}$ & $\mathrm{CC}$ & $\mathrm{CC}$ & $\mathrm{CC}$ & $\mathrm{CC}$ & $\mathrm{CC}$ & $\mathrm{CC}$ & $\mathrm{AC}$ & $\mathrm{CC}$ & $\mathrm{CC}$ \\
\hline $\mathrm{CC}$ & $\mathrm{CC}$ & $\mathrm{CC}$ & $\mathrm{CC}$ & $\mathrm{CC}$ & $\mathrm{CC}$ & $\mathrm{AC}$ & $\mathrm{CC}$ & $\mathrm{CC}$ & $\mathrm{CC}$ & $\mathrm{CC}$ \\
\hline $\mathrm{CC}$ & $\mathrm{CC}$ & $\mathrm{CC}$ & $\mathrm{CC}$ & $\mathrm{CC}$ & $\mathrm{CC}$ & $\mathrm{CC}$ & $\mathrm{CC}$ & $\mathrm{CC}$ & $\mathrm{CC}$ & $\mathrm{CC}$ \\
\hline $\mathrm{CC}$ & $\mathrm{CC}$ & $\mathrm{CC}$ & $\mathrm{CC}$ & $\mathrm{CC}$ & $\mathrm{CC}$ & $\mathrm{CC}$ & $\mathrm{CC}$ & $\mathrm{CC}$ & $\mathrm{CC}$ & $\mathrm{CC}$ \\
\hline $\mathrm{CC}$ & $\mathrm{CC}$ & $\mathrm{CC}$ & $\mathrm{CC}$ & $\mathrm{CC}$ & $\mathrm{CC}$ & $\mathrm{CC}$ & $\mathrm{CC}$ & $\mathrm{CC}$ & $\mathrm{CC}$ & $\mathrm{CC}$ \\
\hline $\mathrm{CC}$ & $\mathrm{CC}$ & $\mathrm{CC}$ & $\mathrm{CC}$ & $\mathrm{CC}$ & $\mathrm{CC}$ & $\mathrm{CC}$ & $\mathrm{CC}$ & $\mathrm{CC}$ & $\mathrm{CC}$ & $\mathrm{CC}$ \\
\hline $\mathrm{CC}$ & $\mathrm{CC}$ & $\mathrm{CC}$ & $\mathrm{CC}$ & $\mathrm{CC}$ & $\mathrm{CC}$ & $\mathrm{CC}$ & $\mathrm{CC}$ & $\mathrm{CC}$ & $\mathrm{CC}$ & $\mathrm{CC}$ \\
\hline $\mathrm{CC}$ & $\mathrm{CC}$ & $\mathrm{CC}$ & $\mathrm{CC}$ & $\mathrm{CC}$ & $\mathrm{CC}$ & $\mathrm{CC}$ & $\mathrm{CC}$ & $\mathrm{CC}$ & $\mathrm{CC}$ & \\
\hline GDsnp00060 & 13 & \multicolumn{2}{|c|}{874638} & $\mathrm{AA}$ & AA & AA & AA & AA & AA & AA \\
\hline AA & $\mathrm{AA}$ & AA & AA & $\mathrm{AA}$ & AA & AA & $\mathrm{AA}$ & $\mathrm{AT}$ & AA & $\mathrm{AA}$ \\
\hline $\mathrm{AA}$ & $\mathrm{AA}$ & $\mathrm{AA}$ & $\mathrm{AA}$ & $\mathrm{AA}$ & $\mathrm{AA}$ & $\mathrm{AT}$ & $\mathrm{AA}$ & $\mathrm{AA}$ & $\mathrm{AA}$ & $\mathrm{AA}$ \\
\hline $\mathrm{AA}$ & $\mathrm{AA}$ & $\mathrm{AA}$ & $\mathrm{AA}$ & $\mathrm{AA}$ & $\mathrm{AA}$ & $\mathrm{AA}$ & $\mathrm{AA}$ & $\mathrm{AA}$ & AA & $\mathrm{AA}$ \\
\hline AA & $\mathrm{AA}$ & $\mathrm{AA}$ & $\mathrm{AA}$ & $\mathrm{AA}$ & $\mathrm{AA}$ & AA & $\mathrm{AA}$ & $\mathrm{AA}$ & $\mathrm{AA}$ & $\mathrm{AA}$ \\
\hline AA & $\mathrm{AA}$ & AA & AA & $\mathrm{AA}$ & AA & AA & $\mathrm{AA}$ & AA & AA & $\mathrm{AA}$ \\
\hline AA & $\mathrm{AA}$ & AA & AA & $\mathrm{AA}$ & $\mathrm{AA}$ & AA & $\mathrm{AA}$ & AA & AA & AA \\
\hline AA & $\mathrm{AA}$ & AA & AA & $\mathrm{AA}$ & $\mathrm{AA}$ & AA & $\mathrm{AA}$ & AA & AA & $\mathrm{AA}$ \\
\hline $\mathrm{AA}$ & $\mathrm{AA}$ & $\mathrm{AA}$ & AA & $\mathrm{AA}$ & AA & AA & $\mathrm{AA}$ & AA & AA & \\
\hline GDsnp00184 & 13 & \multicolumn{2}{|c|}{1600799} & $\mathrm{AA}$ & $\mathrm{TT}$ & $\mathrm{AT}$ & $\mathrm{AA}$ & $\mathrm{AT}$ & $\mathrm{AT}$ & AA \\
\hline TT & $\mathrm{AT}$ & $\mathrm{AA}$ & AA & $\mathrm{AA}$ & $\mathrm{AT}$ & $\mathrm{TT}$ & $\mathrm{AT}$ & $\mathrm{AT}$ & AT & $\mathrm{AA}$ \\
\hline $\mathrm{AT}$ & $\mathrm{AT}$ & $\mathrm{AA}$ & $\mathrm{AT}$ & $\mathrm{TT}$ & $\mathrm{AA}$ & $\mathrm{AT}$ & $\mathrm{AT}$ & AT & $\mathrm{TT}$ & $\mathrm{AA}$ \\
\hline AT & $\mathrm{TT}$ & TT & $\mathrm{AT}$ & $\mathrm{TT}$ & $\mathrm{AT}$ & AA & $\mathrm{AT}$ & AA & AA & AT \\
\hline AT & $\mathrm{AA}$ & $\mathrm{AT}$ & $\mathrm{AT}$ & AT & $\mathrm{AA}$ & $\mathrm{TT}$ & $\mathrm{AT}$ & AA & $\mathrm{AT}$ & TT \\
\hline AT & $\mathrm{AA}$ & AT & $\mathrm{TT}$ & $\mathrm{AA}$ & $\mathrm{AT}$ & AA & $\mathrm{AT}$ & $\mathrm{AT}$ & AA & AT \\
\hline $\mathrm{TT}$ & $\mathrm{AT}$ & $\mathrm{AA}$ & $\mathrm{AT}$ & $\mathrm{AA}$ & $\mathrm{AT}$ & AA & $\mathrm{TT}$ & AT & $\mathrm{TT}$ & AT \\
\hline AT & $\mathrm{AT}$ & TT & AA & $\mathrm{AT}$ & $\mathrm{AT}$ & AT & TT & AT & $\mathrm{AT}$ & AA \\
\hline AT & $\mathrm{AA}$ & AT & AT & $\mathrm{AT}$ & AA & $\mathrm{AT}$ & TT & $\mathrm{TT}$ & AA & \\
\hline GDsnp00098 & 13 & \multicolumn{2}{|c|}{2347048} & GG & $\mathrm{AA}$ & $\mathrm{AG}$ & GG & $\mathrm{AG}$ & $\mathrm{AG}$ & GG \\
\hline AA & GG & GG & GG & GG & $\mathrm{AG}$ & $\mathrm{AG}$ & $\mathrm{AG}$ & $\mathrm{AG}$ & $\mathrm{AG}$ & GG \\
\hline AG & $\mathrm{AG}$ & GG & $\mathrm{AG}$ & $\mathrm{AA}$ & GG & GG & GG & GG & $\mathrm{AG}$ & GG \\
\hline AG & $\mathrm{AG}$ & $\mathrm{AA}$ & $\mathrm{AG}$ & $\mathrm{AG}$ & GG & GG & $\mathrm{AG}$ & GG & $\mathrm{AG}$ & $\mathrm{AG}$ \\
\hline $\mathrm{AG}$ & GG & $\mathrm{AG}$ & $\mathrm{AG}$ & $\mathrm{AG}$ & GG & $\mathrm{AG}$ & $\mathrm{AG}$ & GG & $\mathrm{AG}$ & $\mathrm{AG}$ \\
\hline $\mathrm{AG}$ & GG & GG & AA & GG & $\mathrm{AG}$ & GG & $\mathrm{AG}$ & GG & GG & $\mathrm{AG}$ \\
\hline AA & $\mathrm{AG}$ & $\mathrm{AG}$ & $\mathrm{AG}$ & GG & $\mathrm{AG}$ & GG & $\mathrm{AA}$ & $\mathrm{AG}$ & $\mathrm{AG}$ & $\mathrm{AG}$ \\
\hline $\mathrm{AG}$ & $\mathrm{AG}$ & $\mathrm{AG}$ & GG & $\mathrm{AG}$ & $\mathrm{AG}$ & GG & $\mathrm{AA}$ & $\mathrm{AG}$ & $\mathrm{AG}$ & GG \\
\hline AG & GG & $\mathrm{AG}$ & GG & $\mathrm{AG}$ & GG & $\mathrm{AG}$ & $\mathrm{AA}$ & AA & GG & \\
\hline GDsnp00110 & 13 & \multicolumn{2}{|c|}{2475410} & $\mathrm{CC}$ & GG & $\mathrm{CG}$ & $\mathrm{CC}$ & $\mathrm{CG}$ & $\mathrm{CG}$ & $\mathrm{CC}$ \\
\hline GG & $\mathrm{CC}$ & $\mathrm{CC}$ & $\mathrm{CC}$ & $\mathrm{CC}$ & $\mathrm{CG}$ & $\mathrm{CG}$ & $\mathrm{CG}$ & $\mathrm{CG}$ & $\mathrm{CG}$ & $\mathrm{CC}$ \\
\hline $\mathrm{CG}$ & $\mathrm{CG}$ & $\mathrm{CC}$ & $\mathrm{CG}$ & GG & $\mathrm{CC}$ & $\mathrm{CC}$ & $\mathrm{CC}$ & $\mathrm{CC}$ & $\mathrm{CG}$ & $\mathrm{CC}$ \\
\hline $\mathrm{CG}$ & $\mathrm{CG}$ & GG & $\mathrm{CG}$ & $\mathrm{CG}$ & $\mathrm{CC}$ & $\mathrm{CC}$ & $\mathrm{CG}$ & $\mathrm{CC}$ & $\mathrm{CG}$ & $\mathrm{CG}$ \\
\hline $\mathrm{CG}$ & $\mathrm{CC}$ & $\mathrm{CG}$ & $\mathrm{CG}$ & $\mathrm{CG}$ & $\mathrm{CC}$ & - & $\mathrm{CG}$ & $\mathrm{CC}$ & $\mathrm{CG}$ & $\mathrm{CG}$ \\
\hline $\mathrm{CG}$ & $\mathrm{CC}$ & $\mathrm{CC}$ & GG & $\mathrm{CC}$ & $\mathrm{CG}$ & $\mathrm{CC}$ & $\mathrm{CG}$ & $\mathrm{CC}$ & $\mathrm{CC}$ & $\mathrm{CG}$ \\
\hline GG & $\mathrm{CG}$ & $\mathrm{CG}$ & $\mathrm{CG}$ & $\mathrm{CC}$ & $\mathrm{CG}$ & $\mathrm{CC}$ & GG & $\mathrm{CG}$ & $\mathrm{CG}$ & $\mathrm{CG}$ \\
\hline $\mathrm{CG}$ & $\mathrm{CG}$ & CG & $\mathrm{CC}$ & $\mathrm{CG}$ & $\mathrm{CG}$ & $\mathrm{CC}$ & GG & $\mathrm{CG}$ & $\mathrm{CG}$ & $\mathrm{CC}$ \\
\hline $\mathrm{CG}$ & $\mathrm{CC}$ & $\mathrm{CG}$ & $\mathrm{CC}$ & $\mathrm{CG}$ & $\mathrm{CC}$ & $\mathrm{CG}$ & GG & GG & $\mathrm{CC}$ & \\
\hline
\end{tabular}

Continued next page 
Supplemental Table 2. Continued.

\begin{tabular}{|c|c|c|c|c|c|c|c|c|c|c|}
\hline \multirow{3}{*}{$\begin{array}{l}\text { SNP name } \\
\text { GDsnp00195 } \\
\text { AA }\end{array}$} & \multicolumn{10}{|c|}{ Linkage group Physical location (bp) Individuals } \\
\hline & 13 & \multicolumn{2}{|c|}{2829326} & $\mathrm{CC}$ & $\mathrm{AA}$ & $\mathrm{AC}$ & $\mathrm{CC}$ & $\mathrm{AC}$ & $\mathrm{AC}$ & $\mathrm{CC}$ \\
\hline & $\mathrm{AC}$ & $\mathrm{CC}$ & $\mathrm{CC}$ & $\mathrm{CC}$ & $\mathrm{AC}$ & $\mathrm{AC}$ & $\mathrm{AC}$ & $\mathrm{AC}$ & $\mathrm{AC}$ & $\mathrm{CC}$ \\
\hline $\mathrm{AC}$ & $\mathrm{AC}$ & $\mathrm{CC}$ & $\mathrm{AC}$ & $\mathrm{AA}$ & $\mathrm{CC}$ & $\mathrm{CC}$ & $\mathrm{CC}$ & $\mathrm{CC}$ & $\mathrm{AC}$ & $\mathrm{CC}$ \\
\hline $\mathrm{AC}$ & $\mathrm{AC}$ & AA & $\mathrm{AC}$ & $\mathrm{AC}$ & $\mathrm{CC}$ & $\mathrm{CC}$ & $\mathrm{AC}$ & $\mathrm{CC}$ & $\mathrm{AC}$ & $\mathrm{AC}$ \\
\hline $\mathrm{AC}$ & $\mathrm{CC}$ & $\mathrm{AC}$ & $\mathrm{AC}$ & $\mathrm{AC}$ & $\mathrm{CC}$ & $\mathrm{AC}$ & $\mathrm{AC}$ & $\mathrm{CC}$ & $\mathrm{AC}$ & $\mathrm{AC}$ \\
\hline $\mathrm{AC}$ & $\mathrm{CC}$ & $\mathrm{CC}$ & $\mathrm{AA}$ & $\mathrm{CC}$ & $\mathrm{AC}$ & $\mathrm{CC}$ & $\mathrm{AC}$ & $\mathrm{CC}$ & $\mathrm{CC}$ & $\mathrm{AC}$ \\
\hline $\mathrm{AA}$ & $\mathrm{AC}$ & $\mathrm{AC}$ & $\mathrm{AC}$ & $\mathrm{CC}$ & $\mathrm{AC}$ & $\mathrm{CC}$ & $\mathrm{AA}$ & $\mathrm{AC}$ & $\mathrm{AC}$ & $\mathrm{AC}$ \\
\hline $\mathrm{AC}$ & $\mathrm{AC}$ & $\mathrm{AC}$ & $\mathrm{CC}$ & $\mathrm{AA}$ & $\mathrm{AC}$ & $\mathrm{CC}$ & $\mathrm{AA}$ & $\mathrm{AC}$ & $\mathrm{AC}$ & $\mathrm{CC}$ \\
\hline $\mathrm{AC}$ & $\mathrm{CC}$ & $\mathrm{AC}$ & $\mathrm{CC}$ & $\mathrm{AC}$ & $\mathrm{CC}$ & $\mathrm{AC}$ & $\mathrm{AA}$ & $\mathrm{AA}$ & $\mathrm{CC}$ & \\
\hline GDsnp00922 & 13 & \multicolumn{2}{|c|}{3590826} & $\mathrm{AA}$ & $\mathrm{AA}$ & $\mathrm{AA}$ & $\mathrm{AA}$ & $\mathrm{AG}$ & $\mathrm{AA}$ & $\mathrm{AA}$ \\
\hline AA & $\mathrm{AA}$ & $\mathrm{AG}$ & $\mathrm{AG}$ & $\mathrm{AG}$ & $\mathrm{AA}$ & AA & $\mathrm{AA}$ & AA & $\mathrm{AA}$ & $\mathrm{AG}$ \\
\hline AA & $\mathrm{AG}$ & $\mathrm{AG}$ & AA & $\mathrm{AA}$ & $\mathrm{AG}$ & $\mathrm{AA}$ & $\mathrm{AG}$ & $\mathrm{AG}$ & $\mathrm{AA}$ & $\mathrm{AG}$ \\
\hline AA & $\mathrm{AG}$ & $\mathrm{AA}$ & $\mathrm{AA}$ & $\mathrm{AG}$ & $\mathrm{AG}$ & AA & $\mathrm{AA}$ & $\mathrm{AA}$ & $\mathrm{AA}$ & $\mathrm{AA}$ \\
\hline $\mathrm{AG}$ & $\mathrm{AG}$ & $\mathrm{AG}$ & $\mathrm{AA}$ & $\mathrm{AA}$ & $\mathrm{AG}$ & $\mathrm{AG}$ & $\mathrm{AA}$ & $\mathrm{AA}$ & $\mathrm{AG}$ & $\mathrm{AG}$ \\
\hline $\mathrm{AA}$ & $\mathrm{AA}$ & $\mathrm{AG}$ & $\mathrm{AA}$ & $\mathrm{AA}$ & $\mathrm{AA}$ & $\mathrm{AA}$ & $\mathrm{AA}$ & $\mathrm{AG}$ & $\mathrm{AG}$ & AA \\
\hline AA & $\mathrm{AA}$ & $\mathrm{AA}$ & AA & $\mathrm{AA}$ & $\mathrm{AA}$ & $\mathrm{AG}$ & $\mathrm{AA}$ & AA & $\mathrm{AG}$ & $\mathrm{AA}$ \\
\hline $\mathrm{AG}$ & $\mathrm{AA}$ & $\mathrm{AG}$ & $\mathrm{AA}$ & $\mathrm{AA}$ & $\mathrm{AA}$ & $\mathrm{AG}$ & $\mathrm{AA}$ & $\mathrm{AA}$ & $\mathrm{AA}$ & $\mathrm{AA}$ \\
\hline $\mathrm{AG}$ & $\mathrm{AA}$ & AA & AA & $\mathrm{AA}$ & $\mathrm{AG}$ & AA & $\mathrm{AA}$ & AA & AA & \\
\hline GDsnp00531 & 13 & \multicolumn{2}{|c|}{7368313} & $\mathrm{TT}$ & $\mathrm{TT}$ & $\mathrm{TT}$ & $\mathrm{TT}$ & $\mathrm{TT}$ & $\mathrm{TT}$ & TT \\
\hline AT & $\mathrm{TT}$ & TT & $\mathrm{AT}$ & $\mathrm{TT}$ & $\mathrm{TT}$ & $\mathrm{TT}$ & $\mathrm{TT}$ & $\mathrm{AT}$ & $\mathrm{TT}$ & TT \\
\hline $\mathrm{TT}$ & $\mathrm{TT}$ & AT & TT & $\mathrm{TT}$ & TT & $\mathrm{AT}$ & $\mathrm{TT}$ & $\mathrm{TT}$ & $\mathrm{TT}$ & TT \\
\hline AT & $\mathrm{TT}$ & $\mathrm{TT}$ & $\mathrm{TT}$ & $\mathrm{TT}$ & $\mathrm{TT}$ & $\mathrm{TT}$ & $\mathrm{TT}$ & $\mathrm{TT}$ & $\mathrm{AT}$ & AT \\
\hline $\mathrm{TT}$ & $\mathrm{TT}$ & $\mathrm{TT}$ & $\mathrm{TT}$ & $\mathrm{TT}$ & $\mathrm{TT}$ & $\mathrm{TT}$ & $\mathrm{TT}$ & $\mathrm{TT}$ & $\mathrm{TT}$ & TT \\
\hline $\mathrm{TT}$ & $\mathrm{TT}$ & TT & $\mathrm{TT}$ & $\mathrm{TT}$ & $\mathrm{TT}$ & $\mathrm{TT}$ & $\mathrm{TT}$ & $\mathrm{TT}$ & $\mathrm{TT}$ & TT \\
\hline $\mathrm{TT}$ & $\mathrm{TT}$ & TT & $\mathrm{TT}$ & $\mathrm{TT}$ & $\mathrm{TT}$ & AT & $\mathrm{TT}$ & $\mathrm{TT}$ & $\mathrm{TT}$ & TT \\
\hline AT & $\mathrm{TT}$ & $\mathrm{TT}$ & $\mathrm{TT}$ & $\mathrm{TT}$ & $\mathrm{TT}$ & $\mathrm{TT}$ & $\mathrm{TT}$ & $\mathrm{TT}$ & $\mathrm{TT}$ & TT \\
\hline TT & $\mathrm{TT}$ & $\mathrm{TT}$ & TT & $\mathrm{AT}$ & TT & $\mathrm{TT}$ & $\mathrm{TT}$ & TT & $\mathrm{TT}$ & \\
\hline GDsnp00140 & 13 & \multicolumn{2}{|c|}{10411070} & $\mathrm{CC}$ & $\mathrm{CC}$ & $\mathrm{CC}$ & $\mathrm{CC}$ & $\mathrm{CC}$ & $\mathrm{CC}$ & $\mathrm{CC}$ \\
\hline $\mathrm{AC}$ & $\mathrm{CC}$ & $\mathrm{AC}$ & $\mathrm{CC}$ & $\mathrm{CC}$ & $\mathrm{CC}$ & $\mathrm{CC}$ & $\mathrm{CC}$ & $\mathrm{AC}$ & $\mathrm{CC}$ & $\mathrm{CC}$ \\
\hline $\mathrm{CC}$ & $\mathrm{CC}$ & $\mathrm{AC}$ & $\mathrm{CC}$ & $\mathrm{CC}$ & $\mathrm{CC}$ & $\mathrm{CC}$ & $\mathrm{CC}$ & $\mathrm{CC}$ & $\mathrm{CC}$ & $\mathrm{CC}$ \\
\hline $\mathrm{CC}$ & $\mathrm{CC}$ & $\mathrm{CC}$ & $\mathrm{CC}$ & $\mathrm{CC}$ & $\mathrm{CC}$ & $\mathrm{CC}$ & $\mathrm{CC}$ & $\mathrm{CC}$ & $\mathrm{AC}$ & $\mathrm{AC}$ \\
\hline $\mathrm{CC}$ & $\mathrm{CC}$ & $\mathrm{CC}$ & $\mathrm{CC}$ & $\mathrm{CC}$ & $\mathrm{CC}$ & $\mathrm{CC}$ & $\mathrm{CC}$ & $\mathrm{CC}$ & $\mathrm{CC}$ & $\mathrm{CC}$ \\
\hline $\mathrm{CC}$ & $\mathrm{CC}$ & $\mathrm{CC}$ & $\mathrm{CC}$ & $\mathrm{CC}$ & $\mathrm{CC}$ & $\mathrm{CC}$ & $\mathrm{CC}$ & $\mathrm{CC}$ & $\mathrm{CC}$ & $\mathrm{CC}$ \\
\hline $\mathrm{CC}$ & $\mathrm{CC}$ & $\mathrm{CC}$ & $\mathrm{CC}$ & $\mathrm{CC}$ & $\mathrm{CC}$ & $\mathrm{CC}$ & $\mathrm{CC}$ & $\mathrm{CC}$ & $\mathrm{CC}$ & $\mathrm{CC}$ \\
\hline $\mathrm{CC}$ & $\mathrm{CC}$ & $\mathrm{CC}$ & $\mathrm{CC}$ & $\mathrm{CC}$ & $\mathrm{CC}$ & $\mathrm{CC}$ & $\mathrm{CC}$ & $\mathrm{CC}$ & $\mathrm{CC}$ & $\mathrm{CC}$ \\
\hline $\mathrm{CC}$ & $\mathrm{CC}$ & $\mathrm{CC}$ & $\mathrm{CC}$ & $\mathrm{CC}$ & $\mathrm{CC}$ & $\mathrm{CC}$ & $\mathrm{CC}$ & $\mathrm{CC}$ & $\mathrm{CC}$ & \\
\hline GDsnp02002 & 13 & \multicolumn{2}{|c|}{11871375} & $\mathrm{TT}$ & $\mathrm{TT}$ & $\mathrm{TT}$ & $\mathrm{TT}$ & $\mathrm{TT}$ & $\mathrm{TT}$ & TT \\
\hline $\mathrm{TT}$ & $\mathrm{TT}$ & TT & $\mathrm{AT}$ & $\mathrm{TT}$ & $\mathrm{TT}$ & AT & $\mathrm{AT}$ & $\mathrm{AT}$ & $\mathrm{TT}$ & AT \\
\hline $\mathrm{TT}$ & $\mathrm{AT}$ & AT & $\mathrm{TT}$ & $\mathrm{AT}$ & $\mathrm{AT}$ & $\mathrm{TT}$ & AT & $\mathrm{AT}$ & $\mathrm{TT}$ & AT \\
\hline AT & $\mathrm{AT}$ & $\mathrm{TT}$ & TT & $\mathrm{AT}$ & $\mathrm{AT}$ & AT & $\mathrm{AT}$ & TT & $\mathrm{AT}$ & AT \\
\hline $\mathrm{TT}$ & $\mathrm{AT}$ & TT & $\mathrm{AT}$ & $\mathrm{AT}$ & $\mathrm{AT}$ & $\mathrm{AT}$ & $\mathrm{TT}$ & $\mathrm{TT}$ & $\mathrm{AT}$ & AT \\
\hline $\mathrm{TT}$ & $\mathrm{AT}$ & AT & $\mathrm{AT}$ & $\mathrm{TT}$ & $\mathrm{TT}$ & $\mathrm{TT}$ & $\mathrm{TT}$ & $\mathrm{AT}$ & $\mathrm{AT}$ & TT \\
\hline $\mathrm{TT}$ & $\mathrm{AT}$ & $\mathrm{TT}$ & $\mathrm{TT}$ & $\mathrm{TT}$ & $\mathrm{TT}$ & $\mathrm{AT}$ & AT & $\mathrm{AT}$ & $\mathrm{AT}$ & TT \\
\hline AT & $\mathrm{TT}$ & AT & $\mathrm{TT}$ & $\mathrm{AT}$ & $\mathrm{AT}$ & $\mathrm{AT}$ & $\mathrm{TT}$ & $\mathrm{AT}$ & AT & AT \\
\hline AT & AT & AT & TT & $\mathrm{AT}$ & AT & TT & AT & TT & $\mathrm{AT}$ & \\
\hline GDsnp00340 & 13 & \multicolumn{2}{|c|}{13978602} & $\mathrm{AA}$ & GG & $\mathrm{AA}$ & $\mathrm{AA}$ & $\mathrm{AG}$ & $\mathrm{AG}$ & $\mathrm{AG}$ \\
\hline GG & $\mathrm{AA}$ & $\mathrm{AA}$ & GG & $\mathrm{AA}$ & $\mathrm{AG}$ & AA & $\mathrm{AG}$ & $\mathrm{AG}$ & GG & $\mathrm{AA}$ \\
\hline $\mathrm{AG}$ & $\mathrm{AG}$ & AA & $\mathrm{AG}$ & $\mathrm{AG}$ & $\mathrm{AA}$ & $\mathrm{AG}$ & $\mathrm{AA}$ & AA & $\mathrm{AG}$ & AA \\
\hline $\mathrm{AG}$ & $\mathrm{AA}$ & GG & AA & $\mathrm{AG}$ & $\mathrm{AA}$ & AA & $\mathrm{AG}$ & AA & $\mathrm{AG}$ & AA \\
\hline AG & $\mathrm{AA}$ & $\mathrm{AG}$ & $\mathrm{AA}$ & $\mathrm{AG}$ & $\mathrm{AG}$ & $\mathrm{AG}$ & $\mathrm{AG}$ & $\mathrm{AA}$ & AA & $\mathrm{AG}$ \\
\hline $\mathrm{AG}$ & $\mathrm{AA}$ & $\mathrm{AA}$ & $\mathrm{AG}$ & $\mathrm{AG}$ & GG & $\mathrm{AA}$ & $\mathrm{AG}$ & $\mathrm{AA}$ & $\mathrm{AG}$ & $\mathrm{AG}$ \\
\hline $\mathrm{AG}$ & $\mathrm{AA}$ & $\mathrm{AG}$ & $\mathrm{AG}$ & $\mathrm{AA}$ & $\mathrm{AG}$ & $\mathrm{AA}$ & GG & $\mathrm{AG}$ & $\mathrm{AG}$ & GG \\
\hline $\mathrm{AG}$ & $\mathrm{AG}$ & $\mathrm{AG}$ & GG & $\mathrm{AG}$ & $\mathrm{AG}$ & $\mathrm{AA}$ & $\mathrm{AG}$ & $\mathrm{AG}$ & $\mathrm{AA}$ & $\mathrm{AA}$ \\
\hline $\mathrm{AG}$ & $\mathrm{AA}$ & $\mathrm{AG}$ & $\mathrm{AG}$ & $\mathrm{AA}$ & $\mathrm{AA}$ & $\mathrm{AG}$ & $\mathrm{AA}$ & GG & AA & \\
\hline
\end{tabular}

Continued next page 
Supplemental Table 2. Continued.

\begin{tabular}{|c|c|c|c|c|c|c|c|c|c|c|}
\hline \multirow{3}{*}{$\begin{array}{l}\text { SNP name } \\
\text { GDsnp00890 } \\
\text { AA }\end{array}$} & \multicolumn{10}{|c|}{ Linkage group Physical location (bp) Individuals } \\
\hline & 13 & \multicolumn{2}{|c|}{15261515} & $\mathrm{AA}$ & $\mathrm{AA}$ & $\mathrm{AC}$ & AA & $\mathrm{AA}$ & AA & $\mathrm{CC}$ \\
\hline & AA & AA & AA & AA & AA & $\mathrm{AC}$ & $\mathrm{CC}$ & $\mathrm{CC}$ & AA & $\mathrm{AC}$ \\
\hline AA & $\mathrm{CC}$ & AA & $\mathrm{AA}$ & $\mathrm{CC}$ & $\mathrm{AC}$ & AA & $\mathrm{AC}$ & $\mathrm{AC}$ & AA & $\mathrm{AC}$ \\
\hline $\mathrm{CC}$ & $\mathrm{AC}$ & $\mathrm{CC}$ & $\mathrm{AA}$ & $\mathrm{CC}$ & $\mathrm{AC}$ & $\mathrm{AC}$ & $\mathrm{CC}$ & AA & AA & $\mathrm{AA}$ \\
\hline AA & $\mathrm{AC}$ & $\mathrm{AA}$ & $\mathrm{AC}$ & $\mathrm{CC}$ & $\mathrm{CC}$ & $\mathrm{CC}$ & $\mathrm{AA}$ & $\mathrm{AA}$ & $\mathrm{AC}$ & $\mathrm{CC}$ \\
\hline AA & $\mathrm{AC}$ & $\mathrm{AC}$ & $\mathrm{AA}$ & AA & $\mathrm{AA}$ & AA & AA & $\mathrm{AC}$ & $\mathrm{CC}$ & AA \\
\hline AA & $\mathrm{AC}$ & $\mathrm{AA}$ & $\mathrm{AA}$ & $\mathrm{AA}$ & $\mathrm{AA}$ & $\mathrm{AC}$ & $\mathrm{AA}$ & $\mathrm{CC}$ & $\mathrm{AC}$ & $\mathrm{AA}$ \\
\hline $\mathrm{CC}$ & $\mathrm{AA}$ & $\mathrm{CC}$ & $\mathrm{AA}$ & $\mathrm{AA}$ & $\mathrm{AA}$ & $\mathrm{AC}$ & AA & $\mathrm{CC}$ & $\mathrm{AC}$ & $\mathrm{CC}$ \\
\hline $\mathrm{AC}$ & $\mathrm{AC}$ & $\mathrm{CC}$ & AA & $\mathrm{AC}$ & $\mathrm{AC}$ & AA & $\mathrm{AC}$ & AA & AA & \\
\hline GDsnp01844 & 13 & \multicolumn{2}{|c|}{16496766} & $\mathrm{AG}$ & $\mathrm{AA}$ & $\mathrm{AG}$ & GG & $\mathrm{AG}$ & $\mathrm{AG}$ & AA \\
\hline AA & GG & $\mathrm{AG}$ & $\mathrm{AA}$ & GG & $\mathrm{AA}$ & $\mathrm{AG}$ & $\mathrm{AA}$ & $\mathrm{AA}$ & AA & $\mathrm{AG}$ \\
\hline AG & $\mathrm{AA}$ & $\mathrm{AG}$ & $\mathrm{AG}$ & $\mathrm{AA}$ & $\mathrm{AG}$ & $\mathrm{AG}$ & $\mathrm{AG}$ & $\mathrm{AG}$ & $\mathrm{AG}$ & $\mathrm{AG}$ \\
\hline AA & $\mathrm{AG}$ & $\mathrm{AA}$ & $\mathrm{AG}$ & $\mathrm{AA}$ & $\mathrm{AG}$ & $\mathrm{AG}$ & $\mathrm{AA}$ & GG & AA & GG \\
\hline AG & $\mathrm{AG}$ & $\mathrm{AG}$ & $\mathrm{AG}$ & $\mathrm{AA}$ & AA & AA & - & GG & $\mathrm{AG}$ & $\mathrm{AG}$ \\
\hline $\mathrm{AG}$ & $\mathrm{AG}$ & $\mathrm{AG}$ & $\mathrm{AA}$ & $\mathrm{AG}$ & $\mathrm{AA}$ & GG & $\mathrm{AG}$ & $\mathrm{AG}$ & AA & $\mathrm{AG}$ \\
\hline $\mathrm{AG}$ & $\mathrm{AG}$ & $\mathrm{AG}$ & $\mathrm{AG}$ & GG & $\mathrm{AG}$ & $\mathrm{AG}$ & $\mathrm{AA}$ & $\mathrm{AA}$ & $\mathrm{AG}$ & $\mathrm{AA}$ \\
\hline $\mathrm{AG}$ & $\mathrm{AG}$ & $\mathrm{AA}$ & $\mathrm{AA}$ & $\mathrm{AG}$ & $\mathrm{AG}$ & $\mathrm{AG}$ & $\mathrm{AG}$ & $\mathrm{AA}$ & $\mathrm{AG}$ & $\mathrm{AA}$ \\
\hline $\mathrm{AG}$ & $\mathrm{AG}$ & $\mathrm{AA}$ & $\mathrm{AG}$ & $\mathrm{AG}$ & $\mathrm{AG}$ & $\mathrm{AG}$ & $\mathrm{AG}$ & $\mathrm{AA}$ & $\mathrm{AG}$ & \\
\hline GDsnp01334 & 13 & \multicolumn{2}{|c|}{17723250} & $\mathrm{AG}$ & $\mathrm{AG}$ & $\mathrm{AG}$ & GG & $\mathrm{AG}$ & $\mathrm{AG}$ & $\mathrm{AA}$ \\
\hline AA & GG & $\mathrm{AG}$ & $\mathrm{AA}$ & GG & $\mathrm{AG}$ & $\mathrm{AG}$ & $\mathrm{AA}$ & $\mathrm{AA}$ & AA & $\mathrm{AG}$ \\
\hline GG & $\mathrm{AG}$ & GG & GG & $\mathrm{AA}$ & GG & $\mathrm{AG}$ & GG & GG & $\mathrm{AG}$ & GG \\
\hline AA & GG & AA & $\mathrm{AG}$ & $\mathrm{AG}$ & GG & $\mathrm{AG}$ & $\mathrm{AG}$ & GG & AA & $\mathrm{AG}$ \\
\hline GG & GG & $\mathrm{AG}$ & GG & $\mathrm{AG}$ & $\mathrm{AG}$ & - & - & GG & GG & GG \\
\hline GG & GG & GG & $\mathrm{AG}$ & GG & $\mathrm{AG}$ & GG & $\mathrm{AA}$ & GG & $\mathrm{AG}$ & GG \\
\hline GG & GG & GG & $\mathrm{AG}$ & GG & $\mathrm{AG}$ & $\mathrm{AG}$ & $\mathrm{AG}$ & $\mathrm{AG}$ & GG & $\mathrm{AG}$ \\
\hline $\mathrm{AG}$ & $\mathrm{AA}$ & $\mathrm{AG}$ & $\mathrm{AG}$ & $\mathrm{AG}$ & GG & GG & $\mathrm{AG}$ & $\mathrm{AA}$ & GG & $\mathrm{AA}$ \\
\hline GG & $\mathrm{AG}$ & $\mathrm{AA}$ & GG & $\mathrm{AA}$ & GG & $\mathrm{AG}$ & GG & $\mathrm{AG}$ & GG & \\
\hline GDsnp00532 & 13 & \multicolumn{2}{|c|}{18126371} & GG & GG & $\mathrm{AG}$ & GG & GG & GG & $\mathrm{AG}$ \\
\hline AG & GG & $\mathrm{AG}$ & GG & GG & GG & $\mathrm{AG}$ & $\mathrm{AG}$ & $\mathrm{AG}$ & GG & $\mathrm{AG}$ \\
\hline GG & GG & GG & $\mathrm{AG}$ & $\mathrm{AG}$ & GG & $\mathrm{AG}$ & GG & GG & GG & GG \\
\hline $\mathrm{AG}$ & GG & $\mathrm{AG}$ & GG & GG & GG & $\mathrm{AG}$ & GG & GG & GG & GG \\
\hline GG & GG & GG & GG & GG & GG & GG & $\mathrm{AG}$ & GG & GG & GG \\
\hline GG & GG & GG & GG & GG & GG & GG & GG & GG & GG & GG \\
\hline GG & GG & GG & GG & GG & GG & $\mathrm{AG}$ & $\mathrm{AG}$ & GG & GG & GG \\
\hline $\mathrm{AG}$ & $\mathrm{AG}$ & GG & GG & GG & GG & GG & GG & GG & GG & $\mathrm{AG}$ \\
\hline GG & $\mathrm{AG}$ & $\mathrm{AG}$ & GG & $\mathrm{AG}$ & GG & GG & GG & GG & GG & \\
\hline GDsnp00770 & 13 & \multicolumn{2}{|c|}{20060836} & $\mathrm{CG}$ & $\mathrm{CC}$ & $\mathrm{CG}$ & $\mathrm{CG}$ & GG & $\mathrm{CG}$ & $\mathrm{CC}$ \\
\hline $\mathrm{CC}$ & GG & $\mathrm{CG}$ & $\mathrm{CC}$ & GG & $\mathrm{CG}$ & CG & $\mathrm{CC}$ & $\mathrm{CG}$ & $\mathrm{CC}$ & CG \\
\hline $\mathrm{CG}$ & $\mathrm{CC}$ & GG & $\mathrm{CG}$ & $\mathrm{CC}$ & $\mathrm{CG}$ & $\mathrm{CG}$ & $\mathrm{CG}$ & $\mathrm{CG}$ & $\mathrm{CC}$ & $\mathrm{CG}$ \\
\hline $\mathrm{CC}$ & $\mathrm{CG}$ & $\mathrm{CC}$ & $\mathrm{CG}$ & $\mathrm{CC}$ & $\mathrm{CG}$ & $\mathrm{CG}$ & $\mathrm{CC}$ & $\mathrm{CG}$ & $\mathrm{CC}$ & $\mathrm{CG}$ \\
\hline GG & $\mathrm{CC}$ & $\mathrm{CC}$ & $\mathrm{CG}$ & $\mathrm{CC}$ & $\mathrm{CC}$ & $\mathrm{CC}$ & $\mathrm{CC}$ & $\mathrm{CG}$ & $\mathrm{CG}$ & $\mathrm{CG}$ \\
\hline $\mathrm{CG}$ & $\mathrm{CG}$ & $\mathrm{CG}$ & $\mathrm{CG}$ & $\mathrm{CG}$ & $\mathrm{CC}$ & $\mathrm{CG}$ & $\mathrm{CC}$ & $\mathrm{CG}$ & $\mathrm{CC}$ & $\mathrm{CG}$ \\
\hline $\mathrm{CG}$ & $\mathrm{CG}$ & $\mathrm{CG}$ & $\mathrm{CC}$ & $\mathrm{CG}$ & $\mathrm{CC}$ & $\mathrm{CG}$ & $\mathrm{CG}$ & $\mathrm{CC}$ & $\mathrm{CG}$ & $\mathrm{CC}$ \\
\hline CG & $\mathrm{CC}$ & CG & $\mathrm{CC}$ & CG & CG & CG & CG & CG & CG & $\mathrm{CC}$ \\
\hline CG & CG & $\mathrm{CC}$ & CG & $\mathrm{CC}$ & CG & $\mathrm{CC}$ & CG & CG & CG & \\
\hline GDsnp00744 & 13 & \multicolumn{2}{|c|}{20669641} & $\mathrm{AT}$ & $\mathrm{AA}$ & $\mathrm{AT}$ & AT & $\mathrm{AT}$ & $\mathrm{AT}$ & $\mathrm{AA}$ \\
\hline AA & $\mathrm{TT}$ & $\mathrm{AT}$ & $\mathrm{AA}$ & $\mathrm{TT}$ & $\mathrm{AT}$ & $\mathrm{AT}$ & $\mathrm{AA}$ & $\mathrm{AA}$ & AA & AT \\
\hline AT & $\mathrm{AA}$ & $\mathrm{AT}$ & $\mathrm{AT}$ & $\mathrm{AA}$ & $\mathrm{AT}$ & AT & AT & $\mathrm{AT}$ & $\mathrm{AT}$ & AT \\
\hline AA & $\mathrm{AT}$ & $\mathrm{AA}$ & $\mathrm{AT}$ & $\mathrm{AA}$ & $\mathrm{AT}$ & AT & $\mathrm{AA}$ & TT & $\mathrm{AA}$ & AT \\
\hline TT & $\mathrm{AT}$ & AA & $\mathrm{AT}$ & $\mathrm{AA}$ & AA & AA & $\mathrm{AA}$ & $\mathrm{AT}$ & $\mathrm{AT}$ & TT \\
\hline AT & $\mathrm{AT}$ & $\mathrm{AT}$ & $\mathrm{AA}$ & $\mathrm{AT}$ & $\mathrm{AA}$ & TT & $\mathrm{AA}$ & $\mathrm{TT}$ & AA & AT \\
\hline AT & $\mathrm{AT}$ & $\mathrm{AT}$ & $\mathrm{AT}$ & $\mathrm{TT}$ & $\mathrm{AT}$ & $\mathrm{AT}$ & $\mathrm{AT}$ & $\mathrm{AA}$ & $\mathrm{TT}$ & $\mathrm{AA}$ \\
\hline AT & $\mathrm{AA}$ & AT & $\mathrm{AA}$ & $\mathrm{AT}$ & AT & $\mathrm{AT}$ & $\mathrm{AT}$ & $\mathrm{AA}$ & $\mathrm{AT}$ & $\mathrm{AA}$ \\
\hline AT & $\mathrm{AT}$ & $\mathrm{AA}$ & - & $\mathrm{AA}$ & $\mathrm{AT}$ & AT & $\mathrm{AT}$ & $\mathrm{AA}$ & $\mathrm{AT}$ & \\
\hline
\end{tabular}


Supplemental Table 2. Continued.

\begin{tabular}{|c|c|c|c|c|c|c|c|c|c|c|}
\hline \multirow{3}{*}{$\begin{array}{l}\text { SNP name } \\
\text { GDsnp00164 } \\
\text { AG }\end{array}$} & \multicolumn{10}{|c|}{ Linkage group Physical location (bp) Individuals } \\
\hline & 13 & \multicolumn{2}{|c|}{22891166} & $\mathrm{AG}$ & GG & $\mathrm{AG}$ & $\mathrm{AG}$ & $\mathrm{AG}$ & $\mathrm{AG}$ & GG \\
\hline & AA & $\mathrm{AG}$ & GG & AA & $\mathrm{AG}$ & $\mathrm{AG}$ & GG & GG & GG & $\mathrm{AG}$ \\
\hline $\mathrm{AG}$ & GG & $\mathrm{AG}$ & $\mathrm{AG}$ & GG & $\mathrm{AG}$ & $\mathrm{AG}$ & $\mathrm{AG}$ & $\mathrm{AG}$ & $\mathrm{AG}$ & $\mathrm{AG}$ \\
\hline GG & $\mathrm{AG}$ & GG & $\mathrm{AG}$ & GG & $\mathrm{AG}$ & AG & GG & $\mathrm{AA}$ & GG & $\mathrm{AG}$ \\
\hline AA & $\mathrm{AG}$ & GG & $\mathrm{AG}$ & GG & GG & $\mathrm{AG}$ & GG & $\mathrm{AG}$ & $\mathrm{AG}$ & $\mathrm{AA}$ \\
\hline $\mathrm{AG}$ & $\mathrm{AG}$ & $\mathrm{AG}$ & GG & $\mathrm{AG}$ & GG & AA & GG & $\mathrm{AA}$ & GG & $\mathrm{AG}$ \\
\hline $\mathrm{AG}$ & $\mathrm{AG}$ & $\mathrm{AG}$ & $\mathrm{AG}$ & $\mathrm{AA}$ & $\mathrm{AG}$ & $\mathrm{AG}$ & $\mathrm{AG}$ & GG & $\mathrm{AA}$ & GG \\
\hline $\mathrm{AG}$ & GG & $\mathrm{AG}$ & GG & $\mathrm{AG}$ & $\mathrm{AG}$ & $\mathrm{AG}$ & $\mathrm{AG}$ & GG & $\mathrm{AG}$ & GG \\
\hline $\mathrm{AG}$ & $\mathrm{AG}$ & GG & $\mathrm{AG}$ & GG & $\mathrm{AG}$ & $\mathrm{AG}$ & $\mathrm{AG}$ & GG & $\mathrm{AG}$ & \\
\hline GDsnp02452 & 13 & \multicolumn{2}{|c|}{26647908} & $\mathrm{AG}$ & $\mathrm{AG}$ & $\mathrm{AA}$ & $\mathrm{AG}$ & $\mathrm{AA}$ & $\mathrm{AG}$ & $\mathrm{AG}$ \\
\hline $\mathrm{AG}$ & $\mathrm{AA}$ & $\mathrm{AA}$ & GG & $\mathrm{AA}$ & $\mathrm{AA}$ & AA & $\mathrm{AG}$ & GG & GG & AA \\
\hline AA & $\mathrm{AG}$ & $\mathrm{AG}$ & $\mathrm{AA}$ & $\mathrm{AG}$ & $\mathrm{AA}$ & $\mathrm{AA}$ & $\mathrm{AA}$ & $\mathrm{AA}$ & $\mathrm{AG}$ & $\mathrm{AA}$ \\
\hline $\mathrm{AG}$ & $\mathrm{AA}$ & $\mathrm{AG}$ & $\mathrm{AG}$ & $\mathrm{AG}$ & $\mathrm{AA}$ & AA & $\mathrm{AG}$ & $\mathrm{AA}$ & $\mathrm{AG}$ & $\mathrm{AG}$ \\
\hline AA & $\mathrm{AG}$ & $\mathrm{AG}$ & $\mathrm{AA}$ & $\mathrm{AG}$ & $\mathrm{AG}$ & - & - & $\mathrm{AA}$ & $\mathrm{AA}$ & $\mathrm{AA}$ \\
\hline AA & $\mathrm{AA}$ & $\mathrm{AA}$ & GG & $\mathrm{AA}$ & $\mathrm{AG}$ & $\mathrm{AA}$ & GG & $\mathrm{AA}$ & $\mathrm{AG}$ & AA \\
\hline AA & $\mathrm{AA}$ & $\mathrm{AA}$ & $\mathrm{AG}$ & $\mathrm{AA}$ & $\mathrm{AG}$ & $\mathrm{AA}$ & $\mathrm{AA}$ & $\mathrm{AG}$ & $\mathrm{AA}$ & $\mathrm{AG}$ \\
\hline AA & $\mathrm{AG}$ & $\mathrm{AA}$ & $\mathrm{AG}$ & $\mathrm{AG}$ & $\mathrm{AA}$ & AA & $\mathrm{AG}$ & $\mathrm{AG}$ & $\mathrm{AA}$ & $\mathrm{AG}$ \\
\hline AA & $\mathrm{AA}$ & $\mathrm{AG}$ & $\mathrm{AA}$ & $\mathrm{AG}$ & $\mathrm{AA}$ & $\mathrm{AG}$ & AA & $\mathrm{AG}$ & AA & \\
\hline GDsnp00350 & 13 & \multicolumn{2}{|c|}{27841734} & $\mathrm{AG}$ & $\mathrm{AG}$ & $\mathrm{AA}$ & $\mathrm{AG}$ & $\mathrm{AA}$ & $\mathrm{AG}$ & $\mathrm{AG}$ \\
\hline $\mathrm{AG}$ & $\mathrm{AA}$ & AA & GG & $\mathrm{AA}$ & AA & AA & $\mathrm{AG}$ & GG & GG & $\mathrm{AA}$ \\
\hline AA & $\mathrm{AG}$ & $\mathrm{AA}$ & $\mathrm{AA}$ & $\mathrm{AG}$ & $\mathrm{AA}$ & $\mathrm{AA}$ & $\mathrm{AA}$ & $\mathrm{AA}$ & $\mathrm{AG}$ & $\mathrm{AA}$ \\
\hline $\mathrm{AG}$ & $\mathrm{AA}$ & $\mathrm{AG}$ & $\mathrm{AG}$ & $\mathrm{AG}$ & $\mathrm{AA}$ & $\mathrm{AA}$ & $\mathrm{AG}$ & $\mathrm{AA}$ & $\mathrm{AG}$ & $\mathrm{AG}$ \\
\hline AA & $\mathrm{AG}$ & $\mathrm{AG}$ & $\mathrm{AA}$ & $\mathrm{AG}$ & $\mathrm{AG}$ & $\mathrm{AG}$ & - & $\mathrm{AA}$ & $\mathrm{AA}$ & $\mathrm{AA}$ \\
\hline AA & $\mathrm{AA}$ & AA & $\mathrm{AG}$ & $\mathrm{AA}$ & $\mathrm{AG}$ & AA & GG & $\mathrm{AA}$ & $\mathrm{AG}$ & $\mathrm{AA}$ \\
\hline AA & $\mathrm{AA}$ & AA & $\mathrm{AG}$ & $\mathrm{AA}$ & $\mathrm{AG}$ & $\mathrm{AG}$ & AA & $\mathrm{AG}$ & AA & $\mathrm{AG}$ \\
\hline AA & $\mathrm{AG}$ & AA & $\mathrm{AG}$ & $\mathrm{AG}$ & $\mathrm{AA}$ & AA & $\mathrm{AG}$ & $\mathrm{AA}$ & AA & $\mathrm{AG}$ \\
\hline AA & $\mathrm{AA}$ & $\mathrm{AG}$ & $\mathrm{AA}$ & $\mathrm{AG}$ & $\mathrm{AA}$ & GG & $\mathrm{AA}$ & $\mathrm{AA}$ & AA & \\
\hline GDsnp00748 & 13 & \multicolumn{2}{|c|}{32753515} & $\mathrm{TT}$ & $\mathrm{AT}$ & $\mathrm{AT}$ & $\mathrm{TT}$ & $\mathrm{TT}$ & $\mathrm{TT}$ & AT \\
\hline $\mathrm{TT}$ & $\mathrm{TT}$ & $\mathrm{AT}$ & $\mathrm{TT}$ & $\mathrm{TT}$ & $\mathrm{AT}$ & $\mathrm{AT}$ & AT & $\mathrm{TT}$ & $\mathrm{TT}$ & AT \\
\hline AT & $\mathrm{AT}$ & $\mathrm{TT}$ & $\mathrm{AT}$ & $\mathrm{AT}$ & $\mathrm{AT}$ & $\mathrm{TT}$ & $\mathrm{AT}$ & $\mathrm{AT}$ & $\mathrm{AT}$ & AT \\
\hline AT & $\mathrm{AT}$ & $\mathrm{AT}$ & $\mathrm{TT}$ & AT & $\mathrm{AT}$ & AT & $\mathrm{AT}$ & $\mathrm{AT}$ & $\mathrm{TT}$ & AT \\
\hline $\mathrm{TT}$ & $\mathrm{AT}$ & $\mathrm{AT}$ & $\mathrm{AT}$ & AT & $\mathrm{AT}$ & $\mathrm{AT}$ & $\mathrm{AT}$ & $\mathrm{AT}$ & $\mathrm{AT}$ & AT \\
\hline AT & $\mathrm{AT}$ & $\mathrm{AT}$ & $\mathrm{TT}$ & $\mathrm{AT}$ & $\mathrm{AT}$ & $\mathrm{AT}$ & $\mathrm{TT}$ & $\mathrm{AT}$ & $\mathrm{AT}$ & AT \\
\hline AT & $\mathrm{AT}$ & $\mathrm{AT}$ & $\mathrm{AT}$ & $\mathrm{AT}$ & $\mathrm{AT}$ & $\mathrm{TT}$ & $\mathrm{TT}$ & $\mathrm{AT}$ & $\mathrm{AT}$ & AT \\
\hline AT & $\mathrm{AT}$ & $\mathrm{AT}$ & $\mathrm{AT}$ & TT & $\mathrm{AT}$ & AT & $\mathrm{TT}$ & $\mathrm{AT}$ & $\mathrm{AT}$ & AT \\
\hline AT & $\mathrm{AT}$ & AT & AT & $\mathrm{AT}$ & AT & $\mathrm{AT}$ & AT & $\mathrm{AT}$ & $\mathrm{AT}$ & \\
\hline GDsnp01199 & 13 & \multicolumn{2}{|c|}{33378164} & $\mathrm{AC}$ & AA & $\mathrm{AC}$ & $\mathrm{AC}$ & $\mathrm{CC}$ & $\mathrm{AC}$ & $\mathrm{AA}$ \\
\hline $\mathrm{AC}$ & $\mathrm{CC}$ & $\mathrm{AC}$ & $\mathrm{AA}$ & $\mathrm{CC}$ & $\mathrm{AC}$ & $\mathrm{AC}$ & AA & $\mathrm{AC}$ & AA & $\mathrm{AC}$ \\
\hline $\mathrm{AC}$ & $\mathrm{AA}$ & $\mathrm{AC}$ & $\mathrm{AC}$ & $\mathrm{AA}$ & $\mathrm{AC}$ & $\mathrm{CC}$ & $\mathrm{AC}$ & $\mathrm{AC}$ & AA & $\mathrm{AC}$ \\
\hline AA & $\mathrm{AC}$ & $\mathrm{AA}$ & $\mathrm{AC}$ & $\mathrm{AA}$ & $\mathrm{AC}$ & $\mathrm{AC}$ & $\mathrm{AA}$ & $\mathrm{AC}$ & AA & $\mathrm{AC}$ \\
\hline $\mathrm{CC}$ & $\mathrm{AA}$ & $\mathrm{AA}$ & $\mathrm{AC}$ & $\mathrm{AA}$ & $\mathrm{AA}$ & $\mathrm{AA}$ & $\mathrm{AA}$ & $\mathrm{AC}$ & $\mathrm{AC}$ & $\mathrm{AC}$ \\
\hline $\mathrm{AC}$ & $\mathrm{AC}$ & $\mathrm{AC}$ & $\mathrm{AA}$ & $\mathrm{AC}$ & $\mathrm{AA}$ & $\mathrm{AC}$ & AA & $\mathrm{AC}$ & AA & $\mathrm{AC}$ \\
\hline $\mathrm{AC}$ & $\mathrm{AC}$ & $\mathrm{AC}$ & $\mathrm{AA}$ & $\mathrm{AC}$ & $\mathrm{AA}$ & $\mathrm{AC}$ & $\mathrm{CC}$ & $\mathrm{AA}$ & $\mathrm{AC}$ & $\mathrm{AA}$ \\
\hline $\mathrm{AC}$ & $\mathrm{AA}$ & $\mathrm{AC}$ & $\mathrm{AA}$ & $\mathrm{AC}$ & $\mathrm{AC}$ & $\mathrm{AC}$ & $\mathrm{AC}$ & $\mathrm{AC}$ & $\mathrm{AC}$ & $\mathrm{AA}$ \\
\hline $\mathrm{AC}$ & $\mathrm{AC}$ & AA & $\mathrm{AC}$ & $\mathrm{AA}$ & $\mathrm{AC}$ & AA & $\mathrm{AC}$ & $\mathrm{AC}$ & $\mathrm{AC}$ & \\
\hline GDsnp01888 & 14 & \multicolumn{2}{|c|}{232465} & $\mathrm{AA}$ & $\mathrm{AA}$ & $\mathrm{AA}$ & $\mathrm{AA}$ & $\mathrm{AA}$ & $\mathrm{AA}$ & $\mathrm{AA}$ \\
\hline AA & $\mathrm{AG}$ & $\mathrm{AA}$ & $\mathrm{AA}$ & $\mathrm{AA}$ & $\mathrm{AA}$ & AA & $\mathrm{AA}$ & $\mathrm{AA}$ & $\mathrm{AA}$ & $\mathrm{AA}$ \\
\hline AA & $\mathrm{AA}$ & AA & $\mathrm{AA}$ & $\mathrm{AA}$ & $\mathrm{AA}$ & $\mathrm{AG}$ & AA & $\mathrm{AA}$ & AA & AA \\
\hline AA & $\mathrm{AA}$ & $\mathrm{AA}$ & $\mathrm{AA}$ & $\mathrm{AA}$ & $\mathrm{AA}$ & AA & $\mathrm{AA}$ & $\mathrm{AA}$ & $\mathrm{AA}$ & $\mathrm{AA}$ \\
\hline AA & $\mathrm{AA}$ & $\mathrm{AA}$ & $\mathrm{AA}$ & $\mathrm{AA}$ & $\mathrm{AA}$ & AA & $\mathrm{AA}$ & $\mathrm{AA}$ & AA & $\mathrm{AA}$ \\
\hline AA & $\mathrm{AA}$ & $\mathrm{AA}$ & $\mathrm{AA}$ & $\mathrm{AA}$ & $\mathrm{AA}$ & AA & $\mathrm{AA}$ & $\mathrm{AA}$ & AA & $\mathrm{AA}$ \\
\hline AA & $\mathrm{AA}$ & $\mathrm{AA}$ & $\mathrm{AA}$ & $\mathrm{AA}$ & $\mathrm{AA}$ & $\mathrm{AA}$ & $\mathrm{AA}$ & $\mathrm{AA}$ & $\mathrm{AA}$ & $\mathrm{AA}$ \\
\hline AA & $\mathrm{AA}$ & $\mathrm{AA}$ & $\mathrm{AA}$ & $\mathrm{AA}$ & $\mathrm{AA}$ & $\mathrm{AA}$ & $\mathrm{AA}$ & $\mathrm{AA}$ & $\mathrm{AA}$ & $\mathrm{AA}$ \\
\hline AA & $\mathrm{AA}$ & $\mathrm{AA}$ & $\mathrm{AA}$ & $\mathrm{AA}$ & $\mathrm{AA}$ & AA & $\mathrm{AA}$ & $\mathrm{AA}$ & AA & \\
\hline
\end{tabular}

Continued next page 
Supplemental Table 2. Continued.

\begin{tabular}{|c|c|c|c|c|c|c|c|c|c|c|}
\hline \multirow{3}{*}{$\begin{array}{l}\text { SNP name } \\
\text { GDsnp00207 } \\
\text { AG }\end{array}$} & \multicolumn{10}{|c|}{ Linkage group Physical location (bp) Individuals } \\
\hline & 14 & \multicolumn{2}{|c|}{901694} & $\mathrm{AA}$ & $\mathrm{AG}$ & AA & GG & AA & GG & $\mathrm{AG}$ \\
\hline & AA & $\mathrm{AG}$ & AA & GG & GG & $\mathrm{AG}$ & AG & GG & AA & $\mathrm{AG}$ \\
\hline $\mathrm{AG}$ & $\mathrm{AG}$ & GG & GG & $\mathrm{AA}$ & AA & AA & AA & AA & GG & AA \\
\hline AG & $\mathrm{AG}$ & $\mathrm{AG}$ & AA & $\mathrm{AG}$ & $\mathrm{AG}$ & GG & AG & $\mathrm{AG}$ & $\mathrm{AG}$ & $\mathrm{AA}$ \\
\hline GG & $\mathrm{AG}$ & GG & GG & $\mathrm{AA}$ & $\mathrm{AG}$ & GG & $\mathrm{AG}$ & GG & $\mathrm{AG}$ & GG \\
\hline GG & $\mathrm{AA}$ & GG & $\mathrm{AG}$ & $\mathrm{AG}$ & $\mathrm{AA}$ & $\mathrm{AG}$ & $\mathrm{AG}$ & GG & AA & AA \\
\hline $\mathrm{AA}$ & $\mathrm{AG}$ & $\mathrm{AG}$ & $\mathrm{AA}$ & GG & $\mathrm{AG}$ & GG & AA & GG & $\mathrm{AG}$ & GG \\
\hline GG & $\mathrm{AA}$ & $\mathrm{AA}$ & GG & $\mathrm{AA}$ & $\mathrm{AG}$ & $\mathrm{AG}$ & GG & $\mathrm{AG}$ & $\mathrm{AA}$ & $\mathrm{AA}$ \\
\hline $\mathrm{AG}$ & $\mathrm{AG}$ & GG & $\mathrm{AG}$ & $\mathrm{AA}$ & $\mathrm{AG}$ & $\mathrm{AA}$ & $\mathrm{AG}$ & $\mathrm{AG}$ & AA & \\
\hline GDsnp01846 & 14 & \multicolumn{2}{|c|}{1922227} & $\mathrm{AA}$ & $\mathrm{AA}$ & $\mathrm{AA}$ & AA & $\mathrm{AA}$ & $\mathrm{AA}$ & $\mathrm{AA}$ \\
\hline AA & $\mathrm{AC}$ & $\mathrm{AA}$ & $\mathrm{AA}$ & $\mathrm{AA}$ & $\mathrm{AA}$ & AA & AA & AA & $\mathrm{AA}$ & AA \\
\hline AA & $\mathrm{AA}$ & $\mathrm{AA}$ & AA & $\mathrm{AA}$ & $\mathrm{AA}$ & $\mathrm{AC}$ & AA & AA & $\mathrm{AA}$ & $\mathrm{AA}$ \\
\hline AA & $\mathrm{AA}$ & $\mathrm{AA}$ & $\mathrm{AA}$ & $\mathrm{AA}$ & $\mathrm{AA}$ & AA & AA & $\mathrm{AA}$ & $\mathrm{AA}$ & $\mathrm{AA}$ \\
\hline $\mathrm{AA}$ & $\mathrm{AA}$ & $\mathrm{AA}$ & $\mathrm{AA}$ & $\mathrm{AA}$ & $\mathrm{AA}$ & $\mathrm{AA}$ & AA & $\mathrm{AA}$ & $\mathrm{AA}$ & $\mathrm{AA}$ \\
\hline $\mathrm{AA}$ & $\mathrm{AA}$ & $\mathrm{AA}$ & $\mathrm{AA}$ & $\mathrm{AA}$ & $\mathrm{AA}$ & $\mathrm{AA}$ & AA & $\mathrm{AA}$ & $\mathrm{AA}$ & AA \\
\hline AA & $\mathrm{AA}$ & $\mathrm{AA}$ & AA & $\mathrm{AA}$ & $\mathrm{AA}$ & $\mathrm{AA}$ & AA & AA & $\mathrm{AA}$ & $\mathrm{AA}$ \\
\hline AA & $\mathrm{AA}$ & $\mathrm{AA}$ & $\mathrm{AA}$ & $\mathrm{AA}$ & $\mathrm{AA}$ & AA & AA & $\mathrm{AA}$ & $\mathrm{AA}$ & $\mathrm{AA}$ \\
\hline AA & $\mathrm{AA}$ & AA & AA & $\mathrm{AA}$ & $\mathrm{AA}$ & AA & AA & AA & AA & \\
\hline GDsnp00248 & 14 & \multicolumn{2}{|c|}{3975756} & $\mathrm{CC}$ & $\mathrm{AC}$ & $\mathrm{CC}$ & AA & $\mathrm{CC}$ & $\mathrm{AA}$ & $\mathrm{AC}$ \\
\hline $\mathrm{AC}$ & $\mathrm{CC}$ & $\mathrm{AC}$ & $\mathrm{CC}$ & $\mathrm{AA}$ & $\mathrm{AA}$ & $\mathrm{AC}$ & $\mathrm{AC}$ & AA & $\mathrm{CC}$ & $\mathrm{AC}$ \\
\hline $\mathrm{AC}$ & $\mathrm{AC}$ & $\mathrm{AA}$ & $\mathrm{AA}$ & $\mathrm{CC}$ & $\mathrm{CC}$ & $\mathrm{CC}$ & $\mathrm{CC}$ & $\mathrm{CC}$ & $\mathrm{AA}$ & $\mathrm{CC}$ \\
\hline $\mathrm{AC}$ & $\mathrm{AC}$ & $\mathrm{AC}$ & $\mathrm{CC}$ & $\mathrm{AC}$ & $\mathrm{AC}$ & $\mathrm{AA}$ & $\mathrm{AC}$ & $\mathrm{AC}$ & AA & $\mathrm{AC}$ \\
\hline AA & $\mathrm{AC}$ & $\mathrm{AA}$ & $\mathrm{AA}$ & $\mathrm{CC}$ & $\mathrm{AC}$ & AA & $\mathrm{AC}$ & $\mathrm{AA}$ & $\mathrm{AC}$ & $\mathrm{AC}$ \\
\hline AA & $\mathrm{CC}$ & AA & AA & $\mathrm{AC}$ & $\mathrm{CC}$ & $\mathrm{AC}$ & $\mathrm{AC}$ & AA & $\mathrm{CC}$ & $\mathrm{CC}$ \\
\hline $\mathrm{CC}$ & $\mathrm{AC}$ & $\mathrm{CC}$ & $\mathrm{CC}$ & $\mathrm{AA}$ & $\mathrm{AC}$ & AA & $\mathrm{CC}$ & AA & $\mathrm{CC}$ & AA \\
\hline- & $\mathrm{CC}$ & $\mathrm{CC}$ & AA & $\mathrm{AC}$ & $\mathrm{AC}$ & $\mathrm{AC}$ & AA & $\mathrm{AC}$ & $\mathrm{CC}$ & $\mathrm{CC}$ \\
\hline $\mathrm{AC}$ & $\mathrm{AC}$ & $\mathrm{AC}$ & - & $\mathrm{CC}$ & $\mathrm{AC}$ & $\mathrm{CC}$ & $\mathrm{AC}$ & $\mathrm{AC}$ & $\mathrm{CC}$ & \\
\hline GDsnp02706 & 14 & \multicolumn{2}{|c|}{7488242} & $\mathrm{AG}$ & $\mathrm{AG}$ & $\mathrm{AG}$ & GG & $\mathrm{AG}$ & GG & $\mathrm{AG}$ \\
\hline GG & $\mathrm{AA}$ & GG & $\mathrm{AG}$ & GG & GG & GG & GG & GG & $\mathrm{AA}$ & $\mathrm{AG}$ \\
\hline GG & GG & GG & GG & $\mathrm{AG}$ & $\mathrm{AG}$ & AA & AA & $\mathrm{AA}$ & $\mathrm{AG}$ & $\mathrm{AG}$ \\
\hline $\mathrm{AG}$ & GG & $\mathrm{AG}$ & $\mathrm{AG}$ & $\mathrm{AG}$ & $\mathrm{AG}$ & GG & $\mathrm{AG}$ & $\mathrm{AG}$ & GG & $\mathrm{AG}$ \\
\hline GG & GG & $\mathrm{AG}$ & GG & $\mathrm{AG}$ & GG & GG & - & GG & GG & $\mathrm{AG}$ \\
\hline GG & $\mathrm{AG}$ & GG & GG & GG & GG & $\mathrm{AG}$ & GG & GG & $\mathrm{AG}$ & GG \\
\hline $\mathrm{AG}$ & GG & $\mathrm{AG}$ & $\mathrm{AA}$ & GG & $\mathrm{AG}$ & GG & $\mathrm{AG}$ & GG & $\mathrm{AA}$ & GG \\
\hline GG & $\mathrm{AA}$ & AA & GG & $\mathrm{AA}$ & $\mathrm{AG}$ & $\mathrm{AG}$ & GG & $\mathrm{AG}$ & $\mathrm{AG}$ & $\mathrm{AG}$ \\
\hline GG & GG & GG & GG & $\mathrm{AA}$ & GG & $\mathrm{AG}$ & $\mathrm{AG}$ & $\mathrm{AG}$ & $\mathrm{AG}$ & \\
\hline GDsnp02543 & 14 & \multicolumn{2}{|c|}{12104892} & $\mathrm{AA}$ & $\mathrm{AA}$ & AA & AA & AA & AA & $\mathrm{AA}$ \\
\hline AA & $\mathrm{AA}$ & AA & AA & $\mathrm{AA}$ & $\mathrm{AA}$ & AA & AA & AA & AA & AA \\
\hline AA & $\mathrm{AA}$ & - & AA & $\mathrm{AA}$ & AA & AA & AA & AA & AA & $\mathrm{AA}$ \\
\hline AA & $\mathrm{AA}$ & $\mathrm{AA}$ & $\mathrm{AA}$ & $\mathrm{AA}$ & $\mathrm{AA}$ & AA & AA & $\mathrm{AA}$ & AA & - \\
\hline AA & $\mathrm{AA}$ & $\mathrm{AA}$ & AA & $\mathrm{AA}$ & $\mathrm{AA}$ & $\mathrm{AA}$ & AA & AA & $\mathrm{AA}$ & $\mathrm{AA}$ \\
\hline AA & $\mathrm{AA}$ & AA & AA & $\mathrm{AA}$ & $\mathrm{AA}$ & AA & AA & AA & AA & AA \\
\hline AA & $\mathrm{AA}$ & AA & AA & $\mathrm{AA}$ & AA & AA & AA & AA & AA & $\mathrm{AA}$ \\
\hline $\mathrm{AA}$ & $\mathrm{AA}$ & $\mathrm{AA}$ & $\mathrm{AA}$ & $\mathrm{AA}$ & $\mathrm{AA}$ & $\mathrm{AA}$ & AA & $\mathrm{AA}$ & AA & $\mathrm{AA}$ \\
\hline $\mathrm{AA}$ & AA & AA & - & $\mathrm{AA}$ & AA & AA & AA & AA & AA & \\
\hline GDsnp00165 & 14 & \multicolumn{2}{|c|}{16134804} & $\mathrm{AC}$ & $\mathrm{CC}$ & $\mathrm{AC}$ & $\mathrm{CC}$ & $\mathrm{CC}$ & $\mathrm{CC}$ & $\mathrm{CC}$ \\
\hline $\mathrm{AC}$ & $\mathrm{CC}$ & $\mathrm{CC}$ & $\mathrm{AC}$ & $\mathrm{CC}$ & $\mathrm{CC}$ & $\mathrm{AC}$ & $\mathrm{CC}$ & $\mathrm{AC}$ & $\mathrm{CC}$ & $\mathrm{CC}$ \\
\hline $\mathrm{AC}$ & $\mathrm{CC}$ & $\mathrm{AC}$ & $\mathrm{CC}$ & $\mathrm{AC}$ & $\mathrm{AC}$ & $\mathrm{CC}$ & $\mathrm{CC}$ & $\mathrm{CC}$ & $\mathrm{CC}$ & $\mathrm{AC}$ \\
\hline $\mathrm{AC}$ & $\mathrm{CC}$ & $\mathrm{CC}$ & $\mathrm{CC}$ & $\mathrm{CC}$ & $\mathrm{CC}$ & $\mathrm{CC}$ & $\mathrm{CC}$ & $\mathrm{CC}$ & $\mathrm{AC}$ & - \\
\hline $\mathrm{CC}$ & $\mathrm{CC}$ & $\mathrm{CC}$ & $\mathrm{CC}$ & $\mathrm{CC}$ & $\mathrm{AC}$ & $\mathrm{CC}$ & $\mathrm{AC}$ & $\mathrm{CC}$ & $\mathrm{AC}$ & $\mathrm{CC}$ \\
\hline $\mathrm{CC}$ & $\mathrm{AC}$ & $\mathrm{CC}$ & $\mathrm{AC}$ & $\mathrm{AC}$ & $\mathrm{AC}$ & $\mathrm{CC}$ & $\mathrm{AC}$ & $\mathrm{CC}$ & $\mathrm{AC}$ & $\mathrm{AC}$ \\
\hline $\mathrm{AC}$ & $\mathrm{CC}$ & $\mathrm{AC}$ & $\mathrm{CC}$ & $\mathrm{CC}$ & $\mathrm{CC}$ & $\mathrm{CC}$ & $\mathrm{AC}$ & $\mathrm{AC}$ & $\mathrm{CC}$ & $\mathrm{CC}$ \\
\hline $\mathrm{CC}$ & $\mathrm{CC}$ & $\mathrm{CC}$ & $\mathrm{CC}$ & $\mathrm{AC}$ & $\mathrm{CC}$ & $\mathrm{CC}$ & $\mathrm{CC}$ & $\mathrm{CC}$ & $\mathrm{CC}$ & $\mathrm{AC}$ \\
\hline $\mathrm{CC}$ & $\mathrm{AC}$ & $\mathrm{AC}$ & $\mathrm{AC}$ & $\mathrm{CC}$ & $\mathrm{CC}$ & $\mathrm{CC}$ & $\mathrm{CC}$ & $\mathrm{CC}$ & $\mathrm{AC}$ & \\
\hline
\end{tabular}


Supplemental Table 2. Continued.

\begin{tabular}{|c|c|c|c|c|c|c|c|c|c|c|}
\hline \multirow{3}{*}{$\begin{array}{l}\text { SNP name } \\
\text { GDsnp00062 } \\
\text { AA }\end{array}$} & \multicolumn{10}{|c|}{ Linkage group Physical location (bp) Individuals } \\
\hline & 14 & \multicolumn{2}{|c|}{19851050} & $\mathrm{AA}$ & $\mathrm{AA}$ & AA & AA & $\mathrm{AA}$ & AA & $\mathrm{AA}$ \\
\hline & AA & $\mathrm{AC}$ & AA & AA & AA & AA & AA & AA & AA & AA \\
\hline AA & AA & $\mathrm{AC}$ & $\mathrm{AA}$ & AA & AA & AA & AA & AA & AA & AA \\
\hline AA & AA & AA & $\mathrm{AA}$ & AA & AA & AA & AA & AA & AA & $\mathrm{AC}$ \\
\hline AA & $\mathrm{AA}$ & $\mathrm{AA}$ & $\mathrm{AA}$ & $\mathrm{AA}$ & $\mathrm{AA}$ & $\mathrm{AA}$ & $\mathrm{AC}$ & $\mathrm{AA}$ & $\mathrm{AA}$ & $\mathrm{AA}$ \\
\hline AA & $\mathrm{AA}$ & AA & $\mathrm{AA}$ & AA & $\mathrm{AA}$ & AA & AA & $\mathrm{AA}$ & AA & AA \\
\hline $\mathrm{AA}$ & $\mathrm{AA}$ & $\mathrm{AA}$ & $\mathrm{AA}$ & $\mathrm{AA}$ & $\mathrm{AA}$ & $\mathrm{AA}$ & $\mathrm{AA}$ & $\mathrm{AA}$ & $\mathrm{AA}$ & $\mathrm{AA}$ \\
\hline AA & $\mathrm{AA}$ & AA & $\mathrm{AA}$ & $\mathrm{AC}$ & $\mathrm{AA}$ & AA & AA & $\mathrm{AA}$ & AA & AA \\
\hline $\mathrm{AA}$ & $\mathrm{AA}$ & $\mathrm{AA}$ & $\mathrm{AA}$ & $\mathrm{AA}$ & $\mathrm{AA}$ & $\mathrm{AC}$ & AA & $\mathrm{AA}$ & AA & \\
\hline GDsnp01590 & 14 & \multicolumn{2}{|c|}{20326328} & $\mathrm{CC}$ & $\mathrm{CG}$ & $\mathrm{CC}$ & $\mathrm{CC}$ & $\mathrm{CG}$ & $\mathrm{CG}$ & $\mathrm{CG}$ \\
\hline- & $\mathrm{CC}$ & $\mathrm{CG}$ & $\mathrm{CC}$ & $\mathrm{CC}$ & $\mathrm{CG}$ & $\mathrm{CC}$ & GG & - & $\mathrm{CG}$ & $\mathrm{CG}$ \\
\hline $\mathrm{CG}$ & GG & $\mathrm{CG}$ & GG & $\mathrm{CC}$ & $\mathrm{CC}$ & $\mathrm{CC}$ & $\mathrm{CC}$ & $\mathrm{CC}$ & $\mathrm{CG}$ & $\mathrm{CG}$ \\
\hline $\mathrm{CC}$ & $\mathrm{CG}$ & $\mathrm{CG}$ & $\mathrm{CC}$ & $\mathrm{CG}$ & GG & GG & $\mathrm{CC}$ & $\mathrm{CG}$ & - & $\mathrm{CC}$ \\
\hline $\mathrm{CG}$ & GG & $\mathrm{CC}$ & GG & $\mathrm{CG}$ & $\mathrm{CG}$ & GG & $\mathrm{CC}$ & $\mathrm{CG}$ & $\mathrm{CG}$ & $\mathrm{CG}$ \\
\hline GG & $\mathrm{CC}$ & GG & - & $\mathrm{CG}$ & $\mathrm{CG}$ & GG & $\mathrm{CG}$ & GG & $\mathrm{CC}$ & $\mathrm{CG}$ \\
\hline $\mathrm{CG}$ & $\mathrm{CG}$ & $\mathrm{CC}$ & $\mathrm{CG}$ & GG & $\mathrm{CG}$ & GG & $\mathrm{CG}$ & $\mathrm{CG}$ & $\mathrm{CG}$ & $\mathrm{CG}$ \\
\hline GG & $\mathrm{CG}$ & $\mathrm{CG}$ & GG & $\mathrm{CC}$ & $\mathrm{CG}$ & $\mathrm{CG}$ & $\mathrm{CG}$ & $\mathrm{CC}$ & $\mathrm{CC}$ & $\mathrm{CC}$ \\
\hline GG & GG & $\mathrm{CG}$ & $\mathrm{CC}$ & $\mathrm{CC}$ & GG & $\mathrm{CG}$ & $\mathrm{CG}$ & $\mathrm{CG}$ & $\mathrm{CC}$ & \\
\hline GDsnp00211 & 14 & \multicolumn{2}{|c|}{22601186} & $\mathrm{AG}$ & $\mathrm{AG}$ & GG & $\mathrm{AG}$ & $\mathrm{AA}$ & $\mathrm{AG}$ & $\mathrm{AG}$ \\
\hline AA & $\mathrm{AG}$ & $\mathrm{AG}$ & $\mathrm{AG}$ & $\mathrm{AG}$ & $\mathrm{AG}$ & GG & AA & $\mathrm{AA}$ & $\mathrm{AG}$ & $\mathrm{AG}$ \\
\hline $\mathrm{AG}$ & $\mathrm{AA}$ & $\mathrm{AG}$ & $\mathrm{AA}$ & GG & $\mathrm{AG}$ & $\mathrm{AG}$ & GG & GG & $\mathrm{AG}$ & $\mathrm{AA}$ \\
\hline $\mathrm{AG}$ & $\mathrm{AG}$ & $\mathrm{AG}$ & $\mathrm{AG}$ & $\mathrm{AG}$ & $\mathrm{AA}$ & $\mathrm{AA}$ & $\mathrm{AG}$ & $\mathrm{AG}$ & AA & $\mathrm{AG}$ \\
\hline $\mathrm{AG}$ & $\mathrm{AA}$ & $\mathrm{AG}$ & $\mathrm{AA}$ & $\mathrm{AA}$ & $\mathrm{AA}$ & AA & GG & $\mathrm{AG}$ & $\mathrm{AA}$ & $\mathrm{AG}$ \\
\hline AA & GG & AA & $\mathrm{AA}$ & $\mathrm{AG}$ & $\mathrm{AG}$ & AA & AA & $\mathrm{AA}$ & $\mathrm{AG}$ & $\mathrm{AG}$ \\
\hline $\mathrm{AG}$ & $\mathrm{AA}$ & GG & $\mathrm{AG}$ & $\mathrm{AA}$ & $\mathrm{AG}$ & AA & AA & $\mathrm{AG}$ & $\mathrm{AG}$ & $\mathrm{AG}$ \\
\hline AA & $\mathrm{AG}$ & $\mathrm{AG}$ & $\mathrm{AA}$ & $\mathrm{AG}$ & $\mathrm{AA}$ & $\mathrm{AG}$ & AA & GG & $\mathrm{AG}$ & $\mathrm{AG}$ \\
\hline AA & $\mathrm{AA}$ & GG & GG & GG & AA & AA & $\mathrm{AG}$ & $\mathrm{AG}$ & GG & \\
\hline GDsnp00522 & 14 & \multicolumn{2}{|c|}{23161054} & GG & $\mathrm{AG}$ & GG & GG & $\mathrm{AG}$ & $\mathrm{AG}$ & $\mathrm{AG}$ \\
\hline AA & $\mathrm{AA}$ & $\mathrm{AG}$ & GG & GG & $\mathrm{AG}$ & GG & AA & $\mathrm{AA}$ & $\mathrm{AG}$ & $\mathrm{AG}$ \\
\hline $\mathrm{AG}$ & $\mathrm{AA}$ & $\mathrm{AG}$ & $\mathrm{AA}$ & GG & GG & $\mathrm{AG}$ & GG & GG & $\mathrm{AG}$ & $\mathrm{AA}$ \\
\hline $\mathrm{AG}$ & $\mathrm{AG}$ & $\mathrm{AG}$ & GG & $\mathrm{AG}$ & $\mathrm{AA}$ & AA & GG & $\mathrm{AG}$ & AA & $\mathrm{AG}$ \\
\hline $\mathrm{AG}$ & $\mathrm{AA}$ & GG & $\mathrm{AA}$ & $\mathrm{AA}$ & GG & AA & GG & $\mathrm{AG}$ & $\mathrm{AG}$ & $\mathrm{AG}$ \\
\hline AA & GG & AA & $\mathrm{AA}$ & $\mathrm{AG}$ & $\mathrm{AG}$ & AA & $\mathrm{AG}$ & $\mathrm{AA}$ & GG & $\mathrm{AG}$ \\
\hline $\mathrm{AG}$ & $\mathrm{AG}$ & GG & $\mathrm{AG}$ & $\mathrm{AA}$ & $\mathrm{AG}$ & $\mathrm{AG}$ & $\mathrm{AG}$ & $\mathrm{AG}$ & - & $\mathrm{AG}$ \\
\hline $\mathrm{AG}$ & $\mathrm{AG}$ & $\mathrm{AG}$ & $\mathrm{AA}$ & GG & $\mathrm{AA}$ & $\mathrm{AG}$ & $\mathrm{AG}$ & GG & $\mathrm{AG}$ & GG \\
\hline $\mathrm{AG}$ & $\mathrm{AA}$ & GG & GG & GG & AA & AA & $\mathrm{AG}$ & $\mathrm{AG}$ & GG & \\
\hline GDsnp00048 & 14 & \multicolumn{2}{|c|}{24445979} & $\mathrm{AA}$ & $\mathrm{AG}$ & $\mathrm{AA}$ & $\mathrm{AA}$ & $\mathrm{AG}$ & $\mathrm{AG}$ & $\mathrm{AG}$ \\
\hline $\mathrm{AG}$ & GG & $\mathrm{AG}$ & $\mathrm{AA}$ & $\mathrm{AA}$ & $\mathrm{AG}$ & AA & GG & GG & $\mathrm{AG}$ & $\mathrm{AG}$ \\
\hline AA & GG & $\mathrm{AG}$ & GG & $\mathrm{AA}$ & $\mathrm{AA}$ & $\mathrm{AG}$ & AA & $\mathrm{AA}$ & $\mathrm{AG}$ & GG \\
\hline $\mathrm{AG}$ & $\mathrm{AG}$ & $\mathrm{AG}$ & $\mathrm{AA}$ & $\mathrm{AG}$ & $\mathrm{AG}$ & GG & $\mathrm{AA}$ & GG & GG & $\mathrm{AG}$ \\
\hline $\mathrm{AG}$ & GG & $\mathrm{AA}$ & GG & GG & $\mathrm{AA}$ & GG & $\mathrm{AA}$ & $\mathrm{AG}$ & $\mathrm{AG}$ & $\mathrm{AG}$ \\
\hline GG & $\mathrm{AA}$ & $\mathrm{AG}$ & GG & $\mathrm{AG}$ & $\mathrm{AG}$ & GG & $\mathrm{AG}$ & GG & AA & $\mathrm{AG}$ \\
\hline $\mathrm{AG}$ & $\mathrm{AG}$ & AA & $\mathrm{AG}$ & GG & $\mathrm{AG}$ & $\mathrm{AG}$ & $\mathrm{AG}$ & $\mathrm{AG}$ & $\mathrm{AG}$ & $\mathrm{AG}$ \\
\hline $\mathrm{AG}$ & $\mathrm{AG}$ & $\mathrm{AG}$ & GG & $\mathrm{AG}$ & GG & $\mathrm{AG}$ & $\mathrm{AG}$ & $\mathrm{AA}$ & $\mathrm{AG}$ & $\mathrm{AA}$ \\
\hline AG & GG & AA & AA & $\mathrm{AA}$ & GG & GG & $\mathrm{AG}$ & $\mathrm{AG}$ & AA & \\
\hline GDsnp00948 & 14 & \multicolumn{2}{|c|}{24931458} & $\mathrm{AA}$ & $\mathrm{AG}$ & $\mathrm{AA}$ & $\mathrm{AA}$ & $\mathrm{AG}$ & $\mathrm{AG}$ & $\mathrm{AG}$ \\
\hline GG & GG & $\mathrm{AG}$ & $\mathrm{AA}$ & $\mathrm{AA}$ & $\mathrm{AG}$ & AA & GG & GG & $\mathrm{AG}$ & $\mathrm{AG}$ \\
\hline AA & GG & $\mathrm{AG}$ & GG & $\mathrm{AA}$ & $\mathrm{AA}$ & $\mathrm{AG}$ & AA & $\mathrm{AA}$ & $\mathrm{AG}$ & GG \\
\hline $\mathrm{AG}$ & $\mathrm{AG}$ & $\mathrm{AG}$ & $\mathrm{AA}$ & GG & $\mathrm{AG}$ & GG & AA & GG & $\mathrm{AG}$ & $\mathrm{AG}$ \\
\hline $\mathrm{AG}$ & GG & $\mathrm{AA}$ & GG & GG & $\mathrm{AA}$ & GG & $\mathrm{AG}$ & $\mathrm{AG}$ & $\mathrm{AG}$ & $\mathrm{AG}$ \\
\hline GG & $\mathrm{AA}$ & $\mathrm{AG}$ & GG & $\mathrm{AG}$ & $\mathrm{AG}$ & GG & $\mathrm{AG}$ & GG & $\mathrm{AA}$ & $\mathrm{AG}$ \\
\hline $\mathrm{AG}$ & $\mathrm{AG}$ & $\mathrm{AA}$ & $\mathrm{AG}$ & GG & $\mathrm{AG}$ & $\mathrm{AG}$ & $\mathrm{AG}$ & $\mathrm{AG}$ & $\mathrm{AG}$ & $\mathrm{AG}$ \\
\hline $\mathrm{AG}$ & $\mathrm{AG}$ & $\mathrm{AG}$ & GG & $\mathrm{AG}$ & GG & $\mathrm{AG}$ & $\mathrm{AG}$ & $\mathrm{AA}$ & $\mathrm{AG}$ & $\mathrm{AA}$ \\
\hline $\mathrm{AG}$ & GG & $\mathrm{AA}$ & $\mathrm{AA}$ & $\mathrm{AA}$ & GG & GG & $\mathrm{AG}$ & $\mathrm{AG}$ & AA & \\
\hline
\end{tabular}

Continued next page 
Supplemental Table 2. Continued.

\begin{tabular}{|c|c|c|c|c|c|c|c|c|c|c|}
\hline \multirow{3}{*}{$\begin{array}{l}\text { SNP name } \\
\text { GDsnp00171 } \\
\text { AG }\end{array}$} & \multicolumn{10}{|c|}{ Linkage group Physical location (bp) Individuals } \\
\hline & 14 & \multicolumn{2}{|c|}{25220544} & GG & GG & GG & GG & $\mathrm{AG}$ & $\mathrm{AG}$ & GG \\
\hline & $\mathrm{AA}$ & $\mathrm{AG}$ & GG & GG & $\mathrm{AG}$ & GG & AA & AA & $\mathrm{AG}$ & $\mathrm{AG}$ \\
\hline GG & AA & $\mathrm{AG}$ & AA & GG & GG & AG & GG & GG & $\mathrm{AG}$ & AA \\
\hline AG & $\mathrm{AG}$ & $\mathrm{AG}$ & GG & AA & $\mathrm{AG}$ & AA & GG & AA & $\mathrm{AG}$ & $\mathrm{AG}$ \\
\hline AG & $\mathrm{AA}$ & GG & AA & $\mathrm{AA}$ & GG & AA & GG & $\mathrm{AG}$ & $\mathrm{AG}$ & $\mathrm{AG}$ \\
\hline AA & GG & $\mathrm{AG}$ & AA & $\mathrm{AG}$ & $\mathrm{AG}$ & AA & $\mathrm{AG}$ & AA & GG & $\mathrm{AG}$ \\
\hline AG & $\mathrm{AG}$ & GG & $\mathrm{AG}$ & $\mathrm{AA}$ & $\mathrm{AG}$ & $\mathrm{AG}$ & $\mathrm{AG}$ & $\mathrm{AG}$ & $\mathrm{AG}$ & $\mathrm{AG}$ \\
\hline AG & $\mathrm{AG}$ & $\mathrm{AG}$ & AA & GG & AA & $\mathrm{AG}$ & $\mathrm{AG}$ & GG & $\mathrm{AG}$ & GG \\
\hline $\mathrm{AG}$ & AA & GG & GG & GG & AA & AA & $\mathrm{AG}$ & $\mathrm{AG}$ & GG & \\
\hline GDsnp00213 & 14 & \multicolumn{2}{|c|}{27058274} & GG & GG & GG & GG & GG & GG & GG \\
\hline AG & GG & GG & GG & GG & GG & GG & GG & GG & GG & GG \\
\hline GG & GG & GG & GG & GG & GG & GG & GG & GG & GG & GG \\
\hline GG & GG & GG & GG & GG & GG & GG & GG & GG & GG & GG \\
\hline GG & GG & GG & GG & GG & GG & GG & $\mathrm{AG}$ & GG & GG & GG \\
\hline GG & GG & GG & GG & GG & GG & GG & GG & GG & GG & GG \\
\hline GG & GG & GG & GG & GG & GG & GG & GG & GG & GG & GG \\
\hline GG & GG & GG & GG & GG & GG & GG & GG & GG & GG & GG \\
\hline GG & GG & GG & GG & GG & GG & GG & GG & GG & GG & \\
\hline GDsnp02021 & 14 & \multicolumn{2}{|c|}{27321459} & $\mathrm{AA}$ & $\mathrm{AA}$ & AA & $\mathrm{AA}$ & $\mathrm{AG}$ & $\mathrm{AG}$ & $\mathrm{AA}$ \\
\hline AG & $\mathrm{AG}$ & AA & AA & $\mathrm{AA}$ & $\mathrm{AG}$ & AA & GG & AA & $\mathrm{AG}$ & $\mathrm{AG}$ \\
\hline AA & GG & $\mathrm{AG}$ & GG & $\mathrm{AA}$ & AA & AA & $\mathrm{AA}$ & AA & $\mathrm{AG}$ & GG \\
\hline AG & $\mathrm{AG}$ & $\mathrm{AG}$ & AA & $\mathrm{AG}$ & $\mathrm{AG}$ & GG & AA & GG & $\mathrm{AG}$ & $\mathrm{AG}$ \\
\hline AA & GG & $\mathrm{AA}$ & $\mathrm{AG}$ & GG & $\mathrm{AA}$ & GG & $\mathrm{AA}$ & $\mathrm{AG}$ & $\mathrm{AG}$ & $\mathrm{AG}$ \\
\hline GG & $\mathrm{AA}$ & $\mathrm{AG}$ & $\mathrm{AG}$ & $\mathrm{AA}$ & $\mathrm{AG}$ & GG & $\mathrm{AG}$ & $\mathrm{AG}$ & AA & $\mathrm{AG}$ \\
\hline $\mathrm{AG}$ & $\mathrm{AG}$ & AA & $\mathrm{AG}$ & GG & $\mathrm{AA}$ & $\mathrm{AG}$ & $\mathrm{AG}$ & AA & $\mathrm{AG}$ & $\mathrm{AG}$ \\
\hline AG & $\mathrm{AG}$ & $\mathrm{AG}$ & GG & $\mathrm{AA}$ & GG & AA & $\mathrm{AG}$ & AA & $\mathrm{AG}$ & $\mathrm{AA}$ \\
\hline AG & $\mathrm{AG}$ & $\mathrm{AA}$ & $\mathrm{AA}$ & $\mathrm{AA}$ & $\mathrm{AG}$ & GG & $\mathrm{AG}$ & GG & $\mathrm{AG}$ & \\
\hline GDsnp00261 & 14 & \multicolumn{2}{|c|}{29135091} & GG & GG & GG & $\mathrm{CG}$ & $\mathrm{CG}$ & $\mathrm{CG}$ & GG \\
\hline CG & $\mathrm{CG}$ & GG & GG & GG & $\mathrm{CG}$ & GG & $\mathrm{CG}$ & $\mathrm{CG}$ & $\mathrm{CC}$ & $\mathrm{CC}$ \\
\hline GG & $\mathrm{CC}$ & GG & $\mathrm{CG}$ & GG & $\mathrm{CG}$ & GG & $\mathrm{CG}$ & $\mathrm{CG}$ & $\mathrm{CG}$ & $\mathrm{CC}$ \\
\hline $\mathrm{CG}$ & CG & CG & GG & $\mathrm{CG}$ & CG & $\mathrm{CC}$ & GG & $\mathrm{CC}$ & $\mathrm{CC}$ & CG \\
\hline GG & $\mathrm{CC}$ & - & $\mathrm{CG}$ & $\mathrm{CC}$ & $\mathrm{CG}$ & $\mathrm{CC}$ & - & $\mathrm{CG}$ & $\mathrm{CC}$ & $\mathrm{CG}$ \\
\hline $\mathrm{CG}$ & GG & $\mathrm{CG}$ & $\mathrm{CG}$ & GG & $\mathrm{CG}$ & $\mathrm{CC}$ & $\mathrm{CC}$ & $\mathrm{CG}$ & GG & $\mathrm{CC}$ \\
\hline $\mathrm{CG}$ & $\mathrm{CG}$ & GG & $\mathrm{CG}$ & $\mathrm{CC}$ & GG & $\mathrm{CG}$ & $\mathrm{CG}$ & GG & $\mathrm{CG}$ & $\mathrm{CG}$ \\
\hline GG & $\mathrm{CC}$ & $\mathrm{CG}$ & $\mathrm{CC}$ & $\mathrm{CG}$ & CG & GG & $\mathrm{CG}$ & GG & CG & CG \\
\hline $\mathrm{CC}$ & CG & GG & GG & GG & CG & $\mathrm{CC}$ & $\mathrm{CG}$ & $\mathrm{CC}$ & CG & \\
\hline GDsnp00349 & 15 & \multicolumn{2}{|c|}{701121} & GG & GG & GG & GG & GG & GG & GG \\
\hline GG & $\mathrm{AG}$ & GG & GG & GG & GG & GG & GG & $\mathrm{AG}$ & GG & GG \\
\hline GG & GG & GG & GG & GG & GG & $\mathrm{AG}$ & GG & GG & GG & GG \\
\hline GG & GG & GG & GG & GG & GG & GG & GG & GG & GG & $\mathrm{AG}$ \\
\hline GG & GG & GG & GG & GG & GG & GG & GG & GG & GG & GG \\
\hline GG & GG & GG & GG & GG & GG & GG & GG & GG & GG & GG \\
\hline GG & GG & GG & GG & GG & GG & GG & GG & GG & GG & GG \\
\hline GG & GG & GG & GG & GG & GG & GG & GG & GG & GG & GG \\
\hline GG & GG & GG & - & GG & GG & GG & GG & GG & GG & \\
\hline GDsnp01131 & 15 & \multicolumn{2}{|c|}{1033847} & $\mathrm{AA}$ & $\mathrm{AG}$ & $\mathrm{AG}$ & AA & AA & $\mathrm{AG}$ & $\mathrm{AA}$ \\
\hline $\mathrm{AG}$ & $\mathrm{AG}$ & $\mathrm{AA}$ & AA & $\mathrm{AG}$ & $\mathrm{AA}$ & $\mathrm{AG}$ & $\mathrm{AG}$ & AA & $\mathrm{AG}$ & $\mathrm{AA}$ \\
\hline AA & $\mathrm{AA}$ & $\mathrm{AG}$ & AA & $\mathrm{AA}$ & AA & $\mathrm{AG}$ & $\mathrm{AG}$ & $\mathrm{AG}$ & $\mathrm{AG}$ & AA \\
\hline AA & $\mathrm{AG}$ & $\mathrm{AG}$ & $\mathrm{AA}$ & $\mathrm{AG}$ & $\mathrm{AG}$ & AA & $\mathrm{AG}$ & $\mathrm{AG}$ & $\mathrm{AA}$ & $\mathrm{AA}$ \\
\hline AG & $\mathrm{AA}$ & $\mathrm{AA}$ & $\mathrm{AG}$ & $\mathrm{AG}$ & $\mathrm{AG}$ & $\mathrm{AG}$ & $\mathrm{AA}$ & $\mathrm{AG}$ & $\mathrm{AG}$ & $\mathrm{AG}$ \\
\hline $\mathrm{AG}$ & $\mathrm{AG}$ & $\mathrm{AG}$ & AA & $\mathrm{AG}$ & $\mathrm{AG}$ & $\mathrm{AG}$ & $\mathrm{AG}$ & $\mathrm{AG}$ & $\mathrm{AG}$ & AA \\
\hline $\mathrm{AG}$ & $\mathrm{AA}$ & $\mathrm{AG}$ & $\mathrm{AG}$ & $\mathrm{AG}$ & $\mathrm{AG}$ & $\mathrm{AA}$ & $\mathrm{AG}$ & $\mathrm{AG}$ & $\mathrm{AG}$ & $\mathrm{AG}$ \\
\hline $\mathrm{AA}$ & $\mathrm{AG}$ & $\mathrm{AG}$ & $\mathrm{AG}$ & $\mathrm{AA}$ & $\mathrm{AG}$ & $\mathrm{AG}$ & $\mathrm{AG}$ & $\mathrm{AG}$ & $\mathrm{AG}$ & $\mathrm{AA}$ \\
\hline $\mathrm{AA}$ & $\mathrm{AG}$ & $\mathrm{AA}$ & $\mathrm{AG}$ & $\mathrm{AA}$ & $\mathrm{AG}$ & AA & $\mathrm{AA}$ & $\mathrm{AA}$ & $\mathrm{AG}$ & \\
\hline
\end{tabular}


Supplemental Table 2. Continued.

\begin{tabular}{|c|c|c|c|c|c|c|c|c|c|c|}
\hline \multirow{3}{*}{$\begin{array}{l}\text { SNP name } \\
\text { GDsnp01009 } \\
\text { AG }\end{array}$} & \multicolumn{10}{|c|}{ Linkage group Physical location (bp) Individuals } \\
\hline & 15 & \multicolumn{2}{|c|}{1112703} & $\mathrm{AG}$ & $\mathrm{AG}$ & $\mathrm{AG}$ & $\mathrm{AG}$ & $\mathrm{AG}$ & $\mathrm{AG}$ & GG \\
\hline & $\mathrm{AG}$ & $\mathrm{AG}$ & GG & AA & $\mathrm{AG}$ & AA & AA & $\mathrm{AG}$ & $\mathrm{AG}$ & GG \\
\hline $\mathrm{AG}$ & $\mathrm{AA}$ & $\mathrm{AG}$ & GG & GG & $\mathrm{AG}$ & $\mathrm{AG}$ & $\mathrm{AG}$ & $\mathrm{AG}$ & $\mathrm{AG}$ & $\mathrm{AG}$ \\
\hline AA & $\mathrm{AG}$ & $\mathrm{AG}$ & $\mathrm{AA}$ & $\mathrm{AG}$ & AA & AG & AA & AA & $\mathrm{AG}$ & GG \\
\hline $\mathrm{AG}$ & $\mathrm{AG}$ & $\mathrm{AA}$ & $\mathrm{AG}$ & $\mathrm{AG}$ & $\mathrm{AG}$ & $\mathrm{AA}$ & $\mathrm{AG}$ & $\mathrm{AA}$ & $\mathrm{AG}$ & $\mathrm{AA}$ \\
\hline $\mathrm{AG}$ & $\mathrm{AG}$ & $\mathrm{AG}$ & $\mathrm{AG}$ & $\mathrm{AG}$ & $\mathrm{AG}$ & $\mathrm{AG}$ & $\mathrm{AA}$ & $\mathrm{AG}$ & $\mathrm{AG}$ & GG \\
\hline $\mathrm{AG}$ & $\mathrm{AA}$ & $\mathrm{AG}$ & $\mathrm{AA}$ & $\mathrm{AA}$ & $\mathrm{AG}$ & GG & $\mathrm{AA}$ & $\mathrm{AA}$ & $\mathrm{AA}$ & $\mathrm{AG}$ \\
\hline $\mathrm{AG}$ & $\mathrm{AA}$ & $\mathrm{AG}$ & $\mathrm{AG}$ & GG & $\mathrm{AG}$ & $\mathrm{AG}$ & $\mathrm{AG}$ & $\mathrm{AG}$ & $\mathrm{AG}$ & $\mathrm{AG}$ \\
\hline $\mathrm{AA}$ & $\mathrm{AA}$ & GG & $\mathrm{AA}$ & $\mathrm{AG}$ & $\mathrm{AA}$ & $\mathrm{AG}$ & $\mathrm{AG}$ & $\mathrm{AG}$ & AA & \\
\hline GDsnp01634 & 15 & \multicolumn{2}{|c|}{2111104} & $\mathrm{CG}$ & $\mathrm{CG}$ & $\mathrm{CC}$ & $\mathrm{CG}$ & $\mathrm{CG}$ & $\mathrm{CG}$ & $\mathrm{CC}$ \\
\hline $\mathrm{CG}$ & $\mathrm{CG}$ & CG & $\mathrm{CC}$ & GG & $\mathrm{CG}$ & GG & GG & $\mathrm{CG}$ & $\mathrm{CG}$ & $\mathrm{CC}$ \\
\hline $\mathrm{CG}$ & GG & $\mathrm{CG}$ & $\mathrm{CC}$ & $\mathrm{CC}$ & $\mathrm{CG}$ & $\mathrm{CG}$ & $\mathrm{CG}$ & $\mathrm{CG}$ & $\mathrm{CG}$ & $\mathrm{CG}$ \\
\hline GG & $\mathrm{CG}$ & GG & GG & $\mathrm{CG}$ & GG & $\mathrm{CG}$ & GG & GG & $\mathrm{CG}$ & $\mathrm{CC}$ \\
\hline $\mathrm{CG}$ & $\mathrm{CG}$ & GG & $\mathrm{CG}$ & $\mathrm{CG}$ & $\mathrm{CG}$ & GG & $\mathrm{CG}$ & GG & $\mathrm{CG}$ & GG \\
\hline $\mathrm{CG}$ & $\mathrm{CG}$ & $\mathrm{CG}$ & $\mathrm{CG}$ & $\mathrm{CG}$ & $\mathrm{CG}$ & $\mathrm{CG}$ & GG & $\mathrm{CG}$ & $\mathrm{CG}$ & $\mathrm{CC}$ \\
\hline $\mathrm{CG}$ & GG & $\mathrm{CG}$ & GG & GG & $\mathrm{CG}$ & $\mathrm{CC}$ & GG & GG & GG & $\mathrm{CG}$ \\
\hline $\mathrm{CG}$ & GG & CG & $\mathrm{CG}$ & $\mathrm{CC}$ & GG & $\mathrm{CG}$ & $\mathrm{CG}$ & $\mathrm{CG}$ & $\mathrm{CG}$ & $\mathrm{CG}$ \\
\hline $\mathrm{CG}$ & GG & $\mathrm{CC}$ & GG & $\mathrm{CG}$ & GG & $\mathrm{CG}$ & $\mathrm{CG}$ & $\mathrm{CG}$ & GG & \\
\hline GDsnp00115 & 15 & \multicolumn{2}{|c|}{2652900} & $\mathrm{AA}$ & $\mathrm{AG}$ & $\mathrm{AA}$ & $\mathrm{AA}$ & $\mathrm{AA}$ & $\mathrm{AG}$ & $\mathrm{AA}$ \\
\hline $\mathrm{AG}$ & $\mathrm{AG}$ & AA & $\mathrm{AA}$ & $\mathrm{AG}$ & $\mathrm{AA}$ & $\mathrm{AG}$ & $\mathrm{AG}$ & AA & $\mathrm{AG}$ & $\mathrm{AA}$ \\
\hline $\mathrm{AA}$ & $\mathrm{AA}$ & $\mathrm{AG}$ & $\mathrm{AA}$ & $\mathrm{AA}$ & $\mathrm{AA}$ & $\mathrm{AG}$ & $\mathrm{AG}$ & $\mathrm{AG}$ & $\mathrm{AG}$ & $\mathrm{AA}$ \\
\hline $\mathrm{AA}$ & $\mathrm{AG}$ & $\mathrm{AG}$ & $\mathrm{AA}$ & $\mathrm{AG}$ & $\mathrm{AG}$ & $\mathrm{AA}$ & $\mathrm{AG}$ & $\mathrm{AG}$ & AA & $\mathrm{AA}$ \\
\hline AA & $\mathrm{AA}$ & $\mathrm{AA}$ & $\mathrm{AG}$ & $\mathrm{AG}$ & $\mathrm{AG}$ & $\mathrm{AG}$ & $\mathrm{AA}$ & $\mathrm{AG}$ & $\mathrm{AG}$ & $\mathrm{AG}$ \\
\hline $\mathrm{AG}$ & $\mathrm{AG}$ & $\mathrm{AG}$ & $\mathrm{AA}$ & $\mathrm{AG}$ & $\mathrm{AG}$ & $\mathrm{AG}$ & $\mathrm{AG}$ & $\mathrm{AG}$ & $\mathrm{AG}$ & $\mathrm{AA}$ \\
\hline $\mathrm{AG}$ & $\mathrm{AA}$ & $\mathrm{AG}$ & $\mathrm{AG}$ & $\mathrm{AG}$ & $\mathrm{AG}$ & AA & $\mathrm{AA}$ & $\mathrm{AG}$ & $\mathrm{AG}$ & $\mathrm{AG}$ \\
\hline AG & $\mathrm{AG}$ & $\mathrm{AG}$ & $\mathrm{AG}$ & $\mathrm{AA}$ & $\mathrm{AG}$ & $\mathrm{AG}$ & $\mathrm{AG}$ & $\mathrm{AG}$ & $\mathrm{AG}$ & $\mathrm{AA}$ \\
\hline $\mathrm{AA}$ & $\mathrm{AG}$ & $\mathrm{AA}$ & $\mathrm{AG}$ & $\mathrm{AG}$ & $\mathrm{AG}$ & AA & AA & $\mathrm{AA}$ & $\mathrm{AG}$ & \\
\hline GDsnp00329 & 15 & \multicolumn{2}{|c|}{3601346} & $\mathrm{AT}$ & $\mathrm{AT}$ & $\mathrm{AA}$ & $\mathrm{AT}$ & $\mathrm{AT}$ & $\mathrm{AT}$ & AA \\
\hline AT & $\mathrm{AT}$ & AT & $\mathrm{AA}$ & $\mathrm{TT}$ & $\mathrm{AT}$ & $\mathrm{TT}$ & $\mathrm{TT}$ & $\mathrm{AT}$ & $\mathrm{AT}$ & $\mathrm{AA}$ \\
\hline $\mathrm{AT}$ & $\mathrm{TT}$ & AT & $\mathrm{AA}$ & $\mathrm{AA}$ & $\mathrm{AT}$ & $\mathrm{AT}$ & $\mathrm{AT}$ & AT & $\mathrm{AT}$ & AT \\
\hline $\mathrm{TT}$ & $\mathrm{AT}$ & TT & $\mathrm{TT}$ & AT & $\mathrm{TT}$ & AT & $\mathrm{TT}$ & $\mathrm{TT}$ & $\mathrm{AT}$ & AA \\
\hline AT & $\mathrm{AT}$ & $\mathrm{TT}$ & $\mathrm{AT}$ & AT & $\mathrm{AT}$ & $\mathrm{TT}$ & $\mathrm{AT}$ & $\mathrm{TT}$ & $\mathrm{AT}$ & TT \\
\hline AA & $\mathrm{TT}$ & AT & $\mathrm{AT}$ & $\mathrm{AT}$ & $\mathrm{AT}$ & $\mathrm{AT}$ & $\mathrm{TT}$ & $\mathrm{AT}$ & $\mathrm{AT}$ & $\mathrm{AA}$ \\
\hline $\mathrm{AT}$ & $\mathrm{TT}$ & AT & $\mathrm{TT}$ & $\mathrm{TT}$ & $\mathrm{AT}$ & AA & $\mathrm{TT}$ & TT & $\mathrm{TT}$ & AT \\
\hline AT & $\mathrm{TT}$ & AT & $\mathrm{AT}$ & $\mathrm{AA}$ & $\mathrm{TT}$ & AT & $\mathrm{AT}$ & $\mathrm{AT}$ & $\mathrm{AT}$ & AT \\
\hline AT & $\mathrm{TT}$ & AA & $\mathrm{TT}$ & TT & TT & $\mathrm{AT}$ & AT & AT & $\mathrm{TT}$ & \\
\hline GDsnp00330 & 15 & \multicolumn{2}{|c|}{4387625} & $\mathrm{AG}$ & $\mathrm{AG}$ & GG & $\mathrm{AG}$ & $\mathrm{AG}$ & $\mathrm{AG}$ & GG \\
\hline $\mathrm{AG}$ & $\mathrm{AG}$ & $\mathrm{AG}$ & $\mathrm{AG}$ & $\mathrm{AA}$ & $\mathrm{AG}$ & AA & $\mathrm{AA}$ & $\mathrm{AG}$ & $\mathrm{AG}$ & GG \\
\hline $\mathrm{AG}$ & $\mathrm{AA}$ & $\mathrm{AG}$ & GG & GG & $\mathrm{AG}$ & $\mathrm{AG}$ & $\mathrm{AG}$ & $\mathrm{AG}$ & $\mathrm{AG}$ & $\mathrm{AG}$ \\
\hline AA & $\mathrm{AG}$ & $\mathrm{AA}$ & $\mathrm{AA}$ & $\mathrm{AG}$ & $\mathrm{AA}$ & $\mathrm{AG}$ & $\mathrm{AA}$ & $\mathrm{AA}$ & AA & GG \\
\hline $\mathrm{AG}$ & $\mathrm{AG}$ & $\mathrm{AA}$ & $\mathrm{AG}$ & $\mathrm{AG}$ & $\mathrm{AG}$ & $\mathrm{AG}$ & $\mathrm{AG}$ & AA & $\mathrm{AG}$ & $\mathrm{AA}$ \\
\hline GG & $\mathrm{AA}$ & $\mathrm{AG}$ & $\mathrm{AG}$ & $\mathrm{AG}$ & GG & $\mathrm{AG}$ & $\mathrm{AA}$ & $\mathrm{AG}$ & $\mathrm{AG}$ & GG \\
\hline $\mathrm{AG}$ & $\mathrm{AA}$ & $\mathrm{AG}$ & $\mathrm{AA}$ & $\mathrm{AA}$ & $\mathrm{AG}$ & GG & $\mathrm{AA}$ & AA & AA & $\mathrm{AG}$ \\
\hline GG & $\mathrm{AA}$ & $\mathrm{AG}$ & GG & $\mathrm{AG}$ & $\mathrm{AA}$ & $\mathrm{AG}$ & $\mathrm{AG}$ & $\mathrm{AG}$ & $\mathrm{AG}$ & $\mathrm{AG}$ \\
\hline AG & AA & GG & AA & $\mathrm{AA}$ & AA & $\mathrm{AG}$ & $\mathrm{AG}$ & $\mathrm{AG}$ & AA & \\
\hline GDsnp01454 & 15 & \multicolumn{2}{|c|}{4911763} & $\mathrm{AG}$ & $\mathrm{AG}$ & $\mathrm{AA}$ & $\mathrm{AG}$ & $\mathrm{AG}$ & $\mathrm{AG}$ & $\mathrm{AA}$ \\
\hline $\mathrm{AG}$ & $\mathrm{AG}$ & $\mathrm{AG}$ & $\mathrm{AA}$ & GG & $\mathrm{AG}$ & GG & GG & $\mathrm{AG}$ & $\mathrm{AG}$ & $\mathrm{AG}$ \\
\hline $\mathrm{AG}$ & GG & $\mathrm{AG}$ & $\mathrm{AA}$ & $\mathrm{AA}$ & $\mathrm{AG}$ & $\mathrm{AG}$ & $\mathrm{AG}$ & $\mathrm{AG}$ & $\mathrm{AG}$ & $\mathrm{AG}$ \\
\hline GG & $\mathrm{AG}$ & GG & GG & $\mathrm{AG}$ & GG & $\mathrm{AG}$ & GG & GG & GG & AA \\
\hline AG & $\mathrm{AG}$ & GG & $\mathrm{AG}$ & $\mathrm{AG}$ & $\mathrm{AG}$ & $\mathrm{AG}$ & $\mathrm{AG}$ & GG & $\mathrm{AG}$ & GG \\
\hline $\mathrm{AA}$ & GG & $\mathrm{AG}$ & $\mathrm{AG}$ & $\mathrm{AG}$ & $\mathrm{AA}$ & $\mathrm{AG}$ & GG & $\mathrm{AG}$ & $\mathrm{AG}$ & $\mathrm{AA}$ \\
\hline $\mathrm{AG}$ & GG & $\mathrm{AG}$ & GG & GG & $\mathrm{AG}$ & $\mathrm{AA}$ & GG & GG & GG & $\mathrm{AG}$ \\
\hline AA & GG & $\mathrm{AG}$ & $\mathrm{AA}$ & $\mathrm{AA}$ & GG & $\mathrm{AG}$ & $\mathrm{AG}$ & $\mathrm{AG}$ & $\mathrm{AG}$ & $\mathrm{AG}$ \\
\hline $\mathrm{AG}$ & GG & - & GG & GG & GG & GG & $\mathrm{AG}$ & $\mathrm{AG}$ & GG & \\
\hline
\end{tabular}

Continued next page 
Supplemental Table 2. Continued.

\begin{tabular}{|c|c|c|c|c|c|c|c|c|c|c|}
\hline \multirow{3}{*}{$\begin{array}{l}\text { SNP name } \\
\text { GDsnp00758 } \\
\text { AA }\end{array}$} & \multicolumn{10}{|c|}{ Linkage group Physical location (bp) Individuals } \\
\hline & 15 & \multicolumn{2}{|c|}{4951639} & $\mathrm{AA}$ & $\mathrm{AA}$ & AA & $\mathrm{AA}$ & AA & AA & $\mathrm{AA}$ \\
\hline & $\mathrm{AG}$ & AA & AA & AA & AA & AA & AA & AA & AA & AA \\
\hline AA & AA & AA & AA & AA & AA & AG & AA & AA & AA & AA \\
\hline AA & $\mathrm{AA}$ & AA & AA & $\mathrm{AA}$ & AA & AA & AA & AA & $\mathrm{AA}$ & $\mathrm{AA}$ \\
\hline AA & $\mathrm{AA}$ & $\mathrm{AA}$ & $\mathrm{AA}$ & $\mathrm{AA}$ & $\mathrm{AA}$ & $\mathrm{AA}$ & $\mathrm{AA}$ & $\mathrm{AA}$ & $\mathrm{AA}$ & $\mathrm{AA}$ \\
\hline AA & $\mathrm{AA}$ & AA & AA & AA & $\mathrm{AA}$ & AA & $\mathrm{AA}$ & AA & AA & AA \\
\hline $\mathrm{AA}$ & $\mathrm{AA}$ & $\mathrm{AA}$ & $\mathrm{AA}$ & $\mathrm{AA}$ & $\mathrm{AA}$ & $\mathrm{AA}$ & $\mathrm{AA}$ & $\mathrm{AA}$ & $\mathrm{AA}$ & $\mathrm{AA}$ \\
\hline AA & $\mathrm{AA}$ & AA & AA & $\mathrm{AA}$ & $\mathrm{AA}$ & AA & $\mathrm{AA}$ & AA & AA & AA \\
\hline $\mathrm{AA}$ & $\mathrm{AA}$ & $\mathrm{AA}$ & $\mathrm{AA}$ & $\mathrm{AA}$ & $\mathrm{AA}$ & $\mathrm{AG}$ & $\mathrm{AA}$ & $\mathrm{AA}$ & AA & \\
\hline GDsnp00191 & 15 & \multicolumn{2}{|c|}{5721310} & $\mathrm{TT}$ & $\mathrm{TT}$ & $\mathrm{TT}$ & $\mathrm{TT}$ & $\mathrm{TT}$ & $\mathrm{TT}$ & TT \\
\hline $\mathrm{AT}$ & $\mathrm{AT}$ & $\mathrm{TT}$ & $\mathrm{TT}$ & $\mathrm{TT}$ & $\mathrm{TT}$ & $\mathrm{TT}$ & $\mathrm{TT}$ & $\mathrm{TT}$ & $\mathrm{TT}$ & TT \\
\hline $\mathrm{TT}$ & $\mathrm{TT}$ & $\mathrm{TT}$ & $\mathrm{TT}$ & $\mathrm{TT}$ & $\mathrm{TT}$ & $\mathrm{AT}$ & $\mathrm{TT}$ & $\mathrm{TT}$ & $\mathrm{TT}$ & TT \\
\hline $\mathrm{TT}$ & $\mathrm{TT}$ & $\mathrm{TT}$ & $\mathrm{TT}$ & $\mathrm{TT}$ & $\mathrm{TT}$ & $\mathrm{TT}$ & $\mathrm{TT}$ & TT & $\mathrm{TT}$ & TT \\
\hline TT & $\mathrm{TT}$ & TT & TT & $\mathrm{TT}$ & TT & $\mathrm{TT}$ & $\mathrm{TT}$ & $\mathrm{TT}$ & $\mathrm{TT}$ & TT \\
\hline $\mathrm{TT}$ & $\mathrm{TT}$ & $\mathrm{TT}$ & $\mathrm{AT}$ & $\mathrm{TT}$ & $\mathrm{TT}$ & $\mathrm{TT}$ & $\mathrm{TT}$ & $\mathrm{TT}$ & $\mathrm{TT}$ & TT \\
\hline $\mathrm{TT}$ & $\mathrm{TT}$ & $\mathrm{TT}$ & $\mathrm{TT}$ & $\mathrm{TT}$ & $\mathrm{TT}$ & $\mathrm{TT}$ & $\mathrm{TT}$ & $\mathrm{TT}$ & $\mathrm{TT}$ & TT \\
\hline $\mathrm{TT}$ & $\mathrm{TT}$ & $\mathrm{TT}$ & $\mathrm{TT}$ & $\mathrm{AT}$ & $\mathrm{TT}$ & $\mathrm{TT}$ & $\mathrm{TT}$ & TT & $\mathrm{TT}$ & TT \\
\hline $\mathrm{TT}$ & $\mathrm{TT}$ & TT & $\mathrm{TT}$ & $\mathrm{TT}$ & $\mathrm{TT}$ & AT & $\mathrm{TT}$ & $\mathrm{TT}$ & $\mathrm{TT}$ & \\
\hline GDsnp01820 & 15 & \multicolumn{2}{|c|}{6236008} & $\mathrm{AT}$ & $\mathrm{AT}$ & $\mathrm{TT}$ & $\mathrm{TT}$ & AT & $\mathrm{AT}$ & TT \\
\hline $\mathrm{TT}$ & - & TT & $\mathrm{AT}$ & AT & $\mathrm{TT}$ & $\mathrm{AT}$ & $\mathrm{AT}$ & $\mathrm{TT}$ & $\mathrm{AT}$ & TT \\
\hline $\mathrm{TT}$ & $\mathrm{AT}$ & AT & TT & $\mathrm{TT}$ & TT & - & $\mathrm{AT}$ & $\mathrm{AT}$ & $\mathrm{AT}$ & TT \\
\hline $\mathrm{TT}$ & $\mathrm{AT}$ & AT & $\mathrm{TT}$ & $\mathrm{AT}$ & $\mathrm{AT}$ & $\mathrm{TT}$ & AT & $\mathrm{AT}$ & $\mathrm{AT}$ & AT \\
\hline $\mathrm{TT}$ & $\mathrm{TT}$ & $\mathrm{TT}$ & $\mathrm{AT}$ & $\mathrm{AT}$ & $\mathrm{AT}$ & - & $\mathrm{AT}$ & $\mathrm{AT}$ & $\mathrm{TT}$ & AT \\
\hline $\mathrm{TT}$ & $\mathrm{TT}$ & AT & - & AT & $\mathrm{TT}$ & $\mathrm{AT}$ & $\mathrm{AT}$ & $\mathrm{AT}$ & $\mathrm{AT}$ & TT \\
\hline AT & $\mathrm{TT}$ & AT & $\mathrm{AT}$ & AT & $\mathrm{AT}$ & $\mathrm{TT}$ & $\mathrm{TT}$ & $\mathrm{AT}$ & $\mathrm{AT}$ & AT \\
\hline $\mathrm{TT}$ & $\mathrm{AT}$ & AT & $\mathrm{TT}$ & $\mathrm{AT}$ & $\mathrm{AT}$ & $\mathrm{AT}$ & AT & $\mathrm{AT}$ & $\mathrm{AT}$ & TT \\
\hline TT & $\mathrm{AT}$ & AT & $\mathrm{AT}$ & $\mathrm{AT}$ & $\mathrm{AT}$ & - & $\mathrm{AT}$ & $\mathrm{AT}$ & $\mathrm{AT}$ & \\
\hline GDsnp01130 & 15 & \multicolumn{2}{|c|}{6757592} & GG & GG & GG & GG & GG & GG & GG \\
\hline GG & $\mathrm{AG}$ & GG & GG & GG & GG & GG & GG & GG & GG & GG \\
\hline GG & GG & GG & GG & GG & GG & $\mathrm{AG}$ & GG & GG & GG & GG \\
\hline GG & GG & GG & GG & GG & GG & GG & GG & GG & GG & GG \\
\hline GG & GG & GG & GG & GG & GG & GG & GG & GG & GG & GG \\
\hline GG & GG & GG & GG & GG & GG & GG & GG & GG & GG & GG \\
\hline GG & GG & GG & GG & GG & GG & GG & GG & GG & GG & GG \\
\hline GG & GG & GG & GG & GG & GG & - & GG & GG & GG & GG \\
\hline GG & GG & GG & - & GG & GG & $\mathrm{AG}$ & GG & GG & GG & \\
\hline GDsnp00274 & 15 & \multicolumn{2}{|c|}{7284741} & $\mathrm{AA}$ & $\mathrm{AA}$ & AA & $\mathrm{AA}$ & AA & AA & $\mathrm{AA}$ \\
\hline AA & $\mathrm{AC}$ & AA & AA & $\mathrm{AA}$ & $\mathrm{AA}$ & AA & $\mathrm{AA}$ & AA & AA & AA \\
\hline AA & $\mathrm{AA}$ & AA & AA & $\mathrm{AA}$ & AA & $\mathrm{AC}$ & $\mathrm{AA}$ & AA & AA & $\mathrm{AA}$ \\
\hline AA & $\mathrm{AA}$ & $\mathrm{AA}$ & $\mathrm{AA}$ & $\mathrm{AA}$ & $\mathrm{AA}$ & AA & $\mathrm{AA}$ & $\mathrm{AA}$ & AA & $\mathrm{AA}$ \\
\hline AA & $\mathrm{AA}$ & $\mathrm{AA}$ & AA & $\mathrm{AA}$ & $\mathrm{AA}$ & $\mathrm{AA}$ & $\mathrm{AA}$ & AA & $\mathrm{AA}$ & $\mathrm{AA}$ \\
\hline AA & $\mathrm{AA}$ & AA & AA & $\mathrm{AA}$ & $\mathrm{AA}$ & AA & $\mathrm{AA}$ & AA & AA & AA \\
\hline AA & $\mathrm{AA}$ & AA & AA & $\mathrm{AA}$ & AA & AA & $\mathrm{AA}$ & AA & AA & $\mathrm{AA}$ \\
\hline AA & $\mathrm{AA}$ & AA & AA & $\mathrm{AA}$ & AA & AA & $\mathrm{AA}$ & AA & AA & $\mathrm{AA}$ \\
\hline $\mathrm{AA}$ & AA & AA & AA & $\mathrm{AA}$ & AA & $\mathrm{AC}$ & $\mathrm{AA}$ & AA & AA & \\
\hline GDsnp01488 & 15 & \multicolumn{2}{|c|}{7769721} & $\mathrm{CC}$ & $\mathrm{CC}$ & $\mathrm{CC}$ & $\mathrm{CC}$ & $\mathrm{CC}$ & $\mathrm{CC}$ & $\mathrm{CC}$ \\
\hline $\mathrm{AC}$ & $\mathrm{AC}$ & $\mathrm{CC}$ & $\mathrm{CC}$ & $\mathrm{CC}$ & $\mathrm{CC}$ & $\mathrm{CC}$ & $\mathrm{CC}$ & $\mathrm{CC}$ & $\mathrm{CC}$ & $\mathrm{CC}$ \\
\hline $\mathrm{CC}$ & $\mathrm{CC}$ & $\mathrm{CC}$ & $\mathrm{CC}$ & $\mathrm{CC}$ & $\mathrm{CC}$ & $\mathrm{AC}$ & $\mathrm{CC}$ & $\mathrm{CC}$ & $\mathrm{CC}$ & $\mathrm{CC}$ \\
\hline $\mathrm{CC}$ & $\mathrm{CC}$ & $\mathrm{CC}$ & $\mathrm{CC}$ & $\mathrm{CC}$ & $\mathrm{CC}$ & $\mathrm{CC}$ & $\mathrm{CC}$ & $\mathrm{CC}$ & $\mathrm{CC}$ & $\mathrm{CC}$ \\
\hline $\mathrm{CC}$ & $\mathrm{CC}$ & $\mathrm{CC}$ & $\mathrm{CC}$ & $\mathrm{CC}$ & $\mathrm{CC}$ & $\mathrm{CC}$ & $\mathrm{CC}$ & $\mathrm{CC}$ & $\mathrm{CC}$ & $\mathrm{CC}$ \\
\hline $\mathrm{CC}$ & $\mathrm{CC}$ & $\mathrm{CC}$ & $\mathrm{AC}$ & $\mathrm{CC}$ & $\mathrm{CC}$ & $\mathrm{CC}$ & $\mathrm{CC}$ & $\mathrm{CC}$ & $\mathrm{CC}$ & $\mathrm{CC}$ \\
\hline $\mathrm{CC}$ & $\mathrm{CC}$ & $\mathrm{CC}$ & $\mathrm{CC}$ & $\mathrm{CC}$ & $\mathrm{CC}$ & $\mathrm{CC}$ & $\mathrm{CC}$ & $\mathrm{CC}$ & $\mathrm{CC}$ & $\mathrm{CC}$ \\
\hline $\mathrm{CC}$ & $\mathrm{CC}$ & $\mathrm{CC}$ & $\mathrm{CC}$ & $\mathrm{CC}$ & $\mathrm{CC}$ & $\mathrm{CC}$ & $\mathrm{CC}$ & $\mathrm{CC}$ & $\mathrm{CC}$ & $\mathrm{CC}$ \\
\hline $\mathrm{CC}$ & $\mathrm{CC}$ & $\mathrm{CC}$ & $\mathrm{CC}$ & $\mathrm{CC}$ & $\mathrm{CC}$ & $\mathrm{AC}$ & $\mathrm{CC}$ & $\mathrm{CC}$ & $\mathrm{CC}$ & \\
\hline
\end{tabular}


Supplemental Table 2. Continued.

\begin{tabular}{|c|c|c|c|c|c|c|c|c|c|c|}
\hline \multirow{3}{*}{$\begin{array}{l}\text { SNP name } \\
\text { GDsnp00271 } \\
\text { AA }\end{array}$} & \multicolumn{10}{|c|}{ Linkage group Physical location (bp) Individuals } \\
\hline & 15 & \multicolumn{2}{|c|}{11402005} & $\mathrm{AA}$ & $\mathrm{AA}$ & AA & $\mathrm{AA}$ & AA & AA & $\mathrm{AA}$ \\
\hline & AA & AA & AA & AA & AA & AA & AA & AA & AA & AA \\
\hline AA & AA & AA & $\mathrm{AA}$ & AA & AA & $\mathrm{AC}$ & AA & AA & AA & AA \\
\hline AA & AA & AA & $\mathrm{AA}$ & AA & AA & AA & AA & AA & AA & $\mathrm{AC}$ \\
\hline AA & $\mathrm{AA}$ & $\mathrm{AA}$ & $\mathrm{AA}$ & $\mathrm{AA}$ & $\mathrm{AA}$ & $\mathrm{AA}$ & $\mathrm{AA}$ & $\mathrm{AA}$ & $\mathrm{AA}$ & $\mathrm{AA}$ \\
\hline AA & $\mathrm{AA}$ & AA & $\mathrm{AC}$ & AA & $\mathrm{AA}$ & AA & $\mathrm{AA}$ & AA & AA & AA \\
\hline $\mathrm{AA}$ & $\mathrm{AA}$ & $\mathrm{AA}$ & $\mathrm{AA}$ & $\mathrm{AA}$ & $\mathrm{AA}$ & $\mathrm{AA}$ & $\mathrm{AA}$ & $\mathrm{AA}$ & $\mathrm{AA}$ & $\mathrm{AA}$ \\
\hline AA & $\mathrm{AA}$ & AA & $\mathrm{AA}$ & $\mathrm{AA}$ & $\mathrm{AA}$ & AA & $\mathrm{AA}$ & AA & AA & AA \\
\hline $\mathrm{AA}$ & $\mathrm{AA}$ & $\mathrm{AA}$ & $\mathrm{AA}$ & $\mathrm{AA}$ & $\mathrm{AA}$ & $\mathrm{AC}$ & $\mathrm{AA}$ & $\mathrm{AA}$ & AA & \\
\hline GDsnp01146 & 15 & \multicolumn{2}{|c|}{11945655} & GG & GG & GG & GG & GG & GG & GG \\
\hline GG & $\mathrm{AG}$ & GG & GG & GG & GG & GG & GG & GG & GG & GG \\
\hline GG & GG & GG & GG & GG & GG & $\mathrm{AG}$ & GG & GG & GG & GG \\
\hline GG & GG & GG & GG & GG & GG & GG & GG & GG & GG & $\mathrm{AG}$ \\
\hline GG & GG & GG & GG & GG & GG & - & GG & GG & GG & GG \\
\hline GG & GG & GG & $\mathrm{AG}$ & GG & GG & GG & GG & GG & GG & GG \\
\hline GG & GG & GG & GG & GG & GG & GG & GG & GG & GG & GG \\
\hline GG & GG & GG & GG & GG & GG & GG & GG & GG & GG & GG \\
\hline GG & GG & GG & GG & GG & GG & $\mathrm{AG}$ & GG & GG & GG & \\
\hline GDsnp00816 & 15 & \multicolumn{2}{|c|}{14144051} & GG & $\mathrm{AG}$ & GG & GG & AA & $\mathrm{AG}$ & $\mathrm{AG}$ \\
\hline $\mathrm{AG}$ & $\mathrm{AG}$ & AA & $\mathrm{AG}$ & $\mathrm{AA}$ & $\mathrm{AG}$ & AA & $\mathrm{AG}$ & AA & $\mathrm{AG}$ & $\mathrm{AA}$ \\
\hline $\mathrm{AA}$ & GG & $\mathrm{AG}$ & GG & GG & $\mathrm{AA}$ & $\mathrm{AG}$ & $\mathrm{AG}$ & $\mathrm{AG}$ & $\mathrm{AG}$ & $\mathrm{AG}$ \\
\hline- & $\mathrm{AG}$ & $\mathrm{AG}$ & $\mathrm{AA}$ & $\mathrm{AG}$ & $\mathrm{AA}$ & $\mathrm{AG}$ & $\mathrm{AA}$ & $\mathrm{AA}$ & GG & $\mathrm{AA}$ \\
\hline $\mathrm{AG}$ & $\mathrm{AG}$ & $\mathrm{AA}$ & $\mathrm{AA}$ & $\mathrm{AG}$ & $\mathrm{AG}$ & - & $\mathrm{AG}$ & $\mathrm{AA}$ & $\mathrm{AG}$ & $\mathrm{AA}$ \\
\hline GG & $\mathrm{AG}$ & $\mathrm{AG}$ & $\mathrm{AA}$ & $\mathrm{AG}$ & GG & $\mathrm{AG}$ & $\mathrm{AA}$ & $\mathrm{AG}$ & $\mathrm{AG}$ & GG \\
\hline $\mathrm{AG}$ & $\mathrm{AA}$ & $\mathrm{AG}$ & $\mathrm{AA}$ & $\mathrm{AA}$ & $\mathrm{AG}$ & $\mathrm{AG}$ & $\mathrm{AA}$ & AA & AA & $\mathrm{AG}$ \\
\hline GG & $\mathrm{AA}$ & $\mathrm{AG}$ & GG & $\mathrm{AG}$ & AA & AA & $\mathrm{AG}$ & AA & $\mathrm{AG}$ & $\mathrm{AG}$ \\
\hline $\mathrm{AG}$ & $\mathrm{AA}$ & $\mathrm{AG}$ & $\mathrm{AG}$ & $\mathrm{AA}$ & $\mathrm{AA}$ & AA & $\mathrm{AG}$ & $\mathrm{AA}$ & AA & \\
\hline GDsnp00273 & 15 & \multicolumn{2}{|c|}{14617139} & $\mathrm{AA}$ & $\mathrm{AG}$ & $\mathrm{AA}$ & $\mathrm{AA}$ & GG & $\mathrm{AG}$ & GG \\
\hline $\mathrm{AG}$ & $\mathrm{AG}$ & GG & $\mathrm{AG}$ & GG & $\mathrm{AG}$ & GG & $\mathrm{AG}$ & GG & $\mathrm{AG}$ & GG \\
\hline GG & $\mathrm{AA}$ & $\mathrm{AG}$ & $\mathrm{AA}$ & $\mathrm{AA}$ & GG & $\mathrm{AG}$ & $\mathrm{AG}$ & $\mathrm{AG}$ & $\mathrm{AG}$ & $\mathrm{AG}$ \\
\hline- & $\mathrm{AG}$ & $\mathrm{AG}$ & GG & $\mathrm{AG}$ & GG & $\mathrm{AG}$ & GG & GG & AA & GG \\
\hline $\mathrm{AG}$ & $\mathrm{AG}$ & GG & GG & $\mathrm{AG}$ & $\mathrm{AG}$ & $\mathrm{AG}$ & $\mathrm{AG}$ & GG & $\mathrm{AG}$ & GG \\
\hline AA & $\mathrm{AG}$ & $\mathrm{AG}$ & GG & $\mathrm{AG}$ & AA & $\mathrm{AG}$ & GG & $\mathrm{AG}$ & $\mathrm{AG}$ & $\mathrm{AA}$ \\
\hline $\mathrm{AG}$ & GG & $\mathrm{AG}$ & GG & GG & $\mathrm{AG}$ & $\mathrm{AG}$ & GG & GG & GG & $\mathrm{AG}$ \\
\hline $\mathrm{AA}$ & GG & $\mathrm{AG}$ & $\mathrm{AA}$ & $\mathrm{AG}$ & GG & GG & $\mathrm{AG}$ & GG & $\mathrm{AG}$ & $\mathrm{AG}$ \\
\hline $\mathrm{AG}$ & $\mathrm{AG}$ & $\mathrm{AG}$ & $\mathrm{AG}$ & GG & GG & GG & $\mathrm{AG}$ & GG & GG & \\
\hline GDsnp02029 & 15 & \multicolumn{2}{|c|}{14736474} & $\mathrm{AA}$ & $\mathrm{AT}$ & AA & $\mathrm{AA}$ & $\mathrm{TT}$ & $\mathrm{AT}$ & TT \\
\hline AT & $\mathrm{AT}$ & $\mathrm{TT}$ & $\mathrm{AT}$ & $\mathrm{TT}$ & $\mathrm{AT}$ & $\mathrm{TT}$ & $\mathrm{AT}$ & $\mathrm{TT}$ & $\mathrm{AT}$ & TT \\
\hline $\mathrm{TT}$ & $\mathrm{AA}$ & $\mathrm{AT}$ & $\mathrm{AA}$ & $\mathrm{AA}$ & $\mathrm{TT}$ & $\mathrm{AT}$ & AT & $\mathrm{AT}$ & $\mathrm{AT}$ & AT \\
\hline- & $\mathrm{AT}$ & $\mathrm{AT}$ & $\mathrm{TT}$ & $\mathrm{AT}$ & $\mathrm{TT}$ & AT & TT & TT & AA & TT \\
\hline AT & $\mathrm{AT}$ & $\mathrm{TT}$ & $\mathrm{TT}$ & $\mathrm{AT}$ & $\mathrm{AT}$ & $\mathrm{AT}$ & $\mathrm{AT}$ & $\mathrm{TT}$ & $\mathrm{AT}$ & TT \\
\hline AA & $\mathrm{AT}$ & $\mathrm{AT}$ & $\mathrm{TT}$ & AT & $\mathrm{AA}$ & AT & $\mathrm{TT}$ & $\mathrm{AT}$ & $\mathrm{AT}$ & AA \\
\hline AT & $\mathrm{TT}$ & $\mathrm{AT}$ & $\mathrm{TT}$ & $\mathrm{TT}$ & $\mathrm{AT}$ & $\mathrm{AT}$ & $\mathrm{TT}$ & $\mathrm{TT}$ & $\mathrm{TT}$ & AT \\
\hline $\mathrm{AA}$ & $\mathrm{TT}$ & $\mathrm{AT}$ & $\mathrm{AA}$ & $\mathrm{AT}$ & $\mathrm{TT}$ & $\mathrm{TT}$ & $\mathrm{AT}$ & $\mathrm{TT}$ & AT & AT \\
\hline AT & AT & AT & AT & TT & TT & TT & AT & TT & $\mathrm{TT}$ & \\
\hline GDsnp01229 & 15 & \multicolumn{2}{|c|}{15290673} & GG & $\mathrm{AG}$ & GG & GG & $\mathrm{AA}$ & $\mathrm{AG}$ & $\mathrm{AA}$ \\
\hline GG & GG & $\mathrm{AA}$ & $\mathrm{AG}$ & $\mathrm{AA}$ & $\mathrm{AG}$ & AA & $\mathrm{AG}$ & $\mathrm{AA}$ & $\mathrm{AG}$ & $\mathrm{AA}$ \\
\hline AA & GG & $\mathrm{AG}$ & GG & GG & $\mathrm{AA}$ & GG & $\mathrm{AG}$ & $\mathrm{AG}$ & $\mathrm{AG}$ & $\mathrm{AG}$ \\
\hline- & $\mathrm{AG}$ & $\mathrm{AG}$ & $\mathrm{AA}$ & $\mathrm{AG}$ & $\mathrm{AA}$ & $\mathrm{AG}$ & $\mathrm{AA}$ & AA & $\mathrm{AG}$ & $\mathrm{AG}$ \\
\hline AG & $\mathrm{AG}$ & $\mathrm{AA}$ & $\mathrm{AA}$ & $\mathrm{AG}$ & $\mathrm{AG}$ & $\mathrm{AG}$ & GG & - & $\mathrm{AG}$ & $\mathrm{AA}$ \\
\hline GG & $\mathrm{AG}$ & $\mathrm{AG}$ & $\mathrm{AG}$ & $\mathrm{AG}$ & GG & $\mathrm{AG}$ & $\mathrm{AA}$ & $\mathrm{AG}$ & $\mathrm{AG}$ & GG \\
\hline $\mathrm{AG}$ & $\mathrm{AA}$ & GG & $\mathrm{AA}$ & $\mathrm{AA}$ & $\mathrm{AG}$ & $\mathrm{AG}$ & $\mathrm{AG}$ & $\mathrm{AA}$ & $\mathrm{AA}$ & $\mathrm{AG}$ \\
\hline GG & $\mathrm{AA}$ & $\mathrm{AG}$ & GG & $\mathrm{AG}$ & $\mathrm{AA}$ & $\mathrm{AA}$ & $\mathrm{AG}$ & AA & $\mathrm{AG}$ & $\mathrm{AG}$ \\
\hline $\mathrm{AG}$ & $\mathrm{AG}$ & $\mathrm{AG}$ & $\mathrm{AG}$ & $\mathrm{AA}$ & $\mathrm{AA}$ & $\mathrm{AG}$ & $\mathrm{AG}$ & $\mathrm{AA}$ & AA & \\
\hline
\end{tabular}

Continued next page 
Supplemental Table 2. Continued.

\begin{tabular}{|c|c|c|c|c|c|c|c|c|c|c|}
\hline \multirow{3}{*}{$\begin{array}{l}\text { SNP name } \\
\text { GDsnp01622 } \\
\text { GG }\end{array}$} & \multicolumn{10}{|c|}{ Linkage group Physical location (bp) Individuals } \\
\hline & 15 & \multicolumn{2}{|c|}{18565063} & $\mathrm{AG}$ & $\mathrm{AG}$ & GG & GG & AA & $\mathrm{AG}$ & $\mathrm{AA}$ \\
\hline & $\mathrm{AG}$ & AA & $\mathrm{AG}$ & AA & AG & AA & AG & AA & $\mathrm{AG}$ & AA \\
\hline $\mathrm{AA}$ & $\mathrm{AG}$ & $\mathrm{AG}$ & GG & GG & AA & $\mathrm{AG}$ & AG & $\mathrm{AG}$ & $\mathrm{AG}$ & $\mathrm{AG}$ \\
\hline- & $\mathrm{AG}$ & $\mathrm{AG}$ & AA & $\mathrm{AG}$ & AA & AG & AA & AA & $\mathrm{AG}$ & $\mathrm{AA}$ \\
\hline $\mathrm{AG}$ & $\mathrm{AG}$ & $\mathrm{AA}$ & $\mathrm{AA}$ & $\mathrm{AG}$ & AG & $\mathrm{AG}$ & $\mathrm{AG}$ & $\mathrm{AA}$ & $\mathrm{AG}$ & $\mathrm{AA}$ \\
\hline GG & $\mathrm{AG}$ & $\mathrm{AG}$ & AA & $\mathrm{AG}$ & GG & $\mathrm{AG}$ & AA & $\mathrm{AG}$ & $\mathrm{AG}$ & GG \\
\hline $\mathrm{AG}$ & $\mathrm{AA}$ & GG & $\mathrm{AA}$ & $\mathrm{AA}$ & $\mathrm{AG}$ & $\mathrm{AG}$ & AA & $\mathrm{AA}$ & $\mathrm{AA}$ & $\mathrm{AG}$ \\
\hline GG & $\mathrm{AA}$ & $\mathrm{AG}$ & $\mathrm{AG}$ & $\mathrm{AG}$ & AA & $\mathrm{AA}$ & $\mathrm{AG}$ & $\mathrm{AA}$ & $\mathrm{AA}$ & $\mathrm{AG}$ \\
\hline $\mathrm{AG}$ & $\mathrm{AG}$ & $\mathrm{AA}$ & $\mathrm{AG}$ & $\mathrm{AA}$ & AA & $\mathrm{AG}$ & $\mathrm{AG}$ & $\mathrm{AG}$ & $\mathrm{AG}$ & \\
\hline GDsnp02823 & 15 & \multicolumn{2}{|c|}{20098763} & $\mathrm{AT}$ & AT & $\mathrm{AT}$ & AT & $\mathrm{TT}$ & $\mathrm{TT}$ & TT \\
\hline $\mathrm{AT}$ & $\mathrm{AT}$ & $\mathrm{TT}$ & $\mathrm{AT}$ & $\mathrm{TT}$ & $\mathrm{TT}$ & $\mathrm{AT}$ & AT & $\mathrm{AT}$ & $\mathrm{TT}$ & TT \\
\hline AT & $\mathrm{TT}$ & $\mathrm{AT}$ & AT & $\mathrm{TT}$ & $\mathrm{TT}$ & $\mathrm{AT}$ & $\mathrm{TT}$ & $\mathrm{TT}$ & $\mathrm{TT}$ & TT \\
\hline $\mathrm{TT}$ & $\mathrm{TT}$ & $\mathrm{TT}$ & $\mathrm{TT}$ & $\mathrm{AT}$ & AT & $\mathrm{AT}$ & $\mathrm{TT}$ & TT & $\mathrm{AT}$ & TT \\
\hline TT & $\mathrm{TT}$ & $\mathrm{TT}$ & TT & $\mathrm{TT}$ & $\mathrm{TT}$ & $\mathrm{TT}$ & - & $\mathrm{AT}$ & $\mathrm{TT}$ & TT \\
\hline $\mathrm{TT}$ & $\mathrm{TT}$ & $\mathrm{TT}$ & $\mathrm{TT}$ & $\mathrm{TT}$ & $\mathrm{TT}$ & $\mathrm{TT}$ & $\mathrm{TT}$ & $\mathrm{AT}$ & $\mathrm{AT}$ & TT \\
\hline AT & $\mathrm{TT}$ & $\mathrm{TT}$ & $\mathrm{AT}$ & $\mathrm{TT}$ & $\mathrm{TT}$ & $\mathrm{TT}$ & AT & $\mathrm{AT}$ & $\mathrm{TT}$ & AT \\
\hline $\mathrm{TT}$ & $\mathrm{TT}$ & $\mathrm{TT}$ & $\mathrm{TT}$ & $\mathrm{AT}$ & $\mathrm{TT}$ & $\mathrm{AT}$ & $\mathrm{TT}$ & AT & $\mathrm{TT}$ & TT \\
\hline $\mathrm{TT}$ & $\mathrm{TT}$ & $\mathrm{TT}$ & $\mathrm{TT}$ & $\mathrm{TT}$ & $\mathrm{TT}$ & $\mathrm{TT}$ & AT & $\mathrm{TT}$ & $\mathrm{TT}$ & \\
\hline GDsnp00984 & 15 & \multicolumn{2}{|c|}{20713294} & $\mathrm{AT}$ & $\mathrm{AT}$ & $\mathrm{TT}$ & AT & $\mathrm{AA}$ & $\mathrm{TT}$ & $\mathrm{AA}$ \\
\hline AT & $\mathrm{AT}$ & AA & $\mathrm{AT}$ & AT & AT & AA & AT & AA & $\mathrm{AT}$ & $\mathrm{AA}$ \\
\hline $\mathrm{AA}$ & $\mathrm{AT}$ & AT & TT & $\mathrm{TT}$ & AA & $\mathrm{AT}$ & AT & $\mathrm{AT}$ & $\mathrm{AT}$ & AT \\
\hline $\mathrm{AT}$ & $\mathrm{AT}$ & $\mathrm{AT}$ & $\mathrm{AA}$ & $\mathrm{AT}$ & AA & $\mathrm{AA}$ & AA & $\mathrm{AA}$ & $\mathrm{AT}$ & AT \\
\hline $\mathrm{AT}$ & $\mathrm{AT}$ & $\mathrm{AA}$ & $\mathrm{AA}$ & $\mathrm{AA}$ & AT & $\mathrm{AT}$ & - & $\mathrm{AA}$ & $\mathrm{AT}$ & AT \\
\hline $\mathrm{TT}$ & $\mathrm{AT}$ & AT & $\mathrm{AT}$ & $\mathrm{TT}$ & $\mathrm{TT}$ & $\mathrm{AT}$ & AA & $\mathrm{AT}$ & $\mathrm{AT}$ & TT \\
\hline AT & $\mathrm{AA}$ & $\mathrm{TT}$ & AA & $\mathrm{AA}$ & AT & AT & AA & AA & AA & AA \\
\hline $\mathrm{TT}$ & $\mathrm{AA}$ & $\mathrm{AT}$ & $\mathrm{AT}$ & $\mathrm{AT}$ & AA & AA & AT & AA & AA & AT \\
\hline AT & $\mathrm{AT}$ & $\mathrm{AA}$ & $\mathrm{AT}$ & $\mathrm{AA}$ & AA & AT & AT & $\mathrm{AT}$ & $\mathrm{AT}$ & \\
\hline GDsnp02859 & 15 & \multicolumn{2}{|c|}{21725620} & $\mathrm{AG}$ & $A G$ & GG & $\mathrm{AG}$ & $\mathrm{AA}$ & GG & AA \\
\hline $\mathrm{AG}$ & $\mathrm{AG}$ & $\mathrm{AA}$ & $\mathrm{AG}$ & $\mathrm{AG}$ & $\mathrm{AG}$ & $\mathrm{AA}$ & $\mathrm{AG}$ & AA & $\mathrm{AG}$ & $\mathrm{AA}$ \\
\hline AA & $\mathrm{AG}$ & $\mathrm{AG}$ & $\mathrm{AG}$ & GG & AA & $\mathrm{AG}$ & $\mathrm{AG}$ & $\mathrm{AG}$ & $\mathrm{AG}$ & $\mathrm{AG}$ \\
\hline $\mathrm{AG}$ & $\mathrm{AG}$ & $\mathrm{AG}$ & AA & $\mathrm{AG}$ & AA & AA & AA & AA & $\mathrm{AG}$ & $\mathrm{AG}$ \\
\hline $\mathrm{AG}$ & $\mathrm{AG}$ & AA & AA & $\mathrm{AA}$ & AG & $\mathrm{AG}$ & $\mathrm{AG}$ & AA & $\mathrm{AG}$ & $\mathrm{AG}$ \\
\hline GG & $\mathrm{AG}$ & $\mathrm{AG}$ & $\mathrm{AG}$ & GG & GG & $\mathrm{AG}$ & $\mathrm{AG}$ & AA & $\mathrm{AG}$ & GG \\
\hline AG & $\mathrm{AA}$ & GG & AA & $\mathrm{AA}$ & AG & $\mathrm{AG}$ & AA & AA & AA & $\mathrm{AA}$ \\
\hline GG & $\mathrm{AA}$ & $\mathrm{AG}$ & $\mathrm{AG}$ & $\mathrm{AG}$ & AG & AA & $\mathrm{AG}$ & AA & AA & $\mathrm{AG}$ \\
\hline $\mathrm{AG}$ & $\mathrm{AG}$ & AA & $\mathrm{AG}$ & $\mathrm{AA}$ & AA & $\mathrm{AG}$ & $\mathrm{AG}$ & $\mathrm{AG}$ & $\mathrm{AG}$ & \\
\hline GDsnp00848 & 15 & \multicolumn{2}{|c|}{23555361} & $\mathrm{AG}$ & $\mathrm{AG}$ & GG & $\mathrm{AG}$ & $\mathrm{AA}$ & GG & $\mathrm{AA}$ \\
\hline $\mathrm{AG}$ & $\mathrm{AG}$ & AA & $\mathrm{AG}$ & $\mathrm{AG}$ & AG & AA & $\mathrm{AG}$ & AA & $\mathrm{AG}$ & AA \\
\hline AA & $\mathrm{AG}$ & $\mathrm{AG}$ & $\mathrm{AG}$ & GG & AA & $\mathrm{AG}$ & $\mathrm{AG}$ & $\mathrm{AG}$ & $\mathrm{AG}$ & $\mathrm{AG}$ \\
\hline $\mathrm{AG}$ & $\mathrm{AG}$ & $\mathrm{AG}$ & $\mathrm{AA}$ & $\mathrm{AG}$ & AA & AA & AA & $\mathrm{AA}$ & $\mathrm{AG}$ & $\mathrm{AG}$ \\
\hline $\mathrm{AG}$ & $\mathrm{AG}$ & $\mathrm{AA}$ & AA & $\mathrm{AA}$ & $\mathrm{AG}$ & - & $\mathrm{AG}$ & AA & $\mathrm{AG}$ & $\mathrm{AG}$ \\
\hline GG & $\mathrm{AG}$ & $\mathrm{AG}$ & $\mathrm{AG}$ & GG & GG & $\mathrm{AG}$ & $\mathrm{AG}$ & AA & $\mathrm{AG}$ & GG \\
\hline $\mathrm{AG}$ & $\mathrm{AA}$ & GG & AA & $\mathrm{AA}$ & AG & $\mathrm{AG}$ & AA & AA & AA & $\mathrm{AA}$ \\
\hline GG & $\mathrm{AA}$ & $\mathrm{AG}$ & $\mathrm{AG}$ & $\mathrm{AG}$ & $\mathrm{AG}$ & $\mathrm{AA}$ & $\mathrm{AG}$ & $\mathrm{AA}$ & AA & $\mathrm{AG}$ \\
\hline AG & $\mathrm{AG}$ & $\mathrm{AG}$ & $\mathrm{AG}$ & $\mathrm{AA}$ & AA & $\mathrm{AG}$ & $\mathrm{AG}$ & $\mathrm{AG}$ & GG & \\
\hline GDsnp00241 & 15 & \multicolumn{2}{|c|}{29159671} & $\mathrm{AA}$ & $\mathrm{CC}$ & $\mathrm{CC}$ & AA & $\mathrm{AC}$ & $\mathrm{AA}$ & $\mathrm{AC}$ \\
\hline $\mathrm{AC}$ & $\mathrm{AC}$ & $\mathrm{CC}$ & $\mathrm{AA}$ & $\mathrm{AC}$ & $\mathrm{AC}$ & $\mathrm{CC}$ & $\mathrm{AC}$ & $\mathrm{CC}$ & $\mathrm{AC}$ & $\mathrm{CC}$ \\
\hline $\mathrm{CC}$ & $\mathrm{AC}$ & $\mathrm{AC}$ & $\mathrm{CC}$ & $\mathrm{AA}$ & $\mathrm{AC}$ & $\mathrm{AC}$ & $\mathrm{AC}$ & $\mathrm{AC}$ & $\mathrm{AC}$ & $\mathrm{AC}$ \\
\hline $\mathrm{AC}$ & $\mathrm{AC}$ & $\mathrm{AC}$ & $\mathrm{CC}$ & $\mathrm{AA}$ & AA & $\mathrm{CC}$ & $\mathrm{CC}$ & $\mathrm{CC}$ & $\mathrm{AC}$ & $\mathrm{AC}$ \\
\hline $\mathrm{AC}$ & $\mathrm{AC}$ & $\mathrm{AC}$ & $\mathrm{CC}$ & $\mathrm{CC}$ & $\mathrm{AC}$ & $\mathrm{AC}$ & $\mathrm{AC}$ & $\mathrm{AC}$ & $\mathrm{AC}$ & $\mathrm{AC}$ \\
\hline $\mathrm{AA}$ & $\mathrm{AC}$ & $\mathrm{CC}$ & $\mathrm{CC}$ & $\mathrm{AA}$ & AA & $\mathrm{AC}$ & $\mathrm{AC}$ & $\mathrm{AC}$ & $\mathrm{AC}$ & $\mathrm{AA}$ \\
\hline $\mathrm{AC}$ & $\mathrm{AC}$ & $\mathrm{AC}$ & $\mathrm{CC}$ & $\mathrm{CC}$ & $\mathrm{AC}$ & $\mathrm{AC}$ & AA & $\mathrm{CC}$ & $\mathrm{CC}$ & $\mathrm{CC}$ \\
\hline AA & $\mathrm{AC}$ & $\mathrm{AC}$ & $\mathrm{AC}$ & $\mathrm{AA}$ & $\mathrm{AC}$ & $\mathrm{AC}$ & $\mathrm{AC}$ & $\mathrm{CC}$ & $\mathrm{AC}$ & $\mathrm{AC}$ \\
\hline $\mathrm{AC}$ & $\mathrm{AA}$ & $\mathrm{AC}$ & $\mathrm{AC}$ & $\mathrm{CC}$ & $\mathrm{CC}$ & $\mathrm{AC}$ & $\mathrm{AC}$ & $\mathrm{AC}$ & AA & \\
\hline
\end{tabular}


Supplemental Table 2. Continued.

\begin{tabular}{|c|c|c|c|c|c|c|c|c|c|c|}
\hline \multirow{3}{*}{$\begin{array}{l}\text { SNP name } \\
\text { GDsnp00357 } \\
\text { AG }\end{array}$} & \multicolumn{10}{|c|}{ Linkage group Physical location (bp) Individuals } \\
\hline & 15 & \multicolumn{2}{|c|}{35281901} & $\mathrm{AG}$ & GG & GG & $\mathrm{AG}$ & $\mathrm{AG}$ & $\mathrm{AG}$ & $\mathrm{AG}$ \\
\hline & $\mathrm{AG}$ & GG & AA & AA & $\mathrm{AG}$ & GG & $\mathrm{AG}$ & GG & $\mathrm{AG}$ & GG \\
\hline GG & $\mathrm{AG}$ & AA & GG & $\mathrm{AA}$ & $\mathrm{AG}$ & $\mathrm{AG}$ & $\mathrm{AG}$ & $\mathrm{AG}$ & $\mathrm{AG}$ & $\mathrm{AG}$ \\
\hline AG & $\mathrm{AG}$ & GG & GG & $\mathrm{AG}$ & AA & $\mathrm{AG}$ & GG & GG & AA & $\mathrm{AG}$ \\
\hline $\mathrm{AG}$ & $\mathrm{AG}$ & $\mathrm{AG}$ & $\mathrm{AG}$ & GG & $\mathrm{AG}$ & $\mathrm{AG}$ & $\mathrm{AG}$ & $\mathrm{AG}$ & $\mathrm{AG}$ & $\mathrm{AG}$ \\
\hline AA & $\mathrm{AG}$ & GG & GG & $\mathrm{AG}$ & $\mathrm{AA}$ & $\mathrm{AG}$ & $\mathrm{AG}$ & $\mathrm{AG}$ & $\mathrm{AG}$ & AA \\
\hline $\mathrm{AG}$ & $\mathrm{AG}$ & $\mathrm{AG}$ & GG & GG & GG & $\mathrm{AG}$ & $\mathrm{AA}$ & GG & $\mathrm{AG}$ & GG \\
\hline $\mathrm{AA}$ & GG & $\mathrm{AG}$ & $\mathrm{AG}$ & $\mathrm{AA}$ & $\mathrm{AG}$ & $\mathrm{AG}$ & $\mathrm{AG}$ & GG & $\mathrm{AG}$ & $\mathrm{AG}$ \\
\hline $\mathrm{AA}$ & $\mathrm{AA}$ & $\mathrm{AG}$ & $\mathrm{AG}$ & $\mathrm{AG}$ & GG & $\mathrm{AG}$ & $\mathrm{AG}$ & $\mathrm{AG}$ & AA & \\
\hline GDsnp01805 & 15 & \multicolumn{2}{|c|}{38381924} & $\mathrm{AA}$ & GG & GG & $\mathrm{AA}$ & $\mathrm{AG}$ & $\mathrm{AA}$ & $\mathrm{AG}$ \\
\hline GG & $\mathrm{AG}$ & GG & $\mathrm{AA}$ & $\mathrm{AA}$ & $\mathrm{AG}$ & GG & GG & GG & $\mathrm{AG}$ & GG \\
\hline GG & $\mathrm{AG}$ & $\mathrm{AA}$ & GG & $\mathrm{AA}$ & $\mathrm{AG}$ & $\mathrm{AG}$ & $\mathrm{AG}$ & $\mathrm{AG}$ & $\mathrm{AG}$ & $\mathrm{AG}$ \\
\hline $\mathrm{AG}$ & $\mathrm{AG}$ & $\mathrm{AA}$ & GG & $\mathrm{AG}$ & $\mathrm{AA}$ & $\mathrm{AG}$ & $\mathrm{AG}$ & GG & $\mathrm{AG}$ & $\mathrm{AG}$ \\
\hline $\mathrm{AG}$ & $\mathrm{AG}$ & $\mathrm{AG}$ & $\mathrm{AG}$ & GG & $\mathrm{AG}$ & - & $\mathrm{AG}$ & $\mathrm{AG}$ & $\mathrm{AG}$ & $\mathrm{AG}$ \\
\hline $\mathrm{AA}$ & $\mathrm{AG}$ & GG & GG & $\mathrm{AG}$ & $\mathrm{AA}$ & $\mathrm{AG}$ & $\mathrm{AG}$ & $\mathrm{AG}$ & $\mathrm{AG}$ & AA \\
\hline AG & $\mathrm{AG}$ & $\mathrm{AG}$ & $\mathrm{AG}$ & GG & GG & $\mathrm{AG}$ & $\mathrm{AA}$ & $\mathrm{AG}$ & $\mathrm{AG}$ & GG \\
\hline AA & $\mathrm{AG}$ & $\mathrm{AG}$ & $\mathrm{AG}$ & $\mathrm{AG}$ & $\mathrm{AA}$ & - & $\mathrm{AG}$ & GG & $\mathrm{AG}$ & $\mathrm{AG}$ \\
\hline $\mathrm{AG}$ & $\mathrm{AA}$ & $\mathrm{AG}$ & GG & $\mathrm{AG}$ & GG & GG & $\mathrm{AG}$ & $\mathrm{AG}$ & AA & \\
\hline GDsnp00661 & 15 & \multicolumn{2}{|c|}{43094875} & AT & $\mathrm{TT}$ & $\mathrm{TT}$ & AA & AT & AT & $\mathrm{TT}$ \\
\hline $\mathrm{TT}$ & - & $\mathrm{TT}$ & $\mathrm{AT}$ & $\mathrm{AA}$ & $\mathrm{TT}$ & $\mathrm{TT}$ & $\mathrm{TT}$ & $\mathrm{TT}$ & $\mathrm{AT}$ & TT \\
\hline AT & $\mathrm{AA}$ & AT & $\mathrm{TT}$ & $\mathrm{AA}$ & $\mathrm{AT}$ & $\mathrm{AT}$ & $\mathrm{AT}$ & $\mathrm{AT}$ & $\mathrm{AT}$ & AT \\
\hline $\mathrm{AT}$ & $\mathrm{AT}$ & $\mathrm{AT}$ & $\mathrm{TT}$ & $\mathrm{AT}$ & $\mathrm{AA}$ & $\mathrm{TT}$ & $\mathrm{AT}$ & $\mathrm{TT}$ & $\mathrm{AT}$ & AT \\
\hline $\mathrm{AT}$ & $\mathrm{AT}$ & $\mathrm{AT}$ & $\mathrm{AT}$ & $\mathrm{TT}$ & $\mathrm{AT}$ & $\mathrm{AT}$ & $\mathrm{AT}$ & $\mathrm{AT}$ & $\mathrm{AT}$ & AT \\
\hline AA & $\mathrm{AT}$ & AT & $\mathrm{TT}$ & AT & $\mathrm{AA}$ & $\mathrm{AT}$ & $\mathrm{AT}$ & $\mathrm{AT}$ & $\mathrm{AT}$ & AT \\
\hline AT & $\mathrm{AT}$ & $\mathrm{AT}$ & $\mathrm{AT}$ & $\mathrm{TT}$ & $\mathrm{TT}$ & AT & $\mathrm{AT}$ & $\mathrm{AT}$ & $\mathrm{AT}$ & TT \\
\hline AT & $\mathrm{TT}$ & $\mathrm{TT}$ & $\mathrm{AT}$ & $\mathrm{AT}$ & AA & AA & AT & $\mathrm{TT}$ & $\mathrm{AT}$ & AT \\
\hline AT & $\mathrm{AA}$ & AT & $\mathrm{TT}$ & AT & TT & TT & TT & $\mathrm{AT}$ & $\mathrm{AA}$ & \\
\hline GDsnp00525 & 15 & \multicolumn{2}{|c|}{45537136} & $\mathrm{AC}$ & $\mathrm{CC}$ & $\mathrm{CC}$ & $\mathrm{CC}$ & $\mathrm{CC}$ & $\mathrm{AC}$ & $\mathrm{AC}$ \\
\hline $\mathrm{AC}$ & - & $\mathrm{AC}$ & $\mathrm{AC}$ & $\mathrm{CC}$ & $\mathrm{CC}$ & $\mathrm{CC}$ & $\mathrm{CC}$ & $\mathrm{AC}$ & $\mathrm{CC}$ & $\mathrm{CC}$ \\
\hline $\mathrm{CC}$ & $\mathrm{CC}$ & $\mathrm{CC}$ & $\mathrm{CC}$ & $\mathrm{CC}$ & $\mathrm{CC}$ & - & $\mathrm{CC}$ & $\mathrm{CC}$ & $\mathrm{CC}$ & $\mathrm{CC}$ \\
\hline $\mathrm{CC}$ & $\mathrm{CC}$ & $\mathrm{AC}$ & $\mathrm{CC}$ & $\mathrm{CC}$ & $\mathrm{CC}$ & $\mathrm{CC}$ & $\mathrm{CC}$ & $\mathrm{CC}$ & $\mathrm{AC}$ & $\mathrm{CC}$ \\
\hline $\mathrm{CC}$ & $\mathrm{CC}$ & $\mathrm{CC}$ & $\mathrm{CC}$ & $\mathrm{CC}$ & $\mathrm{CC}$ & $\mathrm{CC}$ & $\mathrm{AC}$ & $\mathrm{CC}$ & $\mathrm{CC}$ & $\mathrm{CC}$ \\
\hline $\mathrm{CC}$ & $\mathrm{CC}$ & $\mathrm{CC}$ & $\mathrm{AC}$ & $\mathrm{CC}$ & $\mathrm{CC}$ & $\mathrm{CC}$ & $\mathrm{CC}$ & $\mathrm{CC}$ & $\mathrm{CC}$ & $\mathrm{CC}$ \\
\hline $\mathrm{CC}$ & $\mathrm{CC}$ & $\mathrm{CC}$ & $\mathrm{CC}$ & $\mathrm{CC}$ & $\mathrm{CC}$ & $\mathrm{AC}$ & $\mathrm{AC}$ & $\mathrm{CC}$ & $\mathrm{CC}$ & $\mathrm{CC}$ \\
\hline $\mathrm{CC}$ & $\mathrm{AC}$ & $\mathrm{CC}$ & $\mathrm{CC}$ & $\mathrm{CC}$ & $\mathrm{CC}$ & $\mathrm{CC}$ & $\mathrm{CC}$ & $\mathrm{CC}$ & $\mathrm{CC}$ & $\mathrm{CC}$ \\
\hline $\mathrm{CC}$ & $\mathrm{CC}$ & $\mathrm{CC}$ & $\mathrm{CC}$ & $\mathrm{CC}$ & $\mathrm{CC}$ & $\mathrm{AC}$ & $\mathrm{CC}$ & $\mathrm{CC}$ & $\mathrm{CC}$ & \\
\hline GDsnp01588 & 16 & \multicolumn{2}{|c|}{1221007} & $\mathrm{CC}$ & $\mathrm{CC}$ & $\mathrm{CC}$ & $\mathrm{CC}$ & AA & $\mathrm{CC}$ & $\mathrm{CC}$ \\
\hline AA & $\mathrm{CC}$ & $\mathrm{CC}$ & $\mathrm{AA}$ & $\mathrm{AA}$ & $\mathrm{CC}$ & AA & $\mathrm{CC}$ & AA & $\mathrm{CC}$ & $\mathrm{CC}$ \\
\hline $\mathrm{CC}$ & $\mathrm{CC}$ & AA & $\mathrm{AA}$ & $\mathrm{CC}$ & $\mathrm{CC}$ & $\mathrm{AC}$ & $\mathrm{AA}$ & AA & $\mathrm{CC}$ & $\mathrm{AA}$ \\
\hline $\mathrm{CC}$ & $\mathrm{CC}$ & $\mathrm{AA}$ & $\mathrm{AA}$ & $\mathrm{CC}$ & $\mathrm{CC}$ & $\mathrm{CC}$ & $\mathrm{AA}$ & $\mathrm{AA}$ & AA & $\mathrm{AA}$ \\
\hline AA & $\mathrm{CC}$ & $\mathrm{AA}$ & $\mathrm{AA}$ & $\mathrm{AA}$ & $\mathrm{CC}$ & $\mathrm{AA}$ & $\mathrm{AC}$ & $\mathrm{CC}$ & $\mathrm{CC}$ & $\mathrm{AA}$ \\
\hline $\mathrm{CC}$ & $\mathrm{CC}$ & $\mathrm{AA}$ & $\mathrm{AA}$ & $\mathrm{AA}$ & $\mathrm{CC}$ & AA & $\mathrm{AA}$ & $\mathrm{CC}$ & $\mathrm{CC}$ & AA \\
\hline $\mathrm{CC}$ & $\mathrm{AA}$ & AA & $\mathrm{AA}$ & $\mathrm{CC}$ & $\mathrm{CC}$ & $\mathrm{CC}$ & $\mathrm{AA}$ & AA & $\mathrm{CC}$ & $\mathrm{CC}$ \\
\hline AA & $\mathrm{CC}$ & $\mathrm{CC}$ & $\mathrm{CC}$ & $\mathrm{CC}$ & $\mathrm{AA}$ & $\mathrm{AA}$ & $\mathrm{AA}$ & $\mathrm{CC}$ & AA & $\mathrm{CC}$ \\
\hline $\mathrm{CC}$ & $\mathrm{CC}$ & $\mathrm{CC}$ & $\mathrm{CC}$ & $\mathrm{AA}$ & $\mathrm{CC}$ & $\mathrm{CC}$ & $\mathrm{CC}$ & $\mathrm{CC}$ & AA & \\
\hline GDsnp01600 & 16 & \multicolumn{2}{|c|}{1344071} & $\mathrm{AG}$ & $\mathrm{AG}$ & $\mathrm{AG}$ & $\mathrm{AG}$ & GG & $\mathrm{AG}$ & $\mathrm{AG}$ \\
\hline GG & $\mathrm{AG}$ & $\mathrm{AG}$ & GG & GG & $\mathrm{AG}$ & GG & $\mathrm{AG}$ & GG & $\mathrm{AG}$ & $\mathrm{AG}$ \\
\hline $\mathrm{AG}$ & $\mathrm{AG}$ & GG & GG & $\mathrm{AG}$ & $\mathrm{AG}$ & $\mathrm{AG}$ & GG & GG & GG & GG \\
\hline $\mathrm{AG}$ & $\mathrm{AG}$ & GG & GG & $\mathrm{AG}$ & $\mathrm{AG}$ & $\mathrm{AG}$ & GG & GG & GG & GG \\
\hline GG & $\mathrm{AG}$ & GG & GG & GG & $\mathrm{AG}$ & GG & $\mathrm{AG}$ & $\mathrm{AG}$ & $\mathrm{AG}$ & GG \\
\hline $\mathrm{AG}$ & $\mathrm{AG}$ & GG & GG & GG & $\mathrm{AG}$ & GG & GG & $\mathrm{AG}$ & $\mathrm{AG}$ & GG \\
\hline $\mathrm{AG}$ & GG & GG & GG & $\mathrm{AG}$ & $\mathrm{AG}$ & $\mathrm{AG}$ & GG & GG & $\mathrm{AG}$ & $\mathrm{AG}$ \\
\hline GG & $\mathrm{AG}$ & $\mathrm{AG}$ & $\mathrm{AG}$ & $\mathrm{AG}$ & GG & GG & GG & $\mathrm{AG}$ & GG & $\mathrm{AG}$ \\
\hline $\mathrm{AG}$ & $\mathrm{AG}$ & $\mathrm{AG}$ & $\mathrm{AG}$ & GG & $\mathrm{AG}$ & $\mathrm{AG}$ & $\mathrm{AG}$ & $\mathrm{AG}$ & GG & \\
\hline
\end{tabular}

Continued next page 
Supplemental Table 2. Continued.

\begin{tabular}{|c|c|c|c|c|c|c|c|c|c|c|}
\hline \multirow{3}{*}{$\begin{array}{l}\text { SNP name } \\
\text { GDsnp01734 } \\
\text { AG }\end{array}$} & \multicolumn{10}{|c|}{ Linkage group Physical location (bp) Individuals } \\
\hline & 16 & \multicolumn{2}{|c|}{1620445} & GG & GG & GG & $\mathrm{AG}$ & $\mathrm{AG}$ & $\mathrm{AG}$ & GG \\
\hline & $\mathrm{AG}$ & GG & AA & $\mathrm{AG}$ & GG & $\mathrm{AG}$ & AG & $\mathrm{AG}$ & GG & GG \\
\hline $\mathrm{AG}$ & GG & AA & $\mathrm{AG}$ & GG & GG & AA & AG & $\mathrm{AG}$ & $\mathrm{AG}$ & $\mathrm{AG}$ \\
\hline GG & $\mathrm{AG}$ & $\mathrm{AG}$ & AA & $\mathrm{AG}$ & $\mathrm{AG}$ & $\mathrm{AG}$ & AG & $\mathrm{AG}$ & $\mathrm{AG}$ & $\mathrm{AG}$ \\
\hline $\mathrm{AG}$ & $\mathrm{AG}$ & $\mathrm{AG}$ & $\mathrm{AG}$ & $\mathrm{AA}$ & $\mathrm{AG}$ & $\mathrm{AA}$ & GG & $\mathrm{AG}$ & GG & $\mathrm{AG}$ \\
\hline $\mathrm{AG}$ & GG & $\mathrm{AA}$ & $\mathrm{AA}$ & $\mathrm{AG}$ & GG & $\mathrm{AG}$ & $\mathrm{AG}$ & GG & GG & $\mathrm{AG}$ \\
\hline GG & $\mathrm{AA}$ & $\mathrm{AG}$ & $\mathrm{AA}$ & GG & $\mathrm{AG}$ & GG & $\mathrm{AG}$ & $\mathrm{AA}$ & GG & $\mathrm{AG}$ \\
\hline $\mathrm{AG}$ & GG & GG & GG & GG & $\mathrm{AG}$ & $\mathrm{AG}$ & $\mathrm{AG}$ & $\mathrm{AG}$ & $\mathrm{AG}$ & $\mathrm{AG}$ \\
\hline $\mathrm{AG}$ & GG & GG & $\mathrm{AG}$ & $\mathrm{AG}$ & $\mathrm{AG}$ & GG & $\mathrm{AG}$ & $\mathrm{AG}$ & AA & \\
\hline GDsnp00292 & 16 & \multicolumn{2}{|c|}{1668613} & $\mathrm{CC}$ & $\mathrm{CC}$ & $\mathrm{CC}$ & $\mathrm{AC}$ & $\mathrm{AC}$ & $\mathrm{AC}$ & $\mathrm{CC}$ \\
\hline $\mathrm{AC}$ & $\mathrm{AC}$ & $\mathrm{CC}$ & $\mathrm{AA}$ & $\mathrm{AC}$ & $\mathrm{CC}$ & $\mathrm{AC}$ & $\mathrm{AC}$ & $\mathrm{AC}$ & $\mathrm{CC}$ & $\mathrm{CC}$ \\
\hline $\mathrm{AC}$ & $\mathrm{CC}$ & $\mathrm{AA}$ & $\mathrm{AC}$ & $\mathrm{CC}$ & $\mathrm{CC}$ & $\mathrm{AC}$ & $\mathrm{AC}$ & $\mathrm{AC}$ & $\mathrm{AC}$ & $\mathrm{AC}$ \\
\hline $\mathrm{CC}$ & $\mathrm{AC}$ & $\mathrm{AC}$ & $\mathrm{AA}$ & $\mathrm{AC}$ & $\mathrm{AC}$ & $\mathrm{AC}$ & $\mathrm{AC}$ & $\mathrm{AC}$ & $\mathrm{AC}$ & $\mathrm{AC}$ \\
\hline $\mathrm{AC}$ & $\mathrm{AC}$ & $\mathrm{AC}$ & $\mathrm{AC}$ & $\mathrm{AA}$ & $\mathrm{AC}$ & $\mathrm{AA}$ & $\mathrm{CC}$ & $\mathrm{AC}$ & $\mathrm{CC}$ & $\mathrm{AC}$ \\
\hline $\mathrm{AC}$ & $\mathrm{CC}$ & $\mathrm{AA}$ & $\mathrm{AA}$ & $\mathrm{AC}$ & $\mathrm{CC}$ & $\mathrm{AC}$ & $\mathrm{AC}$ & $\mathrm{CC}$ & $\mathrm{CC}$ & $\mathrm{AC}$ \\
\hline $\mathrm{CC}$ & $\mathrm{AA}$ & $\mathrm{AC}$ & AA & $\mathrm{CC}$ & $\mathrm{AC}$ & $\mathrm{CC}$ & $\mathrm{AC}$ & AA & $\mathrm{CC}$ & $\mathrm{AC}$ \\
\hline $\mathrm{AC}$ & $\mathrm{CC}$ & $\mathrm{CC}$ & $\mathrm{CC}$ & $\mathrm{CC}$ & $\mathrm{AC}$ & $\mathrm{AC}$ & $\mathrm{AC}$ & $\mathrm{AC}$ & $\mathrm{AC}$ & $\mathrm{AC}$ \\
\hline $\mathrm{AC}$ & $\mathrm{CC}$ & $\mathrm{CC}$ & $\mathrm{AC}$ & $\mathrm{AC}$ & $\mathrm{AC}$ & $\mathrm{CC}$ & $\mathrm{AC}$ & $\mathrm{AC}$ & AA & \\
\hline GDsnp00269 & 16 & \multicolumn{2}{|c|}{1770342} & $\mathrm{AA}$ & AA & AA & $A G$ & $\mathrm{AG}$ & $\mathrm{AG}$ & AA \\
\hline $\mathrm{AG}$ & $\mathrm{AG}$ & AA & GG & $\mathrm{AG}$ & $\mathrm{AA}$ & $\mathrm{AG}$ & AG & $\mathrm{AG}$ & AA & $\mathrm{AA}$ \\
\hline $\mathrm{AG}$ & $\mathrm{AA}$ & GG & $\mathrm{AG}$ & $\mathrm{AA}$ & $\mathrm{AA}$ & GG & $\mathrm{AG}$ & $\mathrm{AG}$ & $\mathrm{AG}$ & $\mathrm{AG}$ \\
\hline $\mathrm{AA}$ & $\mathrm{AG}$ & $\mathrm{AG}$ & GG & $\mathrm{AG}$ & $\mathrm{AG}$ & $\mathrm{AG}$ & $\mathrm{AG}$ & $\mathrm{AG}$ & $\mathrm{AG}$ & $\mathrm{AG}$ \\
\hline $\mathrm{AG}$ & $\mathrm{AG}$ & $\mathrm{AG}$ & $\mathrm{AG}$ & GG & $\mathrm{AG}$ & GG & AA & $\mathrm{AG}$ & $\mathrm{AA}$ & $\mathrm{AG}$ \\
\hline $\mathrm{AG}$ & $\mathrm{AA}$ & GG & GG & $\mathrm{AG}$ & $\mathrm{AA}$ & $\mathrm{AG}$ & AG & AA & AA & $\mathrm{AG}$ \\
\hline AA & GG & $\mathrm{AG}$ & GG & $\mathrm{AA}$ & $\mathrm{AG}$ & AA & $\mathrm{AG}$ & GG & AA & $\mathrm{AG}$ \\
\hline AG & $\mathrm{AA}$ & AA & AA & $\mathrm{AA}$ & $\mathrm{AG}$ & $\mathrm{AG}$ & $\mathrm{AG}$ & $\mathrm{AG}$ & $\mathrm{AG}$ & $\mathrm{AG}$ \\
\hline $\mathrm{AG}$ & $\mathrm{AA}$ & AA & $\mathrm{AG}$ & $\mathrm{AG}$ & AG & AA & $\mathrm{AG}$ & $\mathrm{AG}$ & GG & \\
\hline GDsnp00923 & 16 & \multicolumn{2}{|c|}{2084181} & $\mathrm{AA}$ & $\mathrm{AA}$ & $\mathrm{AG}$ & $\mathrm{AG}$ & $\mathrm{AG}$ & $\mathrm{AG}$ & AA \\
\hline $\mathrm{AG}$ & $\mathrm{AG}$ & $\mathrm{AA}$ & GG & $\mathrm{AG}$ & $\mathrm{AA}$ & $\mathrm{AG}$ & $\mathrm{AG}$ & $\mathrm{AG}$ & $\mathrm{AA}$ & $\mathrm{AA}$ \\
\hline $\mathrm{AG}$ & $\mathrm{AA}$ & GG & $\mathrm{AG}$ & $\mathrm{AA}$ & $\mathrm{AA}$ & GG & $\mathrm{AG}$ & $\mathrm{AG}$ & $\mathrm{AG}$ & $\mathrm{AG}$ \\
\hline AA & $\mathrm{AG}$ & $\mathrm{AG}$ & GG & $\mathrm{AG}$ & $\mathrm{AG}$ & $\mathrm{AG}$ & $\mathrm{AG}$ & $\mathrm{AG}$ & $\mathrm{AG}$ & $\mathrm{AG}$ \\
\hline $\mathrm{AG}$ & $\mathrm{AG}$ & $\mathrm{AG}$ & $\mathrm{AG}$ & GG & $\mathrm{AG}$ & GG & $\mathrm{AG}$ & $\mathrm{AG}$ & AA & $\mathrm{AG}$ \\
\hline AG & $\mathrm{AA}$ & GG & GG & $\mathrm{AG}$ & AA & $\mathrm{AG}$ & $\mathrm{AG}$ & AA & AA & $\mathrm{AG}$ \\
\hline AA & GG & $\mathrm{AG}$ & GG & $\mathrm{AA}$ & $\mathrm{AG}$ & AA & $\mathrm{AG}$ & GG & $\mathrm{AA}$ & $\mathrm{AG}$ \\
\hline $\mathrm{AG}$ & $\mathrm{AA}$ & $\mathrm{AA}$ & AA & $\mathrm{AA}$ & $\mathrm{AG}$ & $\mathrm{AG}$ & $\mathrm{AG}$ & $\mathrm{AG}$ & $\mathrm{AG}$ & $\mathrm{AG}$ \\
\hline $\mathrm{AG}$ & $\mathrm{AA}$ & AA & $\mathrm{AG}$ & $\mathrm{AG}$ & $\mathrm{AG}$ & AA & $\mathrm{AG}$ & $\mathrm{AG}$ & GG & \\
\hline GDsnp00196 & 16 & \multicolumn{2}{|c|}{3023008} & $\mathrm{AA}$ & $\mathrm{AA}$ & AA & AA & AA & AA & $\mathrm{AA}$ \\
\hline AA & $\mathrm{AA}$ & AA & AA & $\mathrm{AA}$ & $\mathrm{AA}$ & AA & AA & AA & AA & AA \\
\hline AA & $\mathrm{AA}$ & AA & AA & $\mathrm{AA}$ & AA & AA & AA & AA & AA & $\mathrm{AA}$ \\
\hline AA & $\mathrm{AA}$ & $\mathrm{AA}$ & $\mathrm{AA}$ & $\mathrm{AA}$ & $\mathrm{AA}$ & AA & AA & $\mathrm{AA}$ & AA & $\mathrm{AA}$ \\
\hline AA & $\mathrm{AA}$ & $\mathrm{AA}$ & AA & $\mathrm{AA}$ & $\mathrm{AA}$ & $\mathrm{AA}$ & $\mathrm{AG}$ & AA & $\mathrm{AA}$ & $\mathrm{AA}$ \\
\hline AA & $\mathrm{AA}$ & AA & AA & $\mathrm{AA}$ & $\mathrm{AA}$ & AA & AA & AA & AA & AA \\
\hline AA & $\mathrm{AA}$ & AA & AA & $\mathrm{AA}$ & AA & AA & AA & AA & AA & $\mathrm{AA}$ \\
\hline $\mathrm{AA}$ & $\mathrm{AA}$ & $\mathrm{AA}$ & $\mathrm{AA}$ & $\mathrm{AA}$ & $\mathrm{AA}$ & $\mathrm{AA}$ & AA & $\mathrm{AA}$ & AA & $\mathrm{AA}$ \\
\hline AA & AA & AA & AA & $\mathrm{AA}$ & AA & AA & AA & AA & AA & \\
\hline GDsnp00069 & 16 & \multicolumn{2}{|c|}{3238583} & GG & GG & GG & $\mathrm{AG}$ & GG & $\mathrm{AG}$ & GG \\
\hline $\mathrm{AG}$ & $\mathrm{AG}$ & GG & $\mathrm{AG}$ & GG & $\mathrm{AG}$ & GG & $\mathrm{AG}$ & GG & GG & GG \\
\hline $\mathrm{AG}$ & GG & $\mathrm{AG}$ & GG & GG & GG & GG & GG & GG & $\mathrm{AG}$ & $\mathrm{AG}$ \\
\hline $\mathrm{AG}$ & $\mathrm{AG}$ & $\mathrm{AG}$ & $\mathrm{AG}$ & $\mathrm{AG}$ & $\mathrm{AG}$ & $\mathrm{AG}$ & GG & GG & $\mathrm{AG}$ & GG \\
\hline GG & $\mathrm{AG}$ & GG & GG & $\mathrm{AG}$ & $\mathrm{AG}$ & $\mathrm{AG}$ & $\mathrm{AG}$ & $\mathrm{AG}$ & GG & GG \\
\hline $\mathrm{AG}$ & GG & $\mathrm{AG}$ & $\mathrm{AG}$ & GG & GG & GG & $\mathrm{AG}$ & GG & $\mathrm{AG}$ & GG \\
\hline GG & $\mathrm{AG}$ & GG & GG & GG & $\mathrm{AG}$ & GG & GG & $\mathrm{AG}$ & GG & $\mathrm{AG}$ \\
\hline GG & GG & GG & GG & GG & GG & GG & GG & $\mathrm{AG}$ & $\mathrm{AA}$ & $\mathrm{AA}$ \\
\hline $\mathrm{AG}$ & GG & GG & $\mathrm{AG}$ & GG & $\mathrm{AG}$ & GG & $\mathrm{AG}$ & $\mathrm{AG}$ & $\mathrm{AG}$ & \\
\hline
\end{tabular}


Supplemental Table 2. Continued.

\begin{tabular}{|c|c|c|c|c|c|c|c|c|c|c|}
\hline \multirow{3}{*}{$\begin{array}{l}\text { SNP name } \\
\text { GDsnp01094 } \\
\text { GG }\end{array}$} & \multicolumn{10}{|c|}{ Linkage group Physical location (bp) Individuals } \\
\hline & 16 & \multicolumn{2}{|c|}{3712687} & GG & GG & GG & GG & GG & GG & GG \\
\hline & GG & GG & GG & GG & GG & GG & GG & GG & GG & GG \\
\hline GG & GG & GG & GG & GG & GG & GG & GG & GG & GG & GG \\
\hline GG & GG & GG & GG & GG & GG & GG & GG & GG & GG & GG \\
\hline GG & GG & GG & GG & GG & GG & GG & GG & GG & GG & GG \\
\hline GG & GG & GG & GG & GG & GG & GG & GG & GG & GG & GG \\
\hline GG & GG & GG & GG & GG & GG & GG & GG & GG & GG & GG \\
\hline GG & GG & GG & GG & GG & GG & GG & GG & GG & GG & GG \\
\hline GG & GG & GG & GG & GG & GG & GG & GG & GG & GG & \\
\hline GDsnp01186 & 16 & \multicolumn{2}{|c|}{3970589} & $\mathrm{AG}$ & $\mathrm{AG}$ & $\mathrm{AA}$ & $A G$ & $\mathrm{AA}$ & $\mathrm{AG}$ & $\mathrm{AG}$ \\
\hline $\mathrm{AG}$ & $\mathrm{AG}$ & $\mathrm{AA}$ & $\mathrm{AG}$ & $\mathrm{AA}$ & GG & AA & GG & AA & $\mathrm{AA}$ & $\mathrm{AG}$ \\
\hline GG & $\mathrm{AA}$ & $\mathrm{AG}$ & $\mathrm{AA}$ & $\mathrm{AG}$ & $\mathrm{AA}$ & $\mathrm{AG}$ & AA & $\mathrm{AA}$ & $\mathrm{AG}$ & $\mathrm{AG}$ \\
\hline $\mathrm{AG}$ & GG & $\mathrm{AG}$ & $\mathrm{AG}$ & GG & $\mathrm{AG}$ & GG & AA & $\mathrm{AA}$ & $\mathrm{AG}$ & $\mathrm{AG}$ \\
\hline $\mathrm{AA}$ & $\mathrm{AG}$ & $\mathrm{AA}$ & $\mathrm{AG}$ & $\mathrm{AG}$ & $\mathrm{AG}$ & $\mathrm{AG}$ & $\mathrm{AG}$ & GG & $\mathrm{AA}$ & $\mathrm{AA}$ \\
\hline GG & $\mathrm{AG}$ & $\mathrm{AG}$ & $\mathrm{AG}$ & $\mathrm{AA}$ & $\mathrm{AG}$ & $\mathrm{AA}$ & $\mathrm{AG}$ & $\mathrm{AG}$ & $\mathrm{AG}$ & AA \\
\hline $\mathrm{AG}$ & $\mathrm{AG}$ & $\mathrm{AG}$ & AA & $\mathrm{AG}$ & GG & $\mathrm{AG}$ & AA & $\mathrm{AG}$ & $\mathrm{AG}$ & GG \\
\hline AA & $\mathrm{AA}$ & $\mathrm{AG}$ & $\mathrm{AG}$ & $\mathrm{AG}$ & $\mathrm{AA}$ & AA & AA & GG & GG & GG \\
\hline $\mathrm{AG}$ & $\mathrm{AG}$ & GG & GG & $\mathrm{AA}$ & $\mathrm{AG}$ & $\mathrm{AG}$ & GG & $\mathrm{AG}$ & $\mathrm{AG}$ & \\
\hline GDsnp01003 & 16 & \multicolumn{2}{|c|}{6056690} & GG & $\mathrm{AG}$ & GG & $\mathrm{AG}$ & $\mathrm{AG}$ & $\mathrm{AG}$ & GG \\
\hline $\mathrm{AG}$ & $\mathrm{AG}$ & GG & GG & GG & $\mathrm{AA}$ & GG & AG & GG & GG & $\mathrm{AG}$ \\
\hline $\mathrm{AG}$ & GG & $\mathrm{AG}$ & $\mathrm{AG}$ & $\mathrm{AG}$ & GG & $\mathrm{AG}$ & GG & GG & $\mathrm{AG}$ & $\mathrm{AG}$ \\
\hline $\mathrm{AG}$ & $\mathrm{AA}$ & $\mathrm{AA}$ & $\mathrm{AG}$ & $\mathrm{AG}$ & GG & $\mathrm{AA}$ & GG & GG & GG & $\mathrm{AA}$ \\
\hline GG & $\mathrm{AG}$ & GG & $\mathrm{AG}$ & $\mathrm{AG}$ & $\mathrm{AG}$ & $\mathrm{AG}$ & $\mathrm{AG}$ & $\mathrm{AA}$ & GG & GG \\
\hline $\mathrm{AG}$ & $\mathrm{AG}$ & $\mathrm{AG}$ & $\mathrm{AG}$ & GG & $\mathrm{AG}$ & GG & AG & GG & $\mathrm{AG}$ & GG \\
\hline $\mathrm{AG}$ & $\mathrm{AA}$ & $\mathrm{AG}$ & GG & $\mathrm{AG}$ & $\mathrm{AA}$ & $\mathrm{AG}$ & GG & $\mathrm{AG}$ & $\mathrm{AG}$ & AA \\
\hline GG & GG & $\mathrm{AG}$ & $\mathrm{AG}$ & $\mathrm{AG}$ & GG & GG & GG & AA & $\mathrm{AG}$ & $\mathrm{AA}$ \\
\hline AG & $\mathrm{AG}$ & $\mathrm{AA}$ & AA & GG & GG & AA & AA & GG & $\mathrm{AG}$ & \\
\hline GDsnp00071 & 16 & \multicolumn{2}{|c|}{6173845} & $\mathrm{AA}$ & $\mathrm{AT}$ & $\mathrm{AA}$ & AA & $\mathrm{AA}$ & $\mathrm{AA}$ & AA \\
\hline $\mathrm{AA}$ & $\mathrm{AA}$ & $\mathrm{AA}$ & AA & $\mathrm{AA}$ & $\mathrm{AT}$ & $\mathrm{AA}$ & AA & AA & $\mathrm{AA}$ & AT \\
\hline AA & $\mathrm{AA}$ & $\mathrm{AA}$ & $\mathrm{AT}$ & $\mathrm{AT}$ & $\mathrm{AA}$ & AA & AA & $\mathrm{AA}$ & $\mathrm{AA}$ & $\mathrm{AA}$ \\
\hline AA & $\mathrm{AT}$ & AA & AA & $\mathrm{AA}$ & $\mathrm{AA}$ & AT & AA & AA & AA & AA \\
\hline AA & $\mathrm{AA}$ & AA & $\mathrm{AT}$ & $\mathrm{AA}$ & AA & AA & AA & $\mathrm{AT}$ & AA & $\mathrm{AA}$ \\
\hline AA & $\mathrm{AT}$ & AA & AA & $\mathrm{AA}$ & $\mathrm{AT}$ & AA & AA & AA & AA & $\mathrm{AA}$ \\
\hline $\mathrm{AT}$ & $\mathrm{AT}$ & AT & $\mathrm{AA}$ & $\mathrm{AT}$ & $\mathrm{AA}$ & AA & AA & $\mathrm{AA}$ & $\mathrm{AT}$ & AT \\
\hline AA & $\mathrm{AA}$ & $\mathrm{AT}$ & AT & $\mathrm{AT}$ & AA & AA & AA & AT & AA & AA \\
\hline $\mathrm{AA}$ & $\mathrm{AT}$ & AT & AT & $\mathrm{AA}$ & AA & $\mathrm{AT}$ & AT & AA & AA & \\
\hline GDsnp01730 & 16 & \multicolumn{2}{|c|}{8722181} & $\mathrm{AG}$ & $\mathrm{AG}$ & AA & AG & AA & AA & $\mathrm{AG}$ \\
\hline AA & $\mathrm{AG}$ & $\mathrm{AG}$ & AA & $\mathrm{AG}$ & $\mathrm{AG}$ & AA & AA & AA & AA & $\mathrm{AG}$ \\
\hline AA & $\mathrm{AG}$ & AA & $\mathrm{AG}$ & $\mathrm{AG}$ & $\mathrm{AG}$ & AA & AA & AA & AA & $\mathrm{AA}$ \\
\hline $\mathrm{AG}$ & $\mathrm{AA}$ & $\mathrm{AA}$ & $\mathrm{AA}$ & $\mathrm{AA}$ & $\mathrm{AA}$ & $\mathrm{AG}$ & $\mathrm{AG}$ & $\mathrm{AA}$ & $\mathrm{AG}$ & $\mathrm{AA}$ \\
\hline AA & $\mathrm{AG}$ & $\mathrm{AA}$ & $\mathrm{AG}$ & $\mathrm{AA}$ & $\mathrm{AG}$ & $\mathrm{AA}$ & AA & $\mathrm{AG}$ & $\mathrm{AG}$ & $\mathrm{AA}$ \\
\hline AA & $\mathrm{AG}$ & AA & AA & $\mathrm{AG}$ & $\mathrm{AG}$ & AA & AA & AA & AA & AA \\
\hline $\mathrm{AG}$ & $\mathrm{AG}$ & $\mathrm{AG}$ & AA & $\mathrm{AG}$ & AA & AA & AA & AA & $\mathrm{AG}$ & $\mathrm{AG}$ \\
\hline $\mathrm{AG}$ & $\mathrm{AA}$ & $\mathrm{AG}$ & $\mathrm{AG}$ & $\mathrm{AG}$ & $\mathrm{AG}$ & $\mathrm{AG}$ & AA & $\mathrm{AG}$ & AA & $\mathrm{AA}$ \\
\hline AG & AA & $\mathrm{AG}$ & $\mathrm{AG}$ & $\mathrm{AG}$ & $\mathrm{AG}$ & AA & $\mathrm{AG}$ & AA & AA & \\
\hline GDsnp01244 & 16 & \multicolumn{2}{|c|}{11556924} & $\mathrm{AG}$ & $\mathrm{AG}$ & GG & $\mathrm{AG}$ & GG & GG & $\mathrm{AG}$ \\
\hline GG & $\mathrm{AG}$ & $\mathrm{AG}$ & GG & $\mathrm{AG}$ & $\mathrm{AG}$ & GG & GG & GG & GG & $\mathrm{AG}$ \\
\hline GG & $\mathrm{AG}$ & GG & $\mathrm{AG}$ & $\mathrm{AG}$ & $\mathrm{AG}$ & $\mathrm{AG}$ & GG & GG & GG & GG \\
\hline $\mathrm{AG}$ & GG & GG & GG & GG & GG & $\mathrm{AG}$ & $\mathrm{AG}$ & GG & $\mathrm{AG}$ & $\mathrm{AG}$ \\
\hline GG & $\mathrm{AG}$ & GG & $\mathrm{AG}$ & GG & $\mathrm{AG}$ & GG & $\mathrm{AG}$ & $\mathrm{AG}$ & $\mathrm{AG}$ & GG \\
\hline GG & $\mathrm{AG}$ & GG & GG & $\mathrm{AG}$ & $\mathrm{AG}$ & GG & GG & GG & GG & GG \\
\hline $\mathrm{AG}$ & $\mathrm{AG}$ & $\mathrm{AG}$ & GG & $\mathrm{AG}$ & GG & GG & GG & GG & $\mathrm{AG}$ & $\mathrm{AG}$ \\
\hline $\mathrm{AG}$ & GG & $\mathrm{AG}$ & $\mathrm{AG}$ & $\mathrm{AG}$ & $\mathrm{AG}$ & $\mathrm{AG}$ & GG & $\mathrm{AG}$ & GG & GG \\
\hline $\mathrm{AG}$ & GG & $\mathrm{AG}$ & $\mathrm{AG}$ & $\mathrm{AG}$ & $\mathrm{AG}$ & GG & $\mathrm{AG}$ & GG & GG & \\
\hline
\end{tabular}

Continued next page 
Supplemental Table 2. Continued.

\begin{tabular}{|c|c|c|c|c|c|c|c|c|c|c|}
\hline \multirow{3}{*}{$\begin{array}{l}\text { SNP name } \\
\text { GDsnp00444 } \\
\text { GG }\end{array}$} & \multicolumn{10}{|c|}{ Linkage group Physical location (bp) Individuals } \\
\hline & 16 & \multicolumn{2}{|c|}{13470183} & $\mathrm{CG}$ & GG & $\mathrm{CG}$ & CG & $\mathrm{CG}$ & GG & $\mathrm{CG}$ \\
\hline & $\mathrm{CG}$ & $\mathrm{CG}$ & GG & GG & GG & $\mathrm{CG}$ & CG & GG & GG & GG \\
\hline $\mathrm{CG}$ & $\mathrm{CG}$ & GG & GG & GG & $\mathrm{CG}$ & $\mathrm{CG}$ & GG & GG & GG & GG \\
\hline CG & GG & GG & $\mathrm{CG}$ & GG & GG & GG & GG & GG & GG & $\mathrm{CG}$ \\
\hline $\mathrm{CG}$ & $\mathrm{CG}$ & $\mathrm{CG}$ & GG & GG & GG & GG & CG & GG & $\mathrm{CG}$ & GG \\
\hline $\mathrm{CG}$ & GG & GG & GG & GG & GG & GG & GG & GG & GG & $\mathrm{CG}$ \\
\hline GG & GG & GG & GG & GG & GG & GG & GG & $\mathrm{CG}$ & GG & GG \\
\hline GG & GG & GG & GG & GG & GG & GG & CG & GG & $\mathrm{CG}$ & GG \\
\hline CG & $\mathrm{CG}$ & GG & GG & GG & $\mathrm{CG}$ & $\mathrm{CG}$ & GG & $\mathrm{CG}$ & $\mathrm{CG}$ & \\
\hline GDsnp00356 & 16 & \multicolumn{2}{|c|}{13548410} & $\mathrm{AG}$ & GG & $\mathrm{AG}$ & $A G$ & $\mathrm{AG}$ & GG & $\mathrm{AG}$ \\
\hline $\mathrm{AG}$ & $\mathrm{AG}$ & $\mathrm{AG}$ & GG & GG & GG & $\mathrm{AG}$ & $\mathrm{AG}$ & GG & GG & GG \\
\hline $\mathrm{AG}$ & $\mathrm{AG}$ & GG & GG & GG & $\mathrm{AG}$ & $\mathrm{AG}$ & GG & GG & GG & GG \\
\hline $\mathrm{AG}$ & GG & GG & $\mathrm{AG}$ & GG & GG & GG & GG & GG & $\mathrm{AG}$ & $\mathrm{AG}$ \\
\hline $\mathrm{AG}$ & $\mathrm{AG}$ & $\mathrm{AG}$ & GG & GG & GG & GG & $\mathrm{AG}$ & GG & $\mathrm{AG}$ & GG \\
\hline $\mathrm{AG}$ & GG & GG & GG & GG & GG & GG & GG & GG & GG & $\mathrm{AG}$ \\
\hline GG & GG & GG & GG & GG & GG & GG & GG & $\mathrm{AG}$ & GG & GG \\
\hline GG & GG & GG & GG & GG & GG & GG & $\mathrm{AG}$ & GG & $\mathrm{AG}$ & GG \\
\hline $\mathrm{AG}$ & $\mathrm{AG}$ & GG & GG & GG & $\mathrm{AG}$ & $\mathrm{AG}$ & GG & $\mathrm{AG}$ & $\mathrm{AG}$ & \\
\hline GDsnp01563 & 16 & \multicolumn{2}{|c|}{14381429} & $\mathrm{AG}$ & $\mathrm{AG}$ & $\mathrm{AG}$ & $\mathrm{AG}$ & $\mathrm{AG}$ & GG & $\mathrm{AG}$ \\
\hline GG & $\mathrm{AG}$ & $\mathrm{AG}$ & $\mathrm{AG}$ & $\mathrm{AG}$ & $\mathrm{AG}$ & $\mathrm{AG}$ & AG & GG & GG & $\mathrm{AG}$ \\
\hline $\mathrm{AG}$ & $\mathrm{AG}$ & GG & $\mathrm{AG}$ & $\mathrm{AG}$ & $\mathrm{AG}$ & $\mathrm{AG}$ & $\mathrm{AG}$ & $\mathrm{AG}$ & GG & GG \\
\hline $\mathrm{AG}$ & GG & GG & $\mathrm{AG}$ & GG & GG & $\mathrm{AG}$ & $\mathrm{AG}$ & GG & GG & GG \\
\hline $\mathrm{AG}$ & $\mathrm{AG}$ & $\mathrm{AG}$ & $\mathrm{AG}$ & GG & GG & GG & $\mathrm{AG}$ & $\mathrm{AG}$ & $\mathrm{AG}$ & GG \\
\hline $\mathrm{AG}$ & $\mathrm{AG}$ & GG & GG & $\mathrm{AG}$ & $\mathrm{AG}$ & $\mathrm{AG}$ & GG & GG & GG & $\mathrm{AG}$ \\
\hline $\mathrm{AG}$ & $\mathrm{AG}$ & $\mathrm{AG}$ & $\mathrm{AG}$ & $\mathrm{AG}$ & GG & GG & GG & $\mathrm{AG}$ & $\mathrm{AG}$ & $\mathrm{AG}$ \\
\hline GG & GG & $\mathrm{AG}$ & $\mathrm{AG}$ & $\mathrm{AG}$ & $\mathrm{AG}$ & $\mathrm{AG}$ & $\mathrm{AG}$ & $\mathrm{AG}$ & $\mathrm{AG}$ & GG \\
\hline AG & $\mathrm{AG}$ & $\mathrm{AG}$ & $\mathrm{AG}$ & GG & AG & $\mathrm{AG}$ & $\mathrm{AG}$ & $\mathrm{AG}$ & $\mathrm{AG}$ & \\
\hline GDsnp00013 & 16 & \multicolumn{2}{|c|}{14991284} & $\mathrm{AG}$ & $\mathrm{AG}$ & $\mathrm{AG}$ & $\mathrm{AG}$ & $\mathrm{AG}$ & $\mathrm{AA}$ & $\mathrm{AG}$ \\
\hline $\mathrm{AA}$ & $\mathrm{AG}$ & $\mathrm{AG}$ & $\mathrm{AG}$ & $\mathrm{AG}$ & $\mathrm{AG}$ & $\mathrm{AG}$ & $\mathrm{AG}$ & AA & $\mathrm{AA}$ & $\mathrm{AG}$ \\
\hline $\mathrm{AG}$ & $\mathrm{AG}$ & $\mathrm{AA}$ & $\mathrm{AG}$ & $\mathrm{AG}$ & $\mathrm{AG}$ & $\mathrm{AG}$ & $\mathrm{AG}$ & $\mathrm{AG}$ & $\mathrm{AA}$ & $\mathrm{AA}$ \\
\hline $\mathrm{AG}$ & $\mathrm{AA}$ & AA & $\mathrm{AG}$ & $\mathrm{AA}$ & $\mathrm{AA}$ & $\mathrm{AG}$ & $\mathrm{AG}$ & AA & AA & AA \\
\hline $\mathrm{AG}$ & $\mathrm{AG}$ & $\mathrm{AG}$ & $\mathrm{AG}$ & $\mathrm{AA}$ & AA & AA & $\mathrm{AG}$ & $\mathrm{AG}$ & $\mathrm{AG}$ & $\mathrm{AA}$ \\
\hline $\mathrm{AG}$ & $\mathrm{AG}$ & AA & AA & $\mathrm{AG}$ & $\mathrm{AG}$ & $\mathrm{AG}$ & $\mathrm{AG}$ & AA & AA & $\mathrm{AG}$ \\
\hline $\mathrm{AG}$ & $\mathrm{AG}$ & $\mathrm{AG}$ & $\mathrm{AG}$ & $\mathrm{AG}$ & $\mathrm{AA}$ & AA & $\mathrm{AG}$ & $\mathrm{AG}$ & $\mathrm{AG}$ & $\mathrm{AG}$ \\
\hline AA & $\mathrm{AA}$ & $\mathrm{AG}$ & $\mathrm{AG}$ & $\mathrm{AG}$ & $\mathrm{AG}$ & $\mathrm{AG}$ & $\mathrm{AG}$ & $\mathrm{AG}$ & $\mathrm{AG}$ & AA \\
\hline $\mathrm{AG}$ & $\mathrm{AG}$ & $\mathrm{AG}$ & $\mathrm{AG}$ & $\mathrm{AA}$ & $\mathrm{AG}$ & $\mathrm{AG}$ & $\mathrm{AG}$ & $\mathrm{AG}$ & $\mathrm{AG}$ & \\
\hline GDsnp02664 & 16 & \multicolumn{2}{|c|}{15523355} & $\mathrm{AA}$ & $\mathrm{AC}$ & $\mathrm{AC}$ & AA & $\mathrm{AC}$ & $\mathrm{AC}$ & $\mathrm{AC}$ \\
\hline AA & $\mathrm{AC}$ & $\mathrm{AC}$ & $\mathrm{AC}$ & $\mathrm{AC}$ & $\mathrm{AC}$ & $\mathrm{AC}$ & $\mathrm{AC}$ & AA & $\mathrm{AC}$ & $\mathrm{AC}$ \\
\hline $\mathrm{AC}$ & $\mathrm{AC}$ & AA & $\mathrm{AC}$ & $\mathrm{AC}$ & $\mathrm{AC}$ & AA & $\mathrm{AC}$ & $\mathrm{AC}$ & AA & $\mathrm{AA}$ \\
\hline $\mathrm{AC}$ & $\mathrm{AA}$ & $\mathrm{AA}$ & $\mathrm{AC}$ & $\mathrm{AA}$ & $\mathrm{AC}$ & $\mathrm{AC}$ & $\mathrm{AC}$ & $\mathrm{AA}$ & AA & $\mathrm{AA}$ \\
\hline $\mathrm{AC}$ & $\mathrm{AC}$ & $\mathrm{AC}$ & $\mathrm{AC}$ & $\mathrm{AA}$ & $\mathrm{AA}$ & $\mathrm{AA}$ & AA & $\mathrm{AC}$ & $\mathrm{AC}$ & $\mathrm{AA}$ \\
\hline $\mathrm{AC}$ & $\mathrm{AC}$ & AA & AA & $\mathrm{AC}$ & $\mathrm{AC}$ & $\mathrm{AC}$ & $\mathrm{AC}$ & AA & AA & $\mathrm{AC}$ \\
\hline $\mathrm{AC}$ & $\mathrm{AC}$ & $\mathrm{AC}$ & $\mathrm{AC}$ & $\mathrm{AC}$ & AA & AA & $\mathrm{AC}$ & $\mathrm{AC}$ & $\mathrm{AC}$ & $\mathrm{AC}$ \\
\hline $\mathrm{AA}$ & $\mathrm{AA}$ & $\mathrm{AC}$ & $\mathrm{AC}$ & $\mathrm{AC}$ & $\mathrm{AC}$ & $\mathrm{AC}$ & $\mathrm{AC}$ & $\mathrm{AC}$ & $\mathrm{AC}$ & $\mathrm{AA}$ \\
\hline $\mathrm{AC}$ & $\mathrm{AC}$ & $\mathrm{AC}$ & $\mathrm{AC}$ & $\mathrm{AA}$ & $\mathrm{AC}$ & $\mathrm{AC}$ & $\mathrm{AC}$ & $\mathrm{AC}$ & $\mathrm{AC}$ & \\
\hline GDsnp01402 & 16 & \multicolumn{2}{|c|}{16845645} & $\mathrm{CG}$ & $\mathrm{CG}$ & $\mathrm{CG}$ & CG & GG & GG & $\mathrm{CG}$ \\
\hline $\mathrm{CG}$ & $\mathrm{CG}$ & $\mathrm{CG}$ & $\mathrm{CG}$ & $\mathrm{CG}$ & GG & GG & GG & GG & $\mathrm{CG}$ & GG \\
\hline GG & GG & $\mathrm{CG}$ & $\mathrm{CG}$ & GG & $\mathrm{CG}$ & $\mathrm{CG}$ & $\mathrm{CG}$ & $\mathrm{CG}$ & $\mathrm{CG}$ & $\mathrm{CG}$ \\
\hline GG & $\mathrm{CG}$ & $\mathrm{CG}$ & $\mathrm{CG}$ & $\mathrm{CG}$ & $\mathrm{CG}$ & $\mathrm{CG}$ & $\mathrm{CG}$ & $\mathrm{CG}$ & $\mathrm{CG}$ & GG \\
\hline GG & GG & $\mathrm{CG}$ & GG & GG & GG & $\mathrm{CG}$ & GG & $\mathrm{CG}$ & GG & $\mathrm{CG}$ \\
\hline GG & $\mathrm{CG}$ & $\mathrm{CG}$ & GG & $\mathrm{CG}$ & $\mathrm{CG}$ & GG & CG & $\mathrm{CC}$ & GG & $\mathrm{CG}$ \\
\hline $\mathrm{CG}$ & GG & $\mathrm{CG}$ & $\mathrm{CG}$ & $\mathrm{CG}$ & $\mathrm{CG}$ & $\mathrm{CC}$ & GG & $\mathrm{CG}$ & $\mathrm{CG}$ & $\mathrm{CG}$ \\
\hline GG & $\mathrm{CG}$ & $\mathrm{CG}$ & $\mathrm{CG}$ & $\mathrm{CG}$ & $\mathrm{CG}$ & $\mathrm{CG}$ & CG & GG & GG & GG \\
\hline GG & $\mathrm{CG}$ & GG & GG & $\mathrm{CG}$ & $\mathrm{CG}$ & GG & GG & $\mathrm{CG}$ & GG & \\
\hline
\end{tabular}


Supplemental Table 2. Continued.

\begin{tabular}{|c|c|c|c|c|c|c|c|c|c|c|}
\hline \multirow{3}{*}{$\begin{array}{l}\text { SNP name } \\
\text { GDsnp00626 } \\
\text { AA }\end{array}$} & \multicolumn{10}{|c|}{ Linkage group Physical location (bp) Individuals } \\
\hline & 16 & \multicolumn{2}{|c|}{19121778} & $\mathrm{AG}$ & $\mathrm{AG}$ & AA & $\mathrm{AG}$ & $\mathrm{AG}$ & GG & $\mathrm{AA}$ \\
\hline & AA & AA & $\mathrm{AG}$ & $\mathrm{AG}$ & GG & $\mathrm{AG}$ & $\mathrm{AG}$ & GG & $\mathrm{AG}$ & GG \\
\hline $\mathrm{AG}$ & $\mathrm{AG}$ & AG & $\mathrm{AG}$ & GG & $\mathrm{AG}$ & AA & $\mathrm{AG}$ & $\mathrm{AG}$ & $\mathrm{AG}$ & $\mathrm{AG}$ \\
\hline $\mathrm{AG}$ & $\mathrm{AG}$ & AG & $\mathrm{AA}$ & $\mathrm{AG}$ & $\mathrm{AG}$ & $\mathrm{AG}$ & $\mathrm{AG}$ & $\mathrm{AG}$ & AA & GG \\
\hline $\mathrm{AG}$ & $\mathrm{AG}$ & AA & GG & GG & GG & $\mathrm{AG}$ & GG & $\mathrm{AG}$ & $\mathrm{AG}$ & $\mathrm{AG}$ \\
\hline $\mathrm{AG}$ & $\mathrm{AG}$ & AG & GG & $\mathrm{AG}$ & $\mathrm{AG}$ & GG & $\mathrm{AG}$ & $\mathrm{AG}$ & GG & AA \\
\hline $\mathrm{AG}$ & $\mathrm{AG}$ & $\mathrm{AG}$ & $\mathrm{AG}$ & $\mathrm{AG}$ & $\mathrm{AG}$ & $\mathrm{AA}$ & GG & $\mathrm{AA}$ & $\mathrm{AG}$ & $\mathrm{AG}$ \\
\hline GG & $\mathrm{AG}$ & $\mathrm{AG}$ & $\mathrm{AA}$ & $\mathrm{AG}$ & GG & $\mathrm{AG}$ & $\mathrm{AA}$ & $\mathrm{AG}$ & $\mathrm{AG}$ & $\mathrm{AG}$ \\
\hline $\mathrm{AG}$ & $\mathrm{AA}$ & GG & GG & $\mathrm{AG}$ & $\mathrm{AA}$ & $\mathrm{AG}$ & GG & $\mathrm{AA}$ & $\mathrm{AG}$ & \\
\hline GDsnp00091 & 17 & 50402 & $\mathrm{AA}$ & $\mathrm{AA}$ & $\mathrm{AA}$ & $\mathrm{AA}$ & $\mathrm{AA}$ & $\mathrm{AA}$ & $\mathrm{AA}$ & $\mathrm{AG}$ \\
\hline AA & $\mathrm{AA}$ & AA & $\mathrm{AA}$ & $\mathrm{AA}$ & $\mathrm{AA}$ & AA & $\mathrm{AA}$ & $\mathrm{AA}$ & $\mathrm{AA}$ & AA \\
\hline AA & $\mathrm{AA}$ & AA & $\mathrm{AA}$ & $\mathrm{AA}$ & $\mathrm{AA}$ & $\mathrm{AA}$ & $\mathrm{AA}$ & $\mathrm{AA}$ & $\mathrm{AA}$ & $\mathrm{AA}$ \\
\hline AA & $\mathrm{AA}$ & AA & $\mathrm{AA}$ & $\mathrm{AA}$ & $\mathrm{AA}$ & AA & $\mathrm{AA}$ & $\mathrm{AG}$ & $\mathrm{AA}$ & $\mathrm{AA}$ \\
\hline AA & $\mathrm{AA}$ & AA & $\mathrm{AA}$ & $\mathrm{AA}$ & - & $\mathrm{AG}$ & $\mathrm{AA}$ & $\mathrm{AA}$ & $\mathrm{AA}$ & $\mathrm{AA}$ \\
\hline AA & $\mathrm{AA}$ & AA & $\mathrm{AA}$ & $\mathrm{AA}$ & $\mathrm{AA}$ & $\mathrm{AA}$ & $\mathrm{AA}$ & $\mathrm{AA}$ & $\mathrm{AA}$ & AA \\
\hline AA & $\mathrm{AA}$ & AA & $\mathrm{AA}$ & $\mathrm{AA}$ & $\mathrm{AA}$ & $\mathrm{AA}$ & $\mathrm{AA}$ & $\mathrm{AA}$ & $\mathrm{AA}$ & $\mathrm{AA}$ \\
\hline AA & $\mathrm{AA}$ & AA & $\mathrm{AG}$ & $\mathrm{AA}$ & $\mathrm{AA}$ & AA & $\mathrm{AA}$ & $\mathrm{AA}$ & $\mathrm{AA}$ & $\mathrm{AA}$ \\
\hline AA & $\mathrm{AA}$ & AA & $\mathrm{AA}$ & $\mathrm{AA}$ & $\mathrm{AG}$ & AA & AA & $\mathrm{AA}$ & & \\
\hline GDsnp00192 & 17 & \multicolumn{2}{|c|}{244794} & $\mathrm{AA}$ & AA & $\mathrm{AA}$ & $\mathrm{AA}$ & $\mathrm{AA}$ & $\mathrm{AA}$ & $\mathrm{AA}$ \\
\hline $\mathrm{AC}$ & $\mathrm{AA}$ & AA & $\mathrm{AA}$ & $\mathrm{AA}$ & AA & AA & AA & $\mathrm{AA}$ & AA & $\mathrm{AA}$ \\
\hline AA & $\mathrm{AA}$ & AA & $\mathrm{AA}$ & $\mathrm{AA}$ & $\mathrm{AA}$ & $\mathrm{AA}$ & $\mathrm{AA}$ & $\mathrm{AA}$ & $\mathrm{AA}$ & $\mathrm{AA}$ \\
\hline AA & $\mathrm{AA}$ & AA & $\mathrm{AA}$ & $\mathrm{AA}$ & $\mathrm{AA}$ & AA & AA & $\mathrm{AA}$ & $\mathrm{AC}$ & $\mathrm{AA}$ \\
\hline AA & $\mathrm{AA}$ & AA & $\mathrm{AA}$ & $\mathrm{AA}$ & $\mathrm{AA}$ & AA & $\mathrm{AA}$ & $\mathrm{AA}$ & $\mathrm{AA}$ & $\mathrm{AA}$ \\
\hline AA & $\mathrm{AA}$ & AA & $\mathrm{AA}$ & $\mathrm{AA}$ & AA & AA & AA & $\mathrm{AA}$ & AA & $\mathrm{AA}$ \\
\hline AA & $\mathrm{AA}$ & AA & $\mathrm{AA}$ & $\mathrm{AA}$ & $\mathrm{AA}$ & AA & AA & $\mathrm{AA}$ & AA & AA \\
\hline AA & $\mathrm{AA}$ & AA & $\mathrm{AA}$ & $\mathrm{AC}$ & $\mathrm{AA}$ & AA & AA & $\mathrm{AA}$ & AA & $\mathrm{AA}$ \\
\hline AA & $\mathrm{AA}$ & AA & $\mathrm{AA}$ & $\mathrm{AA}$ & $\mathrm{AA}$ & $\mathrm{AC}$ & $\mathrm{AA}$ & $\mathrm{AA}$ & AA & \\
\hline GDsnp01841 & 17 & \multicolumn{2}{|c|}{1132464} & $\mathrm{TT}$ & $\mathrm{TT}$ & $\mathrm{TT}$ & $\mathrm{TT}$ & $\mathrm{TT}$ & $\mathrm{TT}$ & TT \\
\hline $\mathrm{TT}$ & $\mathrm{TT}$ & TT & $\mathrm{TT}$ & $\mathrm{TT}$ & $\mathrm{TT}$ & $\mathrm{TT}$ & $\mathrm{TT}$ & $\mathrm{TT}$ & $\mathrm{TT}$ & TT \\
\hline $\mathrm{TT}$ & $\mathrm{TT}$ & TT & $\mathrm{TT}$ & $\mathrm{TT}$ & $\mathrm{TT}$ & $\mathrm{TT}$ & $\mathrm{TT}$ & $\mathrm{TT}$ & $\mathrm{TT}$ & TT \\
\hline $\mathrm{TT}$ & $\mathrm{TT}$ & TT & $\mathrm{TT}$ & $\mathrm{TT}$ & $\mathrm{TT}$ & $\mathrm{TT}$ & $\mathrm{TT}$ & $\mathrm{TT}$ & $\mathrm{TT}$ & TT \\
\hline $\mathrm{TT}$ & $\mathrm{TT}$ & TT & $\mathrm{TT}$ & $\mathrm{TT}$ & $\mathrm{TT}$ & $\mathrm{TT}$ & $\mathrm{AT}$ & $\mathrm{TT}$ & $\mathrm{TT}$ & TT \\
\hline $\mathrm{TT}$ & $\mathrm{TT}$ & TT & $\mathrm{TT}$ & $\mathrm{TT}$ & $\mathrm{TT}$ & $\mathrm{TT}$ & $\mathrm{TT}$ & $\mathrm{TT}$ & $\mathrm{TT}$ & TT \\
\hline $\mathrm{TT}$ & $\mathrm{TT}$ & TT & $\mathrm{TT}$ & $\mathrm{TT}$ & $\mathrm{TT}$ & $\mathrm{TT}$ & $\mathrm{TT}$ & $\mathrm{TT}$ & $\mathrm{TT}$ & TT \\
\hline TT & $\mathrm{TT}$ & TT & TT & TT & $\mathrm{TT}$ & TT & $\mathrm{TT}$ & TT & $\mathrm{TT}$ & TT \\
\hline TT & $\mathrm{TT}$ & TT & $\mathrm{TT}$ & TT & $\mathrm{TT}$ & $\mathrm{AT}$ & TT & TT & $\mathrm{TT}$ & \\
\hline GDsnp00290 & 17 & \multicolumn{2}{|c|}{2077144} & $\mathrm{AG}$ & AA & AA & GG & $\mathrm{AG}$ & $\mathrm{AG}$ & $\mathrm{AA}$ \\
\hline- & GG & $\mathrm{AG}$ & $\mathrm{AA}$ & GG & $\mathrm{AG}$ & $\mathrm{AG}$ & GG & $\mathrm{AA}$ & $\mathrm{AG}$ & AA \\
\hline $\mathrm{AG}$ & $\mathrm{AG}$ & $\mathrm{AG}$ & $\mathrm{AG}$ & $\mathrm{AA}$ & $\mathrm{AG}$ & $\mathrm{AG}$ & GG & GG & AA & $\mathrm{AG}$ \\
\hline $\mathrm{AG}$ & $\mathrm{AA}$ & AA & GG & $\mathrm{AA}$ & GG & GG & $\mathrm{AG}$ & $\mathrm{AG}$ & AA & $\mathrm{AA}$ \\
\hline GG & GG & GG & $\mathrm{AA}$ & $\mathrm{AG}$ & $\mathrm{AG}$ & $\mathrm{AA}$ & $\mathrm{AG}$ & $\mathrm{AG}$ & $\mathrm{AG}$ & $\mathrm{AG}$ \\
\hline GG & GG & AA & $\mathrm{AA}$ & $\mathrm{AG}$ & $\mathrm{AG}$ & AA & $\mathrm{AG}$ & GG & $\mathrm{AG}$ & GG \\
\hline AA & $\mathrm{AG}$ & $\mathrm{AG}$ & $\mathrm{AG}$ & GG & GG & $\mathrm{AG}$ & AA & $\mathrm{AG}$ & $\mathrm{AG}$ & $\mathrm{AG}$ \\
\hline $\mathrm{AG}$ & $\mathrm{AG}$ & GG & $\mathrm{AG}$ & $\mathrm{AG}$ & GG & GG & $\mathrm{AG}$ & $\mathrm{AG}$ & $\mathrm{AG}$ & GG \\
\hline GG & AA & $\mathrm{AG}$ & $\mathrm{AG}$ & GG & $\mathrm{AG}$ & AA & $\mathrm{AA}$ & GG & $\mathrm{AG}$ & \\
\hline GDsnp00202 & 17 & \multicolumn{2}{|c|}{3133119} & $\mathrm{AA}$ & $\mathrm{AA}$ & AA & AA & $\mathrm{AA}$ & AA & $\mathrm{AA}$ \\
\hline AA & $\mathrm{AG}$ & $\mathrm{AA}$ & $\mathrm{AA}$ & $\mathrm{AA}$ & $\mathrm{AA}$ & AA & $\mathrm{AA}$ & $\mathrm{AA}$ & $\mathrm{AA}$ & $\mathrm{AA}$ \\
\hline AA & $\mathrm{AA}$ & AA & $\mathrm{AA}$ & $\mathrm{AA}$ & $\mathrm{AA}$ & $\mathrm{AG}$ & AA & $\mathrm{AA}$ & AA & AA \\
\hline AA & $\mathrm{AA}$ & $\mathrm{AA}$ & $\mathrm{AA}$ & $\mathrm{AA}$ & $\mathrm{AA}$ & AA & $\mathrm{AA}$ & $\mathrm{AA}$ & $\mathrm{AA}$ & $\mathrm{AA}$ \\
\hline AA & $\mathrm{AA}$ & $\mathrm{AA}$ & $\mathrm{AA}$ & $\mathrm{AA}$ & $\mathrm{AA}$ & - & $\mathrm{AA}$ & $\mathrm{AA}$ & AA & $\mathrm{AA}$ \\
\hline AA & $\mathrm{AA}$ & $\mathrm{AA}$ & $\mathrm{AA}$ & $\mathrm{AA}$ & $\mathrm{AA}$ & AA & $\mathrm{AA}$ & $\mathrm{AA}$ & AA & $\mathrm{AA}$ \\
\hline AA & $\mathrm{AA}$ & $\mathrm{AA}$ & $\mathrm{AA}$ & $\mathrm{AA}$ & $\mathrm{AA}$ & $\mathrm{AA}$ & $\mathrm{AA}$ & $\mathrm{AA}$ & $\mathrm{AA}$ & $\mathrm{AA}$ \\
\hline AA & $\mathrm{AA}$ & $\mathrm{AA}$ & $\mathrm{AA}$ & $\mathrm{AA}$ & $\mathrm{AA}$ & $\mathrm{AA}$ & $\mathrm{AA}$ & $\mathrm{AA}$ & $\mathrm{AA}$ & $\mathrm{AA}$ \\
\hline AA & $\mathrm{AA}$ & AA & $\mathrm{AA}$ & $\mathrm{AA}$ & $\mathrm{AA}$ & $\mathrm{AG}$ & $\mathrm{AA}$ & $\mathrm{AA}$ & AA & \\
\hline
\end{tabular}

Continued next page 
Supplemental Table 2. Continued.

\begin{tabular}{|c|c|c|c|c|c|c|c|c|c|c|}
\hline \multirow{3}{*}{$\begin{array}{l}\text { SNP name } \\
\text { GDsnp00088 } \\
\text { AG }\end{array}$} & \multicolumn{10}{|c|}{ Linkage group Physical location (bp) Individuals } \\
\hline & 17 & \multicolumn{2}{|c|}{3613988} & AA & AA & AA & AA & AA & AA & $\mathrm{AA}$ \\
\hline & $\mathrm{AG}$ & $\mathrm{AG}$ & AA & AA & AA & AA & AA & AA & AA & AA \\
\hline AA & AA & AA & $\mathrm{AA}$ & AA & AA & AG & AA & AA & AA & AA \\
\hline AA & $\mathrm{AA}$ & AA & $\mathrm{AA}$ & $\mathrm{AA}$ & $\mathrm{AA}$ & AA & AA & $\mathrm{AA}$ & $\mathrm{AG}$ & $\mathrm{AA}$ \\
\hline AA & $\mathrm{AA}$ & $\mathrm{AA}$ & $\mathrm{AA}$ & $\mathrm{AA}$ & $\mathrm{AA}$ & $\mathrm{AA}$ & $\mathrm{AG}$ & $\mathrm{AA}$ & $\mathrm{AA}$ & $\mathrm{AA}$ \\
\hline AA & $\mathrm{AA}$ & AA & $\mathrm{AA}$ & AA & $\mathrm{AA}$ & AA & AA & $\mathrm{AA}$ & AA & AA \\
\hline $\mathrm{AA}$ & $\mathrm{AA}$ & $\mathrm{AA}$ & $\mathrm{AA}$ & $\mathrm{AA}$ & $\mathrm{AA}$ & $\mathrm{AA}$ & $\mathrm{AA}$ & $\mathrm{AA}$ & $\mathrm{AA}$ & $\mathrm{AA}$ \\
\hline AA & $\mathrm{AA}$ & AA & $\mathrm{AA}$ & $\mathrm{AA}$ & $\mathrm{AA}$ & AA & AA & $\mathrm{AA}$ & AA & AA \\
\hline $\mathrm{AA}$ & $\mathrm{AA}$ & $\mathrm{AA}$ & - & $\mathrm{AA}$ & $\mathrm{AA}$ & $\mathrm{AG}$ & AA & $\mathrm{AA}$ & AA & \\
\hline GDsnp00328 & 17 & \multicolumn{2}{|c|}{4173966} & $\mathrm{AT}$ & $\mathrm{AA}$ & $\mathrm{AA}$ & $\mathrm{TT}$ & $\mathrm{AT}$ & $\mathrm{AA}$ & $\mathrm{AA}$ \\
\hline $\mathrm{AT}$ & $\mathrm{TT}$ & AT & $\mathrm{AT}$ & $\mathrm{TT}$ & $\mathrm{AT}$ & $\mathrm{AT}$ & $\mathrm{AT}$ & - & AT & AA \\
\hline AT & $\mathrm{AT}$ & AT & $\mathrm{AT}$ & $\mathrm{AT}$ & $\mathrm{AT}$ & $\mathrm{AT}$ & $\mathrm{TT}$ & $\mathrm{TT}$ & $\mathrm{AA}$ & AT \\
\hline AT & $\mathrm{AA}$ & $\mathrm{AA}$ & $\mathrm{TT}$ & $\mathrm{AT}$ & $\mathrm{TT}$ & $\mathrm{AT}$ & $\mathrm{AT}$ & $\mathrm{AT}$ & $\mathrm{AA}$ & $\mathrm{AA}$ \\
\hline $\mathrm{TT}$ & $\mathrm{TT}$ & $\mathrm{TT}$ & $\mathrm{AA}$ & $\mathrm{AT}$ & $\mathrm{AT}$ & $\mathrm{AA}$ & $\mathrm{AT}$ & $\mathrm{AT}$ & $\mathrm{AT}$ & AT \\
\hline $\mathrm{TT}$ & $\mathrm{AT}$ & AA & $\mathrm{AA}$ & AT & $\mathrm{AT}$ & AA & $\mathrm{AT}$ & $\mathrm{TT}$ & $\mathrm{AT}$ & TT \\
\hline AA & $\mathrm{AT}$ & AT & $\mathrm{AT}$ & $\mathrm{TT}$ & $\mathrm{TT}$ & $\mathrm{AT}$ & $\mathrm{AT}$ & $\mathrm{AA}$ & AT & AT \\
\hline $\mathrm{TT}$ & $\mathrm{AT}$ & $\mathrm{TT}$ & $\mathrm{AT}$ & $\mathrm{AT}$ & $\mathrm{TT}$ & $\mathrm{TT}$ & $\mathrm{AT}$ & $\mathrm{AT}$ & $\mathrm{AT}$ & TT \\
\hline $\mathrm{TT}$ & $\mathrm{AT}$ & $\mathrm{AT}$ & $\mathrm{AT}$ & $\mathrm{TT}$ & $\mathrm{AT}$ & AA & AA & $\mathrm{TT}$ & $\mathrm{AT}$ & \\
\hline GDsnp02703 & 17 & \multicolumn{2}{|c|}{4713854} & $\mathrm{CC}$ & $\mathrm{CC}$ & $\mathrm{CC}$ & $\mathrm{CC}$ & $\mathrm{CC}$ & $\mathrm{CC}$ & $\mathrm{CC}$ \\
\hline $\mathrm{CC}$ & $\mathrm{CC}$ & $\mathrm{CC}$ & $\mathrm{CC}$ & $\mathrm{CC}$ & $\mathrm{CC}$ & $\mathrm{CC}$ & $\mathrm{CC}$ & $\mathrm{CC}$ & $\mathrm{CC}$ & $\mathrm{CC}$ \\
\hline $\mathrm{CC}$ & $\mathrm{CC}$ & $\mathrm{CC}$ & $\mathrm{CC}$ & $\mathrm{CC}$ & $\mathrm{CC}$ & $\mathrm{CC}$ & $\mathrm{CC}$ & $\mathrm{CC}$ & $\mathrm{CC}$ & $\mathrm{CC}$ \\
\hline $\mathrm{CC}$ & $\mathrm{CC}$ & $\mathrm{CC}$ & $\mathrm{CC}$ & $\mathrm{CC}$ & $\mathrm{CC}$ & $\mathrm{CC}$ & $\mathrm{CC}$ & $\mathrm{CC}$ & $\mathrm{CC}$ & $\mathrm{CC}$ \\
\hline $\mathrm{CC}$ & $\mathrm{CC}$ & $\mathrm{CC}$ & $\mathrm{CC}$ & $\mathrm{CC}$ & $\mathrm{CC}$ & $\mathrm{CC}$ & $\mathrm{CC}$ & $\mathrm{CC}$ & $\mathrm{CC}$ & $\mathrm{CC}$ \\
\hline $\mathrm{CC}$ & $\mathrm{CC}$ & $\mathrm{CC}$ & $\mathrm{CC}$ & $\mathrm{CC}$ & $\mathrm{CC}$ & $\mathrm{CC}$ & $\mathrm{CC}$ & $\mathrm{CC}$ & $\mathrm{CC}$ & $\mathrm{CC}$ \\
\hline $\mathrm{CC}$ & $\mathrm{CC}$ & $\mathrm{CC}$ & $\mathrm{CC}$ & $\mathrm{CC}$ & $\mathrm{CC}$ & $\mathrm{CC}$ & $\mathrm{CC}$ & $\mathrm{CC}$ & $\mathrm{CC}$ & $\mathrm{CC}$ \\
\hline $\mathrm{CC}$ & $\mathrm{CC}$ & $\mathrm{CC}$ & $\mathrm{CC}$ & $\mathrm{CC}$ & $\mathrm{CC}$ & $\mathrm{CC}$ & $\mathrm{CC}$ & $\mathrm{CC}$ & $\mathrm{CC}$ & $\mathrm{CC}$ \\
\hline $\mathrm{CC}$ & $\mathrm{CC}$ & $\mathrm{CC}$ & $\mathrm{CC}$ & $\mathrm{CC}$ & $\mathrm{CC}$ & $\mathrm{AC}$ & $\mathrm{CC}$ & $\mathrm{CC}$ & $\mathrm{CC}$ & \\
\hline GDsnp00262 & 17 & \multicolumn{2}{|c|}{5352180} & - & GG & GG & $\mathrm{CG}$ & GG & GG & GG \\
\hline GG & GG & GG & GG & $\mathrm{CG}$ & GG & GG & GG & $\mathrm{CG}$ & GG & GG \\
\hline GG & GG & GG & GG & GG & $\mathrm{CG}$ & GG & $\mathrm{CG}$ & $\mathrm{CG}$ & GG & GG \\
\hline $\mathrm{CG}$ & GG & GG & $\mathrm{CG}$ & $\mathrm{CG}$ & $\mathrm{CG}$ & GG & $\mathrm{CG}$ & GG & $\mathrm{CG}$ & $\mathrm{CG}$ \\
\hline $\mathrm{CG}$ & $\mathrm{CG}$ & $\mathrm{CG}$ & GG & $\mathrm{CG}$ & $\mathrm{CG}$ & GG & $\mathrm{CG}$ & $\mathrm{CG}$ & GG & $\mathrm{CG}$ \\
\hline GG & GG & GG & $\mathrm{CG}$ & GG & GG & GG & GG & $\mathrm{CG}$ & GG & GG \\
\hline GG & GG & GG & GG & $\mathrm{CG}$ & $\mathrm{CG}$ & GG & GG & GG & $\mathrm{CG}$ & GG \\
\hline GG & $\mathrm{CG}$ & $\mathrm{CG}$ & GG & GG & GG & $\mathrm{CG}$ & GG & GG & $\mathrm{CG}$ & GG \\
\hline $\mathrm{CG}$ & GG & GG & GG & GG & GG & GG & GG & GG & GG & \\
\hline GDsnp02550 & 17 & \multicolumn{2}{|c|}{6479700} & - & - & - & $\mathrm{AG}$ & GG & - & - \\
\hline GG & GG & GG & GG & $\mathrm{AG}$ & GG & GG & GG & GG & - & - \\
\hline GG & - & GG & GG & GG & $\mathrm{AA}$ & GG & $\mathrm{AG}$ & $\mathrm{AG}$ & - & - \\
\hline AA & - & - & $\mathrm{AG}$ & $\mathrm{AA}$ & $\mathrm{AG}$ & GG & GG & GG & GG & GG \\
\hline $\mathrm{AG}$ & $\mathrm{AG}$ & $\mathrm{AG}$ & - & - & $\mathrm{AA}$ & - & GG & - & - & $\mathrm{AA}$ \\
\hline GG & GG & - & GG & GG & GG & - & GG & $\mathrm{AG}$ & GG & GG \\
\hline- & GG & GG & - & $\mathrm{AG}$ & $\mathrm{AG}$ & GG & AA & - & - & GG \\
\hline GG & $\mathrm{AA}$ & $\mathrm{AG}$ & GG & $\mathrm{AG}$ & GG & $\mathrm{AG}$ & GG & - & - & GG \\
\hline AG & GG & - & GG & GG & - & GG & - & GG & - & \\
\hline GDsnp00178 & 17 & \multicolumn{2}{|c|}{8360963} & $\mathrm{AT}$ & $\mathrm{AA}$ & $\mathrm{AA}$ & $\mathrm{AT}$ & $\mathrm{AA}$ & $\mathrm{AA}$ & $\mathrm{AA}$ \\
\hline- & $\mathrm{AA}$ & $\mathrm{AT}$ & $\mathrm{AA}$ & $\mathrm{AA}$ & $\mathrm{AA}$ & AA & $\mathrm{AA}$ & $\mathrm{AA}$ & $\mathrm{AA}$ & AT \\
\hline AA & $\mathrm{AA}$ & AA & $\mathrm{AA}$ & $\mathrm{AA}$ & $\mathrm{AT}$ & AA & $\mathrm{AT}$ & $\mathrm{AT}$ & AA & AA \\
\hline AT & $\mathrm{AA}$ & AA & $\mathrm{AT}$ & AT & $\mathrm{AT}$ & AA & AA & $\mathrm{AA}$ & AA & AA \\
\hline AT & $\mathrm{AT}$ & $\mathrm{AT}$ & $\mathrm{AT}$ & $\mathrm{AT}$ & $\mathrm{AT}$ & AA & $\mathrm{AA}$ & $\mathrm{AT}$ & AA & AT \\
\hline AA & $\mathrm{AA}$ & AA & $\mathrm{AA}$ & $\mathrm{AA}$ & $\mathrm{AA}$ & $\mathrm{AA}$ & $\mathrm{AA}$ & $\mathrm{AT}$ & AA & $\mathrm{AA}$ \\
\hline AA & $\mathrm{AA}$ & $\mathrm{AA}$ & $\mathrm{AA}$ & $\mathrm{AT}$ & $\mathrm{AT}$ & $\mathrm{AA}$ & $\mathrm{AT}$ & $\mathrm{AA}$ & $\mathrm{AT}$ & $\mathrm{AA}$ \\
\hline AT & $\mathrm{AT}$ & AT & $\mathrm{AA}$ & $\mathrm{AT}$ & $\mathrm{AA}$ & $\mathrm{AT}$ & $\mathrm{AA}$ & $\mathrm{AA}$ & $\mathrm{AT}$ & $\mathrm{AA}$ \\
\hline $\mathrm{AT}$ & $\mathrm{AA}$ & $\mathrm{AA}$ & $\mathrm{AA}$ & $\mathrm{AA}$ & $\mathrm{AA}$ & AA & $\mathrm{AA}$ & $\mathrm{AA}$ & AA & \\
\hline
\end{tabular}


Supplemental Table 2. Continued.

\begin{tabular}{|c|c|c|c|c|c|c|c|c|c|c|}
\hline \multirow{3}{*}{$\begin{array}{l}\text { SNP name } \\
\text { GDsnp01269 } \\
\text { TT }\end{array}$} & \multicolumn{10}{|c|}{ Linkage group Physical location (bp) Individuals } \\
\hline & 17 & \multicolumn{2}{|c|}{9745623} & $\mathrm{AT}$ & AA & AT & TT & AT & AA & AT \\
\hline & TT & AT & AT & TT & AT & AT & AT & AT & AA & AT \\
\hline AT & $\mathrm{AA}$ & AT & AT & $\mathrm{AT}$ & AT & AT & $\mathrm{TT}$ & TT & AA & AA \\
\hline TT & $\mathrm{AA}$ & AA & AT & AT & TT & AT & AT & AT & $\mathrm{AT}$ & $\mathrm{AA}$ \\
\hline TT & $\mathrm{AT}$ & $\mathrm{TT}$ & AT & $\mathrm{AT}$ & AT & $\mathrm{AA}$ & $\mathrm{TT}$ & AT & $\mathrm{AT}$ & TT \\
\hline AT & $\mathrm{AT}$ & AA & AA & AT & AT & $\mathrm{AT}$ & AT & $\mathrm{TT}$ & $\mathrm{AT}$ & TT \\
\hline AT & $\mathrm{AT}$ & $\mathrm{AT}$ & $\mathrm{AT}$ & $\mathrm{TT}$ & $\mathrm{TT}$ & $\mathrm{AT}$ & AT & $\mathrm{AA}$ & $\mathrm{AT}$ & $\mathrm{AT}$ \\
\hline $\mathrm{TT}$ & $\mathrm{AT}$ & $\mathrm{TT}$ & $\mathrm{AT}$ & $\mathrm{TT}$ & $\mathrm{TT}$ & $\mathrm{TT}$ & AT & $\mathrm{AT}$ & $\mathrm{AT}$ & AT \\
\hline $\mathrm{AT}$ & $\mathrm{TT}$ & $\mathrm{AA}$ & $\mathrm{AT}$ & $\mathrm{AT}$ & AA & $\mathrm{AA}$ & AA & $\mathrm{TT}$ & AA & \\
\hline GDsnp00287 & 17 & \multicolumn{2}{|c|}{10728484} & GG & GG & $\mathrm{AG}$ & $A G$ & $\mathrm{AG}$ & GG & $\mathrm{AG}$ \\
\hline $\mathrm{AG}$ & $\mathrm{AG}$ & $\mathrm{AA}$ & $\mathrm{AA}$ & $\mathrm{AA}$ & $\mathrm{AG}$ & $\mathrm{AG}$ & $\mathrm{AG}$ & $\mathrm{AG}$ & GG & GG \\
\hline $\mathrm{AG}$ & GG & $\mathrm{AG}$ & $\mathrm{AG}$ & $\mathrm{AG}$ & GG & GG & $\mathrm{AG}$ & $\mathrm{AG}$ & GG & GG \\
\hline $\mathrm{AG}$ & GG & GG & GG & GG & $\mathrm{AG}$ & $\mathrm{AG}$ & $\mathrm{AG}$ & $\mathrm{AG}$ & $\mathrm{AG}$ & GG \\
\hline GG & $\mathrm{AG}$ & $\mathrm{AG}$ & GG & GG & GG & GG & - & $\mathrm{AG}$ & $\mathrm{AG}$ & $\mathrm{AG}$ \\
\hline $\mathrm{AG}$ & $\mathrm{AG}$ & GG & GG & $\mathrm{AA}$ & $A G$ & $\mathrm{AG}$ & $\mathrm{AG}$ & $\mathrm{AG}$ & $\mathrm{AG}$ & AA \\
\hline AG & $\mathrm{AA}$ & $\mathrm{AG}$ & $\mathrm{AG}$ & $\mathrm{AG}$ & $\mathrm{AG}$ & $\mathrm{AG}$ & GG & GG & GG & $\mathrm{AG}$ \\
\hline AA & GG & $\mathrm{AG}$ & $\mathrm{AG}$ & $\mathrm{AG}$ & AA & $\mathrm{AG}$ & $\mathrm{AG}$ & $\mathrm{AG}$ & $\mathrm{AG}$ & $\mathrm{AG}$ \\
\hline GG & $\mathrm{AA}$ & GG & $\mathrm{AG}$ & $\mathrm{AG}$ & GG & $\mathrm{AG}$ & GG & AA & GG & \\
\hline GDsnp00682 & 17 & \multicolumn{2}{|c|}{11471514} & $\mathrm{AG}$ & GG & GG & $\mathrm{AG}$ & GG & GG & GG \\
\hline GG & GG & GG & GG & GG & GG & GG & GG & GG & GG & $\mathrm{AG}$ \\
\hline GG & GG & GG & GG & GG & $\mathrm{AG}$ & GG & $\mathrm{AG}$ & $\mathrm{AG}$ & GG & GG \\
\hline $\mathrm{AG}$ & GG & GG & $\mathrm{AG}$ & $\mathrm{AG}$ & $\mathrm{AG}$ & GG & GG & $\mathrm{AG}$ & GG & GG \\
\hline $\mathrm{AG}$ & GG & $\mathrm{AG}$ & $\mathrm{AG}$ & $\mathrm{AG}$ & $\mathrm{AG}$ & GG & GG & $\mathrm{AG}$ & GG & $\mathrm{AG}$ \\
\hline GG & GG & GG & GG & GG & GG & GG & GG & $\mathrm{AG}$ & GG & GG \\
\hline GG & GG & GG & GG & $\mathrm{AG}$ & AG & GG & $\mathrm{AG}$ & GG & $\mathrm{AG}$ & GG \\
\hline GG & $\mathrm{AG}$ & $\mathrm{AG}$ & GG & $\mathrm{AG}$ & GG & $\mathrm{AG}$ & $\mathrm{AG}$ & GG & GG & GG \\
\hline $\mathrm{AG}$ & GG & GG & GG & GG & $\mathrm{AG}$ & GG & GG & GG & GG & \\
\hline GDsnp01801 & 17 & \multicolumn{2}{|c|}{11678655} & $\mathrm{AG}$ & GG & GG & $\mathrm{AG}$ & GG & GG & GG \\
\hline $\mathrm{AG}$ & GG & GG & GG & GG & GG & GG & GG & GG & GG & $\mathrm{AG}$ \\
\hline GG & GG & GG & GG & GG & $\mathrm{AG}$ & GG & $\mathrm{AG}$ & $\mathrm{AG}$ & GG & GG \\
\hline $\mathrm{AG}$ & GG & GG & $\mathrm{AG}$ & $\mathrm{AG}$ & AG & GG & GG & $\mathrm{AG}$ & GG & GG \\
\hline $\mathrm{AG}$ & GG & $\mathrm{AG}$ & $\mathrm{AG}$ & $\mathrm{AG}$ & AG & $\mathrm{AG}$ & GG & $\mathrm{AG}$ & GG & $\mathrm{AG}$ \\
\hline GG & GG & GG & GG & GG & GG & GG & GG & $\mathrm{AG}$ & GG & GG \\
\hline GG & GG & GG & GG & $\mathrm{AG}$ & $\mathrm{AG}$ & GG & $\mathrm{AG}$ & GG & $\mathrm{AG}$ & GG \\
\hline GG & $\mathrm{AG}$ & $\mathrm{AG}$ & GG & $\mathrm{AG}$ & GG & $\mathrm{AG}$ & $\mathrm{AG}$ & GG & GG & GG \\
\hline $\mathrm{AG}$ & GG & GG & GG & GG & $\mathrm{AG}$ & GG & GG & GG & GG & \\
\hline GDsnp00358 & 17 & \multicolumn{2}{|c|}{13255291} & $\mathrm{CC}$ & $\mathrm{CC}$ & $\mathrm{CC}$ & $\mathrm{CC}$ & $\mathrm{CC}$ & $\mathrm{CC}$ & $\mathrm{AC}$ \\
\hline $\mathrm{AC}$ & $\mathrm{AC}$ & $\mathrm{CC}$ & - & $\mathrm{CC}$ & $\mathrm{CC}$ & $\mathrm{CC}$ & $\mathrm{CC}$ & - & $\mathrm{CC}$ & $\mathrm{AC}$ \\
\hline $\mathrm{CC}$ & $\mathrm{CC}$ & $\mathrm{CC}$ & $\mathrm{CC}$ & $\mathrm{CC}$ & $\mathrm{AC}$ & $\mathrm{AC}$ & $\mathrm{AC}$ & $\mathrm{AC}$ & $\mathrm{CC}$ & $\mathrm{CC}$ \\
\hline $\mathrm{AC}$ & $\mathrm{CC}$ & $\mathrm{CC}$ & $\mathrm{AC}$ & $\mathrm{AC}$ & $\mathrm{AC}$ & $\mathrm{CC}$ & $\mathrm{CC}$ & $\mathrm{AC}$ & $\mathrm{AC}$ & $\mathrm{CC}$ \\
\hline $\mathrm{CC}$ & $\mathrm{CC}$ & $\mathrm{AC}$ & $\mathrm{AC}$ & $\mathrm{AC}$ & $\mathrm{AC}$ & $\mathrm{CC}$ & - & $\mathrm{AC}$ & $\mathrm{CC}$ & $\mathrm{AC}$ \\
\hline $\mathrm{CC}$ & $\mathrm{CC}$ & $\mathrm{AC}$ & - & $\mathrm{CC}$ & $\mathrm{CC}$ & $\mathrm{CC}$ & $\mathrm{CC}$ & $\mathrm{AC}$ & $\mathrm{CC}$ & $\mathrm{CC}$ \\
\hline $\mathrm{CC}$ & $\mathrm{CC}$ & $\mathrm{CC}$ & $\mathrm{CC}$ & $\mathrm{AC}$ & $\mathrm{AC}$ & $\mathrm{CC}$ & $\mathrm{CC}$ & $\mathrm{CC}$ & $\mathrm{AC}$ & $\mathrm{CC}$ \\
\hline $\mathrm{CC}$ & $\mathrm{AC}$ & $\mathrm{AC}$ & $\mathrm{CC}$ & - & $\mathrm{CC}$ & $\mathrm{AC}$ & $\mathrm{AC}$ & $\mathrm{CC}$ & $\mathrm{CC}$ & $\mathrm{AC}$ \\
\hline $\mathrm{AC}$ & $\mathrm{CC}$ & $\mathrm{CC}$ & $\mathrm{CC}$ & $\mathrm{CC}$ & $\mathrm{AC}$ & $\mathrm{CC}$ & $\mathrm{CC}$ & $\mathrm{CC}$ & $\mathrm{CC}$ & \\
\hline GDsnp00058 & 17 & \multicolumn{2}{|c|}{16032466} & $\mathrm{AG}$ & $\mathrm{AG}$ & $\mathrm{AA}$ & GG & $\mathrm{AG}$ & $\mathrm{AA}$ & $\mathrm{AG}$ \\
\hline AA & $\mathrm{AG}$ & $\mathrm{AG}$ & GG & GG & GG & GG & $\mathrm{AG}$ & $\mathrm{AG}$ & $\mathrm{AA}$ & $\mathrm{AA}$ \\
\hline $\mathrm{AG}$ & $\mathrm{AA}$ & $\mathrm{AG}$ & $\mathrm{AG}$ & $\mathrm{AG}$ & AA & AA & $\mathrm{AG}$ & $\mathrm{AG}$ & AA & $\mathrm{AG}$ \\
\hline $\mathrm{AG}$ & $\mathrm{AA}$ & AA & AA & $\mathrm{AA}$ & AG & GG & $\mathrm{AG}$ & $\mathrm{AG}$ & $\mathrm{AG}$ & $\mathrm{AG}$ \\
\hline AG & $\mathrm{AG}$ & $\mathrm{AG}$ & $\mathrm{AA}$ & $\mathrm{AA}$ & AA & AA & GG & $\mathrm{AG}$ & $\mathrm{AG}$ & $\mathrm{AG}$ \\
\hline $\mathrm{AG}$ & $\mathrm{AG}$ & $\mathrm{AA}$ & $\mathrm{AA}$ & GG & $\mathrm{AG}$ & $\mathrm{AA}$ & $\mathrm{AG}$ & $\mathrm{AG}$ & $\mathrm{AG}$ & GG \\
\hline $\mathrm{AG}$ & $\mathrm{AG}$ & $\mathrm{AG}$ & $\mathrm{AG}$ & $\mathrm{AG}$ & $\mathrm{AG}$ & $\mathrm{AG}$ & $\mathrm{AG}$ & $\mathrm{AA}$ & $\mathrm{AA}$ & $\mathrm{AG}$ \\
\hline GG & $\mathrm{AA}$ & $\mathrm{AG}$ & $\mathrm{AG}$ & GG & GG & $\mathrm{AG}$ & $\mathrm{AA}$ & AA & $\mathrm{AG}$ & $\mathrm{AG}$ \\
\hline AA & $\mathrm{AG}$ & $\mathrm{AG}$ & $\mathrm{AG}$ & $\mathrm{AG}$ & AA & $\mathrm{AG}$ & AA & $\mathrm{AG}$ & $\mathrm{AG}$ & \\
\hline
\end{tabular}

Continued next page 
Supplemental Table 2. Continued.

\begin{tabular}{|c|c|c|c|c|c|c|c|c|c|c|}
\hline \multirow{3}{*}{$\begin{array}{l}\text { SNP name } \\
\text { GDsnp01098 } \\
\text { AA }\end{array}$} & \multicolumn{10}{|c|}{ Linkage group Physical location (bp) Individuals } \\
\hline & 17 & \multicolumn{2}{|c|}{22866829} & $\mathrm{AA}$ & AA & AA & AA & AA & AA & $\overline{\mathrm{AA}}$ \\
\hline & $\mathrm{AA}$ & $\mathrm{AG}$ & $\mathrm{AG}$ & AA & AA & AA & AA & AA & AA & AA \\
\hline AA & $\mathrm{AA}$ & AA & $\mathrm{AA}$ & $\mathrm{AA}$ & $\mathrm{AA}$ & AA & AA & $\mathrm{AA}$ & AA & AA \\
\hline AA & $\mathrm{AA}$ & AA & $\mathrm{AA}$ & $\mathrm{AA}$ & $\mathrm{AA}$ & AA & $\mathrm{AA}$ & AA & $\mathrm{AA}$ & AA \\
\hline AA & $\mathrm{AA}$ & AA & $\mathrm{AA}$ & $\mathrm{AA}$ & $\mathrm{AA}$ & AA & $\mathrm{AG}$ & $\mathrm{AA}$ & AA & AA \\
\hline AA & $\mathrm{AA}$ & AA & $\mathrm{AA}$ & $\mathrm{AA}$ & $\mathrm{AA}$ & AA & AA & $\mathrm{AA}$ & AA & AA \\
\hline AA & $\mathrm{AA}$ & AA & $\mathrm{AA}$ & $\mathrm{AA}$ & $\mathrm{AA}$ & AA & AA & $\mathrm{AA}$ & AA & AA \\
\hline AA & $\mathrm{AA}$ & $\mathrm{AA}$ & $\mathrm{AA}$ & $\mathrm{AA}$ & $\mathrm{AA}$ & $\mathrm{AA}$ & $\mathrm{AA}$ & $\mathrm{AA}$ & $\mathrm{AA}$ & AA \\
\hline $\mathrm{AA}$ & $\mathrm{AA}$ & AA & $\mathrm{AA}$ & $\mathrm{AA}$ & $\mathrm{AA}$ & AG & $\mathrm{AA}$ & $\mathrm{AA}$ & AA & \\
\hline GDsnp00265 & 17 & \multicolumn{2}{|c|}{23045622} & $\mathrm{AG}$ & $\mathrm{AG}$ & $\mathrm{AA}$ & $\mathrm{AA}$ & $\mathrm{AG}$ & $\mathrm{AG}$ & $\mathrm{AA}$ \\
\hline GG & $\mathrm{AA}$ & $\mathrm{AG}$ & $\mathrm{AG}$ & GG & $\mathrm{AA}$ & AA & AA & $\mathrm{AG}$ & $\mathrm{AG}$ & $\mathrm{AG}$ \\
\hline $\mathrm{AG}$ & $\mathrm{AG}$ & $\mathrm{AA}$ & $\mathrm{AA}$ & $\mathrm{AG}$ & $\mathrm{AG}$ & $\mathrm{AG}$ & $\mathrm{AA}$ & $\mathrm{AA}$ & $\mathrm{AG}$ & AA \\
\hline AA & $\mathrm{AA}$ & AA & $\mathrm{AG}$ & $\mathrm{AG}$ & $\mathrm{AG}$ & $\mathrm{AG}$ & AA & $\mathrm{AA}$ & $\mathrm{AG}$ & $\mathrm{AG}$ \\
\hline $\mathrm{AG}$ & $\mathrm{AA}$ & $\mathrm{AA}$ & $\mathrm{AA}$ & $\mathrm{AG}$ & $\mathrm{AG}$ & $\mathrm{AA}$ & $\mathrm{AG}$ & $\mathrm{AA}$ & $\mathrm{AA}$ & AA \\
\hline $\mathrm{AG}$ & $\mathrm{AG}$ & $\mathrm{AG}$ & $\mathrm{AG}$ & $\mathrm{AA}$ & $\mathrm{AG}$ & $\mathrm{AG}$ & $\mathrm{AA}$ & $\mathrm{AA}$ & $\mathrm{AA}$ & $\mathrm{AG}$ \\
\hline GG & $\mathrm{AG}$ & $\mathrm{AG}$ & $\mathrm{AA}$ & $\mathrm{AA}$ & $\mathrm{AG}$ & $\mathrm{AA}$ & $\mathrm{AG}$ & $\mathrm{AG}$ & $\mathrm{AG}$ & $\mathrm{AG}$ \\
\hline AA & $\mathrm{AG}$ & AA & $\mathrm{AG}$ & AA & $\mathrm{AA}$ & AA & AA & $\mathrm{AG}$ & AA & AA \\
\hline $\mathrm{AG}$ & $\mathrm{AG}$ & $\mathrm{AA}$ & $\mathrm{AA}$ & $\mathrm{AG}$ & $\mathrm{AG}$ & GG & $\mathrm{AG}$ & $\mathrm{AG}$ & $\mathrm{AA}$ & \\
\hline GDsnp00245 & 17 & \multicolumn{2}{|c|}{23343242} & $\mathrm{AG}$ & $\mathrm{AA}$ & AA & $\mathrm{AG}$ & $\mathrm{AA}$ & AA & $\mathrm{AG}$ \\
\hline AA & $\mathrm{AG}$ & AA & $\mathrm{AA}$ & $\mathrm{AA}$ & $\mathrm{AA}$ & AA & AA & $\mathrm{AA}$ & AA & $\mathrm{AG}$ \\
\hline AA & $\mathrm{AA}$ & AA & $\mathrm{AA}$ & $\mathrm{AA}$ & $\mathrm{AG}$ & AA & $\mathrm{AG}$ & $\mathrm{AG}$ & AA & AA \\
\hline $\mathrm{AG}$ & $\mathrm{AA}$ & $\mathrm{AA}$ & $\mathrm{AA}$ & $\mathrm{AG}$ & $\mathrm{AG}$ & AA & AA & $\mathrm{AG}$ & $\mathrm{AG}$ & AA \\
\hline AA & $\mathrm{AA}$ & AA & $\mathrm{AG}$ & $\mathrm{AG}$ & $\mathrm{AG}$ & $\mathrm{AG}$ & AA & $\mathrm{AG}$ & $\mathrm{AG}$ & AA \\
\hline AA & $\mathrm{AA}$ & $\mathrm{AG}$ & $\mathrm{AA}$ & $\mathrm{AA}$ & $\mathrm{AA}$ & AA & AA & $\mathrm{AG}$ & AA & AA \\
\hline AA & $\mathrm{AA}$ & $\mathrm{AA}$ & $\mathrm{AG}$ & $\mathrm{AG}$ & $\mathrm{AG}$ & $\mathrm{AA}$ & $\mathrm{AA}$ & $\mathrm{AA}$ & $\mathrm{AG}$ & AA \\
\hline AA & $\mathrm{AG}$ & $\mathrm{AA}$ & $\mathrm{AA}$ & $\mathrm{AA}$ & $\mathrm{AA}$ & AA & $\mathrm{AA}$ & $\mathrm{AA}$ & AA & $\mathrm{AG}$ \\
\hline $\mathrm{AG}$ & $\mathrm{AA}$ & $\mathrm{AA}$ & $\mathrm{AA}$ & $\mathrm{AA}$ & $\mathrm{AG}$ & $\mathrm{AA}$ & $\mathrm{AA}$ & $\mathrm{AA}$ & $\mathrm{AA}$ & \\
\hline GDsnp00438 & 17 & \multicolumn{2}{|c|}{24422142} & $\mathrm{AG}$ & $\mathrm{AG}$ & GG & GG & $\mathrm{AG}$ & $\mathrm{AG}$ & GG \\
\hline AA & GG & GG & $\mathrm{AA}$ & $\mathrm{AA}$ & GG & GG & GG & $\mathrm{AA}$ & $\mathrm{AG}$ & $\mathrm{AG}$ \\
\hline GG & $\mathrm{AG}$ & $\mathrm{AA}$ & GG & $\mathrm{AG}$ & GG & $\mathrm{AG}$ & GG & GG & $\mathrm{AG}$ & GG \\
\hline GG & GG & GG & $\mathrm{AG}$ & $\mathrm{AG}$ & $\mathrm{AG}$ & $\mathrm{AG}$ & GG & GG & $\mathrm{AG}$ & AA \\
\hline $\mathrm{AG}$ & GG & GG & GG & $\mathrm{AG}$ & $\mathrm{AG}$ & GG & - & GG & GG & GG \\
\hline $\mathrm{AG}$ & $\mathrm{AG}$ & $\mathrm{AG}$ & $\mathrm{AA}$ & GG & $\mathrm{AG}$ & $\mathrm{AG}$ & GG & $\mathrm{AG}$ & $\mathrm{AA}$ & $\mathrm{AG}$ \\
\hline AA & $\mathrm{AG}$ & $\mathrm{AG}$ & GG & GG & $\mathrm{AG}$ & $\mathrm{GG}$ & $\mathrm{AA}$ & GG & $\mathrm{AG}$ & $\mathrm{AG}$ \\
\hline GG & $\mathrm{AG}$ & GG & $\mathrm{AG}$ & $\mathrm{AA}$ & GG & GG & GG & $\mathrm{AG}$ & GG & $\mathrm{AG}$ \\
\hline $\mathrm{AG}$ & $\mathrm{AG}$ & GG & GG & $\mathrm{AG}$ & GG & $\mathrm{AG}$ & $\mathrm{AG}$ & $\mathrm{AG}$ & GG & \\
\hline
\end{tabular}




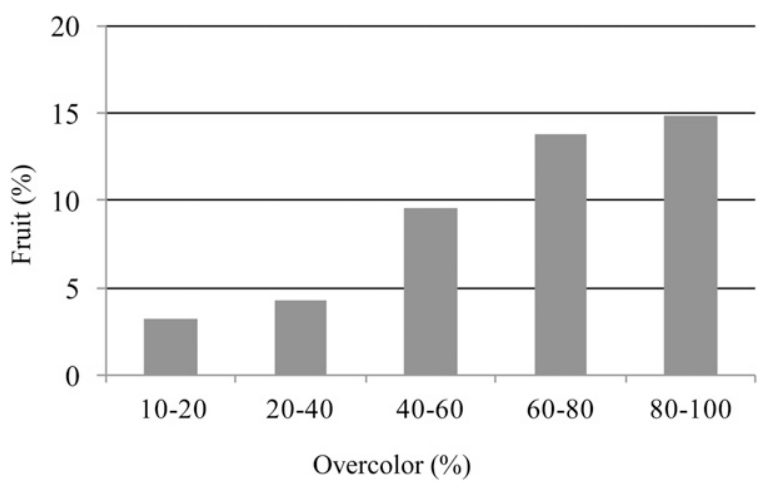

Supplemental Fig. 1. Distribution of surface color for fruit from 94 apple seedlings. Overcolor is expressed as a percentage, averaged over five fruit.

\section{LG_1}

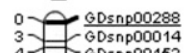

11 - GDsnp00152

$17-\begin{array}{r}\text { GDspo } 1470 \\ G D S p 01500\end{array}$

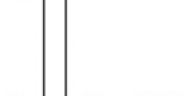

${ }_{43}^{41}$ GDsnp00087

L

$60-$ GDsnp00252

66 GDsnp00361

68- TGDSAD00782

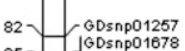

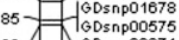

${ }_{90}^{86} \alpha_{\text {GDsnp02092 }}^{\text {GDsnp02371 }}$

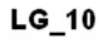

$\left.{ }_{1}^{0}\right\} \frac{\text { GDsnp01731 }}{\text { GDsnp00106 }}$

$11-$ GDsnp01810

$16-$ GDsnp00875

\begin{tabular}{l|l} 
& \\
34 & GDsnp01267
\end{tabular}

- - GDsnp02183

$48-$ GDsnp02072

${ }_{36-1}^{\text {GDsnp00355 }}$

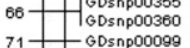

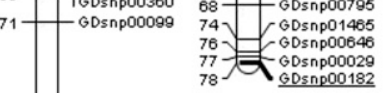

GDsnp01191

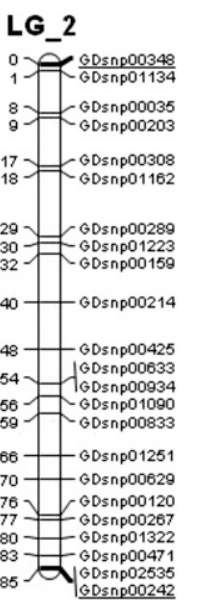

LG_3

$0-$ GDsnp00506

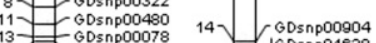

14- CDsnp00588

$21-$ GDsnp02304 $_{\text {GDsnp01493 }}$

33) GDsnp01749

$41-$ GDsnp0182

${ }_{51}-{ }_{\text {GDsnp00955 }}$

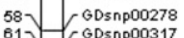

66 GDSnP0196

73 Gos InP01667

$77 \sqrt{\text { GDSnO02030 }}$

81
$86]-$ GDsnp00664

$88=\left\{\begin{array}{l}\text { GDsnp00319 } \\ \text { GDsnp01604 }\end{array}\right.$

${ }_{89} \underline{\mathrm{CH} 3 \mathrm{~g} 12 \mathrm{Y}}$

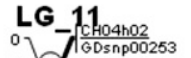

LG_12

${ }_{1}^{0} \mathcal{A} \frac{\text { GDsnp02789 }}{\text { GDsnp00173 }}$

$12-$ GDsnp01690

$16-$ GDsnp00272

24- GDsnp00318

$27-$ Gosnp00034

$31-G D s n p 00240$

$44 \sqrt{\text { GDsnp00743 }}$

$43={ }_{\text {GDsnp00747 }}^{\text {GDsnp01769 }}$

$49-$ GDsnp01426

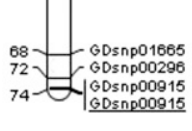

4. Gosnp00063
8- GDsnp00322

$32=\begin{gathered}\text { GDsnp01029 } \\ \text { GDsnp00193 }\end{gathered}$
LG_13

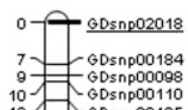

13 GDsnp00195

LG_4

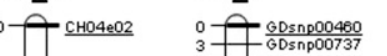

\begin{tabular}{llll} 
& $10-G D s n p 00284$ \\
\hline
\end{tabular}

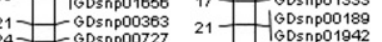

$40-\left.\right|_{\text {GDSnP00148 }} ^{\text {GDSO }}$

Cosit G00881

GDSn01993

- GDsnp0015

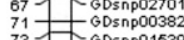

73 GDsnp01539

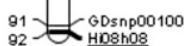

LG_14

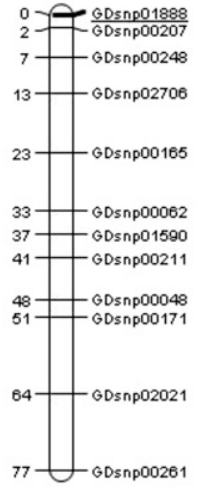

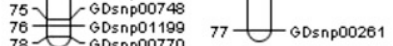

LG_6

${ }_{1}^{0}$ רी GDsnp00347

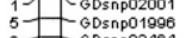

8- GDsnp02464

18- GDsnp00197

$26-H_{G D S n P 00434}^{G D S}$

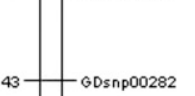

$43-$ GDsnp00282

$56-$ GDsnp01556

63
$65-1$ Gsnp01502
GDsnp01682

73
GDsnp02138

0 GD Gsnp00876

6- GDsnp01994

$10-$ GDsnp00051

22 $17-\begin{aligned} & \text { GDsnp01170 } \\ & \text { GDsnp01872 }\end{aligned}$

$25)$ GDsnp00224

GDsnp01074
GDsnp00247

$32 . y=\begin{gathered}\text { GDsnP02840 } \\ \text { GDSND01848 }\end{gathered}$

GDSnP0025

53 - GDsnp02291

$59-\mathcal{C D D S N D}_{01040}$

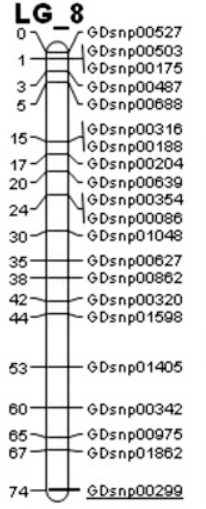

LG_9

0)

1. GDsnp01189

$16-$ GDsnp01911

$20-$ GDsnp00514

26 GDsnp00893

32. GDSAP00337

$34-G D s n p 00343$

$40-$ GDsnp00331

$48-$ GDsnp02460

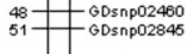

$\begin{aligned} & 68 \\ & 72 \\ & 73\end{aligned}-\begin{gathered}\text { GDsnp00766 } \\ \text { GDsnp00452 } \\ \text { GDsnp00332 }\end{gathered}$

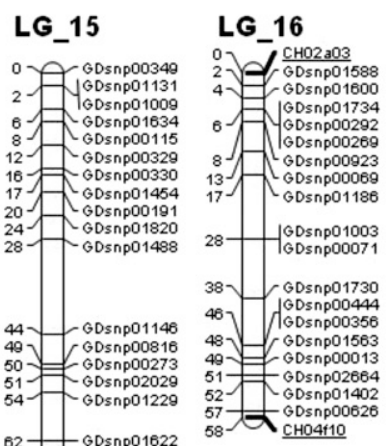

LG_17
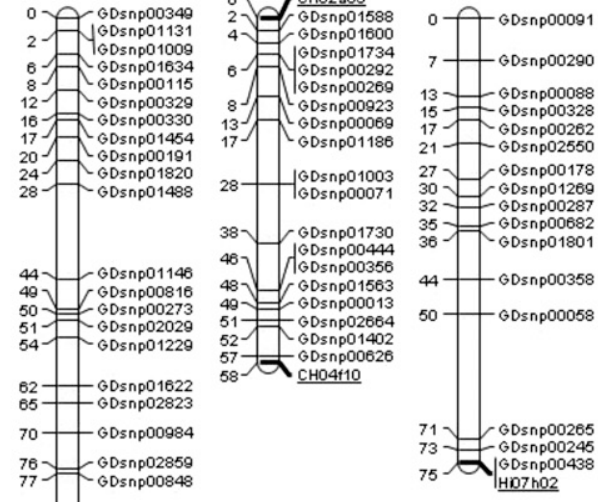

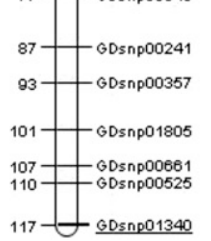

\footnotetext{
0
2
4
6
8
13
17
28
38
46
48
49
51
52
57
58
}

Supplemental Fig. 2. Position of the 295 single nucleotide polymorphism (SNP) markers along the 17 apple linkage groups of the integrated linkage map of Velasco et al. (2010). Underlined markers were not employed in this previous study; however, are reported in this map to indicate the ends of the linkage groups. 


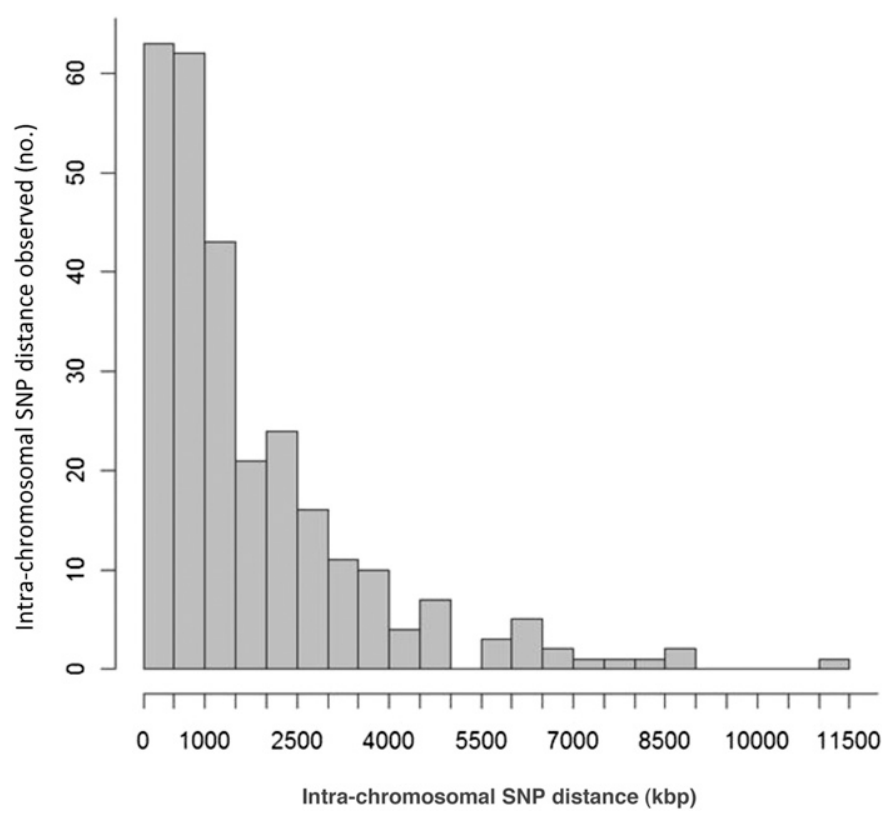

Supplemental Fig. 3. Histogram of intra-chromosomal physical distances between single nucleotide polymorphisms (SNPs) used for association analysis in an apple breeding population of 94 seedlings. 

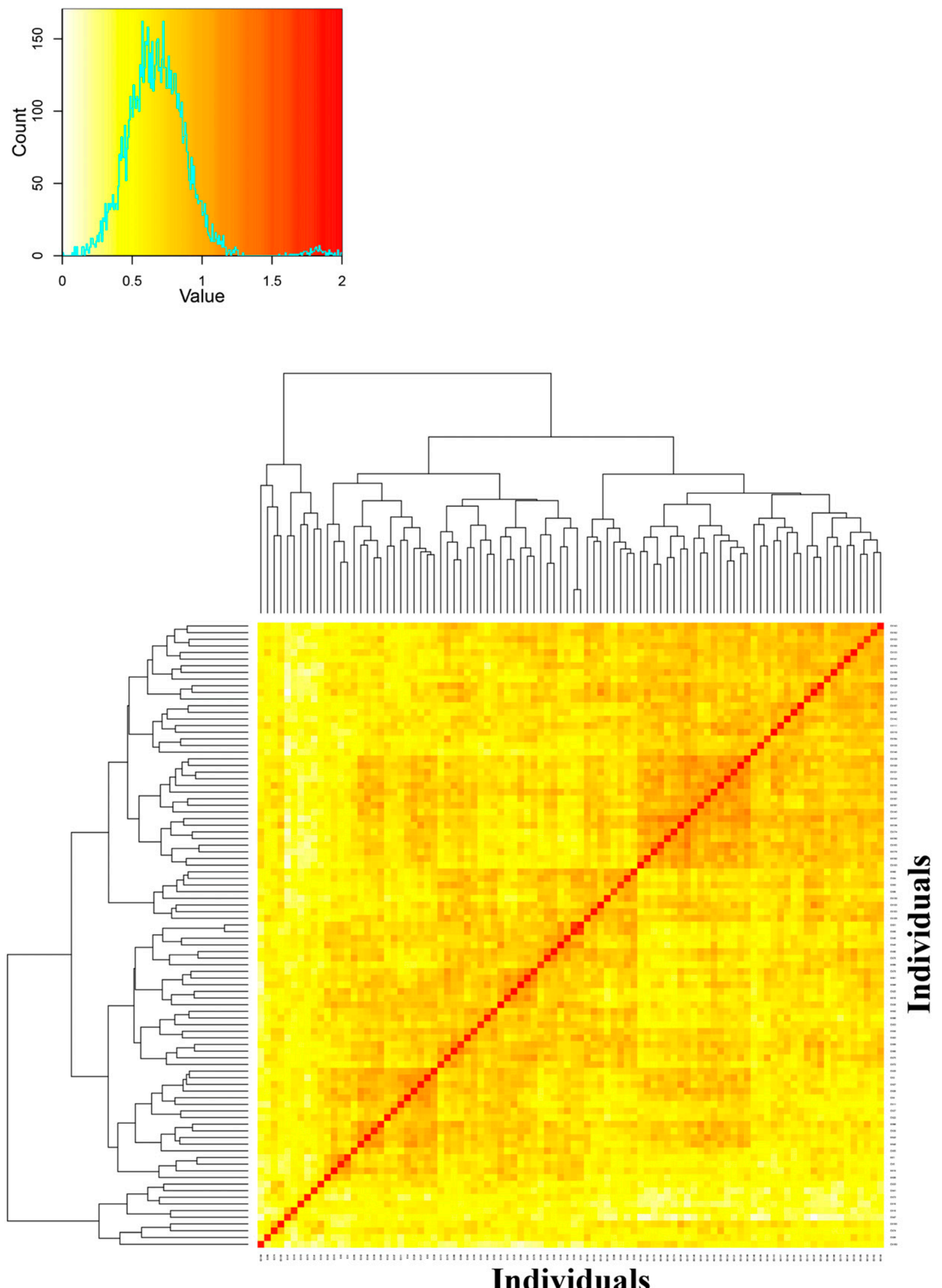

Supplemental Fig. 4. Kinship analysis in an apple breeding populations of 94 seedlings. Hierarchical clustering and heat map of the kinship values from the kinship matrix. 


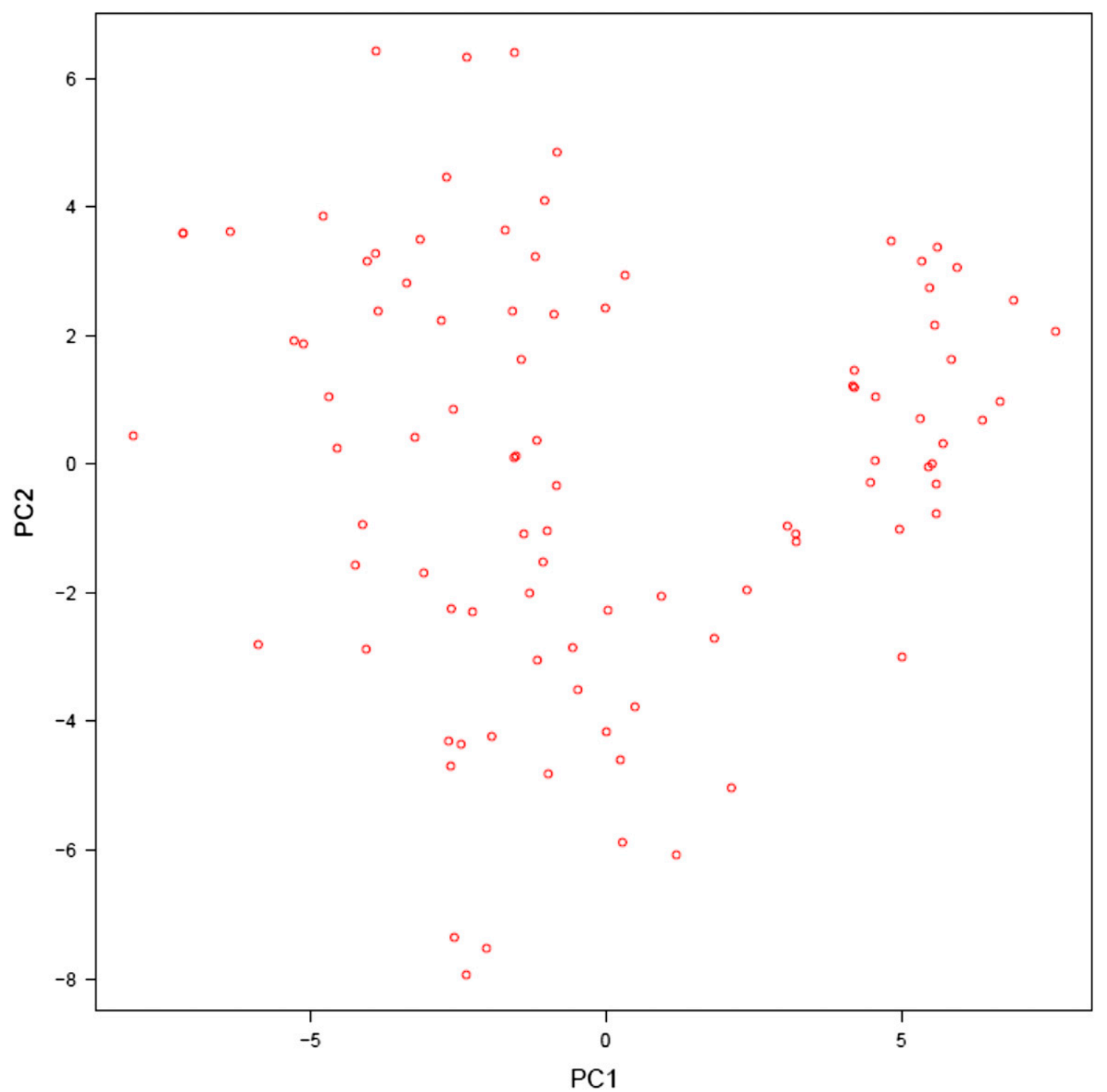

Supplemental Fig. 5. Genetic structure of an apple breeding population of 94 seedlings calculated using principal component (PC) analysis. 